\title{
UC-NRLF
}

C 3 14 B 


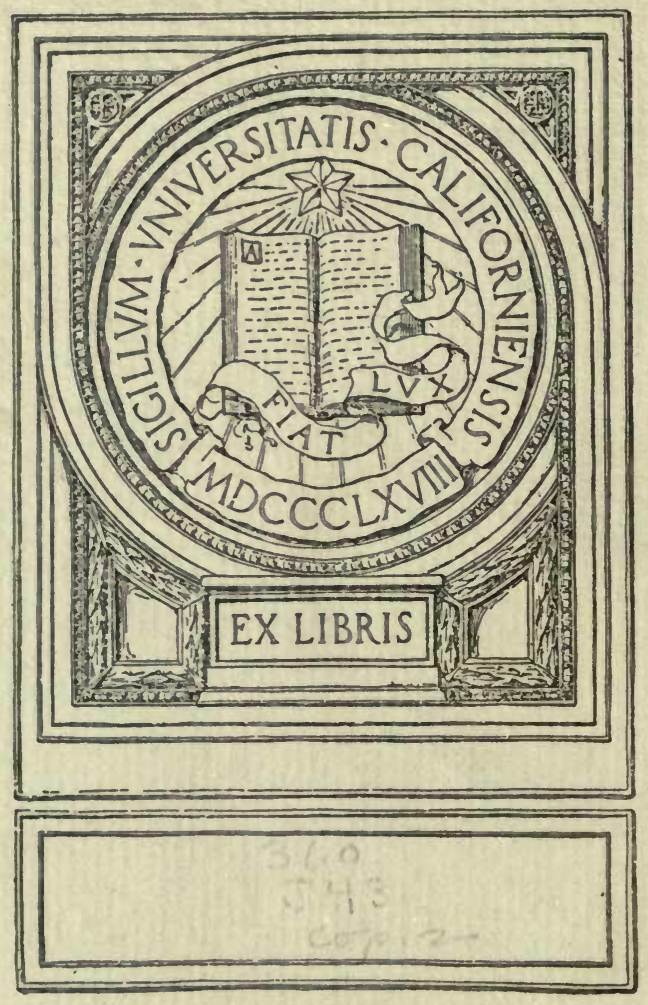



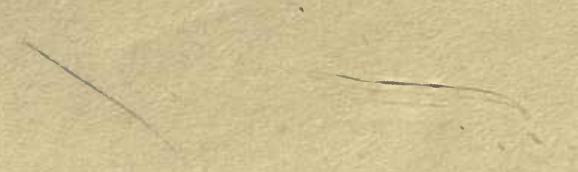


\section{PROBLEMS OF COSMOGONY AND STELLAR DYNAMICS}




\section{CAMBRIDGE UNIVERSITY PRESS}

C. F. CLAY, MANAGER

LONDON : FETTER LANE, E.C. 4 LONDON : H. K. LEWIS AND CO., LTD., ${ }_{3} 6$ Gower Street, W.C. $x$

LONDON : WILLIAM WESLEY AND SON, 28 Essex Street, Strand, W.C. 2

NEW YORK : G. P. PUTNAM'S SONS

BOMBAY

CALCUTTA MACMILLAN AND CO., Ltd. MADRAS

TORONTO: J. M. DENT AND SONS, LTD. TOKYO: MARUZEN-KABUSHIKI-KAISHA

ALL RIGHTS RESERVED 


\title{
PROBLEMS OF COSMOGONY
} AND

\section{STELLAR DYNAMICS}

BY

\author{
J. H. JEANS, M.A., F.R.S.
}

FORMERLY STOKES LECTURER IN APPLIED MATHEMATICS IN THE UNIVERSITY OF CAMBRIDGE ; SOMETIME PROFESSOR OF APPLIED MATHEMATICS IN PRINCETON UNIVERSITY

BEING AN ESSAY TO WHICH THE ADAMS PRIZE OF THE UNIVERSITY OF CAMBRIDGE FOR THE YEAR I9I7 WAS ADJUDGED

\section{CAMBRIDGE}




$$
\begin{aligned}
& \text { QB981 } \\
& J_{4}
\end{aligned}
$$

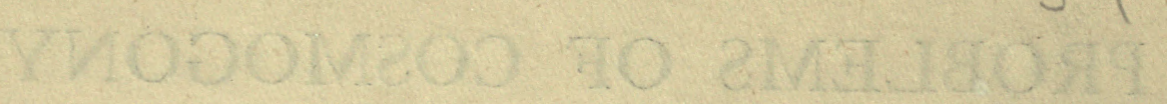
(i) $A$. ROIVAYYY Sh JuAT?

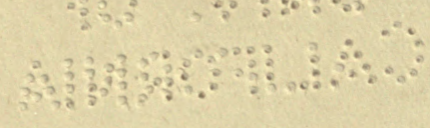

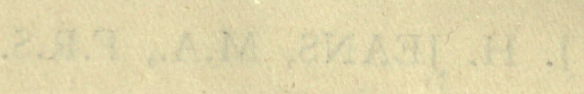

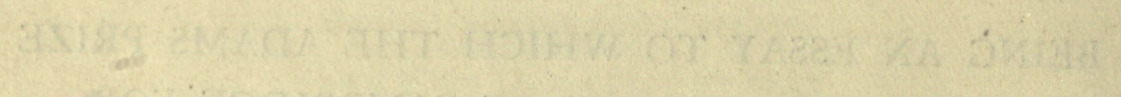

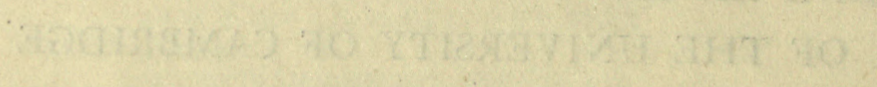

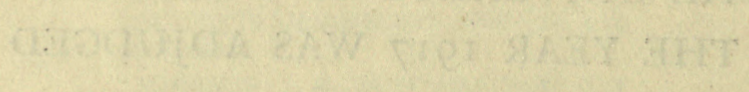

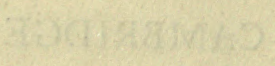

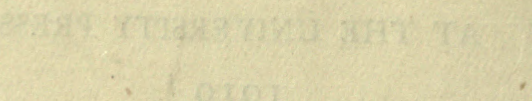




\section{PREFACE}

TTHE present essay is primarily an attempt to follow up a line of research 1 initiated by Laplace and Maclaurin, and extended in various directions by Roche, Lord Kelvin, Jacobi, Poincaré and Sir G. Darwin. Within two years of the close of his life, Darwin remarked that the way to further progress in cosmogony was blocked by our ignorance of the figures of equilibrium of rotating gaseous masses. He wrote as follows (Darwin and Modern Science, p. 563, and Tides, 3rd edition, p. 401):

"As we have seen, the study of the forms of equilibrium of rotating liquids is almost complete, and a good beginning has been made in the investigation of the equilibrium of gaseous stars, but much more remains to be discovered.

"As a beginning we should like to know how a moderate degree of compressibility would alter the results for liquid, and...to understand more as to the manner in which rotation affects the equilibrium and stability of rotating gas. The field for the mathematician is a wide one, and in proportion as the very arduous exploration of that field is attained, so will our knowledge of the processes of cosmical evolution increase....

"Human life is too short to permit us to watch the leisurely procedure of cosmical evolution, but the celestial museum contains so many exhibits that it may become possible, by the aid of theory, to piece together bit by bit the processes through which stars pass in the course of their evolution."

Guided possibly by considerations such as these, the Adjudicators of the Adams Prize announced as the subject for the 1917 Essay:

The course of evolution of the configurations possible for a rotating and gravitating fluid mass, including the discussion of the stabilities of the various forms.

At this time I had for some years been engaged in an attack on this problem. The announcement offered an excuse not only for putting together my own results in essay form but also for welding them on to the earlier results obtained in the classical papers of Darwin, Poincaré and other workers at this problem. After the adjudication of the prize, the essay was enlarged by the addition of some further results which had been obtained in the interval, and the present volume is the result. 
It is hoped that the book will be read in the spirit of the remarks of Darwin just quoted. The main object of the essay is to build a framework of absolute mathematical truth; the backbone of the structure is the theoretical investigation into the behaviour of rotating masses. Of this my own contribution forms only a small part; the book contains also an account of general dynamical theory, and of the researches of Darwin, Poincare and others, in so far as they relate to the main problem in hand. This part of the book has been made as concise as possible, and I have ventured to hope that it will prove of value to those who are embarking on a study of the general problem of cosmic evolution.

I have tried not only to build a skeleton but also to clothe it. When a firm theoretical framework had been constructed, it seemed permissible and proper to try to fit the facts of observational astronomy into their places. If ever a complete mathematical theory is achieved, it will probably be an easy task to trace out the order of evolution of stellar objects, but at present our theoretical knowledge is so incomplete that a large element of speculation must necessarily enter into every attempt to connect up theory and observation. I have tried throughout to keep speculation within reasonable limits, and have applied as many checks and tests as I could to the various conjectural hypotheses brought forward. Many astronomers necessarily will disagree with a number of these conjectures; it is in this way that science advances. To any critic who may think the conjectures ought not to have been brought forward at all, I would reply in the words of Herschel :

"If we indulge a fanciful imagination and build worlds of our own,... these will vanish like the Cartesian vortices, that soon gave way when better theories were offered. On the other hand, if we add observation to observation, without attempting to draw not only certain conclusions but also conjectural views from them, we offend against the very end for which only observations ought to be made. I will endeavour to keep a proper medium; but if I should deviate from that I could not wish to fall into the latter error."

The more speculative chapters fall naturally together at the end of the book. Many readers may find these the most interesting, and I have tried to arrange the book so that they will prove intelligible to those readers who prefer to take mathematical investigations as read. In the present state of our knowledge any attempt to dictate final conclusions on the main problems of cosmogony could be nothing but pure dogmatism; I should not have ventured even to suggest a conclusion except that the various theoretical results obtained seemed to point with considerable unanimity in one particular direction. Consequently a definite scheme of cosmogony has been 
suggested; not in the belief that it will prove to be true, but in the hope that it may in some degree help others ultimately to find the truth. This scheme will be found to contain nothing fundamentally new; it consists only of a patchwork of parts of existing theories. This is perhaps hardly surprising; so many cosmogonical conjectures have been made that it is unlikely that any really novel hypothesis remains to be put forward. In any case a theoretical investigation such as that of the present book is necessarily destructive rather than constructive; primarily it serves to test and eliminate existing theories rather than to indicate new possibilities.

It is a pleasure to thank many friends who have helped me in various ways. First I must thank the great number of astronomers who have allowed me to draw on their stores of astronomical knowledge. I have to express my obligation and cordial thanks to Professor Hale, Professor Ritchey and Mr F. G. Pease of Mount Wilson Observatory for permission to reproduce the very fine photographs which enrich my book. Finally it is a pleasure to express to the officials and staff of the Cambridge University Press my appreciation of their unfailing courtesy and the care they have bestowed on the printing of the work.

J. H. JEANS.

Dec. 18, 1918. 


\section{CONTENTS}

CHAP.

I Introductory Chapter . . . . . . . . . . . 1

II General Dynamical Principles . . . . . . . . 19

III Ellipsoidal Configurations of Equilibrium . . . . . . . 35

IV The Gravitational Potential of a Distorted Ellipsoid . . . 65

V Pear-shaped Configurations of Equilibrium . . . . . . 78

VI Motion when there are no Stable Configurations of Equilibrium . 117

VII The Motion of Compressible and Non-homogeneous Masses . . 139

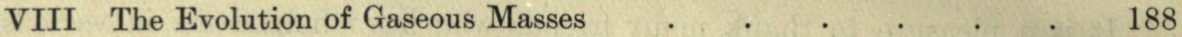

IX The Evolution of Rotating Nebulae . . . . . . . 203

X The Evolution of Star-Clusters . . . . . . . . 220

XI The Evolution of Binary and Multiple Stars . . . . 246

XII The Origin and Evolution of the Solar System . . . . $\quad 269$

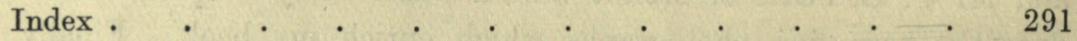

\section{LIST OF ILLUSTRATIONS}

Planetary and Ring Nebulae:

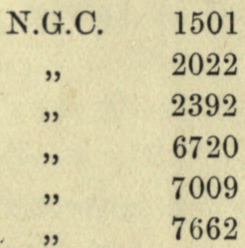

$\begin{array}{cc}\text { Plate } & \text { Facing page } \\ \text { I } & 5 \\ \text { I } & 5 \\ \text { I } & 5 \\ \text { I } & 5 \\ \text { I } & 5 \\ \text { I } & 5\end{array}$

Spiral Nebulae:

$\begin{array}{clcc}\text { N.G.C. } & 3031 \text { (M. 81) } & \text { V } & 289 \\ , & 3115 & \text { III } & 207 \\ , & 4565 \text { (H. v. 24) } & \text { III } & 207 \\ , & 4594 & \text { III } & 207 \\ , & 4828 \text { (M. 64) } & \text { IV } & 215 \\ , & 5194-5 \text { (M. 51) } & \text { II } & 6 \\ , & 5457 \text { (M. 101) } & \text { II } & 6 \\ , & 5746 & \text { III } & 207 \\ , & 5866 & \text { III } & 207 \\ , & 7217 & \text { IV } & 215\end{array}$




\section{CHAPTER I}

\section{INTRODUCTORY CHAP'TER}

\section{SURVEY OF THE PROBLEM}

\section{The Solar System}

1. In 1543 Copernicus published his treatise "De Revolutionibus Orbium Coelestium" in which the apparent motion of the planets was explained by the simple hypothesis that they all described orbits about the Sun at rest. Two thirds of a century later, in the early days of 1610 , Galileo first observed the satellites of Jupiter revolving around their primary, and so obtained what amounted almost to direct visual proof of the truth of the Copernican system of astronomy. But in verifying Copernicus' solution of one problem, Galileo had opened up another. For it now became clear that there were at least two systems of almost exactly similar formation in the universe, and a philosophic mind could not but conclude that they had probably originated from similar causes, and would be impelled to conjecture as to what those causes might be.

In this way the problem of scientific cosmogony had its origin. To the modern astronomer the problem is much richer, wider and more definite, in proportion as the mass of observational material within his knowledge is greater than that with which Galileo was acquainted. In the solar system alone, we know that in addition to the eight great planets, there are upwards of 900 minor planets* or asteroids, and all these 908 or more bodies shew the same regularity in their motion. Their orbits are all nearly circular, they are all approximately in one plane, and they are all described in the same direction. If we assume it to be $\dot{a}$ priori an even chance that a planet should move either from east to west or from west to east, then the chance against 908 planets all moving in the same direction would be $2^{907}-1$ to 1 . But if we regard the problem from the point of view of statistical mechanics, and calculate the odds against these orbits being all of small inclination and of small eccentricity, then we arrive at odds in comparison with which the previously calculated odds of $2^{907}-1$ to 1 are so small as to look approximately like an even chance.

* At the end of 1916 , numbers had been assigned to 826 , and orbits computed for 896 . Of the 520 earliest discovered planets, 13 are regarded as lost, having been seen at no opposition since their discovery. 
A similar uniformity is found in the satellites of the planets. The modern astronomer knows that the system of Saturn as well as that of Jupiter is a small-scale replica of that of the Sun, while the systems of the smaller planets differ only in having fewer satellites. With a few exceptions, it is found that throughout the whole complex system formed by the sun, its satellites, the planets, and the satellites of the planets, the motion is uniformly in the same direction and in nearly circular and nearly coplanar orbits.

The exceptions occur on the outermost edges of the solar system, and on the outermost edges of the systems of Jupiter and Saturn. They are as follows :

Neptune has only one satellite, and this has retrograde motion.

Uranus has four satellites, whose orbits are highly inclined to the plane of the ecliptic.

Saturn has nine satellites*, of which the outermost (Phoebe) revolving at a mean distance of 209 diameters of Saturn, has retrograde motion and high eccentricity of orbit.

Jupiter has nine satellites of which the two outermost move with retrograde motion.

Some of the asteroids also have considerable inclinations and eccentricities. Thus Pallas has an inclination of $34^{\circ} 43^{\prime}$, and Zerline (531) one of $34^{\circ} 33^{\prime}$, these being nearly five times the greatest inclination observed among the planets $\left(7^{\circ} 0^{\prime}\right.$, the inclination of Mercury). Juno has an eccentricity of 0.257 and Pallas one of 0.239 , while a few smaller asteroids are supposed, although with less certainty, to have eccentricities of about $\frac{1}{3}$.

\section{Binary Stars}

2. We do not know whether uniformity of this kind extends to other systems in space, or whether it is a peculiarity of our own system. When it was first realised that the so-called fixed stars were essentially suns more or less similar to our own, it was natural to conjecture that they also might be the centres of planetary systems similar to that of our sun, but the further growth of knowledge has shewn the need for caution in such conjectures.

Of the nineteen stars whose parallaxes are less than $0 \cdot 20^{\prime \prime}-$ i.e. the nineteen stars which happen at the present moment to be within $96 \times 10^{12}$ miles of our sun - no fewer than eight, or 42 per cent. of the whole, are quite certainly binary starst. Although there is no special reason for thinking that these nineteen stars are not likely to be a fair sample of the whole, it is obviously desirable to try to get evidence from other regions of space. Of fifteen stars

\footnotetext{
" Excluding the tenth (Themis) discovered photographically by W. H. Pickering in 1904, but not seen since.

+ Eddington, Stellar Movements, p. 41.
} 
examined by Hertzsprung* in the Ursa Major cluster, nine, or 60 per cent. of the whole, are certainly binary, while Frost $\nmid$ finds that in the Taurus cluster. the corresponding proportion is 50 per cent. Frost also finds that 40 per cent. of stars of B type are binary, while Campbell+ finds that out of 1600 stars considered by him, the spectroscopic binaries alone number 25 per cent., a ratio which must of course be increased by the addition of visual and eclipsing binaries. [Thus there is`every reason to suppose that throughout our universe fully one-third of the stars, and probably more, are binaries.]

To an observer who was so far removed from our system that the light from Jupiter was visible while that from the other planets was not, our system would appear to be a binary system. From observations, either spectroscopic or visual, our imaginary observer might be able to determine the ratio of the masses, and would find it to be 00095 . But when in the same way, we determine the ratio of the masses in the binary systems visible to us, this ratio is found never to be very far from unity. Boss $§$ has found that in ten visual binaries in which the ratio of the masses is well determined, this ratio is never one of greater inequality than 0.33 to 1 , the average being 0.69 to 1 , while Campbell|| finds for nineteen spectroscopic binaries an average massratio $0 \cdot 79$, the greatest inequality of mass being one of ratio 0.39 to one.

Thus it appears that the binary system formed by our sun and Jupiter is of a very different character from the binary systems observed in other parts of the sky, and the same is true of all the planetary systems inside our solar system. In these latter systems the closest approach to equality of masses of primary and satellite is found in our earth-moon system, in which the ratio is 0.0123 to 1 . Next, after a very long interval, come Saturn and Titan having a mass-ratio of the order of 0.0002 to 1 , and Jupiter and its third satellite having a ratio of the order of 0.0001 to 1 .

Thus, although it may be open to question whether or not our moon stands in a class by itself inside the solar system, there appears to be no question at all that the planetary arrangements inside our system stand in a different class from the binary arrangements outside.

Not only binary but also triple and multiple systems are observed. It is stated by Russell 9 that of the double and inultiple stars contained in Burnham's General Catalogue of Double Stars, combined with Lewis' catalogue of the Struve stars, about 800 appear to have common proper motion. And of these 74 are triple or multiple, this number being 9.25 per cent. of the whole. The proportion in.Jonckheere's more recent Catalogue and Measures of Double Stars** which contains 3950 stars is $9 \cdot 7$ per cent. of the whole.

* Astrophys. Journ. 30, p. 139.

† Astrophys. Journ. 29, p. 237.

¥ Stellar Motions, p. 245.

§ Prel. Gen. Catalogue, p. 23.

II Stellar Motions, p. 259, or “Second Catalogue of Binary Stars," Lick Obs. Bull. 181.

I Astrophys. Journ. 31 (1910), p. 199.

** R.A.S. Memoirs, Vol. 71 (1917). 
After allowing statistically for the effects of projection on the celestial sphere, Russell* finds that triple systems consist normally of a close double with a third star revolving at a considerable distance about their centre of gravity, the ratio of the actual separations being about 10 to 1 . The bearing of this on questions of cosmogony will be considered later; for the present it is sufficient to notice that the multiple systems observed in the sky shew no resemblance to our own solar system.

Thus we have found a very definite uniformity of arrangement inside our system, and a very definite uniformity of arrangement outside, but the two arrangements are different, and the question of whether there are other systems arranged like our own has to remain an open one. It may perhaps be mentioned that some astronomers believe that there are irregularities in the motion of binary systems which are too definite to be ascribed merely to errors of observation. These may ultimately be found to point, to the existence of planetary bodies revolving at a great distance round the central binary system, but the evidence is certainly too vague at present for definite conclusions to be drawn.

Our search outside our own system has, however, disclosed the existence of a second uniformity of structure, namely that of binary stars having masses not far from equal.

\section{Spiral and other Nebulae}

3. These two uniformities, namely the planetary formation and the double-star formation, although perhaps the most striking, are by no means the only uniformities which have been discovered by astronomy. Principal among the remaining ones is the spiral nebula formation which appears to be very distinctive and uniform. The characteristic spiral nebula consists invariably of a nucleus with two arms emerging from opposite points; the convolutions of the two arms are similar, the curve of each being approximately an equiangular spiral $\uparrow$. This formation is very freely scattered in space: Keeler and Perrine estimated the number of nebulae easily discoverable with the Crossley reflector to be of the order of half a million, while Keeler found more than half of the nebulae recorded on his plates to be spirals+. Although the spiral nebulae are only special instances of the more general nebular formations found in the sky, they are nevertheless the most frequent and the most distinctive of these formations known; for cosmogony they are the most interesting because the definiteness of their formation must contain a valuable clue to their origin and condition. Besides spiral nebulae there are other types of nebulae, which are commonly described in the following terms.

* Astrophys. Journ. 31 (1910), p. 200.

‡ Campbell, Stellar Motions, p. 36 .

† v. Pahlen, Ast. Nach. No. 4503. 



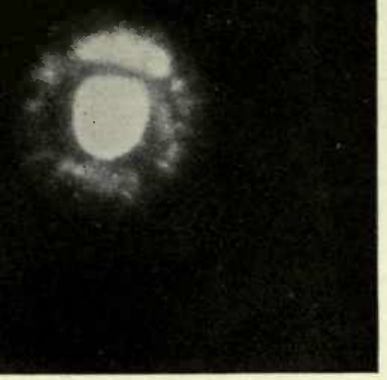

N.G.C. 2392

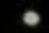

$\bullet$

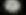

$\boldsymbol{\bullet}$

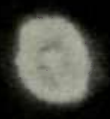

$*$

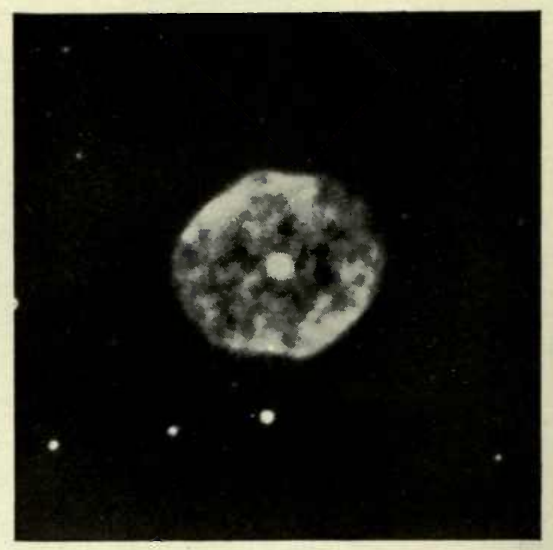

N.G.C. 1501

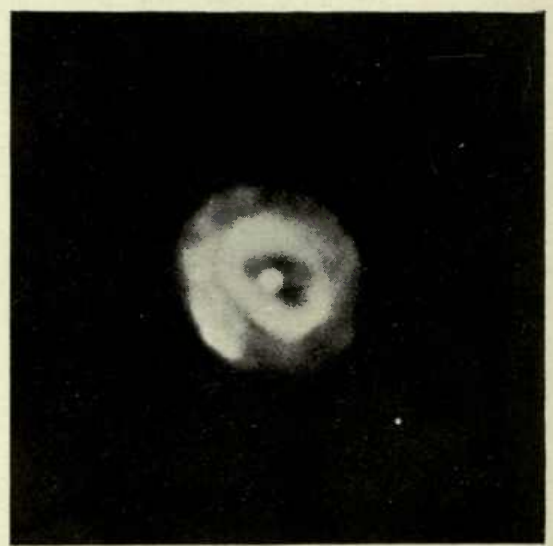

N.G.C. 7662

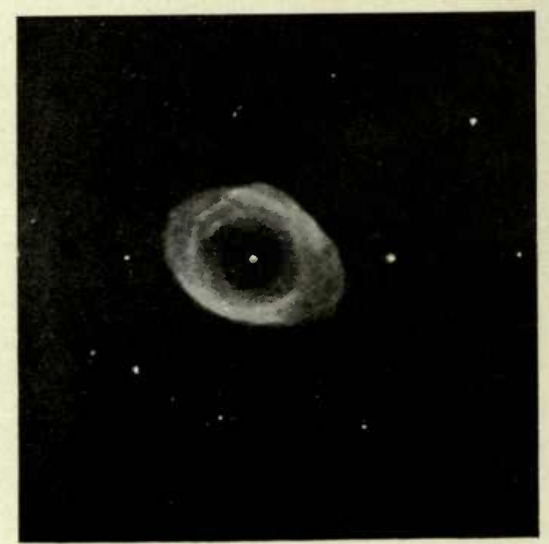

N.G.C. 6720 
(1) Irregular nebulae, such as the great nebula in Orion (N.G.C. 1976).

(2) Planetary nebulae, a class of nebulae of apparently spheroidal or ellipsoidal shape, many shewing detailed features and formations in addition. They are few in number, less than $\mathbf{1 5 0}$ having been discovered out of 15000 nebulae so far investigated*. As a rule they shew bright-line spectra, suggesting that they are masses of hot gas, shining by their own light. Some typical examples of Planetary nebulae will be found illustrated on Plate I.

(3) Ring nebulae, such as the well-known nebula in Lyra (N.G.C. 6720). Many astronomers believe that these are not true rings but ellipsoidal shells seen in projection; the reason for this view is mainly that these formations are never seen edgewise or nearly edgewise (see Plate I).

(4) Elliptical, elongated, lenticular and spindle nebulae. These are terms commonly employed to describe the observed shape of nebular masses. A number of nebulae originally classified as spindle-shaped are probably merely spirals seen edgewise, as has been suggested by Slipher $†$ and others. Descriptions, with excellent photographs of these and other types of nebulae will be found in a recent paper by F. G. Pease + (see also Plate III).

4. Beyond the information obtainable from their appearance and spectra, we have but little knowledge as to the nature, motions or constitutions of these various nebular systems§. Many of the spirals have velocities in space which are enormously greater than any other class of velocities of which we have any experience, a circumstance which gives some support to the view that they may be regarded as "island universes," each comparable in scale to the universe of stars of which our sun is a member.

Thus for the Andromeda nebula there is consistent evidence of a velocity of approach of about $300 \mathrm{kms}$ a second, Slipher $\|$ determining this velocity as 300 kms a second, Wright $\uparrow$ as $304 \mathrm{kms}$ a second and Pease** as $329 \mathrm{kms}$ a second. Many spirals have still greater velocities; thus Pease attributes a velocity of recession of about $1180 \mathrm{kms}$ a second to the nebula in Virgo (N.G.C. 4594) ++ while Slipher finds a velocity of recession of $1120 \mathrm{kms}$ a second for the nebula in Cetus (N.G.C. 1068) ${ }_{++}^{+*}$ The general average velocity is between 300 and $400 \mathrm{kms}$ a second-say twenty times the general average velocity of a star in our universe. Regarding these nebulae as "island universes," it ought of course to be possible to determine the motion of our own galactic system in

* W. W. Campbell, Science (1917), p. 521.

† Lick Obs. Bull. No. 62.

$\ddagger$ Astrophys. Journ. 46 (1917), p. 24. See also W. W. Campbell, Science, 45 (1917), pp. $513-548$.

$\$$ A short summary will be found in the R.A.S. Monthly Notices, 77 (1917), p. 375.

\| Lowell Obs. Bull. No. 58 (1913).

I Popular Ast. 23 (1915), 36.

** Journal Royal Ast. Soc. Canada, Sept. 1915.

†† Astrophys. Journ. 46 (1917), p. 41.

¥¥ Lowell Obs. Bull. 80 (1918). 
space relatively to their centroid. Truman* and Young and Harper f find respectively velocities of $670 \mathrm{kms}$ a second and $598 \mathrm{kms}$ a second.

Not only are large velocities in space revealed by the spectroscope, but also large velocities of rotation. The first discovery of rotation in a nebula was Slipher's $s_{+}^{+}$discovery in 1914 of the rotation of the nebula in Virgo (N.G.C. $4594 \S)$; Pease $\|$ has determined the velocity of rotation to be about $330 \mathrm{kms}$ a second at a distance of $2^{\prime}$ from the centre, the velocity increasing proportionally to the distance from the centre. Velocities of the same order have been found in other nebulae. By a comparison of photographs taken at different dates Van Maanen $\pi$ has found a rotation in the nebula M. 101** in Ursa Major which corresponds to a period of 85,000 years at $5^{\prime}$ from the centre; this nebula does not appear to rotate as a rigid body, the angular velocity being greater near the centre. Van Maanen finds that in this nebula the motion is along the arms and away from the centre, and similar results have been obtained by Kostinsky †+ for the spiral nebula in Canes Venatici (M. $51_{++}^{++}$). Slipher $\S$ suspects similar motion in the nebula N.G.C. 1068||||.

+5 . Very large velocities such as we have been considering are a distinctive property of the spiral nebulae. The large irregular nebulae, such as the Orion and Trifid nebulae are found to be almost at rest relatively to the stars of our system as a whole. The planetary nebulae have radial velocities ranging up to $65 \mathrm{kms}$ a second. The average radial velocity of thirteen measured by Keeler $\pi \uparrow$ is $27.7 \mathrm{kms}$ a 'second. If these velocities are corrected for the solar motion***, their average numerical value is $26.8 \mathrm{kms}$ a second, but their average algebraic value is only $0.9 \mathrm{kms}$ a second. Thus these thirteen planetary nebulae, regarded as a whole, are almost at rest relative to our system, while their individual velocities, although slightly larger than those of ordinary stars, are small compared with the observed velocities of the spiral nebulae.

It must, however, be added that Campbell $++\dagger$ has found quite exceptionally large radial velocities for two planetary nebulae, namely a velocity of approach of $141 \mathrm{kms}$ a second for N.G.C. $4732_{2}$, and a velocity of recession of $202 \mathrm{kms}$ a second for N.G.C. 6644. These velocities are not greater than a few exceptionally high velocities observed for ordinary stars (e.g. $325 \mathrm{kms}$ a sec. for

* Pop. Astronomy, 24, p. 111.

$\ddagger$ Lovell Obs. Bulletin, No. 62.

\| Ast. Soc. Pacific, 28, p. 191.

*" See Plate II.

\#+ See Plate II.

IIII Two fine photographs of this nebula will be found in the paper by Pease already referred to. Astrophys. Journ. 46 (1917), p. 24, Plate IV.

IT Publications of Lick Observatory, 3, 201.

*"* Perrine, Astrophys. Journ. 46 (1917), p. 176. †+† Nat. Acad. Sci. Washington, 1 (1915), No. 9.

+ Journal Royal Ast. Soc. Canada, 10, p. 134.

$\S$ See Plate III.

T. Astrophys. Journ. 44, p. 210.

†† MI. N. Royal Ast. Soc. 77, p. 233.

$\$ \S$ Lowell Obs. Bull. 80 (1918). 


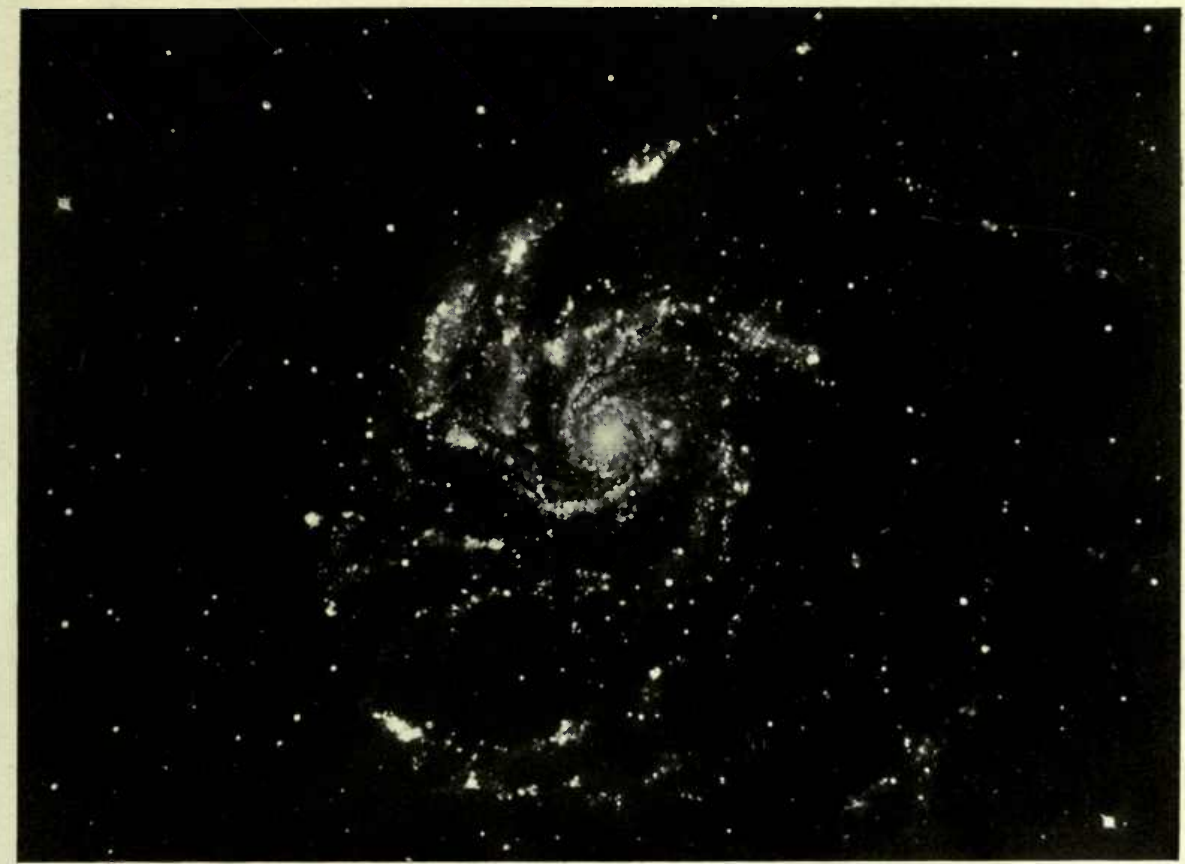

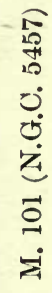

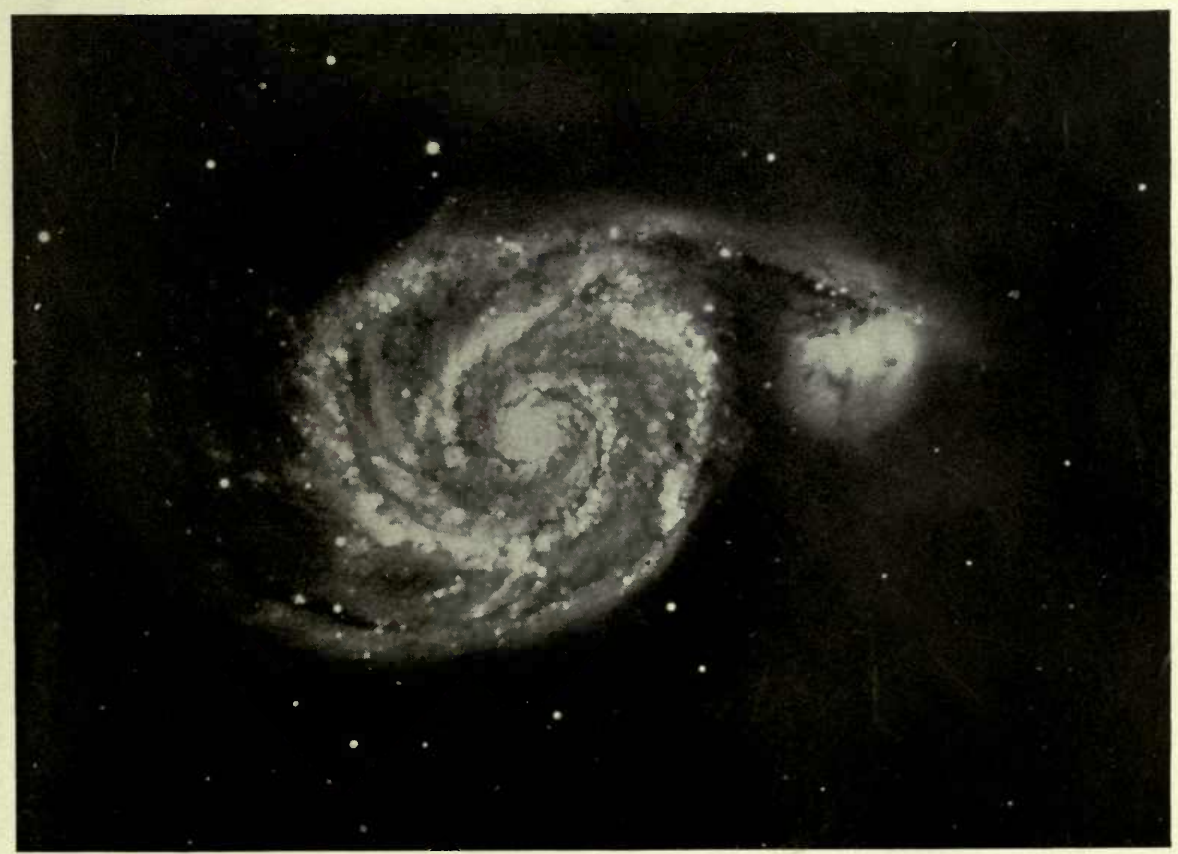



Lalande 1966 and $242 \mathrm{kms}$ a sec. for Cordoba Z. 5. 243), but regarding the problem as a whole, it is clear that they approach nearer to the velocities of the spiral nebulae than to those of ordinary stars.

- With the possible exception of special nebulae such as these last two, it is clear that we may, with good reason, suppose that the irregular and planetary nebulae form a part of our system, and are moving with it, while the spiral nebulae must be supposed to be systems independent of, and outside of, our own system.

Further evidence of this essential difference between the spiral and planetary nebulae is afforded by a study of their positions in the sky. The spiral nebulae are found to be concentrated towards the poles of the milky way, while the planetary nebulae are sparse near the poles of the milky way and shew a very pronounced tendency to collect in the galactic plane. Now there is every reason to believe that our system is of the shape of a coin or watch, our sun being near the middle, and the remote edges being represented by the milky way. Thus the most obvious, although perhaps not the only, explanation of the observed differences of concentration of the spiral and planetary nebulae is this: The planetary nebulae appear to favour the milky way because, being inside our system and intermingled with the other stars of the system, we see most of them in the directions in which we look into the deepest layer of stars, namely directions in the galactic plane. The spirals on the other hand appear to shun the milky way because the absorbing matter of our system blots out or partially obscures such of them as lie in directions near the galactic plane. In confirmation of this view R. F. Sanford* has recently shewn that spirals near the milky way are on the average less bright than those in other parts of the sky. F. G. Brown $\dagger$ has also shewn that the spiral nebulae of larger angular size are in general the brighter, but this is not true of spiral nebulae near the milky way where the visible nebulae are large but faint. All evidence is consistent with the view that the spiral nebulae are uniformly scattered in the sky but are quite outside our system, so that of those which lie in the direction of the galactic plane, the brighter ones are partially, and the fainter ones wholly, obscured by obstructing matter in our own system.

Campbell and Moore $\ddagger$ have recently found that quite a large proportion of planetary nebulae give spectroscopic evidence of internal motion. Of 33 examined, 16 gave definite evidence of internal motion, 12 gave no indications and the remaining 5 were doubtful. In a previous investigation $\S$ internal motions had been found in the two nebulae N.G.C. 7009 and N.G.C. 6543. The motions are believed to consist in most cases of rotations about

* Lick Obs. Bull. No. 297.

+ Monthly Notices R.A.S. 72 (1912), pp. 195 and 718.

$\ddagger$ Nat. Acad. Sci. 2 (1916), p. 566.

§ Lick Obs. Bull. 9 (1916), No. 278. 
axes through the centre, these axes being in general perpendicular to the longest dimensions of the nebulae. In some cases the motion is more complicated than a pure rotation; thus in N.G.C. 6543 the outer portions seem to have velocities much smaller than those of the central parts.

The parallax of the planetary nebula N.G.C. 7662 (Plate I) has been found at Mount Wilson to be $0^{\prime \prime} \cdot 023$, from which its diameter may be calculated to be 19 times that of the orbit of Neptune. That of the ring nebula in Lyra, N.G.C. 6720 (Plate I) has been found to be $0^{\prime \prime} \cdot 004$, but with a probable error comparable to the whole; the corresponding greatest and least diameters are 330 and 250 times those of the orbit of Neptune*.

\section{Star-clusters}

6. "Further uniformities of formation are to be found in star-clusters. The uniformities are not so definite as those we have just been considering, but are quite definite enough to suggest common origins. There are a great variety of star-clusters, shading often imperceptibly into one another, but they may be classified into three broad types: globular clusters, open clusters and moving clusters $\dagger$.

The globular clusters are dense aggregates of stars shewing very great condensation towards the centre. They are approximately globular in form, although Pease and Shapley+ have recently found that out of six supposed globular clusters which it was possible to study in detail, five shewed a pronounced departure from the spherical form, being apparently of a flattened or spheroidal form. A similar absence of complete symmetry in some clusters had been previously noticed by Bailey§. Bailey has also made counts of the stars in some of these globular star-clusters, and it has been shewn by Plummer\| and von Zeipel that the law of distribution is approximately uniform. The procedure has been criticised by Shapley** on the grounds that only a few of the brightest stars are included in such counts, but however this may be, there is no question that there is a uniformity of some kind. The number of known globular clusters is at most about 100: Bailey $+\dagger$ gives the number of. "definitely globular" clusters as 76 , while Melotte estimates the number as 82 . Practically all of these had been discovered by the time of the Herschels.

* Van Maanen, Ast. Soc. Pac. 171 (Oct. 1917).

+ Shapley, Contributions from the Mount Wilson Solar Observatory, No. 115 (1916), where an excellent summary is given; also P. J. Melotte, "A Catalogue of Star-clusters," Mem. R.A.S. 70 (1915), p. 175.

¥ Nat. Acad. of Sciences, 3 (1917), p. 96, and Astrophys. Journ. 45 (1917), p. 225.

$\S$ Harvard Coll. Observatory Annals, 76, No. 4. \# Monthly Notices R.A.S. 76, p. 107.

- K. Svenka. Vetensk. Acad. Handl. Bd. 51, No. 5.

** Observatory, 39 (1916), p. 452.

+† Harvard Coll. Observatory Annals, 76, p. 43. 
The distribution of globular clusters in the sky is somewhat surprising, it being found* that they are practically confined to one hemisphere of the sky. Not only this but there is a very marked condensation about one point in the sky, 42 out of the 82 considered by Melotte lying within $30^{\circ}$ of a point in the galactic plane of latitude $325^{\circ}$.

Slipher has recently measured the radial velocities of ten star clusters, and finds velocities ranging from -410 to $+225 \mathrm{kms}$. a second, the mean of the values, taken without regard to sign being $150 \mathrm{kms}$. a second. It is clear that we have here to deal with velocities of the same order of magnitude as the velocities of the spiral nebulae.

Finally Shapley $\uparrow$ has attempted to estimate the distances of various globular star-clusters, by assuming the absolute magnitudes of the cepheid variables contained in them to be equal to those of similar cepheid variables at known distances. He finds that probably, with one or two exceptions, no globular cluster is nearer than about 30,000 light-years, corresponding to a parallax of $0.00012^{\prime \prime}+$. Thus the globular clusters, like the spiral nebulae, appear to be independent of, and outside, our own system of stars.

The formation of moving star clusters also exhibits a certain, although not very great, degree of uniformity§. A number of stars is said to form a moving cluster when their velocities are sensibly the same, both in magnitude and direction, and also when there is definite evidence of some further real connection between the members of the cluster. The latter condition is important because, by a procedure which is familiar to every student of the Kinetic Theory of Gases, any collection of chaotically moving stars can be resolved into parallel showers. 'Observational astronomy reveals the existence of clusters of stars moving with equal velocities and also having physical characteristics in common which suggest that they have some bond of common origin. The cluster formed by the Pleiades provides perhaps the most superficially obvious instance of a star cluster of this kind. Here we have a group of stars, all of similar spectral type, all of approximately equal brightness, concentrated in one region of space and moving with a common velocity\|. A more thoroughly investigated cluster is the Taurus cluster which consists of the Hyades and other neighbouring stars $\%$. A noteworthy cluster of special interest is the Ursa Major cluster, which contains among other stars, the stars $\beta, \gamma, \delta, \epsilon$ and $\zeta$ Ursae Majoris of the "Plough."** There is a very

* Cf. Melotte, Mem. R.A.S. 70, p. 176, and A. R. Hinks, Monthly Notices R.A.S. 71, p. 693.

+ Proc. Nat. Acad. Sci. 3 (1917), p. 479.

$\mp$ By a similar method Hertzsprung had previously estimated the distance of the lesser Magellanic cloud to be of the same order (parallax $\cdot 0001$ ). Cf. Ast. Nach. 4692.

$\S$ On this subject in general, see Chap. IV of Eddington's Stellar Movements.

II IV. W. Campbell, Stellar Motions, p. 181.

II Boss, Astrophys. Journ. 26 (1908), p. 31.

** Ludendorff, Ast. Nuch. 180 (1909), 265, and W. W. Campbell, Stellar Mutions, p. 175. 
definitely marked cluster in Perseus, and less noteworthy clusters in ScorpiusCentaurus and Cygnus. There seems to be a tendency for clusters to assume a flattened shape, the flattening in the case of the Ursa Major cluster being almost complete*, so that the stars lie almost in a plane.

7. We have now mentioned five different types of structure found in the sky, each of which shews a more or less pronounced uniformity. The aim of a scientific cosmogony must be to trace these and other uniformities to their sources. When we find a formation repeated many times with only slight variations, we may feel fairly confident that its origin is in every case the same. The problem of cosmogony is to discover these origins and to prove that they would lead to the observed formations.

The various uniformities of structure are by no means of equal importance. A purely objective view would perhaps regard the finding of the origins of planetary systems as the least important problem of cosmogony, but, for reasons which can readily be understood, cosmogony has always been more concerned with this special problem than with any of the others. Indeed until quite recent years not enough was known of the universe outside our solar system for the problems of cosmogony to have assumed a definite shape except in reference to our own system. We now proceed to give a short account of some of the various theories of planetary origin which have been propounded.

\section{THEORIES OF COSMOGONY}

\section{The Nebular Hypothesis of Kant and Laplace}

8. Of all theories of cosmogony, the most enduring, and infinitely the most famous, has been the Nebular Hypothesis, commonly associated with the names of Kant and Laplace. Kant's theory was first given in his Allgemeine Naturgeschichte und Theorie des Himmels in 1755; Laplace published the outlines of his theory in 1796 in his Exposition du Système du Monde, developing his ideas further in later editions. Laplace seems to have been quite unacquainted with the earlier speculations of Kant; indeed he specifically states that Buffon was, so far as he knew, the only philosopher who, since the true nature of the solar system had been known, had speculated as to the origin of the planets and their satellites. Thus we have two theories, of distinct and independent origins, trying to explain the same phenomena. Kant's theories, however, attempted to explain the whole stellar universe, while Laplace limited himself to the solar system.

* See a description by H. H. Turner, The Observatory 34 (1911), p. 246. 
9. Kant's Theory. Kant supposed the universe to have developed initially out of a cold nebula at rest. He supposed this nebula to fall in under its own gravitation, and to become hot in so doing owing to the consequent compression. He assumed, of course erroneously, that rotation would be set up in this process. He imagined that the matter would condense into rings, and on superposing the supposed rotation, he arrived at a system of rotating rings similar to the rings of Saturn, to which he appealed as evidence of the truth of his theories. In the second stage of the cosmogonic process, Kant supposes these rings to become unstable and form by agglomeration into planets. The persistence of the rotatory motion results in this system of planets revolving round the sun. The planets continue to contract under their own gravitation, so that the preceding cycle of processes is repeated on a smaller scale, and finally we find the planets also surrounded by rotating satellites*.

10. Laplace's Theory. We turn now to the theory put forward by Laplace. The great French mathematician was not likely to fall into the error of believing that rotation could be generated out of nothing, and so the nebula is assumed to be rotating at the outset. Laplace supposes it to be hot, without attempting in any way to account for the heat, and supposes it to be lens-shaped or flat, without attempting to justify this special choice of shape. The mass is supposed to cool by radiation at the surface, while at the same time falling in upon itself as a result of the action of gravitation, the net result being a heating of the central portion and a general shrinkage of the whole. Since the angular momentum must remain constant throughout the shrinkage, the actual velocity of rotation must increase, and Laplace believed that as this increase of angular velocity took place, the outer ring of matter ceased to be continuous with the main mass. A succession of repetitions of this phenomenon leaves a series of concentric annuli of matter, rotating about a central axis, as imagined by Kant, and from this stage on the hypotheses of the two philosophers are in agreement.

11. It appears that both Kant and Laplace try to develop a theory in which a system such as the rings of Saturn represents a half-way stage between the primitive nebula and the present state of our universe. Neither theory attempts to explain why the supposed ring system should become unstable and agglomerate into planets, and neither theory explains why Saturn's rings have not become unstable.

Perhaps an unbiassed judge, devoid of preconceived ideas, might expect a ring of rotating matter to become unstable. But, as Sir G. Darwin $†$ has

* Fuller accounts of Kant's theory will be found in Poincaré's Leçons sur les Hypothèses Cosmogoniques, Ch. I and Darwin's Tides, Ch. 21. An account is also given in Miss Clerke's Modern Cosmogonies (1905).

† The Tides, p. 410 . 
pointed out, if such a ring agglomerated into a planet, the resulting planet ought to coincide with the centre of gravity of the ring and not with a point on its perimeter. The stability of Saturn's rings was of course explained by Maxwell in his celebrated Adams Prize Essay of 18.57*.

The theory of Laplace, like that of Kant, contained little but pure speculation. It is however obvious that these theories admit of mathematical verification or disproof. With the exception of an investigation by Roche $\dagger$, in which the primitive nebula is represented by a heavy nucleus surrounded by an atmosphere of infinitesimal density, almost all attempts to treat the question mathematically have represented the matter of the supposed nebula by a homogeneous incompressible fluid. This differs by so much from the tenuous gas postulated by Laplace, that we cannot with any certainty regard his theory as being either vindicated or condemned by such researches, whatever their result. It may, however, be remarked that the results obtained from such researches have led to a continual modification of the theory until in its present form it contains little in the way of detail that would be recognised as his own by Laplace, and perhaps nothing that would be recognised by Kant. But two outstanding features of the theory have survived, namely

(i) the supposition that our solar system originated out of a nebulous mass of gas,

(ii) the supposition that the change from the primitive stage to the present stage has been produced mainly by the agency of increasing rotation.

Of these two suppositions, the former receives almost universal acceptance, at any rate as a provisional hypothesis, while the latter can probably claim more adherents than any other theory of planetary origin. In order to avoid the very ambiguous term "Nebular Hypothesis," which in view of the innumerable modifications the hypothesis has undergone might mean almost anything, it will be convenient to refer to these two essential parts of the hypothesis as the "Theory of Nebulous Origin" and the "Rotational Theory." These two theories contain about all of the original "Nebular Hypothesis" which can survive serious criticism; we shall now consider these theories in turn in the light of modern astronomical knowledge.

\section{The Theory of Nebulous Origin}

12. The general belief in the theory of nebulous origin is based mainly upon direct observation of the sky; a reasoned defence of it might rest mainly upon a consideration of the classification of stars according to spectral type; those stars which are believed to be in the earliest stages of development are

* On the Stability of the Motion of Saturn's Rings (Cambridge, 1859).

† "Essai sur la Constitution et l'origine du système solaire," Acad. de Montpellier, Section des Sciences, virI (1873), p. 235. 
observed to be surrounded in general by masses or wisps of nebulous matter, the stars in the Orion nebula and the Pleiades being obvious instances. Whether or not these processes of reasoning are sound, there is no question that the theory of nebulous origin is widely and almost universally held, there being some room for differences of opinion as to whether the primitive nebula ought to be thought of as a mass of gas, or a dust cloud or possibly even a swarm of meteoric stones. For instance Lord Kelvin suggested as the ultimate origin of astronomical bodies, a collection of meteoric stones which were vaporised by repeated collisions and so gave place in time to a gaseous nebula of the Laplacean type. Sir Norman Lockyer suggested that many of the observed nebulae are still in the meteoric state, a view which recent spectroscopic evidence has made untenable as a general explanation of nebular structure.

13. A theory of the order of stellar development recently put forward by H. N. Russell* strikes at the root one of the principal reasons for believing in the nebulous origin of stars. Before the appearance of Russell's theory, the accepted order of stellar evolution, namely through the sequence of spectral types

$$
\text { Nebula, } B, A, F, G, K, M \text {, }
$$

was almost undisputed. In this classification + , the $B$-type stars are the hottest and stars of $M$ type (red stars) are the coolest. The approximate temperatures of the different types, as determined by Wilsing and Scheiner+, are as follows :

$$
\begin{array}{rrrr}
\text { Type } B 0-B 5, & T=9030^{\circ} & \text { Type } G, & T=4450^{\circ} \\
B 8-A 4, & 8880^{\circ} & K & 3970^{\circ} \\
A 5-A 8, & 5780^{\circ} & M & 2960^{\circ}
\end{array}
$$

According to the older view of stellar evolution, the $B$ type was supposed to indicate the stage in which the star was hottest and of lowest density, and so least removed from its original nebular existence $\S$; as the star radiated heat it got cooler, and so passed through the various types in succession; a spectrum of $M$ type was supposed to characterise the oldest stars which were close to extinction.

Russell shewed that the $M$ stars fall into two very clearly differentiated classes which he called "giant" and "dwarf" stars, these names referring to

* For an excellent statement by Prof. Russell himself see Nature, 93, p. 227 (1914); see also The Observatory, 37, p. 165.

+ This is the Draper Classification adopted by Harvard Observatory. A brief but excellent account of spectral classifications is given in Eddington's Stellar Movements, pp. 7-10.

$\ddagger$ Ast. Nach. 183 (1909), p. 87. Here and elsewhere each complete spectral class is divided into ten subdivisions; thus between a $B$ star and an $A$ star are supposed to be nine other stages designated as $B 1, B 2, \ldots B 9$. A spectrum of exact $B$ type is called $B 0$, and so on.

$\S$ To aroid confusiou $I$ have omitted the $O$ or Wolf-Rayet Type from discussion altogether. 
a great difference in absolute brightness, although not necessarily in mass. Any doubt that may have been felt as to the accuracy of this fundamental fact has probably been removed by the investigations of W. S. Adams*, who has found a purely spectroscopic method of determining the absolute brightness of a star. Examining 58 red stars, Adams finds that 48 are of absolute magnitudes between -1.0 and $3 \cdot 4$, while the remaining ten have absolute magnitudes between $9 \cdot 8$ and $10 \cdot 7$, the division between Russell's giant and dwarf stars thus being a clear gap of 6.4 magnitudes. More recently Adams and Joy $\dagger$ have spectroscopically determined the absolute magnitudes of 500 stars of types $F, G, K$ and $M$, and their results confirm Russell's facts completely. Of the 500 stars examined, 42 were of type $M$; of these 29 proved to be of absolute magnitudes brighter than $2: 9$, one was of absolute magnitude $3 \cdot 6$, and the remaining 12 were all of absolute magnitudes fainter than $9 \cdot \bar{\jmath}$. Again there is a clear gap of about 6 magnitudes between "giants" and "dwarfs." A similar, although less pronounced distinction, is found to persist through types $K$ and $G$, but it has almost, if not quite, disappeared for type $F$. It is thus proved beyond doubt that there exist red stars of extraordinary brightness, for which no place could be found in the older scheme of stellar evolution; for it is, as Russell remarks, very improbable that these stars, some of them 100 times as bright as the sun, are on the verge of extinction through old age.

Russell accordingly suggests that a star of $B$ type is not at the beginning of its career, but is half-way through. The star is supposed to have originated as a giant star of $M$ type, to have passed through the series of types $M, K$, $G, F, A$ to the stage $B$, and then to proceed again through the series $A, F, G, K$ until it becomes a dwarf star of type $M$. Only the most massive stars ever attain to the degree of incandescence represented by a $B$-type of spectrum; all others turn backwards before this stage is reached, a hypothesis which gives at once a simple and perfectly acceptable explanation of the known fact that $B$-type stars are of exceptional mass, while at the same time accounting for the gradual disappearance of the gap between giant and dwarf stars in types $K, G$ and $F$.

It will be understood that this brief statement does not give an account of all the details of Russell's theory, neither have we mentioned the many criticisms which have been brought against it $t_{+}^{+}$For our present purpose, it is enough to notice that the indisputable facts on which Russell's theory is based cut away to a large extent the original grounds for the belief that stars originate out of nebulae. It is not proved that they do not, but there is no longer any direct evidence that they do; to retain the theory of nebulous origin, we have now to imagine the nebulous matter to be or become non-

* Proc. Nat. Acad. Washington, March, 1916.

+ Astrophys. Journ. 46 (1917), p. 313.

¥ See for instance W. W. Campbell, Science, 45 (1917), p. 547. 
luminous and remain so until the mass bursts into incandescence as a giant $M$ star. But, as we shall see later, Russell's theory does not destroy, but actually strengthens, the belief that a star starts with a very low density, and this virtually brings us back by a different path to the theory of nebulous origin.

\section{The Rotational Theory}

14. The main outline of what we are now calling the rotational theory has been sufficiently explained already. This theory originated in an effort to explain the origin of the solar system. In its application to this particular problem it has been subjected to many criticisms, one of which in particular has seemed to many to be unanswerable.

In 1861 Babinet* suggested that a criterion as to the tenability of the general rotational theory was provided by a calculation of 'the present total angular momentum of the solar system. He argued that if the planets had been thrown off by rotation the moment of momentum of the original rotating mass must have been exactly equal to the total moment of momentum of the present system. The mass of the original body must also have been equal to the total mass of the present system, so that on assuming a reasonable size for this original body, the dynamical conditions of the mass can to some extent be reconstructed, and in particular we can calculate the amount of rotation with which it must' have been endowed. Babinet pointed out that the aggregate moment of momentum in the solar system is far too small for the original mass to have been broken up by rotation alone.

A simple calculation will shew that the greater part of the present moment of momentum of the solar system resides in the orbital motion of Jupiter. Taking the moment of momentum of the sun's present axial rotation as unity, the moment of momentum of the orbital momentum of Jupiter is found to be about 37, that of Saturn about 14, that of Neptune about 4:8, that of Uranus about $3 \cdot 3$, and the aggregate arising from all the other planets, asteroids, satellites, etc., is less than $0 \cdot 1$. Thus the total is roughly 60 times the present moment of momentum of the sun's rotation $†$.

Now imagine the whole mass of the solar system concentrated in the sun, which can be done with only an inappreciable increase (about 0013) of its mass, and imagine the whole moment of momentum of the present solar system concentrated in this one mass. The moment of momentum being

* Comptes Rendus, 52 (1861), p. 481. See also Moulton, Astrophys. Journ. (1900), p. 103.

+ These are the figures given by T. J. J. See (Ast. Nach. 4053). See makes special assumptions as to the interior constitution of the sun, but any other reasonable assumption would lead to similar figures. Fouché (C.R. 99, p. 903 (24 Nov. 1884)) calculates the total momentum to be 28.2 times that of the sun, but he assumes the sun to be homogeneous. Lord Kelvin has given a well-known estimate (Popular Lectures, I. p. 420) according to which the ratio in question is only 18 , but he assumes the sun to be homogeneous, and also neglects the contributions from Saturn, Uranus, Neptune, etc., this latter procedure being clearly erroneous. 
increased 60 -fold, and the mass remaining substantially the same, it follows that the angular velocity will be about 60 times what it is now, and instead of having a period of rotation of 25 days, the new sun will have a period of about 10 hours-roughly the same as that of Jupiter. The mean density of the sun $(1 \cdot 36)$ is roughly equal to that of Jupiter (1.30) so that the primitive sun reconstituted in this way will be very similar to the present Jupiter, only of greater mass. The mass of a body, as we shall see later, has almost no influence on its tendency to break-up rotationally; this depends almost entirely on its angular velocity and mean density. Now Jupiter shews an ellipticity of only about $\frac{1}{17}$, and is to all appearances very far from breaking up under the influence of its rotation, so that we cannot suppose our primitive sun to have broken up by rotation.

In this we have supposed the primitive sun to be of about the same size as our own sun; it must certainly have been larger, and this makes the result still more certainly true. The rotational theory asserts that shrinkage is the primary cause of the inset of instability which results in the throwing off of a satellite; if the primitive sun, when shrunk to the size of our present sun, does not throw off a satellite, it certainly cannot have thrown off a satellite as the result of rotation before the shrinkage took place, when its dimensions may have been a thousand or a hundred thousand times what they now are.

The discussion of whether or not this criticism of the rotational theory is valid will naturally be deferred until our mathematical investigations have provided evidence on which to base a judgment.

\section{The Tidal-Action Theory}

15. Suggestions have at various times been made that tidal forces may play the preponderating part in effecting the birth of satellites, for it is obvious that, when subjected to tidal forces of sufficient intensity a mass of fluid may reach a breaking point at which it divides into two or more detached masses. The most complete form of tidal-action theory is found in the "Planetesimal Theory" of Chamberlin and Moulton*.

A non-rotating mass will in general assume a spherical shape under the action of its own gravitational forces, but will depart from this form when a second body approaches near enough for its tidal influence to be perceptible. At the approach of a second body, the spherical shape will at first give place to a spheroid of small ellipticity, owing to tides being raised directly under and directly away from the tide-raising body. With the closer approach of this body the tides continually rise in height, and Chamberlin and Moulton suppose that ultimately two jets of matter rush out from the two antiporlal

" A summary of a comprehensive kind will be found in Chamberlin's Origin of the Earth (Univ. of Chicago Press, 1916). 
points at which the tides are highest. But the tide-raising body does not stand still; it is always somewhat ahead of the diameter through the two highest tides, and so exerts not only a tide-generating force, but also a couple which tends to set up rotation in the primary body. The two jets of nebulous matter are therefore being ejected from a slowly rotating body, and instead of forming straight lines, form spiral curves.

The authors of the planetesimal theory claim that these conceptions explain the origin of the spiral nebula formation, which they regard as a halfway stage in the process of planetary formation, just as Kant and Laplace regarded the rings of Saturn. The authors further believe that the ejection of matter will take place by "pulsations"-hence the nuclei observed in the arms of the typical spiral nebula - and that the condensations of these nuclei ultimately form planets by agglomeration. If all this can be shewn to happen according to the authors' programme, then clearly the planetary structure and the spiral nebula structure are explained at one sweep. But whether all this happens or not can only be decided by exhaustive mathematical investigation.

Perhaps the most obvious criticism that can be brought against this and all other tidal theories is that they require the close approach of large astronomical bodies, and that such close approaches are very rare events. Calculations which will be given later seem to shew that this consideration must lead to the abandonment of all tidal theories, including the planetesimal, as explanations of normal cosmogonic processes. It must not of course be asserted that no system has ever been broken up by tidal forces-this would be contrary to all statistical laws-but it will be found that only a small proportion of the stars in the universe are likely to have been broken up in this way.

\section{OTHER TheoRIES}

16. In addition to the theories just mentioned, there are a great number of others in the field which claim to explain the origin of the solar system. Many of these start from a nebulous mass or swarm of meteorites in chaos, and regard the spiral nebula formation as an intermediate stage towards the development of a solar system. Thus in addition to Moulton and Chamberlin, See* and Arrhenius $\dagger$ both contemplate the possibility of spiral nebulae forming out of the collision or near approach of two stars, the condensations in the arms of the spiral being supposed ultimately to form planets circling around a central nucleus. Sutherland + has suggested that Bode's wellknown law of planetary distances is readily explained in terms of a spiral origin; for Bode's law, usually expressed in the form

$$
r=0.4+0.15 \times 2^{n} \quad(n=1,2,3, \ldots) .
$$

* Researches of the Evolution of Stellar Systems, Vol. I.

† Worlds in the Making. (London, 1908.) $\quad \ddagger$ Astrophys. Journ. 34, p. 251.

J. C. 
may be equally expressed in the form

$$
r=r_{0}+r_{1} e^{\theta / a} \quad(\theta=1,2,3, \ldots)
$$

which may be taken to represent the distances of nuclei along the arms of an equiangular spiral.

In a somewhat different class come hypotheses, such as those of Faye* and Ligondèst, which try to prove that our system originated out of a swarm of meteorites in which order has been produced out of disorder by collisions, in opposition to the laws of statistical mechanicst.

Our task in the present essay is not to discuss these and other theories in detail; it is rather to obtain mathematical evidence bearing on the general problem of evolution, incidentally perhaps examining to what extent the speculative theories which have just been described are tenable. Many of these theories, however, have already been condemned by the recent advances in observational astronomy. For in many cases the theories were not based on abstract knowledge of the properties of matter or on dynamical laws; they rather exhibited a tendency to be based on the latest observational knowledge with which their authors were acquainted. Up to the discovery of the spiral nebulae, most theories of cosmogony tried to prove that Saturn's rings (the most sensational astronomical objects then known) formed an intermediate stage in the evolution of planetary systems : since the discovery of spiral nebulae, the tendency has been to try to prove that the spiral nebulae form the link in question. The more scientific method of procedure is to limit the investigation to the abstract problem of the behaviour of masses of astronomical matter under varying dynamical forces; when the solution of this problem has been carried to the limit of our mathematical resources, we shall be in a position to survey the different types of formation that may be expected to be evolved, and possibly not much speculation will be required to identify them with observed forms. Thus the immediate object of the present essay will be to collect and arrange the results of the various researches which have resulted in progress towards the solution of this abstract problem, adding to them and amplifying them wherever we can.

The dynamical forces which can act on astronomical matter are its own gravitation, which must always be taken into account: the gravitational forces from other bodies, which we may for brevity describe as tidal forces; the forces arising from rotation; the forces arising from collisions, impacts, bombardments, etc. Our problem is to find out as much as we can about the behaviour of matter under such forces, paying attention especially to effects of a secular or evolutionary nature.

* Sur l'Origine du Monde. (Paris, Gauthier-Villars, 1884.)

† Formation Mécanique du Système du Monde. (Paris, Gauthier-Villars, 1897.)

¥ Cf. Poincaré, Leçons sur les Hypothèses Cosmogoniques, Chapters IV and V. 


\section{CHAPTER II}

\section{GENERAL DYNAMICAL PRINCIPLES}

17. In general the configuration of a dynamical system can be expressed in terms of Lagrangian coordinates

$$
\theta_{1}, \theta_{2}, \theta_{3}, \ldots \theta_{n}
$$

while its motion at any instant can be specified in terms of the corresponding velocities

$$
\dot{\theta}_{1}, \dot{\theta}_{2}, \dot{\theta}_{3}, \ldots \dot{\theta}_{n}
$$

The potential energy $W$ will be a function of the coordinates of position only, say

$$
W=f\left(\theta_{1}, \theta_{2}, \ldots \theta_{n}\right)
$$

while the kinetic energy $T$ will be a function both of the coordinates of position and of the velocities, say

$$
T=F\left(\theta_{1}, \theta_{2}, \ldots \theta_{n}, \dot{\theta}_{1}, \dot{\theta}_{2}, \ldots \dot{\theta}_{n}\right)
$$

and this function will be quadratic in the velocities $\dot{\theta}_{1}, \dot{\theta}_{2}, \ldots \dot{\theta}_{n}$.

The equations of motion will be the Lagrangian equations

$$
\frac{d}{d t}\left(\frac{\partial T}{\partial \dot{\theta}_{8}}\right)-\frac{\partial T}{\partial \theta_{s}}=-\frac{\partial W}{\partial \theta_{s}}+F_{s} \quad(s=1,2, \ldots n)
$$

where $F_{1}, F_{2}, \ldots F_{n}$ are the "generalised forces" applied from outside.

In a number of cosmogonical problems, we shall be concerned with the motion of astronomical masses, and the equations determining this motion will be equations (5) or some appropriate special form of these equations. But in a much greater number of cosmogonical problems we shall be concerned with astronomical masses which are either in a state of equilibrium or whose motion is so slow that their kinetic energy is negligible. For such configurations, putting $T=0$, equations (5) reduce to

$$
\frac{\partial W}{\partial \theta_{1}}=0, \quad \frac{\partial W}{\partial \theta_{2}}=0, \quad \frac{\partial W}{\partial \theta_{3}}=0, \text { etc. }
$$

These may be regarded either as equations of equilibrium or as equations determining the configuration of a very slowly changing mass. Regarded as equations in $\theta_{1}, \theta_{2}, \theta_{3}, \ldots$, the equations will have a number of solutions of which a typical one may be taken to be

$$
\theta_{1}=\Theta_{1}, \quad \theta_{2}=\Theta_{2} \text {, etc. }
$$


In this solution the quantities $\Theta_{1}, \Theta_{2}, \ldots$ will be functions of the constants which enter into the function $W$ as given by equation (3). But in problems of cosmogony in which changes of a secular or evolutionary nature occur, these constants must themselves be supposed to vary; they are better spoken of as parameters than as constants. When equations such as (5) are satisfied, an astronomical mass has assumed a position of equilibrium for the moment, but with the course of time the physical conditions will change, and the configuration of equilibrium will give place to another. Analytically this process is represented by slow changes in the parameters which occur in the specification of $W$ by equation (3).

\section{STATICAL SYSTEMS}

\section{Linear Series}

18. Let us consider in detail the changes produced in $\Theta_{1}, \Theta_{2}, \ldots$, the coordinates of a configuration of equilibrium, as one of the variable parameters, say $\mu$, is allowed slowly to vary.

A slight change in the value of $\mu$, say from $\mu$ to $\mu+d \mu$, will alter the values of $\Theta_{1}, \Theta_{2}, \ldots$ by quantities which will in general be small quantities of the same order of magnitude as $d \mu$. Thus on making this small change in $\mu$, a configuration of equilibrium such as that given by equations (7) gives place to an adjacent configuration of equilibrium. On continually varying $\mu$ we pass through a whole series of continuous configurations of equilibrium, and these form what Poincaré has called a "linear series*."

We may in imagination construct a generalised space having

$$
\theta_{1}, \theta_{2}, \ldots \theta_{n}, \mu
$$

as coordinates. Any one plane $\mu=$ cons. will be suitable for the representation of all the configurations which are possible for one value of $\mu$, and therefore for all which are possible for one definite physical state of the system. The particular points in this plane determined by equations such as (7) will represent the configurations of equilibrium in this physical state.

The function $W$ must, from its meaning, be a single valued function of $\theta_{1}, \theta_{2}, \ldots$ and $\mu$, so that the surfaces $W=$ cons. in the $(n+1)$-dimensional space are necessarily non-intersecting surfaces. The condition that a configuration shall be one of equilibrium, as expressed by equations (6), is exactly identical with the condition that the tangent to the surface $W=$ cons. shall be perpendicular to the axis of $\mu$. Thus if for convenience we think of the axis of $\mu$ as being vertical, the configurations of equilibrium are represented by points at which the tangents to the surfaces $W=$ cons. are horizontal;

* Poincaré, Acta Math. 7 (1885), p. 259, or F'igures d'Equilibre d'une Masse fuide. (Paris, 1902.) See also Lamb, Hydrodynamic8, p. 680. 
let us for brevity call these "level points." On joining up a succession of level points, such as $P_{1}, P_{2}, P_{3}$ in fig. 1 , we obtain a "linear series."

\section{Points of Bifurcation}

19. The regular succession of such points as we pass along a linear series may be broken in various ways. One obvious way is by a change in the direction of curvature of the $W$-surfaces, resulting in the formation of a kink, such as is shewn occurring at the point $Q$ in fig. 1. On any surface on which this formation has just occurred, there will be three adjacent level points such as $R_{1}, S_{1}, T_{1}$ in the figure. The original linear series $P Q$ will accordingly become replaced by three linear series such as $Q R, Q S$ and $Q T$ as soon as we pass above the point $Q$ at which the kink first forms. It is readily seen that at $Q$ two of the series $Q R$ and $Q T$ must run continuously into one another, and so in effect form a single new series, while the series $Q S$ may be regarded as a

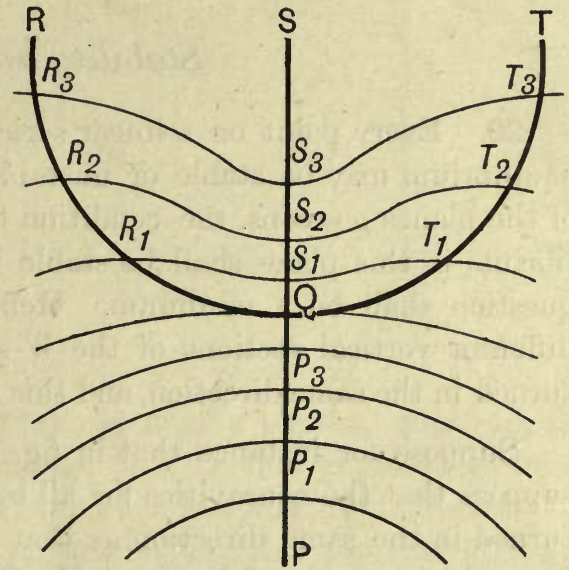

Fig. 1. continuation of $P Q$. We may accordingly suppose that there are two linear series $P Q S$ and $R Q T$ crossing one another at the point $Q$. A point such as $Q$ is called by Poincaré a "point of bifurcation."

Another and more usual way in which the succession of level points can be broken-or rather deviated-is shewn in fig. 2. In this case, as $\mu$ increases,

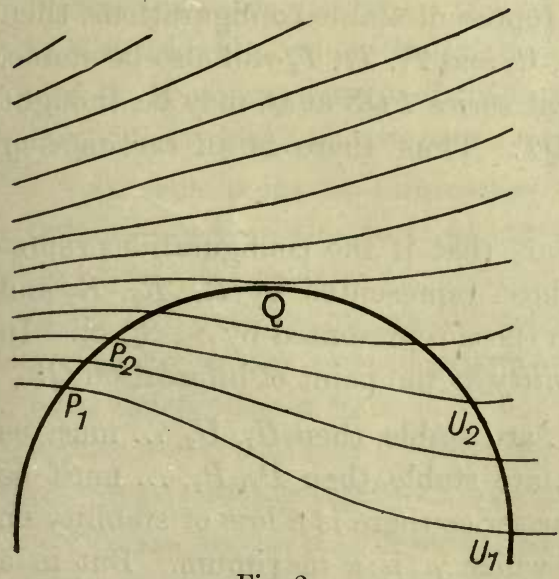

Fig. 2.

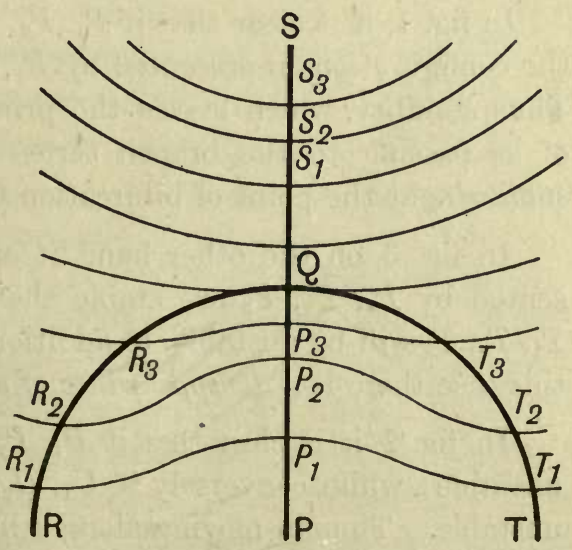

Fig. 3. 
two linear series such as $P_{1} P_{2} Q$ and $U_{1} U_{2} Q$ coalesce in the point $Q$ and then disappear. It will be convenient to refer to a point such as $Q$ in this figure as a "turning point."

Still a third possibility is shewn in fig. 3 ; this however is only a variant of fig. 1, and again leads to two linear series crossing one another in a point of bifurcation $Q$. Other minor variations may occur, but the principal possibilities are those shewn in figures 1, 2 and 3.

\section{Stability and Instability}

20. Every point on a linear series is a configuration of equilibrium; the equilibrium may be stable or unstable. Confining our attention to any one of the planes $\mu=$ cons. the condition that a particular configuration of equilibrium in this plane shall be stable is that the value of $W$ at the point in question shall be a minimum. Hence, for stability, the concavities of the different vertical sections of the $W$-surface through this point must all be turned in the same direction, and this direction must be that of $W$-decreasing.

Suppose for instance that in fig. $1 \mathrm{~W}$ increases as we pass upwards, and suppose that the concavities for all sections of the $W$-surface through $P_{1}$ are turned in the same direction as that shewn in the diagram. Then the configuration represented by the point $P_{1}$ will be one of stable equilibrium.

On passing along a series such as $P Q S$ in fig. 1 or 3 , it is clear that one of the sections must change the direction of its concavity as we pass through the point $Q$ at which a kink is first formed on the $W$-surfaces. Thus configurations which were initially stable give place to unstable configurations on passing through $Q$. It appears that a principal series such as $P Q S$ loses its stability on passing through a point of bifurcation.

In fig. 1, it is clear that if $P_{1}, P_{2}, P_{3}$ represent stable configurations, then the configurations represented by $R_{1}, R_{2}, R_{3}$ and $T_{1}, T_{2}, T_{3}$ will also be stable. Thus stability, which leaves the principal series $P Q S$ at $Q$, may be thought of as passing to the branch series RQT. Thus there is an exchange of stabilities at the point of bifurcation $Q$.

In fig. 3 , on the other hand, it appears that if the configurations represented by $P_{1}, P_{2}, P_{3}$ are stable then those represented by $R_{1}, R_{2}, R_{3}$ and $T_{1}, T_{2}, T_{3}$ will be unstable, in addition to those represented by $S_{1}, S_{2}, S_{3}$. In this case there is a disappearance of stability at the point of bifurcation $Q$.

In fig. 2 , it is clear that if $P_{1}, P_{2}, \ldots$ are stable, then $U_{1}, U_{2}, \ldots$ must be unstable; while conversely if $U_{1}, U_{2}, \ldots$ are stable, then $P_{1}, P_{2}, \ldots$ must be unstable. Thus in moving along a linear series there is a loss of stability on passing through a point such as $Q$ at which $\mu$ is a maximum. But in a physical problem, $\mu$ will continually change in the same direction, and the 
physical phenomenon which will shew itself as $\mu$ passes through its value at $Q$ will be a complete disappearance of two sets of equilibrium configurations.

The results obtained may be shewn diagrammatically in the following figures, in which thick lines represent series of stable configurations, and thin lines series of unstable configurations, the series $P Q$ being assumed to be stable in every case.

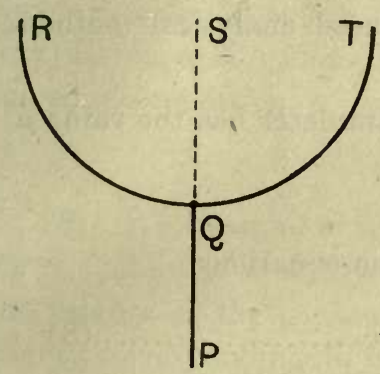

(i)

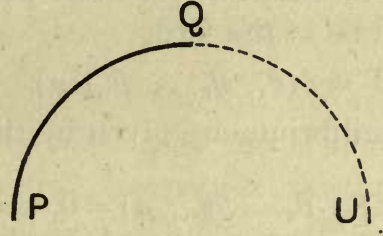

(ii)

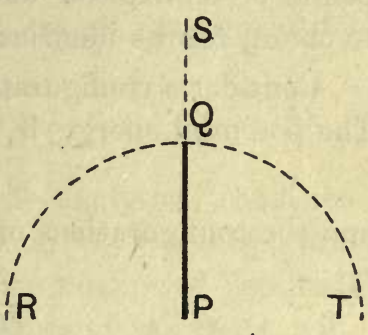

(iii)

Fig. 4.

21. Suppose that $\mu$ changes very slowly in any physical problem, and for definiteness let the direction of change of $\mu$ be that represented by an upward movement in our diagrams. From what has already been said, it is clear that we have the following rule for tracing out the sequence of stable states which will be followed by the system as $\mu$ varies.

Start from a configuration in the diagram which is known to be stable, and follow a path along linear series of equilibrium so as always to move upwards, and so as always to cross over from one series to another at a point of bifurcation. So long as we do this we are following a sequence of configurations which is always stable. When it becomes impossible to do this any longer, a value of $\mu$ has been reached beyond which no stable configurations exist, and when the physical conditions change so that $\mu$ attains to a still higher value, the statical problem gives place to a dynamical one; it is no longer a question of tracing out a sequence of gradual secular changes, but of following up a comparatively rapid motion of a cataclysmic nature.

At each point of bifurcation there is necessarily a certain amount of indefiniteness in the path which will actually be followed. For instance in fig. 4 (i), the system on arriving at $Q$ may proceed either along $Q T$ or along $Q R$, both being equally consistent with the maintenance of stability, and so far as can be seen equally likely. In actual fact there may even be more indefiniteness than this; our figures are two-dimensional diagrammatic representations of $(n+1)$-dimensional spaces, and the line $R Q T$ in our figures may very possibly represent a surface in the $(n+1)$-dimensional space.

These apparent complications cause no difficulty in actual problems. They arise from the obvious circumstance that a general discussion of stability, 
although competent to determine when stability ceases, cannot in general determine what will happen after stability has ceased. In the same way a general discussion will readily shew that a stick standing vertically on its point is in unstable equilibrium, but it cannot determine in which precise direction the stick will fall.

22. In his classical paper* in which the theory of linear series and points of bifurcation was first developed, Poincaré used analytical methods to obtain results identical with those just found.

Consider a configuration in which the variable parameter has the value $\mu$. The potential energy $W$ will be of the form

$$
W=f\left(\theta_{1}, \theta_{2}, \ldots \theta_{n}, \mu\right)
$$

and the configurations of equilibrium are given by the equations

$$
\frac{\partial}{\partial \theta_{1}} f\left(\theta_{1}, \theta_{2}, \ldots \theta_{n}, \mu\right)=0, \text { etc. }
$$

As in $\S 17$, let $\Theta_{1}, \Theta_{2}, \ldots$ be a configuration of equilibrium corresponding to this given value $\mu$ of the parameter, so that at the point $\Theta_{1}, \Theta_{2}, \ldots \Theta_{n}, \mu$,

$$
\frac{\partial W}{\partial \theta_{1}}=\frac{\partial W}{\partial \theta_{2}}=\frac{\partial W}{\partial \theta_{n}}=0
$$

At any adjacent point $\Theta_{1}+\delta \theta_{1}, \Theta_{2}+\delta \theta_{2}, \ldots \mu+\delta \mu$, the value of $W$ may be expressed in the form

$$
\begin{aligned}
W+\frac{1}{2}\left(\delta \theta_{1}\right)^{2} \frac{\partial^{2} W}{\partial \theta_{1}^{2}} & +\left(\delta \theta_{1}\right)\left(\delta \theta_{2}\right) \frac{\partial^{2} W}{\partial \theta_{1} \partial \theta_{2}}+\ldots \\
& +\delta \mu \frac{\partial W}{\partial \mu}+\frac{1}{2}(\delta \mu)^{2} \frac{\partial^{2} W}{\partial \mu^{2}}+(\delta \mu)\left(\delta \theta_{1}\right) \frac{\partial^{2} W}{\partial \theta_{1} \partial \mu}+\ldots \ldots
\end{aligned}
$$

The condition that this new configuration shall be one of equilibrium is, from equations (8),

$$
\delta \theta_{1} \frac{\partial^{2} W}{\partial \theta_{1}^{2}}+\delta \theta_{2} \frac{\partial^{2} W}{\partial \theta_{1} \partial \theta_{2}}+\cdots+\delta \theta_{n} \frac{\partial^{2} W}{\partial \theta_{1} \partial \theta_{n}}+\delta \mu \frac{\partial^{2} W}{\partial \theta_{1} \partial \mu}=0
$$

and similar equations. Writing $W_{12}$ for $\partial^{2} W / \partial \theta_{1} \partial \theta_{2}$ and so on, the solution of these equations is

where $\Delta$ is given by

$$
\mid \frac{\delta \theta_{1}}{\left|\begin{array}{c}
W_{12}, W_{13}, \ldots W_{1 \mu} \\
W_{22}, W_{23}, \ldots W_{2 \mu} \\
\ldots \ldots \ldots \ldots \ldots . \ldots . \ldots
\end{array}\right|}=\frac{\delta \theta_{2}}{|\ldots|}=\ldots=\frac{\delta \mu}{\Delta}
$$

$$
\Delta=\left|\begin{array}{c}
W_{11}, W_{12}, \ldots W_{1 n} \\
W_{21}, W_{22}, \ldots W_{2 n} \\
\ldots \ldots \ldots \ldots \ldots \ldots
\end{array}\right|
$$

and so is the Hessian of $W$ with respect to the variables $\theta_{1}, \theta_{2}, \ldots \theta_{n}$. 
The values of the ratios

$$
\frac{\delta \mu}{\delta \theta_{1}}, \frac{\delta \mu}{\delta \theta_{2}}, \ldots \frac{\delta \mu}{\delta \theta_{n}}
$$

determine the direction of the linear series through the configuration $\Theta_{1}, \Theta_{2}, \ldots \Theta_{n}$ in our diagram. At points such as $Q$ in figs. 4 (i) and 4 (iii) one or more of these ratios must become indeterminate, so that we must have (say) $\delta \mu / \delta \theta_{1}=0 / 0$. At a point such as $Q$ in fig. 4 (ii) we must have (say) $\delta \mu / \delta \theta_{1}=0$. Thus the three points $Q$ in figure 4 are all determined by the single condition

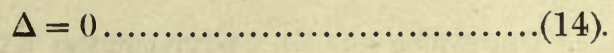

23. We must now try to connect this up with the analytical condition for a change of stability occurring at the configuration $\Theta_{1}, \Theta_{2}, \ldots \Theta_{n}$ and the value $\mu$ of the parameter. Keeping $\mu$ constant, the change of potential energy corresponding to changes $\delta \theta_{1}, \delta \theta_{2}, \ldots$ in the values of $\Theta_{1}, \Theta_{12} \ldots$, will, as in equation (10) be given by

$$
\delta W=\frac{1}{2}\left(\delta \theta_{1}\right)^{2} W_{11}+\left(\delta \theta_{1}\right)\left(\delta \theta_{2}\right) W_{12}+\ldots
$$

in which no terms of degree beyond the second need be written down when $\delta \theta_{1}, \delta \theta_{2} \ldots$ are supposed small.

Let the coordinates $\delta \theta_{1}, \delta \theta_{2} \ldots$ in this quadratic expression for $\delta W$ be changed by a linear transformation to new coordinates $\phi_{1}, \phi_{2} \ldots$, such that $\delta W$ becomes a sum of squares, say

$$
\delta W=\frac{1}{2}\left(b_{1} \phi_{1}{ }^{2}+b_{2} \phi_{2}{ }^{2}+\ldots+b_{n} \phi_{n}{ }^{2}\right)
$$

and let the modulus of transformation be $\lambda$.

Since the discriminant remains invariant through all linear transformations, we have

or

$$
\begin{array}{r}
b_{1}, 0,0, \ldots \\
0, b_{2}, 0, \ldots \\
\ldots \ldots \ldots \ldots \ldots
\end{array}|=\lambda| \begin{gathered}
W_{11}, W_{12}, \ldots W_{1 n} \\
W_{12}, W_{22}, \ldots W_{2 n} \\
\ldots \ldots \ldots \ldots \ldots \ldots
\end{gathered} \mid
$$

The condition that the configuration (17) shall be stable is that $\delta W$ shall be positive for all values of $\delta \theta_{1}, \delta \theta_{2}, \ldots \delta \theta_{n}$, or again that expression (16) shall be positive for all values of $\phi_{1}, \phi_{2}, \ldots \phi_{n}$. This condition is that $b_{1}, b_{2}, \ldots b_{n}$ shall all be positive.

The coefficients $b_{1}, b_{2}, \ldots b_{n}$ are called by Poincaré "coefficients of stability." A change from stability to instability occurs when any one of these coefficients vanishes, and the values of $\mu$ for which this occurs are, from equation (17), given by

$$
\Delta=0
$$


Combining this with the result obtained in the last section, it appears that a change of stability occurs at every point of bifurcation, and at every point on a linear series at which $\mu$ passes through a maximum or a minimum value, agreeing with the result obtained by other means in $\$ 19$ and 20 . The criterion of stability in the branch series at a point of bifurcation is most readily seen by the method already adopted in $\$ 21$; with the conventions there used, it appears that the branch series will be stable if it turns upwards from the point of bifurcation, and unstable if it turns downwards.

\section{ROTATING SYSTEMS}

24. This completes the discussion of the stability of statical systems. The stability of motion of a dynamical system is a very much more complicated question, but assumes a specially simple form when the motion consists mainly of a rigid body rotation. We proceed to discuss the stability of such a system.

Let the system be referred to axes rotating in space with any velocity $\omega$ about the axes of $z$ in the direction from $O x$ to $O y$. Let $x, y, z$ be the coordinates of any point referred to these axes, and let $\dot{x}, \dot{y}, \dot{z}$ denote their rates of increase. The components of velocity in space are then given by

$$
u=\dot{x}-y \omega, \quad v=\dot{y}+x \omega, \quad w=\dot{z} .
$$

so that the kinetic energy $T$ is given by

$$
\begin{aligned}
T & =\frac{1}{2} \Sigma m\left(u^{2}+v^{2}+w^{2}\right) \\
& =\frac{1}{2} \Sigma m\left(\dot{x}^{2}+\dot{y}^{2}+\dot{z}^{2}\right)+\omega \Sigma m(x \dot{y}-y \dot{x})+\frac{1}{2} \omega^{2} \Sigma m\left(x^{2}+y^{2}\right)
\end{aligned}
$$

The total moment of momentum $\mathbf{M}$ about the $z$-axis is given by

$$
\begin{aligned}
\mathbf{M} & =\Sigma m(x v-y u) \\
& =\Sigma m(x \dot{y}-y \dot{x})+\omega \Sigma m\left(x^{2}+y^{2}\right)
\end{aligned}
$$

Put

$$
\begin{aligned}
& T_{R}=\frac{1}{2} \Sigma m\left(\dot{x}^{2}+\dot{y}^{2}+\dot{z}^{2}\right) \\
& U=\Sigma m(x \dot{y}-y \dot{x}) \ldots \\
& I=\Sigma m\left(x^{2}+y^{2}\right) \ldots
\end{aligned}
$$

so that $T_{R}$ is the kinetic energy relative to the rotating axes, $U$ is the moment of momentum relative to the moving axes, and $I$ is the moment of inertia. Then equations (20) and (21) become

$$
\begin{aligned}
& T=T_{R}+\omega U+\frac{1}{2} \omega^{2} I \\
& \mathbf{M}=\quad U+\omega I .
\end{aligned}
$$

Eliminating $U$ we obtain

$$
T=T_{R}+\omega \mathrm{M}-\frac{1}{2} \omega^{2} I
$$


The position of the system may be supposed defined by $\psi$, a coordinate fixing the position of the axes, such that $\dot{\psi}=\omega$, and $n-1$ Lagrangian coordinates $\theta_{1}, \theta_{2} \ldots \theta_{n-1}$ fixing the configuration of the system relative to the axes, so that the system has $n$ degrees of freedom in all.

The equations of motion are (cf. equations (5)),

$$
\begin{array}{r}
\frac{d}{d t}\left(\frac{\partial T}{\partial \omega}\right)-\frac{\partial T}{\partial \psi}=G \ldots \ldots \ldots \ldots \ldots \ldots . . . \ldots . . . \\
\frac{d}{d t}\left(\frac{\partial T}{\partial \dot{\theta}_{8}}\right)-\frac{\partial T}{\partial \theta_{s}}=-\frac{\partial W_{s}}{\partial \theta_{s}}+F_{8} \quad(s=1,2, \ldots n-1)
\end{array}
$$

in which $G$ is the generalised force corresponding to the coordinate $\psi$, and so is the couple about the axis of $z$ which acts upon the system.

From the value of $T$ given by equation (25), we clearly have $\partial T / \partial \psi=0$ and $\partial T / \partial \omega=\mathbf{M}$, so that equation (28) reduces to

$$
\frac{d \mathbf{M}}{d t}=G
$$

expressing simply that the rate of increase of the moment of momentum $\mathbf{M}$ is equal to the couple $G$.

If a mass is rotating freely in space, $G=0$, so that $M$ remains constant.

If a mass is constrained to rotate at a constant angular velocity while $\mathbf{M}$ changes, a couple $G$ will be necessary to maintain the rotation, and the amount of this couple will be determined by equation (30).

\section{Mass rotating with Constant Angular Velocity}

25. Let us first consider the problem when $\omega$ is kept constant. To transform equations (29) we notice that

so that

$$
\frac{d x}{d t}=\Sigma \frac{\partial x}{\partial \theta_{s}} \frac{d \theta_{8}}{d t}
$$

We accordingly have

$$
\frac{\partial \dot{x}}{\partial \dot{\theta}_{s}}=\frac{\partial x}{\partial \theta_{8}} \text {. }
$$

$$
\frac{\partial U}{\partial \dot{\theta}_{s}}=\Sigma m\left(x \frac{\partial \dot{y}}{\partial \dot{\theta}_{s}}-y \frac{\partial \dot{x}}{\partial \dot{\theta}_{s}}\right)=\Sigma m\left(x \frac{\partial y}{\partial \dot{\theta}_{s}}-y \frac{\partial x}{\partial \theta_{s}}\right)
$$

so that

$$
\frac{d}{d t}\left(\frac{\partial U}{\partial \dot{\theta}_{s}}\right)=\Sigma m\left(\dot{x} \frac{\partial y}{\partial \theta_{s}}-\dot{y} \frac{\partial x}{\partial \theta_{s}}\right)+\Sigma m\left[x \frac{d}{d t}\left(\frac{\partial y}{\partial \theta_{s}}\right)-y \frac{d}{d t}\left(\frac{\partial x}{\partial \theta_{s}}\right)\right] .
$$

Also

$$
\frac{\partial U}{\partial \theta_{s}}=\Sigma m\left(\frac{\partial x}{\partial \theta_{s}} \dot{y}-\frac{\partial y}{\partial \theta_{s}} \dot{x}\right)+\Sigma m\left[x \frac{d}{d t}\left(\frac{\partial y}{\partial \theta_{s}}\right)-y \frac{d}{d t}\left(\frac{\partial x}{\partial \theta_{s}}\right)\right] .
$$

so that

$$
\begin{aligned}
\frac{d}{d t}\left(\frac{\partial U}{\partial \dot{\theta}_{s}}\right)-\frac{\partial U}{\partial \theta_{s}} & =2 \Sigma m\left(\dot{x} \frac{\partial y}{\partial \theta_{s}}-\dot{y} \frac{\partial x}{\partial \theta_{s}}\right) \\
& =2 \Sigma m\left[\Sigma\left(\frac{\partial x}{\partial \theta_{r}} \frac{\partial y}{\partial \theta_{s}}-\frac{\partial y}{\partial \theta_{r}} \frac{\partial x}{\partial \theta_{s}}\right) \dot{\theta}_{r}\right]
\end{aligned}
$$


Put

$$
\beta_{r 8}=2 \Sigma m\left(\frac{\partial x}{\partial \theta_{r}} \frac{\partial y}{\partial \theta_{8}}-\frac{\partial y}{\partial \theta_{v}} \frac{\partial x}{\partial \theta_{s}}\right)
$$

so that $\beta_{s r}=-\beta_{r s}$ and $\beta_{r r}=0$. Then

$$
\frac{d}{d t}\left(\frac{\partial U}{\partial \theta_{8}}\right)-\frac{\partial U}{\partial \theta_{8}}=\beta_{18} \dot{\theta}_{1}+\beta_{28} \dot{\theta}_{2}+\ldots
$$

Using the value of $T$ given by equation (24) and keeping $\omega$ constant, we have

$$
\frac{d}{d t}\left(\frac{\partial T}{\partial \dot{\theta}_{8}}\right)-\frac{\partial T}{\partial \theta_{8}}=\frac{d}{d t}\left(\frac{\partial T_{R}}{\partial \dot{\theta}_{8}}\right)-\frac{\partial T_{R}}{\partial \theta_{8}}+\omega\left[\frac{d}{d t}\left(\frac{\partial U}{\partial \dot{\theta}_{8}}\right)-\frac{\partial U}{\partial \theta_{8}}\right]-\frac{1}{2} \omega^{2} \frac{\partial I}{\partial \theta_{8}}
$$

so that the equation of motion (29) becomes, using (31),

$$
\frac{d}{d t}\left(\frac{\partial T_{R}}{\partial \dot{\theta}_{8}}\right)-\frac{\partial T_{R}}{\partial \theta_{s}}+\omega\left(\beta_{18} \dot{\theta}_{1}+\beta_{28} \dot{\theta}_{2}+\ldots\right)=-\frac{\partial}{\partial \theta_{s}}\left(W-\frac{1}{2} \omega^{2} I\right)+F_{8} \ldots(32)
$$

Thus the equations of motion relative to rotating axes differ from the simpler equations appropriate to the case of $\omega=0$ in two respects; first by the presence of what we may call "gyroscopic" terms such as $\beta_{18} \omega \dot{\theta}_{1}$, and second, that $W-\frac{1}{2} \omega^{2} I$ replaces the potential energy $W$ of the simpler equations.

26. The conditions for equilibrium relative to the moving axes are

$$
\dot{\theta}_{1}=\dot{\theta}_{2}=\ldots=0
$$

and so are determined by the equations

$$
\frac{\partial}{\partial \theta_{8}}\left(W^{\prime \prime}-\frac{1}{2} \omega^{2} I\right)=F_{8} \text { etc. }
$$

reducing when there are no externally applied forces, to

$$
\frac{\partial}{\partial \theta_{8}}\left(W-\frac{1}{2} \omega^{2} I\right)=0
$$

The difference between these equations and the simpler ones for a system at rest is merely that $W$ has become replaced by $W-\frac{1}{2} \omega^{2} I$. The configurations of relative equilibrium may accordingly be found just as though the system were at rest under a potential $W-\frac{1}{2} \omega^{2} I$, and these configurations will fall into linear series as before.

27. To discuss the small oscillations of such a system, let us return to the equations of motion (30), and suppose we are considering the oscillations of a configuration which is one of equilibrium under no applied forces, say

$$
\theta_{1}=\Theta_{1} \text {, etc. }
$$

Let the coordinates be replaced by $\theta_{1}-\Theta_{1}$, etc. so that the new values of $\theta_{1}, \theta_{2}, \ldots$ all vanish in the configuration of equilibrium. The values of 
$W-\frac{1}{2} \omega^{2} I$ and of $T_{R}$ for any small displacement may now be expressed in the forms

$$
\begin{aligned}
2 T_{R} & =a_{11} \dot{\theta}_{1}^{2}+2 a_{12} \dot{\theta}_{1} \dot{\theta}_{2}+\ldots \\
2\left(W-\frac{1}{2} \omega^{2} I\right) & =b_{11} \theta_{1}^{2}+2 b_{12} \theta_{1} \theta_{2}+\ldots
\end{aligned}
$$

the condition that equations (32) shall be, satisfied in the configuration of equilibrium requiring the omission of terms of first degree in $\theta_{1}, \theta_{2}, \ldots$ By a linear transformation, $T_{R}$ and $W-\frac{1}{2} \omega^{2} I$ may be simultaneously reduced further to a sum of squares, so that we may assume the still simpler forms

$$
\begin{aligned}
2 T_{R} & =a_{1} \dot{\theta}_{1}{ }^{2}+a_{2} \dot{\theta}_{2}{ }^{2}+\ldots \ldots \ldots \ldots \ldots \ldots \ldots . .(35), \\
2\left(W-\frac{1}{2} \omega^{2} I\right) & =b_{1} \theta_{1}{ }^{2}+b_{2} \theta_{2}{ }^{2}+\ldots \ldots \ldots \ldots \ldots \ldots \ldots \ldots(36) .
\end{aligned}
$$

The equations of motion (32) now reduce to

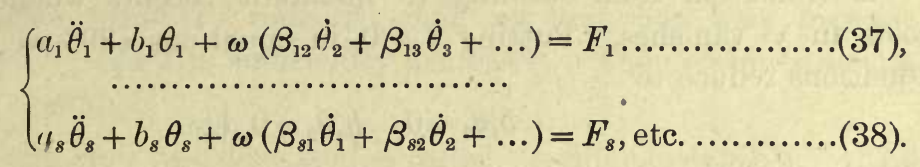

Had the system been at rest, these equations would have reduced to

$$
a_{8} \ddot{\theta}_{8}+b_{8} \theta_{8}=F_{8} \text {, etc. }
$$

and all the properties of "principal coordinates "would have been immediately deducible. But a glance at equations (37) and (38) will shew that these properties no longer persist when the system is in rotation. A disturbance in which $\theta_{1}$ exists alone at first will soon set up oscillations in which $\theta_{2}, \theta_{3} \ldots$ have finite values, and the coordinates $\theta_{1}, \theta_{2}, \ldots$ no longer correspond to independent vibrations.

Since equations (37) and (38) are linear with constant coefficients, it is clear that there will be a system of separate free vibrations. These may be found by putting $F_{1}=F_{2}=\ldots=0$, and assuming $\theta_{1}, \theta_{2}, \ldots$ each proportional to the same time-factor $e^{\lambda t}$. The equations reduce to

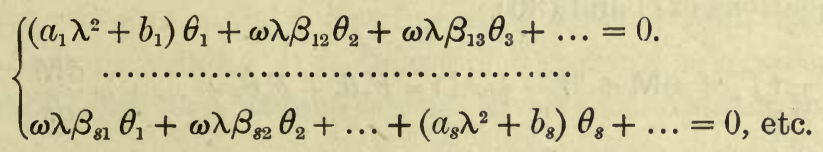

Eliminating the $\theta$ 's, we find as an equation for $\lambda$,

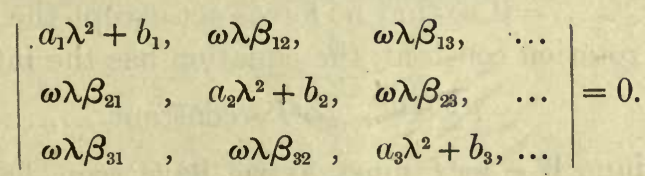

Since $\beta_{r s}=-\beta_{8 r}$, it appears that this equation is unchanged when the sign of $\lambda$ is changed. Thus the equation is an equation in $\lambda^{2}$, just as when the system is at rest. But the roots in $\lambda^{2}$ are no longer all real as they are 
for a system at rest; they will occur in pairs of the form $\lambda^{2}=\rho \pm i \sigma$, and these will, lead to roots for $\lambda$ of the form

$$
\lambda= \pm q \pm i p,
$$

so that the complete time factor for an oscillation is found to be of the form

$$
A e^{q t} \cos (p t-\epsilon)+B e^{-q t} \cos (p t-\eta) \text {. }
$$

If $q$ is different from zero for any vibration, the amplitude of this vibration will continually increase owing to the presence of the factors $e^{ \pm q t}$, and the system will be unstable. Thus the condition for stability is that $q$ shall be zero for every vibration, and this in turn requires that all the roots in $\lambda^{2}$ shall be real and negative-a condition which is the same in form as that for the stability of a non-rotating system.

A transition from stability to instability occurs whenever one of the roots in $\lambda^{2}$ vanishes. Putting $\lambda=0$ in equations (39) we find that these equations reduce to

$$
b_{1} \theta_{1}=0, \quad b_{2} \theta_{2}=0 \text {, etc. }
$$

and the condition for a change from stability to instability is seen to be that one of the coefficients $b_{1}, b_{2}, \ldots$ shall vanish. These coefficients are seen to be precisely the Poincaré "coefficients of stability" calculated for a system of potential energy $W-\frac{1}{2} \omega^{2} I$.

28. Multiply equations (32) by $\dot{\theta}_{1}, \dot{\theta}_{2} \ldots$ and add corresponding sides. We obtain

$$
\frac{d}{d t}\left(T_{R}+W-\frac{1}{2} \omega^{2} I\right)=F_{1} \dot{\theta}_{1}+F_{2} \dot{\theta}_{2}+\ldots
$$

The same result is readily obtained from the equations of energy and angular momentum. The equation of energy is

$$
\frac{d}{d t}(T+W)=F_{1} \dot{\theta}_{1}+F_{2} \dot{\theta}_{2}+\ldots+\omega G
$$

or, using equations (27) and (30),

$$
\frac{d}{d t}\left(T_{R}+\omega \mathrm{M}+W-\frac{1}{2} \omega^{2} I\right)=F_{1} \dot{\theta}_{1}+F_{2} \dot{\theta}_{2}+\ldots+\omega \frac{d \mathrm{M}}{d t}
$$

which is identical with equation (40).

When $F_{1}=F_{2}=\ldots=0$, so that no forces act except the couple $G$ necessary to maintain the rotation constant, the equation has the integral

$$
T_{R}+W-\frac{1}{2} \omega^{2} I=\text { constant }
$$

For equilibrium, $W-\frac{1}{2} \omega^{2} I$ must, as we have seen, be stationary. Consider first what kind of equilibrium obtains when $W-\frac{1}{2} \omega^{2} I$ is an absolute minimum. When any small displacement of the system occurs, $W-\frac{1}{2} \omega^{2} I$ is necessarily increased, so that the constant value of $T_{R}+W-\frac{1}{2} \omega^{2} I$ is greater 
than its value when at rest in the equilibrium configuration by a small constant amount $c$. Thus throughout the subsequent motion $T_{R}$ can never increase beyond the value $c$, so that the motion is absolutely stable. This argument cannot however be reversed to shew that the system is necessarily unstable if $W-\frac{1}{2} \omega^{2} I$ is not an absolute minimum.

Let us examine what happens when the relative motion of the system is affected by dissipative forces, such as viscosity. The right hand of equation (40) will be negative except when the system is relatively at rest, so that $T_{R}+W-\frac{1}{2} \omega^{2} I$ will decrease indefinitely. If $W-\frac{1}{2} \omega^{2} I$ was an absolute minimum in the position of equilibrium, this condition can only be satisfied by $T_{R}$ being reduced to zero, and the system coming to rest in its position of equilibrium. But if $W-\frac{1}{2} \omega^{2} I$ was not an absolute minimum in the configuration of equilibrium, there will be a possible motion in which $W-\frac{1}{2} \omega^{2} I$ continually decreases while $T_{R}$ remains small at first, but may increase beyond limit when $W-\frac{1}{2} \omega^{2} I$ is sufficiently decreased. The system is now in a restricted sense unstable.

Instability of the kind just discussed is called "secular instability." The conception of "secular instability" was first introduced by Thomson and Tait*. It has reference only to rotating systems or systems in a state of steady motion; for systems at rest secular stability become identical with ordinary stability. It is clear that a system which is ordinarily stable may or may not be secularly stable, but a system which is ordinarily unstable is necessarily secularly unstable.

\section{Mass rotating freely in space}

29. As Schwarzschild $\dagger$ has shewn, the conditions of secular stability assume a somewhat different form for a mass rotating freely in space. Here the rate of rotation is not constant but varies with the moment of inertia of the mass; if we refer the motion to axes rotating with a uniform velocity the rotation of the freely rotating mass may lag behind that of the axes and the relative coordinates $x, y, z$ may increase without limit although the configuration remains stable. It is therefore important to express the conditions of stability in a form which does not involve the constancy of $\omega$.

When the mass is rotating freely in space, $G=0$ so that (equation (30)) $M$ is constant. The elimination of $\omega$ from equations (25) and (26) leads to

$$
T=T_{8}+\frac{\mathbf{M}^{2}}{2 I}
$$

where

$$
T_{8}=T_{R}-\frac{U^{2}}{2 I}
$$

* Nat. Phil. 2nd Ed. rr. p. 391.

+ See Schwarzschild; "Die Poincaré'sche Theorie des Gleichgewichts." Neue Annalen. d. Sternwarte München, 3 (1897), p. 275, or Inaugural Dissertation. München (1896). 
Using the values of $T_{R}, U$ and $I$ given by equations (22) to (24),

$$
\begin{aligned}
& 2 I T_{8}=\left[\Sigma m_{1}\left(x_{1}{ }^{2}+y_{1}{ }^{2}\right)\right]\left[\Sigma m_{2}\left(\dot{x}_{2}{ }^{2}+\dot{y}_{2}{ }^{2}+\dot{z}_{2}{ }^{2}\right)\right] \\
& -\left[\sum m_{1}\left(x_{1} \dot{y}_{1}-y_{1} \dot{x}_{1}\right)\right]\left[\Sigma m_{2}\left(x_{2} \dot{y}_{2}-y_{2} \dot{x}_{2}\right)\right] \\
& =\sum \sum m_{1} m_{2}\left[\left(x_{1} \dot{x}_{2}+y_{2} \dot{y}_{1}\right)^{2}+\left(x_{2} \dot{x}_{1}+y_{1} \dot{y}_{2}\right)^{2}\right. \\
& \left.+\left(x_{1} \dot{y}_{2}-x_{2} \dot{y}_{1}\right)^{2}+\left(x_{2} \dot{y}_{1}-x_{1} \dot{y}_{2}\right)^{2}+\dot{z}_{1}{ }^{2}\left(x_{2}{ }^{2}+y_{2}{ }^{2}\right)-\dot{z}_{2}{ }^{2}\left(x_{1}{ }^{2}+y_{1}{ }^{2}\right)\right] \text {. }
\end{aligned}
$$

This expression, being a sum of squares, is always positive. Thus, since $I$ is necessarily positive and independent of $\dot{x}, \dot{y}, \dot{z}$, it appears that $T_{8}$ is always positive and is quadratic in $\dot{x}, \dot{y}, \dot{z}$.

The equation of energy, $T+W=$ cons. now assumes the form

$$
T_{8}+W+\frac{\mathbf{M}^{2}}{2 I}=\text { cons. }
$$

This is of the same form as equation (42), $T_{8}$ replacing $T_{R}$ and $W+\mathrm{M}^{2} / 2 I$ replacing $W-\frac{1}{2} \omega^{2} I$. By the argument already used in $\S 28$, it now appears that configurations for which

$$
W+\frac{M^{2}}{2 I}
$$

is an absolute minimum ( $\mathbf{M}$ being kept constant) will be thoroughly stable, while configurations for which this expression is not an absolute minimum will be secularly unstable, and may or may not be ordinarily unstable.

30. As we pass along a linear series of configurations of equilibrium of a rotating system, starting from a part of the series which is known to be stable, the configurations will become secularly unstable as soon as

or

$$
\begin{gathered}
W-\frac{1}{2} \omega^{2} I \quad(\omega=\text { constant }) \\
W+\frac{1}{2} \mathbf{M}^{2} / I(\mathbf{M}=\text { constant })
\end{gathered}
$$

ceases to be an absolute minimum, the former expression referring to a problem in which the mass is compelled by external forces to rotate at a constant rate $\omega$, while the latter refers to a problem in which the mass is rotating freely in space.

It is now clear that the theory of linear series and stability developed in $\$ \$ 18-20$ will be exactly applicable to the problem of the secular stability of a rotating mass, $W$ being replaced in the argument of those sections by the appropriate one of expressions (45) or (46). Secular stability is lost at a "turning point" or "point of bifurcation." At a turning point stability is lost entirely; at a point of bifurcation it may be lost or may be transferred to the branch series through the point according as the branch series turns downwards or upwards in the appropriate diagram. 


\section{Various forms for the Equations of Equilibrium}

31. The preceding theory has reduced the problem of determining a sequence of stable configurations to the simpler problem of mapping out all configurations of equilibrium. For this latter problem the conditions of equilibrium may be expressed in whatever form is found to be most convenient.

We have already seen that possible forms are

$$
\begin{aligned}
& \delta\left(W-\frac{1}{2} \omega^{2} I\right)=0(\omega=\text { constant }) \\
& \delta\left(W+\frac{1}{2} \mathbf{M}^{2} / I\right)=0(\mathbf{M}=\text { constant })
\end{aligned}
$$

Another form is contained in the ordinary hydrostatic equations of equilibrium

$$
\frac{\partial p}{\partial x}=\rho \frac{\partial V}{\partial x}+\omega^{2} \rho x, \text { etc. }
$$

in which $V$ is the gravitational potential and $p, \rho$ denote the pressure and density respectively.

For a mass of uniform density $\rho$, equations (4.9) have the common integral

$$
\frac{p}{\rho}=V+\frac{1}{2} \omega^{2}\left(x^{2}+y^{2}\right)+\text { cons. }
$$

and so the equations reduce to the single condition that

$$
V+\frac{1}{2} \omega^{2}\left(x^{2}+y^{2}\right)=\text { cons. }
$$

over the boundary of the fluid.

32. In the classical treatment of the rotational problem by Poincaré * and Darwin $\dagger$, the equations of equilibrium are introduced in the form (48); while Liapounoff + treats the same problem by means of equation (50). The method of treatment of the present book finds it convenient to use equation (50) for the incompressible mass, and equation (49) for the compressible mass, this latter case not being discussed at all by Poincaré, Darwin and Liapounoff.

Thus, so far as the treatment of the problems in the present essay is concerned, it was unnecessary to introduce equations of the type (48) for the discussion of figures of equilibrium, but the theory of secular stability could

* Acta Math. l.c. ante, also "Sur la Stabilité de l'Équilibre des Figures Pyriformes affectées par une Masse Fluide en Rotation," Phil. Trans. 198 A (1901), p. 333.

+ "On the Pear-shaped Figure of Equilibrium of a Rotating Mass of Liquid," Phil. Trans. 198 A (1901), p. 301, and subsequent papers. These will be found in Vol. III of Sir George Darwin's Collected Works.

¥ "Sur un Problème de Tchebychef," Mémoires de l'Academie de St Pétersbourg, xvii. 3 (1905), and other papers published by the Academy. 
hardly have been satisfactorily discussed without the help of such equations. Now that this theory has been established we can discard equations (48). We have found that stability can be lost at a turning point or a point of bifurcation on a linear series. The characteristic feature of a turning point is that the variable parameter attains a stationary value at such a point; the characteristic feature of a point of bifurcation is that corresponding to a single value of the parameter, there shall not only be a single configuration of equilibrium, say $\Theta_{1}, \Theta_{2}, \ldots$ but also a small range of configurations of the form

$$
\Theta_{1}+e_{1}, \Theta_{2}, \Theta_{3}, \ldots
$$

for all values of $e$ so small that $e^{2}$ may be neglected. 


\section{CHAPTER III}

\section{ELLIPSOIDAL CONFIGURATIONS OF EQUILIBRIUM}

33. The best-known configurations of equilibrium of a rotating homogeneous mass, namely Maclaurin's spheroids and Jacobi's ellipsoids, are both of the ellipsoidal form, and this form will prove to be of primary importance in all the cosmogonical problems we shall attempt to solve. We accordingly devote a chapter to the subject of ellipsoidal configurations.

Looked at merely from the point of view of convenience in the development of the subject, the ellipsoidal form has the advantage that the potential of an ellipsoidal mass is known and is comparatively simple, and that the ellipsoidal configurations provide admirably clear examples of Poincarés theory of linear series and stability. These reasons alone might justify our studying ellipsoidal configurations in some detail, but there are weightier reasons, as we shall soon see.

Throughout this chapter and the three succeeding chapters the matter under discussion will be supposed homogeneous and incompressible; the more complicated problems presented by non-homogeneous and compressible masses will be attacked in Chapter VII.

We shall deal in turn with three distinct problems-the first, that of a mass of liquid rotating freely under its own gravitational forces; the second, that of a mass devoid of rotation but acted on tidally by another mass; the third that of two masses rotating round one another and acting tidally on one another. The first problem is of course of interest in connection with the rotational theory of planetary evolution; the second is of interest in connection with the tidal theory; while the third is of interest as linking up the two former problems, and also in connection with some double-star problems. In every one of these problems, we shall find ultimately that the only stable configurations are of the ellipsoidal form, or are ellipsoids slightly distorted by tidal inequalities.

34. Notation. When one ellipsoid only is concerned, we shall take $a, b, c$ to be its semi-axes, so that the equation of its boundary will be

$$
\frac{x^{2}}{a^{2}}+\frac{y^{2}}{b^{2}}+\frac{z^{2}}{c^{2}}=1
$$

As in many ellipsoidal problems, it will be convenient to think of the surface (51) as being the surface $\lambda=0$ in the family of confocal ellipsoids

$$
\frac{x^{2}}{a^{2}+\lambda}+\frac{y^{2}}{b^{2}+\lambda}+\frac{z^{2}}{c^{2}+\lambda}=1
$$


We shall write for brevity

$$
\left.\begin{array}{r}
a^{2}+\lambda=A, \quad b^{2}+\lambda=B, \quad c^{2}+\lambda=C \\
{\left[\left(a^{2}+\lambda\right)\left(b^{2}+\lambda\right)\left(c^{2}+\lambda\right)\right]^{\frac{1}{2}}=(A B C)^{\frac{1}{2}}=\Delta}
\end{array}\right\}
$$

We shall take $a b c=r_{0}{ }^{3}$. The matter of which the ellipsoid is formed will be supposed to be homogeneous and of density $\rho$, so that the mass $M$ will be given by

$$
M=\frac{4}{3} \pi \rho a b c=\frac{4}{3} \pi \rho r_{0}^{3} .
$$

The potential $V_{0}$ of this mass at any external point $x, y, z$, is, by a wellknown formula,

$$
V_{0}=-\pi \rho a b c \int_{\lambda}^{\infty}\left(\frac{x^{2}}{A}+\frac{y^{2}}{B}+\frac{z^{2}}{C}-1\right) \frac{d \lambda}{\Delta}
$$

in which the lower limit of integration $\lambda$ is the root of equation (52), and so is the parameter of the confocal ellipsoid on which the point $x, y, z$ lies.

The potential $V_{i}$ of the mass at an internal point $x, y, z$, is

$$
V_{i}=-\pi \rho a b c \int_{0}^{\infty}\left(\frac{x^{2}}{A}+\frac{y^{2}}{B}+\frac{z^{2}}{C}-1\right) \frac{d \lambda}{\Delta}
$$

and so is a quadratic function of $x, y, z$.

35. To simplify the printing of integrals of the type just written down, we shall introduce an abbreviated notation. Let us write

and put further

$$
\int_{0}^{\infty} \frac{d \lambda}{\Delta}=J
$$

$$
\int_{0}^{\infty} \frac{d \lambda}{A^{m} B^{n} C^{p} \Delta}=J_{A^{m} B^{n} C^{p}}
$$

so that, for instance, equation (55) assumes the form

$$
V_{i}=-\pi \rho a b c\left(x^{2} J_{A}+y^{2} J_{B}+z^{2} J_{C}-J\right)
$$

It is easily verified that

$$
J_{A}+J_{B}+J_{C}=\frac{2}{a b c}
$$

or this can be seen from the circumstance that $\nabla^{2} V_{i}$ must be equal to $-4 \pi \rho$. We may also note the formulae

$$
\begin{aligned}
& J_{B}-J_{A}=\left(a^{2}-b^{2}\right) J_{A B} \\
& J_{A^{m} B^{n+1} C^{p}}-J_{A^{m+1} B^{n} C^{p}}=\left(a^{2}-b^{2}\right) J_{A^{m+1} B^{n+1} C^{p}} \\
& J_{A^{n} B}+J_{A^{n} C}+(2 n+1) J_{A^{n+1}}=\frac{2}{a^{2 n} \cdot a b c}
\end{aligned}
$$

all of which are easily verified by algebraic transformations.

With these preliminaries, we proceed to the three problems already specified. 


\section{FREELY ROTATING ELLIPSOIDS}

36. The necessary and sufficient condition that the standard ellipsoid (51) shall be a figure of equilibrium for a homogeneous mass of density $\rho$ freely rotating with angular velocity $\omega$ is that

$$
V_{i}+\frac{1}{2} \omega^{2}\left(x^{2}+y^{2}\right)
$$

shall be constant over the boundary, $V_{i}$ being given by equation (57). Consider the function

$$
V_{i}+\frac{1}{2} \omega^{2}\left(x^{2}+y^{2}\right)+\theta \pi \rho a b c\left(\frac{x^{2}}{a^{2}}+\frac{y^{2}}{b^{2}}+\frac{z^{2}}{c^{2}}-1\right)
$$

where $\theta$ is a constant, as yet undetermined. Operating with $\nabla^{2}$, we find that this function will be a spherical harmonic, if

$$
-4 \pi \rho+2 \omega^{2}+2 \theta \pi \rho a b c\left(\frac{1}{a^{2}}+\frac{1}{b^{2}}+\frac{1}{c^{2}}\right)=0
$$

and this can be satisfied by assigning to $\theta$ the value

$$
\theta=\frac{2\left(1-\frac{\omega^{2}}{2 \pi \rho}\right)}{a b c\left(\frac{1}{a^{2}}+\frac{1}{b^{2}}+\frac{1}{c^{2}}\right)}
$$

Giving this value to $\theta$, expression (63) becomes harmonic. The necessary and sufficient condition that the standard ellipsoid (51) shall be a figure of equilibrium is that this function shall have a constant value over the boundary. The function being harmonic, this is equivalent to the condition that the function shall have a constant value throughout the interior of the ellipsoid. We must accordingly have

$$
\begin{aligned}
-\pi \rho a b c\left(J_{A} x^{2}+J_{B} y^{2}+J_{C} z^{2}-J\right) & +\frac{1}{2} \omega^{2}\left(x^{2}+y^{2}\right) \\
& +\theta \pi \rho a b c\left(\frac{x^{2}}{a^{2}}+\frac{y^{2}}{b^{2}}+\frac{z^{2}}{c^{2}}-1\right)=\text { cons. }
\end{aligned}
$$

where $\theta$ is given by equation (64). Equating coefficients of $x^{2}, y^{2}$ and $z^{2}$, this equation is seen to be equivalent to the three separate equations

$$
\begin{aligned}
J_{A}-\frac{\omega^{2}}{2 \pi \rho a b c} & =\frac{\theta}{a^{2}} \\
J_{B}-\frac{\omega^{2}}{2 \pi \rho a b c} & =\frac{\theta}{b^{2}} \\
J_{C} & =\frac{\theta}{c^{2}}
\end{aligned}
$$

By addition of corresponding sides we again obtain equation (64) which gives the value of $\theta$. Thus the three equations $(65)-(67)$ contain within 
themselves the necessary and sufficient condition that the standard ellipsoid (51) shall be a figure of equilibrium under a rotation $\omega$.

37. On subtracting corresponding sides of equations (65) and (66) we obtain

$$
\left(a^{2}-b^{2}\right) J_{A B}=\left(a^{2}-b^{2}\right) \frac{\theta}{a^{2} b^{2}},
$$

and the elimination of $\theta$ between this and equation (67) leads to

$$
\left(a^{2}-b^{2}\right)\left[a^{2} b^{2} J_{A B}-c^{2} J_{C}\right]=0
$$

It accordingly appears that equations (65) to $(67)$ can be satisfied in two ways ; first by taking

and second by taking

$$
a^{2}=b^{2}
$$

$$
a^{2} b^{2} J_{A B}=c^{2} J_{C}
$$

\section{Maclaurin's Spheroids}

38. Let us examine the former alternative first. When $a=b$, the series. of ellipsoids become a series of spheroids which include the sphere $a=b=c$ for which $\omega^{2}=0$.

Equation (65) now becomes identical with (66). The elimination of $\theta$ between this equation and equation (67) gives

or

$$
\begin{aligned}
& a^{2} J_{A}-c^{2} J_{C}=\frac{\omega^{2} a^{2}}{2 \pi \rho a b c} \\
& \frac{\omega^{2}}{2 \pi \rho a b c}=\frac{a^{2}-c^{2}}{a^{2}} \int_{0}^{\infty} \frac{\lambda d \lambda}{\Delta A C}
\end{aligned}
$$

Since $\omega^{2}$ must be positive, it appears that $a^{2}$ must be greater than $c^{2}$; the spheroids are all oblate. On evaluating the integral in equation (71), the value of $\omega^{2}$ is found to be given by

$$
\frac{\omega^{2}}{2 \pi \rho}=\frac{3-2 c^{2}}{e^{3}}\left(1-e^{2}\right)^{\frac{1}{2}} \sin ^{-1} e-3\left(\frac{1}{e^{2}}-1\right)
$$

where $e$ is the eccentricity, defined by $e^{2}=\left(a^{2}-c^{2}\right) / a^{2}$.

Thus the eccentricity of the spheroid depends only on the ratio of $\omega^{2}$ to $\rho$, as it is apparent from a consideration of physical dimensions that it must. The following table of corresponding values of $\omega^{2} / \rho$ and $e$ is given by Lamb*, being compiled from values calculated by Thomson and Tait $\dagger$ :

\footnotetext{
* Hydrodynamics (4th Ed.), p. 673. I have inserted into this table, Darwin's values for $e=\cdot 81267$, the point of bifurcation.

+ Nat. Phil. $\$ 772$.
} 


\begin{tabular}{|c|c|c|c|c|}
\hline$e$ & $a / r_{0}$ & $c / r_{0}$ & $\frac{\omega^{2}}{2 \pi \rho}$ & Ang. Momentum $/ M^{\frac{3}{2}} r^{\frac{1}{2}}$ \\
\hline $\begin{array}{c}0 \\
\cdot 1 \\
\cdot 2 \\
\cdot 3 \\
.4 \\
\cdot 5 \\
.6 \\
\cdot 7 \\
.8 \\
.81267 \\
\cdot 9 \\
.91 \\
.92 \\
.93 \\
.94 \\
.95 \\
.96 \\
.97 \\
.98 \\
.99 \\
1.00\end{array}$ & $\begin{array}{c}1 \cdot 0000 \\
1 \cdot 0016 \\
1 \cdot 0068 \\
1 \cdot 0159 \\
1 \cdot 0295 \\
1 \cdot 0491 \\
1 \cdot 0772 \\
1 \cdot 1188 \\
1 \cdot 1856 \\
1 \cdot 1972 \\
1 \cdot 3189 \\
1 \cdot 341 \\
1 \cdot 367 \\
1 \cdot 396 \\
1 \cdot 431 \\
1 \cdot 474 \\
1 \cdot 529 \\
1 \cdot 602 \\
1 \cdot 713 \\
1 \cdot 921 \\
\infty\end{array}$ & $\begin{array}{c}1 \cdot 0000 \\
\cdot 9967 \\
\cdot 9865 \\
\cdot 9691 \\
\cdot 9435 \\
\cdot 9068 \\
\cdot 8618 \\
\cdot 7990 \\
\cdot 7114 \\
.6977 \\
\cdot 5749 \\
\cdot 5560 \\
\cdot 5355 \\
.5131 \\
\cdot 4883 \\
\cdot 4603 \\
\cdot 4280 \\
\cdot 3895 \\
\cdot 3409 \\
\cdot 2710 \\
0\end{array}$ & $\begin{array}{c}0 \\
\cdot 0027 \\
\cdot 0107 \\
\cdot 0243 \\
\cdot 0436 \\
\cdot 0690 \\
\cdot 1007 \\
\cdot 1387 \\
\cdot 1816 \\
\cdot 18712 \\
\cdot 2203 \\
\cdot 2225 \\
\cdot 2241 \\
\cdot 2247 \\
\cdot 2239 \\
\cdot 2213 \\
\cdot 2160 \\
\cdot 2063 \\
\cdot 1890 \\
\cdot 1551 \\
0\end{array}$ & $\begin{array}{c}0 \\
\cdot 0255 \\
\cdot 0514 \\
\cdot 0787 \\
\cdot 1085 \\
\cdot 1417 \\
\cdot 1804 \\
\cdot 2283 \\
\cdot 2934 \\
.30375 \\
\cdot 4000 \\
\cdot 4156 \\
\cdot 4330 \\
\cdot 4525 \\
\cdot 4748 \\
\cdot 5008 \\
\cdot 5319 \\
.5692 \\
.6249 \\
\cdot 7121 \\
\infty\end{array}$ \\
\hline
\end{tabular}

\section{Jacobi's Ellipsoids}

39. Let us now examine the second alternative, represented by equation (70) in $\S 37$. For these configurations $a$ is no longer equal to $b$, so that the integrals do not admit of integration in finite terms. They have been discussed by C. O. Meyer*, and also reduced to elliptic integrals and treated numerically by Darwin $†$.

It is found that the ellipsoids form one single continuous series; they are generally known as Jacobian ellipsoids, their existence having been first demonstrated by Jacobi in $1834_{+}^{+}$. The maximum value of $\omega^{2} / 2 \pi \rho$ is found to occur for the particular ellipsoid for which $a=b$; this value is 18712 , and the ellipsoid for which it occurs is one in which $a=b=1 \cdot 7161 \mathrm{c}$. This configuration is also of course a Maclaurin spheroid, and so forms a point of bifurcation on this latter series. It is the configuration printed in heavy type in the table above.

As we pass along the Jacobian series, the ratio $a / b$ may be supposed to vary continuously from 0 to $\infty$, and the point of bifurcation occurs when $a=b$. The two halves of the series are however exactly similar, either one changing into the other on interchanging $a$ and $b$, so that we may legitimately confine our attention to one half, say that for which $a>b$. We now

* Crelle's Journ. 34 (1842).

+ Proc. Roy. Soc. 41 (1887), p. 319 or Coll. Works, Irr. p. 118.

$\ddagger$ Pogg. Ann. 33 (1834), p. 229. 
regard the series of Jacobian ellipsoids as starting at the value $a=b$ (the point of bifurcation), and the ratio $a / b$ continually increases from 1 to $\infty$ as we pass along the series. The following numerical values are given by Darwin*.

\begin{tabular}{|c|c|c|c|c|}
\hline$\frac{a}{r_{0}}$ & $\frac{b}{r_{0}}$ & $\frac{c}{r_{0}}$ & $\frac{\omega^{2}}{2 \pi \rho}$ & $\begin{array}{l}\text { Angular } \\
\text { Momentum }\end{array}$ \\
\hline $1 \cdot 1972$ & $1 \cdot 1972$ & $\cdot 6977$ & •18712 & $\cdot 30375$ \\
\hline $1 \cdot 216$ & $1 \cdot 179$ & 697 & $\cdot 1870$ & $\cdot 304$ \\
\hline $1 \cdot 279$ & $1 \cdot 123$ & $\cdot 696$ & $\cdot 186$ & $\cdot 306$ \\
\hline $1 \cdot 3831$ & $1 \cdot 0454$ & 6916 & $\cdot 1812$ & $\cdot 3134$ \\
\hline $1 \cdot 6007$ & .9235 & $\cdot 6765$ & $\cdot 1659$ & $\cdot 3407$ \\
\hline $1 \cdot 88583$ & •81498 & $\cdot 65066$ & $\cdot 14200$ & $\cdot 3898$ \\
\hline $1 \cdot 899$ & .8111 & $\cdot 6494$ & $\cdot 1409$ & $\cdot 3920$ \\
\hline $2 \cdot 346$ & $\cdot 7019$ & $\cdot 6072$ & $\cdot 1072$ & $\cdot 4809$ \\
\hline $3 \cdot 1294$ & $\cdot 5881$ & 5434 & $\cdot 0661$ & $\cdot 6387$ \\
\hline $5 \cdot 0406$ & $\cdot 4516$ & $\cdot 4393$ & $\cdot 0259$ & $1 \cdot 0087$ \\
\hline$\infty$ & 0 & 0 & 0 & $\infty$ \\
\hline
\end{tabular}

40. We have found that there are two linear series of ellipsoidal configurations-the Maclaurin spheroids and the Jacobian ellipsoids. The stability of these figures can now be investigated by the methods already explained.

\section{Stability when angular velocity is increased, as in Plateau's experiments}

41. In 1842 Plateal devised an experiment in which he attempted to observe directly the sequence of configurations in a rotating mass of fluid with a view to testing whether they were at all similar to those assumed by Laplace as the basis of his nebular hypothesis. Plateau mixed water and alcohol until they were of just the right density to float freely in olive oil. A globule of this mixture was then set in rotation in the oil by spinning a wire through its centre, the globule being kept in position on the wire by a disc round which it clustered. As the speed of rotation increased the globule was observed to flatten itself more and more until finally a dimple formed at the centre, and the globule detached itself from the disc in the form of a perfect ring. The conditions of this experiment were very different from those contemplated in the nebular hypothesis, for the globule was not held together by its own gravitational attractions, but by surface tensions and was not made to shrink while moving freely in space, but had its angular velocity mechanically increased by the medium of the wire and disc. 
42. As a problem suggested by Plateau's experiments, let us examine what would be the sequence of configurations if a mass of gravitating matter had its angular velocity continually increased by some mechanical means such as the spinning at an ever increasing rate of a pole through its centre.

The configurations of equilibrium are those already discussed; so long as the mass is constrained to remain ellipsoidal, they consist of Maclaurin spheroids and Jacobian ellipsoids. To examine the stability of these figures we draw a diagram in which the angular velocity is the vertical coordinate (see fig. 5).

We find at once that the Maclaurin spheroids remain stable until the rotation is given by $\omega^{2} / 2 \pi \rho=\cdot 18712$. At this stage a point of bifurcation occurs, the branch series being the Jacobian ellipsoids: The Maclaurin spheroids accordingly lose their stability, and since the Jacobian ellipsoids turn downwards from the point of bifurcation, these also are unstable. Thus there are no stable configurations of equili-

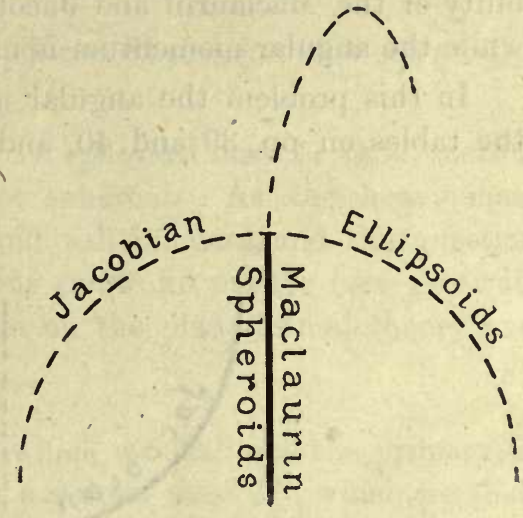

Fig. 5.

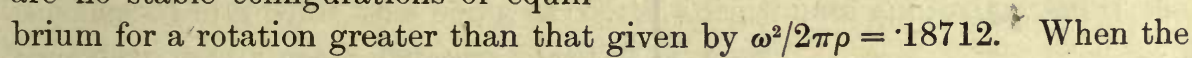
rotation exceeds this amount, the problem ceases to be a statical one and becomes a dynamical one; here we shall not attempt to follow it.

43. Suppose, as an alternative problem, that the mass had been constrained to remain a figure of revolution. The Jacobian series of figures would then have no existence, and the point defined by $\omega^{2} / 2 \pi \rho=\cdot 18712$ on the Maclaurin series would have no physical significance except as being the point at which the newly imposed constraint first came into play. The Maclaurin spheroids now remain stable up to the point defined by $\omega^{2} / 2 \pi \rho=\cdot 225$. This is the maximum value which $\omega$ can have for a spheroidal configuration, and when $\omega$ exceeds this value there are no possible configurations of equilibrium at all subject to the constraints which we have supposed to be imposed. Again the problem becomes a dynamical one, and again we shall not attempt to trace out this part of the motion.

\section{Stability when the angular momentum is increased}

44. The problems just considered are of interest as illustrating the theory of points of bifurcation, but fail entirely to represent the conditions postulated in the rotational theory of planetary evolution. To represent these conditions 
the mass must be supposed to rotate freely in space so that its angular mo mentum remains constant. As it shrinks, its density will continually increase and this may or may not result in an increase of angular velocity. To study the problem by the most direct method, we should have to look for series 0 configurations of constant angular momentum and varying density. It is how ever a convenience to suppose that the density remains constant while the angular momentum increases, and it is easily seen that this leads to exactly the same mathematical problem. We accordingly proceed to study the sta bility of the Maclaurin and Jacobian series, supposing $\rho$ to remain constan while the angular momentum is made continually to increase.

In this problem the angular momentum is given in the last columns 0 the tables on pp. 39 and 40 , and in a diagram in which the angular mo

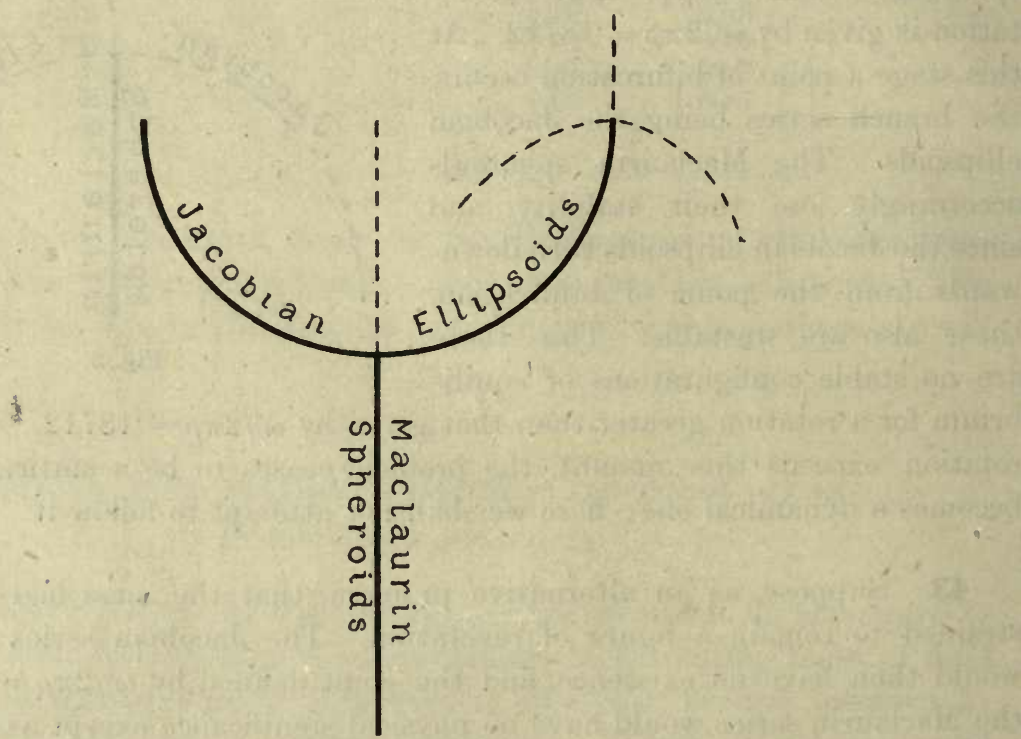

Fig. 6.

mentum is taken for ordinate, the series will be found to be as in fig. 6 . Clearl the Maclaurin spheroids will be stable up to the point at which they meet th Jacobian ellipsoids. At this point of bifurcation they lose their stability, an since the series of Jacobian ellipsoids turns upward at this point, it follow that stability passes to them.

If the mass is constrained to remain ellipsoidal there is no further poin of bifurcation on the Jacobian series, and, as the angular momentum con tinually increases along this series, it follows that all configurations on it ar stable. But it will be found later (Ch. V) that when the constraint to remair ellipsoidal is removed, the Jacobian series loses its stability at a certain stag. by meeting a series of non-ellipsoidal (pear-shaped) configurations. This ha been anticipated in our diagram. 
45. It will be understood that the foregoing discussion of stability has been concerned only with secular stability, this being the only kind of stability which is of interest in problems of cosmogony. The conditions of ordinary stability are quite different; for instance it has been shewn by G. H. Bryan* that Maclaurin's spheroid remains ordinarily stable until its eccentricity is given by $e=9529$.

\section{TIDALLY DISTORTED ELLIPSOIDS}

46. We now pass to a problem in which the distinction between secular and ordinary stability disappears.

A distant heavy mass will raise tides in a spherical mass of fluid, so that the fluid assumes the shape of a prolate spheroid. As the heavy mass approaches, the eccentricity of this spheroid will increase, and the question arises whether the spheroidal form remains stable no matter how great its eccentricity. The bearing of this problem on the planetesimal theory and other tidal-distortion theories is obvious.

47. Suppose that a mass $M$ of fluid which we shall call the primary, is acted on by tidal forces originating from a second mass $M^{\prime}$, which we shall cill the secondary. Let us at first suppose that the mass $M^{\prime}$ of the secondary is collected in a point, this being of course a legitimate approximation if the secondary is at a great distance from the primary.

Let the centre of gravity of the primary be taken for origin, and let the secondary be at a distance $R$, its spherical polar coordinates $(r, \theta, \phi)$ being supposed to be $R, 0,0$. The tide-generating potential at the point $r, \theta, \phi$ will be

$$
\frac{M^{\prime}}{\left(R^{2}+r^{2}-2 R r \cos \theta\right)^{\frac{1}{2}}}=\frac{M^{\prime}}{R}+\frac{M^{\prime} r \cos \theta}{R^{2}}+\frac{M^{\prime} r^{2}}{R^{3}} P_{2}(\cos \theta)+\frac{M^{\prime} r^{3}}{R^{4}} P_{3}(\cos \theta)+\ldots
$$

The first term on the right $M^{\prime} / R$ is a constant and so gives rise to no forces on the primary mass. The second term gives a uniform field of force of intensity $M^{\prime} / R^{2}$, which produces the Newtonian acceleration $M^{\prime} / R^{2}$ in the primary. We can neutralise this term by supposing the axes of reference to move with an acceleration $M^{\prime} / R^{2}$; the centre of gravity of the primary will then always remain at the origin.'

We are left with a tide-generating potential

$$
\frac{M^{\prime} r^{2}}{R^{3}} P_{2}(\cos \theta)+\frac{M^{\prime} r^{3}}{R^{4}} P_{3}(\cos \theta)+\ldots
$$


When $R$ is great the ratio of successive terms is of the order of magnitude of $r / R$, so that when $R$ is very great, the tidal potential reduces to its first term

$$
\frac{M^{\prime} r^{2}}{R^{3}} P_{2}(\cos \theta)
$$

or, writing $\mu$ for $M^{\prime} / R^{3}$ and transforming to Cartesian coordinates,

$$
\mu\left(x^{2}-\frac{1}{2} y^{2}-\frac{1}{2} z^{2}\right)
$$

48. When the tide-generating potential reduces to this simple form, it is at once clear that ellipsoidal configurations are possible for the primary. The condition that the standard ellipsoid (51) shall be a figure of equilibrium under a tide-generating potential (75) is that at every point of the boundary

$$
V_{i}+\mu\left(x^{2}-\frac{1}{2} y^{2}-\frac{1}{2} z^{2}\right)=\text { cons. }
$$

As in $\S 36$, this is equivalent to

$$
V_{i}+\mu\left(x^{2}-\frac{1}{2} y^{2}-\frac{1}{2} z^{2}\right)+\theta \pi \rho a b c\left(\frac{x^{2}}{a^{2}}+\frac{y^{2}}{b^{2}}+\frac{z^{2}}{c^{2}}-1\right)=\text { cons. }
$$

where $\theta$ is a constant.

Equating coefficients of $x^{2}, y^{2}$ and $z^{2}$, we find as the equations to be satisfied:

$$
\begin{aligned}
& J_{A}-\frac{\mu}{\pi \rho a b c}=\frac{\theta}{a^{2}} \\
& J_{B}+\frac{\mu}{2 \pi \rho a b c}=\frac{\theta}{b^{2}} \\
& J_{C}+\frac{\mu}{2 \pi \rho a b c}=\frac{\theta}{c^{2}}
\end{aligned}
$$

The addition of corresponding members of these equations gives

$$
\frac{2}{a b c}=\theta\left(\frac{1}{a^{2}}+\frac{1}{b^{2}}+\frac{1}{c^{2}}\right)
$$

while on subtracting corresponding members of equations (79) and (80), w obtain

$$
\left(b^{2}-c^{2}\right) J_{B C}=\left(b^{2}-c^{2}\right) \frac{\theta}{b^{2} c^{2}} .
$$

The elimination of $\theta$ between this equation and (81) gives

$$
\left(b^{2}-c^{2}\right)\left[\frac{2}{a b^{3} c^{3}}-\left(\frac{1}{a^{2}}+\frac{1}{b^{2}}+\frac{1}{c^{2}}\right) \cdot J_{B C}\right]=0
$$

It is at once clear that as before $(\$ 37)$ there are two sets of ellipsoida configurations, and these are obtained by satisfying respectively the tw equations

$$
\begin{array}{r}
b^{2}=c^{2} \ldots \ldots \ldots \\
\frac{2}{a b^{3} c^{3}}=\left(\frac{1}{a^{2}}+\frac{1}{b^{2}}+\frac{1}{c^{2}}\right) J_{B C}
\end{array}
$$


Obviously these two series of configurations correspond roughly to the series of Maclaurin spheroids and Jacobian ellipsoids in the rotational problem.

49. Eliminating $\theta$ from equations (79) and (80) and dividing out by the factor $b^{2}-c^{2}$, we obtain

$$
\frac{\mu}{2 \pi \rho a b c}=-\int_{0}^{\infty} \frac{\lambda d \lambda}{B C \Delta} .
$$

This does not give the value of $\mu$ on the spheroidal series, for on this series the factor $b^{2}-c^{2}$ vanishes. It gives the value of $\mu$ on the ellipsoidal series, and shews that $\mu$ is necessarily negative throughout this series. Since $\mu$ is positive in the physical problem, it appears that these ellipsoidal configurations cannot actually occur; only the spheroidal series remains as a physical possibility.

To obtain the value of $\mu$ on the spheroidal series we may eliminate $\theta$ between equations (78) and (81) and put $b=c$. We find that the spheroids. corresponding to positive values of $\mu$ are prolate $(a>c)$, and for these

$$
\begin{aligned}
\frac{\mu}{\pi \rho} & =a c^{2} \int_{0}^{\infty} \frac{d \lambda}{\left(a^{2}+\lambda\right)^{\frac{3}{2}}\left(c^{2}+\lambda\right)}-\frac{2 c^{2}}{c^{2}+2 a^{2}} \\
& =\frac{1-e^{2}}{e^{3}} \log \left(\frac{1+e}{1-e}\right)-\frac{6\left(1-e^{2}\right)}{e^{2}\left(3-e^{2}\right)} \cdots \cdots
\end{aligned}
$$

in which $e$ is the eccentricity, given by $e^{2}=\left(a^{2}-c^{2}\right) / a^{2}$.

Equation (85) shews that $\mu=0$ when $e=0$, as it ought to be; it also shews that $\mu=0$ when $e=1$. On treating equation (85) numerically, it is found that $\mu$ continually increases from the value $\mu=0$ at $e=0$, until $e$ has the value 882579 , after which $\mu$ decreases down to the value $\mu=0$ at $e=1$. The maximum value of $\mu$ is $\cdot 125504 \pi \rho$.

Clearly the configurations of equilibrium which correspond to positive values of $\mu$ form a diagram similar to fig. 4 (ii). It follows that all spheroids for which $e<.8826$ are stable, while all others are unstable. So long as $\mu<\cdot 125504 \pi \rho$, there are always two spheroidal configurations, one stable and the other unstable; when $\mu>\cdot 125504 \pi \rho$, there are no spheroidal or ellipsoidal configurations at all.

When a tide-generating mass approaches the primary mass, $\mu$ may be supposed to increase continually. We now see that if $\mu$ changes slowly enough, the primary passes through a series of prolate spheroids of continually increasing eccentricity, until $\mu$ reaches the value $0 \cdot 125504 \pi \rho$. After this value of $\mu$ is passed the problem becomes a dynamical one. We shall give the solution of this dynamical problem in a later chapter. For the present we 
notice that the critical stage at which this dynamical motion begins is determined by

$$
\frac{M^{\prime}}{K^{3}}=0 \cdot 125504 \pi \rho
$$

or, since $M=\frac{4}{3} \pi \rho r_{0}^{3}$, it is determined by

$$
R=2 \cdot 1984\left(\frac{M^{\prime}}{M}\right)^{\frac{1}{3}} r_{0}
$$

When the secondary approaches to a distance less than this, there are no configurations of equilibrium for the primary. When the secondary is at this critical distance, the primary has the greatest eccentricity consistent with stability. This is given by $e=882579$ and the lengths of the semi-axes are

$$
a=1 \cdot 65390 r_{0} ; \quad b=c=\cdot 77757 r_{0},
$$

these lengths being very approximately in the ratio $17: 8: 8$.

\section{THE DOUBLE-STAR PROBLEM}

50. We now proceed to the third problem-that of two bodies rotating round one another without any change of relative position. This problem has been studied in detail by Roche* and Darwint.

Let the two bodies be spoken of as primary and secondary, and let their masses be $M, M^{\prime}$ respectively ; let the distance apart of their centres of gravity be $R$, and let the angular velocity of rotation of the line joining them be $\omega$.

It will be sufficient to fix our attention on the conditions of equilibrium of one of the two masses, say the primary. Let its centre of gravity be taken as origin, let the line joining it to the centre of the secondary be axis of $x$, and let the plane in which the rotation takes place be that of $x y$. Then the equation of the axis of rotation is

$$
x=\frac{M^{\prime}}{M+M^{\prime}}, R, \quad y=0 .
$$

The problem may be reduced to a statical one (cf. $§ 31$ ) by supposing the masses acted on by a field of force of potential

or

$$
\begin{gathered}
\frac{1}{2} \omega^{2}\left[\left(x-\frac{M^{\prime}}{M+M^{\prime}} R\right)^{2}+y^{2}\right], \\
\frac{1}{2} \omega^{2}\left(x^{2}+y^{2}\right)-\frac{M^{\prime}}{M+M^{\prime}} R \omega^{2} x+\text { cons. }
\end{gathered}
$$

" E. Roche, "La Figure d'une Masse fluide soumise à l'attraction d'un Point eloigné." Acad. de Montpellier (Sciences), 1. (1850), p. 243.

+ G. H. Darwin, "On the Figure and Stability of a liquid Satellite." Phil. Trans. 206 A (1906), p. 161, and Coll. Works, III. p. 436. 


\section{ROCHE'S PROBLEM}

51. The simplest problem occurs when the secondary may be treated as a rigid sphere; this is the special problem dealt with by Roche. As in $\S 47$ the tide-generating potential acting on the primary may be supposed to be

$$
\frac{M^{\prime}}{R}+\frac{M^{\prime}}{R^{2}} x+\frac{M^{\prime}}{R^{3}}\left(x^{2}-\frac{1}{2} y^{2}-\frac{1}{2} z^{2}\right)+\ldots
$$

We shall for the present be content to omit all terms beyond those written down. The correction required by the neglect of these terms will be discussed later, and will be found to.be so small that the results now to be obtained are hardly affected.

On omitting these terms, and combining the two potentials (87) and (88), it appears that the primary may be supposed influenced by a statical field of potential

$$
\frac{M^{\prime}}{R^{2}} x\left(1-\frac{\omega^{2} R^{3}}{M+M^{\prime}}\right)+\frac{M^{\prime}}{R^{3}}\left(x^{2}-\frac{1}{2} y^{2}-\frac{1}{2} z^{2}\right)+\frac{1}{2} \omega^{2}\left(x^{2}+y^{2}\right) .
$$

The terms in $x$ may immediately be removed by supposing $\omega$ to have the appropriate value given by

$$
\omega^{2}=\frac{M+M^{\prime}}{R^{3}}
$$

and the condition for equilibrium is now seen to be that we shall have, at every point of the surface,

$$
V_{b}+\mu\left(x^{2}-\frac{1}{2} y^{2}-\frac{1}{2} z^{2}\right)+\frac{1}{2} \omega^{2}\left(x^{2}+y^{2}\right)=\text { cons. }
$$

where $\mu$ again stands for $M^{\prime} / R^{3}$.

52. Equating the left-hand of equation (90), as before, to

$$
-\pi \rho a b c \theta\left(\frac{x^{2}}{a^{2}}+\frac{y^{2}}{b^{2}}+\frac{z^{2}}{c^{2}}-1\right)
$$

we find, as the conditions of equilibrium,

$$
\begin{aligned}
& J_{A}-\frac{\mu}{\pi \rho a b c}-\frac{\omega^{2}}{2 \pi \rho a b c}=\frac{\theta}{a^{2}} \\
& J_{B}+\frac{\mu}{2 \pi \rho a b c}-\frac{\omega^{2}}{2 \pi \rho a b c}=\frac{\theta}{b^{2}} \\
& J_{C}+\frac{\mu}{2 \pi \rho a b c} \quad=\frac{\theta}{c^{2}}
\end{aligned}
$$

It will be seen that these equations are more general than either of the two sets we have considered before, each of which are indeed included as special cases in the present.set. Putting $\mu=0$ we obtain the equations of the rotational problem, while on putting $\omega=0$ we obtain the equations of the tidal problem. 
It is convenient to put $M / M^{\prime}=p$, so that

$$
\omega^{2}=(1+p) \mu
$$

The equations then reduce to

$$
\begin{aligned}
& J_{A}-\frac{(3+p) \mu}{2 \pi \rho a b c}=\frac{\theta}{a^{2}} \\
& J_{B}-\frac{p \mu}{2 \pi \rho a b c}=\frac{\theta}{b^{2}} \\
& J_{C}+\frac{\mu}{2 \pi \rho a b c}=\frac{\theta}{c^{2}}
\end{aligned}
$$

and the special cases are now obtained on putting $p=\infty$ (the rotational problem) and $p=-1$ (the tidal problem).

Eliminating $\theta$ from equations (96) and (97) we obtain

$$
\left(b^{2}-c^{2}\right) \int_{0}^{\infty} \frac{\lambda d \lambda}{\left(b^{2}+\lambda\right)\left(c^{2}+\lambda\right) \Delta}=\left(b^{2} p+c^{2}\right) \frac{\mu}{2 \pi \rho a b c}
$$

while similarly the elimination of $\theta$ from (95) and (97) yields

$$
\left(a^{2}-c^{2}\right) \int_{0}^{\infty} \frac{\lambda d \lambda}{\left(a^{2}+\lambda\right)\left(c^{2}+\lambda\right) \Delta}=\left(a^{2} p+c^{2}+3 a^{2}\right) \frac{\mu}{2 \pi \rho a b c}
$$

These two equations are identical, except for differences of notation, with the two equations which Roche takes as the basis of his discussion*.

53. If we now remove $c$ from equations (98) and (99) by the substitution $c=r_{0}^{3} / a b$, the resulting equations will give $a, b$ in terms of $\mu$ and $p$.

In these equations the value $p=\infty$ has already been fully discussed, and found to give the series of Maclaurin spheroids and Jacobian ellipsoids. For all other values of $p$, the value $\mu=0$ leads at once to $a=b=c$, and so gives a spherical configuration.

For values of $\mu$ other than $\mu=0$, the elimination of $\mu$ from equations (98) and (99) leads to an equation which gives $p$ uniquely in terms of $a$ and $b$, and either equation then gives $\mu$ uniquely. Thus all solutions of equations (98) and (99) may be represented on a graph in which $a$ and $b$, both necessarily positive, are taken as abscissa and ordinate. Each point in this diagram will correspond to one and only one value of $p$ and $\mu$. On drawing the loci $p=$ constant, we obtain the various linear series corresponding to different values of $p$ or $M / M^{\prime}$ in Roche's problem.

Since the value of $p$ for any value of $a$ and $b$ has been seen to be unique, it follows that no two of these linear series corresponding to different values of $p$ can ever intersect. The median line $a=b$ is already occupied by the 
locus $p=\infty$ (Maclaurin spheroids), so that it is impossible for any of the loci ever to cross this line; they all lie completely on one side or the other of it. Moreover the values of $p$ and $\mu$ must obviously vary continuously as we move continuously in the $a, b$ plane.

In fig. 7 such a diagram is represented. The point $S\left(a=b=r_{0}\right)$ represents the spherical configuration; the line $\operatorname{CSM}(a=b)$ is the series of Maclaurin spheroids, and the line $T S T^{\prime}\left(a b^{2}=1\right)$ is the series of tidal spheroids. $B$ is the point of bifurcation on the series of Maclaurin spheroids and $J B J^{\prime}$ is the series of Jacobian ellipsoids.

All points which are on the side $T^{\prime} J^{\prime}$ of the median line OSM represent configurations for which $b>a$, and therefore configurations in which the primary is broadside on to the secondary. It is obvious that all these configurations are unstable, for they would be unstable even if the primary were constrained to remain rigid. These configurations need not trouble us further and we may confine our attention to the right-hand half of the diagram.

Linear series for all values of $p$ pass through $S$. The series for $p=+\infty$ is the broken line $S B J$, that for $p=-1$ is the line $S T$, while that for $p=-\infty$ is the line $S O$. Remembering that two linear series cannot cross, it is clear that the series for a very large positive value of $p$ must be asymptotic to the line $S B J$. All the series from $p=+\infty$ to $p=-1$ accordingly lie within the small area bounded by the lines $J B, B S, S T$. The series in the area OST are of course series for which $p$ is negative and numerically greater than 1 , while those in the area $M B J$ are again series for which $p$ is negative, a second series for $p=-\infty$ coinciding with the line $M B J$.

Let us now confine our attention to the series which lie inside the area $J B S T$, these being as we have seen the only ones of physical interest. Each series starts at $S$ and ends at the point in which the lines $B I$ and $S T$ ultimately meet at infinity. Thus each series begins with a sphere and ends with an infinitely long prolate spheroid. As we pass along any one of these series $\mu$ changes while $p$ remains constant. The value of $\omega^{2}$, which is given by equation (94) accordingly changes also, this giving the value of a real angular velocity when $p$ is positive, and being regarded simply as an algebraic quantity when $p$ is negative. The value of $\omega^{2}$ vanishes only when $p=-1$ or when $\mu=0$; consequently it vanishes at $S$, at $(J T)_{\infty}$ and along the line $S T$. It follows that $\omega^{2}$ is of the same sign everywhere inside the area $S B J T$, and this sign is readily seen to be positive.

Since $\omega^{2}$ vanishes at both the ends $S$ and $(J T)_{\infty}$ of every series, it follows that on passing along each series $\omega^{2}$ at first increases, and then after passing a maximum value decreases. Roche*, treating equations (98) and (99) by a 


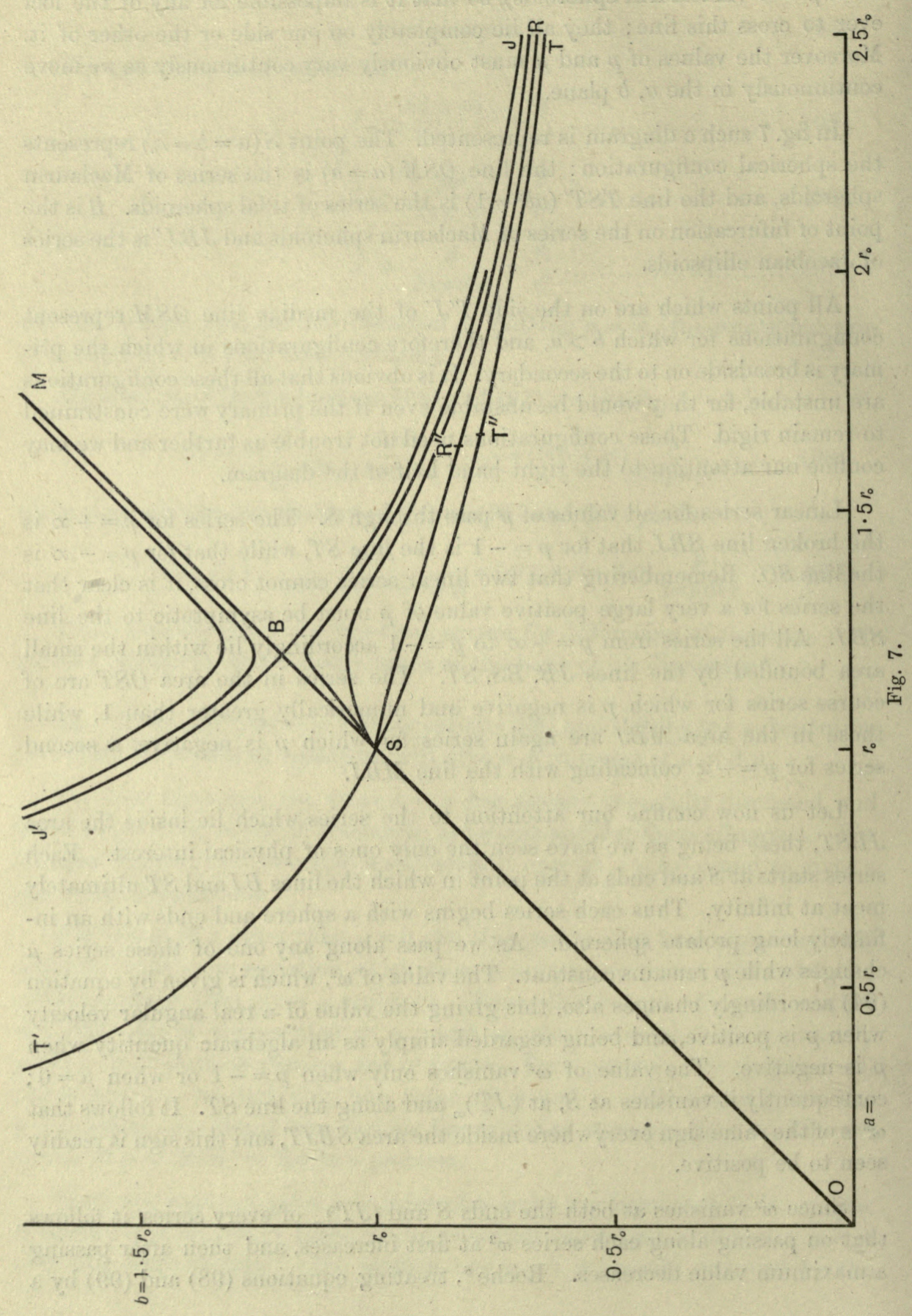


laborious method of numerical calculation, found that there is only one maximum on each series. The maximum on the series $S B J(p=\infty)$ occurs as we have seen at $B$; the maximum value of $\omega^{2} / 2 \pi \rho$ is 0.18712 . Similarly the maximum on the series $S T(p=-1)$ occurs when $\mu$ is a maximum, and so at the configuration of eccentricity 8826 . This is represented by the point $T^{\prime \prime}$ in the diagram, the value of $\omega^{2} / 2 \pi \rho$ at this point is 0 . Roche has calculated the maxima of $\omega^{2} / 2 \pi \rho$ on other series. On the series $p=0$, the series of configurations in which the primary is infinitesimal, he finds the maximum value of $\omega^{2} / 2 \pi \rho$ to be 0.046 , and the configuration at which this maximum occurs is that in which $a=1 \cdot 63 r_{0}, b=\cdot 81 r_{0}$; this is represented by the point $R^{\prime \prime}$ in the diagram. When $p=1$, the maximum value of $\omega^{2} / 2 \pi \rho$ is 0.072 , and Roche finds that the value of this maximum increases continuously from $p=0$ to $p=\infty$.

On connecting the points $B, R^{\prime \prime}, T^{\prime \prime}$ by a continuous line, we get the loci of points at which $\omega^{2}$ is a maximum on the various linear series.

\section{Stability}

54. In a physical problem in which $\omega^{2}$ increased continuously, it would follow, from the principles already discussed, that all configurations on the left of this line would be stable, and all the configurations on the right would be unstable. The stability of the configurations on the left would of course only be stability so long as the configurations were constrained to remain ellipsoidal, although we shall see later that this restriction makes no difference.

In the natural double-star problem, the change in physical conditions is not represented by an increase in $\omega^{2}$. Both masses lose energy by radiation, and shrink accordingly. The rates of shrinkage, and consequent rates of increase in density will in all probability be quite different for the two masses. We can, however, construct an artificial problem in which the density, if supposed uniform to begin with, remains. uniform throughout the shrinkage, or in which the two densities, if not supposed equal to begin with, change so as always to retain the same ratio. The physical conditions are now represented by an increase in the absolute densities, while the moment of momentum remains constant and, exactly as in $\S 44$, these conditions may equally be represented by supposing both densities to remain constant while the moment of momentum increases.

55. Let us first consider the general problem in which the secondary is not regarded merely as a point.

The moment of momentum of the primary about the centre of gravity of the system is

$$
M\left[k^{2}+\left(\frac{M^{\prime}}{M+M^{\prime}}\right)^{2} R^{2}\right] \omega
$$


where $k$ is the radius of gyration of the primary. Adding this to the similar expression for the secondary, we obtain for the total moment of momentum $M$ of the system

$$
\mathbf{M}=\left(M k^{2}+M^{\prime} k^{\prime 2}+\frac{M M^{\prime}}{M+M^{\prime}} R^{2}\right) \omega
$$

or, replacing $R$ by its value $\left(M+M^{\prime}\right)^{\frac{1}{3}} \omega^{-\frac{2}{3}}$,

$$
\mathrm{M}=\left(M k^{2}+M^{\prime} k^{\prime 2}\right) \omega+\frac{M M^{\prime}}{\left(M+M^{\prime}\right)^{\frac{1}{3}}} \omega^{-\frac{1}{3}}
$$

56. When the primary $M$ is infinitely massive compared with the secondary $M^{\prime}$, the total moment of momentum $\mathbf{M}$ has the value $\mathbf{M}=M k^{2} \omega$, and the variation of $M$ is precisely that of a freely rotating mass; it increases steadily from $M=0$ at $S$ to $M=\infty$ at $J$ in fig. 7 .

For finite values of the ratio $M / M^{\prime}$ the value of given $M$ by equation (101) becomes infinite when $\omega=0$-i.e. at the two ends of the linear series of configurations similar to those shewn in fig. 7. Thus on leaving $S$, $\mathbf{M}$ decreases until a minimum is reached, and all configurations beyond this minimum will be unstable. Thus the curved line $B R^{\prime \prime} T^{\prime}$ which divided stable from unstable configurations in fig. 7 must now be replaced by another curved line passing through $S$.

It accordingly appears that when $M / M^{\prime}$ is large the linear series becomes unstable very near to $S$, the range of stability vanishing altogether when $M / M^{\prime}$ is infinite. If both masses are rigid, so that $k^{2}$ and $k^{\prime 2}$ are constants the limit of this range is easily found from equation (101) by making $\delta M=0$. The limit of stability is found to be given by

$$
3 \omega^{\frac{4}{3}}\left(M k^{2}+M^{\prime} k^{\prime 2}\right)=\frac{M M^{\prime}}{\left(M+M^{\prime}\right)^{\frac{1}{3}}}
$$

or, in terms of $R$ (using equation (89)),

$$
R^{2}=3\left(\frac{M+M^{\prime}}{M M^{\prime}}\right)\left(M k^{2}+M^{\prime} k^{\prime 2}\right)
$$

and the range of stability is again seen to be infinitesimal-i.e. limited to very great values of $R$-when $M / M^{\prime}$ is infinite.

The result shews that there can only be secular stability of a large and small mass rotating round one another when the smaller mass is at a very great distance from the larger*. We are dealing, it must be noticed, with secular stability only; the question means nothing except when dissipative forces are present. When there are no dissipative forces, as for instance if both bodies are perfectly rigid, a circular orbit of no matter what radius is thoroughly stable, the orbit $r=a$ giving place when slightly disturbed to

* Cf. Sir G. Darwin. Coll. Works, nI. p. 442. 
the slightly elliptical orbit $r=a(1-e \cos \theta)$. And when frictional forces are introduced, as for instance by making the masses fluid, or by supposing the solid masses covered by shallow oceans, the instability is one of orbital motion only and not one of the configurations of the masses. "When the secondary is supposed wholly fluid so that $k^{\prime 2}$ is variable, the fluidity of the mass modifies the stage at which instability sets in, but introduces no new instability of its own. The mechanism by which this instability is set up is that which has been studied by Darwin under the name of "Tidal Friction"*; it produces a secular increase or decrease in the mean radius of the orbit.

It is important to notice that the case of $M / M^{\prime}=\infty$, in which $M^{\prime}$ is of infinitesimal mass, is not, from our present point of view identical with the case of $p=\infty$ in the diagram shewn in fig. 7 , in which $M^{\prime}$ is supposed to disappear altogether. The former problem is one having one more degree of freedom than the latter, and this one degree of freedom happens to be secularly unstable for all finite values of $r$. In the latter problem, in which the system is supposed to reduce to a single rotating body, the angular momentum increases steadily from 0 to $\infty$ on passing along the path $S B J$ in fig. 7 , so that the configurations on this path are all stable so long as the mass is constrained to remain ellipsoidal.

57. A special problem arises when the rotation of the secondary is not affected by forces exerted on it by the primary. The primary, which is the body whose configurations and stability we are specially considering, may now be a small satellite rotating round a massive planet, which our choice of terms compels us to call the secondary. The term $M^{\prime} k^{\prime 2} \omega$ in equation (101) may now be replaced by $M^{\prime} k^{\prime 2} \omega^{\prime}$, where $\omega^{\prime}$ is the angular velocity of the secondary and neither this nor $k^{\prime 2}$ is subject to variation. The angular momentum is accordingly

$$
\mathrm{M}=M k^{2} \omega+\frac{M M^{\prime}}{\left(M+M^{\prime}\right)^{\frac{1}{3}}} \omega^{-\frac{1}{3}}+\text { cons }
$$

A similar problem occurs when the secondary is treated as a point so that $k^{\prime 2}=0$. This leads back to Roche's problem discussed in $\S 51$. The moment of momentum is again given by equation (104) in which the final constant now vanishes. Let us investigate the stability of systems in which the moment of momentum is given by equation (104).

58. A case of special interest arises when the primary $M$ is infinitesimal. The value of $\mathbf{M}$ now becomes infinitesimal also, but $\mathbf{M} / M$ remains finite, being given by

$$
\frac{M}{M}=\left(M^{\prime}\right)^{\frac{2}{3}} \omega^{-\frac{1}{3}}+\text { cons }
$$


The minimum value of $M$ now coincides with the maximum value of $\omega$. The series of configurations are those represented on the series $(p=0)$ in fig. 7 , and the minimum value of $\omega$ occurs at the point $R^{\prime \prime}$. Thus configurations "on the branch $S R^{\prime \prime}$ are stable, while those beyond $R^{\prime \prime}$ are unstable.

The actual value of $\omega$ at the point $R^{\prime \prime}$ is given by

$$
\omega^{2} / 2 \pi \rho=0.04503^{*} \text {. }
$$

The general value of $\omega^{2} / 2 \pi \rho$ on this series is

$$
\frac{\omega^{2}}{2 \pi \rho}=\frac{M^{\prime}}{2 \pi \rho R^{3}}=\frac{2}{3}\left(\frac{\rho^{\prime}}{\rho}\right) \frac{r_{0}^{\prime 3}}{R^{3}}
$$

so that in the critical configuration we have

$$
R=2 \cdot 4554\left(\frac{\rho^{\prime}}{\rho}\right)^{\frac{1}{3}} r_{0}^{\prime}
$$

Thus a small satellite rotating about a rigid primary of mass enormously greater than its own cannot be in equilibrium in any configuration whatever if its distance from the centre of its planet is less than $2.4554\left(\rho^{\prime} / \rho\right)^{\frac{1}{3}}$ radii of the planet. This distance is commonly spoken of as Roche's limit.

The critical value of $R$ may also be put in the form

$$
R=2.4554\left(\frac{M^{\prime}}{M}\right)^{\frac{3}{3}} r_{0}
$$

This may be compared with equation (86) which determined the limit of closest approach under the tidal forces from a secondary mass when the bodies were not in rotation. The critical value of $R$ just found is about twelve per cent. greater than that found in the former problem; the difference of course represents the disturbing effect of rotation on the primary.

59. In the more general case in which $M$ is not infinitesimal, and the angular momentum is given by the complete equation (104), the maximum value for $\omega$ is not so easily found since $k^{2}$ will vary with $\omega$. It is however clear that $M$ will be infinite when $\omega=0$, and that $\omega$ will again increase to a maximum and again decrease, so that $\mathbf{M}$ will pass through a minimum value which will again divide stable from unstable configurations. Again there will be a limiting value of $R$ similar to Roche's limit, and there will be no configurations of equilibrium at all for smaller values of $R$ than this.

* Roche gave 0.046 ; both here and in equation (105) I quote the more accurate values deduced from Darwin's calculations. (Coll. Works, Irr. p. 436.) 


\section{DARWIN's ProBlem.}

60. In a paper of very great importance, $\operatorname{Sir}^{n}$ G. Darwin* has discussed the double-star problem in the case in which both masses are suppesed fluid so that each is subject to distortion from the tidal forces generated by the other. The discussion falls naturally into two parts - the determination of figures of equilibrium and the determination of the stability or instability of these figures.

In Roche's problem the secondary was assumed to be a rigid sphere, so that its potential could be written down in the form of formula (88),

$$
\frac{M^{\prime}}{R}+\frac{M^{\prime}}{R^{2}} x+\frac{M^{\prime}}{R^{3}}\left(x^{2}-\frac{1}{2} y^{2}-\frac{1}{2} z^{2}\right)+\ldots
$$

In Darwin's problem, the secondary is a mass of fluid of a shape determined by the mutual tidal actions between the two bodies, and an expansion such as the foregoing is no longer permissible. To a first approximation both bodies may be regarded as ellipsoids. Darwin assumes the bodies to be distorted ellipsoids and expresses the distortions in terms of ellipsoidal harmonics. The amount of this distortion is found to be in every case quite small, so that the supposition that the figures are actually ellipsoidal is found to give a tolerably accurate solution. In illustration of this the following figures may be quoted from Darwin's papert; they express the proportional increase $\delta a / a$ in the semi-major axis of the primary which would be produced by the removal of the ellipsoidal constraint when the masses are at the closest distance consistent with stability (cf. $§ 64$ below).

$\begin{array}{lccc}M / M^{\prime}= & 0.4 & 0.7 & 1 \cdot 0 \\ \delta a / a \text { in direction towards secondary } & \frac{1}{17} & \frac{1}{20} & \frac{1}{23} \\ \delta a / a \text { in direction away from secondary } & \frac{1}{25} & \frac{1}{33} & \frac{1}{39} .\end{array}$

The amount of these corrections is shewn by the dotted lines in figures 11,12 and 13 (p. 64 below).

For configurations in which the masses are further apart than this minimum distance the error in the ellipsoidal solution will of course be less, so that the assumption that the figures are ellipsoidal is seen to give a very fair approximation.

* Phil. Trans. 206 A (1906), p. 161, or Coll. Works, III. p. 436. The actual paper occupies 88 pages in each place, so that it will be understood that only the merest outline of it is given here. And, to avoid the complicated methods of ellipsoidal harmonic analysis employed by Darwin, I have substituted a simpler discussion of the fundamental equations, deriving them in a form analogous to the equations of Roche already discussed.

+ l.c. p. 510 . 
61. Our first task must be to evaluate the potential from the secondary, now assumed to be an ellipsoid of mass $M^{\prime}$ and semi-axes $a^{\prime}, b^{\prime}, c^{\prime}$.

Let us momentarily take the centre of the secondary for origin, then the potential of the ellipsoid at any external point $x^{\prime}, y^{\prime}, z^{\prime}$ will be

$$
V=-\frac{3}{4} M^{\prime} \int_{\lambda^{\prime}}^{\infty}\left(\frac{x^{\prime 2}}{a^{\prime 2}+\lambda}+\frac{y^{\prime 2}}{b^{\prime 2}+\lambda}+\frac{z^{\prime 2}}{c^{\prime 2}+\lambda}-1\right) \frac{d \lambda}{\Delta^{\prime}}
$$

where the lower limit $\lambda^{\prime}$ is the root of

and $\Delta^{\prime}$ stands for

$$
\frac{x^{\prime 2}}{a^{\prime 2}+\lambda}+\frac{y^{\prime 2}}{b^{\prime 2}+\lambda}+\frac{z^{\prime 2}}{c^{\prime 2}+\lambda}-1=0
$$

$$
\left[\left(a^{\prime 2}+\lambda\right)\left(b^{\prime 2}+\lambda\right)\left(c^{\prime 2}+\lambda\right)\right]^{\frac{1}{2}}
$$

Differentiating, and bearing in mind that the lower limit $\lambda^{\prime}$ is a function of $x^{\prime}, y^{\prime}$ and $z^{\prime}$, we obtain

$$
\begin{gathered}
\frac{\partial V}{\partial x^{\prime}}=-\frac{3}{2} M^{\prime} x^{\prime} \int_{\lambda^{\prime}}^{\infty} \frac{d \lambda}{\partial x^{\prime 2}}=-\frac{3}{2} M^{\prime}\left[\int_{\lambda^{\prime}}^{\infty} \frac{d \lambda}{\left(a^{\prime 2}+\lambda\right) \Delta^{\prime}}-\frac{2\left(\frac{x}{a^{\prime 2}+\lambda^{\prime}}\right)^{2}}{\left[\left(\frac{x}{a^{\prime 2}+\lambda^{\prime}}\right)^{2}+\left(\frac{y}{b^{\prime 2}+\lambda^{\prime}}\right)+\left(\frac{z}{c^{\prime 2}+\lambda^{\prime}}\right)^{2}\right] \Delta^{\prime}}\right]
\end{gathered}
$$

and similar equations give $\partial V / \partial y^{\prime}, \partial^{2} V / \partial y^{\prime 2}$ etc.

These equations are general. At a point on the axis of $x^{\prime}$, we put $y^{\prime}=z^{\prime}=0$, and the value of $\lambda^{\prime}$ is, from equation (109), $\lambda^{\prime}=x^{\prime 2}-a^{\prime 2}$. The equations become

$$
\frac{\partial V}{\partial x^{\prime}}=-\frac{3}{2} M^{\prime} x^{\prime} \int_{\lambda^{\prime}}^{\infty} \frac{d \lambda}{\left(a^{\prime 2}+\lambda\right) \Delta^{\prime}} ; \frac{\partial V}{\partial y^{\prime}}=\frac{\partial V}{\partial z^{\prime}}=0
$$

$\frac{\partial^{2} V}{\partial x^{\prime 2}}=-\frac{3}{2} M^{\prime}\left[\int_{\lambda^{\prime}}^{\infty} \frac{d \lambda}{\left(a^{\prime 2}+\lambda\right) \Delta^{\prime}}-\frac{2}{\Delta^{\prime}}\right], \quad \frac{\partial^{2} V}{\partial y^{\prime 2}}=-\frac{3}{2} M^{\prime} \int_{\lambda^{\prime}}^{\infty} \frac{d \lambda}{\left(b^{\prime 2}+\lambda\right) \Delta^{\prime}}$, etc. (111).

To obtain the differential coefficients of the potential $V$ at the centre of the primary, we put $x^{\prime}=R$, and of course $\lambda^{\prime}=R^{2}-a^{\prime 2}$. If $V$ denotes the value of the potential at this point, and $\partial V / \partial x$ etc. denote the value of differential coefficients at this point, the general value of the potential of the secondary, referred to the centre of the primary as origin will be

$$
V+x \frac{\partial V}{\partial x}+y \frac{\partial V}{\partial y}+z \frac{\partial V}{\partial z}+\frac{1}{2}\left(x^{2} \frac{\partial^{2} V}{\partial x^{2}}+y^{2} \frac{\partial^{2} V}{\partial y^{2}}+z^{2} \frac{\partial^{2} V}{\partial z^{2}}\right) \cdots
$$

In this expression the terms in $x y, y z, z x$ have been omitted because they vanish on account of the symmetry of figure of the ellipsoid. Terms of degrees three and higher have also been omitted because they would destroy the ellipsoidal shape of the primary. The approximation to which we are now working is one in which the primary is supposed to remain of ellipsoidal shape, so that all tide-generating terms of degrees three and higher must 
be neglected. Or we may, if we please, regard the problem as one in which the primary is constrained to remain ellipsoidal, in which case the forces of constraint must be just sufficient to neutralise the omitted terms in formula (112).

The coefficients in formula (112) are precisely those which have been already evaluated in equations (110) and (111). For we have

$$
\frac{\partial V}{\partial x}=-\frac{\partial V}{\partial x^{\prime}} \text { etc., so that } \frac{\partial V}{\partial y}=\frac{\partial V}{\partial z}=0,
$$

and similarly $\hat{\partial}^{2} V / \partial x^{2}=\partial^{2} V / \partial x^{\prime 2}$.

62. Let $\xi, \xi^{\prime}$ denote for the moment the distances of the centres of the primary and secondary from the axis of rotation. Following our previous procedure, the primary must be in equilibrium under a statical field of force of potential

or

$$
\begin{gathered}
\frac{1}{2} \omega^{2}\left[(x-\xi)^{2}+y^{2}\right] \\
\frac{1}{2} \omega^{2}\left(x^{2}+y^{2}\right)-\omega^{2} \xi x+\text { a constant }
\end{gathered}
$$

and the condition for equilibrium, as in $\S 51$, is that

$$
V_{b}+\frac{1}{2}\left(x^{2} \frac{\partial^{2} V}{\partial x^{\prime 2}}+y^{2} \frac{\partial^{2} V}{\partial y^{\prime 2}}+z^{2} \frac{\partial^{2} V}{\partial z^{\prime 2}}\right)+\frac{1}{2} \omega^{2}\left(\dot{x}^{2}+y^{2}\right)-x \frac{\partial V}{\partial x^{\prime}}-\omega^{2} \xi x=\text { a constant }
$$

over the boundary. The term in $x$ on the right of this equation is removed if

$$
\omega^{2} \xi=-\frac{\partial V}{\partial x^{\prime}}=\frac{3}{2} M^{\prime} R \int_{R^{2}-a^{\prime 2}}^{\infty} \frac{d \lambda}{\left(a^{\prime 2}+\lambda\right) \Delta^{\prime}}
$$

The similar equation for the secondary is

$$
\omega^{2} \xi^{\prime}=\frac{3}{2} M R \int_{R^{2}-a^{2}}^{\infty} \frac{d \lambda}{\left(a^{2}+\lambda\right) \Delta}
$$

Since $\xi+\xi^{\prime}=R$, it is clear that equations (115) and (116) suffice to determine $\xi$, $\xi^{\prime}$ and $\omega^{2}$. The ratio $\xi / \xi^{\prime}$ is obtained at once by division of corresponding sides of the two equations.

These equations, as has been seen, refer to masses which are constrained to remain ellipsoidal by the supposed application of small external forces. Had the bodies been rotating freely in space, the ratio $\xi / \xi^{\prime}$ would have been given directly by the condition that the centre of gravity of the two masses must be on the axis of rotation, namely

$$
\frac{\xi}{M^{\prime}}=\frac{\xi^{\prime}}{M}=\frac{R}{M+M^{\prime}}
$$

The two values of $\xi / \xi^{\prime}$ obtained in these two different ways are not found to be identical, their difference giving a measure of the error introduced in supposing the masses to remain ellipsoidal. If we put

$$
\int_{R^{2}-a^{2}}^{\infty} \frac{d \lambda}{\left(a^{2}+\lambda\right) \Delta}=I ; \int_{R^{2}-a^{\prime 2}}^{\infty} \frac{d \lambda}{\left(a^{\prime 2}+\lambda\right) \Delta^{\prime}}=I^{\prime}
$$


then equation (117) gives $\xi / \xi^{\prime}=M^{\prime} / M$, whereas equations (115) and (116) give

$$
\frac{\xi}{\xi^{\prime}}=\frac{M^{\prime}}{M} \times \frac{I^{\prime}}{I}
$$

The difference between the two values of $\xi / \xi^{\prime}$ consists of a multiplying factor $I^{\prime} / I$.

Now $I$ can be put in the form

$$
I=\int_{R^{2}}^{\infty} \frac{d u}{u^{\frac{3}{2}}\left[u-\left(a^{2}-b^{2}\right)\right]^{\frac{1}{2}}\left[u-\left(a^{2}-c^{2}\right)\right]^{\frac{1}{2}}}
$$

while $I^{\prime}$ is obtained on replacing $a, b, c$ by $a^{\prime}, b^{\prime}, c^{\prime}$. Since $R^{2}$ is large in comparison with $a^{2}-b^{2}$ and $a^{2}-c^{2}$, it is clear that the two integrals do not differ by much from one another, both approximating to

$$
\int_{R^{2}}^{\infty} \frac{d u}{u^{\frac{5}{2}}} \text { or } \frac{2}{3 R^{3}} \text {. }
$$

The integrals agree more closely with one another than with this limiting integral, and when the ellipsoids are nearly equal, $I$ and $I^{\prime}$ become very nearly equal to each other while differing considerably from $2 / 3 R^{3}$. Let us suppose that

$$
\frac{1}{2}\left(I+I^{\prime}\right)=\frac{2}{3 R^{3}}(1+\zeta)
$$

then it is clear that $I$ and $I^{\prime}$ may, without serious error, be taken separately equal to the quantity on the right*.

Using this approximation for $I$, equation (115) becomes

$$
\omega^{2} \xi=\frac{M^{\prime}}{R^{2}}(1+\zeta)
$$

while equation (116) assumes the similar form

$$
\omega^{2} \xi^{\prime}=\frac{M}{R^{2}}(1+\zeta)
$$

These equations are now consistent with equation (117) and by addition we readily find

$$
\omega^{2}=\frac{M+M^{\prime}}{R^{3}}(1+\zeta)
$$

This determines $\omega$, and $\zeta$ is now seen to measure the proportional increase in $\omega^{2}$ produced by the ellipsoidal shape of the bodies.

To balance centrifugal force, the gravitational attraction between the two bodies must be $\omega^{2} R M M^{\prime} /\left(M+M^{\prime}\right)$ or, by equation (121)

$$
\frac{M M^{\prime}}{R^{2}}(1+\zeta)
$$

\footnotetext{
* It will be readily verified that our $\zeta$ is identical with the $\zeta$ used by Darwin.
} 
so that $\zeta$ also measures the proportional increase in the gravitational attraction between the two bodies produced by their ellipsoidal forms.

63. The terms in $\xi$ have now been made to disappear from equation (114) and the condition for equilibrium is seen to be that

$$
V_{b}+\frac{1}{2}\left(x^{2} \frac{\partial^{2} V}{\partial x^{\prime 2}}+y^{2} \frac{\partial^{2} V}{\partial y^{\prime 2}}+z^{2} \frac{\partial^{2} V}{\partial z^{\prime 2}}\right)+\frac{1}{2} \omega^{2}\left(x^{2}+y^{2}\right)=\text { cons. }
$$

over the boundary.

Comparing this with equation (90), it appears that the second degree terms which were used in Roche's problem, namely

$$
\frac{M^{\prime}}{R^{3}}\left(x^{2}-\frac{1}{2} y^{2}-\frac{1}{2} z^{2}\right) \text { or } \mu\left(x^{2}-\frac{1}{2} y^{2}-\frac{1}{2} z^{2}\right)
$$

must now be replaced by

$-\frac{3}{4} M^{\prime}\left[x^{2}\left(\int_{\lambda^{\prime}}^{\infty} \frac{d \lambda}{\left(a^{\prime 2}+\lambda\right) \Delta^{\prime}}-\frac{2}{\Delta^{\prime}}\right)+y^{2} \int_{\lambda^{\prime}}^{\infty} \frac{d \lambda}{\left(b^{\prime 2}+\lambda\right) \Delta^{\prime}}+z^{2} \int_{\lambda^{\prime}}^{\infty} \frac{d \lambda}{\left(c^{\prime 2}+\lambda\right) \Delta^{\prime}}\right]$

in which the lower limit $\lambda^{\prime}$ is put equal to $R^{2}-a^{\prime 2}$.

Following the former procedure ( $\$ 52)$ we find that equation (122) may be replaced by the three separate equations

$$
\begin{array}{rlr}
J_{A}-\frac{M^{\prime}}{M}\left(\int_{\lambda^{\prime}}^{\infty} \frac{d \lambda}{\left(a^{\prime 2}+\lambda\right) \Delta^{\prime}}-\frac{2}{\Delta^{\prime}}\right)-\frac{\omega^{2}}{2 \pi \rho a b c} & =\frac{\theta}{a^{2}} \\
J_{B}-\frac{M^{\prime}}{M} \int_{\lambda^{\prime}}^{\infty} \frac{d \lambda}{\left(b^{\prime 2}+\lambda\right) \Delta^{\prime}} & -\frac{\omega^{2}}{2 \pi \rho a b c}=\frac{\theta}{b^{2}} \\
J_{C}-\frac{M^{\prime}}{M} \int_{-\lambda^{\prime}}^{\infty} \frac{d \lambda}{\left(c^{\prime 2}+\lambda\right) \Delta^{\prime}} & =\frac{\theta}{c^{2}}
\end{array}
$$

These are the equations of equilibrium for the primary, and there is a similar set for the secondary. On solving the set of six equations we obtain a solution of the problem. There is unfortunately no method of solving these equations exactly except by laborious numerical computations. But they are of the same general form as equations (91) to (93) already discussed in connection with Roche's problem, whence it is readily seen that the general arrangement of figures of equilibrium must be the same as that already found in $\S 53$ for Roche's problem.

\section{Stability}

64. The problem of stability demands a more detailed discussion.

The angular momentum of the system is still given by equation (100), namely

$$
\mathrm{M}=\left(M k^{2}+M^{\prime} k^{\prime 2}+\frac{M M^{\prime}}{M+M^{\prime}} R^{2}\right) \omega
$$


but the value of $R$ is now given in terms of $\omega$ by

$$
R=\left(M+M^{\prime}\right)^{\frac{\lambda}{3}}(1+\zeta)^{\frac{1}{2}} \omega^{-\frac{2}{3}}
$$

so that $\mathbf{M}$, expressed in terms of $\omega$ alone, becomes

$$
\mathrm{M}=\left(M k^{2}+M^{\prime} k^{\prime 2}\right) \omega+\frac{M M^{\prime}}{\left(M+M^{\prime}\right)^{\frac{7}{3}}}(1+\zeta)^{\frac{2}{3}} \omega^{-\frac{1}{3}}
$$

This differs from the former value of $M$ obtained in $\$ 55$ only through the occurrence of the factor $(1+\zeta)^{\frac{2}{3}}$, and as this is never far from unity, it is clear that the general discussion of stability given in $\$ \$ 56$ and 57 will remain valid, at least in its general features. Always, except in the special case of $p=\infty$, there is a configuration in which $\mathrm{M}$ is minimum; starting out of this are two series of configurations along each of which $M$ increases indefinitely up to $M=\infty$, these end configurations each being configurations of zero rotation $(\omega=0)$. One of these series ends in two spherical masses rotating infinitely slowly round one another at an infinite distance, and this series is stable throughout. At the end of the other series the primary is an infinitely elongated Jacobian ellipsoid, and this series is unstable throughout.

Instead of eliminating $R$ from equations (127) and (128), and so obtaining $M$ as a function of $\omega$, we might equally well have eliminated $\omega$ from these equations and obtained $\mathrm{M}$ as a function of $R$. The equation obtained in this way is

$$
\mathrm{M}=\left[M k^{2}+M^{\prime} k^{\prime 2}+\frac{M M^{\prime}}{M+M^{\prime}} R^{2}\right](1+\zeta)^{\frac{1}{2}}\left(M+M^{\prime}\right)^{\frac{1}{2}} R^{-\frac{3}{2}} .
$$

When $M$ is not very different from $M^{\prime}$, the value of $\mathrm{M}$ reduces to its last term when $R$ is large. Even for configurations in which the ellipsoids are almost in contact, it is readily seen that by far the greater part of the value of $\mathbf{M}$ comes from this last term, so that $\mathbf{M}$ varies approximately as $R^{\frac{1}{2}}$. It follows that the configuration for which $M$ is a minimum nearly coincides with that for which $R$ is a minimum, this latter being the configuration of closest approach of the centres of the ellipsoids.

Let $R_{0}$ be the distance of closest approach. Then for any value $R_{0}+\delta R$ which is just greater than $R_{0}$ there will be two configurations; in one the ellipsoids are more elongated than in the configuration of closest approach, while in the other they are less elongated. In the former configuration, then, $k^{2}, k^{\prime 2}$ and $\zeta$ are all greater than in the latter, so that, as the values of $R$ are the same in both configurations, it follows that the more elongated configuration has the larger value of $\mathbf{M}$.

Now the diagram of configurations, drawn with $M$ as vertical coordinate, lies as in figure 8. Here $O$ is the configuration of minimum angular momentum, the less elongated configurations $O P$ are stable, while the more 
elongated configurations $O P^{\prime}$ are unstable. We have seen that at the configuration of closest approach $R$, increasing elongation goes with increasing angular momentum; it follows that $R$ is on the unstable branch $O P^{\prime}$ of the series.

Thus the configuration of closest approach is always unstable; it is fairly near to $U$ when $M$ and $M^{\prime}$ are nearly equal, but is far removed from $O$ in other cases. Passing to the limit of configurations of greater elongation, it is easily shewn that in the extreme configuration $P^{\prime}$ in which $M=\infty, \omega=0$, the two bodies

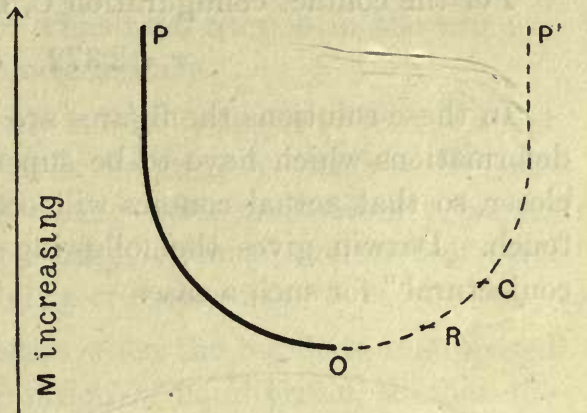

Fig. 8. must overlap; thus this configuration, although satisfying the mathematical equations, is physically impossible: At some stage between $R$ and $P^{\prime}$, there must be a configuration $C$, in which the bodies are just in contact, but without overlapping; this configuration, which we may call the contact configuration, is the last one which is physically possible. It is clear that all contact configurations are necessarily unstable.

Darwin calculates in detail the configurations $C, R$ and $O$ in the case of $p=1$ or $M=M^{\prime}$. In the case of configuration $O$ the calculation is not very accurate, for his series do not give good approximations when the masses are in or close to actual contact.

For the configuration $O$ of limiting stability, Darwin finds in this case

$$
a=a^{\prime}=0.897, \quad b=b^{\prime}=0.771, \quad c=c^{\prime}=0.723, \quad r=2 \cdot 638,
$$

the unit being the radius of the sphere formed by rolling the two masses into one, and the cross-section is shewn in fig. 9 , which is reproduced from Darwin's Collected Works*.

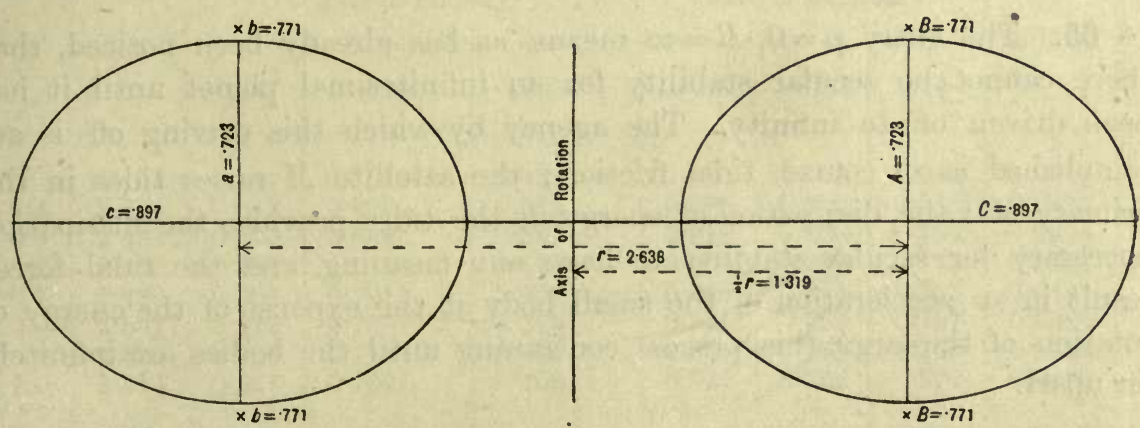

Fig. 9.

* Vol. 1II. p. 513. I am indebted to the Syndics of the Cambridge University Press for permission to reproduce this figure and also figures 10-14 from the original blocks. 
For the configuration $R$ of closest approach, Darwin gives the value of $r$ as $2 \cdot 343$, but he does not compute the axes.

For the contact configuration $C$, Darwin finds*

$$
r=2 \cdot 372, \quad a=a^{\prime}=1 \cdot 186 .
$$

In these solutions the figures are assumed to be ellipsoidal; the harmonic deformations which have to be superposed will of course bring the vertices closer, so that actual contact will occur before the vertices of the ellipsoids touch. Darwin gives the following figure, which he describes as "highly conjectural " for such a case.

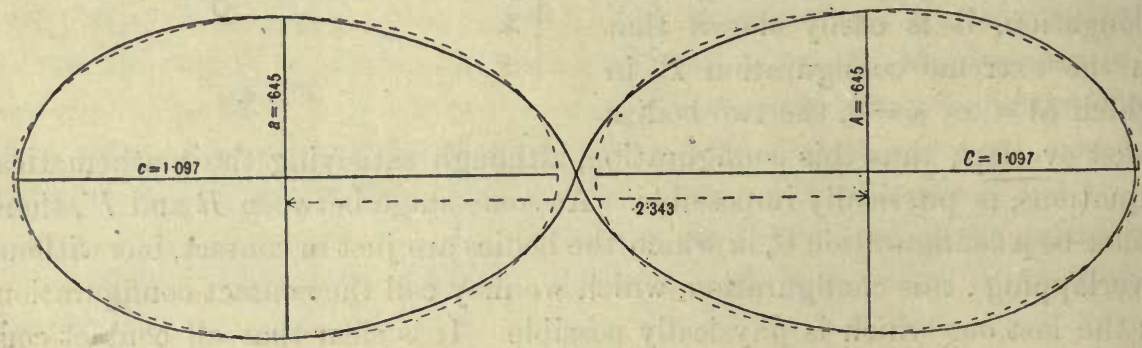

Fig. 10.

Darwin calculates the value of $R$ in the configurations of limiting stability and of closest approach (i.e. the minimum possible value of $R$ ) in some other cases; from his results we can compile the following table:

$$
p=1, \quad 0.8, \quad 0.5, \quad 0.4, \quad 0 .
$$

$R$ (limiting stability) $=2 \cdot 638$,

$2.574,2.59, \infty$.

$R$ (closest approach) $=2 \cdot 343, \quad 2 \cdot 36$,

$2 \cdot 457$.

\section{Partial Stability.}

65. The entry $p=0, R=\infty$ means, as has already been noticed, that there cannot be secular stability for an infinitesimal planet until it has been driven off to infinity. The agency by which this driving off is accomplished is, of course, tidal friction; the satellite $M$ raises tides in the primary $M^{\prime}$; the dissipation of energy in the tides provides the dissipation necessary for secular stability to have any meaning, and the tidal forces result in an acceleration of the small body at the expense of the energy of rotation of the large, this process continuing until the bodies are infinitely far apart.

* Approximately : I have extrapolated to get initial contact in Darwin's table on p. 514 of Coll. Works. Vol. IIr. Stress should not be laid on exact values, as Darwin specially draws attention to the bad convergence of the series used in this and similar calculations. 
On the other hand, if the big body is regarded as a point or rigid sphere, tidal friction cannot operate, and the problem now becomes identical with Roche's problem already discussed. The value of $R$ in limiting stability when $p=0$ is no longer $\infty$, but $2.455 r$. Thus tidal friction in the primary can increase the value of $R$ from this value to infinity.

Darwin describes a system as "partially stable" when it is stable except for the tidal friction arising from the tides in the primary. And he remarks that, inasmuch as tidal friction is a slowly acting cause of instability, partial stability of this kind is from the point of view of cosmical evolution of even greater interest than the full secular stability of the system.

Again, a slightly different problem occurs when the big body is supposed to be an ellipsoid petrified in its configuration of equilibrium, so that the masses are both in equilibrium but tidal friction cannot act on the primary.

Darwin believes that the limit of partial stability of a series of configurations, such as that represented in fig. 8 , can be found by discovering the value at which

$$
\mathbf{M}^{\prime}=\left(M k^{2}+\frac{M M^{\prime}}{M+M^{\prime}} R^{2}\right) \omega
$$

is a minimum, this value $\mathbf{M}^{\prime}$ representing all that part of the moment of momentum which is liable to variation when tides cannot be raised in $M^{\prime}$. A slight modification of the argument of $\$ 64$ will shew that the configuration of closest approach cannot be even "partially stable." It accordingly appears that the configuration of limiting "partial stability" must lie at a point intermediate between $O$ and $R$ in fig. 8. Darwin calculates some configurations of "partial stability," and gives the following table of values for $R$, the closest approach consistent with partial stability, and for the axes of the primary and secondary when in this critical position, a being the mean radius of the combined mass :

\begin{tabular}{|c|c|c|c|c|c|c|c|c|}
\hline \multirow{2}{*}{$p$} & \multirow{2}{*}{$R / \mathfrak{a}$} & \multicolumn{3}{|c|}{ Axes of primary } & \multicolumn{3}{|c|}{ Axes of secondary } & \multirow{2}{*}{$\omega^{2} / 2 \pi \rho$} \\
\hline & & $a / \mathfrak{a}$ & $b / \mathfrak{a}$ & $c / \mathfrak{a}$ & $a^{\prime} / \mathfrak{a}$ & $b^{\prime} / \mathfrak{a}$ & $c^{\prime} / \mathfrak{a}$ & \\
\hline & $2 \cdot 457$ & $1 \div \infty$ & $0.511 \div \infty$ & $0 \cdot 482 \div \infty$ & $1 \cdot 030$ & $1 \cdot 030$ & $0 \cdot 942$ & $0 \cdot 0449$ \\
\hline 0.4 & $2 \cdot 4$ & 0.843 & 0.603 & & & & & 0.0435 \\
\hline 0.5 & $2 \cdot 48$ & 0.8 & & & & & & 0.0434 \\
\hline $0 \cdot 6$ & $2 \cdot 4$ & 0.88 & $0 \cdot 6$ & & & & & 0.0432 \\
\hline $0 \cdot 7$ & $2 \cdot 4$ & 0.9 & & $0 \cdot 6$ & 0.9 & 0.8 & & $0 \cdot 0428$ \\
\hline $0 \cdot 8$ & $2 \cdot 5$ & 0.9 & & & & & & 0.0426 \\
\hline 0.9 & $2 \cdot 508$ & 0.921 & & $0 \cdot 691$ & 0.9 & & & $0 \cdot 0423$ \\
\hline 1.0 & $2 \cdot 514$ & 0.927 & 0.762 & 0.708 & 0.927 & $0 \cdot 762$ & $0 \cdot 708$ & 0.0420 \\
\hline
\end{tabular}

It appears that changes in the ratio of the masses produce surprisingly little change in the critical value of $R / a$. Whatever the ratio of the masses 
it is approximately true that a satellite cannot rotate with partial secular stability round a planet when within a distance less than about $2 \frac{1}{2}$ radii of the combined mass, the densities being the same.

The critical figures for $p=0 \cdot 4,0.7$ and 1.0 are shewn in figures $11,12,13$ which are taken, by permission of the Syndics of the Press, from Sir G. Darwin's Collected Works*.

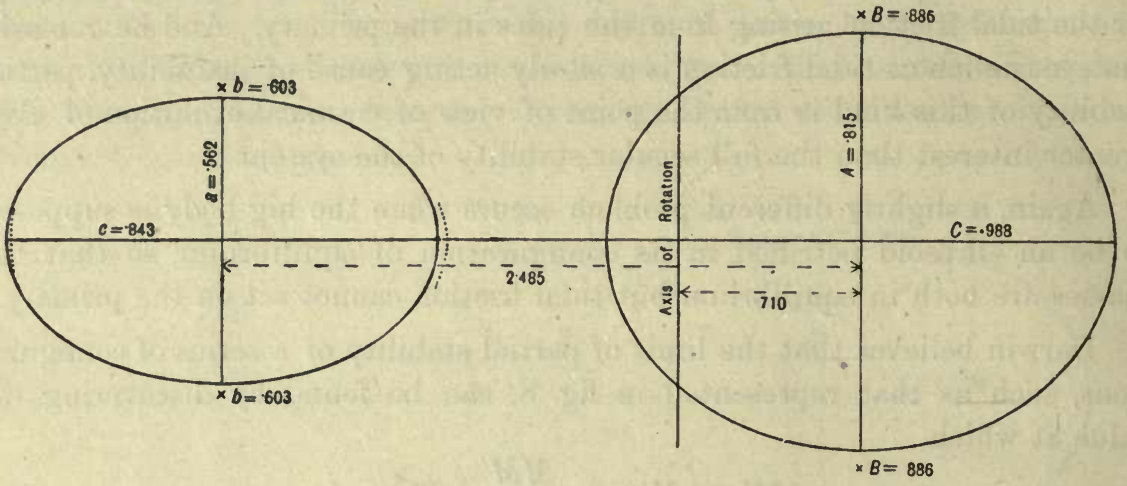

Fig. $11(p=0 \cdot 4)$.

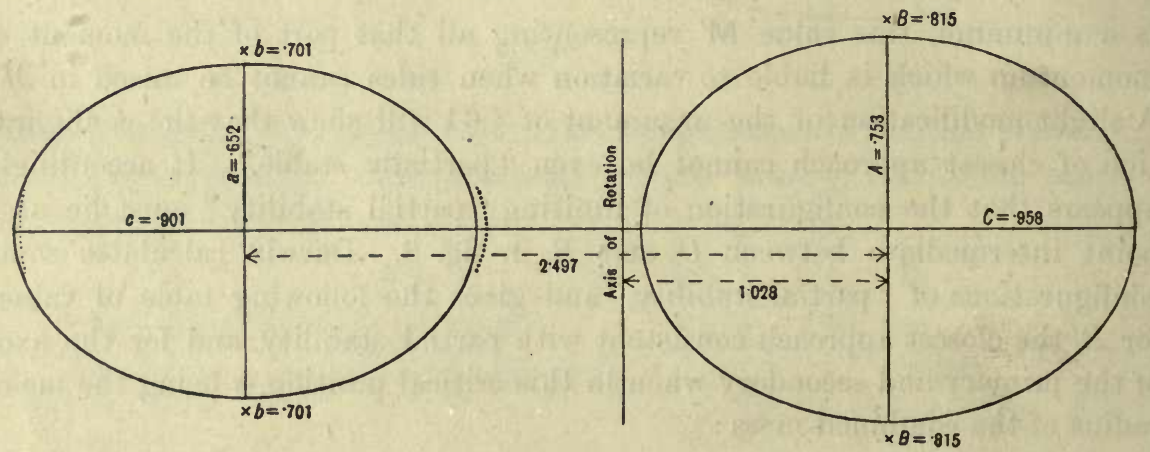

Fig. $12(p=0 \cdot 7)$.

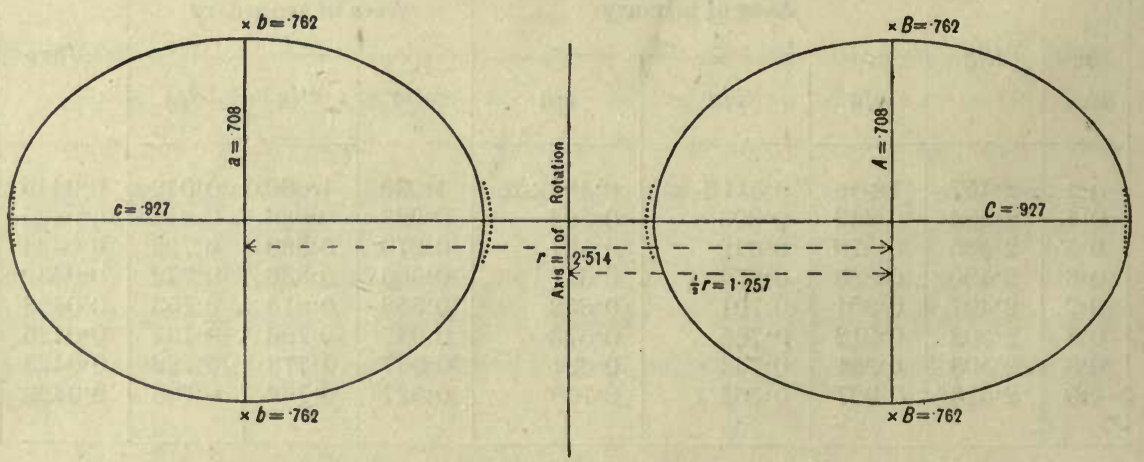

Fig. $13(p=1 \cdot 0)$.

* Vol. IIr. pp. 508, 509. 


\section{CHAPTER IV}

\section{THE GRAVITATIONAL POTENTIAL OF A DISTORTED ELLIPSOID}

66. The last chapter contained a discussion of the ellipsoidal configurations which can occur in the various problems we have had under consideration, and it was found possible to investigate their stability or instability subject to their remaining ellipsoidal. A configuration which is unstable when subject to an ellipsoidal constraint will of course remain unstable when this constraint is removed, but a configuration which is stable before the constraint is removed will not necessarily remain stable. We can only discuss whether such a configuration is stable or not when we have a complete knowledge of all configurations of equilibrium adjacent to the ellipsoidal configurations; we then know the positions of the various points of bifurcation on the ellipsoidal series, and the stability of this series is immediately determined.

A first condition for being able to discover configurations of equilibrium of any type is that we shall be able to write down the potential of the mass when in these configurations. Thus it appears that before being able to discuss in a general way the configurations of equilibrium adjacent to ellipsoidal configurations, we must be able to write down the potential of a distorted ellipsoid.

The method of ellipsoidal harmonics at once suggests itself. It has been used by Poincaré*,'Darwin + , and Schwarzschild to determine configurations of equilibrium adjacent to the equilibrium configurations. In this way the various points of bifurcation on the ellipsoidal series we have had under discussion are readily determined.

After determining the position of points of bifurcation on the ellipsoidal series, the next problem is that of determining whether the branch series through these points are initially stable or unstable, and this, as we have seen, demands a knowledge of the direction of curvature of these branch series at the points of bifurcation. We can only discuss the curvature of this series when the configurations on it are known as far as the second order terms of a parameter $e$, which measures the displacement from the original ellipsoidal configuration. Poincaré§ has devised a method of using ellipsoidal harmonics

* Acta Math. 7, p. 259, and Phil. Trans. 198 A, p. 333.

† Coll. Works, Vol. III. papers 10, 11, 12 and 13.

$\ddagger$ Neue Annalen d. Sternwarte MIünchen, 3 (1897), p. 275, or Inaug. Dissert. München (1896).

$\S$ Phil. Truns. $198 \mathrm{~A}, \mathrm{p} .333$.

J. C. 


\section{The Gravitational Potential of a Distorted Ellipsoid [Cr. IV}

so as to give the potential of a distorted ellipsoid as far as second order terms, but on attempting to apply his method it is found that the second order terms in themselves are inadequate to determine the stability or instability of the branch series; a knowledge of third order terms is demanded.

As Poincarés method does not seem to admit of extension, or at least of easy extension, to the calculation of third order terms, it is found necessary to develop some other method of writing down the third order terms required. Such a method is now given. In the present chapter we confine ourselves entirely to this problem in potential theory; the determination of the configurations of equilibrium being reserved for Chapter $\mathrm{V}$.

\section{GENERAL THEORY}

67. In our discussion of ellipsoidal configurations of equilibrium, the ellipsoid was supposed to be the surface $\lambda=0$ in the family of surfaces

If we write

$$
f \equiv \frac{x^{2}}{a^{2}+\lambda}+\frac{y^{2}}{b^{2}+\lambda}+\frac{z^{2}}{c^{2}+\lambda}-1=0
$$

$$
\psi(\lambda)=-\frac{\pi \rho a b c}{\left\{\left(a^{2}+\lambda\right)^{\frac{1}{2}}\left(b^{2}+\lambda\right)^{\frac{1}{2}}\left(c^{2}+\lambda\right)^{\frac{1}{2}}\right\}}=-\frac{\pi \rho a b c}{\Delta}
$$

then the potential at the point $x, y, z$ of the solid ellipsoid of density $\rho$ is, as in equations (54) and (55), given by

$$
\begin{aligned}
& V_{0}=\int_{\lambda}^{\infty} \psi(\lambda) f d \lambda \\
& V_{i}=\int_{0}^{\infty} \psi(\lambda) f d \lambda
\end{aligned}
$$

where the lower limit $\lambda$ in equation (132) is the positive root of the equation $f=0$.

Suppose the family of surfaces determined by equation (130) to be distorted so that their equation becomes $F=0, F$ being a function of $x, y, z$ and $\lambda$, and let the distortion be such that the surface $\lambda=\infty$ remains at infinity.

We require to find the potential of a homogeneous mass bounded by the surface $\lambda=0$ in the distorted family of surfaces. Let us examine under what conditions it is possible for the external and internal potentials to be given by*

$$
\begin{aligned}
& V_{0}=\int_{\lambda}^{\infty} \psi(\lambda) F d \lambda \\
& V_{i}=\int_{0}^{\infty} \psi(\lambda) F d \lambda
\end{aligned}
$$

* The forms of these equations are of course suggested by analogy with equations (132) and (133). For a direct derivation of equations (134) and (135) see Phil. Trans. 215 A, p. 29. 
the lower limit in equation (134) now being the positive root of the equation

$$
F^{\prime}=0
$$

By differentiation of equation (134) we obtain

$$
\frac{\partial V_{0}}{\partial x}=\int_{\lambda}^{\infty} \psi(\lambda) \frac{\partial F}{\partial x} d \lambda-\psi(\lambda) F \frac{\partial \lambda}{\partial x}
$$

which, in virtue of equation (136), is equivalent to

$$
\frac{\partial V_{0}}{\partial x}=\int_{\lambda}^{\infty} \psi(\lambda) \frac{\partial F}{\partial x} d \lambda
$$

while differentiation of equation (135) gives directly

$$
\frac{\partial V_{i}}{\partial x}=\int_{0}^{\infty} \psi(\lambda) \frac{\partial F}{\partial x} d \lambda
$$

At the boundary, $\lambda=0$; whence it is clear that $V_{0}=V_{i}$ at the boundary and that all the differential coefficients of $V_{0}$ are equal to those of $V_{i}$. At infinity $\lambda=\infty$; whence it appears from equation (134) that $V_{0}=0$ at infinity. It accordingly appears that equations (134) and (135) will give the true values of the potentials provided

$$
\begin{aligned}
& \nabla^{2} V_{0}=0 \\
& \nabla^{2} V_{i}=-4 \pi \rho
\end{aligned}
$$

68. By further differentiation of equation (137), we have

so that

$$
\frac{\partial^{2} V_{0}}{\partial x^{2}}=\int_{\lambda}^{\infty} \psi(\lambda) \frac{\partial^{2} F}{\partial x^{2}} d \lambda-\psi(\lambda) \frac{\partial F}{\partial x} \frac{\partial \lambda}{\partial x}
$$

$$
\nabla^{2} V_{0}=\int_{\lambda}^{\infty} \psi(\lambda) \nabla^{2} F d \lambda-\psi(\lambda) \Sigma \frac{\partial F}{\partial x} \frac{\partial \lambda}{\partial x}
$$

while, for the simpler function $V_{i}$, we have

$$
\nabla^{2} V_{i}=\int_{0}^{\infty} \psi(\lambda) \nabla^{2} F d \lambda .
$$

Now suppose that $F$ is such as to satisfy at all points of space the equation

$$
\int_{0}^{\lambda} \psi(\lambda) \nabla^{2} F d \lambda+\psi(\lambda) \Sigma \frac{\partial F}{\partial x} \frac{\partial \lambda}{\partial x}=-4 \pi \rho
$$

Suppose further that $F$ is such that

at infinity-i.e. when $\lambda=\infty$.

$$
\psi(\lambda) \Sigma \frac{\partial F}{\partial x} \frac{\partial \lambda}{\partial x}=0
$$

Then at infinity equation (143) reduces to

$$
\int_{0}^{\infty} \psi(\lambda) \nabla^{2} F d \lambda=-4 \pi \rho
$$




\section{The Gravitational Potential of a Distorted Ellipsoid [CH. IV}

in which the left-hand member is now a function of $x, y$ and $z$ only. Suppose $F$ to consist of a series of integral algebraic terms in $x, y, z$, then the left-hand member will consist of a series of such terms, and if this expression plus $4 \pi \rho$ vanishes at infinity, it must also vanish at all points of space.

Thus if $F$ is such that equation (143) is satisfied at every point of space, while equation (144) is satisfied at infinity, then equation (145) must also be satisfied at every point of space. Subtracting corresponding sides of these equations, we obtain as a third equation, which must be satisfied at every point of space,

$$
\int_{\lambda}^{\infty} \psi(\lambda) \nabla^{2} F d \lambda-\psi(\lambda) \Sigma \frac{\partial F}{\partial x} \frac{\partial \lambda}{\partial x}=0
$$

By comparison with equations (141) and (142), the last two equations are seen to be equivalent to

$$
\begin{aligned}
& \nabla^{2} V_{0}=0, \\
& \nabla^{2} V_{i}=-4 \pi \rho .
\end{aligned}
$$

Hence it appears that if $F$ is a series of algebraic powers satisfying equation (143), and is such that equation (144) is satisfied at infinity, then the potentials of the homogeneous solid bounded by the surface $\lambda=0$ will be given by equations (134) and (135).

Before leaving this result, we may notice that the limit of integration $\lambda$ is connected with $x, y, z$, the point at which the potential is evaluated, by the relation

$$
F=0
$$

which is true at every point of space. On differentiation we obtain

$$
\frac{\partial F}{\partial x}+\frac{\partial F}{\partial \lambda} \frac{\partial \lambda}{\partial x}=0
$$

which is also true at every point of space. Thus equation (143) which must be satisfied by $F$ may be written in the alternative form

$$
\int_{0}^{\lambda} \psi(\lambda) \nabla^{2} F d \lambda-\psi(\lambda) \frac{\Sigma\left(\frac{\partial F}{\partial x}\right)^{2}}{\frac{\partial F}{\partial \lambda}}=-4 \pi \rho
$$

69. The various possible solutions of this equation, which are such that the second term vanishes at infinity, determine the boundaries of solids whose potentials can be written down in the form of equations (134) and (135). One such solid is already known, namely the ellipsoid. For this $F=f$, so that $F=f$ must be a solution of equation (148) and we must have

$$
\int_{0}^{\lambda} \psi(\lambda) \nabla^{2} f d \lambda-\psi(\lambda) \frac{\Sigma\left(\frac{\partial f}{\partial x}\right)^{2}}{\frac{\partial f}{\partial \lambda}}=-4 \pi \rho
$$


This equation ought to be an identity. That it is so is easily seen with the help of the relation

$$
\Sigma\left(\frac{\partial f}{\partial x}\right)^{2}=-4 \frac{\partial f}{\partial \lambda}
$$

Now assume that a more general solution of equation (148) is

$$
F=f+\phi
$$

in which $\phi$ is any function of $x, y, z$ and $\lambda$. The equation of the family of surfaces $\lambda=$ cons. is now supposed to be

$$
f+\phi=0 \text {. }
$$

Since $\phi$ is so far supposed to be perfectly general, the solution we have under discussion is in reality perfectly general, although written down in a form which is specially applicable to surfaces obtained by distortion from the ellipsoid $f=0$.

Equation (149) is true in any case. If $f+\phi$ is a solution of equation (148) we must also have

$$
\int_{0}^{\lambda} \psi(\lambda) \nabla^{2}(f+\phi)-\psi(\lambda) \frac{\Sigma\left[\frac{\partial}{\partial x}(f+\phi)\right]^{2}}{\frac{\partial}{\partial \lambda}(f+\phi)}=-4 \pi \rho
$$

On subtracting corresponding sides of equations (149) and (152), and simplifying with the help of relation (150), we.obtain

$$
\frac{\partial}{\partial \lambda}(f+\phi) \int_{0}^{\lambda} \psi(\lambda) \nabla^{2} \phi d \lambda=\psi(\lambda)\left\{4\left(\Sigma \frac{x}{A} \frac{\partial \phi}{\partial x}+\frac{\partial \phi}{\partial \lambda}\right)+\Sigma\left(\frac{\partial \phi}{\partial x}\right)^{2}\right\}
$$

\section{SOLUTION OF EQUATIONS}

70. This equation does not admit of direct solution, but may be effectively broken up by assuming a solution

$$
\phi=u+f v
$$

in which $u$ and $v$ are functions of $x, y, z$ and $\lambda$. On substituting this value for $\phi$ and simplifying, equation (153) reduces to

$$
\begin{aligned}
\frac{\partial}{\partial \lambda}(f+\phi) & {\left[\int_{0}^{\lambda} \psi(\lambda) \nabla^{2}(u+f v) d \lambda+4 \psi(\lambda) v\right] } \\
& =\psi(\lambda)\left[4(1+v)\left\{\left(\Sigma \frac{x}{A} \frac{\partial u}{\partial x}+\frac{\partial u}{\partial \lambda}\right)+f\left(\Sigma \frac{x}{A} \frac{\partial v}{\partial x}+\frac{\partial v}{\partial \lambda}\right)\right\}+\Sigma\left(\frac{\partial u}{\partial x}+f \frac{\partial v}{\partial x}\right)^{2}\right]
\end{aligned}
$$

and this will be satisfied if we can satisfy separately the two equations

$$
\begin{array}{r}
\int_{0}^{\lambda} \psi(\lambda) \nabla^{2}(u+f v) d \lambda+4 \Psi(\lambda) v=0 \ldots \ldots . . . . . . \\
4(1+v)\left\{\left(\Sigma \frac{x}{A} \frac{\partial u}{\partial x}+\frac{\partial u}{\partial \lambda}\right)+f\left(\Sigma \frac{x}{A} \frac{\partial v}{\partial x}+\frac{\partial v}{\partial \lambda}\right)\right\}+\Sigma\left(\frac{\partial u}{\partial x}+f \frac{\partial v}{\partial x}\right)^{2}=0
\end{array}
$$




\section{The Gravitational Potential of a Distorted Ellipsoid [CH. Iv}

71. Let us consider equation (155) first. On substituting for $\psi(\lambda)$, this equation reduces to

$$
\int_{0}^{\lambda}\left\{\nabla^{2} u+f \nabla^{2} v+\Sigma \frac{4 x}{A} \frac{\partial v}{\partial x}+2 v\left(\frac{1}{A}+\frac{1}{B}+\frac{1}{C}\right)\right\} \frac{d \lambda}{\Delta}+\frac{4 v}{\Delta}=0
$$

We have, however,

$$
\begin{aligned}
\int_{0}^{\lambda} 2 v\left(\frac{1}{A}+\frac{1}{B}+\frac{1}{C}\right) \frac{d \lambda}{\Delta} & =-\int_{0}^{\lambda} 4 v \frac{\partial}{\partial \lambda}\left(\frac{1}{\Delta}\right) d \lambda \\
& =-\frac{4 v}{\Delta}+\left(\frac{4 v}{\Delta}\right)_{\lambda=0}+\int_{0}^{\lambda} \frac{4}{\Delta} \frac{\partial v}{\partial \lambda} d \lambda
\end{aligned}
$$

so that the equation becomes

$$
\int_{0}^{\lambda}\left\{\nabla^{2} u+f \nabla^{2} v+4\left(\Sigma \frac{x}{A} \frac{\partial v}{\partial x}+\frac{\partial v}{\partial \lambda}\right)\right\} \frac{d \lambda}{\Delta}=-\left(\frac{4 v}{\Delta}\right)_{\lambda=0}
$$

This must be satisfied for all values of $\lambda$, and therefore, in particular, for the value $\lambda=0$. Hence we must have

$$
v=0 \text { when } \lambda=0
$$

It will be remembered that the boundary of the distorted ellipsoid is supposed to be

$$
\frac{x^{2}}{a^{2}}+\frac{y^{2}}{b^{2}}+\frac{z^{2}}{c^{2}}-1+\phi_{\lambda=0}=0
$$

and equation (158) shews that $\phi_{\lambda=0}$ reduces to $u_{\lambda=0}$. Thus different values of $u_{\lambda=0}$ determine different boundaries, and if $u_{\lambda=0}$ can be made perfectly general we can.solve the potential problem for the most general boundary possible.

Since $v$ must vanish when $\lambda=0$, equation (157) becomes

$$
\int_{0}^{\lambda^{\prime}}\left\{\nabla^{2} u+f \nabla^{2} v+4\left(\Sigma \frac{x}{A} \frac{\partial v}{\partial x}+\frac{\partial v}{\partial \lambda}\right)\right\} \frac{d \lambda}{\Delta}=0
$$

in which $\lambda^{\prime}$ is momentarily used to denote the value of $\lambda$ given by the equation $f+\phi=0$.

The most general way of satisfying this equation is to make

$$
\nabla^{2} u+J^{\nabla^{2} v}+4\left(\Sigma \frac{x}{A} \frac{\partial v}{\partial x}+\frac{\partial v}{\partial \lambda}\right)=\Delta \frac{\partial \sigma}{\partial \lambda}
$$

where $\sigma$ may be any function of $x, y, z$ and $\lambda$ which vanishes when $\lambda=\lambda^{\prime}$ and also when $\lambda=0$.

Regarding this as an equation for $v$, let us try a solution

$$
v=w+f w^{\prime}+f^{2} w^{\prime \prime}+\ldots+f^{n} w^{(n)}+\ldots
$$


We find, on differentiating and simplifying,

$$
\begin{gathered}
f \nabla^{2}\left(f^{n} w^{(n)}\right)+4\left(\sum \frac{x}{A} \frac{\partial}{\partial x}+\frac{\partial}{\partial \lambda}\right) f^{n} w^{(n)} \\
=f^{n+1} \nabla^{2} w^{(n)}+4(n+1) f^{n}\left(\sum \frac{x}{A} \frac{\partial}{\partial x}+\frac{\partial}{\partial \lambda}\right) w^{(n)}-4 n \Delta \frac{\partial}{\partial \lambda}\left[\frac{1}{\Delta} f^{n} w^{(n)}\right],
\end{gathered}
$$

so that, on substituting the assumed value (162) for $v$,

$$
\begin{aligned}
\nabla^{2} u & +f \nabla^{2} v+4\left(\Sigma \frac{x}{A} \frac{\partial v}{\partial x}+\frac{\partial v}{\partial \lambda}\right) \\
& =\nabla^{2} u+f \nabla^{2} w+\ldots+f^{n} \nabla^{2} w^{(n-1)}+\ldots \\
& +4\left(\Sigma \frac{x}{A} \frac{\partial w}{\partial x}+\frac{\partial w}{\partial \lambda}\right)+\ldots+4(n+1) f^{n}\left(\Sigma \frac{x}{A} \frac{\partial w^{(n)}}{\partial x}+\frac{\partial w^{(n)}}{\partial \lambda}\right)+\ldots \\
& -\Delta \frac{\partial}{\partial \lambda}\left[\frac{4}{\Delta}\left(f w^{\prime}+2 f^{2} w^{\prime \prime}+\ldots+n f^{n} w^{(n)}+\ldots\right)\right] \ldots \ldots . . . . .(163)
\end{aligned}
$$

Let us now assume $w, w^{\prime}, w^{\prime \prime} \ldots$ to be successively determined by equations of the form

$$
\begin{aligned}
& \left\{\begin{array}{l}
4\left(\Sigma \frac{x}{A} \frac{\partial w}{\partial x}+\frac{\partial w}{\partial \lambda}\right)=-\nabla^{2} u-\Delta \frac{\partial \theta}{\partial \lambda} \\
4\left(\Sigma \frac{x}{A} \frac{\partial w^{\prime}}{\partial x}+\frac{\partial w^{\prime}}{\partial \lambda}\right)=-\frac{1}{2} \nabla^{2} w \ldots \ldots
\end{array}\right. \\
& 4\left(\Sigma \frac{x}{A} \frac{\partial w^{(n)}}{\partial x}+\frac{\partial w^{(n)}}{\partial \lambda}\right)=-\frac{1}{n+1} \nabla^{2} w^{(n-1)}
\end{aligned}
$$

where $\theta$ is as yet undetermined. With the help of these assumed relations, equation (163) becomes

$$
\begin{aligned}
\nabla^{2} u & +f \nabla^{2} v+4\left(\Sigma \frac{x}{A} \frac{\partial v}{\partial x}+\frac{\partial v}{\partial \lambda}\right) \\
& =-\Delta \frac{\partial}{\partial \lambda}\left[\theta+\frac{4}{\Delta}\left(f w^{\prime}+2 f^{2} w^{\prime \prime}+\ldots+n f^{n} w^{(n)}+\ldots\right)\right] \ldots
\end{aligned}
$$

Clearly equation (161) will be satisfied if the quantity in square brackets on the right of equation (167) satisfies the conditions which must be satisfied by $\sigma$ in equation (161). It must therefore be made to vanish when $\lambda=0$ and when $\lambda=\lambda^{\prime}$.

To satisfy this condition, let us now choose for $\theta$ the value

$$
\theta=\frac{4}{\Delta}\left(\frac{u}{1+v}\right)\left[w^{\prime}+2 f w^{\prime \prime}+\ldots+(n+1) f^{n} w^{(n+1)}+\ldots\right] \ldots(168)
$$

then the expression in square brackets on the right of equation (167) reduces to

$$
\frac{4}{\Delta}\left(f+\frac{u}{1+v}\right)\left[w^{\prime}+2 f u^{\prime \prime}+\ldots+(n+1) f^{n} w^{(n+1)}+\ldots\right]
$$




\section{The Gravitational Potential of a Distorted Ellipsvid [CH. Iv}

and this vanishes when $\lambda=\lambda^{\prime}$ through the factor

$$
f+\frac{u}{1+v}=\frac{f+\phi}{1+v}
$$

It must also vanish when $\lambda=0$, and $v$ given by equation (162) must also vanish when $\lambda=0$. These conditions are most easily satisfied by making

$$
w=w^{\prime}=w^{\prime \prime}=\ldots=w^{(n)}=\ldots=0, \text { when } \lambda=0
$$

Thus equations (164)-(166) will contain a complete solution of the original equation if $w, w^{\prime}$, etc. are all chosen so as to vanish when $\lambda=0$, while $\theta$ is given by equation (168).

72. We now turn to equation (156). It is convenient to transform to new variables $\xi, \eta, \zeta, \lambda$ connected with the old variables $x, y, z, \lambda$ by the relations

$$
\xi=\frac{x}{A}, \quad \eta=\frac{y}{B}, \quad \zeta=\frac{z}{C}, \quad \lambda=\lambda
$$

Differentiation with respect to the new variable $\lambda$ is given by

$$
\frac{\partial}{\partial \lambda_{\text {new }}}=\frac{\partial}{\partial \lambda_{\text {old }}}+\Sigma \frac{\partial x}{\partial \lambda} \frac{\partial}{\partial x}=\frac{\partial}{\partial \lambda_{\text {old }}}+\Sigma \frac{x}{A} \frac{\partial}{\partial x}
$$

Expressed in terms of these new coordinates, equation (156) becomes

$$
4(1+v)\left(\frac{\partial u}{\partial \lambda}+f \frac{\partial v}{\partial \lambda}\right)+\Sigma \frac{1}{A^{2}}\left(\frac{\partial u}{\partial \xi}+f \frac{\partial v}{\partial \xi}\right)^{2}=0
$$

In this we may put $f+\phi=0$, or, from equation (169),

$$
f=-\frac{u}{1+v}
$$

and the equation reduces to

$$
4 \frac{\partial}{\partial \lambda}\left(\frac{u}{1+v}\right)+\Sigma \frac{1}{A^{2}}\left\{\frac{\partial}{\partial \xi}\left(\frac{u}{1+v}\right)^{2}\right\}=0
$$

Theoretically, this equation determines $u /(1+v)$, so that a solution of this equation combined with the solution for $v$ obtained in $\$ 71$ will yield a complete solution of the problem. For our present purpose it is convenient to examine solutions in powers of a parameter $e$.

\section{SOLUTION IN POWERS OF A PARAMETER}

73. Equation (174) may readily be solved in powers of a parameter $e$ on assuming a solution of the form

$$
\frac{u}{1+v}=e g_{1}+e^{2} g_{2}+e^{3} g_{3}+\ldots
$$


Inserting this value into equation (174), and equating coefficients of successive powers of $e$, we obtain

$$
\begin{aligned}
& \frac{\partial g_{1}}{\partial \lambda}=0 \\
& \frac{\partial g_{2}}{\partial \lambda}=-\frac{1}{4} \Sigma \frac{1}{A^{2}}\left(\frac{\partial g_{1}}{\partial \xi}\right)^{2} \\
& \frac{\partial g_{3}}{\partial \lambda}=-\frac{1}{4} \Sigma \frac{1}{A^{2}}\left(2 \frac{\partial g_{1}}{\partial \xi} \frac{\partial g_{2}}{\partial \xi}\right), \text { etc. }
\end{aligned}
$$

The first equation shews that $g_{1}$ must be a function of $\xi, \eta, \zeta$ only, say $P$. Write $P_{\xi}$ for $\partial P / \partial \xi$ etc. and put

$$
\mathbf{A}=\frac{1}{a^{2}}-\frac{1}{A} \text { etc. }
$$

so that

$$
\frac{\partial \mathbf{A}}{\partial \lambda}=\frac{1}{A^{2}} \text { etc. }
$$

Then the equation giving $g_{2}$ becomes

$$
\frac{\partial g_{2}}{\partial \lambda}=-\frac{1}{4} \Sigma \frac{\partial \mathbf{A}}{\partial \lambda} P_{\xi}^{2}
$$

so that

$$
g_{2}=-\frac{1}{4}\left(\mathbf{A} P_{\xi^{2}}+\mathbf{B} P_{\eta}{ }^{2}+\mathbf{C} P_{\zeta}{ }^{2}\right)+Q
$$

where $Q$ is a function of $\xi, \eta, \zeta$ only. Similarly we find

$$
g_{3}=\frac{1}{8}\left(A^{2} P_{\xi^{2}} P_{\xi \xi}+\ldots+2 B C P_{\eta} P_{\zeta} P_{\eta \zeta}+\ldots\right)-\frac{1}{2}\left(A P_{\xi} Q_{\xi}+\ldots\right)+R \ldots(178),
$$

where $R$ is another function of $\xi, \eta, \zeta$ only, and so on.

This determines the value of $\frac{u}{1+v}$. To find $u, v$ separately, we return to equations (164)-(166) of p. 71.

Assume for $u, v$ expansions of the form

$$
\begin{aligned}
& u=e u_{1}+e^{2} u_{2}+e^{3} u_{3}+. . \\
& v=e v_{1}+e^{2} v_{2}+e^{3} v_{3}+.
\end{aligned}
$$

The coefficients in these expansions are of course not independent of those in the expansion (175) already assumed for $u /(1+v)$. The relations between them are readily found to be

$$
\begin{aligned}
& u_{1}=g_{1}=P \ldots \ldots \ldots \ldots \ldots \ldots \ldots \ldots \ldots \\
& u_{2}=g_{2}+v_{1} g_{1} \ldots \ldots \ldots \ldots \ldots \\
& u_{3}=g_{3}+v_{1} g_{2}+v_{2} g_{1}, \text { etc. }
\end{aligned}
$$




\section{The Gravitational Potential of a Distorted Ellipsoid [cr. IV}

Let us now assume for $w, w^{\prime}, w^{\prime \prime} \ldots$ expansions of the form

$$
\begin{aligned}
& w=e w_{1}+e^{2} w_{2}+e^{3} w_{3}+\ldots \\
& w^{\prime}=e w_{1}^{\prime}+e^{2} w_{2}^{\prime}+e^{3} w_{3}^{\prime}+\ldots
\end{aligned}
$$

Then the value of $\theta$ given by equation (168) becomes

$$
\begin{aligned}
\theta= & \frac{4}{\Delta}\left[e^{2} g_{1}\left(w_{1}^{\prime}+2 f w_{1}^{\prime \prime}+3 f^{2} w_{1}^{\prime \prime \prime}+\ldots\right)\right. \\
& \left.+e^{3}\left\{g_{2}\left(w_{1}^{\prime}+2 f w_{1}^{\prime \prime}+3 f^{2} w_{1}^{\prime \prime \prime}+\ldots\right)+g_{1}\left(w_{2}^{\prime}+2 f w_{2}^{\prime \prime}+\ldots\right)\right\}+\ldots\right] .
\end{aligned}
$$

Equating coefficients of powers of $e$ in equations (164)-(166) we now obtain

$$
\begin{aligned}
& \left\{\begin{array}{l}
4\left(\Sigma \frac{x}{A} \frac{\partial w_{1}}{\partial x}+\frac{\partial w_{1}}{\partial \lambda}\right)=-\nabla^{2} u_{1} \ldots \ldots \\
4\left(\Sigma \frac{x}{A} \frac{\partial w_{1}^{\prime}}{\partial x}+\frac{\partial w_{1}^{\prime}}{\partial \lambda}\right)=-\frac{1}{2} \nabla^{2} w_{1}, \text { etc. }
\end{array}\right.
\end{aligned}
$$

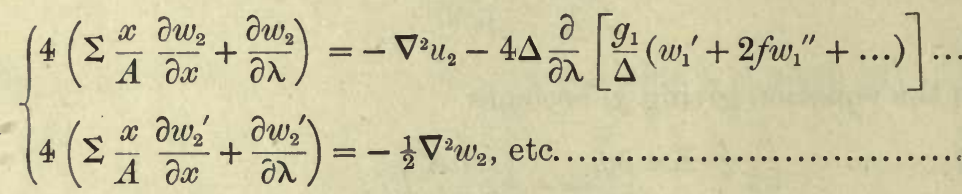

and similar equations.

74. Let us now introduce an operator $D$ defined by

$$
D=\mathbf{A} \frac{\partial^{2}}{\partial \xi^{2}}+\mathbf{B} \frac{\partial^{2}}{\partial \eta^{2}}+\mathbf{C} \frac{\partial^{2}}{\partial \zeta^{2}}
$$

On differentiation with respect to $\lambda$, we find

$$
\frac{\partial D}{\partial \lambda}=\frac{1}{A^{2}} \frac{\partial^{2}}{\partial \xi^{2}}+\frac{1}{B^{2}} \frac{\partial^{2}}{\partial \eta^{2}}+\frac{1}{C^{2}} \frac{\partial^{2}}{\partial \zeta^{2}}
$$

so that $\partial D / \partial \lambda$ is simply $\nabla^{2}$ transformed into $\xi, \eta$, $\zeta$ coordinates.

Transformed into $\xi, \eta, \zeta$ coordinates, equations (184) etc. become (cf. equation (172))

$$
\begin{aligned}
& 4 \frac{\partial w_{1}}{\partial \lambda}=-\frac{\partial D}{\partial \lambda} u_{1} \ldots \ldots . \\
& 4 \frac{\partial w_{1}^{\prime}}{\partial \lambda}=-\frac{1}{2} \frac{\partial D}{\partial \lambda} w_{1} \ldots \ldots \\
& 4 \frac{\partial w_{1}^{\prime \prime}}{\partial \lambda}=-\frac{1}{3} \frac{\partial D}{\partial \lambda} w_{1}^{\prime}, \text { etc. }
\end{aligned}
$$

We have already found that $u_{1}=P$, a function of $\xi, \eta, \xi$ only, so that equation (189) has the integral

$$
w_{1}=-\frac{1}{4} D P,
$$


no constant of integration being added since $w_{1}$ must vanish when $\lambda=0$. Integrating equations (190), (191)... in turn, we find

$$
\begin{aligned}
& w_{1}^{\prime}=\frac{1}{(2 !)^{2} 4^{2}} D^{2} P, \\
& w_{1}^{\prime \prime}=-\frac{1}{(3 !)^{2} 4^{3}} D^{3} P, \\
& \cdots \cdots \cdots \cdots \cdots \cdots \cdots \cdots \cdots \cdots \cdots \cdots \cdots \cdots \\
& w_{1}^{(n)}=\frac{(-1)^{n+1}}{\{(n+1) !\}^{2} 4^{n+1}} D^{n+1} P .
\end{aligned}
$$

If we suppose $\phi$ expanded in powers of the parameter $e$ in the form

we have

$$
\phi=e \phi_{1}+e^{2} \phi_{2}+e^{3} \phi_{3}+\ldots
$$

$$
\begin{aligned}
\phi_{1} & =u_{1}+f w_{1}+f^{2} w_{1}^{\prime}+f^{3} w_{1}^{\prime \prime}+\ldots \\
& =P-\left(\frac{1}{4} f\right) D P+\frac{1}{2^{2}}\left(\frac{1}{4} f\right)^{2} D^{2} P-\frac{1}{2^{2} 3^{2}}\left(\frac{1}{4} f\right)^{3} D^{3} P+\ldots
\end{aligned}
$$

75. To evaluate terms in $e^{2}$, we proceed to equations (186) and (187). For brevity, we shall limit our discussion to that particular type of distortion which ultimately proves to be of importance for the problem immediately in hand. For this, as will appear in the next chapter, $u_{1}$ is of degree 3 in $\xi, \eta, \zeta$. Equations (184) and (185) accordingly. shew that $w_{1}$ must be of degree unity, and $w_{1}^{\prime}$ must vanish. Similarly, $u_{2}$ will be found to be of degree 4 , so that $w_{2}$ is of degree $2, w_{2}^{\prime}$ of degree zero and $w_{2}^{\prime \prime}=0$. Again $u_{3}$ will be of degree $5, w_{3}$ of degree $3, w_{3}^{\prime}$ of degree unity, $w_{3}^{\prime \prime}=0$, and so on.

The value of $v_{1}$ is accordingly

$$
v_{1}=-\frac{1}{4} D P=-\frac{1}{4}\left(\mathbf{A} P_{\xi \xi}+\mathbf{B} P_{\eta \eta}+\mathbf{C} P_{\zeta \zeta}\right)
$$

Proceeding to the determination of second order terms, we have from equations (182) and (177),

$$
\begin{aligned}
& u_{2}=g_{2}+v_{1} g_{1} \\
& =-\frac{1}{4}\left(\mathbf{A} P_{\xi}{ }^{2}+\mathbf{B} P_{\eta}{ }^{2}+\mathbf{C} P_{\zeta}{ }^{2}\right)+Q-\frac{1}{4} P\left(\mathbf{A} P_{\xi \xi}+\mathbf{B} P_{\eta \eta}+\mathbf{C} P_{\zeta \zeta}\right) \\
& =-\frac{1}{8} D P^{2}+Q
\end{aligned}
$$

The value of $w_{2}$ can next be found from equation (186). The right-hand member reduces to $-\nabla^{2} u_{2}$, and the equation, expressed in $\xi, \eta, \zeta$ coordinates, becomes

$$
4 \frac{\partial w_{2}}{\partial \lambda}=-\frac{\partial D}{\partial \lambda} u_{2}=\frac{1}{8} \frac{\partial D}{\partial \lambda} D P^{2}-\frac{\partial D}{\partial \lambda} Q
$$

so that

$$
w_{2}=\frac{1}{64} D^{2} P^{2}-\frac{1}{4} D Q
$$

while similarly equation (136) leads to

$$
w_{2}^{\prime}=-\frac{1}{1536} D^{3} P^{2}+\frac{1}{64} D^{2} Q
$$




\section{The Gravitational Potential of a Distorted Ellipsoid [CH. IV}

Thus collecting results, we find for the second order terms,

$$
u_{2}+f v_{2}=Q-\frac{1}{8} D P^{2}+f\left\{\frac{1}{64} D^{2} P^{2}-\frac{1}{4} D Q\right\}+f^{2}\left\{-\frac{1}{1536} D^{3} P^{2}+\frac{1}{64} D^{2} Q\right\} \text {. }
$$

Proceeding in the same way, we find for the third order terms*,

$$
\begin{aligned}
u_{3}+f v_{3}=u_{3}+ & f\left(w_{3}+f w_{3}^{\prime}\right) \\
=\frac{1}{32} D^{2} & \left(\frac{1}{6} P^{3}\right)-\frac{1}{4} D(P Q)+R \\
& -\frac{1}{4} f\left\{\frac{1}{96} D^{3}\left(\frac{1}{6} P^{3}\right)-\frac{1}{8} D^{2}(P Q)+D R\right\} \\
& +\frac{1}{32} f^{2}\left\{\frac{1}{384} D^{4}\left(\frac{1}{6} P^{3}\right)-\frac{1}{24} D^{3}(P Q)+\frac{1}{2} D^{2} R\right\},
\end{aligned}
$$

and this completes the solution as far as the third order of small quantities.

76. The solution which has been obtained is found, on collecting terms to be

$$
\begin{aligned}
\phi= & e\left(u_{1}+f v_{1}\right)+e^{2}\left(u_{2}+f v_{2}\right)+e^{3}\left(u_{3}+f v_{3}\right) \\
= & e\left[P-\frac{1}{4} f D P\right] \\
& +e^{2}\left[Q-\frac{1}{8} D P^{2}+f\left\{\frac{1}{64} D^{2} P^{2}-\frac{1}{4} D Q\right\}+f^{2}\left\{-\frac{1}{1536} D^{3} P^{2}+\frac{1}{64} D^{2} Q\right\}\right] \\
& +e^{3}\left[R-\frac{1}{4} D P Q+\frac{1}{192} D^{2} P^{3}-\frac{1}{4} f\left\{\frac{1}{56} D^{3} P^{3}-\frac{1}{8} D^{2} P Q+D R\right\}\right. \\
& \left.\left.\quad+\frac{1}{32} f^{2}\left\{\frac{1}{2304} D^{4} P^{3}-\frac{1}{24} D^{3} P Q+\frac{1}{2} D^{2} R\right\}\right] \ldots \ldots \ldots \ldots \ldots \ldots . . . . . . . . .197\right)
\end{aligned}
$$

Putting $\lambda=0$, the value of $\phi$ at the boundary is seen to be

$$
\phi_{0}=e P_{0}+e^{2} Q_{0}+e^{3} R_{0}
$$

and since $P_{0}, Q_{0}, R_{0}$ are entirely at our disposal, this value of $\phi_{0}$ is capable of representing a general distortion of the fundamental ellipsoid as far as the third order of small quantities.

This same distortion might of course have been supposed to be merely

$$
\phi_{0}=e P_{0}
$$

it being at once possible to pass to the general form (198) by replacing $P_{0}$ by $P_{0}+e Q_{0}+e^{2} R_{0}$. We have introduced $Q_{0}$ and $R_{0}$ separately on account of the limitation which has been imposed that $P_{0}$ shall not be of degree above the third.

77. When this limitation is removed, equation (199) may be regarded as representing the most general distortion of any kind which can be experienced by the fundamental ellipsoid. By analogy with equation (197) the corresponding value of $\phi$ is seen to be

$$
\begin{aligned}
\phi= & e\left[P-\frac{1}{4} f D P+\frac{1}{2^{2}}\left(\frac{1}{4} f\right)^{2} D^{2} P-\frac{1}{2^{2} \cdot 3^{2}}\left(\frac{1}{4} f\right)^{3} D^{3} P-\ldots\right] \\
& -\frac{1}{8} e^{2}\left[D P^{2}-\frac{1}{8} f D^{2} P^{2}+\frac{1}{192} f^{2} D^{3} P^{2}-\frac{1}{9216} f^{3} D^{4} P^{2}+\ldots\right] \\
& +\frac{1}{192} e^{3}\left[D^{2} P^{3}-\frac{1}{2} f D^{3} P^{3}+\frac{1}{384} f^{2} D^{4} P^{3}-\ldots\right], \text { etc. } \ldots \ldots
\end{aligned}
$$

* For details of the calculation, see Phil. Trans. 217 A (1916), p. 7. 
I have not succeeded in obtaining a general direct proof of this formula, but it is easy to verify it $\grave{a}$ posteriori for all the cases in which it is used in the present book.

It may be noticed that if

$$
P_{0}=F\left(\frac{x}{a^{2}}, \frac{y}{b^{2}}, \frac{z}{c^{2}}\right)
$$

then the value of $P$ is

$$
P=F^{\prime}\left(\frac{x}{a^{2}+\lambda}, \frac{y}{b^{2}+\lambda}, \frac{z}{c^{2}+\lambda}\right)=F(\xi, \eta, \zeta) .
$$

It appears that a term of degree $n$ in $P_{0}$ gives rise to first order terms of degrees $n, n-2, n-4, \ldots$, second $2 n-2,2 n-4,2 n-6, \ldots$, third $\quad " \quad$ $\quad 3 n-4,3 n-6,3 n-8, \ldots$

and so on. 


\section{CHAPTER V}

\section{PEAR-SHAPED CONFIGURATIONS OF EQUILIBRIUM}

78. In Chapter III we discovered the existence of a number of series of ellipsoidal configurations of equilibrium. We were able to examine the stability of these configurations subject to the restriction that they were constrained to remain ellipsoidal. When it was possible for them to be distorted from the ellipsoidal shape, it was not found feasible to examine their general stability because we had no means of writing down the gravitational potential of a distorted ellipsoid.

The investigation of Chapter IV has now provided us with a formula for the potential of a distorted ellipsoid, and we can proceed to search for configurations of equilibrium which are of the shape of distorted ellipsoids. In this way we discover the points of bifurcation on the ellipsoidal series already discussed, and so obtain a complete knowledge of the stability of these series.

Let us take the equation of the general distorted ellipsoid to be

$$
\frac{x^{2}}{a^{2}}+\frac{y^{2}}{b^{2}}+\frac{z^{2}}{c^{2}}-1+e P_{0}=0
$$

As far as first powers of $e$, the internal potential of the solid whose boundary is given by equation (201) is

where

$$
V_{i}=-\pi \rho a b c \int_{0}^{\infty} \frac{f+e \phi_{1}}{\Delta} d \lambda
$$

$$
\phi_{1}=P-\frac{1}{4} f D P+\frac{1}{4}\left(\frac{1}{4} f\right)^{2} D^{2} P-
$$

and the potential at the boundary, $V_{b}$, is given by the same formula.

\section{GENERAL THEORY.}

79. Let us apply this to the general double-star problem discussed in $\S 50$. So long as we are concerned only with the search for configurations of equilibrium, this problem, as we have seen $(\$ 52)$, includes the rotational and tidal problems as special cases, although the problems become separate when questions of stability are discussed.

The condition that the surface (201) shall be a figure of equilibrium for the primary mass in the double-star problem is (cf. $§ 51)$ that

$$
V_{b}+\frac{M^{\prime}}{R^{3}}\left(x^{2}-\frac{1}{2} y^{2}-\frac{1}{2} z^{2}\right)+\frac{1}{2} \omega^{2}\left(x^{2}+y^{2}\right)=-\pi \rho a b c \theta\left(\frac{x^{2}}{a^{2}}+\frac{y^{2}}{b^{2}}+\frac{\dot{z}^{2}}{c^{2}}-1+e P_{0}\right)
$$


at all points of the boundary. This equation must be satisfied when $e=0$, and for all values of $e$ for which $e^{2}$ may be neglected. Equating coefficients, we obtain the necessary and sufficient conditions of equilibrium,

$$
\begin{array}{ll}
J_{A}-\frac{\mu}{\pi \rho a b c}-\frac{\omega^{2}}{2 \pi \rho a b c} & =\frac{\theta}{a^{2}} . \\
J_{B}+\frac{\mu}{2 \pi \rho a b c}-\frac{\omega^{2}}{2 \pi \rho a b c} & =\frac{\theta}{b^{2}} \\
J_{C}+\frac{\mu}{2 \pi \rho a b c} & =\frac{\theta}{c^{2}} \\
\int_{0}^{\infty} \frac{\phi_{1}}{\Delta} d \lambda & =\theta P_{0}
\end{array}
$$

Equations (205) to (207) are naturally the same as equations (91) to (93) of $\$ 52$; it follows that $\theta$ is the same as before, and that $a, b, c$ as functions of $\mu$ and $\omega^{2}$ are also the same as before.

Since the value of $V_{i}$ given by equation (202) must satisfy $\nabla^{2} V_{i}=-4 \pi \rho$, we at once have

$$
\nabla^{2} \int_{0}^{\infty} \frac{f+e \phi_{1}}{\Delta} d \lambda=\frac{4}{a b c}
$$

giving, on differentiation with respect to $e$,

$$
\nabla^{2} \int_{0}^{\infty} \frac{\phi_{1}}{\Delta} d \lambda=0
$$

so that, in virtue of equation (208), $\nabla^{2} P_{0}=0$, and $P_{0}$ is a spherical harmonic.

80. Not every spherical harmonic will give a possible value for $P_{0}$. For, from the general value of $\phi_{1}$ as given in equation (203), it is clear that a term in $P_{0}$ of degree $n$ in $x, y, z$ will give rise to terms of degrees $n, n-2, n-4, \ldots$ in $\phi_{1}$, and so to terms of similar degrees on the left-hand of equation (208). From the form of equations (208) and (203) it is readily seen that the most general form which will be possible for $P_{0}$ will be a spherical harmonic containing terms of degrees $n, n-2, n-4, \ldots$, these terms only differing from one another by even powers of $x^{2}, y^{2}, z^{2}$. There will be as many values for $P_{0}$ as there are independent spherical harmonics, for when the terms of degree $n$ are given, those of degrees $n-2, n-4$ can always be determined. Thus the values of $P_{0}$ correspond exactly to the different spherical harmonics, although not identical with them.

81. This result can be obtained rather more simply by direct harmonic analysis. Poincaré ${ }^{*}$ has given the requisite analysis for the special rotational problem ; and inasmuch as the form of equations (203) and (208) have nothing to do with special values of $\omega^{2}$ and $\mu$, it is equally true for the more general problem now in hand. 
The condition that equation (208) shall be satisfied for any value of $P_{0}$ is exactly the same as that Poincaré's "coefficient of stability" (cf. § 23) shall vanish, and Poincaré gives, in terms of harmonic analysis, a very full account of the conditions under which this can occur*. The discussion is too long to insert here, and too intricate to summarise; the principal results obtained are the following:

(i) The only type of harmonic for which equation (208) can be satisfied (or Poincaré's "coefficient of stability" vanish) is the zonal type.

(ii) As the ellipsoid lengthens, the first harmonic for which equation (208) can be satisfied is the third zonal harmonic, and beyond this point the equation can be satisfied in turn for zonal harmonics of all orders from 4 to $\infty$.

Poincaré's discussion, being concerned only with the rotational problem, deals only with the Jacobian series of ellipsoids, but for the reasons stated above, is equally applicable to all our ellipsoidal configurations.

It follows that on each of the series represented in fig. 7 (p. 50), as we proceed from $S$ to $\left(J^{\prime}\right)_{\infty}$, we must pass an infinite number of points of bifurcation. Each corresponds to some value of $P_{0}$ in equation (201), and the different values of $P_{0}$ are all zonal harmonics; the first point of bifurcation is that for which $P_{0}$ is of the third degree.

82. The necessity of this result can easily be seen from physical considerations, although it would probably not be easy to construct a rigorous proof.

Let $W$ denote the total potential energy of the fluid mass under its own gravitational forces and the statical field of force (fictitious or otherwise) arising from rotation and tidal action. When a displacement occurs such that the equation of the boundary is altered by the addition of the term $e P_{0}$, as in equation (201), let the new potential energy be $W+\delta W$.

Since the original configuration was one of equilibrium, $\delta W$ will necessarily be of the second order of small quantities, and the original equilibrium will have been unstable if $\delta W$ can be made negative for any value of $P_{0}$.

If $m$ is the mass of any particle of the fluid, and $V$ its potential in the original configuration, the value of $W$ can be put in the form

$$
W=-\frac{1}{2} \Sigma m V .
$$

Now let a displacement occur such that the typical particle of mass $m$ is moved to a position at which the potential in the old configuration was $V^{\prime}$,

* L.c., $\S \S 10$ and 12. See also Schwarzschild, Inaug. Dissert. München (1896), and Darwin, Coll. Works, ril, pp. 302, 307. 
and in the new configuration is $V^{\prime \prime}$. The new potential energy will be $-\frac{1}{2} \Sigma m V^{\prime \prime}$, so that we may write

$$
\delta W=\frac{1}{2} \Sigma m\left(V-V^{\prime}\right)+\frac{1}{2} \Sigma m\left(V^{\prime}-V^{\prime \prime}\right) .
$$

The first term $\frac{1}{2} \Sigma m\left(V-V^{\prime}\right)$ represents the work which would be done in effecting the displacement if the equipotentials remained fixed in space. The boundary originally was an equipotential, so that the work done will be that of moving certain matter from positions inside this equipotential to new positions outside. It is therefore necessarily positive, and the ellipsoidal configuration will be unstable if a displacement can be found such that $\frac{1}{2} \Sigma m\left(V^{\prime}-V^{\prime \prime}\right)$ is negative and numerically greater than the first term.

In the displacement just considered let $\delta n$ denote an average normal depth of matter which may be supposed excavated from one part of the surface and piled up on other parts, so that $\rho \delta n$ is the average mass removed per unit area of surface. The mean change of potential $V^{\prime}-V^{\prime \prime}$ for such matter will have an average value comparable with $(\partial V / \partial n) \delta n$, so that the work done will be of the order of magnitude of

$$
\iint \rho \frac{\partial V}{\partial n}(\delta n)^{2} d S
$$

The integral is only taken over those parts of the surface where the displacement consists of a depression; this may be supposed to be half of the entire surface. Remembering that

$$
\iint \frac{\partial V}{\partial n} d S=-4 \pi M
$$

when the integral is taken over the whole surface we readily find that expression (210) is of the order of magnitude of

$$
-2 \pi \rho M(\delta n)^{2}
$$

This is the negative value of the first term on the right of equation (209). We now proceed to consider the value of the second term.

Values of $P_{0}$ which are of degrees $0,1,2$ in $x, y, z$ result in displacements which give rise only to new ellipsoids, so that we need only concern ourselves with values of $P_{0}$ which are of degrees 3 and higher. Displacements in which $P_{0}$ is of degree higher than 2 produce a furrow or system of furrows in the original ellipsoid. When there are a great number of furrows, either the ellipsoid must be very long or the furrows very close together. In the latter case the second term on the right of equation (209) becomes very small, through the gravitational effects of successive elevations and depressions neutralising one another. No corresponding effect occurs in the first term on the right of equation (209), of which the value is represented by expression (211). Hence it is seen that the ellipsoid can only become unstable through a many-furrowed distortion when it is itself very long. It is easily seen that the more furrows there are in the distortion the longer the ellipsoid has to 
be before instability can set in through this distortion. It follows that the ellipsoid will first become unstable through a distortion in which the number of furrows is the fewest possible, namely one, and this is the third zonal harmonic distortion.:

83. The value of $P$ at the first point of bifurcation on every ellipsoidal series must accordingly be of the form

$$
P=\xi\left(\alpha \xi^{2}+\beta \eta^{2}+\gamma \zeta^{2}+\kappa\right)
$$

and the corresponding value of $P_{0}$ is

$$
P_{0}=\frac{x}{a^{2}}\left(\alpha \frac{x^{2}}{a^{4}}+\beta \frac{y^{2}}{b^{4}}+\gamma \frac{z^{2}}{c^{4}}+\kappa\right)
$$

We obtain at once from equation (203),

$$
\begin{aligned}
\phi_{1}= & \frac{x}{A}\left\{\alpha \frac{x^{2}}{A^{2}}+\beta \frac{y^{2}}{B^{2}}+\gamma \frac{z^{2}}{C^{2}}\right\} \\
& -\frac{1}{2}\left(\frac{x^{2}}{A}+\frac{y^{2}}{B}+\frac{z^{2}}{C}-1\right)\left(\frac{3 \alpha \lambda}{a^{2} A}+\frac{\beta \lambda}{b^{2} B}+\frac{\gamma \lambda}{c^{2} C}\right) \frac{x}{A}+\kappa \frac{x}{A}
\end{aligned}
$$

so that we may write

$$
\int_{0}^{\infty} \frac{\phi_{1}}{\Delta} d \lambda=x\left(a_{1} x^{2}+a_{2} y^{2}+a_{3} z^{2}+a_{4}\right)
$$

Equation (208) can now be satisfied, and on equating coefficients we obtain

$$
\left.\begin{array}{l}
a_{1}=\theta \frac{\alpha}{a^{6}} \\
a_{2}=\theta \frac{\beta}{a^{2} b^{4}} \\
a_{3}=\theta \frac{\gamma}{a^{2} c^{4}} \\
a_{4}=\theta \frac{\kappa}{a^{2}}
\end{array}\right\}
$$

If we introduce new functions of $a, b, c$ defined by

$$
c_{1}=\int_{0}^{\infty} \frac{\lambda d \lambda}{\Delta A B C}, \quad c_{2}=\int_{0}^{\infty} \frac{\lambda d \lambda}{\Delta A^{2} C} ; \quad c_{3}=\int_{0}^{\infty} \frac{\lambda d \lambda}{\Delta A^{2} B}
$$

.then equations (216) are found to assume the form

$$
\begin{aligned}
& \frac{\alpha}{2 a^{2}}\left(c_{2}+c_{3}\right)-\frac{\beta}{2 b^{2}} c_{3}-\frac{\gamma}{2 c^{2}} c_{2}=\theta \frac{\alpha}{a^{6}} \\
& -\frac{3 a}{2 a^{2}} c_{8}+\frac{\beta}{2 b^{2}}\left(3 c_{3}+c_{1}\right)-\frac{\gamma}{2 c^{2}} c_{1}=\theta \frac{\beta}{a^{2} b^{4}} \\
& -\frac{3 \alpha}{2 a^{2}} c_{2}-\frac{\beta}{2 b^{2}} c_{1}+\frac{\gamma}{2 c^{2}}\left(3 c_{2}+c_{1}\right)=\theta \frac{\gamma}{a^{2} c^{4}} \\
& \frac{3 a}{2 a^{2}} \int_{0}^{\infty} \frac{\lambda d \lambda}{\Delta A^{2}}+\frac{\beta}{2 b^{2}} \int_{0}^{\infty} \frac{\lambda d \lambda}{\Delta A B}+\frac{\gamma}{2 c^{2}} \int_{0}^{\infty} \frac{\lambda d \lambda}{\Delta A C}+\kappa \int_{0}^{\infty} \frac{d \lambda}{\Delta A}=\theta \frac{\kappa}{a^{2}} .
\end{aligned}
$$


Mutiplying equations (218) to (220) by $3,1,1$ and adding, we find

$$
\frac{3 \alpha}{a^{4}}+\frac{\beta}{b^{4}}+\frac{\gamma}{c^{4}}=0
$$

which is of course merely the condition that $P_{0}$ shall be harmonic.

The elimination of $\alpha / a^{2}, \beta / b^{2}$ and $\gamma / c^{2}$ from these same three equations gives

$$
\begin{aligned}
a^{2} b^{2} c^{2} & \left(\frac{3}{a^{2}}+\frac{1}{b^{2}}+\frac{1}{c^{2}}\right)\left(c_{1} c_{2}+c_{1} c_{3}+3 c_{2} c_{3}\right) \\
& -\frac{2 \theta}{a^{2}}\left[c_{1}\left(b^{2}+c^{2}\right)+c_{2}\left(3 a^{2}+c^{2}\right)+c_{3}\left(3 a^{2}+b^{2}\right)\right]+\left(\frac{2 \theta}{a^{2}}\right)^{2}=0 \ldots
\end{aligned}
$$

in which we may insert the value of $\theta$ obtained by eliminating $\mu$ and $\omega^{2}$ from equations (205) to (207), namely

$$
\theta=\left(\frac{1}{a^{2}}-\frac{1}{b^{2}}+\frac{3}{c^{2}}\right) /\left(J_{A}-J_{B}+3 J_{C}\right)
$$

Equation (223) now becomes purely an equation in $a, b$ and $c$; it is the equation which determines the first point of bifurcation on any linear series of ellipsoidal configurations.

84. Let us limit our discussion to ellipsoids such that $a b c=r_{0}^{3}$. On replacing $c$ by $r_{0}^{3} / a b$, equation (223) becomes an equation in $a, b$ only, and so may be represented by a curve in a diagram such as that in fig. 7 (p. 50). We may examine in particular the points in which this curve will meet the spheroidal series of tidal figures and the Jacobian series of rotational figures, or, more directly, we may search for the first points of bifurcation on these series.

\section{Tidal Figures}

85. On the tidal series of spheroids, $b=c$, so that $c_{2}=c_{3}$, and for a zonal harmonic distortion we have also $\beta=\gamma$. Thus equations (219) and (220) become identical, each reducing to

$$
-\frac{3 \alpha}{2 a^{2}} c_{2}+\frac{3 \beta}{2 c^{2}} c_{2}=\theta \frac{\beta}{a^{2} c^{4}},
$$

while equation (222) becomes

$$
-\frac{3 \alpha}{a^{4}}=\frac{2 \beta}{c^{4}} .
$$

Eliminating $\alpha, \beta$ from these equations, and inserting the value of $\theta$ from equation (81) we find,

$$
c_{2}=\frac{2 \theta}{a^{2}\left(3 c^{2}+2 a^{2}\right)}=\frac{4}{a\left(3 c^{2}+2 a^{2}\right)\left(c^{2}+2 a^{2}\right)}
$$


In the special case of $b=c$ the integral $c_{2}$ can be integrated in finite terms, and equation (225) is found to assume the form

$$
\frac{4}{3 e^{4}}-\frac{5}{e^{6}}+\frac{5-3 e^{2}}{2 e^{7}} \log \left(\frac{1+e}{1-e}\right)=\frac{4}{\left(3-e^{2}\right)\left(5-3 e^{2}\right)}
$$

where $e$ is the eccentricity, given by $e^{2}=\left(a^{2}-c^{2}\right) / a^{2}$. On numerical treatment, it is found that there is only one root of this equation, namely

$$
e=\cdot 947741
$$

The corresponding values of the semi-axes are

$$
a=2 \cdot 14175 r_{0}, \quad b=c=\cdot 683307 r_{0}
$$

and the value of $\mu$ is $\mu=\cdot 1091311$.

On comparing this with the discussion of the spheroidal series given in $\$ 48-51$, we at once see that this first point of bifurcation is beyond the point at which $\mu$ reached its maximum. It accordingly follows that all configurations on the spheroidal series are stable up to the point at which $\mu$ reaches its maximum $(e=\cdot 882579 ; \mu=\cdot 125504 \pi \rho)$, and all configurations beyond this are unstable.

\section{Rotational Figures}

86. The determination of the first point of bifurcation on the Jacobian series is a much more arduous task. The integrals cannot be evaluated in
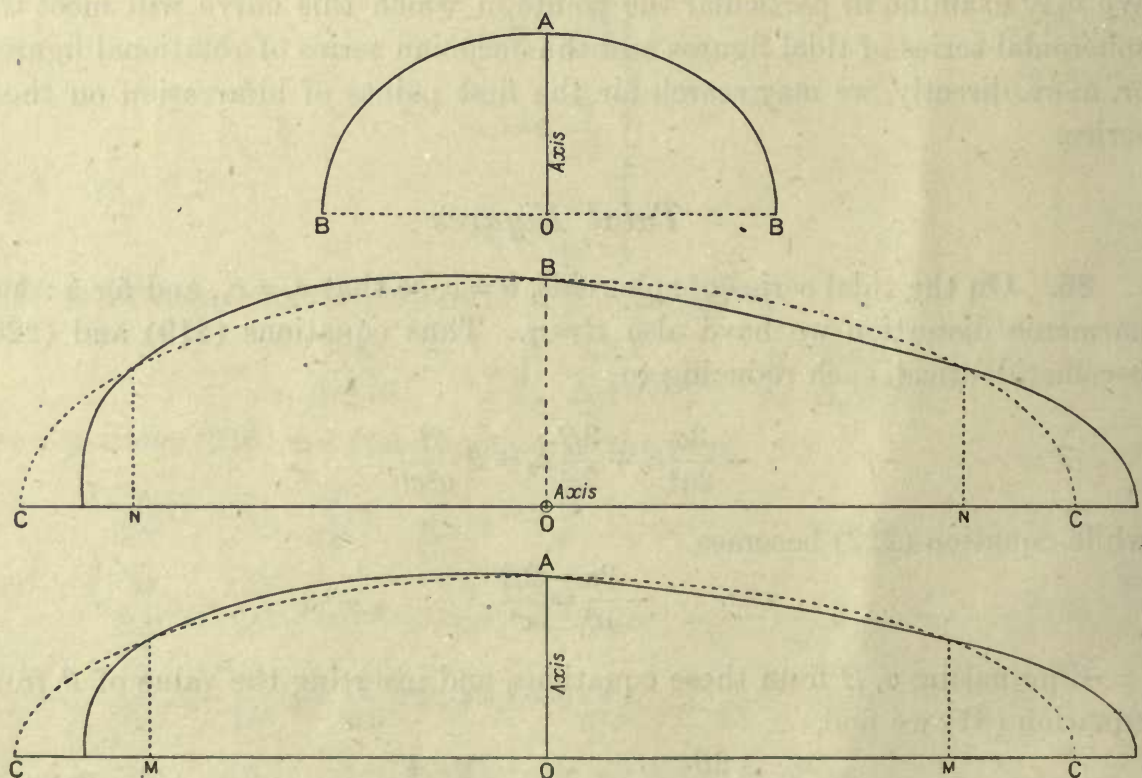

Fig. 14 . 
finite terms, and the equations can only be solved by trial and error. The calculations have been carried through by Sir G. Darwin*, and the-solution he obtains is

$$
a=1.885827 r_{0}, \quad b=\cdot 81,4975 r_{0}, \quad c=\cdot 650659 r_{0}
$$

the corresponding value of $\omega^{2} / 2 \pi \rho$ being 1419990 . The shape of the ellipsoid, together with the pear-shaped figure derived from it, is shewn in fig. 14.

\section{Double-star Figures}

87. The two points which have just been determined enable us to fix with fair approximation the curve given by equation (223), which is the locus of all first points of bifurcation.

For in fig. 15, which reproduces that part of fig. 7 in which the series of ellipsoidal configurations lie, the two points just determined are represented by the points $B^{\prime}$ and $B^{\prime \prime}$. These points are so near to one another that we may regard the straight line $B^{\prime} B^{\prime \prime}$ as a sufficiently good approximation to the position of the locus in this part of the plane.

The curved line $S R^{\prime \prime} T^{\prime \prime}$ represents the locus of points at which the ellipsoidal configurations were found in Chap. III to become unstable through the angular momentum becoming a minimum. It is clear that the line $B^{\prime} B^{\prime \prime}$ cannot cross into the area marked off by this line, so that all configurations on the line $B^{\prime} B^{\prime \prime}$ must already have become unstable in the double-star problem.

88. The results which have been obtained can be now summarised, with reference to fig. 15 (on the next page), as follows :

In the tidal problem, only the part $S T^{\prime \prime}$ of the spheroidal series is stable; the part $T^{\prime \prime} T$ is unstable. The range $T^{\prime \prime} B^{\prime \prime}$ is unstable through a spheroidal displacement only, and the range beyond $B^{\prime \prime}$ is additionally unstable through a pear-shaped displacement.

In the double-star problem, only configurations represented inside an area such as $S R^{\prime \prime} T^{\prime \prime} S$ are stable; all others are unstable. The configurations inside the area $S R^{\prime \prime} T^{\prime \prime} B^{\prime \prime} B^{\prime} B S$ are unstable through ellipsoidal displacements only, while the range beyond $B^{\prime} B^{\prime \prime}$ is additionally unstable through a pear-shaped displacement.

In the rotational problem, the range $S B B^{\prime}$ is stable, while the range $B^{\prime} J$ is unstable through a pear-shaped displacement.

* "On the pear-shaped figure of Equilibrium of a Rotating Mass of Liquid," Phil. Trans. 198 A (1901), p. 301, or Coll. Works, III, p. 288. I have verified that Darwin's solution, which was obtained by harmonic analysis, satisfies my equations (218) to (221). See Phil. Trans. 215 A, p. 53. 


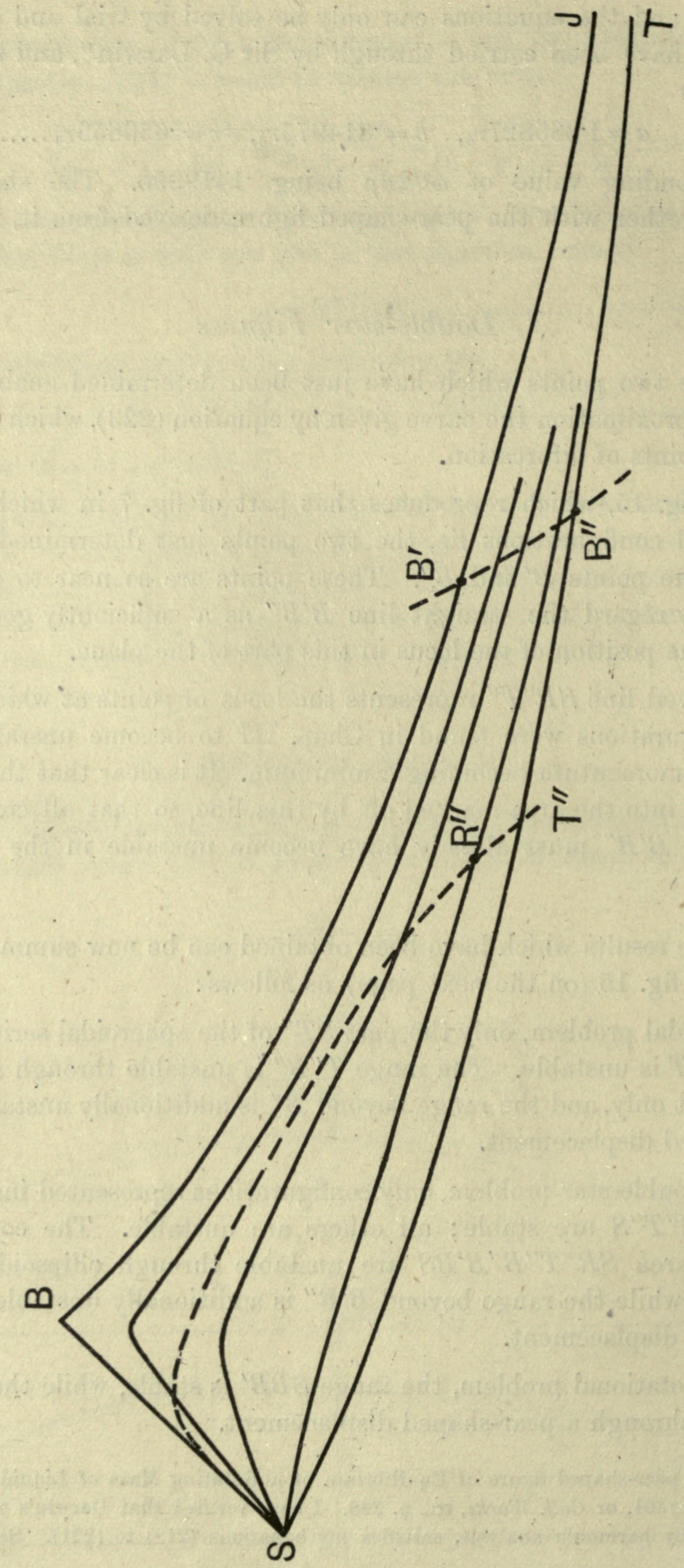

Fig. 15. 


\section{STABILITY OF THE PEAR-SHAPED FIGURES}

89. It is accordingly clear that in the tidal and double-star problems there are no stable configurations beyond the spheroidal and ellipsoidal figures already specified; the pear-shaped configurations are in every case unstable, the mass having become unstable before these configurations are reached. In the rotational problem, on the other hand, the series out of which the pear-shaped series bifurcates is itself stable up to the point of bifurcation, so that the pear-shaped figures, as already explained in $\S 21$, may be either stable or unstable.

The criterion of stability for these pear-shaped figures has already been given in $\S 21$; if on passing along the series from the point of bifurcation, the angular momentum is found initially to increase, then the figures are stable; if on the other hand it is found initially to decrease, then the figures are unstable. As far as first order terms, it is obvious that the angular momentum will be the same as at the point of bifurcation, so that to apply this criterion, we must proceed as far as second order terms in our determination of the series.

This problem has formed the subject of a series of classical papers by Poincaré, Darwin and Liapounoff. The general problem was first opened by Poincarés memoir in Vol. 7 of the Acta Mathematica (1885), to which reference has already been made. The criterion of stability was not accurately . stated here, and the necessary modification was announced by Schwarzschild* in 18.96. The accuracy of Schwarzschild's criterion of stability was admitted by Poincaré in a paper published in $1901+$; in this same paper Poincaré developed a method of carrying ellipsoidal harmonic potentials as far as the second order terms, and reduced the criterion of stability to an algebraic form, without however undertaking the necessary computations. At this stage the problem was taken up by Darwin, who, after preparing the ground by preliminary investigations+, published in 1902 a paper§, "The Stability of the Pear-Shaped Figure of Equilibrium of a Rotating Mass of Fluid." In the paper the equation of the pear-shaped figure was found as far as terms of the second order; and its moment of momentum calculated. This was found to increase on passing along the series, so that the pearshaped figure was announced to be stable.

* K. Schwarzschild, Münchener Inaug. Dissert. (1896).

+ "Sur la Stabilité de l'Equilibre des Figures Pyriformes affectées par une Masse Fluide en rotation." Phil. Trans. 197 A (1901), p. 333.

‡ "Ellipsoidal Harmonic Analysis." Phil. Trans. 197 A (1901), p. 461; “On the Pearshaped figure of Equilibrium of a Rotating Mass of Liquid." Phil. Trans. 198 A (1901), p. 301.

$\S$ Phil. Trans. $200 \mathrm{~A}$ (1902), p. 251 ; see also papers in Phil. Trans. $208 \mathrm{~A}(1908)$, p. 1, and Proc. Roy. Soc. 82 A (1909), p. 188, all combined in one paper in Coll. Scientific Papers, Vol. III, p. 317. 
Darwin's investigation had not been long published when doubt was cast on the accuracy of his conclusions. A paper appeared in 1905 by Iiapounoff* in which he stated that he could prove that the pear-shaped figure was unstable. Liapounoff's method was very different from that of Darwin, and a large part of his investigation appeared in the Russian language; owing perhaps to these circumstances neither investigator was able to announce the exact spot in which the error of the other lay, and the problem remained an open one. The method of treatment given in the present chapter will, it is hoped, shew the source of the divergence of the results obtained by these two investigators.

90. As far as the first order of small quantities, the pear-shaped figure has already $(\S 83)$ been found to be

where

$$
\frac{x^{2}}{a^{2}}+\frac{y^{2}}{b^{2}}+\frac{z^{2}}{c^{2}}-1+e P_{0}=0
$$

so that

$$
P=\xi\left(\alpha \xi^{2}+\beta \eta^{2}+\gamma \zeta^{2}+\kappa\right)
$$

$$
P_{0}=\frac{x}{a^{2}}\left(\alpha \frac{x^{2}}{a^{4}}+\beta \frac{y^{2}}{b^{4}}+\gamma \frac{z^{2}}{c^{4}}+\kappa\right)
$$

The potential of this figure can be found by the method already given in $\S 70$ of Chapter IV. As regards the internal and boundary potentials, the terms in $e$ will be of degrees 3 and 1 , those in $e^{2}$ of degrees $4,2,0$, those in $e^{3}$ will be of degrees $5,3,1$, and so on. It is at once clear that the general equation of equilibrium

$$
V_{b}+\frac{1}{2} \omega^{2}\left(x^{2}+y^{2}\right)=-\pi \rho a b c \theta\left(\frac{x^{2}}{a^{2}}+\frac{y^{2}}{b^{2}}+\frac{z^{2}}{c^{2}}-1+e P_{0}\right) \ldots \ldots
$$

cannot be satisfied as far as $e^{2}$, for terms in $e^{2}$ of degree 4 in $x, y, z$ occur on the left of this equation, and have no balancing terms on the right. To satisfy the equation of equilibrium, it is found to be necessary to add terms in $e^{2}$ of degrees 4, 2 and 0 to the left of equation (230), and such terms then appear also on the right of equation (233).

91. Thus, to calculate the pear-shaped figure as far as second order terms, we assume the boundary of the figure to be

where

$$
\frac{x^{2}}{a^{2}}+\frac{y^{2}}{b^{2}}+\frac{z^{2}}{c^{2}}-1+e P_{0}+e^{2} Q_{0}=0
$$

$$
Q=\frac{1}{4}\left[L \xi^{4}+M \eta^{4}+N \zeta^{4}+2 l \eta^{2} \zeta^{2}+2 m \zeta^{2} \xi^{2}+2 n \xi^{2} \eta^{2}+2\left(p \xi^{2}+q \eta^{2}+r \zeta^{2}\right)+s\right]
$$
(1905). 
so that

$$
\begin{aligned}
Q_{0}=\frac{1}{4}\left[L \frac{x^{4}}{a^{8}}+M \frac{y^{4}}{b^{8}}\right. & +N \frac{z^{4}}{c^{8}}+2 l \frac{y^{2} z^{2}}{b^{2} c^{2}}+2 m \frac{z^{2} x^{2}}{c^{2} a^{2}}+2 n \frac{x^{2} y^{2}}{a^{2} b^{2}} \\
& \left.+2\left(p \frac{x^{2}}{a^{4}}+q \frac{y^{2}}{b^{4}}+r \frac{z^{2}}{c^{4}}\right)+s\right] \ldots \ldots .
\end{aligned}
$$

The constant term $\frac{1}{4} s$ in this value of $Q_{0}$ is not necessary to satisfy the conditions of equilibrium, but is introduced in order to keep the total volume of the distorted surface equal to that of the original ellipsoid.

The potential of the figure determined by equation (234) can be written down by the method already explained. We have for the second order terms

$$
\begin{aligned}
\phi_{2} & =u_{2}+f v_{2} \\
& =Q-\frac{1}{8} D P^{2}+f\left\{\frac{1}{64} D^{2} P^{2}-\frac{1}{4} D Q\right\}+f^{2}\left\{-\frac{1}{1536} D^{3} P^{2}+\frac{1}{64} D^{2} Q\right\} \ldots
\end{aligned}
$$

If we now put

$$
\begin{array}{r}
\int_{0}^{\infty} \frac{\phi_{2}}{\Delta} d \lambda=c_{11} x^{4}+c_{22} y^{4}+c_{33} z^{4}+c_{12} x^{2} y^{2}+c_{23} y^{2} z^{2}+c_{31} z^{2} x^{2} \\
+d_{1} x^{2}+d_{2} y^{2}+d_{3} z^{2}+d_{4} \ldots
\end{array}
$$

in which the coefficients $c_{11}, \ldots$ are determined by comparison with equation (237), then the potential at the boundary of the figure (234) will, as far as $e^{2}$, be given by

$$
\begin{aligned}
-\frac{V_{b}}{\pi \rho a b c}= & x^{2} J_{A}+y^{2} J_{B}+z^{2} J_{C}-J \\
& +e x\left(a_{1} x^{2}+a_{2} y^{2}+a_{3} z^{2}+a_{4}\right) \\
& +e^{2}\left[c_{11} x^{4}+c_{22} y^{4}+c_{33} z^{4}+c_{12} x^{2} y^{2}+c_{23} y^{2} z^{2}+c_{31} z^{2} x^{2}\right. \\
& \left.\quad+d_{1} x^{2}+d_{2} y^{2}+d_{3} z^{2}+d_{4}\right] \ldots \ldots
\end{aligned}
$$

The value of $\omega^{2}$ in this configuration is not necessarily the same as in the ellipsoidal figure, although it must obviously differ only by terms in $e^{2}$. Let us assume the new value to be $\omega^{2}+e^{2} \delta \omega^{2}$, where the first term refers to the value of $\omega^{2}$ in the ellipsoidal configuration. Then the equation of equilibrium becomes

$$
V_{b}+\frac{1}{2}\left(\omega^{2}+e^{2} \delta \omega^{2}\right)\left(x^{2}+y^{2}\right)=-\pi \rho a b c \theta\left(\frac{x^{2}}{a^{2}}+\frac{y^{2}}{b^{2}}+\frac{z^{2}}{c^{2}}-1+e P_{0}+e^{2} Q_{0}\right)
$$

and this must be satisfied for all values of $x, y, z$ and for all values of $e^{*}$.

Equating terms independent of $e$ we obtain merely equations (65)-(67) of $\S 36$. These are of course simply the equations which determine the

* We might have obtained an appearance of greater generality by replacing $\theta$ in this equation by an expression of the form $\theta+e \theta^{\prime}+e^{2} \theta^{\prime \prime}$, but it would have been only an appearance. On equating coefficients we should have immediately been forced to put $\theta^{\prime}=0$, and the generality introduced by the undetermined $\theta^{\prime \prime}$ adds nothing to that already involved in the presence of the coefficients $p, q, r$ and $s$. 
series of Jacobian ellipsoids. Equating terms in $e$ we obtain again equations (216) which determine the position of the point of bifurcation and the ratio of the coefficients $\alpha, \beta, \gamma$ and $\kappa$. On finally equating terms in $e^{2}$ we obtain the system of equations:

$$
\left.\begin{array}{r}
c_{11}=\frac{1}{4} \frac{\theta L}{a^{8}}, \quad c_{12}=\frac{1}{4} \frac{\theta M}{b^{8}}, \quad c_{13}=\frac{1}{4} \frac{\theta N}{c^{8}} \\
c_{23}=\frac{1}{2} \frac{\theta l}{b^{4} c^{4}}, \quad c_{31}=\frac{1}{2} \frac{\theta m}{c^{4} a^{4}}, \quad c_{12}=\frac{1}{2} \frac{\theta n}{a^{4} b^{4}}
\end{array}\right\}
$$

92. In starting computations, we may first determine the ratios $\alpha: \beta: \gamma: \kappa$ from equations (218) $-(221)$, which are equivalent to equations (216). Assigning to $\alpha$ the arbitrary value $\alpha=-a^{2}$, the values of $\alpha, \beta, \gamma, \kappa$ are found to be

$\alpha=-3.556343, \quad \beta=0.204689, \quad \gamma=0.0679189, \quad \kappa=0.506278 \ldots(243)$.

The potential coefficients $c_{11}, c_{12}, \ldots$ may now be evaluated in equation (238). These coefficients cannot be completely determined, but they reduce to linear functions of the still unknown coefficients $L, M, N, l, m, n$, so that equations (241) become a series of six simultaneous linear equations in the six variables $L, M, N, l, m, n$.

Solving these equations, the values of these six coefficients are found to $b^{*}$

$$
\left.\begin{array}{lll}
L=-11 \cdot 71505, & M=-0.00583504, & N=-0.000808592 \\
l=-0.00214448, & m=0.232659, & n=0.653198
\end{array}\right\}
$$

The values of $d_{1}, d_{2}$ and $d_{3}$ may now be evaluated from equation (238), and expressed as linear functions of $p, q$ and $r$. Equations (242) now become three linear equations connecting the three variables $p, q, r$, and on solving these, we find

$$
\begin{aligned}
& p=2 \cdot 278950+113.9697 \frac{\delta \omega^{2}}{2 \pi \rho} \ldots \\
& q=-0.044997-7.83600 \frac{\delta \omega^{2}}{2 \pi \rho} \cdots \\
& r=-0.0140132-\cdot 1647351 \frac{\delta \omega^{2}}{2 \pi \rho}
\end{aligned}
$$

* Details of this computation and of the checks on its accuracy will be found in a paper already referred to. Phil. Trans. 215 A (1915), p. 27. 
When the coefficients have these values, all the conditions for equilibrium are satisfied. The value of $s$ may still be anything we please, but only one value of $s$ will keep the volume of the figure equal to the initial volume, and this value is found to be

$$
s=-0 \cdot 1586814-13 \cdot 239893 \frac{\delta \omega^{2}}{2 \pi \rho}
$$

93. Equations (24.5)-(248) may be written in the form

$$
p=p^{\prime}+p^{\prime \prime} \zeta \text {, etc. }
$$

where

$$
\zeta=\frac{\delta \omega^{2}}{2 \pi \rho} e^{2}
$$

and the value of $Q_{0}$ given by equation (236) may similarly be expressed in the form

$$
Q_{0}=Q_{0}^{\prime}+\zeta Q_{0}^{\prime \prime}
$$

The equation of the boundary (equation (234)) now becornes

$$
\frac{x^{2}}{a^{2}}+\frac{y^{2}}{b^{2}}+\frac{z^{2}}{c^{2}}-1+e P_{0}+e^{2} Q_{0}^{\prime}+\zeta Q_{0}^{\prime \prime}
$$

This will be a figure of equilibrium whatever the values of $e$ and $\zeta$, provided only that they are sufficiently small. If we put $e=0$ but retain $\zeta$ the equation becomes

$$
\frac{x^{2}}{a^{2}}\left(1+\frac{1}{2} \zeta \frac{p_{1}}{a^{2}}\right)+\frac{y^{2}}{b^{2}}\left(1+\frac{1}{2} \zeta \frac{q_{1}}{b^{2}}\right)+\frac{z^{2}}{c^{2}}\left(1+\frac{1}{2} \zeta \frac{r_{1}}{c^{2}}\right)=1-\frac{1}{4} \zeta s_{1}
$$

and this is an ellipsoid whose semi-axes $a^{\prime}, b^{\prime}, c^{\prime}$ are given by

$$
\left\{\begin{array}{l}
\frac{a^{\prime 2}}{a^{2}}=1-\frac{1}{2} \zeta\left(\frac{p_{1}}{a^{2}}+\frac{1}{2} s_{1}\right)=1-12 \cdot 71347 \zeta, \\
\frac{b^{\prime 2}}{b^{2}}=1-\frac{1}{2} \zeta\left(\frac{q_{1}}{b^{2}}+\frac{1}{2} s_{1}\right)=1+9 \cdot 20894 \zeta \\
\frac{c^{\prime 2}}{c^{2}}=1-\frac{1}{2} \zeta\left(\frac{r_{1}^{\circ}}{c^{2}}+\frac{1}{2} s_{1}\right)=1+3 \cdot 50453 \zeta .
\end{array}\right.
$$

Clearly then, as $\zeta$ varies with $e=0$, the figure of equilibrium coincides with the various Jacobian ellipsoids near to the point of bifurcation.

On putting $\zeta=0$ but retaining $e$ in equation (2.51) we obtain a series of figures of equilibrium for all of which the angular velocity is the same as that at the point of bifurcation.

The two series of configurations obtained by putting $e=0$ and $\zeta=0$ in equation (251) may be represented by two intersecting straight lines such as $P O P^{\prime}, Q O Q^{\prime}$ in fig. 16 , the point $O$ being of course the point of bifurcation. But the general figure of equilibrium represented by equation (251), in which $e$ and $\zeta$ are limited only by the condition that $e^{3}$ and $\zeta^{\frac{3}{2}}$ shall be 
negligible, will be represented by all points inside a certain rectangle $A B C D$ surrounding the point $O$ in fig. 16 . They do not fall into linear series, as it was assumed by Poincaré and Darwin that they would.

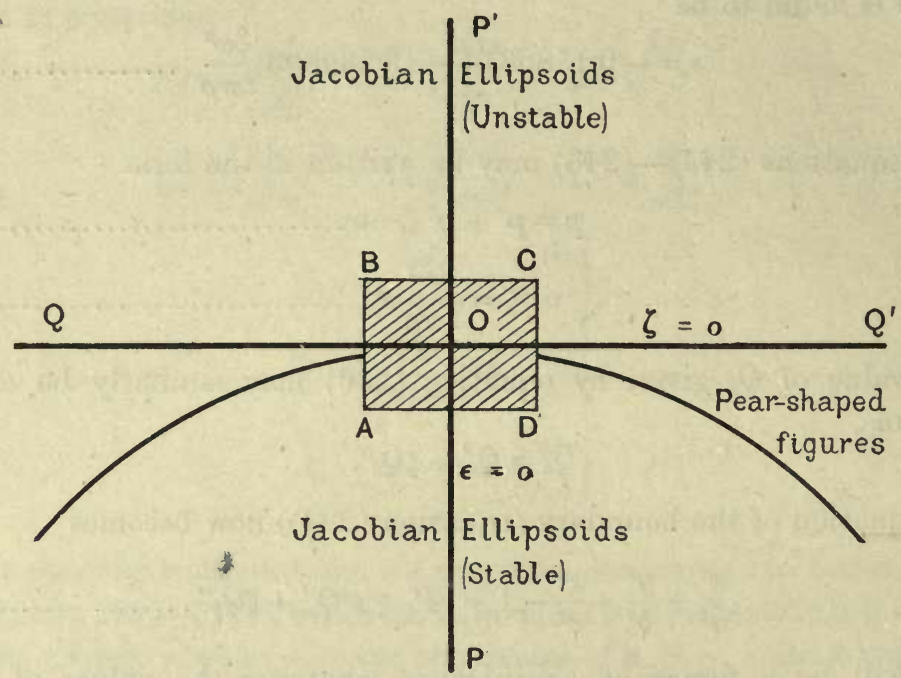

Fig. 16.

That the two linear series will lose their identity and give place to a two-dimensional area seems to be predicted by Poincarés analysis of which an account has already been given in $\$ \S 22,23$ of Chap. II. For the condition that a point of bifurcation shall occur at $O$, namely $\Delta=0$, is also the condition that the direction of the linear series shall be indeterminate at $O$, or, what is the same thing, that the two linear series shall become merged into an area as they approach the point of bifurcation.

Thus it now appears that an expansion as far as $e^{2}$ is not adequate to reveal the direction in which the second linear series turns on starting out from the point of bifurcation 0 . The difficulty is introduced by the artificial method of expansion in powers of the parameter $e$; the linear series are in reality completely determinate, but an expansion as far as $e^{2}$ only does not suffice to determine them. A precisely similar complication occurs in considering the direction in which lines of force start out from a point of equilibrium in an electrostatic field*.

94. Sir G. Darwin seems to have carried out his investigation under the impression that there would be a unique configuration of equilibrium when the calculations were carried as far as $e^{2}$, and this led him to introduce a spurious condition of equilibrium, the effect of which was to limit him to one of the doubly infinite series we have discovered $t$. In point of fact,

+ For details, see Phil. Trans. 215 A, p. 76. 
Darwin's extra condition of equilibrium could only be satisfied by assigning to $\zeta$ a special value, namely $\zeta=-0.015988 e^{2}$, and this value gives a figure whose angular momentum is greater than that of the undistorted ellipsoid. Darwin accordingly announced the pear-shaped figure to be stable.

But we shall now see that this special value for $\zeta$ makes it impossible to carry the linear series on to third order terms at all. The condition that it shall be possible to carry on the series to third order terms requires that $\zeta$ shall have a special value, but this special value is not the one assumed by Darwin; it is a value which shews the pear-shaped figure to be unstable, as we shall now see.

95. We proceed to calculate the pear-shaped series as far as the third order terms.

An argument similar to that of $\S 90$ shews that the boundary (234) can only be made a figure of equilibrium as far as third order terms by including in it additional terms of degrees 5, 3 and 1 . We accordingly assume for the boundary of the distorted ellipsoid,

$$
\frac{x^{2}}{a^{2}}+\frac{y^{2}}{b^{2}}+\frac{z^{2}}{c^{2}}-1+e P_{0}+e^{2} Q_{0}+e^{3} R_{0}=0 .
$$

where $P_{0}$ and $Q_{0}$ have the values already given in equations (232) and (236), and

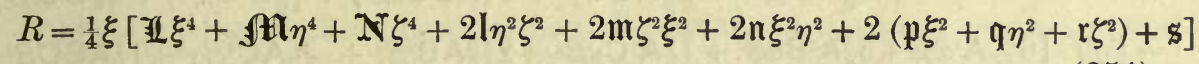

so that

$$
\begin{aligned}
R_{0}=\frac{1}{4} \frac{x}{a^{2}}\left[\mathfrak{I} \frac{x^{4}}{a^{8}}+\sin \frac{y^{4}}{b^{8}}+\mathfrak{N} \frac{z^{4}}{c^{8}}+2 \mathrm{I} \frac{y^{2} z^{2}}{b^{4} c^{4}}+2 \mathfrak{m} \frac{z^{2} x^{2}}{c^{4} a^{4}}\right. \\
\left.+2 \mathfrak{n} \frac{x^{2} y^{2}}{a^{4} b^{4}}+2\left(\mathfrak{p} \frac{x^{2}}{a^{4}}+\mathfrak{q} \frac{y^{2}}{b^{4}}+\mathfrak{r} \frac{z^{2}}{c^{4}}\right)+\$\right]
\end{aligned}
$$

96. We can calculate the potential of this figure as far as $e^{3}$ by the formulae given in the last chapter. Calculating the terms in $e^{3}$ in formula (197) in terms of the values which have now been assigned to $P, Q$ and $R$ we find as the value of $\phi_{3}$,

$$
\begin{aligned}
\phi_{3}= & \xi^{5}\left[15 \frac{3}{4} A^{2} \alpha^{3}+2 \frac{5}{8} A B \alpha^{2} \beta+2 \frac{5}{8} A C \alpha^{2} \gamma+\frac{3}{8} B^{2} \alpha \beta^{2}+\frac{1}{4} B C \alpha \beta \gamma+\frac{3}{8} C^{2} \alpha \gamma^{2}\right] \\
& +\xi^{3} \eta^{2}\left[13 \frac{1}{8} A^{2} \alpha^{2} \beta+7 \frac{1}{2} A B \alpha^{2} \beta+2 \frac{1}{2} A C \alpha \beta \gamma+1 \frac{7}{8} B^{2} \beta^{3}+\frac{3}{4} B C \beta^{2} \gamma+\frac{3}{8} C^{2} \beta \gamma^{2}\right] \\
& +\xi^{3} \zeta^{2}\left[13 \frac{1}{8} A^{2} \alpha^{2} \gamma+2 \frac{1}{2} A B \alpha \beta \gamma+7 \frac{1}{2} A C \alpha \gamma^{2}+\frac{3}{8} B^{2} \beta^{2} \gamma+\frac{3}{4} B C \beta \gamma^{2}+1 \frac{7}{8} C^{2} \gamma^{3}\right] \\
& +\xi \eta^{4}\left[1 \frac{7}{8} A^{2} \alpha \beta^{2}+1 \frac{7}{8} A B \beta^{3}+\frac{3}{8} A C \beta^{2} \gamma\right] \\
& +\xi \zeta^{4}\left[1 \frac{7}{8} A^{2} \alpha \gamma^{2}+\frac{3}{8} A B \beta \gamma^{2}+1 \frac{7}{8} A C \gamma^{3}\right] \\
& +\xi \eta^{2} \zeta^{2}\left[3 \frac{3}{4} A^{2} \alpha \beta \gamma+2 \frac{1}{4} A B \beta^{2} \gamma+2 \frac{1}{4} A C \beta \gamma^{2}\right]
\end{aligned}
$$


$+\xi^{3} \kappa\left[13 \frac{1}{8} A^{2} \alpha^{2}+2 \frac{1}{2} A B \alpha \beta+2 \frac{1}{2} A C \alpha \gamma+\frac{3}{8} B^{2} \beta^{2}+\frac{1}{4} B C \beta \gamma+\frac{3}{8} C^{2} \gamma^{2}\right]$

$+\xi \eta^{2} \kappa\left[3 \frac{3}{4} \mathrm{~A}^{2} \alpha \beta+2 \frac{1}{4} \mathrm{AB} \beta^{2}+\frac{3}{4} \mathrm{AC} \beta \gamma\right]$

$+\xi \zeta^{2} \kappa\left[3 \frac{3}{4} A^{2} \alpha \gamma+\frac{3}{4} A B \beta \gamma+2 \frac{1}{4} A C \gamma^{2}\right]$

$+\xi \kappa^{2}\left[1 \frac{7}{8} A^{2} \alpha+\frac{3}{8} A B \beta+\frac{3}{8} A C \gamma\right]$

$-\xi^{3}\left[2 \frac{5}{8} \mathrm{~A} L \alpha+\frac{1}{8} \mathrm{~B}(L \beta+2 n \alpha)+\frac{1}{8} \mathrm{C}(L \gamma+2 m \alpha)\right]$

$-\xi^{3} \eta^{2}\left[1 \frac{1}{4} \mathrm{~A}(L \beta+2 n \alpha)+\frac{3}{4} \mathbf{B}(M \alpha+2 n \beta)+\frac{1}{4} \mathbf{C}(l \alpha+m \beta+n \gamma)\right]$

$-\xi^{3} \xi^{2}\left[1 \frac{1}{4} \mathrm{~A}(L \gamma+2 m \alpha)+\frac{1}{4} \mathrm{~B}(l \alpha+m \beta+n \gamma)+\frac{3}{4} \mathrm{C}(N \alpha+2 n \gamma)\right]$

$-\xi \eta^{4}\left[\frac{3}{8} \mathrm{~A}(M \alpha+2 n \beta)+1 \frac{7}{8} \mathrm{~B} M \beta+\frac{1}{8} \mathrm{C}(M \gamma+2 l \beta)\right]$

$-\xi \zeta^{4}\left[\frac{3}{8} \mathrm{~A}(N \alpha+2 n \gamma)+\frac{1}{8} \mathrm{~B}(N \beta+2 l \gamma)+1 \frac{7}{8} \mathrm{C} N \gamma\right]$

$-\xi \eta^{2} \zeta^{2}\left[\frac{3}{4} \mathrm{~A}(l \alpha+m \beta+n \gamma)+\frac{3}{4} \mathrm{~B}(M \gamma+2 l \beta)+\frac{3}{4} \mathrm{C}(N \beta+2 l \gamma)\right]$

$-\xi^{3} \quad\left[1 \frac{1}{4} \mathrm{~A}(L \kappa+2 \alpha p)+\frac{1}{4} \mathrm{~B}(n \kappa+\alpha q+\beta p)+\frac{1}{4} \mathrm{C}(m \kappa+\gamma p+\alpha r)\right]$

$-\xi \eta^{2} \quad\left[\frac{3}{4} \mathrm{~A}(n \kappa+\alpha q+\beta p)+\frac{3}{4} \mathrm{~B}(M \kappa+2 \beta q)+\frac{1}{4} \mathrm{C}(l \kappa+\gamma q+\beta r)\right]$

$-\xi \zeta^{2} \quad\left[\frac{3}{4} \mathrm{~A}(m \kappa+\gamma p+\alpha r)+{ }_{4}^{1} \mathrm{~B}\left(l_{\kappa}+\gamma q+\beta r\right)+\frac{3}{4} \mathrm{C}(N \kappa+2 \gamma r)\right]$

$-\xi \quad\left[\frac{3}{8} \mathrm{~A}(2 p \kappa+s \alpha)+\frac{1}{8} \mathrm{~B}(2 q \kappa+s \beta)+\frac{1}{8} \mathrm{C}(2 r \kappa+s \gamma)\right]$

$+\frac{1}{4} \xi \quad\left[\mathcal{I} \xi^{4}+\mathfrak{A} \lambda \eta^{4}+\mathfrak{N} \zeta^{4}+2 l \eta^{2} \zeta^{2}+2 \mathfrak{m} \xi^{2} \xi^{2}+2 \mathfrak{n} \xi^{2} \eta^{2}+2\left(p \xi^{2}+q \eta^{2}+r \xi^{2}\right)+\$\right]$

$-\xi 3 f \quad\left[26 \frac{1}{4} \mathrm{~A}^{3} \alpha^{3}+\frac{5}{16} \mathrm{~B}^{3} \beta^{3}+\frac{5}{16} \mathrm{C}^{3} \gamma^{3}+6 \frac{9}{16} \mathrm{~A}^{2} \mathrm{~B} \alpha^{2} \beta+6 \frac{9}{16} \mathrm{~A}^{2} \mathrm{C} \alpha^{2} \gamma\right.$

$\left.+1 \frac{7}{8} A B^{2} \alpha \beta^{2}+1 \frac{7}{8} A C^{2} \alpha \gamma^{2}+1 \frac{1}{4} A B C \alpha \beta \gamma+\frac{3}{16} B^{2} C \beta^{2} \gamma+\frac{3}{16} B C^{2} \beta \gamma^{2}\right]$

$-\xi \eta^{2} f\left[6 \frac{9}{16} A^{3} \alpha^{2} \beta+5 \frac{5}{8} A^{2} B \alpha \beta^{2}+1 \frac{7}{8} A^{2} C \alpha \beta \gamma+2 \frac{13}{16} A B^{2} \beta^{3}+\frac{9}{16} A C^{2} \beta \gamma^{2}+1 \frac{1}{8} A B C \beta^{2} \gamma\right]$

$-\xi \zeta^{2} f\left[6 \frac{9}{16} A^{3} \alpha^{2} \gamma+1 \frac{7}{8} A^{2} B \alpha \beta \gamma+5 \frac{5}{8} A^{2} C \alpha \gamma^{2}+\frac{9}{16} A B^{2} \beta^{2} \gamma+2 \frac{13}{16} A C^{2} \gamma^{3}+1 \frac{1}{8} A B C \beta \gamma^{2}\right]$

$-\xi f \quad\left[6 \frac{9}{16} \mathrm{~A}^{3} \alpha^{2} \kappa+1 \frac{7}{8} \mathrm{~A}^{2} \mathrm{~B} \alpha \beta \kappa+1 \frac{7}{8} \mathrm{~A}^{2} \mathrm{C} \alpha \gamma \kappa+\frac{9}{16} \mathrm{AB}^{2} \beta^{2} \kappa+\frac{9}{16} \mathrm{~A} \mathrm{C}^{2} \gamma^{2} \kappa+{ }_{8}^{3} \mathrm{ABC} \beta \gamma \hat{\kappa}\right]$

$+\xi^{3} f\left[6 \frac{9}{16} \mathrm{~A}^{2} L \alpha+\frac{3}{16} \mathrm{~B}^{2}(M \alpha+2 n \beta)+\frac{3}{16} \mathrm{C}^{2}(N \alpha+2 n \gamma)+\frac{5}{8} \mathrm{AB}(L \beta+2 n \alpha)\right.$

$\left.+\frac{5}{8} \mathrm{AC}(L \gamma+2 m \alpha)+\frac{1}{8} \mathrm{BC}(l \alpha+m \beta+n \gamma)\right]$

$+\xi \eta^{2} f\left[\frac{15}{16} \mathrm{~A}^{2}(L \beta+2 n \alpha)+2 \frac{13}{16} \mathrm{~B}^{2} M \beta+\frac{3}{16} \mathrm{C}^{2}(N \beta+2 l \gamma)+1 \frac{1}{8} \mathrm{AB}(M \alpha+2 n \beta)\right.$

$\left.+\frac{3}{8} \mathrm{AC}(l \alpha+m \beta+n \gamma)+\frac{3}{8} \mathrm{BC}(M \gamma+2 l \beta)\right]$

$+\xi \zeta^{2} f\left[\frac{15}{16} \mathrm{~A}^{2}\left(L_{\gamma}+2 m \alpha\right)+\frac{3}{16} \mathrm{~B}^{2}(M \gamma+2 l \beta)+2 \frac{13}{16} \mathrm{C}^{2} N \gamma+\frac{3}{8} \mathrm{AB}(l \alpha+m \beta+n \gamma)\right.$

$\left.+1 \frac{1}{8} \mathrm{AC}(N \alpha+2 n \gamma)+\frac{3}{8} \mathrm{BC}(N \beta+2 l \gamma)\right]$

$+\xi f \quad\left[\frac{15}{16} \mathrm{~A}^{2}(L \kappa+2 \alpha p)+\frac{3}{16} \mathrm{~B}^{3}(M \kappa+2 \beta q)+\frac{3}{16} \mathrm{C}^{2}(N \kappa+2 \gamma r)+\frac{3}{8} \mathrm{AB}(n \kappa+\alpha q+\beta p]\right.$ $\left.+\frac{3}{8} \mathrm{AC}(m \kappa+\gamma p+\alpha r)+\frac{1}{8} \mathrm{BC}\left(l_{\kappa}+\gamma q+\beta r\right)\right]$

$-\xi^{3} f \quad\left[1 \frac{1}{4} \mathbb{I} \mathrm{A}+\frac{1}{4} m B+\frac{1}{4} m C\right]$

$-\xi \eta^{2} f\left[\frac{3}{4} \| A+\frac{3}{4} \sqrt{\mathfrak{A}} \mathrm{R}+\frac{1}{4}\right.$ lC $]$

$-\xi \zeta^{2} f\left[\frac{3}{4} m A+\frac{1}{4} \mid B+\frac{3}{4} \mathrm{NC}\right]$

$-\xi f \quad\left[\frac{3}{4} p A+\frac{1}{4} q B+\frac{1}{4} \mathrm{rC}\right]+\xi f^{2} G$ 
where $f$ stands for $A \xi^{2}+B \eta^{2}+C \zeta^{2}-1$, and $G$ is given by

$$
\begin{aligned}
& G=\mathrm{A}\left\{\frac{315}{64} \mathrm{~A}^{3} \alpha^{3}+\frac{105}{64} \mathrm{~A}^{2} \mathrm{~B} \alpha^{2} \beta+\frac{105}{64} \mathrm{~A}^{2} \mathrm{C} \alpha^{2} \gamma+\frac{45}{64} \mathrm{~A} \mathrm{~B}^{2} \alpha \beta^{2}\right. \\
& +\frac{45}{64} A C^{2} \alpha \gamma^{2}+\frac{15}{32} A B C \alpha \beta \gamma+\frac{15}{64} B^{3} \beta^{3}+\frac{15}{64} C^{3} \gamma^{3} \\
& \left.+\frac{9}{64} \mathrm{~B}^{2} \mathrm{C} \beta^{2} \gamma+\frac{9}{64} \mathrm{BC}^{2} \beta \gamma^{2}\right\} \\
& -\frac{105}{64} A^{3} L \alpha-\frac{15}{64} A^{2} B(L \beta+2 n \alpha)-\frac{15}{64} A^{2} C(L \gamma+2 m \alpha) \\
& -\frac{9}{64} \mathrm{AB}^{2}(M \alpha+2 n \beta)-\frac{9}{64} \mathrm{AC}^{2}(N \alpha+2 n \gamma)-\frac{3}{32} \mathrm{ABC}(l \alpha+m \beta+n \gamma) \\
& -\frac{15}{64} \mathrm{~B}^{3} M \beta-\frac{15}{64} \mathrm{C}^{3} N_{\gamma}-\frac{3}{64} \mathrm{~B}^{2} \mathrm{C}(M \gamma+2 l \beta)-\frac{3}{64} \mathrm{BC}^{2}(N \beta+2 l \gamma) \\
& +\frac{15}{32} A^{2} \mathbf{Z} \mathbf{L}+\frac{3}{32} B^{2} \mathfrak{A t}+\frac{3}{32} C^{2} \mathfrak{N}+\frac{1}{16} B C l+\frac{3}{16} A C m+\frac{3}{16} A B \mathfrak{m}
\end{aligned}
$$

With this value for $\phi_{3}$, let us put

$\int_{0}^{\infty} \frac{\phi_{3}}{\Delta} d \lambda=x\left(\mathfrak{c}_{11} x^{4}+\mathfrak{c}_{22} y^{4}+\mathfrak{c}_{33} z^{4}+\mathfrak{c}_{12} x^{2} y^{2}+\mathfrak{c}_{31} z^{2} x^{2}+\mathfrak{c}_{23} y^{2} z^{2}+\mathfrak{d}_{1} x^{2}+\mathfrak{d}_{2} y^{2}+\mathfrak{d}_{3} z^{2}+\mathfrak{\searrow}_{4}\right)$

The value of $V_{b}$ the potential at the boundary is

$$
V_{b}=-\pi \rho a b c \int_{0}^{\infty}\left[f+e \phi_{1}+e^{2} \phi_{2}+e^{3} \phi_{3}\right] \frac{d \lambda}{\Delta} ;
$$

the condition that the figure (253) shall be a figure of equilibrium is, as in equation (240),

$$
V_{b}+\frac{1}{2}\left(\omega^{2}+e^{2} \delta \omega^{2}\right)\left(x^{2}+y^{2}\right)=-\pi \rho a b c \theta\left(\frac{x^{2}}{a^{2}}+\frac{y^{2}}{b^{2}}+\frac{z^{2}}{c^{2}}-1+e P_{0}+e^{2} Q_{0}+e^{3} R_{0}\right)
$$

On equating coefficients of terms independent of $e$, and of terms in $e$ and $e^{2}$ in this equation we obtain precisely the systems of equations which have been already obtained and discussed; on equating terms in $e^{3}$ we obtain

$$
\begin{aligned}
& x\left(\mathfrak{c}_{11} x^{4}+\mathfrak{c}_{22} y^{4}+\mathfrak{c}_{33} z^{4}+\mathfrak{c}_{12} x^{2} y^{2}+\mathfrak{c}_{31} z^{2} x^{2}+\mathfrak{c}_{23} y^{2} z^{2}+\mathfrak{d}_{1} x^{2}+\mathfrak{d}_{2} y^{2}+\mathfrak{d}_{3} z^{2}+\mathfrak{\searrow}_{4}\right) \\
& =\frac{1}{4} \theta \frac{x}{a^{2}}\left[\mathfrak{Z} \frac{x^{4}}{a^{8}}+\mathfrak{A t} \frac{y^{4}}{b^{8}}+\mathfrak{N} \frac{z^{4}}{c^{8}}+2 \mathrm{l} \frac{y^{2} z^{2}}{b^{4} c^{4}}+2 \mathfrak{m} \frac{z^{2} x^{2}}{c^{4} a^{4}}\right. \\
& \left.+2 \mathfrak{n} \frac{x^{2} y^{2}}{a^{4} b^{4}}+2\left(\mathfrak{p} \frac{x^{2}}{a^{4}}+\mathfrak{q} \frac{y^{2}}{b^{4}}+\mathfrak{r} \frac{z^{2}}{c^{4}}\right)+s\right] \text {. }
\end{aligned}
$$

On equating coefficients, this is found to be equivalent to the separate equations :

$$
\left.\begin{array}{lll}
\mathfrak{l}_{11}=\frac{1}{4} \frac{\theta}{a^{10}} \mathfrak{I} ; & \mathfrak{c}_{22}=\frac{1}{4} \frac{\theta}{a^{2} b^{8}} \mathfrak{A t} ; & \mathfrak{c}_{33}=\frac{1}{4} \frac{\theta}{a^{2} c^{8}} \mathfrak{N} \\
\mathfrak{c}_{23}=\frac{1}{2} \frac{\theta}{a^{2} b^{4} c^{4}} \mathfrak{l} ; & \mathfrak{c}_{31}=\frac{1}{2} \frac{\theta}{a^{6} c^{4}} \mathfrak{m} ; & \mathfrak{c}_{12}=\frac{1}{2} \frac{\theta}{a^{8} b^{4}} \mathfrak{n}
\end{array}\right\}
$$


and these equations, in combination with the first and second order equations already discussed, express the condition that the third order figure (253) shall be a configuration of equilibrium.

97. The numerical discussion of these equations proves long and tedious*. We first write down the values of $\mathfrak{c}_{11}, \mathfrak{l}_{12}$, etc. by a comparison of equations (256) and (258). As a typical coefficient may be given the value of $\mathfrak{c}_{12}$ which is found to be

$$
\begin{aligned}
& \mathfrak{l}_{12}=\int_{0}^{\infty} \frac{1}{\Delta A^{3} B^{2}}\left[13 \frac{1}{8} \mathrm{~A}^{2} \alpha^{2} \beta+7 \frac{1}{2} \mathrm{AB} \alpha \beta^{2}+2 \frac{1}{2} \mathrm{AC} \alpha \beta \gamma+1 \frac{7}{8} \mathrm{~B}^{2} \beta^{3}+\frac{3}{4} \mathrm{BC} \beta^{2} \gamma\right. \\
& \left.+\frac{3}{8} \mathrm{C}^{2} \beta \gamma^{2}-1 \frac{1}{4} \mathrm{~A}(L \beta+2 n \alpha)-\frac{3}{4} \mathrm{~B}(M \alpha+2 n \beta)-\frac{1}{4} \mathrm{C}(l \alpha+m \beta+n \gamma)+\frac{1}{2} n\right] d \lambda \\
& -\int_{0}^{\infty} \frac{1}{\Delta A^{3} B}\left[26 \frac{1}{4} \mathrm{~A}^{3} \alpha^{2}+\frac{5}{16} \mathrm{~B}^{3} \beta^{3}+\frac{5}{16} \mathrm{C}^{3} \gamma^{3}+6 \frac{9}{16} \mathrm{~A}^{2} \mathrm{~B} a^{2} \beta+6 \frac{9}{16} \mathrm{~A}^{2} \mathrm{C} \alpha^{2} \gamma\right. \\
& \left.+1 \frac{7}{8} A B^{2} \alpha \beta^{2}+1 \frac{7}{8} A C^{2} \alpha \gamma^{2}+1 \frac{1}{4} A B C \alpha \beta \gamma+\frac{3}{16} B^{2} C \beta^{2} \gamma+\frac{3}{16} B C^{2} \beta \gamma^{2}\right] d \lambda \\
& -\int_{0}^{\infty} \frac{1}{\Delta A^{2} B^{2}}\left[6 \frac{9}{16} A^{3} \alpha^{2} \beta+5 \frac{5}{8} A^{2} B \alpha \beta^{2}+1 \frac{7}{8} A^{2} C \alpha \beta \gamma+2 \frac{13}{16} A B^{2} \beta^{3}\right. \\
& \left.+\frac{9}{16} A C^{2} \beta \gamma^{2}+1 \frac{1}{8} A B C \beta^{2} \gamma\right] d \lambda \\
& +\int_{0}^{\infty} \frac{1}{\Delta A^{2} B^{2}}\left[\frac{15}{16} \mathrm{~A}^{2}(L \beta+2 n \alpha)+2 \frac{13}{16} \mathrm{~B}^{2} M \beta+\frac{3}{16} \mathrm{C}^{2}(N \beta+2 l \gamma)\right. \\
& \left.+1 \frac{1}{8} \mathrm{AB}(M \alpha+2 n \beta)+\frac{3}{8} \mathrm{AC}(l \alpha+m \beta+n \gamma)+\frac{3}{8} \mathrm{BC}(M \gamma+2 l \beta)\right] d \lambda \\
& +\int_{0}^{\infty} \frac{1}{\Delta A^{3} B}\left[6 \frac{9}{16} \mathrm{~A}^{2} L \alpha+\frac{3}{16} \mathrm{~B}^{2}(M \alpha+2 n \beta)+\frac{3}{16} \mathrm{C}^{2}(N \alpha+2 n \gamma)\right. \\
& \left.+\frac{5}{8} \mathrm{AB}(L \beta+2 n \alpha)+\frac{5}{8} \mathrm{AC}\left(L_{\gamma}+2 m \alpha\right)+\frac{1}{8} \mathrm{BC}(l \alpha+m \beta+n \gamma)\right] d \lambda \\
& -\int_{0}^{\infty} \frac{1}{\Delta A^{2} B^{2}} \frac{3}{4}\left[\mathrm{An}+\frac{3}{4} \mathrm{Bm}+\frac{1}{4} \mathrm{Cl}\right] d \lambda \\
& -\int_{0}^{\infty} \frac{1}{\Delta A^{3} B}\left[1 \frac{1}{4} \mathrm{~A} Z \mathbf{I}+\frac{1}{4} \mathrm{Bn}+\frac{1}{4} \mathrm{Cm}\right] d \lambda+2 \int_{0}^{\infty} \frac{G}{\Delta A^{2} B} d \lambda
\end{aligned}
$$

and on computing the numerical values of the various terms this reduces to

$$
\begin{aligned}
\mathfrak{c}_{12}= & -0.0002799 \mathfrak{Z} \mathbf{L}-0.0093206 \mathfrak{A t}+0.0103775 \mathfrak{N} \\
& -0.0045815 \mathrm{l}-0.0016151 \mathfrak{m}+0.0040268 \mathfrak{N}+0.0042388 .
\end{aligned}
$$

The remaining $\mathfrak{c}$-coefficients may be similarly evaluated, and equations (260) then become a system of six linear equations from which to determine the six unknowns $\mathfrak{Z}, \mathfrak{A} \mathfrak{t}, \mathfrak{N}, \mathfrak{l}, \mathfrak{m}, \mathfrak{n}$. The solution of these equations is found to be

$$
\begin{aligned}
\mathfrak{Z} \mathfrak{L}=-12.6275, & \mathfrak{I} \mathfrak{t}=-0.0307056, & \mathfrak{N}=-0.0044636, \\
\mathfrak{I}=-0.0116194, & \mathfrak{m}=0.42602, & \mathfrak{I}=1.15365 .
\end{aligned}
$$

* For fuller details than are given here see Phil. Trans. 217 A, p. 20. 
98. We proceed next to the three equations (261). On comparison of equations (256) and (258) it is found that the values of $\grave{D}_{1}, \mathfrak{d}_{2}, \mathfrak{d}_{3}$ are of the forms

$$
\begin{aligned}
& \mathfrak{J}_{1}=\delta_{1}-\int_{0}^{\infty} \frac{1}{\Delta A^{2}}\left(\frac{3}{4} \mathrm{pA}+\frac{1}{4} \mathrm{qB}+\frac{1}{4} \mathrm{rC}\right) d \lambda+\frac{1}{2} \mathrm{p} \int_{0}^{\infty} \frac{d \lambda}{\Delta A^{3}}-2 \int_{0}^{\infty} \frac{G}{\Delta A^{2}} d \lambda, \\
& \mathfrak{\nabla}_{2}=\delta_{2}-\int_{0}^{\infty} \frac{1}{\Delta A B}\left(\frac{3}{4} \mathrm{pA}+\frac{1}{4} \mathfrak{q} \mathrm{B}+\frac{1}{4} \mathrm{rC}\right) d \lambda+\frac{1}{2} \mathfrak{q} \int_{0}^{\infty} \frac{d \lambda}{\Delta A B^{2}}-2 \int_{0}^{\infty} \frac{G}{\Delta A B} d \lambda \text {, } \\
& \mathfrak{J}_{3}=\delta_{3}-\int_{0}^{\infty} \frac{1}{\Delta A C}\left(\frac{3}{4} \mathrm{pA}+\frac{1}{4} \mathfrak{q B}+\frac{1}{4} \mathrm{rC}\right) d \lambda+\frac{1}{2} \mathfrak{r} \int_{0}^{\infty} \frac{d \lambda}{\Delta A C^{2}}-2 \int_{0}^{\infty} \frac{G}{\Delta A C} d \lambda,
\end{aligned}
$$

in which $\delta_{1}, \delta_{2}, \delta_{3}$ are quantities which do not depend on $\mathfrak{p}, \mathfrak{q}, \mathfrak{r}, \boldsymbol{s}$. The values of $\delta_{1}$ and $\delta_{2}$ are as follows:

$$
\begin{aligned}
& \delta_{1}=\int_{0}^{\infty} \frac{1}{\Delta A^{3}}\left[\kappa\left(13 \frac{1}{8} \mathrm{~A}^{2} \alpha^{2}+2 \frac{1}{2} \mathrm{AB} \alpha \beta+2 \frac{1}{2} \mathrm{AC} \alpha \gamma+\frac{3}{8} \mathrm{~B}^{2} \beta^{2}+\frac{1}{4} \mathrm{BC} \beta \gamma+\frac{3}{8} \mathrm{C}^{2} \gamma^{2}\right)\right. \\
& -1 \frac{1}{4} \mathbf{A}(L \kappa+2 \alpha p)-\frac{1}{4} \mathbf{B}(n \kappa+\alpha q+\beta p)-\frac{1}{4} \mathbf{C}(m \kappa+p \gamma+r \alpha) \\
& +26 \frac{1}{4} \mathbf{A}^{3} \alpha^{3}+\frac{5}{16} \mathbf{B}^{3} \beta^{3}+\frac{5}{16} \mathbf{C}^{3} \gamma^{3}+6 \frac{9}{16} \mathbf{A}^{2} \mathbf{B} \alpha^{2} \beta+6 \frac{9}{16} \mathrm{~A}^{2} \mathbf{C} \alpha^{2} \gamma \\
& \left.+1 \frac{7}{8} A B^{2} \alpha \beta^{2}+1 \frac{7}{8} A C^{2} \alpha \gamma^{2}+1 \frac{1}{4} A B C \alpha \beta \gamma+\frac{3}{16} B^{2} C \beta^{2} \gamma+\frac{3}{16} B C^{2} \beta \gamma^{2}\right] d \lambda \\
& -\int_{0}^{\infty} \frac{\kappa}{\Delta A^{2}}\left[6 \frac{9}{16} \mathrm{~A}^{3} \alpha^{2}+1 \frac{7}{8} \mathrm{~A}^{2} \mathrm{~B} \alpha \beta+1 \frac{7}{8} \mathrm{~A}^{2} \mathrm{C} \alpha \gamma+\frac{9}{16} \mathrm{AB}^{2} \beta^{2}+\frac{3}{16} \mathrm{AC}^{2} \gamma^{2}+\frac{3}{8} \mathrm{ABC} \beta \gamma\right] d \lambda \\
& -\int_{0}^{\infty} \frac{1}{\Delta A^{3}}\left[6 \frac{9}{16} \mathrm{~A}^{2} L \alpha+\frac{3}{16} \mathrm{~B}^{2}(M a+2 n \beta)+\frac{3}{16} \mathrm{C}^{2}(N \alpha+2 m \gamma)\right. \\
& \left.+\frac{5}{8} \mathrm{AB}(L \beta+2 n \alpha)+\frac{5}{8} \mathrm{AC}(L \gamma+2 m \alpha)+\frac{1}{8} \mathrm{BC}(l \alpha+m \beta+n \gamma)\right] d \lambda \\
& +\int_{0}^{\infty} \frac{1}{\Delta A^{2}}\left[\frac{15}{16} \mathrm{~A}^{2}\left(L_{\kappa}+2 \alpha p\right)+\frac{3}{16} \mathrm{~B}^{2}(M \kappa+2 \beta q)+\frac{3}{16} \mathrm{C}^{2}\left(N_{\kappa}+2 \gamma r\right)\right. \\
& \left.+\frac{3}{8} \mathrm{AB}(n \kappa+\alpha q+\beta p)+\frac{3}{8} \mathrm{AC}(m \kappa+\gamma p+\alpha r)+\frac{1}{8} \mathrm{BC}\left(l_{\kappa}+\gamma q+\beta r\right)\right] d \lambda \\
& +\int_{0}^{\infty} \frac{1}{\Delta A^{3}}\left[1 \frac{1}{4} \mathrm{~A} \mathbb{Z}+\frac{1}{4} \mathrm{Bn}+\frac{1}{4} \mathrm{Cm}\right] d \lambda \text {. } \\
& \delta_{2}=\int_{0}^{\infty} \frac{1}{\Delta A B^{2}}\left[\kappa\left(3 \frac{3}{4} A^{2} \alpha \beta+2 \frac{1}{4} A B \beta^{2}+\frac{3}{4} A C \beta \gamma\right)\right. \\
& -\frac{3}{4} \mathrm{~A}(n \kappa+\alpha q+\beta p)-\frac{3}{4} \mathrm{~B}(M \kappa+2 \beta q)-\frac{1}{4} \mathrm{C}(l \kappa+\gamma q+\beta r) \\
& +6 \frac{9}{16} A^{3} \alpha^{2} \beta+5 \frac{5}{8} A^{2} B \alpha \beta^{2}+1 \frac{7}{8} A^{2} C \alpha \beta \gamma \\
& \left.+2 \frac{13}{16} A B^{2} \beta^{3}+\frac{9}{16} A C^{2} \beta \gamma^{2}+\frac{9}{8} A B C \beta^{2} \gamma\right] d \lambda \\
& -\int_{0}^{\infty} \frac{\kappa}{\Delta A B}\left[6 \frac{9}{16} \mathbf{A}^{3} \alpha^{2}+1 \frac{7}{8} A^{2} B \alpha \beta+1 \frac{7}{8} A^{2} C \alpha \beta+\frac{9}{16} A^{2} B^{2} \beta^{2}+\frac{9}{16} A C^{2} \gamma^{2}+\frac{3}{8} A B C \beta \gamma\right] d \lambda \\
& -\int_{0}^{\infty} \frac{1}{\Delta A B^{2}}\left[\frac{15}{16} \mathrm{~A}^{2}(L \beta+2 n \alpha)+2 \frac{13}{16} \mathrm{~B}^{2} M \beta+\frac{3}{16} \mathrm{C}^{2}(N \beta+2 l \gamma)\right. \\
& \left.\int_{0}^{\infty} 1+1 \frac{1}{8} \mathrm{AB}(M \alpha+2 n \beta)+\frac{3}{8} \mathrm{AC}(l \alpha+m \beta+n \gamma)+\frac{3}{8} \mathrm{BC}(M \gamma+2 l \beta)\right] d \lambda \\
& +\int_{0}^{\infty} \frac{1}{\Delta A B}\left[\frac{15}{16} \mathrm{~A}^{2}(L \kappa+2 \alpha p)+\frac{3}{16} \mathrm{~B}^{2}(M \kappa+2 \beta q)+\frac{3}{16} \mathrm{C}^{2}(N \kappa+2 \gamma r)\right.
\end{aligned}
$$

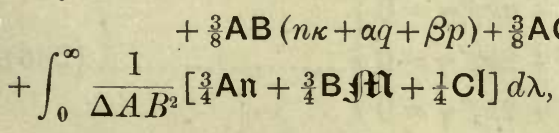

$$
\begin{aligned}
& \text { J. C. }
\end{aligned}
$$


while the value of $\delta_{3}$ may be at once written down by interchange of letters in $\delta_{2}$.

The three equations (261) can now be written in the form

$$
\begin{aligned}
& \left.\begin{array}{rl}
\mathfrak{p}\left(\frac{1}{2} \frac{\theta}{a^{6}}+\frac{3}{4} \int_{0}^{\infty} \frac{\mathrm{A} d \lambda}{\Delta A^{2}}-\frac{1}{2} \int_{0}^{\infty} \frac{d \lambda}{\Delta A^{3}}\right)+\frac{1}{4} \mathfrak{q} \int_{0}^{\infty} \frac{\mathrm{B} d \lambda}{\Delta A^{2}} & +\frac{1}{4} \mathfrak{r} \int_{0}^{\infty} \frac{\mathrm{C} d \lambda}{\Delta A^{2}} \\
& =\delta_{1}-2 \int_{0}^{\infty} \frac{G}{\Delta A^{2}} d \lambda
\end{array}\right) \\
& \frac{3}{4} \mathfrak{p} \int_{0}^{\infty} \frac{\mathrm{A} d \lambda}{\Delta A B}+\mathfrak{q}\left(\frac{1}{2} \frac{\theta}{a^{2} b^{4}}+\frac{1}{4} \int_{0}^{\infty} \frac{\mathrm{B} d \lambda}{\Delta A B}-\frac{1}{2} \int_{0}^{\infty} \frac{d \lambda}{\Delta A B^{2}}\right)+\frac{1}{4} \mathfrak{r} \int_{0}^{\infty} \frac{\mathrm{C} d \lambda}{\Delta A B} \\
& =\delta_{2}-2 \int_{0}^{\infty} \frac{G}{\Delta A B} d \lambda \\
& \frac{3}{4} \mathfrak{p} \int_{0}^{\infty} \frac{\mathrm{A} d \lambda}{\Delta A C}+\frac{1}{4} \mathfrak{⿰} \int_{0}^{\infty} \frac{\mathrm{B} d \lambda}{\Delta A C}+\mathfrak{r}\left(\frac{1}{2} \frac{\theta}{a^{2} c^{4}}+\frac{1}{4} \int_{0}^{\infty} \frac{\mathrm{C} d \lambda}{\Delta A C}-\frac{1}{2} \int_{0}^{\infty} \frac{d \lambda}{\Delta A C^{2}}\right) \\
& \left.=\delta_{3}-2 \int_{0}^{\infty} \frac{G}{\Delta A C} d \lambda\right)
\end{aligned}
$$

and $\mathfrak{p}, \mathfrak{q}, \mathfrak{r}$ do not occur in the right-hand members of these equations.

99. These equations appear at first sight to be a system of three simple linear equations determining $\mathfrak{p}, \mathfrak{q}, \mathfrak{r}$, but this proves not to be the case. Let the equations be written for brevity in the form

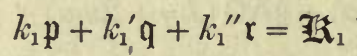

$$
\begin{aligned}
& k_{2} \mathfrak{p}+k_{2}^{\prime} \mathfrak{q}+k_{2}^{\prime \prime} \mathrm{r}=\mathbf{Z}_{2} \\
& k_{3} p+k_{3}^{\prime} \mathfrak{q}+k_{3}^{\prime \prime} \mathfrak{r}=\mathbf{z i}_{3}
\end{aligned}
$$

Then, by simple transformation of the integrals, the values of the coefficients $k_{1}, k_{1}{ }^{\prime}, \ldots$ are found to be

$$
\left.\begin{array}{lll}
k_{1}=\frac{1}{4 a^{2}}\left(\frac{2 \theta}{a^{4}}-c_{2}-c_{3}\right), \quad k_{1}^{\prime}=\frac{1}{4 b^{2}} c_{3}, & k_{1}^{\prime \prime}=\frac{1}{4 c^{2}} c_{2} \\
k_{2}=\frac{3}{4 a^{2}} c_{3}, & k_{2}^{\prime}=\frac{1}{4 b^{2}}\left(\frac{2 \theta}{a^{2} b^{2}}-3 c_{3}-c_{1}\right), \quad k_{2}^{\prime \prime}=\frac{1}{4 c^{2}} c_{1} \\
k_{3}=\frac{3}{4 a^{2}} c_{2}, & k_{3}^{\prime}=\frac{1}{4 b^{2}} c_{1}, \quad k_{3}^{\prime \prime}=\frac{1}{4 c^{2}}\left(\frac{2 \theta}{a^{2} c^{2}}-3 c_{2}-c_{1}\right)
\end{array}\right\}
$$

in which $c_{1}, c_{2}, c_{3}$ are the integrals already specified in equations (217).

With these values assigned to $k_{1}, k_{1}^{\prime}, \ldots$ it will be found that equations (218) - (220), which are the equations determining the third-harmonic displacement at the point of bifurcation, assume the form

$$
\left.\begin{array}{l}
2 k_{1} \alpha+2 k_{1}^{\prime} \beta+2 k_{1}^{\prime \prime} \gamma=0 \\
2 k_{2} \alpha+2 k_{2}^{\prime} \beta+2 k_{2}^{\prime \prime} \gamma=0 \\
2 k_{3} \alpha+2 k_{3}^{\prime} \beta+2 k_{3}^{\prime \prime} \gamma=0
\end{array}\right\}
$$


On eliminating $\alpha, \beta, \gamma$ we have the relation

$$
\left|\begin{array}{lll}
k_{1}, & k_{1}^{\prime}, & k_{1}^{\prime \prime} \\
k_{2}, & k_{2}^{\prime}, & k_{2}^{\prime \prime} \\
k_{3}, & k_{3}^{\prime}, & k_{3}^{\prime \prime}
\end{array}\right|=0
$$

which determines the condition for a point of bifurcation.

On inspecting equations (264) in the light of relation (267), it becomes clear that in general the solution for $\mathfrak{p}, \mathfrak{q}, \mathfrak{r}$ is $\mathfrak{p}=\mathfrak{q}=\mathfrak{r}=\infty$, the ratio of these quantities, from equations (266), being that of $\alpha: \beta: \gamma$. With this solution the third order terms $R_{0}$ become identical with the previously found first order terms $P_{0}$, and the attempt to extend the solution to the third order of small quantities has failed entirely.

It is, however, easy to find the condition that equations (264) shall have a finite solution. For, assuming $\mathfrak{p}, \mathfrak{q}, \mathfrak{r}$ to be finite, and multiplying the three equations (264) by the minors of $k_{1}{ }^{\prime \prime}, k_{2}^{\prime \prime}, k_{3}^{\prime \prime}$ in the determinant (267) and adding, we obtain

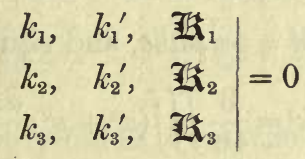

When, and only when, this relation is satisfied, there is a solution such that $\mathfrak{p}, \mathfrak{q}, \mathfrak{r}$ are finite, and there is a genuine third order solution.

After some transformation, this equation can be put in the simpler form

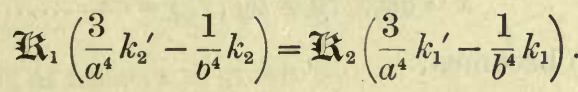

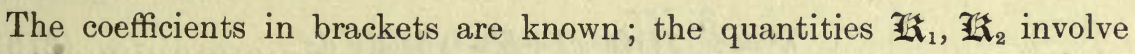
$p, q, r$ and so also $\delta \omega^{2}$ linearly (cf. equations (245) etc.), and the equation is seen to be a linear equation for $\delta \omega^{2}$. Carrying out the necessary computations, the solution of the equation is found to be

$$
\frac{\delta \omega^{2}}{2 \pi \rho}=0.0074231
$$

This gives the value of $\delta \omega^{2}$ on the true linear series; if we attempt to carry the solution beyond terms of the second order with any other value of $\delta \omega^{2}$, the solution simply lapses back to the first order solution already found. We notice that $\omega^{2}$ increases initially as we pass along the pear-shaped series.

100. On inserting into equations $(245)-(248)$ the value of $\delta \omega^{2}$ given by equation (270), we obtain

$$
\begin{aligned}
& p=3 \cdot 124954 \\
& q=-0 \cdot 103164 \\
& r=-0 \cdot 015236 \\
& s=-0 \cdot 256962
\end{aligned}
$$


thus completing the figure as far as second order terms. We have now a full knowledge of the second order pear-shaped figure, and so are in a position to determine whether or not it is stable.

\section{Calculation of the Moment of Inertia}

101. The question of stability of the pear-shaped figures turns, as we have seen, on whether or not the angular momentum $\mathbf{M}$ of these figures increases or decreases as we pass from the critical Jacobian ellipsoid along the series of pear-shaped figures.

The moment of inertia $M k^{2}$ of the pear-shaped figure about its axis of rotation is given by

$$
M k^{2}=\iiint \rho\left(x^{2}+y^{2}\right) d x d y d z
$$

We have determined the coefficient $s$ so that the mass of the pear-shaped figure shall remain always equal to the mass of the original ellipsoid.

We accordingly have $M=\frac{4}{3} \pi \rho a b c$, and equation (275) reduces to

$$
k^{2}=\frac{3}{4 \pi} \iiint\left(x^{2}+y^{2}\right) \frac{d x d y d z}{a b c}
$$

the integral being taken throughout the pear-shaped figure.

Transform to new variables $x^{\prime}, y^{\prime}, z^{\prime}$ given by

$$
x=a x^{\prime}, \quad y=b y^{\prime}, \quad z=c z^{\prime}
$$

then equation (276) becomes

$$
k^{2}=\frac{3}{4 \pi} \iiint\left(a^{2} x^{\prime 2}+b^{2} y^{\prime 2}\right) d x^{\prime} d y^{\prime} d z^{\prime}
$$

and the integral is now to be taken through the volume bounded by the surface

$x^{\prime 2}+y^{\prime 2}+z^{\prime 2}-1+e \frac{x^{\prime}}{a}\left(\alpha \frac{x^{\prime 2}}{a^{2}}+\beta \frac{y^{\prime 2}}{b^{2}}+\gamma \frac{z^{\prime 2}}{c^{2}}+\kappa\right)+\frac{1}{4} e^{2}\left[\frac{L x^{\prime 4}}{a^{4}}+\frac{M y^{\prime 4}}{b^{4}}+\ldots+s\right]=0$

which is a distorted sphere of unit radius.

Let $r$ denote the radius vector to this distorted sphere in any direction, so that $r^{2}=x^{\prime 2}+y^{\prime 2}+z^{\prime 2}$. Let us again transform to coordinates $\mathrm{x}, \mathrm{y}, \mathrm{z}$, given by

$$
x^{\prime}=r \mathrm{x}, \quad y^{\prime}=r \mathrm{y}, \quad z^{\prime}=r \mathrm{z},
$$

so that $\mathrm{x}, \mathrm{y}, \mathrm{z}$ are coordinates of points on a sphere of unit radius. Equation (278) becomes

$$
k^{2}=\frac{3}{4 \pi} \iiint\left(a^{2} \mathrm{x}^{2}+b^{2} \mathrm{y}^{2}\right) r^{4} d r d S
$$

where $d S$ is an element of surface on this unit sphere. 
Equation (279) becomes

$$
r^{2}-1+e r^{3} \frac{\mathrm{x}}{a}\left(\alpha \frac{\mathrm{x}^{2}}{a^{2}}+\beta \frac{\mathrm{y}^{2}}{b^{2}}+\gamma \frac{\mathrm{z}^{2}}{c^{2}}\right)+e r \frac{\mathrm{x} \kappa}{a}+\frac{1}{4} e^{2}\left[\frac{L \mathrm{x}^{4} r^{4}}{a^{4}}+\frac{M \mathrm{y}^{4} r^{4}}{b^{4}}+\ldots+s\right]=0
$$

and this may be regarded as an equation to determine $r$. Let its solution be supposed to be

$$
r=1+e f+e^{2} g+e^{3} h+\ldots
$$

Then, on carrying out the integration with respect to $r$ in equation (280) we obtain

$$
k^{2}=\frac{3}{20 \pi} \iint\left(a^{2} x^{2}+b^{2} y^{2}\right)\left(1+5 e f+5 e^{2} g+10 e^{2} f^{2}\right) d S
$$

Clearly the term $\left(a^{2} \mathrm{x}^{2}+b^{2} \mathrm{y}^{2}\right)(5 e f)$ vanishes on integration, so that $k^{2}$ may be put in the form $k_{0}^{2}+\Delta k^{2}$, where

$$
\begin{aligned}
k_{0}^{2} & =\frac{1}{5}\left(a^{2}+b^{2}\right), \\
\Delta k^{2} & =\frac{3 e^{2}}{4 \pi} \iint\left(a^{2} x^{2}+b^{2} y^{2}\right)\left(g+2 f^{2}\right) d S .
\end{aligned}
$$

The values of $f, g$ are readily found from the condition that the solution (282) shall satisfy equation (281). Carrying out the necessary computations, we obtain

$$
\begin{gathered}
k_{0}^{2}=0.844105, \\
\Delta k^{2}=-0.079156 e^{2},
\end{gathered}
$$

so that the moment of inertia is given by

$$
M k^{2}=M k_{0}{ }^{2}\left(1-0.09378 e^{2}\right)
$$

\section{The Stability Criterion}

102. Collecting results, we have now found for the pear-shaped figure

$$
\begin{aligned}
\frac{\omega^{2}}{2 \pi \rho} & =0.14200\left(1+0.05227 e^{2}\right) \\
k^{2} & =0.8441\left(1-0.09378 e^{2}\right) \ldots
\end{aligned}
$$

whence it is readily found that the moment of momentum $M$ is connected with the moment of momentum $\mathbf{M}_{0}$ of the critical Jacobian ellipsoid by the relation

$$
M=M_{0}\left(1-0.06765 e^{2}\right)
$$

Thus it appears that $\mathbf{M}<\mathbf{M}_{0}$, the moment of momentum decreases as we pass along the pear-shaped series. This series is accordingly proved to be unstable. 
103. The fact that the pear-shaped series is initially unstable shews that a rotating mass cannot evolve by slow secular changes through a series of pear-shaped figures. This somewhat diminishes the interest of the pearshaped series in the problem of cosmogony, but nevertheless it remains important to obtain as clear an idea as we can of the nature of this series. For we shall find, when we come to the discussion of dynamical motions, that the unstable series are of the utmost importance in directing the course of dynamical or cataclysmal motions such as occur when statical evolution is no longer possible.

There is nothing in abstract theory to prevent us following out the configuration of the pear-shaped series as far as we like, but the labour of computation would be so great as to make this course impracticable.

A problem which admits of very much easier solution is the two-dimensional problem of tracing out the sequence of configurations of a rotating cylinder of liquid. So far as the three-dimensional case has been solved, the analogy between the two-dimensional and three-dimensional cases is so very close that we may reasonably hope that it will persist beyond. If this is so, we can discover the general nature of the solution to the three-dimensional problem by examining that of the much simpler two-dimensional problem. We accordingly turn to a discussion of the two-dimensional problem.

\section{The Configurations of Equilibrium of Rotating Liquid Cylinders}

104. Let $F(x, y)=0$ be the equation of a cylindrical boundary in the plane of $x, y$, and for simplicity let us assume the axis of $x$ to be one of symmetry.

Let us change to complex variables $\xi$, $\eta$ defined by

$$
\xi=x+i y, \quad \eta=x-i y,
$$

and let the equation of the curve become

$$
f(\xi, \eta)=0
$$

If the original curve was symmetrical about the axis of $x$, the function $f$ must of course be symmetrical in $\xi$ and $\eta$.

To write down the potential of a homogeneous cylindrical mass having (287) as the equation of its cross-section, we solve the equation explicitly for $\xi$; let the solution be

$$
\xi=\Phi(\eta) \equiv \phi(\eta)+\psi(\eta)
$$

where $\phi(\eta)$ and $\psi(\eta)$ are terms in ascending and descending powers of $\eta$ respectively, say

$$
\begin{aligned}
& \phi(\eta)=p_{0}+p_{1} \eta+p_{2} \eta^{2}+\ldots \\
& \psi(\eta)=p_{-1} \eta^{-1}+p_{-2} \eta^{-2}+\ldots
\end{aligned}
$$


Then it can be verified ${ }^{*}$ that the potentials of the cylinder, assumed composed of homogeneous matter of density $\rho$, are given by

$$
\begin{aligned}
& V_{i}=\pi \rho\left\{\int_{0}^{\xi} \phi(\xi) d \xi+\int_{0}^{\eta} \phi(\eta) d \eta-\xi \eta\right\}+\text { cons. } \\
& V_{0}=\pi \rho\left\{\int_{\xi}^{\infty} \psi(\xi) d \xi+\int_{\eta}^{\infty} \psi(\eta) d \eta\right\}+\text { cons. } . . .
\end{aligned}
$$

105. Now suppose that a cylinder of matter of density $\rho$ has for its equation $x^{2}+y^{2}=a^{2}$ or

$$
\xi \eta=a^{2}
$$

when there is no rotation, and that under a rotation $\omega$, this gives place to a boundary of equation

$$
\xi \eta=a^{2}+a_{1}(\xi+\eta)+a_{2}\left(\xi^{2}+\eta^{2}\right)+
$$

or, in polar coordinates,

$$
r^{2}=a^{2}+2 a_{1} r \cos \theta+2 a_{2} r^{2} \cos 2 \theta+\ldots
$$

The condition that the surface (294) can be a figure of equilibrium is that, at every point of the boundary,

$$
V_{i}+\frac{1}{2} \omega^{2} r^{2}=\text { a cons. }
$$

Since the value of $r^{2}$ at the boundary is given by equation (294), this condition readily transforms into

$$
V_{i}+\pi \rho r^{2}-\pi \rho\left(1-\frac{\omega^{2}}{2 \pi \rho}\right)\left(a^{2}+2 a_{1} r \cos \theta+2 a_{2} r^{2} \cos 2 \theta+\ldots\right)+\text { cons. }=0
$$

This expression must vanish at every point of the boundary; it is readily seen to be harmonic, and so must vanish at every point inside the boundary.

From equation (291) the potential $V_{i}$ must be of the form

$$
V_{i}=\pi \rho\left[\sum_{n=1}^{n=\infty} b_{n}\left(\xi^{n}+\eta^{n}\right)-\xi \eta\right]+\text { a cons. }
$$

where $b_{1}, b_{2}, \ldots$ are functions of $a_{1}, a_{2}, \ldots$. Hence equation (296) assumes the form

$\Sigma b_{n}\left(\xi^{n}+\eta^{n}\right)-\left(1-\frac{\omega^{2}}{2 \pi \rho}\right)\left[a^{2}+a_{1}(\xi+\eta)+a_{2}\left(\xi^{2}+\eta^{2}\right)+\ldots\right]=$ a cons....

This must be satisfied at every point inside the boundary; equating coefficients we obtain

$$
\left.\begin{array}{l}
b_{1}=\left(1-\frac{\omega^{2}}{2 \pi \rho}\right) a_{1} \\
b_{2}=\left(1-\frac{\omega^{2}}{2 \pi \rho}\right) a_{2}, \text { etc. }
\end{array}\right\}
$$

*Phil. Trans. 200 A (1902), p. 67. 
These are the conditions that the surface (294) shall be a possible figure of equilibrium under a rotation $\omega$.

The points of bifurcation and points at which $\omega^{2}$ reaches a turning point will be determined by the Hessian of this system of equations*, namely

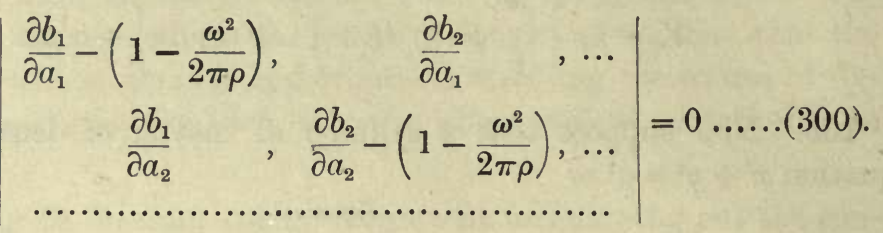

If it were possible to calculate the $b$ 's in terms of the $a$ 's and solve equations (299) and (300) in the most general case, we should obtain a complete knowledge of all the linear series and their points of bifurcation. As this is not possible, we start from a known configuration and trace out configurations by following the different series.

106. The simplest configuration is the circular one, for which

$$
a_{1}=a_{2}=a_{3}=\ldots=0 \text {. }
$$

With these values all the $b$ 's vanish, and equations (299) are satisfied for all values of $\omega$. Thus there is a linear series of circular configurations, along which $\omega$ increases from zero upwards, and this is obviously the two-diment sional analogue of the series of Maclaurin's spheroids.

To search for points of bifurcation on this series we examine configurations in which $a_{1}, a_{2}, \ldots$ are all small. Neglecting squares of these small quantities equation (293) becomes

$$
\xi \eta=a^{2}+a_{1}\left(\eta+a^{2} \eta^{-1}\right)+a_{2}\left(\eta^{2}+a^{4} \eta^{-2}\right)+\ldots
$$

so that, by comparison with equation (288),

and, from (291),

$$
\phi(\eta)=a_{1}+a_{2} \eta+a_{3} \eta^{2}+\ldots
$$

$$
V_{i}=\pi \rho\left\{a_{1}(\xi+\eta)+\frac{1}{2} a_{2}\left(\xi^{2}+\eta^{2}\right)+\frac{1}{3} a_{3}\left(\xi^{3}+\eta^{3}\right)+\ldots-\xi \eta\right\}+\text { cons. }
$$

Comparing with equation (297), the value of $b_{n}$ is seen to be $a_{n} / n$. Thus the determinant in equation (300) reduces to its leading diagonal, and the points of bifurcation on the circular series are given by

$$
\left[1-\left(1-\frac{\omega^{2}}{2 \pi \rho}\right)\right]\left[\frac{1}{2}-\left(1-\frac{\omega^{2}}{2 \pi \rho}\right)\right]\left[\frac{1}{3}-\left(1-\frac{\omega^{2}}{2 \pi \rho}\right)\right] \ldots=0 .
$$

As a typical solution we have

$$
\frac{\omega^{2}}{2 \pi \rho}=1-\frac{1}{n}
$$


corresponding to a distortion of the circular section such that $a_{n}$ alone occurs in equation (293). The different points of bifurcation correspond to the different integral values of $n$.

107. The value $n=1$ may be rejected at once, since the corresponding displacement is merely a rigid-body displacement of the cylinder when at rest. Thus the first real point of bifurcation is given by $n=2$. At this point

$$
\frac{\omega^{2}}{2 \pi \rho}=\frac{1}{2},
$$

and here the series of circular configurations loses its stability. The branch series has for its equation initially;

$$
r^{2}=a^{2}+2 a_{2} r^{2} \cos \theta
$$

so is of elliptical cross-section.

When $a_{2}$ is small, the value of $b_{2}$ has been seen to be $\frac{1}{2} a_{2}$. But when the boundary is determined by equation (301), the values of the b's are easily determined, whether $\alpha_{2}$ is small or not. Equation (293) reduces to

$$
\xi \eta=a^{2}+a_{2}\left(\xi^{2}+\eta^{2}\right),
$$

of which the solution is of the form

where $\alpha$ is a root of

$$
\xi=\alpha \eta+q_{-1} \eta^{-1}+q_{-3} \eta^{-3}+\ldots,
$$

$$
\alpha=a_{2}\left(1+\alpha^{2}\right)
$$

Thus the general value of $b_{2}$ is $\frac{1}{2} \alpha$ and all the other $b$ 's vanish. The equations of equilibrium (299) can accordingly be satisfied by a surface of boundary (301) for all values of $a_{2}$. Thus the branch series through the point of bifurcation just found is a series such that $a_{2}$ varies from 0 to $\infty$ in equation (301). The configurations form a series of elliptic cylinders, which are obviously the two-dimensional analogue of the Jacobian ellipsoids.

The conditions of equilibrium (299) reduce to the single equation

$$
\frac{1}{2} \alpha=\left(1-\frac{\omega^{2}}{2 \pi \rho}\right) a_{2}
$$

which on combination with (302) gives

$$
\frac{\omega^{2}}{2 \pi \rho}=\frac{1}{2}-\frac{1}{2} \alpha^{2}
$$

Thus as we pass along this elliptic series, $\omega^{2}$ decreases from $\pi \rho$ to 0 . The angular momentum is however found to increase, so that the series is initially stable. 
108. To search for points of bifurcation on this series, we have to examine configurations for which all the $a$ 's are small except $a_{2}$. We readily find that $b_{n}$ must be of the form

$$
b_{n}=\frac{1+\alpha^{n}}{1-2 \alpha a_{2}} \frac{a_{n}}{n}+\text { terms linear in } a_{n+2}, a_{n+4}, \ldots
$$

Hence when $m<n, \partial b_{n} / \partial a_{m}=0$, and when $m=n$,

$$
\frac{\partial b_{n}}{\partial a_{n}}=\frac{1+a^{n}}{n\left(1-2 \alpha a_{2}\right)} .
$$

Thus in equation (300) all terms below the leading diagonal vanish; the determinant reduces to the product of the terms in its leading diagonal, and the equation for points of bifurcation reduces to the separate equations

$$
\frac{1+\alpha^{n}}{n\left(1-2 \alpha a_{2}\right)}=1-\frac{\omega^{2}}{2 \pi \rho}(n=3,4,5, \ldots)
$$

Simplified with the help of equations (303) and (304), this equation is found to reduce to

$$
\frac{1-a^{2}}{2}=\frac{1+\alpha^{n}}{n}
$$

This equation is readily solved by graphical methods. In fig. 17 the curve which is concave to the axis of $\alpha$ is the parabola $y=\frac{1}{2}\left(1-\alpha^{2}\right)$, while

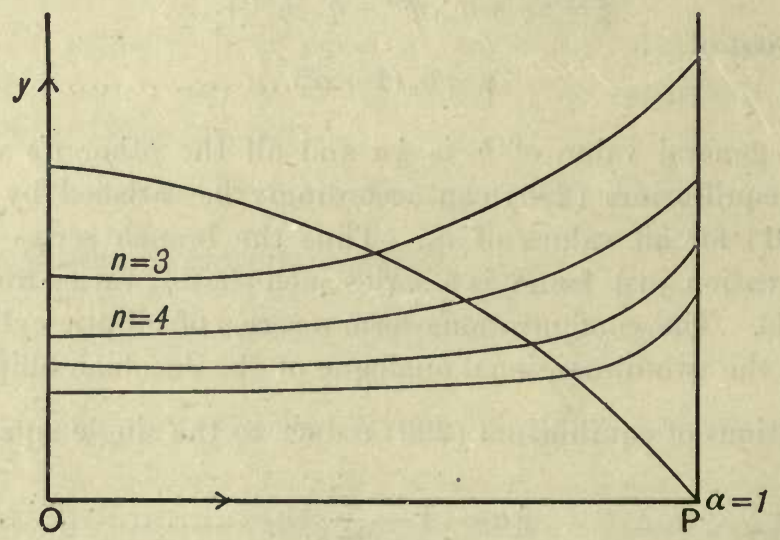

Fig. 17.

the remaining curves are the graphs of

$$
y=\frac{1+a^{n}}{n}
$$

for the values $n=3,4, \ldots$. As we pass along the elliptic series, starting from the point of bifurcation with the circular series, we may suppose that we pass along the axis $O P$ in fig. 17 from $\alpha=0$ to $\alpha=1$. 
The different intersections between the parabola and the other curves will represent the different points of bifurcation. And, just as Poincaré has shewn to be the case in the three-dimensional problem, so we see here that there is one point of bifurcation of each of the orders $n=3,4,5, \ldots$, and that they occur in this order.

The elliptical series accordingly loses its stability at the point of bifurcation $n=3$. The position of this point is obtained by solving equation (306) with $n$ put equal to 3 , and the solution is readily found to be $\alpha=\frac{1}{2}$.

From equations (303) and (304) we find that at this point of bifurcation $\omega^{2}=\frac{3}{4} \pi \rho$, and $a_{2}=\frac{2}{5}$. The configuration at the point of bifurcation is accordingly the elliptic cylinder

or, in Cartesian Coordinates,

$$
\xi \eta=a^{2}+\frac{2}{5}\left(\xi^{2}+\eta^{2}\right)
$$

$$
x^{2}+9 y^{2}=5 a^{2}
$$

109. Near the point of bifurcation, the configuration of the new linear series will be determined by an equation of the form

$$
\xi \eta=a^{2}+\frac{2}{5}\left(\xi^{2}+\eta^{2}\right)+a_{3}\left(\xi^{3}+\eta^{3}\right)+a_{1}(\xi+\eta)
$$

and this is at once seen to be analogous to the pear-shaped series in threedimensions. The problem before us is to extend this series as far as possible in the hope, which will be found to be fully justified by the event, that the series will be found to be closely analogous to the three-dimensional series.

Let us assume for the general configuration an expansion of the form

$$
\xi \eta=a^{2}+\frac{2}{5}\left(\xi^{2}+\eta^{2}\right)+\sum_{s=1}^{s=\infty} \theta^{s}\left\{{ }_{8} C_{0}+\sum_{n=1}^{n=\infty}{ }_{8} C_{n}\left(\xi^{n}+\eta^{n}\right)\right\}
$$

where $\theta$ is a parameter which vanishes at the point of bifurcation and continually increases as we pass along the series. The corresponding value of $\omega$ may be supposed given by

$$
1-\frac{\omega^{2}}{2 \pi \rho}=\delta_{0}+\delta_{2} \theta^{2}+\delta_{4} 6^{4}+\delta_{6} \theta^{6}+\ldots
$$

it being immediately found that terms in $\theta, \theta^{3}, \ldots$ are unnecessary. It has already been seen that $\delta_{0}$, which is the value of $1-\omega^{2} / 2 \pi \rho$ at the point of bifurcation, is equal to $\frac{5}{8}$.

Let us suppose that equation (310), solved explicitly for $\xi$, has the solution

$$
\xi=\left(1-\frac{\omega^{2}}{2 \pi \rho}\right)\left(\frac{8}{5} \xi_{0}+\xi_{1} \theta+\xi_{2} \theta^{2}+\xi_{3} \theta^{3}+\ldots\right)
$$

where $\xi_{0}$ is the already known value of $\xi$ when $\theta=0$, and $\xi_{8}$ is a general series of ascending and descending powers of $\eta$, say

$$
\xi_{8}=s_{0}+s_{1} \eta+s_{2} \eta^{2}+\ldots+s_{-1} \eta^{-1}+s_{-2} \eta^{-2}+\ldots
$$


From this expansion for $\xi$, the value of $V_{i}$ can at once be written down; it will agree with equation (297) if we take

$$
b_{n}=\left(1-\frac{\omega^{2}}{2 \pi \rho}\right) \sum_{s=1}^{s=\infty} \frac{s_{n-1}}{n} \theta^{s} \quad(n=1,2, \ldots) \ldots
$$

The equations of equilibrium (299) which must be satisfied are however of the type

$$
b_{n}=\left(1-\frac{\omega^{2}}{2 \pi \rho}\right) a_{n}=\left(1-\frac{\omega^{2}}{2 \pi \rho}\right) \sum_{s=1}^{s=\infty} C_{s} C_{n} \theta^{s}
$$

so that the conditions for equilibrium are that

$$
{ }_{8} C_{n}=\frac{s_{n-1}}{n} \quad(n=1,2,3, \ldots)
$$

for all $s$ 's. Thus in order to satisfy the equation of equilibrium it is merely necessary that $\xi_{8}$ shall be of the form (cf. equation (313))

$$
\xi_{8}={ }_{8} C_{1}+2{ }_{8} C_{2} \eta+3{ }_{8} C_{3} \eta^{2}+\ldots+s_{-1} \eta^{-1}+s_{-2} \eta^{-2}+\ldots
$$

To introduce the limitation that the curve shall remain of constant area we must have $s_{-1}=0$, as is at once evident on considering the form assumed by $V_{0}$ at infinity. To keep the centre of gravity at the origin we must further have $s_{-2}=0$. If we replace $s_{-2}, s_{-3}, \ldots$ by new symbols ${ }_{8} C_{-1}{ }_{8} C_{-2}, \ldots$ we may write equation (316) in the symmetrical form

$$
\xi_{8}=\sum_{n=-\infty}^{n=+\infty} n_{8} C_{n} \eta^{n-1}
$$

in which there is no term in ${ }_{8} C_{0}$ and we know that ${ }_{8} C_{-1}$ must ultimately be zero in order that the centre of gravity may remain on the axis of rotation.

Thus we have found that the assumed equation (310) will represent a configuration of equilibrium provided the explicit solution for $\xi$ is of the form (312) in which $\xi_{8}$ etc. are given by equations of the type of (317).

110. Let us introduce $p_{0}, p_{1}, p_{2}, \ldots$ defined by

$$
\begin{aligned}
& p_{0}=\frac{8}{5} \delta_{0} \xi_{0}=\xi_{0}, \\
& p_{1}=\delta_{0} \xi_{1}, \\
& p_{2}=\delta_{0} \xi_{2}+\frac{8}{5} \delta_{2} \xi_{0}, \\
& p_{3}=\delta_{0} \xi_{3}+\delta_{2} \xi_{1}, \\
& p_{4}=\delta_{0} \xi_{4}+\delta_{2} \xi_{2}+\frac{8}{5} \delta_{4} \xi_{0}, \text { etc. }
\end{aligned}
$$

Then on substituting for $1-\omega^{2} / 2 \pi \rho$ from equation (311), the supposed solution (312) assumes the form

$$
\xi=\xi_{0}+p_{1} \theta+p_{2} \theta^{2}+p_{3} \theta^{3}+\ldots
$$


This is to be a solution of equation (310), so that the following equation must be an identity:

$$
\begin{aligned}
\eta\left(\xi_{0}+p_{1} \theta\right. & \left.+p_{2} \theta^{2}+p_{3} \theta^{3}+\ldots\right) \\
& =a^{2}+\frac{2}{5} \eta^{2}+\frac{2}{5}\left(\xi_{0}+p_{1} \theta+p_{2} \theta^{2}+p_{3} \theta^{3}+\ldots\right)^{2} \\
& +\sum_{s=1}^{s=\infty} \theta^{s}\left\{C_{s}+\sum_{n=1}^{n=\infty}{ }_{s} C_{n}\left[\eta^{n}+\left(\xi_{0}+p_{1} \theta+p_{2} \theta^{2}+\ldots\right)^{n}\right]\right\}
\end{aligned}
$$

To avoid the useless printing of terms which would ultimately be found to vanish, we shall at once strike out all coefficients ${ }_{8} C_{n}$ of which the true value is zero. Accordingly, in place of the general equation (317), we assume separate equations of the form

$$
\begin{aligned}
& \xi_{1}=3 c_{3} \eta^{2}+c_{1}-c_{-1} \eta^{-2}-3 c_{-3} \eta^{-4}-5 c_{-5} \eta^{-6}-7 c_{-7} \eta^{-8} \\
& \xi_{2}=4 d_{4} \eta^{3}+2 d_{2} \eta+0 . d_{0} \eta^{-1}-2 d_{-2} \eta^{-3}-4 d_{-4} \eta^{-5}-6 d_{-6} \eta^{-7} \\
& \xi_{3}=5 e_{5} \eta^{4}+3 e_{3} \eta^{2}+e_{1}-e_{-1} \eta^{-2}-3 e_{-3} \eta^{-4}-5 e_{-5} \eta^{-6} \ldots \ldots \ldots \ldots \\
& \xi_{4}=6 f_{6} \eta^{5}+4 f_{4} \eta^{3}+2 f_{2} \eta+0 . f_{0} \eta^{-1}-2 f_{-2} \eta^{-3}-4 f_{-4} \eta^{-5} \ldots \ldots
\end{aligned}
$$

and so on. In these equations terms such as $0 . d_{0} \eta^{-1}$ have no value but are written in for completeness. The quantities $d_{0}, f_{0}$ etc. do not themselves vanish but represent the quantities ${ }_{2} C_{0}{ }_{4} C_{0}$ etc. which may have finite values. Each of the series $\xi_{1}, \xi_{2}, \xi_{3}, \xi_{4}, \ldots$ extend to infinity, but we shall not require more than the six first terms written down to give the approximation to which we are working. We shall assume three similar series for $\xi_{5}, \xi_{6}$ and $\xi_{7}$, the coefficients being denoted by the letters $g, h, i$ respectively.

111. Since equation (319) is to be an identity for all values of $\theta$ and $\eta$, we may equate the coefficients of $\theta$ and shall obtain a system of equations which must be true for all values of $\eta$. The equations obtained by equating coefficients of $\theta^{0}, \theta, \theta^{2}, \theta^{3}, \ldots$ are found to be as follows :

$$
\begin{aligned}
& \eta \xi_{0}=a^{2}+\frac{2}{5}\left(\eta^{2}+\xi_{0}^{2}\right) \\
& \left(\eta-\frac{4}{5} \xi_{0}\right) p_{1}=c_{1}\left(\eta+\xi_{0}\right)+c_{3}\left(\eta^{3}+\xi_{0}^{3}\right) \\
& \left(\eta-\frac{4}{5} \xi_{0}\right) p_{2}=\frac{2}{5} p_{1}^{2}+c_{1} p_{1}+c_{3}\left(3 p_{1} \xi_{0}{ }^{2}\right)+d_{0}+d_{2}\left(\eta^{2}+\xi_{0}{ }^{2}\right)+d_{4}\left(\eta^{4}+\xi_{0}^{4}\right) . \\
& \left(\eta-\frac{4}{5} \xi_{0}\right) p_{3}=\frac{2}{5}\left(2 p_{1} p_{2}\right)+c_{1} p_{2}+c_{3}\left(3 p_{2} \xi_{0}^{2}+3 p_{1}^{2} \xi_{0}\right) \\
& +d_{2}\left(2 \xi_{0} p_{1}\right)+d_{4}\left(4 \xi_{0}^{3} p_{1}\right)+e_{1}\left(\eta+\xi_{0}\right)+e_{3}\left(\eta^{3}+\xi_{0}^{3}\right)+e_{5}\left(\eta^{5}+\xi_{0}^{5}\right) \\
& \left(\eta-\frac{4}{5} \xi_{0}\right) p_{4}=\frac{2}{5}\left(2 p_{1} p_{3}+p_{2}{ }^{2}\right)+c_{1} p_{3}+c_{3}\left(3 p_{3} \xi_{0}{ }^{2}+6 p_{2} p_{1} \xi_{0}+p_{1}{ }^{3}\right) \\
& +d_{2}\left(2 \xi_{0} p_{2}+p_{1}^{2}\right)+d_{4}\left(4 p_{2} \xi_{0}^{3}+6 p_{1}^{2} \xi_{0}^{2}\right) \\
& +e_{1} p_{1}+e_{3}\left(3 p_{1} \xi_{0}^{2}\right)+e_{5}\left(5 p_{1} \xi_{0}^{4}\right) \\
& +f_{0}+f_{2}\left(\eta^{2}+\xi_{0}^{2}\right)+f_{4}\left(\eta^{4}+\xi_{0}^{4}\right)+f_{6}\left(\eta^{6}+\xi_{0}^{6}\right)
\end{aligned}
$$

and similar equations. 
112. Equation (324) is a quadratic equation giving $\xi_{0}$ in terms of $\eta$. Writing $a=1$ for convenience, the solution is

$$
\begin{aligned}
\xi_{0} & =\frac{5}{4} \eta-\frac{1}{2}\left(\frac{9}{4} \eta^{2}-10\right)^{\frac{1}{2}} \ldots \ldots \ldots \ldots \ldots \ldots \ldots \ldots \ldots \ldots \ldots \ldots \ldots \ldots \ldots \ldots \ldots \ldots \ldots \ldots \\
& =\frac{1}{2} \eta+\frac{5}{3} \eta^{-1}+\frac{50}{27} \eta^{-3}+\frac{1000}{.243} \eta^{-5}+\frac{25000}{3^{7} \eta^{7}}+\frac{700,000}{3^{9} \eta^{9}}+\ldots
\end{aligned}
$$

Inserting this value for $\xi_{0}$ into equation (325), this equation becomes an equation in $\eta^{\prime}$ having $c_{3}, c_{1}, c_{-1}, \ldots$ as coefficients. Since this equation must be an identity we may equate the coefficients of different powers of $\eta$ and obtain

$$
\begin{aligned}
\frac{9}{8} c_{3} & =\frac{9}{8} c_{3} \\
-\frac{5}{2} c_{3}+\frac{3}{8} c_{1} & =\frac{5}{4} c_{3}+\frac{3}{2} c_{1}, \\
-\frac{25}{9} c_{3}-\frac{5}{6} c_{1}-\frac{3}{8} c_{-1} & =\frac{50}{9} c_{3}+\frac{5}{3} c_{1} \\
-\frac{500}{81} c_{3}-\frac{25}{27} c_{1}+\frac{5}{6} c_{-1}-\frac{9}{8} c_{-3} & =\frac{1375}{81} c_{3}+\frac{50}{27} c_{1}, \text { etc. }
\end{aligned}
$$

The first equation is satisfied automatically, as it ought to be. We may assign any value we please to $c_{3}$, this merely determining the scale on which the parameter $\theta$ is measured. Taking $c_{3}=1$ we find in succession

$$
c_{3}=1, \quad c_{1}=-\frac{10}{3}, \quad c_{-1}=0, \quad c_{-3}=-\frac{1000}{81} .
$$

The vanishing of $c_{-1}$ shews that the centre of gravity of the curve is, as it ought to be, at the origin.

The value of $\xi_{1}$ is now given in the form

$$
\dot{\xi}_{1}=3 \eta^{2}-\frac{10}{3}+\frac{1000}{27} \eta^{-4}+\ldots
$$

Inserting this value for $\xi_{1}$ in equation (326) and equating coefficients, we obtain the equations

$$
\begin{aligned}
\frac{3}{4} d_{4} & =\frac{45}{16}+\frac{17}{16} d_{4} \\
-\frac{10}{3} d_{4}+\frac{3}{4} d_{2}+\frac{12}{25} \delta_{2}= & -\frac{25}{16}+\frac{5}{6} d_{4}+\frac{5}{4} d_{2} \\
-\frac{100}{27} d_{4}-\frac{5}{3} d_{2}+\frac{8}{15} \delta_{2}= & \frac{875}{36}+\frac{275}{54} d_{4}+\frac{5}{3} d_{2}+d_{0} \\
-\frac{2000}{243} d_{4}-\frac{50}{27} d_{2}-\frac{80}{27} \delta_{2}-\frac{3}{4} d_{-2}= & \frac{4375}{54}+\frac{5000}{243} d_{4}+\frac{125}{27} d_{2}
\end{aligned}
$$

Solving, we obtain in succession,

$$
\left.\begin{array}{ll}
d_{4}=\frac{45}{7}, & d_{2}=-\frac{2825}{56}+\frac{24}{25} \delta_{2} \\
d_{0}=\frac{5500}{63}-\frac{8}{3} \delta_{2}, & d_{-2}=\frac{4375}{54}-\frac{992}{81} \delta_{2}
\end{array}\right\}
$$

This completes the solution as far as second order terms, and we find, precisely as in the three-dimensional problem, that there is an ambiguity in the solution, in that $\delta_{2}$ has not been determined and cannot be until we proceed to terms of higher order. 
The third order terms are determined by equation (327). Equating coefficients as before, we obtain the equations

$$
\begin{aligned}
\frac{15}{8} e_{5} & =\frac{6075}{128}+\frac{33}{32} e_{5} \\
-\frac{25}{6} e_{5}+\frac{9}{8} e_{3}-\frac{9}{2} \delta_{2} & =-\frac{158,625}{896}+\frac{25}{48} e_{5}+\frac{9}{8} e_{3} \\
-\frac{125}{27} e_{5}-\frac{5}{2} e_{3}+\frac{3}{8} e_{1}-16 \delta_{2} & =\frac{5625}{28}+\frac{875}{216} e_{5}+\frac{5}{4} e_{3}+\frac{3}{2} e_{1} \\
-\frac{2500}{243} e_{5}-\frac{25}{9} e_{3}-\frac{5}{6} e_{1}-\frac{620}{9} \delta_{2}-\frac{3}{8} e_{-1} & =\frac{440,625}{1008}+\frac{5000}{243} e_{5}+\frac{50}{9} e_{3}+\frac{5}{8} e_{1}
\end{aligned}
$$

in which of course the unknown quantity $\delta_{2}$ still appears. To satisfy the first of these equations we must take

$$
e_{5}=\frac{225}{4} \text {. }
$$

The second equation is not, as might at first have been expected, an equation for $e_{3}$. If $\delta_{2}$ has a certain value, it is satisfied by any value of $e_{3}$, but if $\delta_{2}$ has a value different from this, there is no solution other than $e_{3}=\infty$. An examination of this equation will elucidate at once the whole of the difficulty that was encountered in determining the true second order solution in the three-dimensional problem (cf. $\$ \$ 93-99$ ).

For $e_{3}$ to have a finite value, $\delta_{2}$ must have the value

$$
\delta_{2}=-\frac{8625}{448}
$$

The third equation now does not become an equation for $e_{1}$ but for $e_{3}+\frac{10}{3} e_{1}$. It is satisfied by

$$
e_{3}=-\frac{17.075}{168}+\lambda, \quad e_{1}=-\frac{10}{3} \lambda,
$$

where $\lambda$ may have any value. Finally, the fourth equation does not determine $\lambda$; it reduces merely to $e_{-1}=0$, and so merely provides a check on the accuracy of our work (cf. $\S 109$ ).

113. Collecting the values of the various constants, we find as the equation to the surface (equation 293),

$$
\begin{aligned}
\xi \eta=1 & +\frac{2}{5}\left(\xi^{2}+\eta^{2}\right)+\left\{\left(\xi^{3}+\eta^{3}\right)-\frac{10}{3}(\xi+\eta)\right\}\left(\theta+\lambda \theta^{3}\right) \\
& +\theta^{2}\left\{\frac{45}{7}\left(\xi^{4}+\eta^{4}\right)-\frac{965}{14}\left(\xi^{2}+\eta^{2}\right)+\frac{69,875}{504}\right\} \\
& +\theta^{3}\left\{\frac{225}{4}\left(\xi^{5}+\eta^{5}\right)-\frac{17,075}{168}\left(\xi^{3}+\eta^{3}\right)\right\}+\text { terms in } \theta^{4}, \theta^{5}, \text { etc. }
\end{aligned}
$$

The occurrence of the indeterminate quantity $\lambda$ can easily be accounted for. For if we have a solution

$$
\xi \eta=a^{2}+\theta f_{1}+\theta^{2} f_{2}+\theta^{3} f_{3}+\ldots
$$

corresponding to a parameter $\theta$ which is connected with the rotation by the relation

$$
1-\omega^{2} / 2 \pi \rho=\delta_{0}+\delta_{2} \theta^{2}+\delta_{3} \theta^{3}+\cdots
$$

then we can obtain precisely the same solution in another form on replacing the parameter $\theta$ by $\theta+\lambda \theta^{3}$. It accordingly appears that the quantity $\lambda$ is 
entirely at our disposal, and we shall accordingly take $\lambda=0$. We shall, as a matter of numerical convenience, replace the parameter $\theta$ by a new parameter $\theta^{\prime}$ such that $1000 \theta^{2}=\theta^{\prime 2}$.

As far as terms in $\theta^{\prime}$, the pear-shaped figure determined by equation (333) is now found to be

$$
\frac{x^{2}}{5}+\frac{y^{2}}{0 \cdot 555}+\theta^{\prime}\left(-0 \cdot 063 x^{2}+0 \cdot 190 x y^{2}+0 \cdot 211 x\right)=1 .
$$

The corresponding pear-shaped figure in three-dimensions was

$$
\frac{x^{2}}{3 \cdot 55}+\frac{y^{2}}{0 \cdot 664}+\frac{z^{2}}{0 \cdot 424}+e\left(-0 \cdot 079 x^{3}+0 \cdot 127 x y^{2}+0 \cdot 106 x z^{2}+0 \cdot 142 x\right)=1,
$$

and we see that the two figures agree as closely as possible if we take $\theta^{\prime}=e$. Thus our new choice of parameter results in $\theta^{\prime}$ having the same meaning as $e$ has in the three-dimensional problem.

The second order solution now assumes the definite form

$$
\begin{aligned}
r^{2}=1+\frac{4}{5} r^{2} \cos 2 \phi & +2 \theta^{\prime} \cdot 10^{-\frac{3}{2}}\left(r^{3} \cos 3 \phi-\frac{10}{3} r \cos \phi\right) \\
& +10^{-3} \theta^{\prime 2}\left(\frac{90}{7} r^{4} \cos 4 \phi-\frac{965}{7} r^{2} \cos 2 \phi+\frac{69,875}{504}\right) \ldots(336),
\end{aligned}
$$

while the value of $\omega^{2}$ is given by

$$
\frac{\omega^{2}}{2 \pi \rho}=0.3750\left(1+0.0513 \theta^{\prime 2}\right)
$$

We may notice that this rate of increase of $\omega^{2}$ is closely analogous to that in the three-dimensional problem

$$
\frac{\omega^{2}}{2 \pi \rho}=0 \cdot 14200\left(1+0.05227 e^{2}\right) \text {. }
$$

On calculating the moment of inertia of the curve defined by equation (336), we find

which compares with

$$
\begin{aligned}
& M k^{2}=M k_{0}{ }^{2}\left(1-0 \cdot 1679 \theta^{\prime 2}\right), \\
& M k^{2}=M k_{0}{ }^{2}\left(1-0.09378 e^{2}\right)
\end{aligned}
$$

in the three-dimensional problem.

Calculating the moment of momentum in the cylindrical problem, we find

$$
M k^{2} \omega=M k_{0}{ }^{2} \omega_{0}\left(1-0 \cdot 1423 \theta^{\prime 2}\right) \text {; }
$$

This shews that $M k^{2} \omega$ diminishes as we proceed along the two-dimensional pear-shaped series, and therefore that the series is initially unstable.

114. The agreement between the two-dimensional and three-dimensional problems has so far been so marked that it may be hoped that it will persist into those regions in which the three-dimensional figure cannot be calculated. On the assumption that this is the case, we may infer the advanced stages of the three-dimensional problem from those of the two-dimensional problem. 
Writing $e$ for $\theta^{\prime}$, and calculating the curve in the two-dimensional problem as far as $e^{5}$, we find for the equation to its surface expressed in polar coordinates

$$
\begin{aligned}
r^{2}=(1 & \left.+\cdot 139 e^{2}+\cdot 023 e^{4}+\ldots\right)-\cdot 211 e r \cos \phi \\
& +\left(\cdot 8-\cdot 138 e^{2}-\cdot 069 e^{4}+\ldots\right) r^{2} \cos 2 \phi \\
& +\left(\cdot 063 e-\cdot 0064 e^{3}-\cdot 0031 e^{5}\right) r^{3} \cos 3 \phi \\
& +\left(\cdot 013 e^{2}+\cdot 0008 e^{4}+\ldots\right) r^{4} \cos 4 \phi+\left(\cdot 0036 e^{3}+\cdot 00093 e^{5}+\ldots\right) r^{5} \cos 5 \phi \\
& +\left(\cdot 0011 e^{4}+\ldots\right) r^{6} \cos 6 \phi+\left(\cdot 00043 e^{5}+\ldots\right) r^{7} \cos 7 \phi \\
& +\left(\cdot 0001 e^{6}+\ldots\right) r^{8} \cos 8 \phi+\ldots
\end{aligned}
$$

while the equation determining $\omega^{2}$ is

$$
1-\frac{\omega^{2}}{2 \pi \rho}=\cdot 625-\cdot 019 e^{2}-\cdot 016 e^{4}-\ldots
$$

115. The intersections of the curve with its longest axis are given by

where

$$
\Phi(r, e)=0,
$$

$$
\begin{aligned}
& \Phi(r, e)=1+\left(\cdot 139 e^{2}+\cdot 023 e^{4}+\ldots\right)-\cdot 211 e r \\
& -\left(\cdot 2+\cdot 138 e^{2}+\cdot 069 e^{4}+\ldots\right) r^{2}+\left(\cdot 063 e-0064 e^{3}-\cdot 0031 e^{5}+\ldots\right) r^{3} \\
& +\left(\cdot 013 e^{2}+.0008 e^{4}+\ldots\right) r^{4}+\left(\cdot 0036 e^{3}+.00093 e^{5}+\ldots\right) r^{5} \\
& +\left(\cdot 0011 e^{4}+\ldots\right) r^{6}+\left(\cdot 00043 e^{5}+\ldots\right) r^{7}+\ldots \ldots \ldots \ldots \ldots \ldots \ldots . . .(340)
\end{aligned}
$$

In this equation only a few terms are written down of the doubly infinite series which represents the true value of $\Phi$. For small values of $r$ and $e$ these terms will give the value of $\Phi$ with considerable accuracy, but for larger values the approximation may fail. We require to determine over what region of values of $r$ and $e$ the terms actually written down will give a good approximation to the whole.

The coefficient of each power of $r$ is an infinite series, of which terms up to $r^{5}$ have been calculated. The approximation provided by these terms is seen to be tolerably good so long as $e<1$, but fails when $e$ exceeds a unit value.

When some definite value less than unity has been assigned to $e$, the value of $\Phi$ will be given by an infinite series of powers of $r$ of which the first seven only are known. For small values of $r$ these first seven terms will give a good approximation; for higher values of $r$ the approximation will be poor, while for still greater values the series will become divergent, and the first few terms will give no approximation at all. Inspection of equation (340) shews that the approximation will be tolerably good so long as $r^{2}<1 / e^{2}$. 


\section{4}

In fig. 18, values of $r$ are represented by abscissae and values of $e$ by ordinates, so that $\Phi(r, e)$ is a function of position in the plane, and the equation $\Phi(r, e)=0$ will be that of a curve in this plane. The values of $r$ and $e$ for which equation (340) has been seen to give a tolerable approximation are those within the area, shaded in the figure, which is bounded by the curve $e= \pm 1, r= \pm 1 / e$.

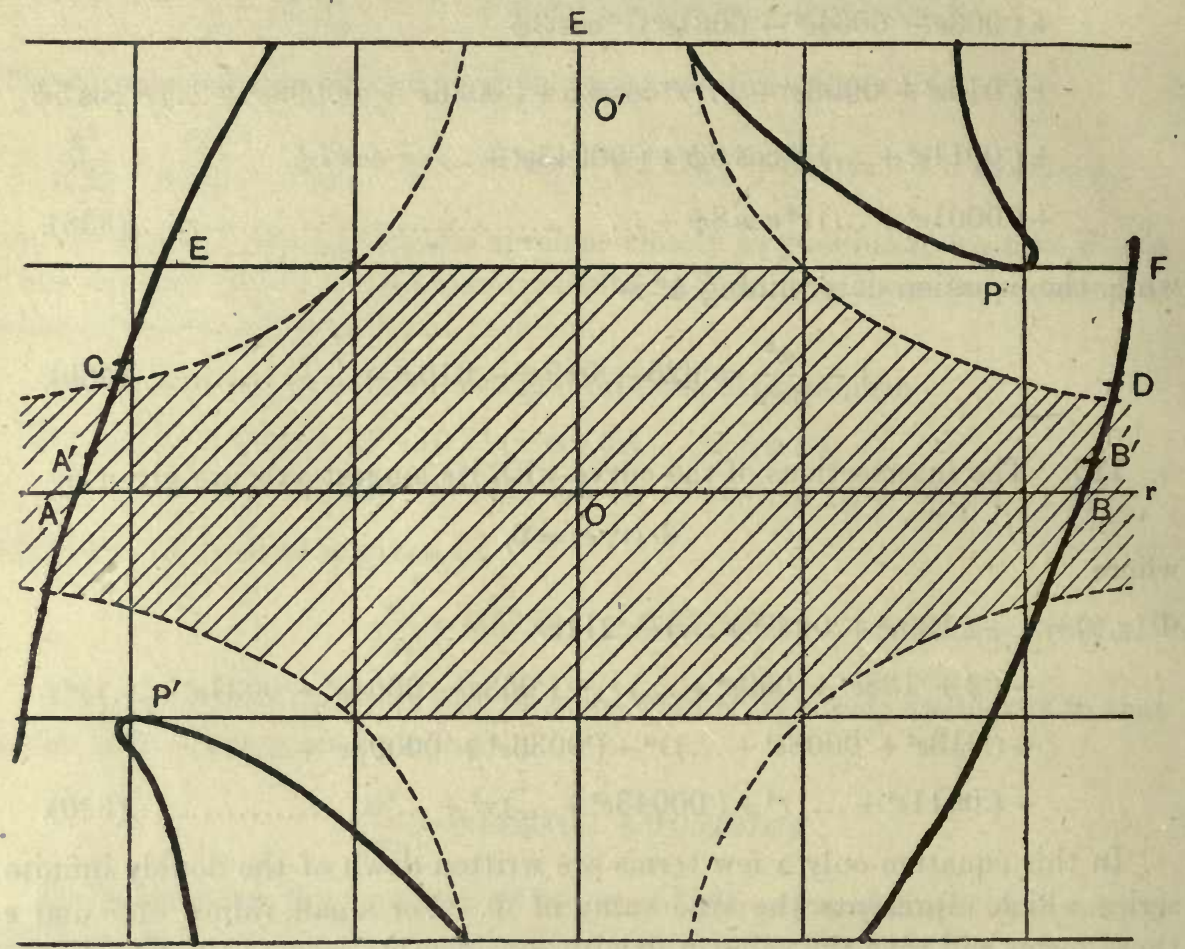

Fig. 18.

The thick curves in this figure represent the locus $\Phi(r, e)=0$ calculated from the terms actually written down in equation (340). So long as we do not pass far beyond the shaded area, this curve will give a fair representation of the position of the true curve $\Phi(r, e)=0$ which would be obtained by the inclusion of all terms in the series of equation (340). The most important points on this curve are those at which $d e / d r=0$. These are the points $P, P^{\prime}$ in the figure, and they may, with sufficient accuracy for our present purpose, be taken to be $r=2, e=1$ and $r=-2, e=-1$.

116. The diagram given in fig. 18 enables us to watch the changes in the lengths of the intercepts on the principal axis of the rotating cylinder as $e$ increases-i.e. as we pass along the linear series of pear-shaped figures.

When $e=0$, the intercepts are represented by the line $A B, O$ being the centre of gravity. The complete figure is of course the elliptic cylinder whose equation is (308), and $O A, O B$ are each equal to the semi-major axis $\sqrt{ } 5$. 
When $e=\frac{1}{5}$, the intercepts are represented by the line $A^{\prime} B^{\prime}$; the centre of gravity still being on the line $O O^{\prime}$. Thus there is a slight elongation at one end of the figure and a corresponding contraction at the other end. In fig. 19 on the next page the chain curve shews the complete figure for $e=\frac{1}{5}$, the curve being of course calculated from the complete equation (338), while the continuous curve represents the undisturbed elliptic cylinder $e=0$.

The intercepts of the figure $e=\frac{1}{2}$ are represented by the line $C D$ in fig. 18, and the complete figure is shewn in fig. 20. We are still within the limits within which equation (340) gives a good approximation.

For the value $e=1$, the approximate intercepts are represented by the line $E F$ with a new double intercept at $P$. For values of $e$ greater than unity, there are four intersections of the surface with its axis, so that the surface consists of two detached parts. At $e=1$ this detachment is just beginning; there are two parts represented by $E P, P F$, but these are still in contact at $P$. The curve calculated from equation (340) for the case of $e=1$ is shewn in fig. 21. For values of $\phi$ other than zero the convergence is considerably better than for $\phi=0$, and this circumstance enables us to determine the greater part of this curve with better accuracy than the points $E, F$ in fig. 18*. It appears that the curve has not yet quite divided, but it is obvious that it is just on the point of doing so.

Finally fig. 22 shews two ellipses which, with an approximation similar to that used in $\$ \$ 60-65$, may be regarded as figures of equilibrium in rotation about one another. The axes of the greater are in the ratio $2: 1$ which corresponds to a rotation

$$
\frac{\omega^{2}}{2 \pi \rho}=\cdot 455
$$

A glance will suggest the probability that this figure gives a good representation of the stage succeeding that shewn in fig. 21 . If so the value (341) ought to represent the value of $\omega^{2} / 2 \pi \rho$ given by equation (339) when $e$ is just greater than 1. The series is not convergent enough for us to determine this limit from equation (339) directly, but it is clear that the value (341) is a perfectly possible value.

Thus we may with fair confidence assert that the two-dimensional series ends by fission into two detached masses, and in view of the close parallelism which we have discovered between the two-dimensional and the threedimensional problems, it seems highly probable that the three-dimensional series also will end by a similar fission into detached masses.

* For greater detail, see Phil. Trans. 200 A, p. 100. 
116 Pear-shaped Configurations of Equilibrium [CH. v

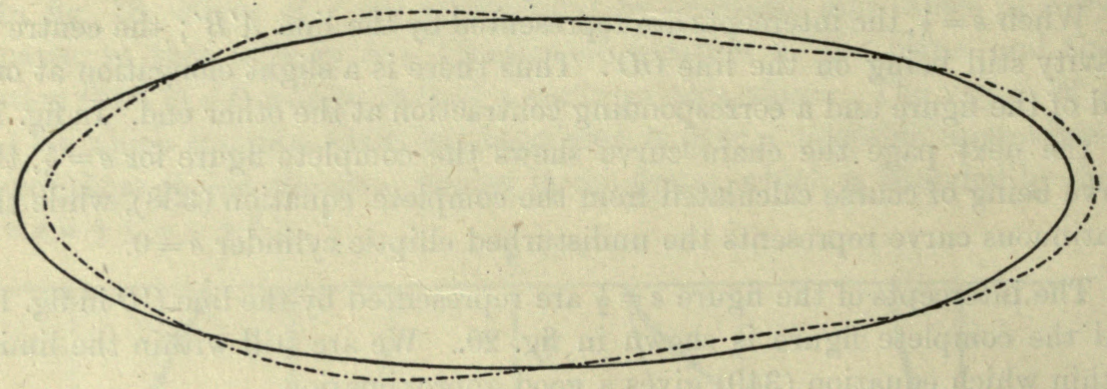

Fig. 19.

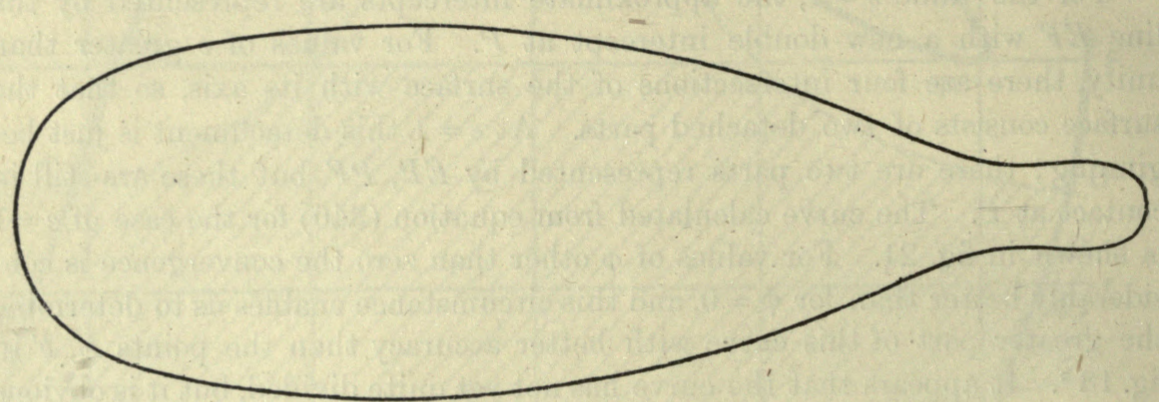

Fig. 20.

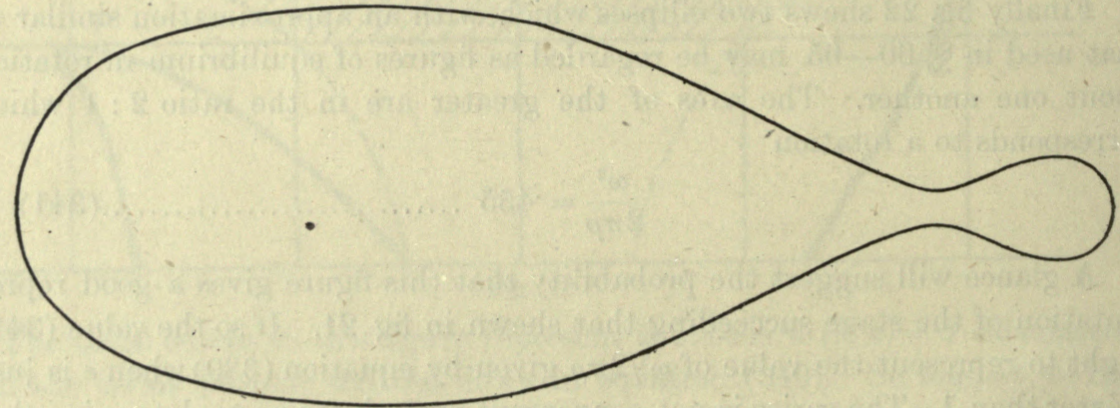

Fig. 21.

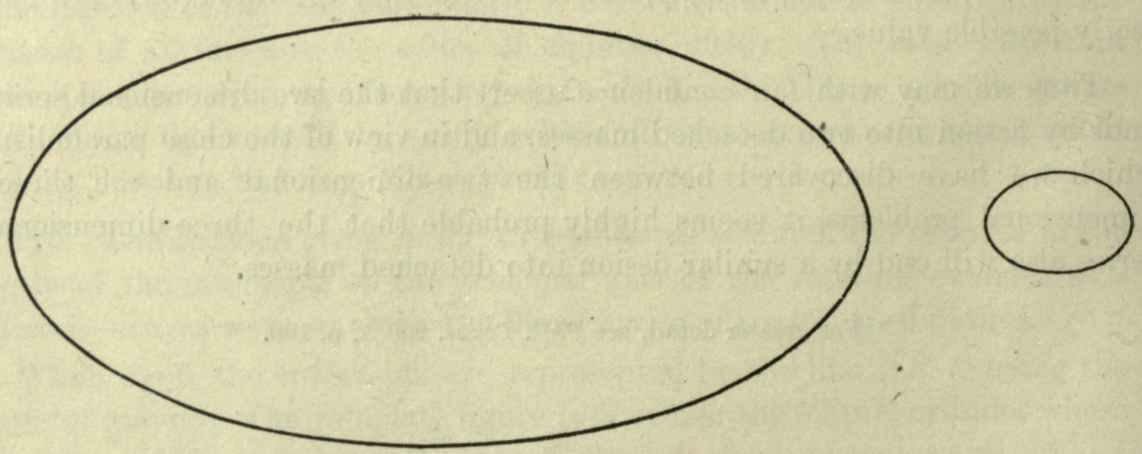

Fig. 22. 


\section{CHAPTER VI}

\section{MOTION WHEN THERE ARE NO STABLE CONFIGURATIONS OF EQUILIBRIUM}

117. The result obtained in the last chapter for the rotational problem combined with those previously obtained in Chapter III for the tidal and double-star problems, has now established that

In all the three problems under consideration there are no figures of stable equilibrium except ellipsoids and spheroids.

In each of these problems the succession of states has been determined by the continuous variation of a parameter-the angular momentum in the rotational and double-star problems, and the distance $R$ in the tidal problem. And in each case it is quite possible for this parameter to vary to beyond the limits within which stable configurations are possible. We must accordingly try to obtain what information we can as to the changes to be expected after this limit is passed.

Poincaré*, writing with special reference to the rotational problem, remarks that if the pear-shaped figure proved to be unstable, "la masse fluide devrait se dissoudre par un cataclysme subit." The pear-shaped figure has now been proved to be unstable, and we must examine the nature of the cataclysm. The situation is similar in the two other problems; when the two masses concerned in either approach one another to within less than a certain distance no configurations of stable equilibrium are possible, and a cataclysm occurs.

The term cataclysm provides a convenient name for the events which take place when stable equilibrium becomes impossible, but we must notice that mathematically nothing more sensational happens than that a statical problem gives place to a dynamical one. A statical problem may or may not admit of solution, but a dynamical problem must always have a solution. Equations of motion which cannot be satisfied with the accelerations put equal to zero, necessarily admit of solution when the acceleration terms are restored.

We now consider the three problems in turn, beginning with the tidal problem.

* Letter to Sir G. Darwin, quoted in the latter's Coll. Works, IIr. p. 315. 


\section{THE TIDAL PROBLEM}

118. In the tidal problem there is no point of bifurcation at the stage at which instability sets in. We have seen that there is a series of spheroidal configurations which are thoroughly stable for eccentricities from 0 to 882579 , but are unstable beyond. The vibration for which instability sets in is one in which the figure remains spheroidal but its eccentricity varies.

We shall now find that when, as assumed in Chapter III, the tide generating potential reduces to the simple form

$$
\mu\left(x^{2}-\frac{1}{2} y^{2}-\frac{1}{2} z^{2}\right)
$$

there is a possible motion in which the boundary remains spheroidal throughout; the question of whether this motion is stable, as well as that of what modifications are introduced when the tidal potential does not reduce to this simple form, will be discussed later.

119. Let us consider the possibility of the general ellipsoid

$$
\frac{x^{2}}{a^{2}}+\frac{y^{2}}{b^{2}}+\frac{z^{2}}{c^{2}}-1=0
$$

being a boundary for the fluid mass when in motion. The rates of change of $a, b, c$ will be denoted by $\dot{a}, \dot{b}, \dot{c}$. At every stage of the motion we must have

so that we necessarily have

$$
a b c=r_{0}^{3}
$$

$$
\frac{\dot{a}}{a}+\frac{\dot{b}}{b}+\frac{\dot{c}}{c}=0
$$

And on again differentiating with respect to the time we obtain

$$
\frac{\ddot{a}}{a}+\frac{\ddot{b}}{b}+\frac{\dot{c}}{c}=\frac{\dot{a}^{2}}{a^{2}}+\frac{\dot{b}^{2}}{b^{2}}+\frac{\dot{c}^{2}}{c^{2}}
$$

The velocity-potential of the motion, if the fluid is still assumed incompressible, is given by*

$$
\Phi=-\frac{1}{2}\left(\frac{\dot{a}}{a} x^{2}+\frac{\dot{b}}{b} y^{2}+\frac{\dot{c}}{c} z^{2}\right)
$$

this satisfying the requisite condition $\nabla^{2} \Phi=0$ in virtue of equation (34:3). The velocity $v$ at any point is accordingly

$$
v^{2}=\left(\frac{\dot{a}}{a}\right)^{2} x^{2}+\left(\frac{\dot{b}}{b}\right)^{2} y^{2}+\left(\frac{\dot{c}}{c}\right)^{2} z^{2}
$$

* Lamb, Hydrodynamics (4th Edition), § 110. 
and the pressure at any point will be given by

$$
\begin{aligned}
\frac{p}{\rho} & =\frac{\partial \Phi}{\partial t}+V_{i}+\mu\left(x^{2}-\frac{1}{2} y^{2}-\frac{1}{2} z^{2}\right)-\frac{1}{2} v^{2}+F(t) \\
& =-\frac{1}{2}\left(\frac{\ddot{a}}{a} x^{2}+\frac{\ddot{b}}{b} y^{2}+\frac{\ddot{c}}{c} z^{2}\right)+V_{i}+\mu\left(x^{2}-\frac{1}{2} y^{2}-\frac{1}{2} z^{2}\right)+F(t)
\end{aligned}
$$

The function

$$
\frac{p}{\rho}+\theta^{\prime} \pi \rho a b c\left(\frac{x^{2}}{a^{2}}+\frac{y^{2}}{b^{2}}+\frac{z^{2}}{c^{2}}-1\right)
$$

is a linear function of $x^{2}, y^{2}$ and $z^{2}$. It can accordingly be made a spherical harmonic by assigning a suitable value to $\theta^{\prime}$, and on operating on the function with $\nabla^{2}$, the requisite value for $\theta^{\prime}$ is found to be given by

$$
\frac{2}{a b c}+\frac{1}{2 \pi \rho a b c}\left(\frac{\ddot{a}}{a}+\frac{\ddot{b}}{b}+\frac{\ddot{c}}{c}\right)=\theta^{\prime}\left(\frac{1}{a^{2}}+\frac{1}{b^{2}}+\frac{1}{c^{2}}\right)
$$

Now during the motion of the spheroid, the pressure over the surface will be uniform at each instant*, so that the function (348) must be constant over the surface at each instant. If we assign to $\theta^{\prime}$ the value given by equation (349), the function (348) is a spherical harmonic, so that being constant over the surface of the ellipsoid, it must also be constant throughout its volume, and hence the coefficients of $x^{2}, y^{2}$ and $z^{2}$ must vanish separately. Equating these to zero, we obtain the system of equations

$$
\begin{aligned}
& -\frac{1}{2 \pi \rho a b c} \frac{\ddot{a}}{a}=J_{A}-\frac{\mu}{\pi \rho a b c}-\frac{\theta^{\prime}}{a^{2}} . \\
& -\frac{1}{2 \pi \rho a b c} \frac{\ddot{b}}{b}=J_{B}+\frac{1}{2} \frac{\mu}{\pi \rho a b c}-\frac{\theta^{\prime}}{b^{2}} \\
& -\frac{1}{2 \pi \rho a b c} \frac{\ddot{c}}{c}=J_{C}+\frac{1}{2} \frac{\mu}{\pi \rho a b c}-\frac{\theta^{\prime}}{c^{2}} .
\end{aligned}
$$

On adding corresponding sides of these three equations, we again obtain equation (349) which determines $\theta^{\prime}$, so that the three equations (350) to (352) contain within themselves the necessary and sufficient condition that the pressure shall remain uniform (or zero) over the boundary throughout the motion. The equations are equations expressing $\ddot{a}, \ddot{b}$ and $\ddot{c}$ in terms of the configuration at any instant; they may accordingly be regarded as equations of motion for the ellipsoid (343). Naturally they reduce to the statical equations $(78)$ to $(80)$ when the ellipsoid is at rest.

Clearly the relation between $\theta$, as given by equation ( 81$)$, and $\theta^{\prime}$, given by equation (349), is

$$
\pi \rho a b c\left(\theta^{\prime}-\theta\right)\left(\frac{1}{a^{2}}+\frac{1}{b^{2}}+\frac{1}{c^{2}}\right)=\frac{1}{2}\left(\frac{\ddot{a}}{a}+\frac{\ddot{b}}{b}+\frac{\ddot{c}}{c}\right)=\frac{1}{2}\left(\frac{\dot{a}^{2}}{a^{2}}+\frac{\dot{b}^{2}}{b^{2}}+\frac{\dot{c}^{2}}{c^{2}}\right)
$$

by equation (344). Thus $\theta^{\prime}$ becomes identical with $\theta$ when there is no motion, or when the figure is instantaneously at rest, so that $\dot{a}=\dot{b}=\dot{c}=0$

* It will be seen that the method we follow is that of Dirichlet, Gott. Abhand. 8 (1860), p. 3. or Coll. Works, rr. p. 263. See also Lamb's Hydrodynamics (4th Edition), p. 689. 
120. Multiplying equations (351) and (352) by $b, c$ and subtracting,

$$
-\frac{(\ddot{b}-\ddot{c})}{2 \pi \rho a b c}=(b-c)\left[\int_{0}^{\infty} \frac{(\lambda-b c) d \lambda}{\left(b^{2}+\lambda\right)\left(c^{2}+\lambda\right) \Delta}+\frac{1}{2} \frac{\mu}{\pi \rho a b c}+\frac{\theta^{\prime}}{b c}\right]
$$

The integral in this expression may be positive or negative, but the integral plus $\theta / b c$ is found to be always positive.

Since equation (353) has shewn that $\theta^{\prime}-\theta$ is always positive, it follows that the expression in square brackets in equation (354) is always positive, so that this equation assumes the form

$$
\frac{d^{2}}{d t^{2}}(b-c)=-(b-c) \times(\text { a positive quantity })
$$

This shews that any initial inequality in $b$ and $c$ gives rise only to oscillations about the value $b-c=0$. We may therefore suppose henceforth that $b=c$ throughout the motion.

121. Putting $b=c$, equation (351) becomes identical with equation (352). From equation (35̃3) we obtain

$$
\frac{1}{2} \frac{\ddot{a}}{a}-\frac{\pi \rho a b c\left(\theta^{\prime}-\theta\right)}{a^{2}}=-\left[\frac{\ddot{c}}{c}-\frac{2 \pi \rho a b c\left(\theta^{\prime}-\theta\right)}{c^{2}}\right] .
$$

Denoting each member of this equation by $\eta$, equations (350) and (352) assume the forms

$$
\begin{aligned}
& J_{A}-\frac{\mu-\eta}{\pi \rho a b c}=\frac{\theta}{a^{2}} \\
& J_{C}+\frac{1}{2} \frac{\mu-\eta}{\pi \rho a b c}=\frac{\theta}{c^{2}}
\end{aligned}
$$

which are exactly identical with the statical equations (78) and (80) of Chapter III except that $\mu$ has become replaced by $\mu-\eta$.

Hence, exactly as in equation (85), it follows that

where

$$
\frac{\mu-\eta}{\pi \rho}=F(e)
$$

$$
F(e)=\frac{1-e^{2}}{e^{8}} \log \left(\frac{1+e}{1-e}\right)-\frac{6\left(1-e^{2}\right)}{e^{2}\left(3-e^{2}\right)}
$$

The values of the semi-axes of the spheroid are

$$
a=\left(1-e^{2}\right)^{-\frac{1}{3}} r_{0}, \quad c=\left(1-e^{2}\right)^{\frac{1}{6}} r_{0},
$$

so that the value of $\eta$ is found from equation (356) to be

$$
\eta=\frac{a \ddot{a}-c \ddot{c}}{2 a^{2}+c^{2}}=\frac{1}{3\left(1-e^{2}\right)}\left[e \ddot{e}+\dot{e}^{2}\left(1+\frac{21-5 e^{2}}{3\left(1-e^{2}\right)\left(3-e^{2}\right)}\right)\right] \ldots(361) .
$$


Equation (359) may now be put in the form

$$
e \ddot{e}+\dot{e}^{2}\left(1+\frac{21-\check{5} e^{2}}{3\left(1-e^{2}\right)\left(3-e^{2}\right)}\right)=3 \pi \rho\left(1-e^{2}\right)\left(\frac{\mu}{\pi \rho}-F(e)\right) \ldots(362) .
$$

When $\mu$ is given as a function of the time, this becomes an equation of motion for $e$, and so enables us to follow the changes in the shape of the spheroid. The equation cannot be completely solved, but the general character of the motion is easily traced out.

122. To represent roughly the approach and passage of a second star, we may suppose $\mu$ to start from a zero value and increase-slowly at first but afterwards more rapidly. The initial conditions will be $e=0$ and $\dot{e}=0$. So long as $\dot{e}$ is so small that its square may be neglected, equation (362) reduces to

$$
e \ddot{e}=3 \pi \rho\left(1-e^{2}\right)\left(\frac{\mu}{\pi \rho}-F(e)\right) .
$$

Provided that $\mu$ changes slowly, the solution will remain very close to that of

$$
\frac{\mu}{\pi \rho}-F(e)=0
$$

so long as this equation has a solution-i.e. so long as $\mu$ is less than $\cdot 125 \pi \rho$. But as soon as $\mu$ exceeds this critical value, $\mu / \pi \rho-F(e)$ can no longer vanish or remain small, so that no matter how slowly $\mu$ increases, $\ddot{e}$ becomes finite and $\dot{e}^{2}$ necessarily becomes appreciable. The eccentricity now increases rapidly, its changes being given by equation (362). This determines the dynamical motion which occurs when statical configurations of equilibrium are no longer possible, and we see that it consists of a passage along the unstable series of spheroids, the rate of motion being determined by equation (362).

When $\mu$ increases more rapidly, there will be no sharp change in the character of the motion on passing the critical value $\mu=\cdot 125 \pi \rho$; the statical and dynamical parts of the motion merge imperceptibly into one another.

In either case equation (362) shews that the motion may or may not pass the value $e=1$. In the latter case $e$ increases until a "turning point" is reached, defined by $\dot{e}=0$, after which it decreases, ultimately coming back to rest at the value $e=0$ when dissipative forces are present. At the turning point $\ddot{e}$ is negative, so that equation (362) shews that $\mu$ must be less than $\pi \rho F(e)$ and therefore $\dot{a}$ fortiori less than the maximum value of $\pi \rho F(e)$ which is $125 \pi \rho$. Thus $e$ goes on increasing not merely while $\mu$ is increasing but also through the whole period in which $\mu>\cdot 125 \pi \rho$.

123. A case in which the motion can be fully determined, and is moreover of great importance, is that in which $\mu$ increases and decreases with great rapidity, so that the primary is "impulsively" set into motion before 
it has departed far from its original spherical shape. Thus during the short interval in which $\mu$ is finite, we have approximately $e=0$ and $a=c$.

It follows, from equations (359) and (361), that at any instant during this interval,

$$
\mu=\eta=\frac{\ddot{a}-\ddot{c}}{3 r_{0}},
$$

where $r_{0}$ is the mean radius. Integrating through the interval in which $\mu$ is appreciable, say from 0 to $\tau$, we obtain

$$
\dot{a}-\dot{c}=3 r_{0} \int_{0}^{\tau} \mu d t
$$

where $\dot{a}$ and $\dot{c}$ are the velocities at the end of the interval in question. We also have $\dot{a}+2 \dot{c}=0$ from the condition of constancy of volume, so that

$$
\frac{\dot{a}}{r_{0}}=-\frac{2 \dot{c}}{r_{0}}=2 \int_{0}^{\tau} \mu d t
$$

Each fraction is equal to $\frac{2}{3} e \dot{e}$, so that these equations give the value of $\dot{e}$, and therefore the kinetic energy, at the instant $t=\tau$. The subsequent motion is of course one under no applied forces, with this assigned amount of energy, and so can be completely determined.

The energy equation is readily found to be*

$$
\frac{2}{9}\left(3-e^{2}\right)\left(1-e^{2}\right)^{-\frac{8}{3}} e^{2} \dot{e}^{2}=6\left[\int \mu d t\right]^{2}-4 \pi \rho\left\{2-\frac{\left(1-e^{2}\right)^{\frac{1}{3}}}{e} \log \frac{1+e}{1-e}\right\}
$$

and the turning points, at which $\dot{e}=0$, are accordingly determined by

$$
2-\frac{\left(1-e^{2}\right)^{\frac{1}{3}}}{e} \log \frac{1+e}{1-e}=\frac{3}{2 \pi \rho}\left[\int \mu d t\right]^{2}
$$

We may notice that if $\int \mu d t>\left(\frac{4}{3} \pi \rho\right)^{\frac{1}{2}}$, there will be no turning point and the motion will overshoot the value $e=1$. But we shall immediately see that such motions as this cannot occur, for instability will be set up before the value $e=1$ is reached.

124. We have seen that a motion which satisfies the dynamical equations is one in which the figure remains always spheroidal, the velocity potential being given by

$$
\Phi=-\frac{1}{2}\left(\frac{\dot{a}}{a} x^{2}+\frac{\dot{b}}{b} y^{2}+\frac{\dot{c}}{c} z^{2}\right)
$$

in which $b$ and $c$ remain equal. This motion has not however been shewn to be stable. 
To examine the stability of the motion, we compare it with a slightly varied motion in which the boundary varies slightly from the ellipsoidal shape. Let us assume at any instant that the boundary is

$$
\frac{x^{2}}{a^{2}}+\frac{y^{2}}{b^{2}}+\frac{z^{2}}{c^{2}}+\psi-1
$$

and that the velocity potential is $\Phi+\Psi$, where $\Phi$ is still given by equation (365).

Assuming $\Psi$ and $\psi$ to be small, we find, from a consideration of the normal velocity at a point on the boundary,

$$
\dot{\psi}=2\left(\frac{x}{a^{2}} \frac{\partial \Psi}{\partial x}+\frac{y}{b^{2}} \frac{\partial \Psi}{\partial y}+\frac{z}{c^{2}} \frac{\partial \Psi}{\partial z}\right)
$$

so that $\dot{\psi}$ is algebraically of the same degree as $\Psi$.

Let us use $P_{1}, P_{2}, \ldots$ to denote products of powers of $x, y$, $z$, say

$$
P_{1}=x^{\alpha_{1}} y^{\beta_{1}} z^{\gamma_{1}} \text { etc., }
$$

and suppose that values for $\Psi$ and $\psi$ are

$$
\begin{aligned}
& \Psi=p_{1} P_{1}+p_{2} P_{2}+\ldots \ldots \ldots \ldots \ldots \ldots \ldots \ldots(368), \\
& \psi=q_{1} P_{1}+q_{2} P_{2}+\ldots \ldots \ldots \ldots \ldots \ldots \ldots \ldots . . .(369),
\end{aligned}
$$

then, on substituting into equation (367) and equating coefficients, we obtain

where

$$
\dot{q}_{1}=2 s_{1} p_{1} \text { etc. }
$$

$$
s_{1}=\frac{\alpha_{1}}{a^{2}}+\frac{\beta_{1}}{b^{2}}+\frac{\gamma_{1}}{c^{2}}
$$

The components of the total velocity $v$ at $x, y, z$ are

so that

$$
\frac{\dot{a}}{a} x-\frac{\partial \Psi}{\partial x} \text { etc., }
$$

$$
v^{2}=v_{0}^{2}-2\left(\frac{\dot{a}}{a} x \frac{\partial \Psi}{\partial x}+\frac{\dot{b}}{b} y \frac{\partial \Psi}{\partial y}+\frac{\dot{c}}{c} z \frac{\partial \Psi}{\partial z}\right)
$$

where $v_{0}$ is the velocity when $\Psi=0$, given by equation (346). Just as in $\S 119$, the pressure can be made constant over the surface by satisfying

$$
\begin{aligned}
\dot{\Phi}+\dot{\Psi}+V_{b}+ & \mu\left(x^{2}-\frac{1}{2} y^{2}-\frac{1}{2} z^{2}\right)-\frac{1}{2} v^{2} \\
& =-\pi \rho a b c \theta^{\prime \prime}\left(\frac{x^{2}}{a^{2}}+\frac{y^{2}}{b^{2}}+\frac{z^{2}}{c^{2}}+\psi-1\right)+a \text { cons. }
\end{aligned}
$$

where $\theta^{\prime \prime}$ is a new constant.

We have already seen $(\$ 120)$ that the motion is stable as regards ellipsoidal displacement, so that we may suppose that $\Psi$ and $\psi$ are free from 
second degree terms. The potential $V_{b}$ at the boundary may be put in the form

$$
V_{b}=\left(V_{b}\right)_{0}+\left(V_{b}\right)_{\psi}
$$

where $\left(V_{b}\right)_{0}$ is the potential when $\psi=0$, and $\left(V_{b}\right)_{\psi}$ represents the terms in $\psi$ which are of degrees $1,3,4, \ldots$.

Equating terms of degree 2 in equation (372), we obtain

$$
\begin{aligned}
\dot{\Phi}+\left(V_{b}\right)_{0}+\mu\left(x^{2}-\frac{1}{2} y^{2}-\frac{1}{2} z^{2}\right)-\frac{1}{2} v_{0}^{2} & \\
& =-\pi \rho a b c \theta^{\prime \prime}\left(\frac{x^{2}}{a^{2}}+\frac{y^{2}}{b^{2}}+\frac{z^{2}}{c^{2}}\right)
\end{aligned}
$$

On equating separate coefficients of $x^{2}, y^{2}$ and $z^{2}$ we obtain precisely our previous equations $(350)-(352)$ except that $\theta^{\prime \prime}$ replaces $\theta^{\prime}$. On adding corresponding sides we obtain equation (349), with $\theta^{\prime \prime}$ replacing $\theta^{\prime}$. Hence $\theta^{\prime \prime}$ must be the same as our former $\theta^{\prime}$, and it appears that the changes in the fundamental spheroid will be just the same as in the former problem in which $\psi$ was absent.

On subtracting corresponding sides of equations (373) and (372), we find

$$
\dot{\Psi}+\left(V_{b}\right)_{\psi}+\left(\frac{\dot{a}}{a} x \frac{\partial \Psi}{\partial x}+\frac{\dot{b}}{b} y \frac{\partial \Psi}{\partial y}+\frac{\dot{c}}{c} z \frac{\partial \Psi}{\partial z}\right)=-\pi \rho a b c \theta^{\prime} \Psi \ldots(374)
$$

Using the values of $\Psi$ and $\psi$ already assumed in equations (368) and (369), we have

$$
\begin{gathered}
\dot{\Psi}=\Sigma \dot{p}_{1} P_{1}, \\
\frac{\dot{a}}{a} x \frac{\partial \Psi}{\partial x}+\ldots=\Sigma\left(\frac{\dot{a}}{a} \alpha_{1}+\frac{\dot{b}}{b} \beta_{1}+\frac{\dot{c}}{c} \gamma_{1}\right) p_{1} P_{1} .
\end{gathered}
$$

In $\left(V_{b}\right)_{\psi}$ the coefficient of $P_{1}$ may be supposed to be

$$
c_{1} q_{1}+c_{2} q_{2}+\ldots
$$

Thus on equating coefficients in equation (374) we obtain a system of equations of which a typical one is

$$
\dot{p}_{1}+c_{1} q_{1}+c_{2} q_{2}+\ldots+\left(\frac{\dot{a}}{a} \alpha_{1}+\frac{\dot{b}}{b} \beta_{1}+\frac{\dot{c}}{c} \gamma_{1}\right) p_{1}=-\pi \rho a b c \theta^{\prime} q_{1} \ldots(375) .
$$

This and equations such as (370) are the equations of motion giving changes in the $p$ 's and $q$ 's. From them the stability of the motion may be determined.

Eliminating $p_{1}$ from equations (370) and (375) we find $\frac{d}{d t}\left[\frac{\dot{q}_{1}}{2 s_{1}}\right]+c_{1} q_{1}+c_{2} q_{2}+\ldots+\left(\frac{\dot{a}}{a} \alpha_{1}+\frac{\dot{b}}{b} \beta_{1}+\frac{\dot{c}}{c} \gamma_{1}\right)\left[\frac{\dot{q}_{1}}{2 s_{1}}\right]=-\pi \rho a b c \theta^{\prime} q_{1} \ldots(376)$

125. In general these equations are so complicated that no progress can be made. We have, however, seen that the changes in $\dot{a}, \dot{b}, \dot{c}$ are the 
same as if the figure had remained strictly spheroidal, so that the motion may be supposed to be accompanied by an increase in the eccentricity $e$ until a turning point is reached at which $\dot{e}=0$ and $\dot{a}=\dot{b}=\dot{c}=0$. At this point equation (376) assumes the simple form

$$
\frac{1}{2 s_{1}} \frac{d^{2} q_{1}}{d t^{2}}=-c_{1} q_{1}-c_{2} q_{2}-\ldots-\pi \rho a b c \theta q_{1}
$$

since we have seen that $\theta^{\prime}$ becomes identical with $\theta$ at the turning point.

The stability or instability of the motion at the turning point depends on the signs of $d^{2} q_{1} / d t^{2}$, and since the factor $s_{1}$ is, by its definition, always positive, these signs are those of the right-hand members of equations such as (377). But the right-hand members of equations (377) are exactly the quantities of which the vanishing determines the points of bifurcation on the spheroidal series. They are all negative so long as no point of bifurcation has been passed on the spheroidal series, and one of them changes sign at each point of bifurcation.

The first point of bifurcation, as we have seen $(\$ 85)$, occurs when $e=947741$, and corresponds to a third harmonic deformation. If the turning point, at which $\dot{e}=0$, occurs before $e$ has reached the value 947741 , then the right-hand members of all the equations such as (377) will be negative at the turning point, so that the dynamical motion will be stable up to the turning point and also in returning, and the mass will sink back into a spherical configuration.

But if the turning point occurs just after $e$ has reached the value $\cdot 947741$, the dynamical motion at the turning point will be unstable through a third harmonic displacement. Thus after passing the point of bifurcation a third harmonic displacement will appear and will increase very rapidly, at least until after the eccentricity has again diminished to below 947741 , at which stage the third harmonic vibration will again become stable, so that whatever third harmonic displacement there may be will oscillate and finally disappear. The condition that the mass shall depart from the spheroidal form is thus seen to be that the turning point shall occur for a value of $e$ greater than 947741 .

The intensity of tidal action necessary for this to occur can be determined accurately in two cases-(i) when $\mu$ changes very slowly, (ii) when $\mu$ changes so rapidly that the tidal action may be treated as "impulsive."

When $\mu$ changes very slowly, any value of $\mu$ greater than $125504 \pi \rho$ will suffice to set up dynamical motion and $e$ will continue to increase until after $\mu$ has again receded below the value $125504 \pi \rho$. Hence when $\mu$ changes very slowly the eccentricity will pass above $\cdot 947741$ if $\mu$ exceeds $\cdot 125504 \pi \rho$. In this $\mu$ stands for $M^{\prime} / R^{3}$, where $M^{\prime}$ is the mass of the tide-raising body 
and $R$ its distance. The critical value of $R$, as we have already seen (equation (86)), is

$$
R=2 \cdot 1984\left(\frac{M^{\prime}}{M}\right)^{\frac{1}{3}} r_{0}
$$

When $\mu$ changes so rapidly that the forces may be regarded as impulsive; the turning point is determined by equation (364), and it is found that this will lie beyond the critical eccentricity 947741 if

$$
\int \mu d t>0.675 \rho^{\frac{1}{2}}
$$

126. In general we must contemplate not only cases in which the spheroid runs over one point of bifurcation before reaching the turning point, but also cases in which it runs over several points of bifurcation. Somewhere near eccentricity $e=9477$, a third harmonic displacement will become unstable, and the spheroid will give place to a pear-shaped figure. A furrow will develop near the middle plane of the spheroid and this will increase rapidly (approximately exponentially) with the time. But meanwhile the eccentricity of the spheroid may continue to increase, and it may be that before the pear-shape is much developed, a second point of bifurcation will be reached, namely that corresponding to a fourth harmonic displacement. At this stage two new furrows begin to form, but these, like the former pear-shaped furrow, will be forming in a spheroid which may simultaneously be elongating itself with considerable velocity. When, or if, the next point of bifurcation is reached, three more new furrows may begin to form, and so on.

Fig. 23 shews rough drawings (partly conjectural) of spheroids with the furrows produced on passing the earlier points of bifurcation. Little doubt will be felt that such figures will in time break up into a number of separate detached pieces.

127. So far we have been considering only an idealised mathematical problem; in nature there will be innumerable complications, and we must try to calculate the effect of the more important of these.

We have supposed the tidal potential to be $M^{\prime}\left(x^{2}-\frac{1}{2} y^{2}-\frac{1}{2} z^{2}\right) / R^{3}$, which is the potential either of a spherical mass, or of a mass of any shape at a great distance. In an actual problem the potential will be more complicated than this, for not only will the secondary mass not be spherical but the shapes of the primary and secondary will influence one another, as in Darwin's problem considered in $\S 60$. It is however not difficult to shew* that in the most general case the motion is, in its main characteristics, entirely similar to that just discussed. The numerical results are slightly altered,

* The question is discussed in detail in a paper "The Motion of Tidally Distorted Masses," R.A.S. Memoirs, Vol. 72. 


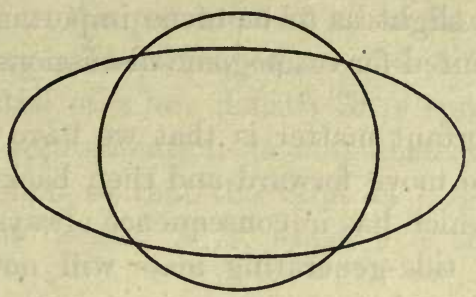

(a)

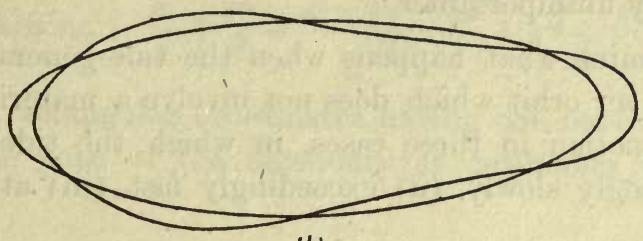

(b)

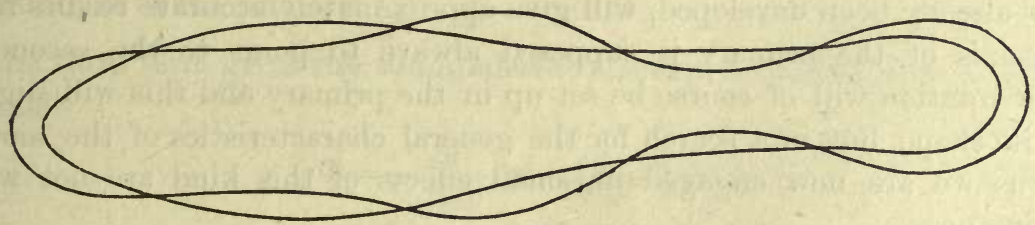

(C)
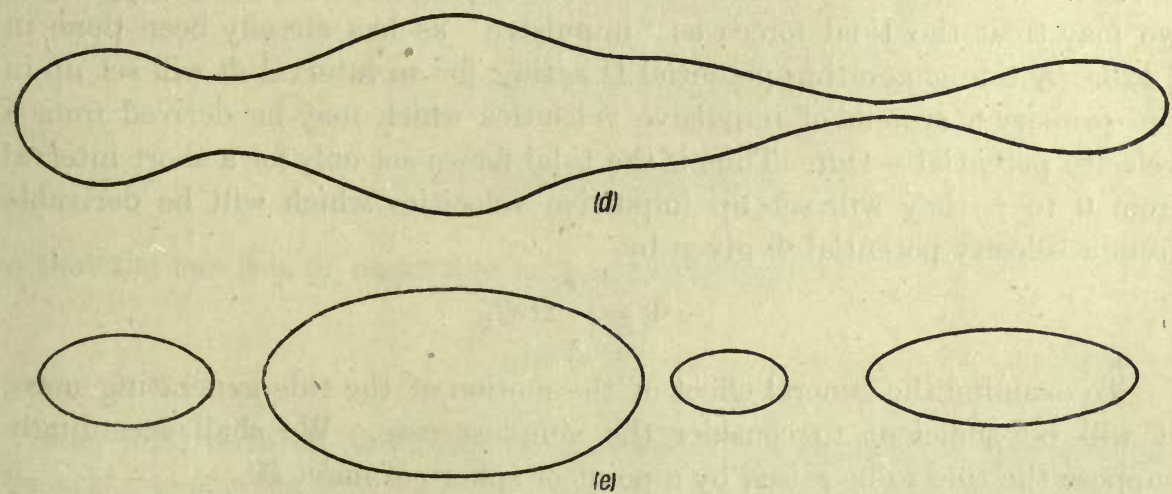

Fig. 23.

\section{Explanation.}

(a) Undistorted sphere, and longest spheroid which is statically stable.

(b) Longest spheroid which is dynamically stable, and pear-shaped figure derived by third harmonic displacement.

(c) More elongated pear-shaped figure, and figure derived by fourth harmonic displacement.

(d) The last figure more elongated, and with fifth harmonic displacement surperposed.

(e) Conjectural drawing of subsequent configuration. 
but the alteration is so slight as to be of no importance within the limits of accuracy which are required for cosmogonic discussions.

128. A more important matter is that we have in effect supposed the tide-generating mass to move forward and then backward along a line $(O x)$ through the primary, which has in consequence always been an axis of symmetry. In nature the tide-generating mass will not in general approach the primary along a line through its centre; indeed if it did a material collision would occur, and the course of events before this collision took place would be relatively unimportant.

We must examine what happens when the tide-generating mass passes by the primary in an orbit which does not involve a material collision. Let us examine the motion in three cases, in which the tide-generating body moves (i) exceedingly slowly, (ii) exceedingly fast, (iii) at an intermediate rate.

129. When the motion is exceedingly slow an equilibrium theory, such as has already been developed, will give approximately accurate results if the major-axis of the primary is supposed always to point to the secondary. A slow rotation will of course be set up in the primary and this will slightly alter its shape, but in a search for the general characteristics of the motion, such as we are now engaged in, small effects of this kind are not worth delaying over.

130. When the motion of the tide-generating mass is exceedingly rapid, we may treat the tidal forces as "impulsive" as has already been done in $\S 123$. A tide-generating potential $\Omega$ acting for an interval $d t$ will set up in the primary a system of impulsive velocities which may be derived from a velocity potential $-\Omega d t$. Thus if the tidal forces act only for a short interval from 0 to $\tau$, they will set up impulsive velocities which will be derivable from a velocity potential $\Phi$ given by

$$
-\Phi=\int_{0}^{\tau} \Omega d t
$$

To examine the general effect of the motion of the tide-generating mass, it will be sufficient to consider the simplest case. We shall accordingly suppose the tide to be raised by a point or sphere of mass $M^{\prime}$.

Let $v$ denote the velocity of the secondary mass at any instant. Then in any interval $d t$, the secondary moves over a distance of path $d s=v d t$. The contribution to $-\Phi$ is

but may also be expressed as

$$
\frac{M^{\prime}}{R} d t
$$

$$
\frac{M^{\prime}}{v R} d s,
$$


and so may be regarded as the potential of a line density $M^{\prime} / v$ spread along the path of the secondary. The whole value of $-\Phi$ is accordingly equal to the gravitational potential of a line density $M^{\prime} / v$ spread along the orbit of the secondary. To an approximation we may neglect all parts of the orbit except that near perihelion, so that the value of $-\Phi$ will be the potential of a straight rod of line density $M^{\prime} / v$, where $v$ is the velocity at or near perihelion.

If $R_{0}$ denote the distance at perihelion we readily find

$$
\Phi=-\int_{0}^{\tau} \Omega d t=\text { cons. }-\frac{M^{\prime}}{v}\left[\frac{2 x}{R_{0}}+\frac{x^{2}-\frac{1}{2} y^{2}-\frac{1}{2} z^{2}}{R_{0}{ }^{2}}+\frac{1}{2} \frac{y^{2}-z^{2}}{R_{0}^{2}}+\ldots\right] \ldots
$$

where $x, y, z$ are rectangular coordinates having the centre of the primary as origin, and the path of the secondary at perihelion is along the line $x=R_{0}, z=0$.

In this velocity potential the constant term does not affect the motion; the second term sets up a uniform velocity $2 M^{\prime} / R_{0} v$ which does not alter the configuration of the primary.

The third term gives rise to impulsive velocities deducible from a velocity potential

$$
\Phi=-\frac{M^{\prime}}{R_{0}^{2} v}\left(x^{2}-\frac{1}{2} y^{2}-\frac{1}{2} z^{2}\right)
$$

Now the impulsive velocities discussed in $\S 123$ were deducible from a velocity potential (cf. equations (345) and (363))

$$
\begin{aligned}
\Phi & =-\frac{1}{2}\left(\frac{\dot{a}}{a} x^{2}+\frac{\dot{b}}{b} y^{2}+\frac{\dot{c}}{c} z^{2}\right) \\
& =-\int_{0}^{\tau} \mu d t\left[x^{2}-\frac{1}{2} y^{2}-\frac{1}{2} z^{2}\right]
\end{aligned}
$$

so that the two sets of impulsive velocities will agree if

$$
\int_{0}^{\tau} \mu d t=\frac{M^{\prime}}{R_{0}^{2} v}
$$

The final term on the right of equation (380) indicates a tendency for the axis $c$ to shorten while the axis $b$ lengthens, just as would happen if the system were in rotation. There cannot ultimately be rotation in this case for the tidal couples from the two halves of the orbit of the secondary exactly neutralise one another; it therefore appears that the values of $b$ and $c$ will oscillate about the value $b=c$, as in $\S 120$, and under the influence of viscosity the figure will ultimately resume its spheroidal form.

Thus, neglecting terms in $1 / R_{0}{ }^{3}$ etc. it appears that the motion will be the same, except for the preliminary oscillations in the values of $b$ and $c$, as 
that already discussed ( $(123)$ in which $\mu$ was supposed to act impulsively, and the motions will agree quantitively if $\int \mu d t$ is supposed given by equation (382). From equation (379). it appears that the spheroid will lengthen to beyond the critical eccentricity $\cdot 947741$, and so finally depart from the spheroidal shape, if

$$
\frac{M^{\prime}}{R_{0}^{2} v}>0.675 \rho^{\frac{1}{2}}
$$

This criterion only holds in the special case in which the tidal forces satisfy the condition we have described as "impulsive." This requires that the tidal forces shall come and go before the spheroid is much different from a sphere. From equations (363) and (382) it is clear that at the end of the action of the tidal forces, the velocity $\dot{a}$ of the end of the major-axis is given by

$$
\frac{\dot{a}}{a}=\frac{2 M^{\prime}}{R_{0}{ }^{2} v} .
$$

The time during which the tidal forces are appreciable will be of the order of $2 R_{0} / v$, so that if $r_{0}+\delta a$ is the length of the semi-major-axis at the end of the encounter we have, as regards order of magnitude,

$$
\frac{\delta a}{r_{0}}=\frac{1}{2}\left(\frac{2 M^{\prime}}{R_{0}{ }^{2} v}\right)\left(\frac{2 R_{0}}{v}\right)=\frac{2 M^{\prime}}{R_{0} v^{2}} .
$$

Let us now agree conventionally to define the action of tidal forces as "impulsive" when $\delta a$ is less than $\frac{1}{5} r_{0}$, so that $\delta b$ and $\delta c$ are of course less than $\frac{1}{10} r_{0}$. With this conventional definition it appears that an encounter will be impulsive if

$$
R_{0}>10 M^{\prime} / v^{2}
$$

We see that all encounters at great distances satisfy the condition of the tidal forces being impulsive. Considering in detail an encounter in which $M^{\prime}$ is equal to the sun's mass $\left(2 \times 10^{33}\right.$ grammes $)$ and in which the two stars pass with a relative velocity of $40 \mathrm{kms}$. a second, we find that the action will be impulsive if the distance of closest approach $R_{0}$ is greater than $8 \times 10^{13} \mathrm{cms}$., which is about the distance of Jupiter from the sun. Having regard to astronomical scales of length we may say that all encounters of stars having masses comparable with that of the sun are impulsive except the very closest ones.

131. Nevertheless we cannot advance far in our cosmogonic problem so long as we consider only purely transitory encounters, and we must try to examine the effect resulting from the actual finite duration of tidal forces.

It is difficult to obtain definite or exact results, but the general nature of the motion can best be seen by thinking of the tidal body as moving too 
quickly for the series of equilibrium configurations discussed in $\$ 122$ to be able to keep pace with its motion.

There will be a lag in the orientation of the primary so that its majoraxis may be expected to point to some position such as $T^{\prime}$ in fig. 24 , when the tide-raising mass is actually in a position such as $T$ at a distance $l$ ahead of $T^{\prime}$.

The potential of the tide-generating mass at $T^{\prime}$ would be $\Omega=M^{\prime} / r$ where $r$ is the distance $O T^{\prime}$, but the true potential produced by the mass actually at $T$ is

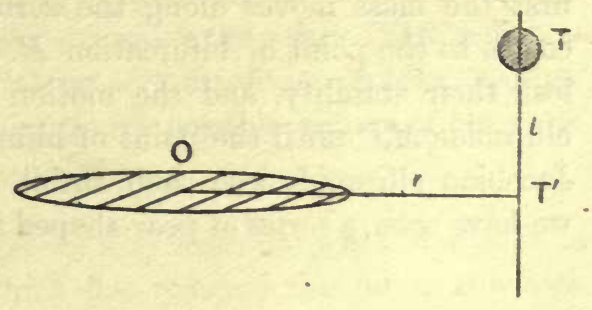

Fig. 24.

$$
\Omega+l \frac{\partial \Omega}{\partial y}=\frac{M^{\prime}}{r}+\frac{M^{\prime} l y}{r^{3}} .
$$

The correction which has to be introduced is that arising from the last term. For simplicity the primary may be regarded as a chain of matter lying along the axis of $x$, and the effect of the correction is to introduce a force of amount $M^{\prime} l / r^{s}$ per unit mass perpendicular to the axis of $x$.

Replacing $r$ by its value $R-x$, we find

$$
\frac{M^{\prime} l}{r^{3}}=\frac{M^{\prime} l}{R^{3}}\left(1+\frac{3 x}{R}+\frac{6 x^{2}}{R^{2}}+\frac{10 x^{3}}{R^{3}}+\ldots\right)
$$

The first term will produce a uniform acceleration $M^{\prime} l / R^{3}$ along the axis of $y$. Combining this with the acceleration $M^{\prime} / R^{2}$ towards $T^{\prime}$ which the primary has so far been supposed to have, we obtain a resultant acceleration $M^{\prime} / R^{2}$ towards $T$.

The remaining terms on the right of equation (385) set up various distortions. The second term sets up a uniform rotation at a rate $3 M I^{\prime} l / R^{4}$ per unit time; the third twists the major-axis of the primary into a piece of a parabola, the next superposes a cubical distortion, and so on. It can be readily seen from equation (385) that the combined effect of all these distortions will be to set up such a motion that initially the axis of the spheroid is bent to the shape of a piece of the curve $y=1 / x^{3}$, a curve shaped somewhat like a boomerang; there seems to be no tendency for the axis of the primary to assume the shape of a logarithmic spiral, which is the observed shape of the spiral nebulae.

This last result has an obvious bearing on the tenability of the "Planetesimal Theory" of Chamberlin and Moulton, described in $\$ 15$. 


\section{THE ROTATIONAL PROBLEM}

132. The diagram of the equilibrium configurations of a rotating mass of incompressible liquid has been seen to be of the type shewn in fig. 25. At first the mass moves along the series of Maclaurin spheroids $S M$ until it comes to the point of bifurcation $M$. At this point the Maclaurin spheroids lose their stability, and the motion proceeds along the series of Jacobian ellipsoids $M J^{\prime}$ until the point of bifurcation $J$ is reached. At this point the Jacobian ellipsoids lose their stability. The second series through $J$ is, as we have seen, a series of pear-shaped figures such as $J P$ in the diagram. The

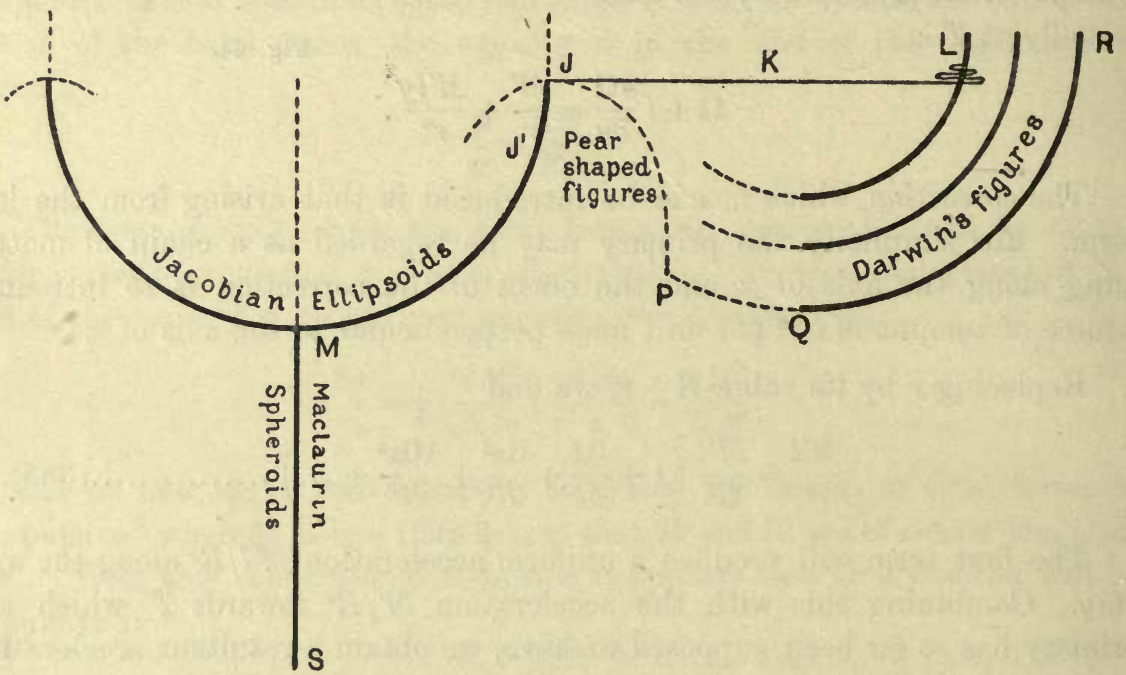

Fig. 25.

angular momentum of these figures decreases as we proceed along the series from $J$, so that the series is unstable and the curve $J P$ turns downwards in the diagram after leaving $J$. Thus there is no stable configuration beyond $J$, and dynamical motion of some kind must occur as soon as shrinkage has proceeded so far that the angular momentum is greater than that represented by the point $J$.

In the tidal problem we saw that the dynamical motion, when it occurred, was along the unstable series through the point at which the dynamical motion commenced. In the present problem such a solution is impossible, since the angular momentum must remain constant through the dynamical motion and equal to that at $J$.

Judging from the analogy of the two-dimensional problem, we may be fairly confident that the series of pear-shaped figures $J P$ ends in a configuration $P$ at which the mass divides into two parts, and so may be regarded as 
two separate masses just in contact. This configuration belongs not only to the pear-shaped series of figures but also to one of the double-star figures investigated by Darwin ( $\$ 60-65$ ). It forms one of a series of figures $P Q R$ of which the remaining members consist of double-stars rotating at different distances apart. If $Q$ is the figure of least angular momentum, we know that configurations on the branch $P Q$ including $P$ are unstable, while configurations on the other branch $Q R$ are stable. Besides the series $P Q R$, which is continuous with the pear-shaped series, there are an infinite number of other series of double-star figures, corresponding to all possible values of the ratio of the masses.

133. In the light of this knowledge we may examine what motion is to be expected in a Jacobian ellipsoid which has reached the point at which secular instability sets in.

In fig. 26 let $J J^{\prime}$ represent the series of stable Jacobian ellipsoids in the neighbourhood of the point of bifurcation $J$. For any configuration within the range $J J^{\prime}$, the third harmonic (pear-shaped) vibration is stable both ordinarily and secularly. Thus if any small pear-shaped vibration is set up when the mass is in a configuration such as $A$, the representative point will oscillate backwards and forwards through some small range such as $A^{\prime} A A^{\prime \prime}$ until the vibration is damped by viscosity. If the vibration is set up when the representative point is at some point $B$ close to $J$, there may still be oscillation through a small

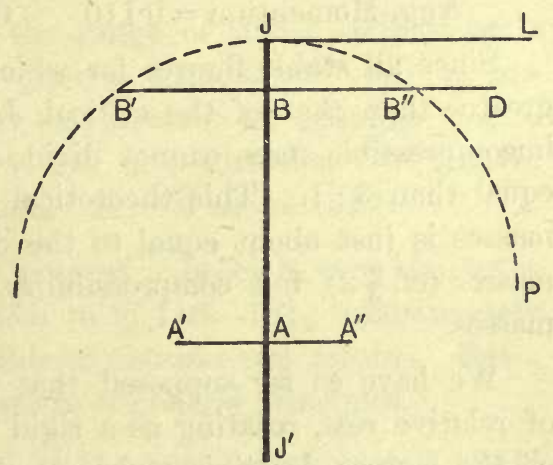

Fig. 26. range, but the motion can only be stable if this range is less than the range $B^{\prime} B^{\prime \prime}$ in fig. 26. For the point $B^{\prime \prime}$ represents a secularly unstable configuration, so that if the representative point once passes beyond $B^{\prime \prime}$, on the line $B B^{\prime \prime} D$, it will not return but will describe some path such as $B B^{\prime \prime} D$ in the plane through $B$.

As the point $B$ approaches $J$ the range of vibration which is possible without instability setting in becomes smaller and smaller and finally vanishes altogether, so that in the limit any disturbance, no matter how slight, causes the representative point to move permanently away from the line $J^{\prime} J$. The path of this point is necessarily in the horizontal plane through $J$, and we know that the direction of this path initially is that of the tangent $J L$ at $J$ to the pear-shaped series $J B^{\prime \prime}$. In other words the motion is one in which a furrow first forms on the ellipsoid, as in fig. 14, and this furrow continually deepens. 
It seems likely that this furrow will deepen until the mass divides into two parts. If so the motion, which must be in the plane $J L$ in fig. 25 , may end by the representative point coming to rest at some point such as $L$ on one of the stable double-star figures which consist of two stars revolving about one another. Thus the "cataclysm" which must occur when the representative point reaches the configuration $J$ may be represented by a jump of this point from $J$, the highest stable configuration on the ellipsoidal series, to $L$, a configuration of equal angular momentum on one of the double-star series.

Some of these double-star series do not possess stable configurations having angular momentum equal to that of the critical Jacobian ellipsoid $J$. Denoting this latter angular momentum by 0.3898 , so as to conform to the measurement of angular momentum used in our tables on pp. 39 and 40, I find the following angular momenta for Darwin's figures of limiting partial stability tabulated on p. $63 *$.

$$
\begin{array}{rrrlll}
M^{\prime} / M & 0 & 0.33 & 0.4 & 0.5 & 1.0 \\
\text { Ang. Momentum }= & 0.110 & 0.390 & 0.413 & 0.440 & 0.481
\end{array}
$$

Since all stable figures for which $M^{\prime} / M>0.33$ have angular momentum greater than that of the critical Jacobian ellipsoid, it is evident that an imcompressible mass cannot divide by fission into two masses more nearly equal than 3:1. This theoretical upper limit of $M^{\prime} / M$ for incompressible masses is just about equal to the observed lower limit of $M^{\prime} / M$ for actual masses (cf. $\S 2$ ), but compressibility may tend to equalise the ratio of the masses.

We have so far supposed that the two masses will assume a position of relative rest, rotating as a rigid body. Other possibilities, such as that of the masses describing non-circular orbits about one another or of the periods of rotation and revolution not coinciding, ought also to be considered; for convenience this is deferred to Chap. XI.

\section{THE DOUBLE-STAR PROBLEM}

134. We found in $\S 58$ that in Roche's problem of an infinitesimal satellite revolving in a circular orbit about a massive primary, there is a limit of closest approach within which no stable configurations of equilibrium exist for the satellite. Thus if a small satellite falls, or is in any way driven, into a certain sphere surrounding its primary, its configuration will become unstable and dynamical or cataclysmic motion must ensue. We have further seen in $\$ 60-65$ that in the more general double-star problem a precisely similar situation arises, and it will be clear that the dynamical motion in

* The last figures cannot be guaranteed as I have assumed for $1+\zeta$ the uniform value 1.06 from $M^{\prime} / M=0.4$ to $M^{\prime} / M=1$. The entry corresponding to $M^{\prime} / M=0.33$ is obtained by interpolation. 
this more general problem must in its general features be very similar to that occurring in the simpler problem of Roche.

135. The general nature of the motion can be seen from considerations precisely similar to those brought forward in considering the dynamical motion in the rotational problem $(\$ 133)$.

Let $P O P^{\prime}$ (fig. 27) represent the series of configurations possible for the satellite, the branch $P O$ being stable, the branch $O P^{\prime}$ being unstable, and the point $O$ representing the configuration of limiting stability.

When the configuration is represented by a point such as $A$ on the stable branch, a small displacement will result in stable oscillations through some small range $A^{\prime} A A^{\prime \prime}$.

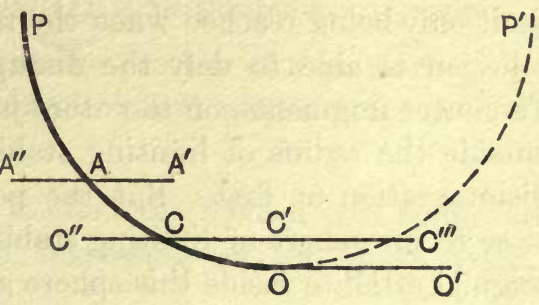

Fig. 27. When the representative point is at $C$ the range of stable oscillations is very small, and an oscillation of range greater than $C^{\prime} C C^{\prime \prime}$ will be unstable. Finally at $O$ any oscillation at all will result in an unstable motion which will initially be represented by motion in a direction $00^{\prime}$, and so will consist of an elongation of the ellipsoidal figure of the satellite.

The tracing out of this motion must present a problem very similar to that already discussed in the tidal problem in $\$ \S 118-131$; unfortunately the presence of rotation makes it impossible to obtain exact results. But a good deal of the motion is disclosed by a study of general principles.

The radius of the orbit is determined by the same equation as it would be if the whole mass of the satellite were concentrated at its centre of mass. The satellite may be thought of as consisting of two halves $H$ and $H^{\prime}$, the former being nearer to the primary than the centre of mass and the latter further away. If it were not for the presence of $H^{\prime}$, the half $H$ would be too near the primary for a circular orbit to be possible under the prescribed rotation; equilibrium is maintained by the gravitational pull from $H^{\prime}$ which neutralises part of the attraction of the primary on $H$. Similarly it is only the gravitational attraction of $H$ which makes a circular orbit possible for $H^{\prime}$.

When the configuration reaches limiting stability at the point $O$, a rapid elongation of figure begins, and this lessens to gravitational attraction between $H$ and $H^{\prime}$. The immediate result is that $H$ is drawn in closer to the primary, while $H^{\prime}$ is driven further away. At first this motion is only another representation of the elongation of the figure of the satellite, but it is clear that this elongation cannot continue for ever-a long thin filament of matter must be unstable under all conditions. Thus the satellite must before long 
break up into detached masses, much as it was seen to do in the tidal problem, and of these the innermost will fall in towards the primary while the outermost will recede from it. If we think of these fragments as ultimately describing elliptic orbits, the point at which instability sets in will approximately coincide with the aphelia of the inner pieces and with the perihelia of the outer ones.

If no change of density takes place in the matter of the satellite, the orbits of the inner fragments will all be within the radius of limiting stability, so that for each fragment the same process must repeat itself indefinitely, a limit only being reached when the fragments are so small that their chemical cohesion is able to defy the disruptive effects of gravitation and rotation. The outer fragments, on the other hand, will describe orbits which will all lie outside the radius of limiting stability, and so they will not suffer further disintegration at first. But the perihelia of these orbits are already very close to the sphere of limiting stability, and if the agencies which drove the original satellite inside this sphere are still operative, it may be expected that before long the new satellites also will be driven in and broken up in turn.

136. If the matter of the satellite is even slightly compressible, and therefore liable to changes of density, an entirely new feature presents itself. For the initial elongation of the satellite when the configuration of limiting stability is reached will be accompanied by a rapid diminution of pressure in the interior of the satellite, and therefore by a rapid diminution of average density. The radius of the sphere of limiting stability is however a function of the density $\rho$ of the satellite (cf. equation (65)), its radius varying as $\rho^{-\frac{1}{3}}$. Thus the elongation of the satellite will be accompanied by a rapid expansion of the sphere of limiting stability; when the satellite breaks into fragments all these will be within the new sphere of limiting stability, and the process of breaking up will repeat itself indefinitely.

Whichever way we approach the problem, the final result of the motion must be a ring of broken fragments, each fragment being so small that its forces of cohesion can resist the mechanical tendency to disintegration. Roche has suggested that Saturn's rings may have formed in this way, a suggestion borne out by the following figures:

Radius of Saturn's outermost ring $=2 \cdot 30$ radii of Saturn.

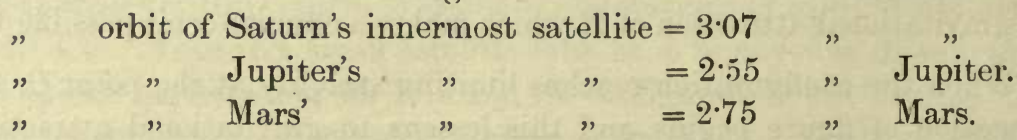

Roche's critical radius, it will be remembered, has been found equal to 2.45 radii of the primary when the densities of primary and satellite are the same. 


\section{SUMMARY OF RESULTS}

137. At this stage we leave the problem of the motions of an incompressible homogeneous mass of fluid. Before passing on to the next problem it may be of value to summarise, in the briefest and broadest manner possible, the results which have been obtained.

We have had three distinct problems under discussion-I. The Tidal Problem, II. The Rotational Problem, and III. The Double-star Problem.

In the Tidal Problem we have studied the motion of a primary mass as tides are raised in it by the continued approach and ultimate recession of a secondary mass.

In the Rotational Problem we have studied the motion of a single mass rotating freely in space, the rotation increasing as the mass cools by radiation.

In the Double-star Problem we have studied the motion of two stars revolving round one another, a secular change being supposed to occur in their distance apart.

In all three problems we have found that the motion will consist of two parts. The first may be described as "statical" or "secular"; the second may be described as "dynamical " or "cataclysmic."

In the Rotational Problem and in the Double-star Problem, there is a quite precise demarcation between the two types of motion. In the Tidal Problem, the two motions may gradually merge into one another, although here also there may be a precisely defined point of transition.

In all three problems, the statical motion has been found to consist of a slow secular change of shape in which the body under consideration remains always of a spheroidal or ellipsoidal shape, except that in the tidal and double-star problems (in which two masses are involved) the spheroidal or ellipsoidal shape of the primary may be slightly distorted by tides of third and higher orders raised by the secondary mass. In the Tidal Problem, the motion is through a series of prolate spheroids; in the Rotational Problem the motion is first through a series of oblate spheroids (Maclaurin's spheroids), and then through a series of ellipsoids (Jacobi's ellipsoids); in the Doublestar Problem the motion is through a series of ellipsoids.

In all three problems, dynamical motion supervenes when the prolate spheroid or ellipsoid reaches a certain elongation. The motion results in the formation of a furrow or system of furrows on the elongated mass. In the Tidal Problem the furrowing process does not commence immediately, and there inay be any number of furrows formed. In the two other problems, the furrows start to form at once and only one furrow is formed. 
The final result of the dynamical motion appears in every case to be fission into detached masses, although a rigorous mathematical proof of this has not been obtained. In the Tidal Problem any finite number of detached masses may result (cf. fig. 23, p. 127); in the Rotational Problem the mass appears to divide into two bodies of unequal size (cf. figures on p. 116); in the Double-star Problem the mass breaks up into a very great number of small masses.

Hence it appears highly probable that tidal-action may produce systems such as are seen in our own solar system and in the systems of Jupiter, Saturn, etc.; that increasing rotation may produce systems such as are seen in ordinary binary stars; and that the close approach of two stars revolving about one another may produce systems such as Saturn's rings and possibly the asteroids also. We shall investigate these conjectures more fully in subsequent chapters; before doing so we attempt to gain some knowledge of the motion of compressible and non-homogeneous masses. 


\section{CHAPTER VII}

\section{THE MOTION OF COMPRESSIBLE AND NON-HOMOGENEOUS MASSES}

\section{GENERAL THEORY}

138. So far we have discussed only the behaviour of masses of perfectly homogeneous and incompressible matter. In so doing we have followed the classical line of development, based upon the researches of Maclaurin, Jacobi, Poincaré and Darwin. Astronomical matter must however be highly compressible and far from homogeneous, so that the question of how far we are justified in attributing to real astronomical matter the behaviour which is found to occur in ideal incompressible masses is obviously one of great importance. In the present chapter we shall develop a general theory of the configurations of equilibrium of compressible masses, and shall in particular attempt to examine in a general way the effect of compressibility in introducing departures from the motion predicted by the incompressible model which we have so far had under consideration.

139. If $p$ is the pressure at any point $x, y, z$ of a mass rotating with angular velocity $\omega$ about the axis of $z$, the equations of equilibrium will be

$$
\begin{aligned}
& \frac{\partial p}{\partial x}=\rho \frac{\partial V}{\partial x}+\omega^{2} \rho x \\
& \frac{\partial p}{\partial y}=\rho \frac{\partial V}{\partial y}+\omega^{2} \rho y \\
& \frac{\partial p}{\partial z}=\rho \frac{\partial V}{\partial z} \quad \ldots . . .
\end{aligned}
$$

in which $V$ is the potential of the whole gravitational field of force, including tidal forces if any are present. Thus we may write

$$
V=V_{M}+V_{T}
$$

where $V_{M}$ is the gravitational potential of the rotating mass under consideration, and $V_{T}$ is the potential of the tidal field.

Writing

$$
\Omega=V+\frac{1}{2} \omega^{2}\left(x^{2}+y^{2}\right)
$$


these equations of equilibrium become

$$
\begin{aligned}
& \frac{\partial p}{\partial x}=\rho \frac{\partial \Omega}{\partial x} \\
& \frac{\partial p}{\partial y}=\rho \frac{\partial \Omega}{\partial y} \\
& \frac{\partial p}{\partial z}=\rho \frac{\partial \Omega}{\partial z}
\end{aligned}
$$

On equating two values of $\partial^{2} p / \partial y \partial z$ we obtain

so that

$$
\frac{\partial \rho}{\partial z} \frac{\partial \Omega}{\partial y}=\frac{\partial \rho}{\partial y} \frac{\partial \Omega}{\partial z}
$$

$$
\frac{\frac{\partial \rho}{\partial x}}{\frac{\partial \Omega}{\partial x}}=\frac{\frac{\partial \rho}{\partial y}}{\frac{\partial \Omega}{\partial y}}=\frac{\frac{\partial \rho}{\partial z}}{\frac{\partial \Omega}{\partial z}}
$$

It follows at once that the surfaces $\rho=$ cons. necessarily coincide with the equipotentials $\Omega=$ cons., and it further follows from equations (391)-(393) that these surfaces also coincide with the surfaces of constant pressure $p=$ cons. The boundary of the fluid must of course be one of this family of surfaces, say $\rho=\sigma$, and the necessity for the condition that $\Omega$ shall be constant over the boundary, which has so far been used as the condition for equilibrium, is at once obvious.

The condition that $\Omega$ shall be constant over the boundary will however no longer be sufficient to ensure equilibrium; it is still necessary but not sufficient, and equations of equilibrium must be satisfied throughout the mass.

\section{Masses of Uniform Composition}

140. The simplest case arises when the matter is of uniform composition throughout, so that the pressure is a function of the density, say

$$
p=f(\rho) \text {. }
$$

Equations (391) etc. now assume the form

If $\phi(\rho)$ is defined by

$$
\frac{\partial f(\rho)}{\partial \rho} \frac{\partial \rho}{\partial x}=\rho \frac{\partial \Omega}{\partial x} \text { etc. }
$$

these become

$$
\phi(\rho)=\int \frac{1}{\rho} \frac{\partial f(\rho)}{\partial \rho} d \rho
$$

$$
\frac{\partial \phi(\rho)}{\partial x}=\frac{\partial \Omega}{\partial x} \text { etc. }
$$


so that the equations of equilibrium have the common integral

$$
\phi(\rho)=\Omega+C .
$$

where $C$ is a constant. At any point inside the mass,

so that, from equation (390), $\nabla^{2} V_{T}=0$

$$
\begin{aligned}
& \nabla^{2} V_{M}=-4 \pi \rho, \\
& \nabla^{2} V_{T}=0, \\
& \nabla^{2} \Omega=2 \omega^{2}-4 \pi \rho
\end{aligned}
$$

Thus on operating on equation (396) with $\nabla^{2}$, we obtain

$$
\nabla^{2} \phi(\rho)+4 \pi \rho=2 \omega^{2}
$$

the differential equation which must be satisfied by $\rho$ for equilibrium to be possible.

From equation (396) we can obtain $\rho$ in the form

$$
\rho=\psi(\Omega)
$$

and equation (397) now becomes

$$
\nabla^{2} \Omega+4 \pi \psi(\Omega)=2 \omega^{2}
$$

the differential equation which must be satisfied by $\Omega$ for equilibrium to be possible.

141. Let $P$ be any point inside the mass, and let $R$ denote the distance from $P$ to a variable point $x, y, z$ inside the mass; let $d S^{\prime}$ be an element of surface of a small sphere surrounding $P$, and let $d S$ be an element of surface of the boundary. Then for the value of $V_{M}$ at the point $P$ we have

$$
\begin{aligned}
V_{M} & =\iiint \frac{\rho}{R} d x d y d z \\
& =-\frac{1}{4 \pi} \iiint \frac{\nabla^{2} V}{R} d x d y d z \\
& =\frac{1}{4 \pi} \iint\left\{\frac{\partial V}{\partial n}\left(\frac{1}{R}\right)-V \frac{\partial}{\partial n}\left(\frac{1}{R}\right)\right\}\left(d S+d S^{\prime \prime}\right) \\
& =V+\frac{1}{4 \pi} \iint\left\{\frac{\partial V}{\partial n}\left(\frac{1}{R}\right)-V \frac{\partial}{\partial n}\left(\frac{1}{R}\right)\right\} d S
\end{aligned}
$$

so that

$$
V_{T}=-\frac{1}{4 \pi} \iint\left\{\left(\frac{\partial V}{\partial n}\right) \frac{1}{R}-V \frac{\partial}{\partial n}\left(\frac{1}{R}\right)\right\} d S
$$

The integral on the right is of course the potential of a Green's equivalent stratum; it is a known theorem that the potential of this together with that of external masses has a constant value inside the surface. 
Since $V+\frac{1}{2} \omega^{2}\left(x^{2}+y^{2}\right)$ is constant over the boundary in a configuration of equilibrium, the value of $V_{T}$ becomes

$$
V_{T}=-\frac{1}{4 \pi} \iint \frac{\partial V}{\partial n} \frac{1}{R} d S-\frac{\omega^{2}}{8 \pi} \iint\left(x^{2}+y^{2}\right) \frac{\partial}{\partial n}\left(\frac{1}{R}\right) d S+\text { a cons. }
$$

For a problem in which $V_{T}, \omega$ and the shape of the boundary are given, the value of

$$
V_{T}+\frac{\omega^{2}}{8 \pi} \iint\left(x^{2}+y^{2}\right) \frac{\partial}{\partial n}\left(\frac{1}{R}\right) d S
$$

is known at every point inside the boundary. It follows that

$$
\iint \frac{\partial V}{\partial n} \frac{1}{R} d S
$$

must be given at all points inside the boundary except for a constant, and this determines $\partial V / \partial n$ at all points of the boundary, except for a constant. But

$$
\iint \frac{\partial V}{\partial n} d S
$$

is given, being equal to $4 \pi$ times the total mass of the rotating body, so that $\partial V / \partial n$ is uniquely determined at every point of the boundary.

It follows from equation (396) that under the conditions now contemplated, $\rho$ and $\partial \rho / \partial n$ are determined at every point of the boundary, and from this and equation (398) it is easy to see that the solution for $\rho$ is unique.*

It follows that configurations of equilibrium may be specified by their boundaries alone, but a more important result also follows. When $V_{T}$ and $\omega^{2}$ are given and the boundary is given, there will be an endless number of possible vibrations in which the internal particles move, while those at the boundary remain in position. The result just obtained shews that none of these can ever be of zero frequency, so that no points of bifurcation can occur, and the internal vibrations, if stable in the initial configuration of the mass, must always remain stable.

From the circumstance that configurations of equilibrium may be specified by their boundaries alone, it will be clear that the various configurations must fall into linear series much in the same way as in the incompressible problem. The configuration for no rotation and no tidal forces will of course be spherical.

142. In the rotational problem there will obviously be a series, analogous to the Maclaurin spheroids, in which the boundary is a figure of revolution.

* It is difficult to construct a rigorous proof, for complications of a mathematical nature arise. See Proc. Roy. Soc. 93 (1917), p. 416. 
The configurations near to the spherical one are spheroids of small ellipticity, but the series will not remain spheroidal throughout its length. But the far end of this series is again spheroidal, being in fact identical with the Maclaurin spheroid for a mass of uniform density $\sigma$. Just as in the incompressible problem, this is unstable for all displacements specified by sectorial harmonic deformations of its boundary*. It follows that, on the series we are considering, there must be points of bifurcation corresponding to all sectorial harmonics. The general physical principles explained in $\S 82$ lead us to expect with confidence that the first of these to occur will be that corresponding to the second harmonic. At this point the circular crosssection of the figure gives place at first to an elliptic cross-section of small ellipticity, and the configurations on the new series are analogous to the Jacobian ellipsoids.

Further, the far end of the series analogous to the Jacobian series is again identical with that in the incompressible problem, both as regards configuration and stability, so that again this series must have the same points of bifurcation as the Jacobian series.

143. Almost identical remarks apply to the tidal problem. Again there is a principal series of figures of revolution analogous to the tidal spheroids examined in $\$ 49$, and again these figures are strictly spheroidal at the two extreme ends of the series. The stability of the end configuration of this series-a long drawn out line of matter-is plainly the same as for the incompressible mass, so that the same points of bifurcation must occur on the series.

144. All these statements obviously require slight modification in the extreme case of $\sigma=0$, but except for this case it is clear that the general arrangement of series and points of bifurcation will be very similar to that in the incompressible problem. It ought again to be possible to construct a diagram similar to that of fig. 7 (p. 50); the general arrangement will be the same but the numerical values different, and the shape of the figures will of course be different except at the extreme ends of the various series.

Figures of equilibrium which take the place of the spheroidal figures of the incompressible problem, whether rotational or tidal, may conveniently be referred to as "pseudo-spheroids." Similarly figures which take the place of ellipsoidal figures of equilibriurn may be referred to as "pseudo-ellipsoids"; these of course do not enter in the tidal problem, but occur in the rotational and double-star problems.

145. This general discussion does not touch the question of the stability of the various branch series; this can only be determined by detailed 
calculations in individual problems. Thus in the rotational problem it is not possible, from a consideration of general principles, to predict whether the pseudo-ellipsoidal series will initially be stable or unstable. If in any problem it is unstable, cataclysmic motion will begin as soon as the first point of bifurcation on the pseudo-spheroidal series is reached. This motion will consist at first of an ellipsoidal elongation of the pseudo-spheroid, the circular cross-sections giving place to elliptical ones, and the points of bifurcation on the pseudo-ellipsoidal series will be replaced by "dynamical points of bifurcation" in this motion. In such a case, if ever it occurs, it seems to be quite possible that the rotating mass may divide up into a number of detached masses (instead of into only two) very much as in the tidal problem.

It will, however, be remembered that the angular momentum of the pseudo-ellipsoidal series is infinite at its far end, so that much the most likely event is that it increases all along the length of this series; in this case the pseudo-ellipsoidal series would initially be stable. But no such general consideration can be brought forward in the case of the pear-shaped series which branches off at the first point of bifurcation, and nothing justifies us in predicting whether this will in general be stable or unstable. Indeed it appears to be at least possible that in some problems this series may be initially stable, a possibility which has been mentioned by Poincaré.*

\section{Masses of non-uniform Composition}

146. From $\S 140$ on, we have assumed the astronomical matter to be of uniform composition throughout, the pressure being a function of the density only. When this restriction is removed, the discussion of equilibrium configurations is naturally more difficult.

Suppose that we are dealing with a mass of different types of matter $a, b, c, \ldots$, these letters referring either to chemically distinct types of matter or to mixtures of such types in varying proportions.

Consider a special problem in which the values of $V_{T}$ and $\omega$ are given, and in which it is also given that the shells of matter occur in an assigned order $a, b, c, \ldots$ from the boundary inwards.

The external boundary will of course be one of the equipotentials $\Omega=$ cons. The surfaces of transition between the different types of matter will be surfaces at which the density changes abruptly. Thus these surfaces will coincide with surfaces of constant density and hence, by equations (394), they will coincide with equipotentials $\Omega=$ cons.

* “Sur la Stabilité de l’Équilibre des Figures Pyriformes affectées par une Masse Fluide en Rotation," Phil. Trans. 198 A (1901), p. 335. 
As in $\S 141$, the values of $\rho$ and $\partial \rho / \partial n$ are determined at the boundary. It follows from equation (398) that the arrangement of density is determined throughout the layer of matter of type $a$. At the surface of transition to the next layer, $p$ and $\partial \Omega / \partial n$ are continuous, so that $\rho$ and $\partial \rho / \partial n$ are determined at the boundary of layer $b$, and so also through this layer. In this way the configuration can be built up layer by layer, the configuration being uniquely determined when the order of the layers is determined.

The order of the layers is determined by conditions of stability. We shall return to a discussion of this matter in the next chapter. For the present we may notice that any arrangement will be unstable if energy can be gained by an interchange of any two layers, the instability shewing itself by the creation of convection currents which result in the actual interchange of the layers in question. Thus the only arrangement of layers which can be stable is that for which the potential energy is a minimum. For this arrangement the results already obtained for a homogeneous compressible mass remain true; in particular the configuration is uniquely determined, and is stable as regards internal vibrations, when the values of $V_{T}$ and $\omega$ and the shape of the boundary are given.

\section{The Two Mechanisms of breaking up}

147. There are two conditions that must be satisfied by a configuration of equilibrium; the equations of equilibrium must be satisfied, and also $p$ must be positive everywhere. Now the linear series so far discussed have been series of configurations such that the conditions of equilibrium have been satisfied everywhere, but we have not introduced the condition that $p$ must be positive everywhere. Any region on these series in which $p$ is anywhere negative will represent configurations in which the equations of equilibrium are satisfied but which are physically impossible through negative pressures being demanded. Hence if at any point on a linear series the pressure becomes negative, the series may be supposed to be abruptly terminated at that point: the configurations beyond are of no physical interest.

It is easily seen that $p$ cannot change sign at a point in the interior of the mass, for $p$ can only change sign by first vanishing, and the points at which $p$ vanishes determine the boundary. But close to the boundary $p$ can change sign by passing through a zero value; when this happens $\partial p / \partial n$ vanishes at the point of the boundary in question. Thus the normal force $\partial \Omega / \partial n$ vanishes, which means that the gravitational attraction of the mass is just neutralised, and ultimately outbalanced, at this point by centrifugal and tidal forces. When this happens a stream of matter will be thrown off from the point in question. 
Clearly it is of the utmost importance to cosmogony to know under what conditions streams of matter will be thrown off in this way. We have found two ways in which a mass can break up - the one by fission, and the other by the ejection of streams of matter from a point or points on its boundary. In an actual astronomical mass, which will happen first?

This question is one which can only be solved by detailed analysis in special cases. Now there is an infinite variety of arrangements of compressible matter possible, while the solution of even a single case is a problem of considerable difficulty and complexity. It therefore behoves us to choose the special cases which we attempt to solve with skill and care, so as to economise labour as much as possible.

148. Compressibility of matter is of course associated with variations of density in the compressible mass, and the greater the compressibility of the matter, the greater these variations of density will be. In Chapters III-VI we have solved the problem in the special case of a mass having no compressibility and so having no variations of density.

This problem formed in a sense a limiting case of the problem of the motion of a compressible mass. At the other end of the general problem there will be another limiting case in which the compressibility is so great that infinite variations of density may be expected. Mathematically this limiting case may be specified by the condition that the density is infinite or zero at different places. Physically, as we shall now see, this limiting case is not so artificial as its mathematical specification might lead us to suppose.

149. For a mass of gas at rest in isothermal equilibrium, the density at great distances from the centre falls off as $1 / r^{2}$. The general law of density has been obtained by Darwin* and otherst. But without detailed analysis it is clear that, at a sufficient distance from the centre, the law of density must become+

$$
\rho=\rho_{0}\left(a^{2} / r^{2}\right),
$$

so that, when viewed from a very great distance, the density may be regarded as infinite at the centre and zero everywhere else. The total mass is however infinite, so that a finite mass of gas in isothermal equilibrium will be of zero density everywhere.

Similarly for a mass of gas in adiabatic equilibrium with the ratio of the specific heats $\gamma$ equal to $1 \frac{1}{5}$, the law of density is

$$
\rho=\rho_{0}\left(1+r^{2} / a^{2}\right)^{-\frac{5}{2}} \text {. }
$$

" "On the Mechanical Conditions of a Swarm of Meteorites and on Theories of Cosmogony." Phil. Trans. 180 A (1889), p. 1, and Coll. Works, rv. p. 362.

$\uparrow$ For detailed references see Darwin's paper.

$\ddagger$ L. c. p. 377.

§ A. Schuster, Brit. A88. Report, 1883, p. 428. 
Again, when the mass of gas is viewed from a sufficient distance, the value of $\rho$ becomes infinite at the centre and zero everywhere else. The same is true for any value of $\gamma$ from 1 to $1 \frac{1}{5}$. The mass is infinite when $\gamma<1 \frac{1}{5}$, but becomes finite when $\gamma=1 \frac{1}{5}$.

This same model, in which the density is infinite or very great over a point or small concentrated area but zero everywhere else, has been largely utilised by Roche* in his researches on cosmogony. For convenience we may refer to it as "Roche's model." Roche interpreted it physically as referring to a small and intensely dense solid nucleus surrounded by an atmosphere of negligible density. In Roche's model, the whole of the mass is supposed concentrated at the centre; in this respect it differs from a mass of gas in isothermal equilibrium, although giving a faithful representation of an adiabatic mass for which $\gamma=1 \frac{1}{5}$.

\section{ROCHE'S MODEL}

150. We have seen that Roche's model and the incompressible model form the two limiting cases of the general compressible mass. The latter has already been studied in detail ; it is natural to begin our investigation of the compressible problem with a discussion of the former.

Roche's model has one great advantage over the incompressible model. For in studying the configurations and motion of an incompressible mass, one of the main difficulties was found to lie in the determination of the gravitational potential. Now in Roche's model no such difficulty occurs; the mass is supposed collected at one or more points and the gravitational potential reduces to $M / r$, or to a sum of such terms in cases where there is more than one nucleus. Thus, when there is only one nucleus involved, the quantity which has been denoted by $\Omega$ assumes the simple form

$$
\begin{aligned}
\Omega & =V+\frac{1}{2} \omega^{2}\left(x^{2}+y^{2}\right) \\
& =\frac{M}{r}+V_{T}+\frac{1}{2} \omega^{2}\left(x^{2}+y^{2}\right)
\end{aligned}
$$

For given values of $V_{T}$ and $\omega$, the surfaces $\Omega=$ cons. will be a system of equipotentials of the usual type ; since $\Omega$ is uniquely determined as a function of $x, y$ and $z$, two different equipotentials can never intersect. Of the system of equipotentials only one is suitable for the boundary of the gravitating mass, this being picked out by the condition that the volume enclosed by it shall be just adequate to contain the whole amount of the compressible matter.

* "Essai sur la Constitution et l'Origine du Système solaire" (1873). Acad. de Montpellier, Section des Sciences, viIr. p. 235̃. See also Poincaré, Leçons sur les Hypothèses Cosmogoniques, Chap. III. 
When either $V_{T}$ or $\omega^{2}$ are allowed to vary, we obtain a linear series of configurations by picking out the appropriate equipotential surface from each set. When $V_{T}$ and $\omega^{2}$ both vanish, the equipotentials are spheres and the boundary is therefore spherical; as we pass along the linear series the boundary will depart more and more from the spherical shape.

One property of Roche's model may be noticed at once. There can be no points of bifurcation or turning points on any linear series. For when $V_{T}$ and $\omega$ are given, the value of $\Omega$ is uniquely determined by equation (400) and hence the boundary is uniquely determined. But the condition for a point of bifurcation or a turning point is that there shall be two adjacent configurations of equilibrium, and hence (by $\S 141$ ) two different boundaries, possible for the same value of $V_{T}$ and $\omega$.

It follows that all possible configurations for a Roche's model lie on one linear series, and this may in every case be supposed to originate in the spherical configuration for which $V_{T}$ and $\omega$ both vanish. As we proceed along this series, the different boundaries are equipotentials which differ more and more from spheres, until finally it may happen that the equipotential which forms the boundary coincides with one which marks a transition from closed to open equipotentials. On moving one step further along the linear series we shall find that there is no closed equipotential capable of containing the whole mass. There is therefore no equilibrium configuration consistent with values of $\omega^{2}$ and $V_{T}$ beyond a certain limit, and as soon as this limit is exceeded, a cataclysm of some kind must occur.

151. The transition from an open equipotential to a closed one must necessarily be through one which intersects itself, and therefore through an equipotential on which a point of equilibrium occurs. Such a point is determined by the equations

$$
\frac{\partial \Omega}{\partial x}=\frac{\partial \Omega}{\partial y}=\frac{\partial \Omega}{\partial z}=0
$$

Since $\Omega$ is necessarily constant over the surface of every equipotential, including the boundary, this condition may be put in the alternative form

$$
\frac{\partial \Omega}{\partial n}=0
$$

or, again, from equations (391)-(393),

$$
\frac{\partial p}{\partial n}=0
$$

We are now back to the point of view of $\S 147$; the series terminates as soon as $\partial p / \partial n$ vanishes at any point of the boundary. But we have now seen that this will occur at a point at which the equipotential which 
forms the boundary intersects itself, so that at such a point the boundary must have the shape either of a sharp edge or of a conical point.

Let us now examine the various types of problem in detail, beginning with the rotational problem.

\section{The Rotational Problem}

152. To discuss the problem of a freely-rotating mass, we put $V_{T}=0$, so that

$$
\Omega=\frac{M}{r}+\frac{1}{2} \omega^{2}\left(x^{2}+y^{2}\right) .
$$

The condition for a point of equilibrium will obviously first be satisfied in the plane of $x y$. It will be satisfied at $x, 0,0$ if

$$
-\frac{M x}{r^{3}}+\omega^{2} x=0
$$

and so is first satisfied when

$$
M=\omega^{2} \varpi_{0}{ }^{3},
$$

where $\varpi_{0}$-is the radius of the cross-section in the plane of $x y$. The particular equipotential on which this point of equilibrium occurs is found to be

$$
\frac{M}{r}+\frac{1}{2} \omega^{2}\left(x^{2}+y^{2}\right)=\frac{3}{2}(M \omega)^{\frac{2}{3}} \text {. }
$$

Since $\varpi_{0}{ }^{3}=M / \omega^{2}$, this equation may be written in the form

$$
\frac{1}{r}+\frac{1}{2} \frac{\varpi^{2}}{\varpi_{0}^{3}}=\frac{3}{2} \frac{1}{\varpi_{0}}
$$

where $\varpi^{2}$ stands for $x^{2}+y^{2}$.

The general equipotentials are found to lie as in fig. 28 , the critical equipotential being drawn thick.

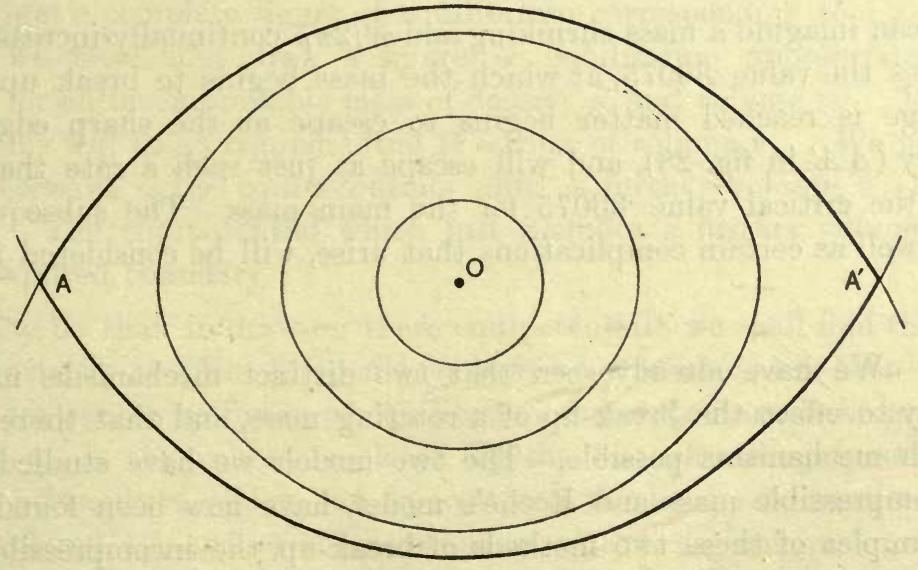

Fig. 28. 
The volume of the critical equipotential determined by equation (401) is easily found. Putting $r^{2}=z^{2}+\varpi^{2}$, the equation becomes

$$
z^{2}=\frac{4 \varpi_{0}^{6}}{\left(3 \varpi_{0}^{2}-\varpi^{2}\right)^{2}}-\varpi^{2}=\frac{\left(\varpi_{0}^{2}-\varpi^{2}\right)^{2}}{\left(3 \varpi_{0}^{2}-\varpi^{2}\right)^{2}}\left(4 \varpi_{0}^{2}-\varpi^{2}\right)
$$

If we put $\varpi=2 \varpi_{0} \sin \theta$,

$$
z=\frac{1-4 \sin ^{2} \theta}{3-4 \sin ^{2} \theta} 2 \varpi_{0} \cos \theta
$$

so that the volume is

$$
\begin{aligned}
4 \pi \int_{z=0}^{z=\varpi_{0}} z \varpi d \varpi & =32 \pi \varpi_{0}^{3} \int_{0}^{\pi / 6} \frac{4 \cos ^{2} \theta-3}{4 \cos ^{2} \theta-1} \cos ^{2} \theta d(\cos \theta) \\
& =32 \pi \varpi_{0}^{3}\left|\frac{1}{3} p^{3}-\frac{1}{2} p-\frac{1}{8} \log \frac{2 p-1}{2 p+1}\right|_{p=\frac{1}{3} \sqrt{ } 3}^{p=1} \\
& =32 \pi \varpi_{0}^{3} \times 0225466 .
\end{aligned}
$$

The mass is equal to $\bar{\rho}$ times this, where $\bar{\rho}$ is the mean density, hence

$$
\frac{\omega^{2}}{2 \pi \bar{\rho}}=\frac{M}{2 \pi \bar{\rho} \varpi_{0}{ }^{3}}=\frac{\text { volume }}{2 \pi \varpi_{0}{ }^{3}}=\cdot 36075 \ldots \ldots \ldots \ldots \ldots . .(402) \text {. }
$$

Thus the series of configurations possible for the mass in question form a single linear series, starting from $\omega=0$ and ending abruptly when

$$
\omega^{2} / 2 \pi \bar{\rho}=\cdot 36075 \text {. }
$$

For greater values of $\omega$ there is no equilibrium configuration possible.

As a homogeneous mass shrinks, keeping its moment of momentum constant, it is easy to shew that $\omega^{2} / \bar{\rho}$ will continually increase. It cannot be rigorously proved that the same is necessarily true for a non-homogeneous mass, but obviously the normal event will be for shrinkage to be accompanied by an increase of $\omega^{2} / \bar{\rho}$.

We can imagine a mass shrinking and $\omega^{2} / 2 \pi \bar{\rho}$ continually increasing until it reaches the value 36075 , at which the mass begins to break up. When this stage is reached matter begins to escape at the sharp edge of the boundary $\left(A A^{\prime}\right.$ in fig. 28$)$, and will escape at just such a rate that $\omega^{2} / 2 \pi \bar{\rho}$ retains the critical value 36075 for the main mass. The subsequent motion, as well as certain complications that arise, will be considered in a later chapter.

153. We have already seen that two distinct mechanisms may come into play to effect the break-up of a rotating mass, and that there are only two such mechanisms possible. The two models we have studied, namely the incompressible mass and Roche's model, have now been found to provide examples of these two methods of break-up, the incompressible model breaking up by fission into two parts, and Roche's model breaking up by 
the ejection of streams of matter from the equator. It is obviously very desirable to bridge over, if possible, the wide gap between these two extreme cases, and this is to some extent effected by the consideration of a third model, which combines some of the properties of both of the two models so far discussed.

154. Roche's model consisted of a nucleus of finite mass but infinitesimal volume, surrounded by an atmosphere of zero mass but finite volume. The density of the nucleus was accordingly infinite while that of the atmosphere was zero.

In the new model we take the nucleus to be of finite extent, and therefore of finite density, while supposing the atmosphere to remain of finite extent but of infinitesimal density. Thus the potential of the mass $V_{M}$ may no longer be put equal to $M / r$ but becomes equal to the potential of the nucleus. The nucleus will be supposed to be incompressible and of uniform density $\rho_{0}$, and the atmosphere will be supposed to exert no appreciable pressure on the nucleus.

Let $v_{N}$ denote the volume of the nucleus and $v_{A}$ that of the atmosphere. The mass $M$ is equal to $\rho_{0} v_{N}$, so that the mean density $\bar{\rho}$ is given by

$$
\bar{\rho}=\frac{v_{N}}{v_{A}+v_{N}} \rho_{0} .
$$

Under a rotation $\omega$ each particle of the nucleus will be subjected to exactly the same forces as though the nucleus alone were rotating with angular velocity $\omega$, the atmosphere being entirely non-existent. This determines the configurations of the nucleus; they consist of Maclaurin spheroids, Jacobian ellipsoids, etc.

The boundary of the atmosphere must be one of the equipotentials $\Omega=$ cons.; it must moreover be an equipotential of total volume $v_{A}+v_{N}$. Thus to get a complete figure of equilibrium corresponding to a given rotation $\omega$, we must first draw a figure of equilibrium appropriate to this rotation for an incompressible mass of density $\rho_{0}$ and volume $v_{N}$. The boundary of this will be an equipotential $\Omega=$ cons. of volume $v_{N}$. We must then draw successive outer equipotentials until a further volume $v_{A}$ has been enclosed. The equipotential which just includes a further volume $v_{A}$ will be the required boundary.

It may be that, in drawing these equipotentials, we shall find that closed equipotentials give place to open ones before a volume $v_{A}$ has been enclosed. If so, there can be no figure of equilibrium corresponding to the given rotation. If $v_{A}{ }^{\prime}$ is the volume enclosed by the last closed equipotential, the greatest atmosphere which can be retained at the given rotation will be one of volume $v_{A}{ }^{\prime}$, and of the atmosphere of the original model, a volume $v_{A}-v_{A}{ }^{\prime}$ must already have been thrown off at the equator. 
For a spherical configuration $(\omega=0)$ there is obviously no limit to the value of $v_{A}$ : the critical value $v_{A}{ }^{\prime}$ is infinite. But as $\omega$ increases $v_{A}^{\prime}$ will diminish, and matter will be ejected from the equator as soon as $v_{A}=v_{A}{ }^{\prime}$. Let us examine the value of the critical value $v_{A}{ }^{\prime}$.

Let the spheroidal or ellipsoidal figure of the nucleus be supposed to be the standard ellipsoid

$$
\frac{x^{2}}{a^{2}}+\frac{y^{2}}{b^{2}}+\frac{z^{2}}{c^{2}}=1
$$

the axis of $z$ being the axis of rotation. At a point $x$ on the prolongation of the major-axis, the gravitational attraction is

$$
X=2 \pi \rho_{0} a b c x \int_{x^{2}-a^{2}}^{\infty} \frac{d \lambda}{\left(a^{2}+\lambda\right)^{\frac{3}{2}}\left(b^{2}+\lambda\right)^{\frac{1}{2}}\left(c^{2}+\lambda\right)^{\frac{1}{2}}} .
$$

For a Maclaurin spheroid, in which $a=b$, the integration can be effected, and we find

$$
X=2 \pi \rho_{0} a b c x\left[\frac{1}{\alpha^{3}} \sin ^{-1} \frac{\alpha}{x}-\frac{\left(x^{2}-\alpha^{2}\right)^{\frac{1}{2}}}{a^{2} x^{2}}\right] .
$$

where $\alpha=\left(a^{2}-c^{2}\right)^{\frac{1}{2}}$. The ratio of centrifugal force to gravity at any point on the $x$-axis is $\omega^{2} x / X$. At a point on the boundary of the nucleus, this ratio is always less than unity, but it increases as we pass outwards, and the point at which it attains the value unity is the critical point at which $\partial \Omega / \partial x=0$. Hence to obtain this critical point, we must equate the right-hand member of equation (403) to $\omega^{2} x$; the resulting equation is

$$
\frac{\omega^{2}}{2 \pi \rho_{0}}=a b c\left[\frac{1}{\alpha^{3}} \sin ^{-1} \frac{\alpha}{x}-\frac{\left(x^{2}-\alpha^{2}\right)^{\frac{1}{2}}}{\alpha^{2} x^{2}}\right]
$$

The value of $x$ which satisfies this equation determines the radius of the equator of the limiting equipotential.

In the special case in which the nucleus is a Maclaurin spheroid at its ellipsoidal point of bifurcation, the value of $\omega^{2} / 2 \pi \rho_{0}$ is $0 \cdot 18712$, and the root of equation (404) is found to be

$$
x=1.64 .36 \alpha=1.5990(a b c)^{\frac{1}{2}} \text {. }
$$

The critical equipotential is drawn in fig. 29 ; it is clear that the value of $v_{A}^{\prime}$ here is quite small, being in point of fact rather less than one-third of $v_{N}$.

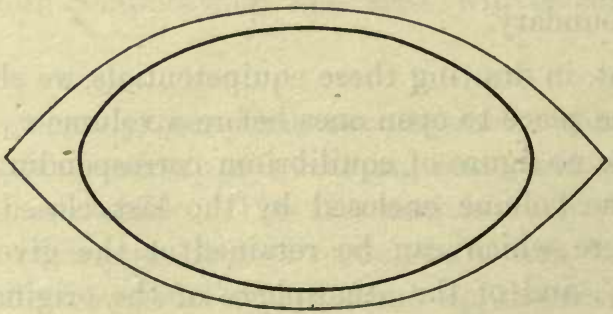

Fig. 29. 
Thus it will be seen that the value of the ratio $v_{A}{ }^{\prime} / v_{N}$ steadily decreases from $\infty$ to about $\frac{1}{3}$ as we pass along the Maclaurin series to the point of bifurcation, and it is readily found that it decreases still further as we pass along the Jacobian series. Its value at the pear-shaped point of bifurcation is about $\frac{1}{8}$.

We can now describe the series of equilibrium configurations assumed by this model as its angular momentum continually increases. Suppose first that the ratio $v_{A} / v_{N}$ is greater than $\frac{1}{3}$.

For small values of $\omega$, the boundary of the nucleus and the atmosphere will both be spheroids of small eccentricity. For larger values of $\omega$ the boundary of the nucleus will remain spheroidal, while that of the atmosphere will be a pseudo-spheroid coinciding with one of the external equipotentials. As $\omega$ still increases this pseudo-spheroid will develop a sharp edge, this occurring when the critical volume $v_{A}{ }^{\prime}$ is equal to $v_{A}$. After this, matter will be ejected from the sharp edge on the equatorial plane of the mass. By the time the rotation is given by $\omega^{2} / 2 \pi \rho_{0}=\cdot 18712$, the atmosphere is reduced to about $\frac{1}{3} v_{N}$ in volume. Thus $\bar{\rho}=\frac{3}{4} \rho_{0}$, and $\omega^{2} / 2 \pi \bar{\rho}=\cdot 2496$. At this stage the figure loses its symmetry, being no longer a figure of revolution. The nucleus becomes ellipsoidal, while the boundary becomes a pseudo-ellipsoidal figure having two sharp pointed ends, and as the rotation still increases, two streams of matter will be ejected from these ends. Gradually the nucleus becomes more elongated and the atmosphere diminishes more and more, until the pear-shaped point of bifurcation is reached. After this the nucleus will divide into detached masses, each of which will be surrounded by a thin atmosphere.

If the original atmosphere were of volume less than $\frac{1}{3} v_{N}$, the course of events would be the same except that none of the atmosphere would be thrown off until after the symmetry of revolution had been lost. In this case the sequence of figures would be-spheroids of small eccentricity, pseudo-spheroids, pseudo-ellipsoids, pseudo-ellipsoids with pointed ends and a stream of matter emerging from each, finally ending in detached masses surrounded by thin atmospheres.

\section{The Tidal Problem}

155. In the tidal problem $\omega$ vanishes but $V_{T}$ does not, so that equation (400) becomes

$$
\Omega=\frac{M}{r}+V_{T}
$$

where $M$, as before, is the mass of the primary, and its centre of gravity is taken as origin. 
Let us suppose that the tidal forces originate from a secondary mass $M^{\prime}$, which may be treated as a point at a distance $R$. If $r^{\prime}$ denotes distance from this mass, the whole tidal potential is $M^{\prime} / r^{\prime}$, but of this a part is effective only in producing the acceleration $M^{\prime} / R^{2}$ of the primary, and this part may be taken to be a potential $M^{\prime} x / R^{2}$, where the axis of $x$ is taken to be the line joining the two masses. This part of the potential of the second mass must be supposed neutralised by the acceleration of the axes to which the primary is referred, so that the effective tide-generating potential may be taken to be

$$
V_{T}=\frac{M^{\prime}}{r^{\prime}}-\frac{M^{\prime} x}{R^{2}}
$$

The value of $\Omega$ is now

$$
\Omega=\frac{M}{r}+\frac{M^{\prime}}{r^{\prime}}-\frac{M^{\prime} x}{R^{2}}
$$

and the boundary of the primary must be one of the surfaces $\Omega=$ constant.

On parts of the $x$-axis which lie between the two masses, we put $r=x$, $r^{\prime}=R-x$, and find

$$
\begin{gathered}
\Omega=\frac{M}{x}+\frac{M^{\prime}}{R-x}-\frac{M^{\prime} x}{R^{2}} \\
\frac{\partial \Omega}{\partial x}=-\frac{M}{x^{2}}+\frac{M^{\prime}}{(R-x)^{2}}-\frac{M^{\prime}}{R^{2}}
\end{gathered}
$$

It is easily found that $\partial \Omega / \partial x$ vanishes once and once only on this part of the $x$-axis. On parts of the $x$-axis which lie outside the two masses, between $x=0$ and $x=-\infty$, the first term in $\partial \Omega / \partial x$ must be taken to be $+M / x^{2}$, and it is easily found that in this range also $\partial \Omega / \partial x$ vanishes once and only once.

Each of the points at which $\partial \Omega / \partial x$ vanishes on the axis of $x$ is a point at which one of the equipotentials intersects itself, and so represents a possible transition from closed to open equipotentials. But it is readily shewn that, except in the limiting case in which $M^{\prime} / M$ is infinite, the equipotentials first open out at the intersection which lies between the two masses.

Thus the arrangement of equipotentials is as follows: For the highest values of $\Omega$ the equipotentials are spheres round the nucleus $M$. As $\Omega$ decreases these give place to elongated but still closed figures which persist until $\Omega$ reaches a critical value $\Omega_{1}$, which is the value at the point at which $\partial \Omega / \partial x$ first vanishes. After this the surfaces are open at the end towards the secondary until $\Omega$ reaches a second critical value $\Omega_{2}$, for which $\partial \Omega / \partial x$ vanishes on the negative axis of $x$. For still lower values of $\Omega$ the equipotentials are open at both ends. 
156. In illustration of this the equipotentials when $M^{\prime}=2 M$ are shewn in fig. 30. The values of $x$ for which $\partial \Omega / \partial x$ vanishes are found to be $x=.457 R$ and $x=-.85 R$, while the critical values of $\Omega$ are $\Omega_{1}=4.957 \mathrm{M} / R$ and $\Omega_{2}=3.96 \mathrm{M} / R$. The last entirely closed equipotential is the curve $\Omega=4.957 M / R$ which is drawn thick in the figure. From a rough quadrature it is found that the whole volume of this equipotential is equal to that of a sphere of radius $: 348 R$.

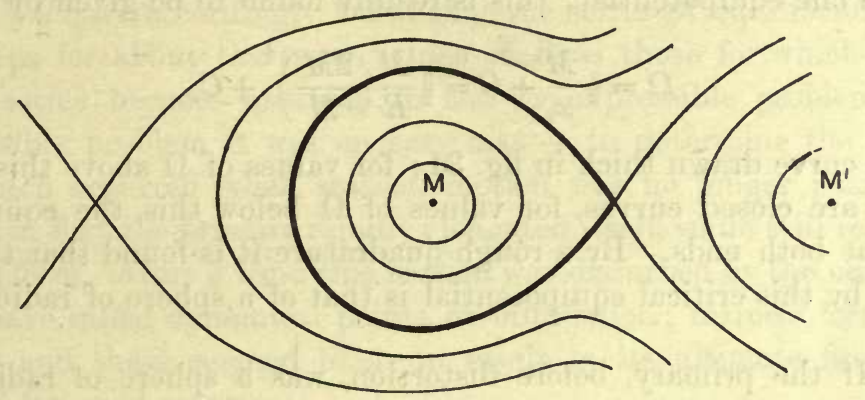

Fig. 30 .

157. As a second illustration, the equipotentials when $M^{\prime} / M$ is infinite are shewn in fig. 31. The value of $\Omega$ is now given by equation (406), in which $M^{\prime}$ and $R$ are both infinite. Thus

$$
\Omega=\frac{M}{r}+M^{\prime}\left[\frac{1}{R}+\frac{x}{R^{2}}+\frac{\left(x^{2}-\frac{1}{2} y^{2}-\frac{1}{2} z^{2}\right)}{R^{3}}+\ldots\right]-\frac{M^{\prime} x}{R^{2}},
$$

or, replacing the infinite constant $M^{\prime} / R$ by $C$,

$$
\Omega=\frac{M}{r}+\frac{M^{\prime}}{R^{3}}\left(x^{2}-\frac{1}{2} y^{2}-\frac{1}{2} z^{2}\right)+C .
$$

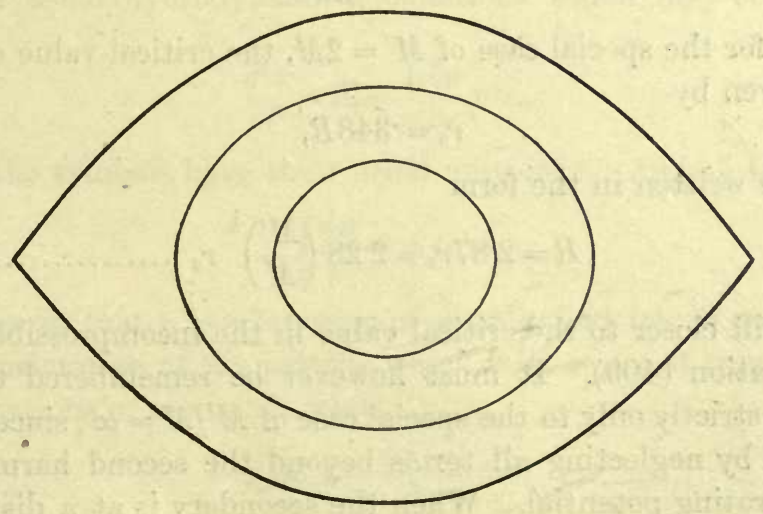

Fig. 31 . 
On the positive part of the $x$-axis,

$$
\Omega=\frac{M}{x}+\frac{M^{\prime}}{R^{3}} x^{2}+C,
$$

so that $\partial \Omega / \partial x$ vanishes for a value $x=x_{0}$, where $x_{0}=\left(\frac{M}{2 M^{\prime}}\right)^{\frac{1}{3}} R$. It is readily seen that $\partial \Omega / \partial x$ vanishes for an equal negative value of $x$. Thus in this special case of $M^{\prime} \mid M=\infty$ it appears that the two critical equipotentials coincide in one equipotential; this is readily found to be given by

$$
\Omega=\frac{3}{2} \frac{M}{x_{0}}+C=\frac{3}{2} \frac{M}{R}\left(\frac{2 M^{\prime}}{M}\right)^{\frac{1}{3}}+C,
$$

and is the curve drawn thick in fig. 31 ; for values of $\Omega$ above this the equipotentials are closed curves, for values of $\Omega$ below this, the equipotentials are open at both ends. By a rough quadrature it is found that the volume contained by this critical equipotential is that of a sphere of radius $72 x_{0}$.

158. If the primary, before distortion, was a sphere of radius $r_{0}$, the limit of statical stability, in the case $M^{\prime} \mid M=\infty$, will be reached when it is distorted to the shape of the thick curve in fig. 31. Thus it is reached when $M^{\prime}$ approaches to a distance $R$ such that

$$
r_{0}=\cdot 72 x_{0}=\cdot 72\left(\frac{M}{2 M^{\prime}}\right)^{\frac{1}{3}} R
$$

This critical value of $R$ may be put in the form

$$
R=1 \cdot 75\left(\frac{M^{\prime}}{M}\right)^{\frac{1}{3}} r_{0}
$$

This may be compared with the critical value of $R$ found in the incompressible problem (p. 46), namely

$$
R=2 \cdot 1984\left(\frac{M^{\prime}}{M}\right)^{\frac{1}{3}} r_{0}
$$

Similarly for the special case of $M^{\prime}=2 M$, the critical value of $R$ has been seen to be given by

$$
r_{0}=\cdot 348 R,
$$

which may be written in the form

$$
R=2 \cdot 87 r_{0}=2 \cdot 28\left(\frac{M^{\prime}}{M}\right)^{\frac{1}{3}} r_{0}
$$

This is still closer to the critical value in the incompressible problem, as given by equation (409). It must however be remembered that equation (409) applies strictly only to the special case of $M^{\prime} / M=\infty$, since the equation was obtained by neglecting all terms beyond the second harmonic term in the tide-generating potential. When the secondary is at a distance of only $2 \cdot 87 r_{0}$ away from the primary, the third harmonic terms may not legitimately 
be neglected and equation (409) requires to be modified accordingly. We can, however, see that the correction to this equation cannot be large, being of the order of 5 per cent. at most*, so that equation (409) may be regarded as giving a tolerably good approximation for all ratios of $M^{\prime}$ to $M$.

Thus both when $M^{\prime}=2 M$ and when $M^{\prime} / M=\infty$, it appears that heterogeneity, even of the very extreme kind now under consideration, has only a very slight influence upon the critical value of $R$.

159. We have accordingly found that the series of equilibrium configurations stops for about the same values of $R$ as those for which the corresponding series became unstable in the incompressible problem. In the incompressible problem it was an easy matter to determine the dynamical motion which occurred when statical motion was no longer possible. We found that at first the primary rapidly elongated itself, while still retaining its spheroidal form. After a time this motion was disturbed by the occurrence of what we have called dynamical points of bifurcation; furrows formed round the figure and these seemed likely to result in its ultimate fission into a number of detached masses.

In the compressible problem now under consideration the dynamical motion is, as we shall see, very similar to that just described. Consider first the simplest case in which $M^{\prime} / M=\infty$. In this case, as soon as the critical point is reached, the equipotential by which the mass is bounded opens symmetrically at both ends and matter is ejected. This matter will form two long symmetrical jets or arms and the elongation of these arms corresponds fairly closely to the elongation of the spheroid in the incompressible figure of equilibrium. We shall now see that, during this process of elongation, dynamical points of bifurcation will oecur, very much in the same way as in the incompressible problem.

160. The motion of the ejected streams of matter will of course be determined by the usual hydrodynamical equations which may be expressed in the form

$$
\frac{\partial^{2} x}{\partial t^{2}}=X-\frac{1}{\rho} \frac{\partial p}{\partial x} \text { etc. }
$$

in which all the symbols have their usual meanings. As in $\S 140$, let us put

$$
\int \frac{d p}{\rho}=\phi(\rho)
$$

thereby assuming that $p$ is a function of $\rho$ only, and let us denote the components of acceleration of the particle which is at $x, y, z$ at time $t$ by $f_{x}, f_{y}, f_{z}$. Then the equations of motion become

$$
f_{x}=X-\frac{\partial \phi(\rho)}{\partial x} \text { etc. }
$$

* Cf. Mem. R.d.S. 72 , pp. $10-14$. 
With a view to discovering dynamical points of bifurcation on the sequence of configurations determined by equations (411), let us compare this motion with a slightly varied motion in which the particle which in the original motion was at $x, y, z$ at time $t$ is, in the varied rnotion, at the point

at time $t$.

$$
x+\xi, y+\eta, z+\zeta
$$

In the varied motion the particle just specified will have components of acceleration

$$
f_{x}+\frac{\partial^{2} \xi}{\partial t^{2}} \text { etc. }
$$

so that the particle which is at $x, y, z$ at time $t$ in the varied motion will have components of acceleration

$$
f_{x}-\xi \frac{\partial f_{x}}{\partial x}-\eta \frac{\partial f_{x}}{\partial y}-\zeta \frac{\partial f_{x}}{\partial z}+\frac{\partial^{2} \xi}{\partial t^{2}} \text { etc. }
$$

Let the values of $X$ and $\rho$ at the point $x, y, z$ be changed, in the varied motion, to $X+\delta X$ and $\rho+\delta \rho$. Then the equations by which the varied motion is governed will be

$$
f_{x}-\xi \frac{\partial f_{x}}{\partial x}-\eta \frac{\partial f_{x}}{\partial y}-\zeta \frac{\partial f_{x}}{\partial z}+\frac{\partial^{2} \xi}{\partial t^{2}}=X+\delta X-\frac{\partial}{\partial x} \phi(\rho+\delta \rho)
$$

On subtracting corresponding sides of equations (411) and (412) we obtain

$$
\frac{\partial^{2} \xi}{\partial t^{2}}-\xi \frac{\partial f_{x}}{\partial x}-\eta \frac{\partial f_{x}}{\partial y}-\zeta \frac{\partial f_{x}}{\partial z}=\delta X-\frac{\partial}{\partial x}\left[\frac{\partial \phi}{\partial \rho} \delta \rho\right]
$$

which is an equation of motion for all small displacements which can be superposed on to the original motion while still conforming to the laws of dynamics. The original motion must be determined from the three equations (411). Equation (413) and its two companions will then determine the dynamical points of bifurcation on this motion.

Equations (411) cannot be solved in detail, so that an exact knowledge of the dynamical points of bifurcation determined by equation (413) cannot be obtained. But a knowledge of the general nature of the solution of equation (413) can be obtained from a consideration of the simple case in which $f_{x}, f_{y}$ and $f_{z}$ are all constants, so that the jet is supposed to move with uniform acceleration-e.g. as though moving under a uniform gravitational field.

Equation (413) now reduces to

$$
\frac{\partial^{2} \xi}{\partial t^{2}}=\delta X-\frac{\partial}{\partial x}\left[\frac{\partial \phi}{\partial \rho} \delta \rho\right]
$$

and there are two similar equations. Differentiating with respect to $x, y, z$ and adding, we obtain

$$
\frac{\partial^{2}}{\partial t^{2}}\left(\frac{\partial \xi}{\partial x}+\frac{\partial \eta}{\partial y}+\frac{\partial \zeta}{\partial z}\right)=\frac{\partial \delta X}{\partial x}+\frac{\partial \delta Y}{\partial y}+\frac{\partial \delta Z}{\partial z}-\nabla^{2}\left[\frac{\partial \phi}{\partial \rho} \delta \rho\right]
$$


Let $s$ denote $\delta \rho / \rho$, the excess condensation of the varied motion, so that the bracket on the left-hand side of the above equation is equal to $-s$. We have

$$
\frac{\partial \phi}{\partial \rho} \delta \rho=\frac{1}{\rho} \frac{\partial p}{\partial \rho} \delta \rho=\frac{\partial p}{\partial \rho} s .
$$

Using $\gamma$ temporarily to denote the gravitation constant, we have

so that

$$
\frac{\partial X}{\partial x}+\frac{\partial Y}{\partial y}+\frac{\partial Z}{\partial z}=-4 \pi \gamma \rho
$$

$$
\frac{\partial \delta X}{\partial x}+\frac{\partial \delta Y}{\partial y}+\frac{\partial \delta Z}{\partial z}=-4 \pi \gamma \rho s
$$

Equation (415) accordingly becomes

$$
\frac{\partial^{2} s}{\partial t^{2}}=4 \pi \gamma \rho s+\nabla^{2}\left(\frac{\partial p}{\partial \rho} s\right)
$$

and is now seen to be a differential equation determining the condensation $s$ in the varied motion.

Putting $\gamma=0$ in this equation, and thereby neglecting the effects of gravitation, we are left with the well-known equation

$$
\frac{\partial^{2} s}{\partial t^{2}}=\nabla^{2}\left(\frac{\partial p}{\partial \rho} s\right)
$$

which simply expresses that any excess condensation $s$ is propagated as a wave with a velocity $\sqrt{ }(\partial p / \partial \rho)$ relative to the moving jet. In this case any displacement from the original motion can only give rise to small oscillations about this motion, so that the motion is thoroughly stable.

Restoring $\gamma$ and assuming for simplicity that $\rho$ and $\partial p / \partial \rho$ are uniform throughout the jet, we find that a solution of the full equation (416) can be obtained by taking $s$ proportional to an exponential factor

$$
e^{i(q t-2 \pi x / \lambda)},
$$

this solution representing waves of wave-length $\lambda$ projected with a velocity $q \lambda / 2 \pi$. Certain boundary conditions must be satisfied in addition to the differential equation (416). These may be taken to be that $s$ shall vanish at the two ends of the jet, say at $x=0$ and $x=l$.

On substituting the exponential factor into the differential equation (416), we find that

$$
q^{2}=\left(\frac{2 \pi}{\lambda}\right)^{2} \frac{d p}{d \rho}-4 \pi \gamma \rho
$$

while the boundary conditions are satisfied if

$$
l=\frac{1}{2} n \lambda,
$$


where $n$ is any integer. Thus the different values of $q$ are given by

$$
q^{2}=n^{2}\left(\frac{\pi}{l}\right)^{2} \frac{d p}{d \rho}-4 \pi \gamma \rho
$$

The vibration of lowest frequency is given by $n=1$, and $q^{2}$ becomes negative when $l$ first reaches the value

$$
l=\frac{1}{2} \sqrt{\frac{\pi}{\gamma \rho} \frac{d p}{d \rho}}
$$

This fixes the first dynamical point of bifurcation; as $l$ increases more and more points of bifurcation occur, the complete set being given by

$$
l=\frac{1}{2} n \sqrt{\frac{\pi}{\gamma \rho} \frac{d p}{d \rho}} \quad(n=1,2,3 \ldots) \ldots(4,20) .
$$

Thus as $l$ increases, one vibration after another loses its stability. The initial unstable motion of any vibration is one in which the matter of the jet tends to collect into nuclei or bunches at the nodes of the wave. Or, alternatively, we may consider that a series of furrows tends to form in the jet, and that these get continually deeper. After passing the first point of bifurcation one furrow tends to form, namely a furrow between the jet and the main body; after passing the next point of bifurcation two furrows begin to form, and so on.

161. Clearly the formation of $1,2,3 \ldots$ furrows in succession in this problem is very closely analogous to the formation of $1,2,3 \ldots$ furrows in succession which occurs when the incompressible mass passes points of bifurcation corresponding to harmonics of orders $3,4,5 \ldots$

Although the formation of furrows in these two problems is closely analogous, it would be a mistake to suppose that the two solutions we have obtained merge gradually into one another as the compressibility of the primary mass gradually changes. We may notice that the breaking up of an incompressible mass takes place independently of its size, whereas the breaking up of the jet of matter formed from the atmosphere in Roche's model will only take place when the system is beyond a certain size.

162. Further insight into the motion will be obtained from a consideration of the composite model already discussed in $\$ 154$. We suppose the primary to consist of an incompressible nucleus of volume $v_{N}$ and density $\rho_{0}$, surrounded by an atmosphere of volume $v_{\mathcal{A}}$ and negligible density. Under tidal forces the configuration of the nucleus will be exactly the same as if the atmosphere were non-existent, while the atmosphere will be bounded by one of the equipotentials surrounding this nucleus.

It will be sufficient to consider the simplest case in which $M^{\prime} / M$ is infinite. In this case the total gravitational potential is

$$
\Omega=V_{M}+\mu\left(x^{2}-\frac{1}{2} y^{2}-\frac{1}{2} z^{2}\right),
$$


where $V_{M}$ is the potential of the spheroidal primary, and $\mu$ stands as usual for $M^{\prime} / R^{3}$. The point of equilibrium on the $x$-axis is determined by $\partial \Omega / \partial x=0$ or (cf. $§ 154)$

$$
2 \pi \rho_{0} a b c x \int_{x^{2}-a^{2}}^{\infty} \frac{d \lambda}{\left(a^{2}+\lambda\right)^{\frac{3}{2}}\left(c^{2}+\lambda\right)}=2 \mu x .
$$

The integral can be evaluated in finite terms, so that the equation becomes

where $\alpha^{2}=a^{2}-c^{2}$.

$$
\frac{\pi \rho_{0} a b c}{\alpha^{3}}\left[\log \frac{x+\alpha}{x-\alpha}-\frac{2 \alpha}{x}\right]=\mu
$$

In the special case in which the nucleus of the primary is on the verge of instability, the value of $\mu$ is $0 \cdot 125504 \pi \rho_{0}$, while

$$
\alpha=\left(a^{2}-c^{2}\right)^{\frac{1}{2}}=1 \cdot 45970(a b c)^{\frac{1}{3}} .
$$

Thus equation (421) reduces to

$$
\log \frac{x+\alpha}{x-\alpha}-\frac{2 \alpha}{x}=390343
$$

of which a root is readily found to be

$$
x=1.37578 \alpha=2.00822 r_{0} \text {. }
$$

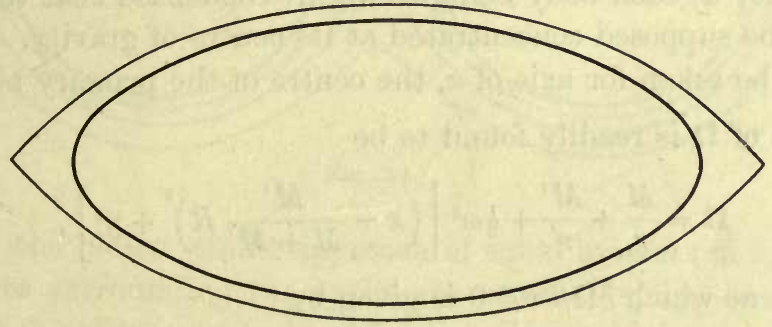

Fig. 32.

The corresponding figure of equilibrium is shewn in fig. 32. The thick curve is the boundary of the nucleus, and the thin outer curve that of the greatest atmosphere which can be retained by this nucleus. The volume $v_{A}$ of this atmosphere is only about a tenth of $v_{N}$ the volume of the nucleus. Thus

$$
\bar{\rho}=\frac{10}{1} \frac{0}{1} \rho_{0}, \text { and } R=1 \cdot 70\left(\frac{M^{\prime}}{M}\right)^{\frac{1}{3}} r_{0} .
$$

Hence we arrive at the following conception of the series of configurations of this model. At first, when the tidal forces are inappreciable, the figure of equilibrium is spherical, this giving place to a spheroidal figure where the tidal forces become appreciable but small. As the tidal forces increase, the boundary of the nucleus remains spheroidal, but that of the atmosphere is a pseudo-spheroid. If the volume of the atmosphere is greater than about a tenth of that of the nucleus, this pseudo-spheroid develops two conical pointed ends at the extremities of its major-axis, and a further increase in the tidal forces results in matter streaming out from these two ends. (This is in the 
special case of $M^{\prime} / M=\infty$; in the more natural but less simple case in which $M^{\prime} / M$ is finite, the boundary of the atmosphere will be a distorted pseudospheroid; a conical point will develop at one end only, and matter will stream out only from this end, which is the end nearest to the tide-generating mass.) Of the matter which streams out, some will fall into the tide-generating mass, and some will fall back on to the primary. The general effect may be thought of as the creation of an outer atmosphere in which the subsequent motion will take place. With a still further increase of tidal forces, the nucleus will attain the critical shape shewn in fig. 32, the retained atmosphere now being reduced to about a tenth of $v_{N}$. After this the motion both in the nucleus and the atmosphere will be dynamical ; the motion of the nucleus will be the same as that already considered in $\$ \$ 118-126$ except in so far as this may be altered by the presence of a resisting outer atmosphere.

\section{The Double-Star Problem}

163. To form a double-star problem on Roche's model, suppose we have two masses $M, M^{\prime}$ rotating in steady motion at a distance $R$ apart with angular velocity $\omega$, each body being so highly condensed that the whole mass of each may be supposed concentrated at its centre of gravity. Let the line joining them be taken for axis of $x$, the centre of the primary being origin.

The value of $\Omega$ is readily found to be

$$
\Omega=\frac{M}{r}+\frac{M^{\prime}}{r^{\prime}}+\frac{1}{2} \omega^{2}\left[\left(x-\frac{M^{\prime}}{M+M^{\prime}} R\right)^{2}+y^{2}\right],
$$

and the point at which $\partial \Omega / \partial x=0$ is given by

$$
-\frac{M}{x^{2}}+\frac{M^{\prime}}{(R-x)^{2}}+\omega^{2}\left(x-\frac{M^{\prime}}{M+M^{\prime}} R\right)=0
$$

The value of $\omega$ is given by the usual relation $\omega^{2} R^{3}=\left(M+M^{\prime}\right)$, whence it, appears that equation (422) can be expressed in the symmetrical form

$$
M\left(\frac{R^{2}}{x^{2}}-\frac{x}{R}\right)=M^{\prime}\left(\frac{R^{2}}{y^{2}}-\frac{y}{R}\right),
$$

where $y=R-x$. The graphs of the two similar functions in brackets are of the shape shewn in fig. 33, whence it appears that the root of the equation is $x=O P$ where $P$ is so chosen that

$$
M \times P S=M^{\prime} \times P S^{\prime} .
$$

There is therefore one and only one root of equation (422), and the critical equipotential intersects the axis of $x$ at a point distant $O P$ from

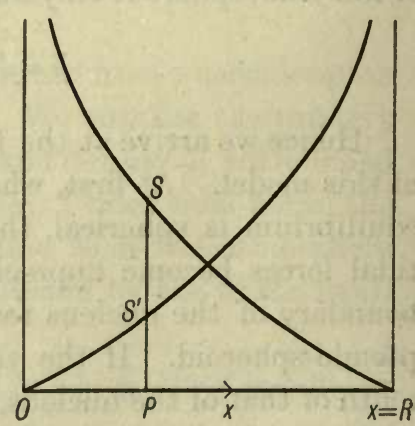

Fig. 33. the origin. If the volume of either component of the double star is greater 
than that of the corresponding branch of this equipotential, dynamical motion must have occurred before the components approach to within a distance $R$ of one another, and this motion will consist of matter streaming out of the conical end of the critical equipotential.

164. Other features of interest in the double-star problem are revealed by a study of the composite model in which the nuclei are homogeneous masses of finite size. The different forms which the two nuclei can assume are precisely those which appear in the double-star problem of Darwin already discussed in Chapter III ( $\$ 60-65)$. The boundaries of these masses are equipotential surfaces, and are surrounded by other equipotentials, any closed one of which may form the boundary of a possible atmosphere.

For instance in fig. 34, the thick curves form the boundaries determined

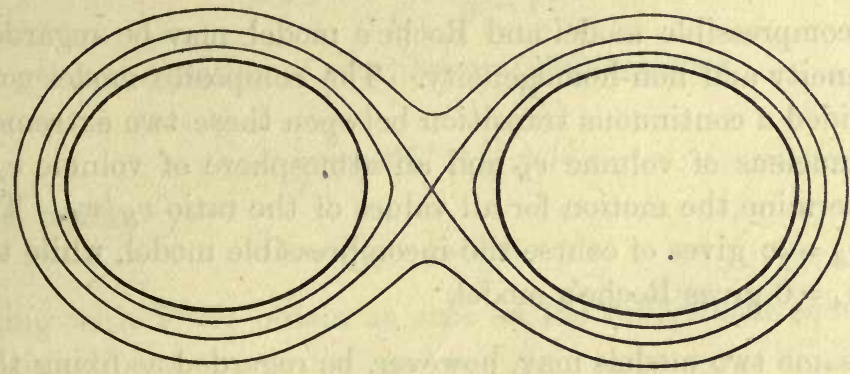

Fig. 34 .

by Darwin for the closest stable approach of equal masses (cf. fig. 13, p. 64). The thin curves surrounding the nuclei are external equipotentials, and the atmospheres of the stars may be bounded by any one of these.

Darwin's figures were at the closest distance which was consistent with stability for homogeneous masses, but it is at once apparent that the boundaries of the atmospheres may be closer than this. They may be in actual contact without stability being violated, or the two atmospheres inay be merged into a single atmosphere which will now be bounded by a single closed equipotential surrounding boch stars. Thus our investigation suggests that heterogeneity will in general lessen the distance of closest approach found by Darwin for the incompressible mass.

165. The models just considered may be regarded as marking the limit of non-homogeneity in one direction, the limit in the other direction being provided by the perfectly homogeneous model studied in Chapters III to VI.

In both the tidal and double-star problems, the motion of the nonhomogeneous models has been found to be very similar in its broad outlines to that already discovered for the perfectly homogeneous model. In each problem we found in both models a single series of configurations of 
equilibrium of approximately spheroidal or ellipsoidal shape, these remaining stable until the two masses concerned reached a certain critical distance from one another, after which dynamical motion was found to occur. And the general features of this dynamical motion were broadly the same for each model in both of the two problems.

In the rotational problem the situation is very different. So long as the rotation is slow the figures of equilibrium for every model are necessarily spheroidal in shape, but for more rapid rotations the shapes of the figures of equilibrium have been found to vary greatly. In the incompressible model, we found a sequence of figures, spheroidal, ellipsoidal, pear-shaped, ending with fission into two detached masses. In Roche's model, on the other hand, we found a pseudo-spheroidal series which ended abruptly by matter being thrown off from the equator.

The incompressible model and Roche's model, may be regarded as limits of homogeneity and non-homogeneity. The composite model considered in $\$ 154$ provided a continuous transition between these two extremes. In this we had a nucleus of volume $v_{N}$ and an atmosphere of volume $v_{A}$, and were able to determine the motion for all values of the ratio $v_{N} / v_{A}$. The limiting value $v_{N} / v_{A}=\infty$ gives of course the incompressible model, while the limiting value $v_{N} / v_{A}=0$ gives Roche's model.

These same two models may, however, be regarded as fixing the limits of compressibility and non-compressibility, and when they are regarded in this light the composite model does not provide a gradual transition from one to the other. A convenient sequence of figures of varying compressibility is provided by masses obeying the pressure-density law

$$
p=k \rho^{\gamma}+p_{0}
$$

where $\gamma$ varies from one mass to another. The value $\gamma=\infty$ provides a completely incompressible mass, while the value $\gamma=1 \frac{1}{5}$ provides, as we have already seen ( $(149)$, a model in which the mass is entirely concentrated at the centre, as in Roche's model.

Thus a general study of figures obeying the law (423) for values of $\gamma$ from $1 \frac{1}{5}$ to $\infty$ will provide a continuous transition from Roche's model to the incompressible model, through a series of figures of continually varying compressibility. To such a study we now proceed, limiting ourselves, for reasons already explained, to the rotational problem. 


\section{THE CONFIGURATIONS OF ROTATING COMPRESSIBLE MASSES}

166. It will be convenient to write the pressure-density law (423) in the form

$$
p=\frac{c}{\gamma} \rho^{\gamma}+p_{0}
$$

We may notice that this law includes as special cases Laplace's law $\left[p=\frac{1}{2} c\left(\rho^{2}-\sigma^{2}\right)\right]$ and also the law obeyed by a gas in adiabatic or convective equilibrium $\left[p_{0}=0\right]$.

We find at once that $\phi(\rho)$, defined by equation (395), is given by

$$
\phi(\rho)=\int \frac{d p}{\rho}=\frac{c}{\gamma-1} \rho^{\gamma-1},
$$

so that the general equation of equilibrium (396) becomes

in which, as before,

$$
\frac{c}{\gamma-1} \rho^{\gamma-1}=\Omega+C
$$

$$
\Omega=V_{M}+\frac{1}{2} \omega^{2}\left(x^{2}+y^{2}\right)
$$

Operating with $\nabla^{2}$ we obtain at once as the differential equation which must be satisfied by $\rho$,

$$
\frac{c}{\gamma-1} \nabla^{2} \rho^{\gamma-1}=-4 \pi \rho+2 \omega^{2}
$$

Taking the point of maximum density $\rho_{0}$ as origin, it will be possible to expand $\rho$ in the form

$$
\rho=\rho_{0}-\rho_{2}-\rho_{3}-\rho_{4}-\ldots
$$

where $\rho_{2}, \rho_{3}, \rho_{4} \ldots$ are functions of $x, y, z$, of degrees $2,3,4 \ldots$ respectively. The value of $\rho_{2}$ is

$$
\rho_{2}=-\frac{1}{2}\left(x^{2} \frac{\partial^{2} \rho}{\partial x^{2}}+2 x y \frac{\partial^{2} \rho}{\partial x \partial y}+\ldots\right),
$$

the differential coefficients being evaluated at the origin. Since the origin is supposed to be the point of maximum density, $\rho_{2}$ must be negative for all values of $x, y$ and $z$. Changing axes, it must be possible to put $\rho_{2}$ in the form

$$
\rho_{2}=-\left(\rho_{0}-\sigma\right)\left(\frac{x^{2}}{a^{2}}+\frac{y^{2}}{b^{2}}+\frac{z^{2}}{c^{2}}\right)
$$

where $\sigma$ denotes the density at the boundary of the mass.

If we further put

$$
\rho_{3}+\rho_{4}+\ldots=-e\left(\rho_{0}-\sigma\right) P_{0}
$$


then the general value of the density given by equation (426) becomes

$$
\rho=\rho_{0}-\left(\rho_{0}-\sigma\right)\left(\frac{x^{2}}{a^{2}}+\frac{y^{2}}{b^{2}}+\frac{z^{2}}{c^{2}}+e P_{0}\right)
$$

and the boundary, which is defined by the condition $\rho=\sigma$ has for its equation

$$
\frac{x^{2}}{a^{2}}+\frac{y^{2}}{b^{2}}+\frac{z^{2}}{c^{2}}-1+e P_{0}=0
$$

If $e P_{0}$ is small, this represents a distorted ellipsoid. Now for a perfectly incompressible mass, the boundaries of all stable configurations have been seen to be spheroids and ellipsoids, and so are all included in equation (428) with $P_{0}=0$. Moreover the general argument of $\$ \$ 142-144$ has shewn that the stable configurations of compressible masses can be derived from these spheroidal or ellipsoidal configurations by continuous distortion. Thus it appears that the boundaries of compressible rotating masses may be supposed given by an equation of the form of $(428)$; in this equation $e P_{0}$ will be small if the matter is only slightly compressible, but may become comparable with the other terms of the equation for highly compressible matter.

A preliminary problem must accordingly be the determination of the potential of a mass whose boundary is determined by equation (428), while the density at any point $x, y, z$ in its interior is given by equation (427).

\section{The potential of a non-homogeneous distorted ellipsoid}

167. Let $q$ be a function of the density $\rho$, defined by

$$
q^{2}=\frac{\rho_{0}-\rho}{\rho_{0}-\sigma}
$$

As we pass from the centre to the boundary, $\rho$ will vary continuously from $\rho_{0}$ to $\sigma$, so that $q$ will vary continuously from 0 to 1 . The surface of constant density $\rho$ has for its equation

$$
\frac{x^{2}}{a^{2}}+\frac{y^{2}}{b^{2}}+\frac{z^{2}}{c^{2}}+e P_{0}=q^{2}
$$

and this may be regarded as arrived at by distortion from an ellipsoid of semi-axes $q a, q b, q c$. Equation (428) is a special case of (430), arrived at by taking $q=1$.

In Chapter IV we found how to write down the potential of a distorted ellipsoid such as that determined by equation (4.30), the density being supposed uniform. Let the potential of a homogeneous mass of unit density bounded by the surface $(4: 30)$ be denoted by $V_{0}(q)$ when evaluated at a point outside the surface, and by $V_{i}(q)$ when evaluated at a point inside the surface. 
Then it is readily seen that the potential of the whole heterogeneous mass whose density is given by equation (427), and whose boundary is given by $\rho=\sigma$, will be

$$
\begin{aligned}
& V_{0}=\sigma V_{0}(1)+\int_{\sigma}^{\rho_{0}} V_{0}(q) d \rho \\
& V_{i}=\sigma V_{i}(1)+\int_{\sigma}^{\rho^{\prime}} V_{i}(q) d \rho+\int_{\rho^{\prime}}^{\rho_{0}} V_{0}(q) d \rho
\end{aligned}
$$

the first formula giving the potential at a point outside the mass, and the second formula giving the potential at an internal point at which the density is $\rho^{\prime}$.

168. As in $\S 77$, let us suppose that $P_{0}$ is put in the form

$$
P_{0}=F\left(\frac{x}{a^{2}}, \frac{y}{b^{2}}, \frac{z}{c^{2}}\right) \text {. }
$$

Introduce new coordinates $\xi^{\prime}, \eta^{\prime}, \zeta^{\prime}$ such that

and let

$$
\xi^{\prime}=\frac{x}{q^{2} a^{2}+\mu} \text { etc. }
$$

$$
P=F\left(\frac{q^{2} x}{q^{2} a^{2}+\mu}, \frac{q^{2} y}{q^{2} b^{2}+\mu}, \frac{q^{2} z}{q^{2} c^{2}+\mu}\right)=F\left(q^{2} \xi^{\prime}, q^{2} \eta^{\prime}, q^{2} \xi^{\prime}\right)
$$

so that $P$ reduces to $P_{0}$ when $\mu=0$.

Suppose further that $f$ and $D$ are given by

$$
\begin{aligned}
f=\frac{x^{2}}{q^{2} a^{2}+\mu} & +\frac{y^{2}}{q^{2} b^{2}+\mu}+\frac{z^{2}}{q^{2} c^{2}+\mu}-1 \\
& =\left(q^{2} a^{2}+\mu\right) \xi^{\prime 2}+\left(q^{2} b^{2}+\mu\right) \eta^{\prime 2}+\left(q^{2} c^{2}+\mu\right) \zeta^{2}-1
\end{aligned}
$$

$D=\left(\frac{1}{q^{2} a^{2}}-\frac{1}{q^{2} a^{2}+\mu}\right) \frac{\partial^{2}}{\partial \xi^{\prime 2}}+\left(\frac{1}{q^{2} b^{2}}-\frac{1}{q^{2} b^{2}+\mu}\right) \frac{\partial^{2}}{\partial \eta^{\prime 2}}+\left(\frac{1}{q^{2} c^{2}}-\frac{1}{q^{2} c^{2}+\mu}\right) \frac{\partial^{2}}{\partial \zeta^{\prime 2}}$

Let $\phi(q)$ be given by our former equation (200), namely

$$
\begin{aligned}
\phi(q)= & e\left[P-\frac{1}{4} f D P+\frac{1}{2^{2}}\left(\frac{1}{4} f\right)^{2} D^{2} P-\frac{1}{2^{2} .3^{2}}\left(\frac{1}{4} f\right)^{3} D^{3} P-\ldots\right] \\
& -\frac{1}{8} e^{2}\left[D P^{2}-\frac{1}{8} f D^{2} P^{2}+\frac{1}{92} f^{2} D^{3} P^{2}-\frac{1}{9216} f^{3} D^{4} P^{2}+\ldots\right] \\
& +\frac{1}{192} e^{3}\left[D^{2} P^{3}-\frac{1}{2} f D^{3} P^{3}+\frac{1}{384} f^{2} D^{4} P^{3}-\ldots\right] \text { etc. } \ldots \ldots . .
\end{aligned}
$$

in which $f$ and $D$ are now supposed defined by equations (433) and (434). When $\mu=0$ the equation $f=0$ represents an ellipsoid of semi-axes $q \alpha$, $q b, q c$. Moreover, when $\mu=0, D$ reduces to zero and $P$ to $P_{0}$, so that $\phi(q)$ reduces to $e P_{0}$, and

$$
f+\frac{\phi(q)}{q^{2}}=\frac{1}{q^{2}}\left(\frac{x^{2}}{a^{2}}+\frac{y^{2}}{b^{2}}+\frac{z^{2}}{c^{2}}+e P_{0}-q^{2}\right) .
$$


Thus when $\mu=0$, the equation

$$
f+\frac{\phi(q)}{q^{2}}=0
$$

becomes identical with equation (430), which is the equation of the surface of constant density $\rho$, where $\rho$ is connected with $q$ by equation (429).

The value of $V_{0}(q)$, the external potential of a uniform mass of unit density filling this surface of constant density $\rho$, is at once seen, by the methods of Chapter IV, to be

$$
V_{0}(q)=-\pi \int_{\mu^{\prime}}^{\infty}\left(f+\frac{\phi(q)}{q^{2}}\right) \frac{q^{3} a b c d \mu}{\left[\left(q^{2} a^{2}+\mu\right)\left(q^{2} b^{2}+\mu\right)\left(q^{2} c^{2}+\mu\right)\right]^{\frac{1}{2}}} \ldots
$$

where the lower limit of integration $\mu^{\prime}$ is the root of equation (436) at the external point $x, y, z$ at which the potential is being evaluated. The internal potential is given by precisely the same formula (437) with $\mu^{\prime}$ put equal to zero.

169. The formulae for the potential may be simplified by introducing a new variable $\lambda$ equal to $\mu / q^{2}$. If we further put

we find that

$$
\xi=q^{2} \xi^{\prime}=\frac{x}{a^{2}+\lambda} \text { etc., }
$$

and

$$
\begin{array}{r}
P=F(\xi, \eta, \zeta) \ldots \ldots \ldots \ldots \\
q^{2} f=\frac{x^{2}}{a^{2}+\lambda}+\frac{y^{2}}{b^{2}+\lambda}+\frac{z^{2}}{c^{2}+\lambda}-q^{2} \\
\frac{D}{q^{2}}=\left(\frac{1}{a^{2}}-\frac{1}{a^{2}+\lambda}\right) \frac{\partial^{2}}{\partial \xi^{2}}+\ldots \ldots \ldots
\end{array}
$$

$$
V_{0}(q)=-\pi a b c \int_{\lambda^{\prime}}^{\infty}\left[q^{2} f+\phi(q)\right] \frac{d \lambda}{\Delta}
$$

where $\Delta$ has its usual meaning $\left[\left(a^{2}+\lambda\right)\left(b^{2}+\lambda\right)\left(c^{2}+\lambda\right)\right]^{\frac{1}{2}}$, and the lower limit $\lambda^{\prime}$ is now a root of

$$
\frac{x^{2}}{a^{2}+\lambda}+\frac{y^{2}}{b^{2}+\lambda}+\frac{z^{2}}{c^{2}+\lambda}+\phi(q)=q^{2}
$$

The same formula (441), with the lower limit put equal to zero, will give the value of the internal potential $V_{i}(q)$.

170. Having evaluated $V_{0}(q)$ and $V_{i}(q)$, we are in a position to attack equations (431) and (432). Only the second of these equations is of immediate importance to our problem. 
Using relation (429) we find

$$
V_{i}=\sigma V_{i}(1)+\left(\rho_{0}-\sigma\right)\left[\int_{0}^{q^{\prime}} V_{0}(q) d q^{2}+\int_{q^{\prime}}^{1} V_{i}(q) d q^{2}\right]
$$

where $q^{\prime}$ is the value of $q$ at the point $x, y, z$ at which the potential is being evaluated. The sum of the two integrals in square brackets is found, on integrating by parts, to become

$$
\left|q^{2} V_{i}(q)\right|_{q^{\prime}}^{1}+\left|q^{2} V_{0}(q)\right|_{0}^{q^{\prime}}-\int_{0}^{q^{\prime}} q^{2} \frac{d V_{0}(q)}{d q^{2}} d q^{2}-\int_{q^{\prime}}^{1} q^{2} \frac{d V_{i}(q)}{d q^{2}} d q^{2}
$$

Since $V_{i}\left(q^{\prime}\right)=V_{0}\left(q^{\prime}\right)$, the sum of the first two terms in this expression reduces at once to $V_{i}(1)$, so that equation (443) can be put in the form

where

$$
V_{i}=\rho_{0} V_{i}(1)-\left(\rho_{0}-\sigma\right) E
$$

$$
E=\int_{0}^{q^{\prime}} q^{2} \frac{d V_{0}(q)}{d q^{2}} d q^{2}+\int_{q^{\prime}}^{1} q^{2} \frac{d V_{i}(q)}{d q^{2}} d q^{2}
$$

The first term on the right hand of equation (444) is the internal potential of a homogeneous solid of uniform density $\rho_{0}$; the second term accordingly represents the effect of the falling off of density from $\rho_{0}$ at the centre to $\sigma$ at the boundary.

The value of $V_{0}(q)$, as given by equation (441) is a function of $q$ and also of $\lambda^{\prime}$, which is connected with $q$ by equation (442). Thus

$$
\frac{d V_{0}(q)}{d q^{2}}=\frac{\partial V_{0}(q)}{\partial q^{2}}+\frac{\partial V_{0}(q)}{\partial \lambda^{\prime}} \frac{\partial \lambda^{\prime}}{\partial q^{2}}
$$

In this equation we have

$$
\frac{\partial V_{0}(q)}{\partial \lambda^{\prime}}=\pi a b c\left[\frac{q^{2} f+\phi(q)}{\Delta}\right]_{\lambda=\lambda^{\prime}}
$$

and this vanishes from the definition of $\lambda^{\prime}$ (equation (442)). We accordingly have, from equations (441) and (439),

$$
\frac{d V_{0}(q)}{d q^{2}}=\frac{\partial V_{0}(q)}{\partial q^{2}}=\pi a b c \int_{\lambda^{\prime}}^{\infty}\left(1-\frac{\partial \phi(q)}{\partial q^{2}}\right) \frac{d \lambda}{\Delta},
$$

while similarly by direct differentiation,

$$
\frac{d V_{i}(q)}{d q^{2}}=\pi a b c \int_{0}^{\infty}\left(1-\frac{\partial \phi(q)}{\partial q^{2}}\right) \frac{d \lambda}{\Delta} .
$$

Thus the value of $E$ given by equation (445) becomes

$$
E=\pi a b c\left\{\int_{0}^{q^{\prime}} q^{2}\left[\int_{\lambda^{\prime}}^{\infty}\left(1-\frac{\partial \phi(q)}{\partial q^{2}}\right) \frac{d \lambda}{\Delta}\right] d q^{2}+\int_{q^{\prime}}^{1} q^{2}\left[\int_{0}^{\infty}\left(1-\frac{\partial \phi(q)}{\partial q^{2}}\right) \frac{d \lambda}{\Delta}\right] d q^{2}\right.
$$


This value of $E$ may be regarded as being obtained by a double integration with respect to $q^{2}$ and $\lambda$. In fig. 35 , let $O A, O B$ represent axes of $q$ and $\lambda$ respectively, and let the thick curve $P Q$ represent the relation between $q$ and $\lambda$ expressed by equation (442). This curve meets the axis of $q$ at the value $q=q^{\prime}$, for by the definition of $q^{\prime}$, we have

$$
\frac{x^{2}}{a^{2}+\lambda}+\frac{y^{2}}{b^{2}+\lambda}+\frac{z^{2}}{c^{2}+\lambda}+\phi\left(q^{\prime}\right)=q^{\prime 2}
$$

where $\lambda=0$. It clearly meets $q=0\left(\rho=\rho_{0}\right)$ at $\lambda=\infty$.

Thus it appears that the first integral in the value of $E$ is represented by an integration over the area $B Q R$ while the second

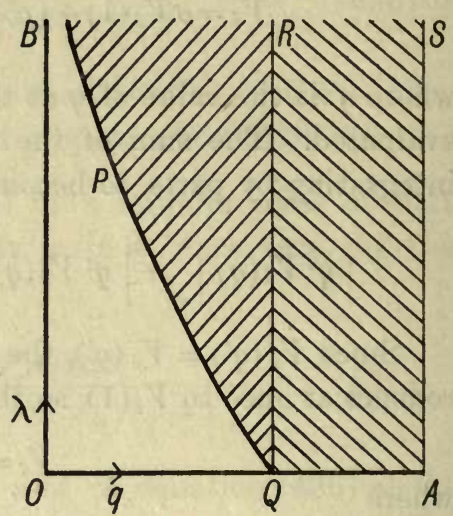

Fig. 35. integral is represented by integration over the area $R Q A S$. Thus the whole integration is over the area which is shaded in the figure, and on changing the order of integration we find

$$
E=\pi a b c\left\{\int_{0}^{\infty}\left[\int_{q}^{1} q^{2}\left(1-\frac{\partial \phi(q)}{\partial q^{2}}\right) d q^{2}\right]\right\} \frac{d \lambda}{\Delta}
$$

where the lower limit $q$ is now determined as a function of $\lambda$ by equation $(442)$.

This completes the evaluation of $V_{i}$. The external potential can be evaluated in a similar way*, but is not required in the present problem.

\section{Configurations of Equilibrium}

171. We may now turn to the conditions of equilibrium, which as we have seen ( $\$ 166)$, are expressed by the single equation (424), namely

$$
\frac{c}{\gamma-1} \rho^{\gamma-1}=\Omega+c
$$

In this equation $\rho$ has the value

$$
\rho=\rho_{0}-\left(\rho_{0}-\sigma\right)\left(\frac{x^{2}}{a^{2}}+\frac{y^{2}}{b^{2}}+\frac{z^{2}}{c^{2}}+e P_{0}\right)
$$

Expanding $\rho^{\gamma-1}$ by the binomial theorem, we find that equation (448) assumes the form

$$
\begin{gathered}
\frac{c}{\gamma-1} \rho_{0}^{\gamma-1}\left[1-\frac{(\gamma-1)\left(\rho_{0}-\sigma\right)}{\rho_{0}}\left(\Sigma \frac{x^{2}}{a^{2}}+e P_{0}\right)+\frac{(\gamma-1)(\gamma-2)\left(\rho_{0}-\sigma\right)^{2}}{2 \rho_{0}^{2}}\left(\Sigma \frac{x^{2}}{a^{2}}+e P_{0}\right)^{2}\right. \\
\left.-\frac{(\gamma-1)(\gamma-2)(\gamma-3)\left(\rho_{0}-\sigma\right)^{3}}{6 \rho_{0}^{3}}\left(\Sigma \frac{x^{2}}{a^{2}}+e P_{0}\right)^{3}+\ldots\right]=\Omega+C \ldots(450),
\end{gathered}
$$

\footnotetext{
* See Bakerian Lecture for 1917. Royal Society Phil. Trans. A (not yet published).
} 
in which of course

$$
\begin{aligned}
\Omega & =V_{i}+\frac{1}{2} \omega^{2}\left(x^{2}+y^{2}\right) \\
& =\rho_{0} V_{i}(1)-\left(\rho_{0}-\sigma\right) E+\frac{1}{2} \omega^{2}\left(x^{2}+y^{2}\right)
\end{aligned}
$$

Equation (450) contains the solution for all compressible masses, and so must include the solution for the incompressible problem in which $\rho_{0}-\sigma$ vanishes.

In this solution of the incompressible problem, the figure is known to be ellipsoidal, so that $P_{0}=0$, while $V_{i}(1)$ becomes the potential of a homogeneous ellipsoid of unit density, and so is given by

$$
V_{i}(1)=-\pi a b c\left(J_{A} x^{2}+J_{B} y^{2}+J_{C} z^{2}-J\right)
$$

Thus, omitting all terms which disappear when the mass is incompressible, equation (450) reduces to

$$
\begin{aligned}
\frac{c}{\gamma-1} \rho_{0}^{\gamma-1} & {\left[1-\frac{(\gamma-1)\left(\rho_{0}-\sigma\right)}{\rho_{0}}\left(\Sigma \frac{x^{2}}{a^{2}}\right)\right] } \\
= & -\pi \rho_{0} a b c\left(J_{A} x^{2}+J_{B} y^{2}+J_{C} z^{2}-J\right)+\frac{1}{2} \omega^{2}\left(x^{2}+y^{2}\right)+C \ldots(453) .
\end{aligned}
$$

The term in $c\left(\rho_{0}-\sigma\right)$ on the left-hand has been retained because it is obvious that the equation can only be satisfied by supposing $c\left(\rho_{0}-\sigma\right)$ to remain finite when $\left(\rho_{0}-\sigma\right)$ vanishes. We know that in any case $c$ must become infinite when the mass is incompressible, for the value of $\partial p / \partial \rho$ then is infinite.

In the general problem, let us put

$$
\theta=\frac{c \rho_{0}^{\gamma-3}\left(\rho_{0}-\sigma\right)}{\pi a b c}
$$

this equation defining $\theta$. Then equation (453) becomes

$$
\theta \Sigma \frac{x^{2}}{a^{2}}=J_{A} x^{2}+J_{B} y^{2}+J_{C} z^{2}-\frac{\omega^{2}}{2 \pi \rho_{0} a b c}\left(x^{2}+y^{2}\right)
$$

and on equating coefficients of $x^{2}, y^{2}$ and $z^{2}$, we find

$$
\left.\begin{array}{l}
J_{A}-\frac{\omega^{2}}{2 \pi \rho_{0} a b c}=\frac{\theta}{a^{2}} \\
J_{B}-\frac{\omega^{2}}{2 \pi \rho_{0} a b c}=\frac{\theta}{b^{2}} \\
J_{C}=\frac{\theta}{c^{2}}
\end{array}\right\}
$$

These are the conditions that an ellipsoid of semi-axes $a, b, c$ shall be a figure of equilibrium for a mass of uniform density $\rho_{0}$ rotating with angular velocity $\omega$. It is at once seen that they are identical with the three equations $(65)-(67)$ which were found to determine the solution of this problem in $\S 36$. 
Thus if we assign to $\theta$ the value given by equation (4.54), it is clear that our general equation (450) will reduce, when the mass is incompressible, to the equation from which the solution for the incompressible problem was previously obtained. With this meaning for $\theta$, the general equation (450) becomes

$$
\begin{aligned}
-\theta & {\left[\left(\Sigma \frac{x^{2}}{a^{2}}+e P_{0}\right)-\frac{1}{2}(\gamma-2)\left(\frac{\rho_{0}-\sigma}{\rho_{0}}\right)\left(\Sigma \frac{x^{2}}{a^{2}}+e P_{0}\right)^{2}\right.} \\
& \left.+\frac{1}{6}(\gamma-2)(\gamma-3)\left(\frac{\rho_{0}-\sigma}{\rho_{0}}\right)^{2}\left(\Sigma \frac{x^{2}}{a^{2}}+e P_{0}\right)^{3}+\ldots\right] \\
& =\frac{1}{\pi a b c}\left[V_{i}(1)-\left(\frac{\rho_{0}-\sigma}{\rho_{0}}\right) E\right]+\frac{\omega^{2}}{2 \pi \rho_{0} a b c}\left(x^{2}+y^{2}\right)+\text { cons. ... }
\end{aligned}
$$

The solution for the incompressible mass is derived from the equation

$$
-\theta\left[\Sigma \frac{x^{2}}{a^{2}}\right]=-\left[J_{A} x^{2}+J_{B} y^{2}+J_{C} z^{2}\right]+\frac{\omega^{2}}{2 \pi \rho_{0} a b c}\left(x^{2}+y^{2}\right) \ldots(458),
$$

which is a special case of the above.

172. On equating coefficients in this last equation we shall obtain three equations (456) and the solution of these equations will consist of sets of values of $a, b, c$ and $\omega^{2}$.

Similarly the solution of equation (457) will consist of sets of values of $a, b, c, \omega^{2}$ and $P_{0}$. In the incompressible problem, $P_{0}$ is always zero, and the sets of values for $a, b, c$ and $\omega^{2}$ coincide with those found from equation (458). But in the more general problem, this is not the case.

Let us now agree, as a matter of convenience, that the symbols $a, b, c$ shall be reserved to refer only to solutions of the incompressible problem. A solution of the compressible problem may now be designated by symbols such as $a+\Delta a, b+\Delta b, c+\Delta c$. Strictly speaking, the equation of the boundary ought no longer to be taken to be

$$
\Sigma \frac{x^{2}}{a^{2}}+e P_{0}=1
$$

it must be taken to be

$$
\Sigma \frac{x^{2}}{(a+\Delta a)^{2}}+e P_{0}=1
$$

This however may be re-written in the form (459) if we permit of $P_{0}$ containing terms of degree 2 as well as those of degrees $3,4, \ldots$ of which it has so far been supposed to consist. Thus, in what follows, we shall suppose $P_{0}$ to include second degree terms and $a, b, c$ will be supposed to have the meaning just agreed upon.

Let the general value of $V_{i}(1)$ corresponding to the boundary (459) in which $P_{0}$ consists of terms of degrees $2,3,4 \ldots$ be supposed to be

$$
V_{i}(1)=-\pi a b c\left(J_{A} x^{2}+J_{B} y^{2}+J_{C} z^{2}-J\right)+\Delta V_{i}(1),
$$


so that $\Delta V_{i}(1)$ represents the change produced in $V_{i}(1)$ by the additional term $e P_{0}$ in the equation to the boundary (459).

Let us further agree that $\omega_{0}$ is to be used to denote the value of $\omega$ in the incompressible problem, the general value of $\omega^{2}$ being henceforth denoted by $\omega_{0}^{2}+\Delta \omega^{2}$.

With these conventions equation (457) becomes

$$
\begin{aligned}
& -\theta\left[\left(\Sigma \frac{x^{2}}{a^{2}}+e P_{0}\right)-\frac{1}{2}(\gamma-2)\left(\frac{\rho_{0}-\sigma}{\rho_{0}}\right)\left(\Sigma \frac{x^{2}}{a^{2}}+e P_{0}\right)^{2}\right. \\
& \left.+\frac{1}{6}(\gamma-2)(\gamma-3)\left(\frac{\rho_{0}-\sigma}{\rho_{0}}\right)^{2}\left(\Sigma \frac{x^{2}}{a^{2}}+e P_{0}\right)^{3}-\ldots\right] \\
& =-\left[J_{A} x^{2}+J_{B} y^{2}+J_{C} z^{2}\right]+\frac{1}{\pi a b c}\left[\Delta V_{i}(1)-\left(\frac{\rho_{0}-\sigma}{\rho_{0}}\right) E\right] \\
& +\frac{\omega^{2}+\Delta \omega^{2}}{2 \pi \rho_{0} a b c}\left(x^{2}+y^{2}\right)+\mathrm{cons}
\end{aligned}
$$

With the meaning now assigned to the symbols $a, b, c$ and $\omega^{2}$, equation (458) is also true. On subtraction of corresponding sides of the two equations, we obtain

$$
\begin{aligned}
& -\theta\left[e P_{0}-\frac{1}{2}(\gamma-2)\left(\frac{\rho_{0}-\sigma}{\rho_{0}}\right)\left(\Sigma \frac{x^{2}}{a^{2}}+e P_{0}\right)^{2}\right. \\
& \left.\quad+\frac{1}{6}(\gamma-2)(\gamma-3)\left(\frac{\rho_{0}-\sigma}{\rho_{0}}\right)^{2}\left(\Sigma \frac{x^{2}}{a^{2}}+e P_{0}\right)^{3}+\ldots\right] \\
& =\frac{1}{\pi a b c}\left[\Delta V_{i}(1)-\left(\frac{\rho_{0}-\sigma}{\rho_{0}}\right) E\right]+\frac{\Delta \omega^{2}}{2 \pi \rho_{0} a b c}\left(x^{2}+y^{2}\right)+\text { cons. }
\end{aligned}
$$

Equation (458) determines a solution when the mass is incompressible; equation (461) determines the relation between the new quantities introduced by compressibility. More definitely, it connects $P_{0}$, the distortion of the boundary from the shape suited to an incompressible mass, with $\rho_{0}-\sigma$, the range of density, and with $\Delta \omega^{2}$, the change in $\omega^{2}$.

173. The simplest solution occurs when all the changes from incompressibility are measured by quantities so small that their squares may be neglected. In this case $\left(\rho_{0}-\sigma\right) / \rho_{0}, e P_{0}$ and $\Delta \omega^{2}$ are all of the first order of small quantities, and equation (461) assumes the simple form

$$
\begin{aligned}
& -\theta\left[e P_{0}-\frac{1}{2}(\gamma-2)\left(\frac{\rho_{0}-\sigma}{\rho_{0}}\right)\left(\Sigma \frac{x^{2}}{a^{2}}\right)^{2}\right] \\
& \quad=\frac{1}{\pi a b c}\left[\Delta V_{i}(1)-\left(\frac{\rho_{0}-\sigma}{\rho_{0}}\right) E\right]+\frac{\Delta \omega^{2}}{2 \pi \rho_{0} a b c}\left(x^{2}+y^{2}\right)+\text { cons. (462). }
\end{aligned}
$$

In this equation we notice that $E$ is multiplied by the small quantity $\left(\rho_{0}-\sigma\right) / \rho_{0}$, so that in evaluating $E$ we may neglect all further small 
quantities. With this simplification, equation (447) gives as the value of $E$,

$$
\begin{aligned}
E & =\pi a b c \int_{0}^{\infty} \int_{g}^{1} q^{2} d q^{2} \frac{d \lambda}{\Delta} \\
& =\frac{1}{2} \pi a b c \int_{0}^{\infty}\left(1-q^{4}\right) \frac{d \lambda}{\Delta}
\end{aligned}
$$

in which $q$ is given, now that small quantities of the first order may be neglected, by

$$
q^{2}=\frac{x^{2}}{a^{2}+\lambda}+\frac{y^{2}}{b^{2}+\lambda}+\frac{z^{2}}{c^{2}+\lambda} .
$$

Substituting this value for $q^{2}$ in equation (463), we obtain, to our present order of approximation,

$$
E=\frac{1}{2} \pi a b c\left[J-\Sigma\left(x^{4} J_{A A}+2 x^{2} y^{2} J_{A B}\right)\right]
$$

where $J_{A A}$, etc. denote integrals defined by equation (56) of p. 36 .

In calculating $V_{i}(1)$ we must of course retain small quantities of the first order. The whole potential of a solid of unit density whose boundary is determined by equation (459) is

so that

$$
V_{i}(1)+\Delta V_{i}(1)=-\pi a b c \int_{0}^{\infty}[f+\phi(1)] \frac{d \lambda}{\Delta}
$$

$$
\Delta V_{i}(1)=-\pi a b c \int_{0}^{\infty} \phi(1) \frac{d \lambda}{\Delta},
$$

in which, neglecting small quantities of the second order, we may put

$$
\phi(1)=e\left[P-\frac{1}{4} f D P+\frac{1}{4}\left(\frac{1}{4} f\right)^{2} D^{2} P-\ldots\right] .
$$

Collecting terms, equation (462) becomes

$$
\begin{aligned}
& -\theta\left[e P_{0}-\frac{1}{2}(\gamma-2)\left(\frac{\rho_{0}-\sigma}{\rho_{0}}\right)\left(\Sigma \frac{x^{4}}{a^{4}}+2 \Sigma \frac{x^{2} y^{2}}{a^{2} b^{2}}\right)\right] \\
& =-\int_{0}^{\infty} \phi(1) \frac{d \lambda}{\Delta}-\frac{1}{2}\left(\frac{\rho_{0}-\sigma}{\rho_{0}}\right)\left[\Sigma x^{4} J_{A A}+2 \Sigma x^{2} y^{2} J_{A B}\right] \\
& +\frac{\Delta \omega^{2}}{2 \pi \rho_{0} a b c}\left(x^{2}+y^{2}\right)+\text { cons. }
\end{aligned}
$$

Clearly there will be a solution in which $P_{0}$ consists solely of terms of degrees 4 and 2 in $x, y, z$. Let us assume for $P_{0}$ the value

so that

$$
\begin{aligned}
e P_{0}=\left(\frac{\rho_{0}-\sigma}{\rho_{0}}\right)\left[\frac{L x^{4}}{a^{8}}+\frac{M y^{4}}{b^{8}}+\frac{N z^{4}}{c^{8}}\right. & +\frac{2 l y^{2} z^{2}}{b^{4} c^{4}}+\frac{2 m z^{2} x^{2}}{c^{4} a^{4}} \\
& \left.+\frac{2 n x^{2} y^{2}}{a^{4} b^{4}}+\frac{2 p x^{2}}{a^{4}}+\frac{2 q y^{2}}{b^{4}}+\frac{2 r z^{2}}{c^{4}}\right] \ldots(466)
\end{aligned}
$$

$$
\begin{aligned}
e P=\left(\frac{\rho_{0}-\sigma}{\rho_{0}}\right)\left[L \xi^{4}+M \eta^{4}+N \zeta^{4}\right. & +2 l \eta^{2} \zeta^{2}+2 m \zeta^{2} \xi^{2} \\
& \left.+2 n \xi^{2} \eta^{2}+2 p \xi^{2}+2 q \eta^{2}+2 r \zeta^{2}\right]
\end{aligned}
$$


Then we may assume*

$\int_{0}^{\infty} \phi \frac{d \lambda}{\Delta}=4\left(\frac{\rho_{0}-\sigma}{\rho_{0}}\right)\left[c_{11} x^{4}+c_{22} y^{4}+c_{33} z^{4}+2 c_{12} x^{2} y^{2}+\ldots+d_{1} x^{2}+d_{2} y^{2}+d_{3} z^{2}+d_{4}\right]$

Inserting these values into equation (465) and equating coefficients, we obtain

$$
\left.\begin{array}{c}
\theta\left(\frac{L}{a^{8}}-\frac{1}{2} \frac{\gamma-2}{a^{4}}\right)=4 c_{11}-\frac{1}{2} J_{A A} \\
\theta\left(\frac{l}{b^{4} c^{4}}=\frac{1}{2} \frac{\gamma-2}{b^{2} c^{2}}\right)=4 c_{23}-\frac{1}{2} J_{B C}
\end{array}\right\}
$$

and four similar equations; there are also three equations such as

$$
\theta\left(\frac{2 p}{a^{4}}\right)=4 d_{1}-\frac{\Delta \omega^{2}}{2 \pi\left(\rho_{0}-\sigma\right) a b c}
$$

In these equations the six coefficients $c_{11}, c_{22}, \ldots c_{23}$ are linear functions of the six coefficients $L, M, N, l, m, n$ only, but the coefficients, $d_{1}, d_{2}, d_{3}$ are linear functions of $L, M, N, l, m, n, p, q$ and $r$.

It follows that the six equations (468) form a set of linear equations for the determination of $L, M, N, l, m, n$. The solution of these equations, if written down directly in analytical form, would be too complicated to convey any definite meaning to the mind. Fortunately there is an approximate solution of a very simple type, namely $\dagger$

$$
\left.\begin{array}{l}
L=\frac{1}{2}(\gamma-2) a^{4}-\frac{1}{2} \frac{a^{8}}{\theta} J_{A A} \text { etc. } \\
l=\frac{1}{2}(\gamma-2) b^{2} c^{2}-\frac{1}{2} \frac{b^{4} c^{4}}{\theta} J_{B C} \text { etc. }
\end{array}\right\}(\text { approximation } A) \ldots \ldots(470) .
$$

To understand the meaning of this approximation, we may notice that it satisfies equations $(468)$ if $c_{11}, c_{22}, \ldots c_{33}$ are neglected. Thus the approximation is arrived at by neglecting terms of degree 4 in $\Delta V_{i}(1)$, and is therefore equivalent to treating the boundary $q=1$ as ellipsoidal when calculating its gravitational potential.

174. To obtain some idea of the amount of error involved in this approximate solution, I have worked out exactly the true solution in two special cases.

It will be remembered that the configurations for an incompressible mass consist of spheroids, ellipsoids and pear-shaped figures. The corresponding configurations for the compressible mass are derived from the foregoing by distortion and consist of pseudo-spheroids, pseudo-ellipsoids and pear-shaped

* The numerical multiplier 4 is introduced in order to facilitate comparison with the corresponding analysis in Chapter $\mathrm{V}$.

+ Bakerian Lecture for 1917 (not yet published). 
figures. There are points of bifurcation on the sequence of compressible figures; at the first of these the pseudo-spheroidal shape gives place to a pseudo-elliptical shape, and at the second the pseudo-elliptical shape gives place to a pear shape. The configurations at these points of bifurcation are arrived at by distortion from the corresponding configurations for the incompressible mass, and it is these configurations for which $I$ have calculated the exact values of $L, M, N, l, \dot{m}, n$.

At the first point of bifurcation

$$
a=b=1 \cdot 1972, \quad c=69766, \quad \frac{\omega^{2}}{2 \pi \rho_{0}}=\cdot 18712, \quad \theta=47125,
$$

the scale of length, which is at our disposal, being chosen so as to make $r_{0}=(a b c)^{\frac{3}{3}}=1$. The exact solution is found, by direct solution of equations (468) to be

$$
\left.\begin{array}{rl}
L=M=n & =1.0273 \quad(\gamma-2)-1.0466 \\
l=m & =0.3488 \\
N & =0.11845(\gamma-2)-0.23784 \\
& (\gamma-2)-0.06328
\end{array}\right\} \text { (exact) }
$$

while the approximate solution (470) is found to be

$$
\left.\begin{array}{rl}
L=M=n & =1.0273(\gamma-2)-1.0273 \\
l=m & =0.3488(\gamma-2)-0.2467 \\
N & =0.11845(\gamma-2)-0.0626
\end{array}\right\} \text { (approx. A) ...(472). }
$$

It will be seen that the error is of the order of two per cent. in the terms which do not involve $\gamma$.

175. At the second point of bifurcation, at which the pseudo-ellipsoid gives place to the pear-shaped figure, I find for the exact solution

$$
\begin{aligned}
& L=6.3238(\gamma-2)-15.4353 \\
& M=0.22057(\gamma-2)-0.15560 \\
& N=0.08962(\gamma-2)-0.04733 \\
& l=0.14059(\gamma-2)-0.08468 \\
& m=0.75280(\gamma-2)-0.49850 \\
& n=1 \cdot 18103(\gamma-2)-0.95103)
\end{aligned}
$$

while the approximate solution is found to be

$$
\begin{aligned}
& L=6.3238(\gamma-2)-10 \cdot 1768 \\
& M=0.22057(\gamma-2)-0.15329 \\
& N=0.08962(\gamma-2)-0.04677 \\
& l=0.14059(\gamma-2)-0.08424 \\
& m=0.75280(\gamma-2)-0.62860 \\
& n=1 \cdot 18103(\gamma-2)-1 \cdot 18103)
\end{aligned}
$$


Again, as must in general be the case, there is no error in the terms multiplied by $\gamma$. In the remaining terms, the errors in $l, M$ and $N$, the coefficients which determine the smallest cross-section, are seen to be less than two per cent. but the errors in the remaining coefficients $L, m$ and $n$ are very much greater, being respectively 34,26 and 24 per cent.

176. Having determined $L, M, N, l, m, n$, we complete the solution by finding $p, q, r$ from equations (469), which, written out in full, become

$$
\begin{aligned}
& \frac{2 p \theta}{a^{4}}=4 d_{1}-\frac{\Delta \omega^{2}}{2 \pi\left(\rho_{0}-\sigma\right) a b c} \\
& \frac{2 q \theta}{b^{4}}=4 d_{2}-\frac{\Delta \omega^{2}}{2 \pi\left(\rho_{0}-\sigma\right) a b c} \\
& \frac{2 r \theta}{c^{4}}=4 d_{3} \ldots \ldots \ldots \ldots \ldots \ldots \ldots \ldots \ldots \ldots . . .
\end{aligned}
$$

At this stage it is convenient to extend the notation already introduced in $\S 35$. By analogy with the integrals $J$ defined in equation (56) we shall writite

$$
\int_{0}^{\infty} \frac{\lambda d \lambda}{A^{m} B^{n} C^{p} \Delta}=I_{A^{m} B^{n} C^{p}} ; \int_{0}^{\infty} \frac{\lambda^{2} d \lambda}{A^{m} B^{n} C^{p} \Delta}=H_{A^{m} B^{n} C^{p}}
$$

With this notation the value of $4 d_{1}$ is readily found to be

$$
4 d_{1}=2 p J_{A A}-\frac{p}{a^{2}} I_{A A}-\frac{q}{b^{2}} I_{A B}-\frac{r}{c^{2}} I_{A C}+4 \delta_{1}
$$

in which

$$
\begin{gathered}
4 \delta_{1}=-\frac{3}{4}\left[\frac{L}{a^{4}} H_{A A A}+\frac{M}{b^{4}} H_{A B B}+\frac{N}{c^{4}} H_{A C C}\right]-\frac{1}{2}\left[\frac{l}{b^{2} c^{2}} H_{A B C}+\frac{m}{c^{2} a^{2}} H_{A A C}+\frac{n}{a^{2} b^{2}} H_{A A B}\right] \\
+\frac{3 L}{a^{2}} I_{A A A}+\frac{n}{b^{2}} I_{A A B}+\frac{m}{c^{2}} I_{A A C} \ldots \ldots \ldots \ldots \ldots \ldots \ldots \ldots \ldots \ldots \ldots
\end{gathered}
$$

The value of $\delta_{1}$ can be determined as soon as the values of the coefficients $L, M, N, l, m, n$ have been found, and equations (475) etc. assume the form

$$
\frac{2 p \theta}{a^{4}}-2 p J_{A A}+\frac{p}{a^{2}} I_{A A}+\frac{q}{b^{2}} I_{A B}+\frac{r}{c^{2}} I_{A C}=4 \delta_{1}-\frac{\Delta \omega^{2}}{2 \pi\left(\rho_{0}-\sigma\right) a b c} \text { etc. }(480) \text {. }
$$

These linear equations determine $p, q$ and $r$. The solution may be regarded as the sum of two solutions, the first arising from the terms $4 \delta_{1}, 4 \delta_{2}$ and $4 \delta_{3}$ on the right, and the second from the terms in $\Delta \omega^{2}$. The second solution represents merely a step along the ellipsoidal (or spheroidal) series corresponding to a small change $\Delta \omega^{2}$ in the value of $\omega^{2}$. To obtain a compressible solution we may give any value to $\Delta \omega^{2}$, the zero value, which is of course most convenient, giving a solution which corresponds to the same rotation as the incompressible figure from which it is derived. 
But to obtain all the compressible solutions adjacent to a given incompressible solution, we must retain $\Delta \omega^{2}$. In particular, the retention of $\Delta \omega^{2}$ will be necessary in searching for points of bifurcation in the compressible problem. A point of bifurcation in the compressible problem will be adjacent to the corresponding point of bifurcation in the incompressible problem, but will not in general have the same rotation.

Thus in searching for the points of bifurcation in the incompressible problem, we retain the so far undetermined quantity $\Delta \omega^{2}$ in our equations. The three equations (475)-(4.77) determine three relations between $p, q, r$ and $\Delta \omega^{2}$, but to determine these four quantities fully a further equation is needed, this equation of course expressing the condition for a point of bifurcation.

177. Let us confine our attention to the particular point of bifurcation at which the pseudo-spheroidal figure gives place to a pseudo-ellipsoidal figure. The corresponding point in the incompressible problem is the point of bifurcation at which the Jacobian ellipsoids join the Maclaurin spheroids. At this point equations (69) and (70) of $\S 37$ are both satisfied, as well as the equations of equilibrium (65)-(67). Combining equations (69) and (70), we obtain as the equation determining the position of this point of bifurcation,

$$
a^{4} J_{A A}=c^{2} J_{C} \ldots \ldots \ldots \ldots \ldots \ldots \ldots \ldots \ldots \ldots \ldots \ldots \ldots \ldots(481),
$$

or, using the equation of equilibrium (67),

$$
a^{5} b c J_{A A}=\theta a b c
$$

The actual values of $a, b, c, \theta$ and $\omega^{2}$ are those already given in $\S 174$.

In the compressible problem, the equation of the boundary has been taken to be

$$
\frac{x^{2}}{a^{2}}+\frac{y^{2}}{b^{2}}+\frac{z^{2}}{c^{2}}-1+\left(\frac{\rho_{0}-\sigma}{\rho_{0}}\right)\left[\frac{2 p x^{2}}{a^{4}}+\frac{2 q y^{2}}{b^{4}}+\frac{2 r z^{2}}{c^{4}}+F_{4}\right],
$$

where $F_{4}$ stands for the fourth-degree terms $L x^{4} / a^{8}+\ldots$, which have already been determined. This may be put in the form

where

$$
\frac{x^{2}}{a^{\prime 2}}+\frac{y^{2}}{b^{\prime 2}}+\frac{z^{2}}{c^{\prime 2}}+\left(\frac{\rho_{0}-\sigma}{\rho_{0}}\right) F_{4}=1
$$

$$
a^{\prime 2}=a^{2}-2 p\left(\frac{\rho_{0}-\sigma}{\rho_{0}}\right) \text { etc. }
$$

The condition determining a point of bifurcation in the incompressible problem is readily seen to be

$$
a^{\prime s} b^{\prime} c^{\prime} J_{A A}^{\prime}=\theta^{\prime} a^{\prime} b^{\prime} c^{\prime}
$$

where $\theta^{\prime}, J_{A A}^{\prime}$ refer to an ellipsoid of semi-axes $a^{\prime}, b^{\prime}, c^{\prime}$. 
From equation (445) it is at once seen that $\theta^{\prime} a^{\prime} b^{\prime} c^{\prime}=\theta a b c$, so that the point of bifurcation is determined by

$$
a^{\prime 5} b^{\prime} c^{\prime} J_{A A}^{\prime}-a^{5} b c J_{A A}=0 .
$$

Using relations (484), this may be put in the form

$$
\frac{p}{a^{2}}\left(5 I_{A A A}\right)+\frac{q}{b^{2}}\left(I_{A A B}\right)+\frac{r}{c^{2}}\left(I_{A A C}\right)=0
$$

'This equation, together with equations (475)-(477) determine the values of $p, q, r$ and $\Delta \omega^{2}$ at the point of bifurcation.

178. In these equations $a=b$, so that $p=q$, and the system of four equations reduces to the three equations

$$
\begin{aligned}
& \frac{2 p \theta}{a^{4}}=4 d_{1}-\frac{\Delta \omega^{2}}{2 \pi\left(\rho_{0}-\sigma\right) a b c}, \\
& \frac{2 r \theta}{c^{4}}=4 d_{3}, \\
& 6 I_{\triangle A A} \frac{p}{a^{2}}+I_{A A C} \frac{r}{c^{2}}=0,
\end{aligned}
$$

in which $d_{1}$ is given by equation (478) and $d_{3}$ is given by a similar equation, or may be more readily derived from the relation

$$
2 d_{1}+d_{3}=0
$$

which is necessarily satisfied since the potential $\int_{0}^{\infty} \phi \frac{d \lambda}{\Delta}(\S 173)$ is harmonic.

The value of $\delta_{1}$ for the configuration in question calculated from equation (479) is found to be -0.00851 , whence the solution of the equations is found to be

$$
\frac{p}{a^{2}}=-0.016037, \quad \frac{r}{c^{2}}=0.056337, \quad \frac{\Delta \omega^{2}}{2 \pi\left(\rho_{0}-\sigma\right)}=-04400 \ldots(486) .
$$

179. It now appears that at the point of bifurcation the rotation $\omega$ is given by

$$
\frac{\omega^{2}}{2 \pi}=\frac{\omega_{0}^{2}+\Delta \omega^{2}}{2 \pi}=\cdot 18712 \rho_{0}-\cdot 04400\left(\rho_{0}-\sigma\right)
$$

This relation is exact only as far as first powers of $\left(\rho_{0}-\sigma\right)$. To the same degree of accuracy, the mean density $\bar{\rho}$ of the figure is given by

$$
\bar{\rho}=\rho_{0}-\frac{3}{5}\left(\rho_{0}-\sigma\right),
$$

so that equation (487) may also be put in the form

$$
\frac{\omega^{2}}{2 \pi}=\cdot 18712 \bar{\rho}+\cdot 06827\left(\rho_{0}-\sigma\right)
$$


We may notice that although terms in $\gamma$ occur in the equation to the boundary, yet no such terms occur in equations (487) and (488). This is necessarily the case, as can be at once seen on considering the incompressible mass for which $\gamma=\infty$. For such a mass, we have already seen $(\$ 171)$ that $c\left(\rho_{0}-\sigma\right)$ and therefore also $\gamma\left(\rho_{0}-\sigma\right)$ remain finite, while the value of $\omega^{2} / 2 \pi \bar{\rho}$ must necessarily reduce to $\cdot 18712$.

Equation (488) indicates that compressibility increases the value of $\omega^{2} / 2 \pi \bar{\rho}$ which is necessary for the pseudo-spheroidal form to give place to a pseudoellipsoidal form. Stated in another way, a compressible mass retains the form of a figure of revolution up to higher values of the rotation than does an incompressible mass, rotation being measured with reference to the mean density $\bar{\rho}$.

180. We have so far obtained a solution which is accurate as far only as the first order of the small quantity $\rho_{0}-\sigma$. The method we have used admits of extension as far as any power of $\rho_{0}-\sigma$, but the labour of computation makes it almost impossible to carry the calculations beyond second order terms.

To obtain a second order solution, we may replace $e P$ by $e P+e^{2} Q$, and $\Delta \omega^{2}$ by $\Delta \omega^{2}+\delta \omega^{2}$, where $\delta \omega^{2}$ is of the second order. Similarly we replace $\Delta V_{i}$ by $\Delta V_{i}+\delta V_{i}$. We are accordingly assuming a boundary of the form (cf. $§ 172$ )

$$
\Sigma \frac{x^{2}}{a^{2}}+e P_{0}+e^{2} Q_{0}=1
$$

corresponding to a rotation $\omega$ given by

$$
\omega^{2}=\omega_{0}^{2}+\Delta \omega^{2}+\delta \omega^{2}
$$

The general equation (461), written down as far as second order terms, now becomes

$$
\begin{aligned}
& -\theta\left[e P_{0}+e^{2} Q_{0}-\frac{1}{2}(\gamma-2)\left(\frac{\rho_{0}-\sigma}{\rho_{0}}\right)\left\{\left(\Sigma \frac{x^{2}}{a^{2}}\right)^{2}+2 e P_{0} \Sigma \frac{x^{2}}{a^{2}}\right\}\right. \\
& \left.+\frac{1}{6}(\gamma-2)(\gamma-3)\left(\frac{\rho_{0}-\sigma}{\rho_{0}}\right)^{2}\left(\Sigma \frac{x^{2}}{a^{2}}\right)^{3}\right] \\
& =\frac{1}{\pi a b c}\left[\Delta V_{i}(1)+\delta V_{i}(1)-\left(\frac{\rho_{0}-\sigma}{\rho_{0}}\right)(E+\Delta E)\right] \\
& +\frac{\Delta \omega^{2}+\delta \omega^{2}}{2 \pi \rho_{0} a b c}\left(x^{2}+y^{2}\right)+\text { cons }
\end{aligned}
$$

On equating the first order terms in this equation we of course obtain equation (462). On equating the second order terms we obtain

$$
\begin{array}{r}
-\theta\left[e^{2} Q_{0}-(\gamma-2)\left(\frac{\rho_{0}-\sigma}{\rho_{0}}\right) e P_{0} \Sigma \frac{x^{2}}{a^{2}}+\frac{1}{6}(\gamma-2)(\gamma-3)\left(\frac{\rho_{0}-\sigma}{\rho_{0}}\right)^{2}\left(\Sigma \frac{x^{2}}{a^{2}}\right)^{3}\right] \\
\quad=\frac{1}{\pi a b c}\left[\delta V_{i}(1)-\left(\frac{\rho_{0}-\sigma}{\rho_{0}}\right) \Delta E\right]+\frac{\delta \omega^{2}}{2 \pi \rho_{0} a b c}\left(x^{2}+y^{2}\right)+\text { cons. ...(492). }
\end{array}
$$


It is readily seen that the appropriate form to assume for $Q$ is one consisting of terms of degrees 6,4 and 2 in $\xi, \eta, \zeta$.

A general discussion of the solution of this equation will be found elsewhere* ${ }^{*}$ For our immediate purpose we may consider the particutar solution at the point of bifurcation at which the pseudo-spheroidal form becomes unstable, thus extending the solution already obtained in $\S 174$ to the second order of small quantities.

181. In this solution $x$ and $y$ enter symmetrically. Let us write $\varpi^{2}$ for $\xi^{2}+\eta^{2}$, and assume for $Q$ the value

$$
\begin{array}{r}
e^{2} Q=\left(\frac{\rho_{0}-\sigma}{\rho_{0}}\right)^{2}\left[\mathrm{R} \varpi^{6}+3 \mathrm{~S} \varpi^{4} \zeta^{2}+3 \mathrm{~T} \varpi^{2} \zeta^{4}\right. \\
+\mathrm{U} \zeta^{6}+\mathrm{r} \varpi^{4}+2 \mathrm{~s} \varpi^{2} \zeta^{2}+\mathrm{t} \zeta^{4} \\
\left.+2 u \varpi^{2}+2 v \zeta^{2}\right] \ldots \ldots \ldots \ldots \ldots
\end{array}
$$

On carrying out the necessary calculations and solving equation (492) we find

$$
\begin{aligned}
& \mathrm{R}=0.4155-1.7799(\gamma-2)+0.4908(\gamma-2)(2 \gamma-3) \\
& 3 \mathrm{~S}=0.1894-1.4585(\gamma-2)+0.5000(\gamma-2)(2 \gamma-3) \\
& 3 \mathrm{~T}=0.0506-0.4124(\gamma-2)+0.1698(\gamma-2)(2 \gamma-3) \\
& \mathrm{U}=0.00346-0.0375(\gamma-2)+0.01922(\gamma-2)(2 \gamma-3) \\
& \mathrm{r}=0.08755-0.10962(\gamma-2) \\
& \mathrm{s}=-0.01727+0.04871(\gamma-2) \\
& \mathrm{t}=-0.007862+0.02511(\gamma-2) \\
& \mathrm{u}=-0.00550-0.02651(\gamma-2) \\
& \mathrm{v}=0.00778+0.03195(\gamma-2) \\
& \frac{\delta \omega^{2}}{2 \pi \rho_{0}}=-\left(\frac{\rho_{0}-\sigma}{\rho_{0}}\right)^{2}[0.01292+0.05495(\gamma-2)]
\end{aligned}
$$

This completes the determination of the equation of the boundary as far as the second order of small quantities.

182. The lengths of the intercept on the $x$-axis are determined by the equation $\sqrt{f}=1$, where

$$
\mathfrak{f F}=\frac{x^{2}}{a^{2}}+\left(\frac{\rho_{0}-\sigma}{\rho_{0}}\right)\left[\frac{L x^{4}}{a^{8}}+\frac{2 p x^{2}}{a^{4}}\right]+\left(\frac{\rho_{0}-\sigma}{\rho_{0}}\right)^{2}\left[\frac{\mathrm{R} x^{6}}{a^{12}}+\frac{\mathrm{r} x^{4}}{a^{8}}+\frac{2 u x^{2}}{a^{4}}\right]+\ldots
$$

and the solution of this equation is found to be

$$
\frac{x^{2}}{a^{2}}=1-\left(\frac{\rho_{0}-\sigma}{\rho_{0}}\right)\left[\frac{L}{a^{4}}+\frac{2 p}{a^{2}}\right]-\left(\frac{\rho_{0}-\sigma}{\rho_{0}}\right)^{2}\left[\frac{\mathrm{R}}{a^{6}}+\frac{\mathrm{r}}{a^{4}}+\frac{2 u}{a^{2}}-\left(\frac{L}{a^{4}}+\frac{2 p}{a^{2}}\right)\left(\frac{2 L}{a^{4}}+\frac{2 p}{a^{2}}\right)\right]+\ldots
$$

\footnotetext{
* Bakerian Lecture 1917. Phil. Trans. R. S. (not yet published).
} 
From equation (450), the value of $\Omega$ at a point on the $x$-axis is given by

$$
\begin{array}{r}
\Omega+C=\frac{c}{\gamma-1} \rho_{0}^{\gamma-1}\left[1-(\gamma-1)\left(\frac{\rho_{0}-\sigma}{\rho_{0}}\right) \mathfrak{f f}+\frac{1}{2}(\gamma-1)(\gamma-2)\left(\frac{\rho_{0}-\sigma}{\rho_{0}}\right)^{2} \cdot \mathbb{f}^{2}\right. \\
\left.-\frac{1}{6}(\gamma-1)(\gamma-2)(\gamma-3)\left(\frac{\rho_{0}-\sigma}{\rho_{0}}\right)^{3} \mathbb{f}^{3}+\ldots\right]
\end{array}
$$

$=F(\sqrt[f]{ })$, say.

The points on the $x$-axis at which $\partial \Omega / \partial x=0$ are given by $\frac{\partial}{\partial x} F(\sqrt{f})=0$, or

$$
F^{\prime}(\sqrt{f}) \frac{\partial \sqrt{f}}{\partial x}=0
$$

This condition can be satisfied either by making $F^{\prime}(\sqrt{f})=0$ or $\partial \mathfrak{f f} / \partial x=0$. The first condition cannot be satisfied except when $\left(\rho_{0}-\sigma\right) / \rho_{0}=1$; in this case $\sigma=0$ and the equation merely reduces an equation which is automatically satisfied when $\sigma=0$. Thus the true points at which $\partial \Omega / \partial x$ vanishes are given by $\partial \sqrt[f f]{ } / \partial x=0$.

The condition that centrifugal force just balances gravity at the equator is therefore that $\partial \mathfrak{f} / \partial x=0$ when $\mathfrak{f f}=1$, and this is also the condition that the surface $\mathfrak{f}=1$ shall have a double point on the axis of $x$. That the two conditions must necessarily be identical is of course shewn by the analysis of $§ 151$.

The equation $\partial \mathfrak{f} / \partial x=0$ becomes

$$
1+\left(\frac{\rho_{0}-\sigma}{\rho_{0}}\right)\left[\frac{2 L}{a^{4}}\left(\frac{x^{2}}{a^{2}}\right)+\frac{2 p}{a^{2}}\right]+\left(\frac{\rho_{0}-\sigma}{\rho_{0}}\right)^{2}\left[\frac{3 \mathrm{R}}{a^{6}}\left(\frac{x^{4}}{a^{4}}\right)+\frac{2 \mathrm{r}}{a^{4}}\left(\frac{x^{2}}{a^{2}}\right)+\frac{2 \mathrm{u}}{a^{2}}\right]+\ldots=0 .
$$

Using the value of $x^{2} / a^{2}$ provided by equation (495), we find as the condition that $\partial \mathfrak{f} / \partial x$ shall vanish on the boundary $(\mathfrak{f}=1)$,

$1+\left(\frac{\rho_{0}-\sigma}{\rho_{0}}\right)\left[\frac{2 L}{a^{4}}+\frac{2 p}{a^{2}}\right]+\left(\frac{\rho_{0}-\sigma}{\rho_{0}}\right)^{2}\left[\frac{3 \mathrm{R}}{a^{6}}+\frac{2 \mathrm{r}}{a^{4}}+\frac{2 \mathrm{u}}{a^{2}}-\frac{2 L}{a^{4}}\left(\frac{L}{a^{4}}+\frac{2 p}{a^{2}}\right)\right]+\ldots=0$,

or, inserting numerical values,

$$
\begin{aligned}
& 1+\left(\frac{\rho_{0}-\sigma}{\rho_{0}}\right)[(\gamma-2)-1 \cdot 0509] \\
& \quad+\left(\frac{\rho_{0}-\sigma}{\rho_{0}}\right)^{2}\left[\frac{1}{2}(\gamma-2)^{2}-4063(\gamma-2)-0510\right]+\ldots=0 \ldots \ldots(496) .
\end{aligned}
$$

For a given value of $\gamma$, this equation determines a value of $\left(\rho_{0}-\sigma\right) / \rho_{0}$ such that centrifugal force just balances gravity at the instant at which the pseudo-spheroidal form is giving place to the pseudo-ellipsoidal.

We may alternatively regard the equation as determining a critical value of $\gamma$ when $\left(\rho_{0}-\sigma\right) / \rho_{0}$ is assigned. It is this latter use of the equation which is of primary interest to us, the important case being $\left(\rho_{0}-\sigma\right) / \rho_{0}=1$. It seems probable that the full series will be fairly rapidly convergent up to 
this value of $\left(\rho_{0}-\sigma\right) / \rho_{0}$, so that the critical value derived from the terms actually calculated may be expected to be tolerably near to the true value.

Putting $\left(\rho_{0}-\sigma\right) / \rho_{0}=1$, the critical value of $\gamma$ derived from the first two terms of the equation is seen to be

$$
\gamma=2.0509
$$

while if all the terms written down are used, the value is found to be

$$
\gamma=2 \cdot 1521
$$

We cannot state with great accuracy the value of $\gamma$ to which these values are converging, but there is not likely to be any very great error in taking it to be $\gamma=2 \cdot 2$. Assuming this value, it appears that a mass of gas for which $\gamma=2 \cdot 2$ will begin to lose matter equatorially at precisely the moment at which the pseudo-spheroidal form becomes unstable and gives place to the pseudo-ellipsoidal form.

183. The value of $\delta \omega^{2}$ has already been obtained in $\S 181$. From this we find that equation (488) extended as far as the second order of small quantities becomes

$$
\frac{\omega^{2}}{2 \pi \bar{\rho}}=0.18712+0.06827\left(\frac{\rho_{0}-\sigma}{\rho_{0}}\right)+[0.01602+0.07098(\gamma-2)]\left(\frac{\rho_{0}-\sigma}{\rho_{0}}\right)^{2}
$$

When $\gamma=2 \cdot 2$, this becomes

$$
\frac{\omega^{2}}{2 \pi \bar{\rho}}=0.18712+0.06827\left(\frac{\rho_{0}-\sigma}{\rho_{0}}\right)+0.03022\left(\frac{\rho_{0}-\sigma}{\rho_{0}}\right)^{2} \quad \ldots(500) .
$$

The general series of which the first three terms are here written down is probably convergent right up to the limiting value $\left(\rho_{0}-\sigma\right) / \rho_{0}=1$, but it is not easy to determine the value to which it converges. At a guess the value of $\omega^{2} / 2 \pi \bar{\rho}$ appears to converge to about 0.31 .

The critical figure for $\gamma=2 \cdot 2$ is shewn in fig. 36 , but it is not possible to draw the figure with much accuracy in the neighbourhood of the sharp edge. The interior curves are equiputentials and so are also surfaces of constant density and temperature.

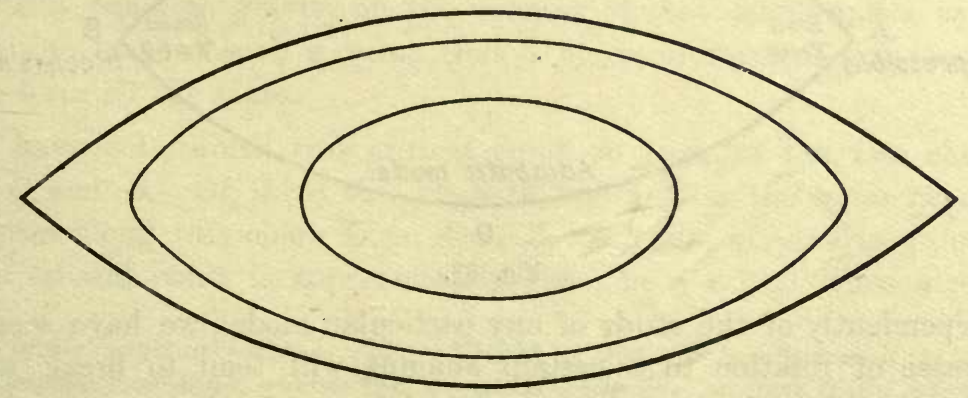

Fig. 36. 


\section{SUMMARY OF RESULTS}

184. Let us now recapitulate and summarise the results which have been obtained in the present and preceding chapters. We have been attempting to obtain an idea of the configurations which will be assumed by astronomical matter under the influence of its own rotation and under the action of tidal forces. Some results have been obtained which are applicable to all matter, but in general the investigation has had to be limited to certain simplified model masses. The models we have had under consideration have been four in number:

(A) The incompressible model, consisting of a mass of homogeneous incompressible matter of uniform density.

(B) Roche's model, consisting of a point nucleus of very great density, surrounded by an atmosphere of negligible density.

(C) The generalised Roche's model, consisting of a homogeneous incompressible mass of finite size and of finite density, surrounded by an atmosphere of negligible density.

(D) The adiabatic model, consisting of a mass of gas in adiabatic equilibrium, so that the pressure and density are connected at every point by the relation $p=\kappa \rho^{\gamma}$, where $\kappa$ and $\gamma$ retain the same values throughout the mass.

Of these four models $A$ and $B$ are limiting cases of the more general models $C$ and $D$. If $s$ denote the ratio of the volume of the atmosphere to that of the nucleus in the generalised Roche's model $C$, then model $C$ degenerates into model $A$ when $s=0$, and degenerates into model $B$ when $s=\infty$. Similarly the adiabatic model $D$ degenerates into model $A$ when $\gamma=\infty$ and into model $B$ when $\gamma=1 \frac{1}{5}$ (cf. $\S 149$ ). The relation between the four models is represented diagrammatically in fig. 37 .

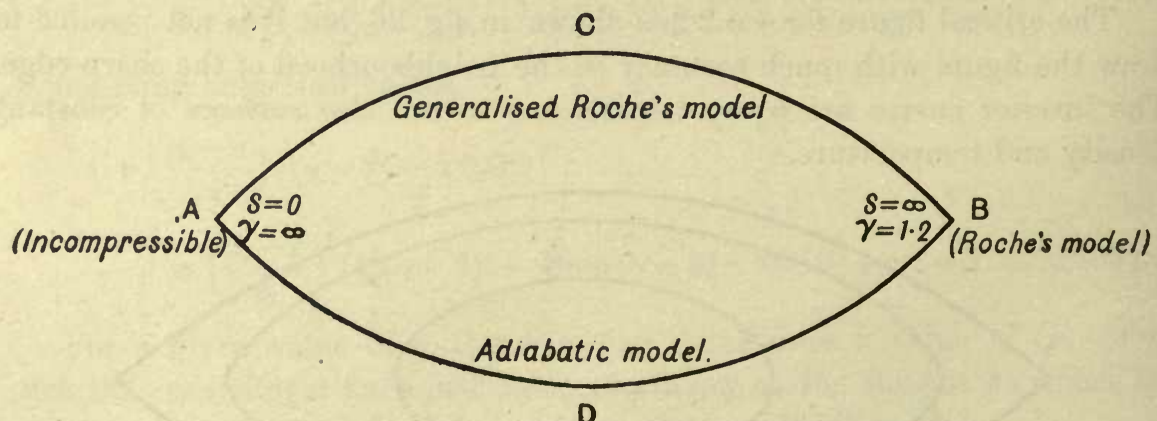

Fig. 37.

Independently of the study of any particular model, we have seen that an increase of rotation to a certain amount will tend to break up the original mass, while the same is true of tidal forces of sufficient intensity. 


\section{The Rotational Problem}

185. Let us consider the rotational problem first.

For the incompressible model $A$, the mechanism of breaking up is very fully known to us, thanks mainly to the investigations of Maclaurin, Jacobi, Kelvin, Darwin and Poincaré. For sinall rotations the mass will be spheroidal in shape, but as soon as the angular velocity exceeds a value $\omega$ given by $\omega^{2} / 2 \pi \rho=0 \cdot 18712$, the spheroidal form no longer remains stable, but gives place to an ellipsoidal form. With still further increasing rotation*, the mass elongates until a furrow begins to form across a section of the ellipsoid, giving it a pear-shaped appearance. After this furrow has once started, the motion is cataclysmal until the mass divides into two detached parts.

For Roche's model $B$, the mechanism of break-up is also fully known. As the rotation gradually increases, the equator of the mass bulges more and more, until finally a sharp edge forms on the equator, so that the whole figure becomes lens-shaped (see fig. 28, p. 149). Any further increase of rotation now results in matter being thrown off from the equator in a continuous stream, owing to centrifugal force outweighing gravity on the equator.

Thus models $A$ and $B$ both break up with increasing rotation, but they break up in very different ways. We have been able to shew quite generally that there are only these two distinct ways of breaking up; the method of breaking up of any other mass must be a variant of one or other of these two. It will be convenient to refer to the first method of break-up, that of the incompressible mass, as fissional break-up; and to the second method of break-up, that of Roche's model, as equatorial break-up.

It follows that as we pass along either of the chains of models $C$ and $D$ which connect $A$ and $B$, or along any other chain of models connecting $A$ and $B$, there must be some point on each at which fissional break-up gives place to rotational break-up. At such a point, the two methods of break-up must be about to begin simultaneously with the same rotation. Thus the condition determining such a point is that centrifugal force shall be precisely equal to gravity on the equator of that configuration at which the rotation reaches such a value that a figure of revolution is no longer a stable form for the mass.

We have determined this critical point on each of the two chains of models $C$ and $D$. Of these the adiabatic chain $D$ is the more important. As we pass along this chain from $A$ to $B$, the value of $\gamma$ varies from $\infty$ to $1 \cdot 2$; the critical point is approximately given by $\gamma=2 \cdot 2$. Thus a mass of

* The critical angular velocity is $\omega^{2} / 2 \pi \rho=0 \cdot 14200$, so that $\omega / \rho^{\frac{1}{2}}$ has decreased, but the constancy of angular momentum requires that $\rho$ shall have increased so much that $\omega$ is found also to have increased (cf. G. H. Darwin, Tides, p. 371). 
gas or other compressible matter in adiabatic equilibrium will break up by fission if $\gamma$ is greater than $2 \cdot 2$; it will break up equatorially if $\gamma$ lies between 1.2 and 2.2. This latter range of course includes the values of $\gamma$ for all gases whose density is so low that Boyle's law is approximately satisfied; for these $\boldsymbol{\gamma}$ is always less than $1 \cdot 66$.

Similarly as we pass along the chain $C$ of generalised Roche's models, the value of $s$, the ratio of the volume of the nucleus to that of the atmosphere, varies from $\infty$ to 0 . The critical point is found to occur at about $s=\frac{1}{3}$. Thus when the atmosphere is less than a third of the volume of the nucleus, the mass will break up by fission; when the atmosphere is greater than this the mass will break up equatorially.

These various results may be exhibited diagrammatically as in fig. 38 .

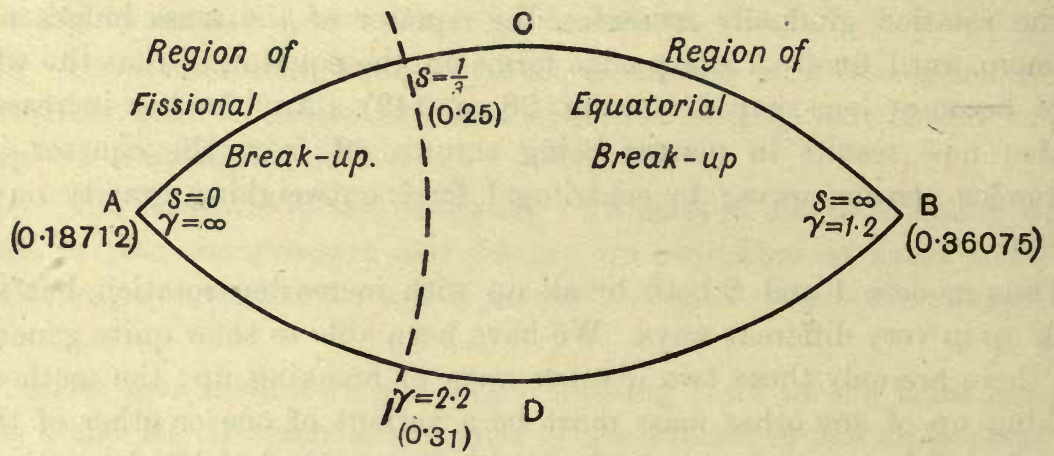

Fig. 38. Rotational break-up. [The figures in brackets denote the values of $\omega^{2} / 2 \pi \bar{\rho}$.]

\section{The Tidal Problem}

186. In the tidal problem we have found precisely similar results, the incompressible model breaking up by a process very closely analogous to that of fissional break-up in the rotational problem, and Roche's model breaking up by a process which is at least suggestive of the equatorial break-up of a rotating mass.

Going further into detail, we have found that the incompressible mass will, under small tidal forces, have the shape of a prolate spheroid. As the tidal forces increase, the elongation of this spheroid increases. When the elongation reaches a stage such that the axes are approximately in the ratio $17: 8: 8$, this spheroidal figure becomes unstable. Dynamical motion ensues, the elongation at first increasing rapidly until finally furrows form on the mass and it breaks up into several detached masses (cf. fig. 23, p. 127).

Roche's model also will assume the shape of a prolate spheroid so long as the tidal forces in action are small. As the tidal forces increase the boundary of the figure departs from a true spheroidal form; conical points form and 
finally jets of matter stream out from these cones, just as they streamed off at the equator in the rotational problem.

On the chain $C$ of generalised Roche's models, we have found that the former method of break-up gives place to the latter when $s$, the ratio of the volume of the atmosphere to that of the central mass, has a value approximately equal to $\frac{1}{10}$. The chain $D$ of adiabatic models has not been studied in detail, but it seems safe to suppose that at some point on this chain the one method of break-up gives place to the other. Assuming this, the results obtained for the tidal problem are those exhibited diagrammatically in fig. 39 ; the region to the left of the broken line represents configurations in which the mass, when broken up tidally, divides into a number of masses of comparable size, while the region on the right represents configurations in which one or two jets of matter will be thrown off from the mass.

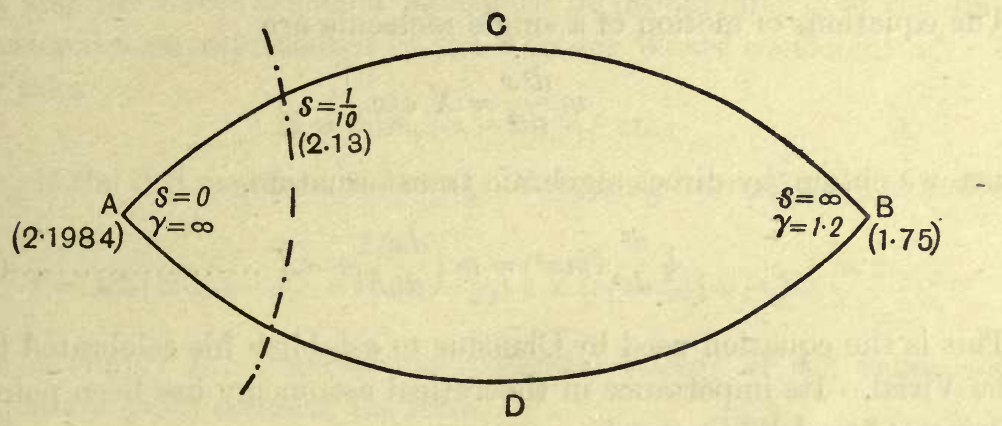

Fig. 39. Tidal break-up. $\left[\right.$ The figures in brackets denote the values of $\left.\frac{R}{r_{0}}\left(\frac{M^{\prime}}{M}\right)^{\frac{1}{3}} \cdot\right]$

\section{The Double-star Problem}

187. The results obtained for the double-star problem are so similar to those obtained for the tidal problem that it is hardly worth recapitulating them in detail. In the double-star problem, as in the tidal problem, there are two masses concerned, and we have been studying the mutual gravitational action of these two bodies on one another. From the mathematical point of view the double-star problem is little more than the tidal problem with a rotation set up just adequate to keep the masses permanently at a given distance from one another, and this explains the general similarity of the mathematical results obtained.

We proceed now to apply the abstract results obtained to actual problems of astronomy and cosmogony. 


\section{CHAPTER VIII}

\section{THE EVOLUTION OF GASEOUS MASSES}

\section{GENERAL THEORY}

188. We may begin with a consideration of the general motion of a cloud of nebulous astronomical matter. This may be supposed constituted either of gaseous molecules or of dust particles; for convenience we shall speak of the separate particles as molecules.

The equations of motion of a single molecule are

$$
m \frac{d^{2} x}{d t^{2}}=X \text { etc., }
$$

whence we obtain, by direct algebraic transformation,

$$
\frac{1}{2} \frac{d^{2}}{d t^{2}}\left(m x^{2}\right)=m\left(\frac{d x}{d t}\right)^{2}+x X
$$

This is the equation used by Clausius to establish his celebrated theorem of the Virial. Its importance in theoretical astronomy has been pointed out by Poincaré* and Eddington $t$.

Summing the three equations such as (501), we find

$$
\frac{1}{2} \frac{d^{2}}{d t^{2}}\left[m\left(x^{2}+y^{2}+z^{2}\right)\right]=m\left[\left(\frac{d x}{d t}\right)^{2}+\left(\frac{d y}{d t}\right)^{2}+\left(\frac{d z}{d t}\right)^{2}\right]+(x X+y Y+z Z) \text {. }
$$

On further summing this equation for all the molecules, or other particles of the mass under consideration, we obtain

$$
\frac{1}{2} \frac{d^{2} I}{d t^{2}}=2 T+\Sigma(x X+y Y+z Z)
$$

where $I$ is the moment of inertia about the origin, defined by

$$
I=\Sigma m\left(x^{2}+y^{2}+z^{2}\right)
$$

and $T$ is the kinetic energy of translation of the molecules of the gas. The last term $\Sigma(x X+y Y+z Z)$ is the Virial of Clausius; call it $V$.

To evaluate the virial, we fix our attention on two particles of masses $m_{1}, m_{2}$ at the points $x_{1}, y_{1}, z_{1}$ and $x_{2}, y_{2}, z_{2}$ respectively. Let the force exerted by the second on the first have components $\Xi, \mathrm{H}, \mathrm{Z}$, so that the force exerted 
on the first by the second will have components $-\Xi,-H,-Z$. The contribution of this pair of forces to the virial will be

$$
\Xi\left(x_{1}-x_{2}\right)+\mathrm{H}\left(y_{1}-y_{2}\right)+\mathrm{Z}\left(z_{1}-z_{2}\right)
$$

and the whole virial will be

$$
V=\Sigma \Sigma\left[\Xi\left(x_{1}-x_{2}\right)+\mathrm{H}\left(y_{1}-y_{2}\right)+\mathrm{Z}\left(z_{1}-z_{2}\right)\right]
$$

the summation being over all pairs of particles.

Forces such as $\Xi, \mathrm{H}, \mathrm{Z}$ will consist of the molecular forces between pairs of molecules in collision or in propinquity, and of the gravitational forces between pairs of molecules at all distances. For a gas of density so low that the ideal gas laws may be assumed to hold, all these forces may be neglected except those of gravitation. For the forces between molecules in collision give rise only to the Van der Waals' coefficient $b$ in the equation of state of the gas, and the forces between molecules in propinquity give rise only to the cohesional term, represented by the Van der Waals' coefficient $a^{*}$. Thus we may take

$$
\Xi=m_{1} m_{2}\left(x_{2}-x_{1}\right) / r_{12}{ }^{3} \text { etc., }
$$

where $r_{12}$ is the distance from $m_{1}$ to $m_{2}$. Summing over all pairs of molecules we find

$$
V=\Sigma \Sigma\left[\Xi\left(x_{1}-x_{2}\right)+\mathrm{H}\left(y_{1}-y_{2}\right)+Z\left(z_{1}-z_{2}\right)\right]=-\Sigma \Sigma \frac{m_{1} m_{2}}{r_{12}}
$$

This is simply the gravitational potential energy of the mass, say $W$. Thus equation (502) assumes the form

$$
\frac{1}{2} \frac{d^{2} I}{d t^{2}}=2 T+W
$$

an equation first given by Eddington $\dagger$ for the motion of a star-cluster, to which it is also applicable.

189. Let the axes be supposed to move with the Centre of Gravity of the mass. If the mass has neither appreciable mass-motion relative to its Centre of Gravity nor rotation in space, $T$ becomes the kinetic energy of translation of the molecular motion. The energy of internal molecular motion may be supposed to be $\beta T$ where $\beta$ is the usual coefficient of the Kinetic Theory of Gases. In the case of a perfect gas, this is connected with $\gamma$, the ratio of the specific heats of the gas, by the relation

$$
\beta=\frac{2}{3(\gamma-1)}-1
$$

The whole heat-content of the mass of gas, say $H$, is now given by

$$
H=(1+\beta) T,
$$

* Jeans, Dynamical Theory of Gases, 2nd ed. \$§ 181-187.

+ l.c. ante, p. 527. 
and the value of $E$, the total energy, is

$$
E=H+W=(1+\beta) T+W
$$

From equation (504), a condition to be satisfied by a mass of gas in a steady state is $2 T+W=0$, or, in virtue of the equation just obtained,

$$
T(\beta-1)=E \ldots \ldots \ldots \ldots \ldots \ldots \ldots \ldots \ldots(506) .
$$

The special case of $\beta=1$ or $\gamma=\frac{4}{3}$ demands attention. For a mass of gas for which $\gamma=\frac{4}{3}$ in a steady state it appears that $E=0$ independently of the radius of the mass. A small radial expansion of the mass can accordingly take place, the mass passing from one configuration of equilibrium to an adjacent configuration of equilibrium, without change of energy. Thus in any configuration of equilibrium the frequency of one radial vibration is zero.

It follows that on any linear series of configurations of equilibrium along which $\gamma$ varies, there will be a change from stability to instability at the value $\gamma=\frac{4}{3}$, instability setting in through a radial vibration. Gases for which $\gamma=\infty$ are readily found to be stable, whence it appears that masses of gas are radially stable when $\gamma>\frac{4}{3}$ and are radially unstable when $\gamma<\frac{4}{3} *$.

In illustration may be mentioned the period of radial vibration for a mass of gas found by Ritter, subject to certain simplifying assumptions, to be $\dagger$

$$
2 \pi \sqrt{\frac{1}{(3 \gamma-4) \rho}}
$$

where $\rho$ is the mean density in gravitational units.

As a particular case of our result, it appears that a mass of gas for which $\gamma<\frac{4}{3}$ cannot rest in a state of stable equilibrium except when in a state of infinite rarity. This result has been obtained only on the supposition that the ideal gas laws are obeyed throughout. There will be other states of equilibrium in which the density is so great that the ideal gas laws do not hold. A mass of gas for which $\gamma<\frac{4}{3}$ and the total energy $E$ is negative must necessarily fall into one of these latter states of equilibrium.

For a mass of gas for which $\gamma>\frac{4}{3}$, equation (506) requires that $E$ shall be negative; in a steady state the energy is less than that in a state of diffusion at intinity. As such a mass loses energy by radiation, there will be a slow secular decrease of $E$, and therefore a secular increase of $T$. Thus the mass will contract as it gets older and will get hotter at the same time, this process of course continuing until the ideal gas laws are no longer obeyed.

The energy lost by radiation during contraction is equal to the decrease in $E$. This, from equation (506), is equal to $(1-\beta)$ times the increase in $T$,

* Emden has obtained this result by a slightly different method (Gaskiigeln, 1907, Chapter viri).

† Emden, Gaskügeln, p. 481. 
or to $(1-\beta) /(1+\beta)$ times the increase in $H$, the total heat-content of the gas. The total heat generated by contraction falls into two parts - the first part is radiated away; the second is stored up in the gas and goes to increase the total heat-content $H$. We have seen that these two parts are in the ratio $(1-\beta)$ to $(1+\beta)$. Hence

Of the total heat generated by contraction, a fraction $\frac{1}{2}(1-\beta)$ is radiated away, while a fraction $\frac{1}{2}(1+\beta)$ is stored up in the gas.

To form an idea of the way in which the mass gets hotter we suppose that the contraction is a uniform one, so that after an interval of time each length in the mass is reduced by the same fraction $\theta$. The potential energy $W$, which was initially $\Sigma m m^{\prime} / r$, becomes changed to $\Sigma m m^{\prime} / r \theta$, so that the contraction increases $W$ to $1 / \theta$ times its initial value. Since $2 T+W=0$, both before and after the contraction, it follows that $T$ must also have increased to $1 / \theta$ times its initial value. The total heat-content $H$ or $T(1+\beta)$ must have similarly changed. Thus

If a mass of gas contracts uniformly, its density being so low that the ideal gas laws may be assumed to hold, then its heat-content varies inversely as its linear dimensions*.

\section{SPHERICAL MASS OF GAS}

190. To consider secular changes more in detail we shall suppose the mass of gas to have assumed a spherical form, its boundary being a sphere of radius $a$.

Let $T, p, \rho$ denote the temperature, pressure and density at a distance $r$ from the centre, and let the density be everywhere so small that these may be supposed connected by the ordinary gas equation

$$
p=\frac{R}{m} \rho T
$$

where $R$ is the universal gas-constant, and $m$ is the mass of a molecule of the gas. When the matter consists of a mixture of different types of molecules, ions, atoms, electrons, etc. $m$ may be supposed defined by equation (507).

Let $M_{r}$ stand for $4 \pi \int_{0}^{r} \rho r^{2} d r$, the mass inside a sphere of radius $r$; then the condition for mechanical equilibrium is

$$
\frac{d p}{d r}=-\frac{M_{r}}{r^{2}} \rho
$$

Instead of using $r$ as a coordinate, we may more conveniently use $q$, defined by $r=a q$, so that $q$ increases from zero at the centre to unity at the

* This result is obtained much in the form in which I have given it by Poincaré (Leçons sur les Hypothèses Cosmogoniques, p. 95 (footnote) and p. 227) ; it may be obtained more rapidly from a consideration of physical dimensions, $H$ necessarily varying as $\gamma M^{2} / r$. 
surface. In a uniform shrinkage such as we considered in the last section, each element of the gas will retain the same value of $q$ throughout the shrinkage. Changing the variable from $r$ to $q$, equation (508) becomes

$$
a q^{2} \frac{d p}{d q}=-M_{q} \rho
$$

where $M_{q}$ is the mass inside a sphere of radius $r=a q$; this of course remains unaltered throughout shrinkage.

Now let the mass shrink uniformly in a ratio $\theta$ to a new configuration of radius $a^{\prime}$, so that $a^{\prime}=a \theta$. Let $p^{\prime}, \rho^{\prime}, T^{\prime}$ be the new values of $p, \rho, T$. Since the shrinkage is supposed uniform, the density $\rho^{\prime}$ at each point will be $1 / \theta^{3}$ times the old density $\rho$, so that

$$
\rho^{\prime} a^{\prime 3}=\rho a^{3}
$$

Multiplying both sides of equation (509) by $a^{3}$, it appears that the new configuration will be one of equilibrium if

$$
a^{\prime 4} \frac{d p^{\prime}}{d q}=a^{4} \frac{d p}{d q}
$$

at every point. Integrating with respect to $q$, we have as the condition of equilibrium

$$
a^{\prime 4} p^{\prime}=a^{4} p
$$

Dividing by corresponding sides of equation (510) and comparing with equation (507) we find

$$
a^{\prime} T^{\prime}=a T
$$

Thus if a spherical mass of gas shrinks uniformly from an equilibrium configuration, the new configuration will also be one of equilibrium provided the temperature at every point is made to vary inversely as the radius of the sphere.

This is commonly called Lane's law*. The analysis has not shewn that the natural flow of heat will be such that a uniform shrinkage will take place--it merely shews that if, for any reason, a uniform shrinkage does occur, and the new configuration is one of equilibrium, then relation (512) must be satisfied at every point of the mass.

It must be noticed that in this law $T$ is the temperature of a given internal element of the gas, corresponding to an assigned value of $q$. The emission of radiation from the mass comes, not from a single layer corresponding to a single value of $q$, but from a number of layers near to the surface. Thus the law (512) has no application to the temperature of a star as determined from its emission of radiation-this is a different question altogether, to which we shall turn our attention later.

"J. Homer Lane, "On the Theoretical Temperature of the Sun." Amer. Journ. Sci. 53 (1870), p. 57. 
191. The ratio of the specific heats $\gamma$ being assumed uniform throughout the mass, let us introduce a new quantity $k$ defined by

$$
p=k \rho^{\gamma}
$$

Combining this relation with the general equation (507) obtain

$$
\frac{R}{m} T=k \rho^{\gamma-1}
$$

If a configuration is one of adiabatic equilibrium, $k$ will of course have a uniform value throughout. We shall now see that only those configurations are mechanically stable in which $k$ either stays constant or increases at every point as we pass from the centre to the surface.

To see this, let us fix our attention on any two small elements of gas in different parts of the mass. Let the first be of mass $M_{1}$, volume $\delta v_{1}$ and density $\rho_{1}$, and let it be at a point $P_{1}$ at which the pressure is $p_{1}$ and the value of $k$ is $k_{1}$. Then

$$
M_{1}=\rho_{1} \delta v_{1} ; p_{1}=k_{1} \rho_{1}{ }^{\gamma} .
$$

Let the same symbols with suffix 2 refer to the second element.

Let us consider the process of interchanging these two elements, the remainder of the gas remaining undisturbed. To do this, the element $M_{1}$ must be compressed or expanded to a volume $\delta v_{2}$, so that its new density will be $M_{1} / \delta v_{2}$. Let us suppose this contraction or expansion to take place adiabatically, then the final pressure will be

$$
p=k_{1}\left(\frac{M_{1}}{\delta v_{2}}\right)^{\gamma}
$$

If this element can be placed in the cavity $\delta v_{2}$ at $P_{2}$ without creating a disturbance in the remainder of the gas, the pressure just calculated must be equal to the equilibrium pressure at $P_{2}$, and this is given by

$$
p=k_{2} \rho_{2}{ }^{\gamma}=k_{2}\left(\frac{M_{2}}{\delta v_{2}}\right)^{\gamma} \text {. }
$$

The pressures are accordingly equal if

$$
k_{1} M_{1}^{\gamma}=k_{2} M_{2}^{\gamma}
$$

This is the condition that $M_{1}$ can be fitted into the place of $M_{2}$ without disturbance; since it is symmetrical, it is also the condition that $M_{2}$ can be fitted into the place of $M_{1}$ without disturbance. Thus if $M_{1}$ and $M_{2}$ are chosen so that this condition is satisfied, the two elements can be interchanged without any work being done except that done against the gravitational field. Of the two masses, let $M_{1}$ be the one originally nearer to the centre. A condition for the stability of the original configuration is clearly that the work done in any interchange such as that just considered shall be positive, and 
this requires that $M_{1}$ shall be greater than $M_{2}$, or again, from equation (516), that $k_{1}$ shall be less than $k_{2}$.

Thus the necessary and sufficient condition for stability as regards interchange of places of the different elements or layers of the gas is that $k$ shall increase continuously from the centre to the surface.

If, in any configuration, $\partial k / \partial r$ is negative over any range, convective currents will be set up and the various layers will change places, until a new configuration is formed in which $\partial k / \partial r$ will be positive or zero everywhere. And if steady agencies are at work tending to depress the value of $\partial k / \partial r$ over any range to a value below zero, a steady system of convection currents will be set up of amount just sufficient to prevent $\partial k / \partial r$ from falling below zero. The value of $\partial k / \partial r$ will be kept permanently equal to zero over this range, so that $k$ will be constant, and the equilibrium will be adiabatic.

\section{Homologous Series}

192. Uniform contraction of the kind considered in $\S 190$ may be spoken of as "homologous" contraction, the initial and final configurations being homologous. A series of configurations of equilibrium, each of which may be derived from the preceding by homologous contraction, may be called a homologous series.

On a homologous series, the relations (510), (511) and (512) hold for every pair of configurations. Combining these with equation (513) we readily. obtain the further relation

$$
k^{\prime} a^{\prime(4-3 \gamma)}=k a^{(4-3 \gamma)}
$$

Let us examine how many of these homologous series there are. Using the relation $p=k \rho^{\gamma}$, the equation of equilibrium (509) may be put in the form

$$
a q^{2} p^{-1 / \gamma} \frac{\partial p}{\partial q}=-M_{q} k^{-1 / \gamma}
$$

If $M$ the mass and $a$ the radius are given, and $k$ is given as a function of $q$, we are able to start from the surface (at which $p=0, q=1$ and $M_{q}=M$ ) and determine step by step successive values of $p$ up to the centre $q=0$. These values of $p$, since $k$ is given, suffice to determine $\rho$ and $T$ uniquely. Thus given values of $M, a$ and $k$ determine uniquely one equilibrium configuration. We cannot, by this means, ensure that the total mass obtained by integrating $4 \pi \rho r^{2}$ shall be equal to the assumed value of the mass; this difficulty can be met by admitting as configurations of equilibrium a set of configurations having point-masses, positive or negative, at the centre. With this convention, it appears that there are just as many equilibrium configurations as there are sets of values of $a$ and $k, k$ being a function of $q$. 
The different equilibrium configurations may accordingly be specified by relations of the type

$$
k=a^{(3 \gamma-4)} f(q) .
$$

Different configurations are got by varying $a$ and $f(q)$. From equation (517) it is clear that a single homologous series will be obtained by varying $a$ while keeping $f(q)$ unaltered, while the different homologous series correspond to the different functions of $q$. Thus there are just as many homologous series as there are functions of $q$, but only those series are stable for which $\partial k / \partial q$ is everywhere positive.

\section{The Condition for Homologous Contraction}

193. The contraction of a configuration under natural conditions will not in general be homologous; it will be determined by the flow of heat inside the mass. Starting from any assigned real configuration we can calculate the natural changes produced in a mass of gas by the flow of heat and consequent radiation in the following way.

Imagine that each element of the gas is held at rest, and let the natural flow of heat take place for an interval $d t$. the temperature of the different elements being changed thereby, and therefore the pressures also. Next imagine that each element of heat is constrained to remain attached to its particular element of gas, so that the elements of gas can only change adiabatically, and allow these different elements to arrange themselves in equilibrium under their own gravitation. The final configuration will be identical with that which would naturally be reached after a time $d t$.

During the first process, in which heat flows while the gas is held at rest, the flow of heat per unit area across any sphere may be taken to be $-\kappa \partial T / \partial r$, where $\kappa$ is a coefficient which must always be positive, from the second law of thermodynamics. When the whole transfer of heat is by pure conduction, $\kappa$ will of course be the ordinary coefficient of thermal conduction.

The aggregate outward flow of heat per unit time across a sphere of radius $r$, say $E_{r}$, will be

$$
E_{r}=-4 \pi \kappa r^{2} \frac{\partial T}{\partial r}
$$

so that the rise of temperature at a distance $r$ from the centre will be given by

$$
r^{2} \rho C_{v} \frac{\partial T}{\partial t}=\frac{\partial}{\partial r}\left(\kappa r^{2} \frac{\partial T}{\partial r}\right)
$$

This change of temperature will be accompanied by a change in the value of $k$, and since $\rho$ is kept constant this will, from equation (j14), be given by

$$
\frac{1}{k} \frac{\partial k}{\partial t}=\frac{1}{T} \frac{\partial T}{\partial t}
$$


In the second part of the motion, the various elements all change adiabatically, so that $k$ remains the same for each. Thus the whole rate of change of $k$ for the element initially at a distance $r$ from the centre will be given by equations (520) and (519), or by the single equation

$$
\frac{1}{k} \frac{\partial k}{\partial t}=\frac{1}{r^{2} \rho C_{v} T} \frac{\partial}{\partial r}\left(\kappa r^{2} \frac{\partial T}{\partial r}\right)
$$

This equation accordingly determines the rate of change of $k$, in the actual motion, for any element of the mass. Knowing the rate of change of $k$ we know the values of $k$ at the end of the interval $d t$, and these suffice to determine the whole configuration.

194. The final configuration will be homologous with the original one if the initial and final values of $k$ for every element are connected by a relation such as (517). Thus the condition that the contraction shall be homologous may be put in the differential form

$$
\frac{1}{k} \frac{\partial k}{\partial t}=(3 \gamma-4) \frac{1}{a} \frac{d a}{d t}=-\zeta \text { say }
$$

where $\zeta$ is a constant throughout the mass. Comparing with equation (521) we obtain the condition for homologous contraction in the form

$$
\frac{\partial}{\partial r}\left(\kappa r^{2} \frac{\partial T}{\partial r}\right)=-\zeta r^{2} \rho C_{v} T
$$

The total heat-content inside a sphere of radius $r$ may be taken to be

$$
H_{r}=\int_{0}^{r} 4 \pi \rho r^{2} C_{v} T d r
$$

Using this relation and (518), equation (523) becomes

$$
\frac{\partial E_{r}}{\partial r}=\zeta \frac{\partial H_{r}}{\partial r}
$$

At the centre $E_{r}$ and $H_{r}$ both vanish; at the surface they become equal to $E$ and $H$. Hence, eliminating $\zeta$, the condition for homologous contraction may be put in the form

$$
\frac{E_{r}}{E}=\frac{H_{r}}{H}
$$

or, replacing $E_{r}$ by its value,

$$
-4 \pi \kappa r^{2} \frac{\partial T}{\partial r}=\frac{H_{r}}{H} E
$$

\section{The Permanent Homologous Series}

195. If equation (525) holds throughout the life of a mass of gas, its whole motion will be along a single homologous series. The equation inust of course be true for all values of $a$ and $q$. Now during homologous contraction $r^{2} \partial T / \partial r$ is, from equation (512), a function of $q$ only, as of course is also the fraction $H_{r} / H$. The total emission $E$ is a function of $a$ only. 
Thus equation (525) can only be satisfied if $\kappa$ is of the form

$$
\kappa=(\text { a function of } a) \times(\text { a function of } q)
$$

This requires that as the gas contracts homologously, $\kappa$ shall change in the same ratio at all points of the mass.

In a mass of hot gas it seems highly probable that the transfer of heat is effected mainly by radiation rather than by ordinary gaseous conduction*. Except close to the surface of a gaseous mass it is found that, corresponding to a temperature gradient $\partial T / \partial x$, there is a flow of radiant energy per unit area of ainount $\dagger$

$$
-\frac{16 \sigma T^{3}}{3 c \rho} \frac{\partial T}{\partial x}
$$

where $\sigma$ is Stefan's constant $\left(5.32 \times 10^{-5}\right)$ and $c$ is the coefficient of opacity of the gas, this being such that on passing a distance $x$ through the medium at density $\rho$, a beam of light is diminished in intensity in the ratio $e^{-c \rho x}$. This flow of heat may be put in the form

$$
-\kappa \frac{\partial I}{\partial x}
$$

where the value of $\kappa$ in heat units is

$$
\kappa=6 \cdot 8 \times 10^{-12} \frac{T^{3}}{c \rho}
$$

This value of $\kappa$ is so much greater than any known coefficient of ordinary gaseous conduction, that it appears to be legitimate to assume, as an approximation, that the whole transfer of heat is radiative.

As homologous contraction proceeds, $T^{3}$ and $\rho$ each vary as $1 / a^{3}$, so that $T^{3} / \rho$ remains constant. Thus if we assume $c$ to be independent of the density and temperature, $\kappa$ will be unaffected by homologous contraction, and equation (526) is satisfied through $\kappa$ being a function of $q$ only.

A permanent homologous series now becomes possible; it is defined by equation (525). In this equation $\kappa, r^{2} \partial T / \partial r$ and $H_{r} / H$ are unaffected by homologous contraction. It follows that $E$ is unaffected by homologous contraction-the emission of radiation remains always the same.

196. It will naturally be suspected that the permanent homologous series whose existence has now been demonstrated represents a stable final state in the sense that a configuration not initially on this series will gradually approach it as contraction proceeds. A rigorous formal proof of this is not easily constructed, but the general truth of the proposition can be seen as follows. 
Consider a configuration in which equation (525), the condition for homologous contraction, is satisfied everywhere except in the neighbourhood of some point $P$. Let there be an excess of heat to the right of $P$ and a deficiency of equal amount to the left of $P$. Then the temperature gradient from right to left at $P$ will be in excess of that determined by equation (525), so that the flow of heat from right to left will be greater than that in the permanent homologous series. This flow may be regarded as made up of two parts : first a flow of amount given by equation (525), and, second, a flow in the neighbourhood of the point $P$, this latter flow being necessarily from right to left. The first flow results in a homologous contraction of the whole mass; the second flow reduces the excess of heat to the right of $P$ and reduces also the deficiency to the left. Thus the final state of the mass is nearer to the permanent series than was the original state.

By an obvious extension of this argument it can be seen, although not by strict mathematical proof, that any configuration not on the permanent homologous series always moves towards that series as contraction proceeds. Thus a mass of gas which has been contracting for a sufficient length of time may be assumed to be on the permanent homologous series.

\section{Stellar Radiation}

197. We have considered the mechanism by which heat is brought to the surface of a star, but have not yet considered the mechanism by which it is radiated away.

To an approximation which will prove to be good enough for our present purpose, the radiation from a gaseous mass may be thought of as the free radiation into space from a definite " photosphere," this being roughly identical with the deepest layer of gas to which we can see from outside*.

As a mass of gas contracts, the depth of the photosphere below the surface will naturally diminish. On account of the increase of density produced by the lateral contraction of the surface layers, the depth of the photosphere must decrease more rapidly than the radius $a$. Thus when a mass of gas moves through a series of homologous configurations, the various photospheres will not form homologous points on this series.

The position of the photosphere may be supposed to be determined by the condition that the mass per unit area between it and the surface of the star is always the same quantity $\mu$. Thus if $g$ is the value of gravity at the surface, the pressure at the photosphere will be $\mu g$, and the temperature will be given by

$$
\frac{R}{m_{1}} T_{1} \rho_{1}=\mu g
$$

\footnotetext{
* For a more exact treatment, see Monthly Notices R.A.S. 78 (1918), p. 28.
} 
the quantities with suffix 1 referring to the photosphere. The total emission of radiation is now

$$
E=4 \pi r_{1}^{2} \sigma T_{1}^{4}=4 \pi \sigma M \mu\left(\frac{m_{1}}{R}\right) \frac{T_{1}^{3}}{\rho_{1}}
$$

On the permanent homologous series, $E$ must remain unaltered by contraction, so that $m_{1} T_{1}^{3} / \rho_{1}$ must also remain unaltered by contraction. In a homologous contraction $T^{3} / \rho$ remains unaltered for homologous points, and $m$ may be supposed to remain unaltered. In a mixture of gases, there must be a certain amount of rearrangement when a mass contracts, and the increase of temperature must alter the degree of ionisation when any is present; these complications prevent a strictly homologous contraction occurring at all, but we may, as an approximation, neglect them and suppose that the mass can contract homologously, so that $m$ remains always the same function of $q$. Assuming this, $m T^{3} / \rho$ will be unaltered by contraction, whence it follows that $m_{1} T_{1}^{3} / \rho_{1}$ will remain unaltered for the photosphere if, and only if, $m T^{T^{3}} / \rho$ has initially a uniform value throughout the range within which the photosphere moves.

This range may be regarded as infinitesimal in comparison with the radius of the star, so that the condition just found may be put in the form of a boundary condition, namely that at the boundary of the star

$$
\frac{d}{d r}\left(\frac{m T^{3}}{\rho}\right)=0
$$

This boundary condition together with the differential equation (525) suffice to determine uniquely the series of permanent homologous configurations.

\section{Mechanical Stability}

198. We have not so far discussed the question of mechanical stability of the permanent homologous series.

From the fundamental equations $p=k \rho^{\gamma}$ and $p=(R / m) T \rho$, we readily find that

$$
\frac{m T^{3}}{\rho}=\frac{m^{4}}{R^{3}} k^{4 / \gamma} p^{(3-4 / \gamma)} .
$$

We are only considering masses for which $\gamma>\frac{4}{3}$, so that $p^{(3-4 / \gamma)}$ will increase with $p$ as we pass inwards. It follows that on the permanent series $\mathrm{km}^{\gamma}$ will decrease as we pass inwards.

Strictly speaking, a permanent homologous series only exists when $m$ remains constant throughout contraction-i.e. when no ionisation or chemical change occurs. In such a case $m$ will increase as we pass inwards, so that $k$ must decrease as we pass inwards. Thus $\partial k / \partial r$ will be positive everywhere, which is the condition for mechanical stability without convection. 
When ionisation is present, the value of $m$ may decrease as we pass inwards to the more highly ionised layers, and convection currents may be set up near the surface.

The investigation of the mechanical stability of the inner layers presents a more difficult problem. It can however be shewn* that in general the permanent series of homologous configurations will satisfy the conditions for mechanical stability without convection currents being set up except near the surface, an exception possibly arising when $\gamma$ is very close to the value $\gamma=\frac{4}{3}$.

\section{SUMMARY}

199. We may now summarise the changes which are to be expected in a mass of gas in consequence of the continual emission of radiation from its surface, making for the moment the somewhat illegitimate assumptions that the mass obeys the laws of a perfect gas, that $\gamma$, the ratio of the specific heats, has a uniform value throughout, and that the opacity $c$ is constant throughout.

So long as the ideal gas laws are supposed to be obeyed, masses of gas for which $\gamma<\frac{4}{3}$ cannot condense into spherical masses in stable equilibrium. Masses for which $\gamma>\frac{4}{3}$ contract and become hotter as radiation proceeds. We have seen, although by something short of strict proof, that they are likely to approach to a definite series of homologous configurations, on which, subject to the assumptions just mentioned, the emission of radiation $E$ remains constant as contraction proceeds.

Our hypothetical mass has been assumed to obey the ideal gas laws throughout, so that the laws we have discovered must only be expected to describe the changes in a star so long as its density remains small. There will be a stage later than those we have considered in which the laws are not obeyed owing to the gas laws being substantially departed from. Still later there will come a stage when the mass has contracted so far that further contraction becomes impossible; its temperature will now fall steadily without contraction taking place. The emission is still given by equation (530), but $r_{1}$ is now approximately constant, so that $E$ falls as $T_{1}^{4}$.

In fig. 40 , let the temperature $T_{1}$ of the photosphere be represented by the abscissa and the total emission of energy by the ordinate. In the earliest stages in which the ideal gas laws hold, the temperature $T_{1}$ goes on increasing as contraction proceeds, while the emission remains constant. Thus the relation between $T_{1}$ and $E$ is represented by a horizontal line such as $P Q$ described in the direction of $T_{1}$ increasing. In the last stages in which contraction can proceed no further the relation between $T_{1}$ and $E$ is that $E \propto T_{1}^{4}$. 
and this relation may be represented by a curve such as $R O$ described in the direction of $T_{1}$ decreasing. In the intermediate stage in which the gas does not obey the gas laws, but is still highly compressible, the law relating $E$ and $T_{1}$ cannot be precisely specified. Clearly, however, the curve by which it is represented in fig. 40 must depart asymptotically from $P Q$ and approach asymptotically to $R O$. Thus the actual sequence of changes in $E$ and $T_{1}$ will

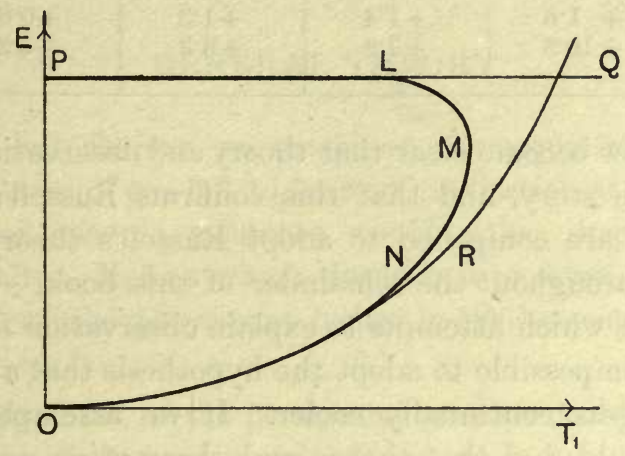

Fig. 40.

be represented by a curve such as PLMNO in fig. 40. The mass of gas starts at a low temperature, increases to a maximum temperature and cools again. Meanwhile the emission of energy will remain constant until approximately the stage at which the maximum temperature is attained, after which it falls steadily and rapidly to zero.

200. It will at once be seen that this theoretical result describes exactly Russell's theory of the order of stellar evolution, of which a brief account was given in $§ 13$. Russell, while pointing out that his theory was in accordance with theoretical principles, based the evidence for it mainly upon a diagram of observed absolute magnitudes of stars*. In this diagram the spectral class, giving a rough measure of the temperature, was taken as abscissa while the ordinate measured the absolute magnitude. The stars in the redder spectral classes $(M, K, G)$ were found to fall into two detached, or nearly detached, groups. In an upper group the absolute magnitude was approximately independent of spectral type; in the lower group it varied rapidly with spectral type, falling off towards the red end of the scale. These two groups of stars form what Russell calls "giant" and "dwarf" stars respectively.

Clearly Russell's diagram provides powerful confirmation of our theoretical diagram shewn in fig. 40, the stars along the branch $P L$ being giant stars, and those along the branch $O N$ being dwarfs. Further confirmation has recently been afforded by the investigations of Adams and Joy † already referred to in $\S 13$. Here 500 stars are considered and these are again found to fall into

* Nature, 93, p. 242 (May 7, 1914), and Popular Astron. 22 (1914), p. 11.

+ Astrophys. Journ. 46 (1917), p. 334. 
two classes-Russell's giant and dwarf stars. In each class the absolute magnitudes are found to cluster fairly closely about a maximum of frequency, the absolute magnitudes of these maxima being as follows:

\begin{tabular}{|c|c|c|c|c|c|}
\hline Spectral type & $M a-M d$ & $K 9-K 4$ & $K 3-K 0$ & $G 9-G 0$ & $F 9-F 0$ \\
\hline $\begin{array}{ll}\text { Giant } & \ldots \\
\text { Dwarf } & \ldots\end{array}$ & $\begin{array}{l}+1.6 \\
+10.8\end{array}$ & $\begin{array}{l}+1 \cdot 4 \\
+7 \cdot 8\end{array}$ & $\begin{array}{l}+1 \cdot 3 \\
+6 \cdot 3\end{array}$ & $\begin{array}{l}+0 \cdot 6 \\
+5 \cdot 3\end{array}$ & $\begin{array}{l}+1 \cdot 1 \\
+4 \cdot 1\end{array}$ \\
\hline
\end{tabular}

201. It has now become clear that theory and observation agree in telling precisely the same story, and that this confirms Russell's theory entirely. This being so we are compelled to adopt Russell's theory of the order of stellar evolution throughout the remainder of this book. Indeed in a book such as the present, which attempts to explain observation in terms of theory, it would be quite impossible to adopt the hypothesis that a mass of gas starts its life hot and gets continually cooler. If we attempted to adopt this hypothesis we should find that theory and observation would be pulling in different directions at every stage of our investigation.

At the same time we are not compelled altogether to abandon the older view of stellar evolution. Our theoretical results have applied to a mass of gas starting from a condition of extreme diffusion, not necessarily to an actual star; they have traced out the path along which the evolution of a star must progress but nothing has shewn that a star may not be born half-way along this path. So far as our theoretical results go, it is still quite possible for every star in the universe to have been born as a $B$-type star; so far as the observations of Russell and of Adams and Joy go, it is still quite possible for ninety-nine per cent. of the stars in the universe to have been born as $B$-type stars. We shall return to these questions in the next chapter.

As regards the main evolutionary problem, we have learnt that a mass of gas, at least so long as it is of low density, will increase in density and also in temperature. The earliest matter is that of lowest density. We have accordingly been led by a theoretical path to the view which we described as the "Theory of nebulous origin" in our Introductory Chapter, but the theory must now be supposed to refer to the gas out of which the star originates rather than to the star itself in the form of a star. 


\section{CHAPTER IX}

\section{THE EVOLUTION OF ROTATING NEBULAE}

\section{GENERAL THEORY}

202. In the last chapter we examined the sequence of changes which would occur in a mass of gas left to its own gravitation at rest in space. We found that matter once in existence would either disperse into space or contract continually. Masses which disperse into space would have but a transitory existence; the permanent bodies in the heavens must be supposed to be contracting.

We accordingly think of the permanent astronomical bodies as beginning existence in a state of extreme rarity. If one such mass existed alone in the universe, it would tend to assume a spherical form if devoid of rotation, or a spheroidal or pseudo-spheroidal form if endowed with a small amount of rotation. Observation, however, does not encourage the view that the whole universe originated out of a single mass of gas ; we shall find it more profitable to think of a number of separate and detached nebular masses as forming the earliest stage in the process of cosmic evolution.

Whether these masses ought to be thought of as being originally endowed with motion, either of translation or of rotation, we do not know. In any case they must in time be set into motion by their mutual gravitational attractions. As the masses move under these attractions there will be occasions in which two of the masses will pass fairly close to one another, and the tidal couples raised in this way will necessarily set both masses into slow rotation, the mechanism being that which has already been considered in $\$ 131$. Rotations set up in this way would doubtless be of very small amount at first, but they will increase with the shrinkage of the mass in accordance with the law of conservation of angular momentum.

In this way we are led quite naturally to the consideration of a number of separately-moving and rotating gaseous masses as providing the initial material for our problem of cosmogony. The problem of tracing out the history of the astronomical universe is seen to be closely related to the abstract problem of following out the sequence of events in a rotating mass of gas.

203. This abstract problem has already been solved in certain ideal cases. Primarily it has been solved for a mass of gas in adiabatic equilibrium, this being determined by the relation $p=k \rho^{\gamma}$ in which $k$ and $\gamma$ are supposed constant throughout the mass. 
In the chapter preceding this we found that an actual mass of gas would not arrange itself in adiabatic equilibrium while contracting under its own gravitation. In adiabatic equilibrium the quantity denoted by $k$ is everywhere constant; in an actual contracting mass, we found that $k$, defined by the equation $p=k \rho^{\gamma}$, would increase continually from the centre to the edge. Except possibly in a comparatively shallow surface layer, there is no convection, and the result of this must be that the heavier elements tend to congregate at and near the centre, while the lighter elements form a surrounding atmosphere. That this actually occurs is made probable by the results of spectroscopic examination of nebulous masses. Campbell* found that in a number of nebulae the different gases are not uniformly distributed throughout the nebular structure. In some, as for example the Orion and Trifid nebulae, the hydrogen is definitely found to extend further out than the other chemical elements. Wright has found that in the planetary nebulae the helium always favours the central nucleus more than the hydrogen and nebulium do; in some cases the helium is entirely confined to the central nucleus. Campbell again has found that in a slitless spectrum of the small planetary nebula N.G.C. $418_{2}$, the $H_{\beta}$ line of hydrogen forms a circle of 14:" diameter, while the first and second green nebulium lines form circles of diameters only $11^{\prime \prime}$ and 9 " respectively.

Thus both theory and observation agree in suggesting that the "adiabatic" model does not altogether give a faithful representation of actual conditions. The quantity $k$ is not constant throughout the mass but increases from centre to edge, while the different chemical constituents are not thoroughly mixed up; the heavier elements have sunk towards the centre.

We are accordingly led to inquire to what extent the theoretical results which were obtained from a study of the "adiabatic" model may be expected to require modification for an actual mass of gas.

204. In our study of the adiabatic model, $p$ and $\rho$ were supposed connected by the relation

$$
p=k \rho^{\gamma},
$$

and $k$ and $\gamma$ were supposed constant throughout the mass, but it was nowhere found necessary to attach any special physical meanings to $k$ and $\gamma$. From: this general relation we readily find that

$$
\frac{\partial \log p}{\partial \log \rho}=\gamma
$$

since $k$ was supposed kept constant. Thus $\gamma$ might have been regarded merely as a symbol for $\partial \log p / \partial \log \rho$. 
In an actual mass of gas in which $G$ denotes the ratio of the specific heats at any point, $p$ and $\rho$ may be supposed connected by the relation

$$
p=k \rho^{G},
$$

in which $k$ will vary from point to point. We accordingly find

$$
\frac{\partial \log p}{\partial \log \rho}=G+\frac{\partial \log k}{\partial \log \rho} .
$$

If we use the symbol $\gamma$ to denote $\partial \log p / \partial \log \rho$, this becomes

$$
\gamma=G+\frac{\partial \log k}{\partial \log \rho}
$$

We have seen that, as we pass along the radius of an actual mass of gas from the centre to the edge, $k$ will continually increase, or at most remain constant, while $\rho$ will continually decrease. Thus $k$ and $\rho$ change in opposite senses, so that $\partial \log k / \partial \log \rho$ will be negative at every point of the gas, and the effect of the non-constancy of $k$ will be to decrease the value of $\gamma$.

The same result may be obtained by noticing that the equilibrium of an isothermal mass of gas is the same as that of a gas in adiabatic equilibrium with $\gamma=1$, while in an actual mass of gas conditions are such that the equilibrium is intermediate between adiabatic and isothermal equilibrium.

In our study of the "adiabatic" model, we found that the series of equilibrium configurations was of the same general type for all values of $\gamma$ less than $2 \frac{1}{5}$. In an actual mass of gas, $G$, the true ratio of the specific heats must be less than $1 \frac{2}{3}$, and the value of $\gamma$, as determined by equation (533) must be still less on account of the non-constancy of $k$. Thus it seems permissible to assume that the sequence of configurations in an actual gas would be of the same type as those in an "adiabatic" mass in which $\gamma<2 \frac{1}{5}$. And this series of configurations, as we saw, consisted of spheroids when the rotation was small, these giving place to pseudo-spheroids for larger rotations, and ultimately giving place to a lenticular figure with a sharp edge from which matter was thrown off.

The effect of the sinking of the heavier chemical elements to the centre is easily allowed for. It results in an excess of central condensation of mass, and this may be allowed for by supposing the mass to approach more nearly to Roche's model than would be the case if the gas were of uniform composition. We have just seen that the motion of the gas, even without allowing for this excess of central condensation, will in its main features be the same as that of Roche's model. The resemblance of the motion to that of Roche's model will be still closer when central condensation of mass is taken into account. 
205. In two cases we have calculated the value of $\omega^{2} / 2 \pi \bar{\rho}$ at which the sharp edge forms on the equator of a rotating mass of gas. In $§ 152$, discussing Roche's model, or an adiabatic gas for which $\gamma=1 \frac{1}{5}$, we found the critical value of $\omega^{2} / 2 \pi \bar{\rho}$ to be given by

$$
\frac{\omega^{2}}{2 \pi \bar{\rho}}=0.36075
$$

And in $§ 183$, discussing a mass of adiabatic gas for which $\gamma$ had the highest value consistent with the formation of a sharp edge, namely $2 \frac{1}{5}$, we found the critical value of $\omega^{2} / 2 \pi \bar{\rho}$ to be given, approximately, by

$$
\frac{\omega^{2}}{2 \pi \bar{\rho}}=0.31 \text {. }
$$

These limits for $\gamma$ are much wider than those which can occur in an actual mass of gas, for which $\gamma$ must be greater than $1 \frac{1}{3}$ and less than $1 \frac{2}{3}$. The critical values of $\omega^{2} / 2 \pi \bar{\rho}$ are so comparatively close together that it seems safe to assume that for an actual gas the critical value must be somewhere between the two theoretical critical values, and probably considerably nearer to the former than to the latter. For purposes of rough calculation we shall suppose that the critical value is given by

$$
\frac{\omega^{2}}{2 \pi \bar{\rho}}=0.35
$$

\section{Comparison with Observation}

206. The course of events in our typical nebulous mass of gas may now be briefly recapitulated. It has been supposed to come into. existence in an entirely unknown way, probably forming at first an irregular mass of comparatively cold gas at a very low density. This will contract under its own gravitation and would in time assume a spherical form except that it is repeatedly being disturbed by tidal forces from passing masses. The effect of these is to set up a slow rotation which continually increases as the mass contracts. The mass assumes at first a spheroidal form, then a pseudospheroidal form, until, when the rotation reaches an amount given by equation (534), a sharp edge is formed round the equator. The figure of the mass is now lenticular in shape, and any further contraction results in matter being thrown off from the periphery or equator of the lens.

Fig. 41 shews the theoretical cross-sections which have been found for two rotating masses of gas at the instant at which the sharp edge is first formed, the two figures corresponding to the two extreme values of $\gamma, \gamma=1 \frac{1}{5}$ and $\gamma=2 \frac{1}{5}$ respectively. The next stage in the motion will consist of the ejection of streams of matter from the sharp edge.

On comparing these figures with those of actual nebulae shewn on Plate III, we at once notice the similarity in the cross-sections of the two sets of figures, 


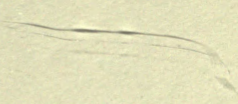

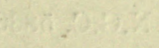




\section{Plate III}

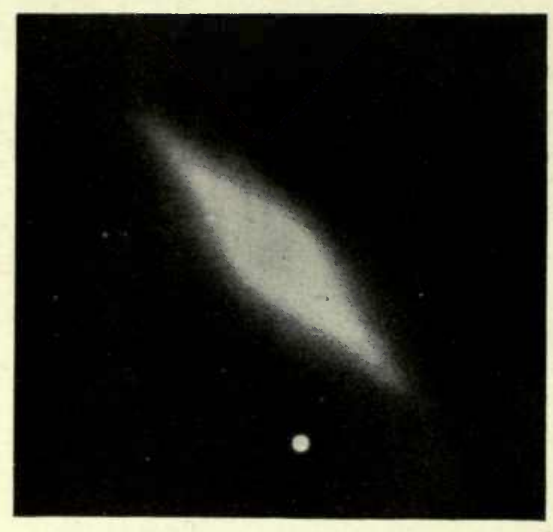

N.G.C. 3115

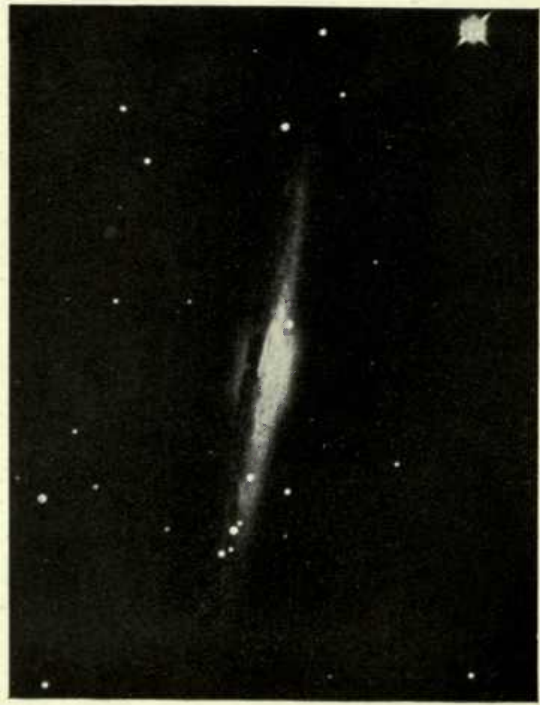

N.G.C. 5746

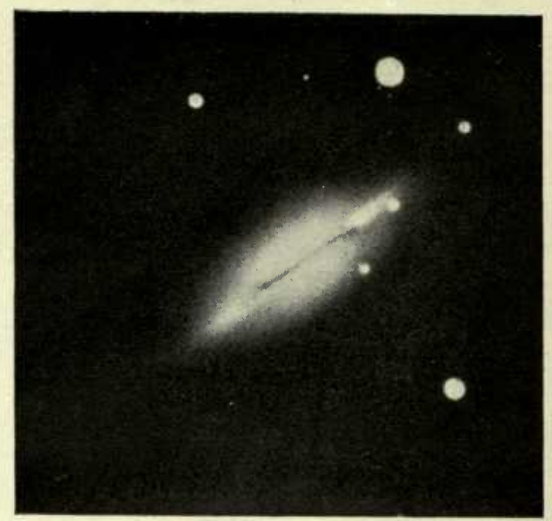

N.G.C. 5866

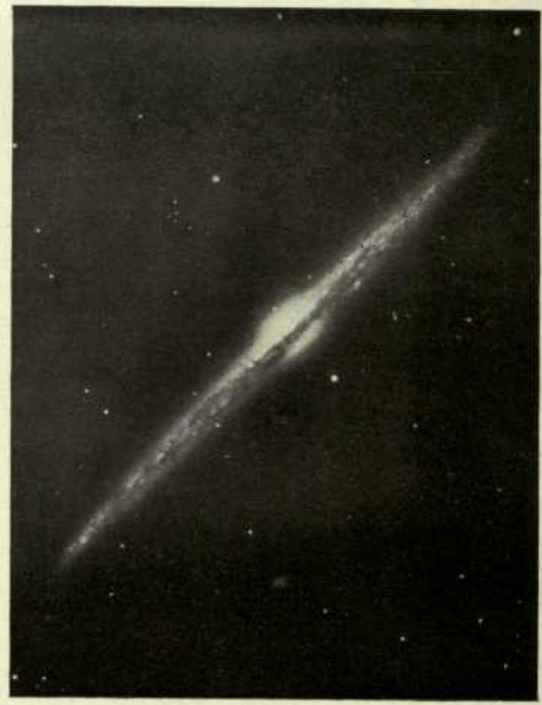

N.G.C. 4565 (H. v. 24)

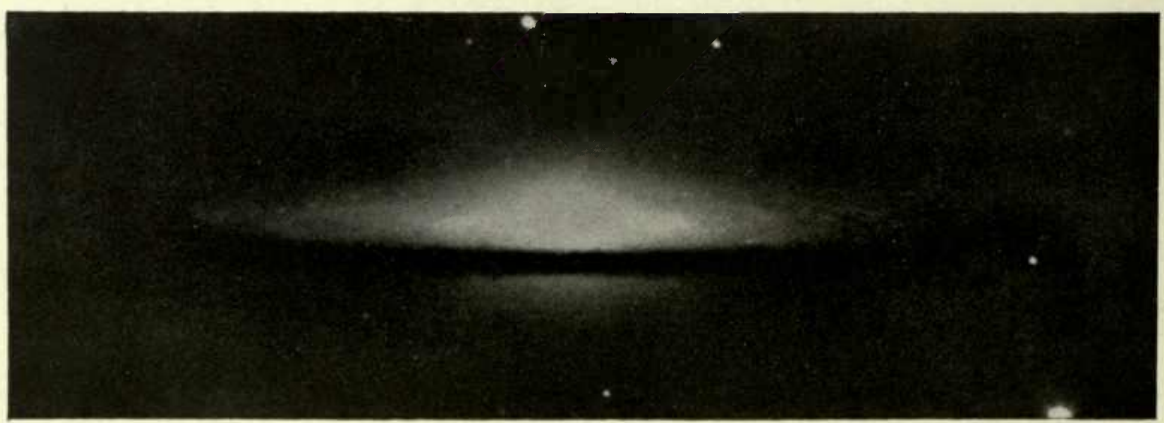


and it seems permissible to identify these actual figures, at least conjecturally and tentatively, with the theoretical figures shewn in fig. 41. On this suggested interpretation then, the nebulae shewn on Plate III are masses of gas, or possibly clouds of dust, in rotation. Rotation has actually been
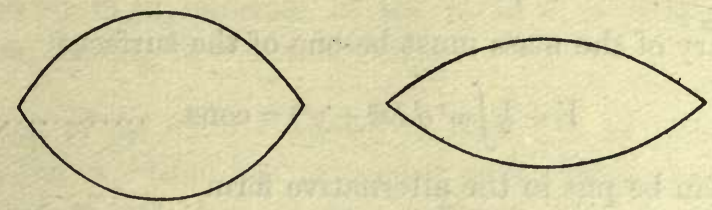

Fig. 41.

observed spectroscopically in some nebulae, as for instance the last nebula shewn on Plate III, namely N.G.C. 4594 and the Andromeda Nebula M. 31, while it would be difficult to imagine any cause other than rotation which could account for the flattened symmetrical shape of the remainder.

One point of difference perhaps appears between the theoretical and the actual curves. The photographs shew curves which are somewhat less blunt near the equatorial edge than the theoretical curves; in some of the photographic curves the boundary appears to become convex to its equatorial section at points near this edge.

The theoretical curves have been obtained on the supposition that the angular velocity has everywhere the same value; the mass has been assumed to rotate as a rigid body. If shrinkage were an infinitely slow process, or if the action of viscosity were infinitely rapid, a rotating and shrinking mass would rotate at every instant like a rigid body, but in nature viscosity acts so slowly in a mass of gas that we have to contemplate the possibility of uniform rotation never becoming established*.

To examine the effect of non-uniform rotation, we return to the fundamental equations (386) to (388) of Chapter VII. Assuming the pressure to be a function of the density, these may be expressed in the form

$$
\frac{\partial}{\partial x} \int \frac{d p}{\rho}=\frac{\partial V}{\partial x}+\omega^{2} x, \text { etc. }
$$

and these will be the equations of relative equilibrium even when $\omega^{2}$ varies from point to point in the mass.

Differentiating the $x, y$ equations with respect to $y, x$ respectively and subtracting, and treating the two other pairs similarly, we find

$$
x \frac{\partial \omega^{2}}{\partial y}=y \frac{\partial \omega^{2}}{\partial x} ; \frac{\partial \omega^{2}}{\partial z}=0
$$

so that $\omega^{2}$ must be a function of $x^{2}+y^{2}$. Thus the surfaces of constant

\footnotetext{
* Cf. Poincaré, Leçons sur les Hypothèses Cosmogoniques, p. 28.
} 
angular velocity must be circular cylinders about the axis of rotation*. This being the case, the three equations of equilibrium (535) have the common integral

$$
\int \frac{d p}{\rho}=V+\frac{1}{2} \int \omega^{2} d\left(x^{2}+y^{2}\right)
$$

and the boundary of the mass must be one of the surfaces

$$
V+\frac{1}{2} \int \omega^{2} d\left(x^{2}+y^{2}\right)=\text { cons. }
$$

This equation can be put in the alternative form

$$
V+\frac{1}{2} \overline{\omega^{2}}\left(x^{2}+y^{2}\right)=\text { cons. }
$$

where $\overline{\omega^{2}}$ is used to denote the mean value of $\omega^{2}$ at all points of the equatorial plane inside a circle of radius $\left(x^{2}+y^{2}\right)^{\frac{1}{2}}$.

A nebula shrinking homologously in the way described in the last chapter would increase its angular velocity at the same rate throughout, so that uniformity of angular velocity, if once established, would not be disturbed by homologous shrinkage. But if a nebula has shrunk from an approximately isothermal condition to one in which there is a rapid temperature gradient from surface to centre, then the outer parts will have fallen in much more than the inner parts. In the absence of any viscosity at all the conservation of angular momentum would require that the parts furthest from the axis of rotation should have a greater angular velocity than the parts nearer the centre. In such a case $\omega^{2}$ would increase with $x^{2}+y^{2}$, and when viscosity acts, but without sufficient power to produce absolutely uniform rotation, this increase of $\omega^{2}$ with $x^{2}+y^{2}$ will still persist to some extent.

Thus in a natural nebula, or other rotating mass of gas, we should expect $\omega^{2}$ to increase as we pass from the centre outwards. This is the type of motion observed in the sun, while the measurements of Pease $\dagger$ on the rotation of the Andromeda nebula suggest a similar increase of angular velocity with distance from the centre.

It will be readily seen that the effect of such a variation in $\omega^{2}$ is to change the critical equipotential from the theoretical curves shewn in fig. 41 in the direction towards the photographic curves exhibited in Plate III. The general principle is sufficiently illustrated by a consideration of Roche's model $(\$ 152)$. Let $\varpi^{2}$ stand for $x^{2}+y^{2}$ and let $\varpi_{0}$ be the value of $\varpi$ at the sharp edge of the critical equipotential. The equation of this equipotential is seen from equation (536) to be

- Poincaré, l.c. p. 32.

$$
M\left(\frac{1}{r}-\frac{1}{\varpi_{0}}\right)=\frac{1}{2} \int_{\varpi}^{\varpi_{0}} \omega^{2} d \varpi^{2} .
$$

+ Nat. Acad. Sciences, 4 (1918), p. 21. It would perhaps be straining the evidence to regard the variation of $\omega$ with distance as being definitely established, the more so as Pease himself does not interpret his measurements in this way. 
The value of $\varpi_{0}$ depends only on the value of $\omega^{2}$ at the boundary of the figure, being determined by the relation $M=\omega^{2} \omega_{0}^{3}$, where $\omega$ is the angular velocity at the sharp edge $\sigma=\varpi_{0}$. Clearly a lessening of $\omega^{2}$ as we pass inwards from the sharp edge $\omega=\varpi_{0}$ will result in a lessening of the value of the integral, and so in an increase in the value of $r$ corresponding to any given value of $\varpi$, leading to the result stated.

207. In most of the nebulae shewn in Plate III, as indeed in almost all known nebulae, the evolution has proceeded somewhat beyond the formation of the sharp edge; matter has, on our interpretation, already been ejected from this edge. Thus before attempting a fuller interpretation of observed nebulae, we ought to return to the theoretical problem, and attempt to trace out the motion which is to be expected after the formation of the sharp edge.

Let us consider first what may be expected to happen to the main mass. The ejected matter, as soon as it is of sufficient amount, will exert gravitational forces on the remainder of the mass, but in the earliest stages of the motion, so long as the total mass of ejected matter is still small, the gravitational field set up by this ejected matter may be neglected, and the main mass may be supposed to be acted on solely by its own gravitation. As the mass slowly shrinks, the radius of the critical circle on which centrifugal force just balances gravity will also slowly shrink. Matter will be gradually thrust across this circle much in the same way in which water would gradually drip over the edge of a slowly shrinking cup*.

During this early stage, it is impossible for the sharp edge ever to disappear. For if at any instant it did so, the main mass would at once become again a mass rotating freely in space under its own gravitation; the slightest amount of further shrinkage would produce an increase of rotation which would again result in the formation of a new sharp edge, and the ejection of more matter. Thus the motion is one in which the main mass shrinks, keeping always a sharp edge. Just enough matter must be ejected through this edge for the main mass to remain always of the form of the critical figure of equilibrium, the condition for this being that equation (534) shall always remain satisfied. In the motion before the sharp edge was formed, the angular momentum of the mass remained constant, so that $\omega^{2} / 2 \pi \bar{\rho}$ increased

* Poincaré (Leçons, p. 25) appears to follow Roche in believing that fairly violent oscillations might be set up in the main mass, so that the rate of ejection of matter would periodically overshoot the amount necessary for equilibrium. Thus after an eruption there ought to be a period of quiescence until the angular velocity has again overtaken the ejection of matter; after this another period of eruption, another period of quiescence and so on.

Although every opinion expressed by Poincaré must be considered with the greatest respect, it is extremely difficult to find any dynamical justification for these supposed violent oscillations. The oscillations of the main mass appear to be thoroughly stable (this has been rigorously proved for Roche's model in $\$ 150$ ), and it is hard to find any agency capable of forcing oscillations of any but infinitesimal amplitude.

J. C. 
steadily. In the motion after the sharp edge has formed, both the angular momentum and the mass decrease owing to loss from the sharp edge. The lost particles are those for which the angular momentum is greatest, so that a result of this loss is that the ratio of angular momentum to mass decreases. The decrease has to be at just such a rate that $\omega^{2} / 2 \pi \bar{\rho}$ remains constant; this condition determines the rate at which matter is thrown off from the edge of the main mass.

During the later stages of the motion it will not be legitimate to neglect the gravitational field set up by the ejected matter. The ejected matter will exert gravitational forces on the main mass, in directions such as to reinforce centrifugal force and to neutralise the gravitational attraction of the main mass. Thus each element of matter which is ejected provides in itself a force tending to increase the rate of ejection of matter. It is at once clear that the motion of ejection must ultimately become of a "cataclysmic" nature; it cannot cease until either the main central mass has become entirely disintegrated, or until the physical conditions of the problem change in some way.

\section{The Theory of Laplace and Roche}

208. Laplace and Roche both imagined the mass to throw off matter for a time and then to stop. The ejected matter was supposed to form an annulus or ring out of which planets were ultimately formed. Roche went further and imagined a continual series of alternations in the physical condition of the mass, so that the mass threw off a succession of detached rings at distinct intervals *.

Clearly the ejection of matter at the sharp edge can cease only when the condition for the existence of a sharp edge and the condition of the constancy of angular momentum (without ejection of matter) become identical. So long as the ideal gas laws are obeyed, the former condition requires that $\omega^{2} / \bar{\rho}$ shall remain constant, while the latter requires that $\omega^{2} / \bar{\rho}$ shall vary as $(\bar{\rho})^{\frac{1}{3}}$. Thus it is quite clear that the ejection of matter, when once it has started, can never cease so long as the ideal gas laws are obeyed. When these laws are substantially departed from, the ejection of matter may cease, but so long as we regard our nebulous mass as being approximately a perfect gas, the emission of matter from the sharp edge will be a continuous process.

209. Let us now examine the motion of the matter thrown off from the sharp edge. Each particle or molecule, as it leaves the edge, will have a tangential velocity in space equal to $\omega r$, arising from the rotation of the mass in space, and superposed on to this velocity there may be a velocity of ejection 
arising from the motion of the matter inside the mass, and also a velocity of molecular motion when the nebula is gaseous.

As the problem is one of great complexity, it will be well to separate the difficulties, and consider first the motion to be expected when the velocity $\omega r$ exists alone or preponderates enormously over the other velocities. This condition would be approximately satisfied if we could regard the matter of the nebula as fluid, although it must be remembered that in actual fact a sharp edge could not form on a fluid mass in rotation unless there was a considerable central condensation of mass.

Since the sharp edge is determined by the condition that centrifugal force shall exactly balance gravity, it is clear that the tangential velocity $\omega r$ will be exactly that required for the description of a circular orbit. Thus at first the ejected particles will form a chain of infinitesimal satellites in contact with the main mass.

As the main mass shrinks, this contact will of course be broken. Moreover, with the shrinkage of the main mass, the gravitational field will change, so that the velocity of the satellites will no longer be that appropriate to the description of circular orbits. Clearly a shrinkage of the main mass will result in a lessening of the radial gravitational force at a fixed distance $r$, so that the tangential velocity $\omega r$ will become greater than the velocity for a circular orbit at distance $r$, and the ring will begin to expand. Throughout this motion the ring will remain circular, its angular velocity being determined by the constancy of its angular momentum; $\omega r^{2}$ will always be equal to the value at the instant of projection.

The ring will not expand indefinitely. To a close approximation each separate particle will describe an elliptic orbit, the loci of these particles at each instant being a circle. Thus it appears that the ring may be expected to expand and contract rhythmically.

Immediately after the ejection of the first ring, a second ring may be thought of as being ejected with approximately equal values of $\omega$ and $r$, and this will be followed by a continuous succession of other rings. In the process of expansion and contraction these rings will collide and interfere with one another's motion.

210. To follow out the train of thought of Laplace and Roche, we should have to imagine these rings to coalesce into a single ring of finite size which would rotate with a uniform angular velocity $\omega$ about the main mass. This ring will be held together by its own gravitations.l cohesion but, like the main mass, it will be subject to the disruptive effects of rotation. Clearly it will only be matter of very considerable density that will possess sufficient gravitational cohesion to form a definite ring. Poincaré*, by a very simple

* Leçons sur les Hypothèses Cosmogoniques, p. 22. 
proof, has shewn that when a ring is formed, the mean density $\overline{\boldsymbol{\rho}}_{A}$ of the ring must always be greater than $\omega^{2} / 2 \pi$. By a simple extension of Poincaré's method, a much more general result may be obtained.

Consider the equilibrium of any mass whatever which is rotating approximately as a rigid body with angular velocity $\omega$. Let the motion be referred to axes rotating with uniform velocity $\omega$, and let $u, v, w$ be the velocity relative to these axes at any point of the mass. Let us assume that $u, v, w$ are small compared with $\omega r$, the velocity of rotation.

Then the equations of motion are of the form

$$
\frac{d u}{d t}=\frac{\partial V}{\partial x}+\omega^{2} x-\frac{1}{\rho} \frac{\partial p}{\partial x} .
$$

Differentiating with respect to $x, y, z$ and adding, we obtain, on using Poisson's relation $\nabla^{2} V=-4 \pi \rho$,

$$
\frac{d}{d t}\left(\frac{\partial u}{\partial x}+\frac{\partial v}{\partial y}+\frac{\partial w}{\partial z}\right)=-4 \pi \rho+2 \omega^{2}-\left[\frac{\partial}{\partial x}\left(\frac{1}{\rho} \frac{\partial p}{\partial x}\right)+\frac{\partial}{\partial y}\left(\frac{1}{\rho} \frac{\partial p}{\partial y}\right)+\frac{\partial}{\partial z}\left(\frac{1}{\rho} \frac{\partial p}{\partial z}\right)\right] .
$$

Let us multiply by $d x d y d z$, and integrate throughout the whole volume of any detached mass; let us further transform the first and last integrals by Green's Theorem. We obtain

$$
\frac{d}{d t} \iint(l u+m v+n w) d S=\iiint\left(2 \omega^{2}-4 \pi \rho\right) d x d y d z+\iint \frac{1}{\rho} \frac{\partial p}{\partial \nu} d S,
$$

where $\partial / \partial \nu$ denotes differentiation with respect to the inward normal.

The integral on the left measures the rate of expansion of the volume $A$ of the mass under consideration, so that the equation may be put in the form

$$
\frac{d^{2} A}{d t^{2}}=\left(2 \omega^{2}-4 \pi \bar{\rho}\right) A+\iint \frac{1}{\rho} \frac{\partial p}{\partial \nu} d S
$$

Now $p$ vanishes at the boundary of the mass and must, if disintegration is not to occur, be positive at all points inside. It follows that $\partial p / \partial \nu$ must be positive at every point of the boundary, and hence that the final term in equation (537) must be positive. Thus if $\bar{\rho}<\omega^{2} / 2 \pi$, the whole right-hand member of the above equation is positive and $d^{2} A / d t^{2}$ must therefore be positive. If the mass is relatively at rest, it starts expanding; if it is already expanding, it expands still more rapidly; if it is in process of contraction, the contraction is checked. A condition for a steady state is that $d^{2} A / d t^{2}$ shall vanish, and this clearly requires that $\bar{\rho}$ shall be greater than $\omega^{2} / 2 \pi$, which is Poincaré's result.

211. Returning now to the particular problem in hand, $\omega$ will be supposed to be the angular velocity of the Laplacian ring. In the earliest stages of the motion this must be very approximately equal to that of the main 
mass. Thus this angular velocity is connected with $\bar{\rho}$, the mean density of the matter of the main mass, by equation (534),

$$
\omega^{2}=0.35 \times 2 \pi \bar{\rho},
$$

so that Poincare's result takes the form that $\vec{\rho}_{A}$, the mean-density of the matter in the ring, must be not less than 0.35 times $\bar{\rho}$, the mean density of the main mass-otherwise the ring cannot form at all.

To appreciate the full meaning of this result, we must remember that it presupposes the formation of a sharp edge on the main mass, and that this sharp edge cannot form at all unless the matter of the main mass has a degree of compressibility comparable with that of a perfect gas. The ring, if ever formed, will be a structure in equilibrium under gravitation and its own pressure. Clearly the matter of a ring of small mass will expand under its own pressure until its density becomes very small, and the condition that the mean density shall be as great as $0.35 \bar{\rho}$ cannot be satisfied at all-the ring will be disintegrated by its own rotation. Thus we see that a ring of this type, if it forms at all, must have a mass comparable with that of the main central body-for a ring of mass much less than that of the central body would have a mean density much less than that of the central body. This result seems to dispose of Laplace's theory of the formation of the solar system, for this supposed the planets to have formed out of a ring whose mass must have been small compared with that of the central mass.

\section{EJECTION IN FILAMENTS}

212. The formation of Laplace's ring required perfect symmetry of the mass about its axis of rotation. To ensure this the mass was supposed to be rotating freely in space, unaffected by the presence of any other masses. The distances of adjacent masses in space will in general be so great that their gravitational influence will be extremely small. For most problems there would be no question that this gravitational influence might legitimately be neglected, but the problem we now have under consideration is peculiar in that even the slightest external gravitational field is sufficient to alter entirely the nature of the solution.

In the neighbourhood of the mass of gas under consideration the gravitational potential of all external masses will be a spherical harmonic, and therefore will be capable of expansion in the form

$$
V_{T}=S_{0}+S_{1}+S_{2}+\ldots
$$

where $S_{0}, S_{1}, S_{2}, \ldots$ are harmonics of degrees $0,1,2, \ldots$ respectively. As in previous discussions of the value of $V_{T}$ (cf. for instance, $\S 47$ ), the constant term $S_{0}$ may be omitted as giving rise to no forces, the term $S_{1}$ may be neutralised by supposing the areas of reference to have the same acceleration 
as the centre of gravity of the nebula, and terms beyond $S_{2}$ may be omitted as being numerically small in comparison with $S_{2}$. Thus $V_{T}$ may be supposed to reduce to the single term $S_{2}$, and the total potential $\Omega$ (cf. equation (390)) assumes the form

$$
\Omega=V_{M}+S_{2}+\frac{1}{2} \omega^{2}\left(x^{2}+y^{2}\right) \ldots \ldots \ldots \ldots \ldots \ldots . .(538) .
$$

When the external potential $S_{2}$ is omitted, the condition for a break-up $(\partial \Omega / \partial n=0)$ becomes

$$
-\frac{\partial V_{M}}{\partial r}=\omega^{2} r
$$

and the break-up commences simultaneously at all points of the cross-section in the plane of $x y$. But when the term $S_{2}$ is included in $\Omega$ the cross-section in the plane of $x y$ can no longer be circular; it becomes slightly elliptical and obviously the break-up will occur first at the two ends of the major-axis of this ellipse. Thus instead of a ring of matter being thrown off, we see that matter will be thrown off initially only at two antipodal points.

The first elements of matter thrown off from these two points form in themselves a tide-generating system whose potential must now be included in the general tide-generating potential $V_{T}$. The effect of this addition will clearly be to reinforce the value of the second harmonic term in $V_{T}$, so that when matter has once started coming off from two antipodal points, the region of ejection will concentrate more and more at two points as the motion proceeds. Under ideal conditions we may expect to have matter thrown off uniformly from all round the equator; under actual conditions we must expect two streams of matter issuing from antipodal points.

213. At this stage it will be profitable to pause again in our theoretical investigation to compare theory with astronomical observation.

Theory predicts the existence of rotating masses of gas of a lenticular form having sharp edges in their equatorial plane. These we have already ( $\$ 207$ ) found reasons for identifying, at least provisionally, with the so-called "lenticular" nebulae of which examples are illustrated in Plate III.

Theory further predicts that an emission of matter ought to take place in the equatorial plane: most of the examples illustrated in Plate III shew an extension of figure in the equatorial plane which may very reasonably be interpreted as matter ejected from this plane. The dark band in nebula N.G.C. 5866, lying as it does along the equator*, strongly suggests darker and cooler matter which has cooled after ejection, while still more pronounced dark bands are shewn in the three subsequent nebulae on Plate III. Theory predicts that the ejection of matter from the equator ought to continue almost indefinitely, so that the extensions in the equatorial plane ought to extend further and further as the evolution of the nebulae proceeds. Theory

* Pease (l.c. ante) describes the dark streak as making an angle of $3^{\circ}$ with the major-axis of the nebula. 


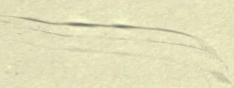




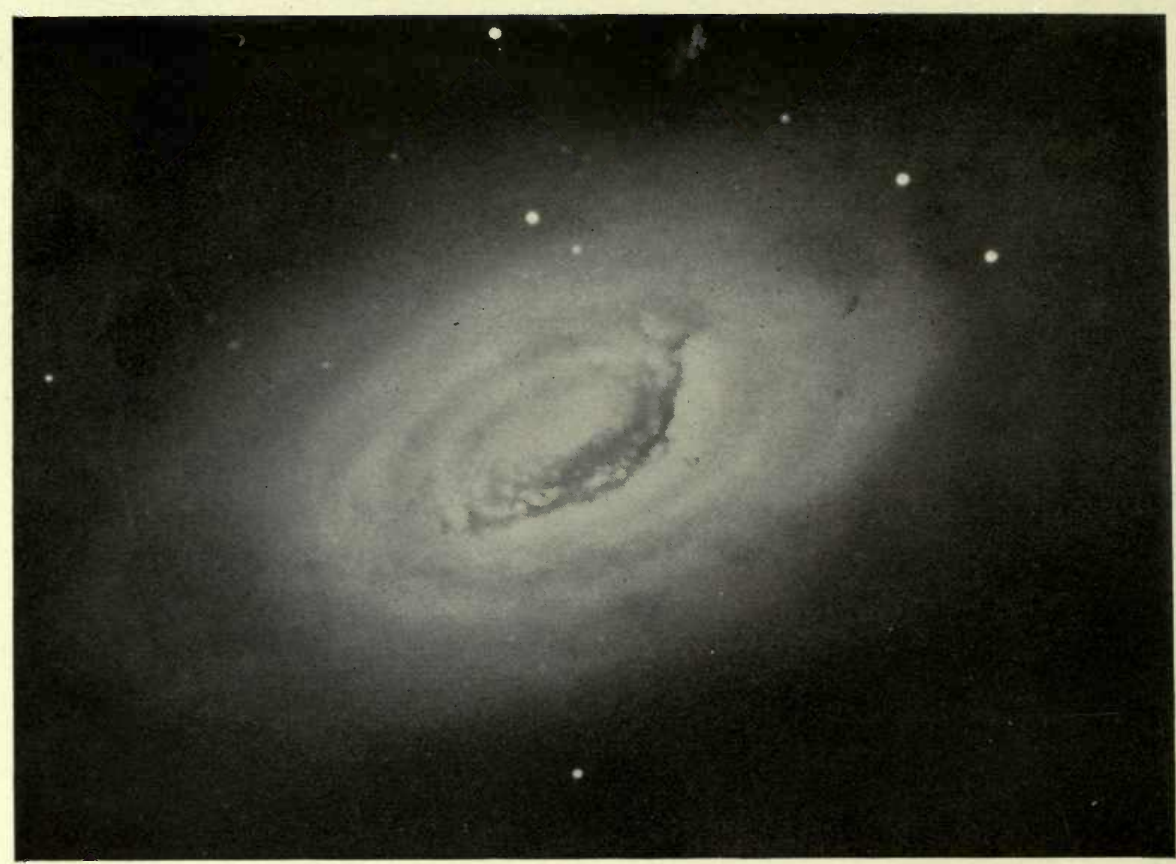

N.G.C. 4828 (M. 64)

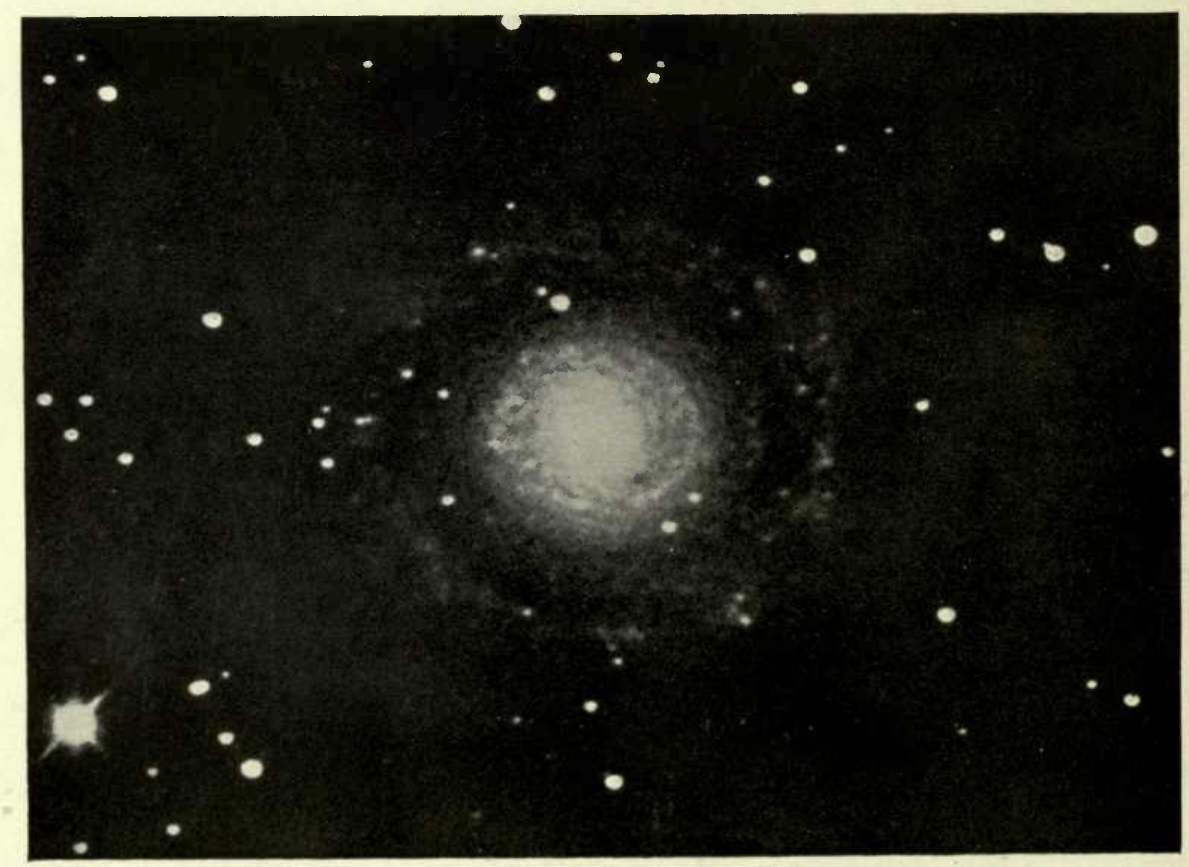

N.G.C. 7217 
further predicts that the emitted matter ought to proceed mainly from two antipodal points on the equator of the nebula.

To examine whether these latter predictions of theory are verified, we must obviously study our nebulae from a line of sight. near to the axis of rotation; the photographs shewn in Plate III, all being taken edgewise, cannot be expected to give any information on the question. But there is no reasonable doubt that the last three nebulae on Plate III are nothing but ordinary spiral nebulae seen edgewise; although we cannot study the crosssections of these by their equatorial planes, there are innumerable other spiral nebulae whose orientation in space is such that we look almost directly on to their equatorial planes. Typical examples will be found on Plates II and V; other types will be found in any collection of photographs of nebulae. The general characteristic of all these nebulae is that the two arms proceed approximately from antipodal points on the equator. That these arms really represent an ejection of matter from the central nucleus is almost proved by the two instances of M. 51 and M. 101 already discussed in $\S 4$. All this is quite in accordance with theory.

Two non-typical spiral nebulae are illustrated on Plate IV. The peculiarity of the first is that the spiral arms have given place to an almost continuous cloud of gas or dust, the separation of the arms being shewn only by the faint rifts or lanes between them. The peculiarity of the second is that the spiral arms are almost circular in shape, so that the whole figure is nearly symmetrical about its axis of revolution. In this nebula we appear to have a close approach to the manner of evolution imagined by Laplace. It will be seen that our tentative hypothesis is able to account for these exceptional nebulae as well as for those of more normal type.

Thus our conjectural interpretation of all spiral nebulae is that they are masses of gas or clouds of dust in rotation, this rotation being so rapid that no figure of statical equilibrium is possible. In the earlier stages of their evolution, they must have passed through a series of figures of equilibrium of the pseudo-spheroidal type discovered in Chapter VII, until a sharp edge was formed. After this, matter was ejected along two arms originating from this sharp edge. At first the points of origin of the arms were determined by the infinitesimal tidal forces set up by the rest of the universe; subsequently the tidal forces from the symmetrical arms themselves would suffice to confine the emission to two antipodal points.

214. It is worth noticing that on this interpretation of the spiral nebulae the mean density of the nucleus can be approximately determined when the period of rotation is known, the angular velocity $\omega$ being connected with the mean density $\bar{\rho}$ of the nucleus by the approximate relation

$$
\omega^{2}=0.35 \times 2 \pi \gamma \bar{\rho}=2 \cdot 2 \gamma \bar{\rho},
$$

$\gamma$ being the gravitation constant. 
For instance (cf. $\S 6$ ) van Maanen has found the period of rotation of the Ursa Major nebula M. 101 to be about 85,000 years for a number of points at average distance of $5^{\prime}$ from the centre. The angular velocity is not quite uniform, increasing somewhat as the nucleus is approached, but if, for purposes of a rough calculation, we assume the period of rotation of the nucleus to be 85,000 years, we find that the mean density of the nucleus must be about $3.8 \times 10^{-17}$ grammes per cubic centimetre. At this density there will be about a million atoms or molecules of atomic or molecular weight 20 per cubic centimetre, the mean free path being of the order of two thousand kilometres.

215. There remain some characteristic features of spiral nebulae which have not so far been predicted or explained by our theory, and which will accordingly provide further tests of the tenability of this theory. In particular may be mentioned the characteristic shape of the arms (cf. $\S 3$ ) and the condensations or nuclei in these arms. The determination of the shape of the arms to be expected on our theory seems at present to be beyond the reach of mathematical analysis, but the formation of condensations admits of discussion.

In examining the ejection of streams of gaseous or other compressible matter under tidal forces $(\$ 160)$ we found that a long stream of gas must become longitudinally unstable and will tend to break up into condensations or nuclei under its own gravitational attraction. Exactly similar effects are naturally to be expected in the present problem, and these seem to provide a very natural and satisfactory explanation of the nuclei observed in the arms of spiral nebulae.

In a stream of compressible matter of uniform density $\rho$, the distance apart of successive nuclei was found to be (approximately)

$$
l=\frac{1}{2} \sqrt{\frac{\pi}{\gamma \rho} \frac{d p}{d \rho}}
$$

For a gas, or quasi-gas formed of dust or meteorites, $p=\frac{1}{3} \rho C^{2}$, where $C$ is the molecular velocity, so that

$$
l=\frac{1}{2} C \sqrt{\frac{\pi}{3 \gamma \rho}}=\frac{1}{2} C \gamma^{-\frac{1}{2}} \rho^{-\frac{1}{2}} \text { (say) }
$$

Here $\rho$ may be taken to be the mean density in the nebular arms. The mean density of the nucleus, which we have so far denoted by $\bar{\rho}$, will probably be somewhat greater : let us denote it by $\theta \rho$. Then equation (534) becomes

$$
\omega^{2}=0.35 \times 2 \pi \gamma \theta \rho=2 \theta \gamma \rho \text { (say), }
$$

so that equation (539) gives

$$
l=\sqrt{\frac{\bar{\theta}}{2}} \cdot \frac{C}{\omega} .
$$


Some idea of the value of $\theta$ can be obtained from the result of $\S 211$. If the matter had formed a ring instead of spiral arms, $\theta$ could not have been greater than $(0.35)^{-1}$, or say 3 . The conditions in spiral arms are slightly, but not widely different, so that $\theta$ does not appear likely to be much greater than 3, neither does it appear likely to be less than 1 . If at a guess we put $\theta=2$ our equation becomes

$$
C=l \omega .
$$

This has a simple physical interpretation - the difference of velocity of rotation of two adjacent nuclei on the same radius must be approximately equal to the molecular (i.e. temperature) velocity in the arms.

For the rotation of the Andromeda nebula, M. 31, Pease* has found the velocity in $\mathrm{kms}$. per second along the major-axis to be given approximately by the formula

$$
-0.48 x-316
$$

where $x$ is measured in seconds of arc. The distance of successive condensation is perhaps about $3^{\prime \prime}$, so that $l \omega=1.44 \mathrm{kms}$. a second about. On our theory this ought to be at least comparable with the molecular velocity. We might of course invert the argument. Assume a molecular velocity in the arms of $1.44 \mathrm{kms}$. a second, which is a reasonable value to assume, and our formula (539) would at once give a value for $l$ just about equal to the observed distance apart of adjacent condensations.

To take another instance, the period of rotation of the Ursa Major nebula M. 101 has been found by van Maanen to be about 85,000 years. This gives $\omega=2.35 \times 10^{-12}$ so that if at a guess we put $C=1.6 \mathrm{kms}$. a sec., we obtain $l=7 \times 10^{11} \mathrm{kms}$. (about $\frac{1}{45}$ parsec), and this must be the distance of adjacent nuclei. Since these appear to be at distances of about $5^{\prime \prime}$ apart, the distance of the nebula ought to be about 1000 parsecs (parallax 0.001"). We have already estimated the mean density of the nucleus $\bar{\rho}$ to be about $4 \times 10^{-17}$ whence it appears that the mass of the nucleus must be of the order of $10^{37}$ gms., equal to 5000 times the mass of our sun.

If we conjecturally suppose the values of $C$ and $\bar{\rho}$ to be the same for the Andromeda nebula as for the nebula M. 101 just discussed, we find that the Andromeda nebula ought to have a parallax of about 0.0006 and a mass of the order of $10^{42}$ guns., which is of the same order as the probable mass of the whole universe of stars of which our sun forms a member.

No stress ought to be laid on any of these numbers except as shewing that our conjectural interpretation of the nuclear condensations in the arms would predict effects of the right order of magnitude. The figures indicate, however, that our conjecture commits us to supposing that the mass of the big Andromeda nebula (M. 31) is comparable with that of the whole galactic

* Nat. Acad. Science, 4 (1918), p. 21. 
universe-the "island universe" in which we live-while the smaller nebulae are of masses equal to thousands of that of our sun. The masses of the individual condensations in the arms appear to be probably about comparable with that of our sun - a conclusion which we shall again arrive at, in a more precise form, by a different path.

216. So far the ejected matter has been supposed to form a definite filament. Now it is clear that a jet of gas ejected into a vacuum will merely scatter into space under its own expansive forces, except when the mass is so large that its own gravitational coherence is sufficient to outbalance the expansive forces produced by molecular velocity. We must examine under what conditions a jet will condense into a filament.

Consider first the simpler problem of the conditions under which a filament, in existence, can continue in existence without scattering into space. Let $\tau$ be the line density, or mass per unit length of a uniform long filament. The potential of this filament at a point near its surface will be of the order of magnitude of $\gamma \tau$, so that a molecule moving with velocity $C$ will escape altogether if

$$
\frac{1}{2} C^{2}>\gamma \tau \text {. }
$$

Thus if $C$ is the mean-square molecular velocity near the surface of the filament, the filament will scatter into space unless (approximately)

$$
\tau>C^{2} / 2 \gamma
$$

If the filament is assumed to be in isothermal equilibrium a more precise result can be obtained. Let $p=k \rho$, so that $k=\frac{1}{3} C^{2}$, then the potential must satisfy the differential equation

$$
\nabla^{2} V+4 \pi e^{\nabla / k}=0
$$

of which the appropriate solution for a long filament is

$$
\rho=e^{V / k}=\frac{k c^{2}}{2 \pi r^{2}} \frac{A r^{c}}{\left(1+A r^{c}\right)^{2}},
$$

where $c$ and $A$ are constants of integration. The mass of gas per unit length is readily found to be $\tau=k c$.

The density is finite at the origin only if $c=2$; in all other cases there is found to be a nucleus at the origin of line density $k\left(1-\frac{1}{2} c\right)$, and the mass of this nucleus together with that of the surrounding gas will give a total mass per unit length equal to $k\left(1+\frac{1}{2} c\right)$.

Interpreted physically, this means that a filament of line density $2 k$ can rest in equilibrium with finite density at the centre and no tendency to scatter into space. A filament of line density greater than $2 k$ can rest in equilibrium with no tendency to scatter into space, but mathematically there will be zero density at the centre and a line charge repelling the gas; in 
nature this would mean that equilibrium could be established only when $A$ had become so great, and therefore the density so great, that the ordinary gas laws would be departed from. A filament of line density less than $2 k$ cannot rest in equilibrium at all under natural conditions; it would scatter into space. Thus for an isothermal filament the exact critical value of $\tau$ is seen to be

$$
\tau=\frac{2 k}{\gamma}=\frac{2 C^{2}}{3 \gamma}
$$

in which the gravitation-constant $\gamma$ has been restored. This exact value differs by a factor $\frac{4}{3}$ from the general approximate value obtained in equation (540).

217. Thus the lowest line density for which condensation can occur at all will be comparable with that given by equation (541). Multiplying this by expression (539) which gives the length of filament which goes to form a single condensation in the nebular arms, we find that the minimum mass in one such condensation must be comparable with

$$
\frac{1}{3} C^{3} \sqrt{\frac{\pi}{3 \gamma^{3} \rho}} \text {, or say } \frac{1}{3} C^{3} \gamma^{-\frac{3}{2}} \rho^{-\frac{1}{2}}
$$

For line densities much greater than this, the filament may become transversely as well as longitudinally unstable, the mechanism again being that already explained in $\$ 160$. If the filament is of $n$ times the critical line density $2 C^{2} / 3 \gamma$, the linear dimensions of its cross-section will be of the order of $n^{\frac{1}{2}} C_{\gamma}{ }^{-\frac{1}{2}} \rho^{-\frac{1}{2}}$, which is $2 n$ times the critical length (539) at which wave-motion becomes unstable. Thus when a filament has a line density much beyond the critical line density $2 C^{3} / 3 \gamma$, the motion may be supposed to be one in which nuclei of condensation form both laterally and transversely, their average distance being comparable with that given by formula (539), namely $\frac{1}{2} C \gamma^{-\frac{1}{2}} \rho^{-\frac{1}{2}}$. The average mass surrounding each nucleus will be $\rho$ times the cube of this expression or

$$
\frac{1}{8} C^{3} \gamma^{-\frac{3}{2}} \rho^{-\frac{1}{2}}
$$

which is comparable with our former expression (542), although less by a factor $\frac{3}{8}$.

Inserting our previous conjectural values $C=1.6 \times 10^{5}$ and $\rho=1.5 \times 10^{-17}$, the numerical value of expression (542) is found to be $1.6 \times 10^{34}$, or eight times the mass of our sun, while the numerical value of expression (543) is about three times the mass of our sun.

Thus we are again led to the conclusion, reached rather more vaguely in $\S 215$, that the nuclear condensations in the arms of spiral nebulae are of mass comparable with our sun. 


\section{CHAPTER X}

\section{THE EVOLUTION OF STAR-CLUSTERS}

218. From a purely theoretical discussion of the evolution of a mass of rotating gas we have been led to the hypothesis that the spiral nebulae are merely masses of rotating gas which have reached a stage of disintegration, the rotation having become so great through shrinkage that configurations of equilibrium are no longer possible. It would be of the utmost interest to follow out dynamically the different processes of this disintegration but unfortunately the mathematical difficulties have so far proved to be too great.

We have, however, found that the masses of these spirals must be supposed to be enormously greater than that of our sun, and the general nature of the disintegration has been seen to consist of the formation in the nebular arms of condensing nuclei each of mass just about comparable with that of our sun. Thus the hypothesis which has already been adopted seems to lead irresistibly to the conclusion that the final result of the process of disintegration which we see going on in the spiral nebulae must be the formation of star-clusters.

As to the features to be expected in these final star-clusters our dynamical analysis has so far told us almost nothing. It seems not unreasonable to expect that the star-clusters will be of the type we have described as "globular" thus we may conjecture that the observed spiral nebulae are forming starclusters similar to observed globular star-clusters and that the observed globular clusters have originated out of spiral nebulae.

For the present we shall regard this conjecture merely as a hypothesis whose truth is to be tested. The hypothesis commits us to what is commonly called the "island universe" theory-just as the spiral nebulae are distinct independent objects in space, so the star-clusters formed from them may be expected to be distinct independent objects in space. The "island universe" theory need not, however, be accepted in any extreme or categorical form. Twin nebulae are known to exist in the sky, and these may easily be imagined to form overlapping or intermingling star-clusters. Thus the "island universes " may overlap or intermingle-one "island " may be entirely enclosed inside another and larger "island." These, however, will be exceptional phenomena; normally we regard the stars as falling into detached clusters or separate "island universes." The particular cluster of which our sun is a member will be spoken of as the galactic universe. 
219. Some features of similarity between spiral nebulae and star-clusters have already been noted; these fall in naturally with the hypothesis we are now considering. We have already mentioned $(\$ 5)$ that the velocities in space of the star-clusters are approximately of the same order of magnitude as those of the spiral nebulae. The masses again are of the same order of magnitude. At a reasonable estimate the mass of the galactic universe is that of 1500 million stars each of mass equal to 1.7 times that of our sun $\left(2 \times 10^{33} \mathrm{gms}\right.$.), giving a total mass of $5 \times 10^{42}$ gms., which is of the same order of magnitude as our estimate of the mass of the Andromeda nebula M. 31 (p. 217). This nebula is perhaps the biggest of known nebulae, just as our galactic universe is the biggest of known star-clusters. Shapley inclines to an estimate of about 100,000 * or possibly more f for the mean number of stars in more typical clusters. This may correspond to a mass of the order of $10^{38}$ which is comparable with the mass of the nebula M. 101 conjecturally determined on p. 217. Finally according to Pease and Shapley $(\$ 6)$ many of the so-called globular clusters are in reality of a flattened shape, suggesting that the plane of the spiral nebula persists as the plane of symmetry of the resulting starcluster, this being of course the galactic plane in our own universe.

On the other hand before the hypothesis can be finally accepted some obvious features of dissimilarity between the spiral nebulae and star-clusters will demand explanation. The known star-clusters are very few in number compared with the spiral nebulae, and their observed distribution in space is different $(\$ 55,6)$. Also the star-clusters are on the whole probably more distant than the spiral nebulae. Curtis + gives $0.033^{\prime \prime}$ as the average annual proper motion of 66 large spiral nebulae, whence, the order of magnitude of their linear velocities being known, Curtis suggests an average parallax of the order of $0.0003^{\prime \prime}$. On the other hand Shapley $(\$ 6)$ has estimated the nearest star-clusters to have a parallax of only about 0.00012 ; a later study $\S$ of the distances of 69 globular clusters has led him to the conclusion that the nearest clusters of all, $\omega$ Centauri and 47 Toucanae, are distant just under 7000 parsecs $(\varpi=0.00014)$, the furthest, N.G.C. 7006 , is distant 67,000 parsecs $(\varpi=0.000015)$, while the mean distance of 69 is 23,000 parsecs $(\varpi=0.000044)$. These facts suggest problems which have to be solved rather than fatal difficulties. For the present we may confine ourselves to discussing the general theoretical problem of the possibility of nebulae evolving. into star-clusters. As we have found it impossible to progress in a forward direction from nebula to star-cluster, we must attempt to pass backwards from star-cluster to nebula.

220. Of all the star-clusters known to us our own universe is naturally the best known. Let us try to reconstruct the nebula out of which, on our present hypothesis, it must have formed. 
Some evidence can be obtained from the masses of the stars and their distribution, but, except for our own sun there is no means of determining the mass of a star except when it happens to be a binary. Eddington* has given a list of all the stars whose masses he believes to be really well determined. They are only seven in number, their masses in terms of that of our sun being $0 \cdot 7,1 \cdot 0,1 \cdot 3,1 \cdot 8,1 \cdot 9,2 \cdot 5$ and $3 \cdot 4$. Including our sun, this gives eight stars, all of fairly equal mass, the average of these masses being $1 \cdot 7$ times that of our sun, or $10^{33 \cdot 53} \mathrm{gms}$. The circumstance that the masses of the stars are fairly uniform is not unfavourable to our hypothesis as to their origin; we should expect the condensations in the nebular arms, if formed in the way we have imagined, to be all about equal in mass.

On equating the average mass of the stars to the mass of a nebular condensation, as given by formula (543), we can determine the density of the primitive nebular arms. The equation is

$$
\frac{1}{8} C^{3} \gamma^{-\frac{3}{2}} \rho^{-\frac{1}{2}}=10^{33 \cdot 53}
$$

Unfortunately the value of $\rho$ depends largely on the unknown molecular velocity $C$, varying as $C^{6}$. On taking $C=1.6 \times 10^{5}$ we find $\rho=4 \times 10^{-17}$. For cold gas, or gas mixed with solid dust particles the value of $C$ might perhaps be only a quarter of that just used, and the calculated value of $\rho$ would then be only one four-thousandth part of that calculated, say $\rho=10^{-20}$.

If $l$ is the mean distance of the condensations in the primitive nebular arms, each member of equation (544) is equal to $\rho l^{3}$, so that $l=\rho^{-\frac{1}{3}} \times 10^{11 \cdot 18} \mathrm{cms}$. Taking $\rho=4 \times 10^{-17}$, we find $l=10^{18 \cdot 64} \mathrm{cms}$. $=\frac{1}{75}$ parsec. Taking $\rho=10^{-20}$, we find $l=10^{17 \cdot 84} \mathrm{cms} .=\frac{1}{5}$ parsec.

Compare these figures with the present density and distances in our galactic universe. Eddington $†$ estimates that there are probably between 30 and 40 stars within a distance of 5 parsecs of our sun. The higher estimate gives a stellar density of one star per 13 cubic parsecs, or per $10^{56 \cdot 54}$ cubic cms., and an average stellar distance of $(13)^{\frac{3}{3}}$ or 2.3 parsecs. Introducing our former estimate of $10^{33 \cdot 53}$ for the average stellar mass, this gives an average density of matter of about $10^{-23}$ in the neighbourhood of our sun.

It is more difficult to estimate the mean-density in the universe as a whole. At a rough guess, our universe may be supposed to be a lensshaped figure of equatorial radius 2000 parsecs and transverse radius 600 parsecs. The volume of such a figure is $4 \times 10^{9}$ cubic parsecs or $10^{65}$ cubic cms. Thus a total mass of $5 \times 10^{42}$ grammes would require a mean density of $5 \times 10^{-23}$, or five times the density just estimated for the neighbourhood of the sun.

Both these estimates evaluate the density of the matter in the bright stars only; the dark stars, of which it is impossible even to guess at the 
number, will increase the density to a quite unknown extent, so that the estimates only provide lower limits to the true density.

Assuming these estimates to be somewhere near to the truth, a comparison with our previous estimate of the density of the primitive nebular arms shews that the system must have expanded very largely in its passage from nebula to star-cluster. Even the lower estimate of $\rho=10^{-20}$ requires an expansion of about six linear diameters. The necessity for some such expansion can be seen without detailed calculations. Our estimate of p. 217 has already suggested that the mass of the Andromeda nebula is about equal to that of our galactic universe, so that we may think of it as a picture of the primaeval nebula out of which we are conjecturing that our universe has been formed. But the Andromeda nebula subtends an angle of less than $2^{\circ}$ from the centre of our universe, whereas our universe probably subtends about $30^{\circ}$ when seen from the Andromeda nebula.

The idea of such an expansion will probably present no difficulties to the observational astronomer. The general appearance of the spiral nebulae is certainly not unfavourable to the view that they are in an expanding state, and this view is confirmed by the measurements of van Maanen and Kostinsky already referred to $(\$ 4)$; the matter in the nebular arms appears to be moving away from the nucleus with no inconsiderable velocity.

221. For further calculations, let us assume the density of the original nucleus to have been $10^{-17}$, corresponding $(\$ 214)$ to a period of rotation of 160,000 years. The mass of the whole system being supposed to be $5 \times 10^{42} \mathrm{gms}$., the volume of the nucleus before disintegration commenced must have been $5 \times 10^{59}$ cubic centimetres - say a figure of radius 30 parsecs in its equatorial plane and of radius 10 parsecs perpendicular to this.

We suppose that this figure has expanded until its equatorial radius is about 2000 parsecs - say 66 times that of the original nucleus. During this expansion the angular momentum $M k^{2} \omega$ must remain constant. The value of $k^{2}$ may be supposed to have increased about $(66)^{2}$ times or say 4000 times, so that the mean value of $\omega$ will have decreased to one four-thousandth of its former value, and the final period of rotation will be about $640,000,000$ years. Thus our whole system may be expected to average one rotation in $640,000,000$ years, or about $0.0020^{\prime \prime}$ per annum. The rotation calculated in this way depends only very slightly on the initially assumed value of $\bar{\rho}$, being in point of fact proportional to $\bar{\rho}^{\frac{1}{6}}$.

In this connection it may be mentioned that Charlier* has found that the node of the invariable plane of the Solar System has a direct motion on the plane of the Milky Way amounting to $0.003528^{\prime \prime}$ per year, or a revolution in about 370,000,000 years. This, as Eddington $\dagger$ has remarked, might equally well be interpreted as a retrograde rotation of the Milky Way in space. 


\section{General Stellar Dynamics}

222. After this brief consideration of the possible origin of our own universe, let us proceed to a general discussion of the motions of stars in clusters. In searching for numerical data we may be guided by the figures we have conjecturally obtained for the past history of our own universe. We have a number of stars, or, at first, gaseous condensations, moving in space under their own attractions and possibly also under the attraction of a central nucleus. We require to find as much as possible about the nature of the motion and the evolution of the system.

The motion of the stars may to some extent he compared to the motion of the molecules of a gas, and certain formulae may be borrowed from the kinetic theory of gases which will give approximately true results when applied to a star-cluster.

223. Let us begin by considering the frequency of actual material collisions in a cluster of stars.

At first let us regard the stars as being uniformly spheres of diameter $\sigma$, and let us treat the problem as a purely geometrical one, the gravitational attractions of the stars being momentarily neglected. By the familiar methods of the kinetic theory of gases, it is readily shewn that the number of collisions experienced by any one star in time $d t$ will be

$$
\pi \nu \sigma^{2} V d t
$$

where $\nu$ is the number of stars per unit volume, and $V$ is their mean relative velocity.

Thus the mean time between successive collisions of a single star will be

$$
\frac{1}{\pi \nu \sigma^{2} V}
$$

For our universe in its present state we may take $\nu=10^{-56 \cdot 5}$ and $V=40 \mathrm{kms}$. a sec. $=4 \times 10^{6}$ C.G.S. units. Thus formula (545) gives for the mean interval between collisions $10^{41 \cdot} / \sigma^{2}$ years. Even if we assign to the average star a diameter equal to that of Neptune's orbit, say $\sigma=4.5 \times 10^{14} \mathrm{cms}$., this gives a mean interval of $4 \times 10^{12}$ years, a period which is so large compared with any reasonable estimate of the age of the universe, that it is at once clear that the chance of material collisions may be disregarded entirely.

224. This calculation has neglected the effect of gravitational attraction, which naturally increases the chances of collision. Not only this but stars will act on one another gravitationally, and so influence one another's motion, at distances far beyond those at which material collisions can occur. The event of two stars coming so close to one another that their gravitational 
attraction appreciably influences their motion may conveniently be referred to as an "encounter." It now becomes necessary to estimate the frequency of stellar encounters.

225. In fig. 42 let $O P Q$ be a slightly curved orbit described by a star of mass $M$ about $G$ the centre of gravity of a second star of mass $M^{\prime}$ and itself.

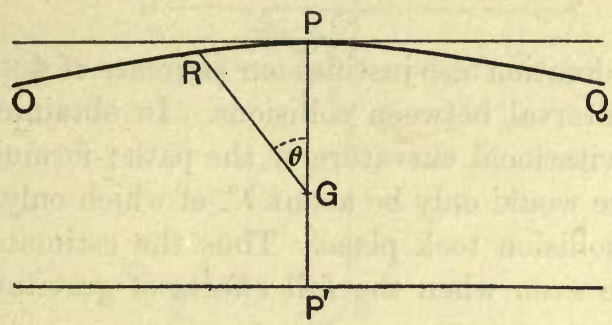

Fig. 42.

The distance of closest approach $P G P^{\prime}$ will be denoted by $\sigma$. The velocity of $M$ relative to $G$ will be $M^{\prime} V /\left(M+M^{\prime}\right)$, where $V$ is the relative velocity of the pair of stars.

When the star $M$ is at $R$, the acceleration along $G R$ is $\gamma M^{\prime} \cos ^{2} \theta / \sigma^{2}$, $\gamma$ being the gravitation constant. Thus the rate of change of velocity along $P G$ is $\gamma M^{\prime} \cos ^{3} \theta / \sigma^{2}$, and the total change of velocity along $P G$ will be

$$
\int_{-\infty}^{+\infty} \frac{\gamma M^{\prime} \cos ^{3} \theta}{\sigma^{2}} d t=\int_{-\frac{1}{2} \pi}^{\frac{1}{2} \pi} \frac{\gamma M^{\prime} \cos \theta}{\sigma V} d \theta=\frac{2 \gamma M^{\prime}}{\sigma V}
$$

This total change of velocity must however be equal to

$$
\frac{M^{\prime} V}{M+M^{\prime}} \psi
$$

where $\psi$ is the total deviation of either orbit; thus

$$
\psi=\frac{2 \gamma\left(M+M^{\prime}\right)}{\sigma V^{2}}
$$

It must be remembered that this formula may only be used when $\psi$ is small.

A certain value of $\psi$ will correspond to each value of closest approach $\sigma$, and conversely. The value of $\sigma$ which corresponds to a deviation $\psi$ of $1^{\circ}$ is

$$
\sigma=\frac{360 \gamma\left(M+M^{\prime}\right)}{\pi V^{2}}
$$

Taking, as values appropriate to our present universe, $M+M^{\prime}=6 \cdot 8 \times 10^{33} \mathrm{gms}$., $V=4 \times 10^{6} \mathrm{cms}$., we find as the value of $\sigma$ about $3 \cdot 2 \times 10^{15} \mathrm{cms}$., or about seven times the radius of Neptune's orbit.

J. c. 
Encounters for which $\sigma$ is equal to, or less than, this value-i.e. encounters in which the deviation is $1^{\circ}$ or more-may be expected from formula $(545)$ to occur only about once in $10^{11}$ years. It is true that formula (545) was obtained only by neglecting the curvature of the stars' paths produced by gravitational attraction, but it is quite legitimate to apply it to determine the present critical value of $\sigma$, in which by hypothesis the total deflection of path is only $1^{\circ}$.

A similar consideration also justifies our estimate of $4 \times 10^{12}$ years obtained in $\$ 223$ for the interval between collisions. In obtaining this estimate we neglected the gravitational curvature of the path; formula (547) now shews that this curvature would only be about $7^{\circ}$, of which only half, or $3 \frac{1}{2}^{\circ}$, would occur before the collision took place. Thus the estimate will remain very approximately true even when the full effects of gravitation are taken into account.

226. We have now, allowing fully for gravitation, obtained two estimates applicable to a system of stars in the state in which our universe now is. For the frequency of actual collisions, even assuming the stars to have a diameter equal to that of Neptune's orbit, we have found $4 \times 10^{12}$ years, and obviously for smaller stars collisions would be still more infrequent. For encounters producing a total deflection of path of $1^{\circ}$ or more, we have obtained a frequency of one in $1.4 \times 10^{11}$ years. These intervals of time are both so long in comparison with astronomical times that it is clear that, in statistical calculations dealing with our universe as it now is, we may neglect altogether the possibility of collisions and of encounters in which $\psi$ is as large as $1^{\circ}$, and confine our attention to encounters for which $\psi$ is less than $1^{\circ}$. For such encounters formulae (546) and (547) may be regarded as accurate.

227. We proceed now to study the cumulative effect of these feeble encounters.

It will be remembered that we have so far been concerned only with motion relative to the centre of gravity of two stars. In fig. 4.3 let $O P, O Q$ represent the velocities in space of the two stars $M, M^{\prime}$; let these velocities be denoted by $v_{1}, v_{2}$ and be inclined at an angle $\alpha$. Let $G$ divide $P Q$ in the ratio $M^{\prime}: M$, then $O G$ will represent the velocity of the centre of gravity of the two stars.

The direction of the line of closest approach may be supposed to be $S P$. This is necessarily perpendicular to the direction of relative velocity $P Q$. Let the angle $S P O$ be $\beta$.

The effect of the encounter is to superpose on to $v_{1}$, the velocity of $M$, a 
new velocity $2 \gamma M^{\prime} / \sigma V$ along $S P$. Thus after encounter the components of the velocity of $M$ along and perpendicular to $O P$ will be

$$
v_{1}+\frac{2 \gamma M^{\prime}}{\sigma V} \cos \beta, \frac{2 \gamma M^{\prime}}{\sigma V} \sin \beta
$$

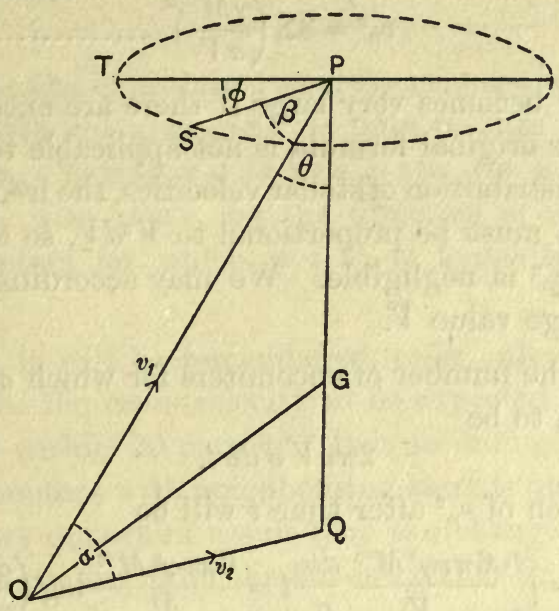

Fig. 43.

228. Let $T P$ be the intersection of the plane $P O Q$ with the plane perpendicular to $P Q$ in which the direction of closest approach must lie. Let the angle $O P Q$ be $\theta$, and the angle $T P S$ be $\phi$. Then $\cos \beta=\sin \theta \cos \phi$.

In a series of encounters all directions in the plane TPS are equally likely for the direction of closest approach $P S$, so that all values are equally likely for $\phi$. Thus, using a bar to denote mean values over a series of encounters,

$$
\begin{aligned}
& \overline{\cos \beta}=0, \\
& \overline{\cos ^{2} \beta}=\frac{1}{2} \sin ^{2} \theta=\frac{1}{2} \frac{v_{2}^{2} \sin ^{2} \alpha}{V^{2}} .
\end{aligned}
$$

Returning to formula (549) it is clear that the expectation of the component along $O P$ is simply $v_{1}$; the velocity along the original path remains unaltered. The component of velocity perpendicular to this, say $v_{n}$, can be in any direction perpendicular to $O P$. After any number of encounters the expectation of the value of $v_{n}^{2}$ will be

Now

$$
v_{n}^{2}=\Sigma\left(\frac{2 \gamma M^{\prime}}{\sigma V}\right)^{2} \overline{\sin ^{2} \beta}
$$

$$
\overline{\sin ^{2} \beta}=1-\frac{1}{2} \frac{v_{2}^{2} \sin ^{2} \alpha}{V^{2}},
$$

and since $V^{2}=v_{1}^{2}+v_{2}^{2}-2 v_{1} v_{2} \cos \alpha$, this can be expressed as

$$
\overline{\sin ^{2} \beta}=\frac{1}{2}+\frac{\left(V^{2}+v_{1}^{2}-v_{2}^{2}\right)^{2}}{8 V^{2} v_{1}^{2}} \text {. }
$$


A comparison of these expressions shews that, whatever the values of $v_{1}$ and $v_{2}, \sin ^{2} \beta$ must always be between $\frac{1}{2}$ and 1 . For rough numerical estimates, which can at best only be accurate as regards order of magnitude, we may take $\overline{\sin ^{2} \beta}$ uniformly equal to $\frac{3}{4}$, giving

$$
v_{n}^{2}=3 \Sigma\left(\frac{\gamma M^{\prime}}{\sigma V}\right)^{2}
$$

This value of $v_{n}{ }^{2}$ becomes very large if there are encounters for which $V$ is very small, but our original formula is not applicable to these. Moreover, whatever the law of distribution of stellar velocities, the frequency of encounters for which $V$ is small must be proportional to $V^{3} d V$, so that the influence of such encounters on $v_{n}{ }^{2}$ is negligible. We may accordingly suppose $V$ to be replaced by its average value $\vec{V}$.

In an interval $t$, the number of encounters for which $\sigma$ lies between $\sigma$ and $\sigma+d \sigma$ may be taken to be

$$
2 \pi \nu \bar{V} \sigma d \sigma t,
$$

so that the expectation of $v_{n}^{2}$ after time $t$ will be

$$
v_{n}^{2}=\int_{\sigma_{1}}^{\sigma_{2}} \frac{6 \pi \nu \gamma^{2} M^{\prime 2}}{\bar{V}} \frac{d \sigma}{\sigma} t=\frac{6 \pi \nu \gamma^{2} M^{\prime 2}}{\bar{V}} \log \left(\frac{\sigma_{2}}{\sigma_{1}}\right) t
$$

In this evaluation of $v_{n}{ }^{2}$ we have assumed $M^{\prime}$ to be about the same for all encountering stars; if it is not we need only replace $M^{\prime 2}$ by its mean value.

Further, as the limits indicate, we consider only encounters for which $\sigma$ lies between two values $\sigma_{1}, \sigma_{2}$. We notice at once that the expression on the right would become infinite both for $\sigma_{1}=0$ and for $\sigma_{2}=\infty$. We are not entitled to put $\sigma_{1}=0$ because by doing so we should be taking into account the effect of violent encounters, for which our formula does not apply. We have seen that encounters for which $\psi>1^{\circ}$ will be of extremely rare occurrence. Reserving these for separate discussion, we may give to $\sigma_{0}$ the minimum value for which $\psi<1^{\circ}$, which we have seen to be about $10^{15 \cdot 5} \mathrm{cms}$.

The circumstance that expression (551) becomes infinite when $\sigma_{2}=\infty$ shews that encounters with very distant stars contribute greatly to the value of $v_{n}{ }^{2}$. We may, however, use our formula to find the effect of encounters with fairly near stars, say stars within 20 parsecs, and to do this we put

$$
\sigma_{2}=20 \text { parsecs }=10^{19 \cdot 8} \mathrm{cms} \text {. }
$$

With these values, we have

$$
\log _{e}\left(\frac{\sigma_{2}}{\sigma_{1}}\right)=\log _{e} 10^{4 \cdot 8}=9 \cdot 9
$$

Thus we find for the expectation of $v_{n}{ }^{2}$ produced by non-violent encounters with stars within 20 parsecs,

$$
v_{n}^{2}=\frac{6 \pi \nu \gamma^{2} M^{\prime 2}}{\bar{V}} 9 \cdot 9 t=187 \frac{\nu \gamma^{2} M^{\prime 2}}{\bar{V}} t .
$$


With the numerical values already assumed $\left(\nu=10^{-56 \cdot 54} ; M^{\prime}=10^{33 \cdot 53}\right.$, $\left.\bar{V}=4 \times 10^{6}\right)$, this gives $v_{n}^{2}=7 \times 10^{-9} t$.

Here $t$ is measured in seconds. If $t$ is measured in years, the formula becomes, nearly enough,

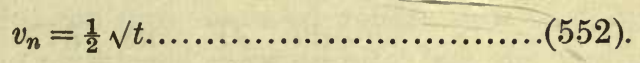

Thus in one year the expectation of cross-velocity is $\frac{1}{2} \mathrm{~cm}$. a second; in a million years it is $.005 \mathrm{~km}$. a second. It only reaches $1 \mathrm{~km}$. a second after 40,000 million years. Taking $v=25 \mathrm{kms}$. a sec., the time required for $v_{n}{ }^{2}$ to become comparable with $v^{2}$, i.e. for the direction of motion to be entirely changed by encounters for which $\psi<1^{\circ}$, is found to be of the order of $10^{13 \cdot 5}$ years*.

These numbers, it will be remembered, refer only to our universe as it now is, and measure the cross-velocity to be expected from encounters with neighbouring stars within 20 parsecs. It is accordingly clear that the velocity set up by encounters with neighbouring stars is quite negligible. Thus we arrive at the very important result that the changes in stellar velocities may be regarded as coming from the forces exerted by the main body of the universe: near stars need not be taken into the calculation at all.

229. It now appears that, for our present universe, the problem of stellar dynamics is the same as the problem of the kinetic theory of gases with the collisions left out. This being so, stellar dynamics is naturally very much simpler than gas-dynamics. As Eddington $†$ has remarked, it may, in virtue of the result just obtained, be regarded as a quite different study from gasdynamics, or from that of the motion of any type of system that has yet been investigated. For the action of contiguous units, which becomes gradually simpler as we pass in succession through rigid dynamics, hydrodynamics and gas-dynamics, disappears entirely when we come to stellar dynamics.

230. Just as in gas-dynamics, the units in any small region of space may be classified according to their velocities into a system of showers of parallelmoving units. But there is the essential difference between the two cases, that in stellar dynamics these showers retain their identity through very long periods of time, whereas in gas-dynamics they do not.

Suppose that in any small region of space $d x d y d z$, the number of stars which have velocities lying within a small range $d u d v d w$ surrounding the values $u, v, w$ at time $t$ is

$$
f(u, v, w, x, y, z, t) d u d v d w d x d y d z
$$

* This time corresponds to the "time of relaxation" in a gas. For this same time, Prof. Charlier has obtained, by a somewhat different process, a value of the order of $10^{16}$ years (Lund Meddel. Ir. No. 15).

† Stellar Movements, p. 256. 
Within the small range $d x d y d z$, the gravitational forces arising from the universe as a whole will be sensibly constant; suppose them derived from a potential $V$. Then the motion of every star included in formula (553) must be determined by the equations of motion,

$$
\frac{d u}{d t}=\frac{\partial V}{\partial x} ; \quad \frac{d v}{d t}=\frac{\partial V}{\partial y} ; \quad \frac{d w}{d t}=\frac{\partial V}{\partial z}
$$

After an interval $d t$, this group of stars must have velocity components lying within a small range $d u d v d w$ surrounding the values

$$
u+\frac{\partial V}{\partial x} d t, \quad v+\frac{\partial V}{\partial y} d t, \quad w+\frac{\partial V}{\partial z} d t
$$

while its position will be confined to a small region of space of extent $d x d y d z$ surrounding the point

$$
x+u d t, \quad y+v d t, \quad z+w d t .
$$

Hence, with the notation already introduced in formula (553), the number of stars in this group must be

$f\left(u+\frac{\partial V}{\partial x} d t, v+\frac{\partial V}{\partial y} d t, w+\frac{\partial V}{\partial z} d t, x+u d t, y+v d t, z+w d t, t+d t\right)$ $d u d v d w d x d y d z$...(555),

so that this expression must be equal to expression (553).

Expanding expression (555) as far as first powers of $d t$, and equating to expression (553), we obtain

$$
\frac{d f}{d t}+\frac{\partial V}{\partial x} \frac{\partial f}{\partial u}+\frac{\partial V}{\partial y} \frac{\partial f}{\partial v}+\frac{\partial V}{\partial z} \frac{\partial f}{\partial w}+u \frac{\partial f}{\partial x}+v \frac{\partial f}{\partial y}+w \frac{\partial f}{\partial z}=0
$$

This is the differential equation which must be satisfied by the distribution function $f$ in every problem of stellar dynamics*.

231. Being a linear equation in $f$, this equation may be solved in accordance with Lagrange's rule. This rule directs us to find as many integrals as possible of the system of equations

$$
d t=\frac{d u}{\frac{\partial V}{\partial x}}=\frac{d v}{\frac{\partial V}{\partial y}}=\frac{d w}{\frac{\partial V}{\partial z}}=\frac{d x}{u}=\frac{d y}{v}=\frac{d z}{w}
$$

If $E_{1}=$ cons., $E_{2}=$ cons., ... are all the integrals of these equations, then the solution of the original equation (556) is simply

$$
f=\phi\left(E_{1}, E_{2}, \ldots\right)
$$

* The student of the Kinetic Theory will recognise that it is simply Boltzmann's well-known equation with the collisions left out. Cf. Boltzmann, Vorlesungen Uber Gastheorie, r. p. 132, or Jeans, Dynamical Theory of Gases (2nd Ed.), p. 226. 
where $\phi$ is any arbitrary function. It is however clear that equations (557) are merely the equations of motion of a star or other particle in the universe, so that $E_{1}, E_{2}, \ldots$ are the first integrals of the equations of motion*.

232. The general solution (558) contains the most general law of distribution which is consistent with the conditions of continuity, but the finding of $f$ is only the first step in the solution of a given problem. The potential $V$ may in general be supposed to be of the form

$$
V=V_{M}+V_{T}
$$

where $V_{M}$ is the potential of the mass of stars under consideration and $V_{T}$ is that of any extraneous forces. Thus $\nabla^{2} V_{M}=-4 \pi \rho$ and $\nabla^{2} V_{T}=0$ at all points inside the star-cluster, so that

$$
\nabla 2 V=-4 \pi \rho
$$

We need not assume all the stars to be of equal mass or type. Let us assume them, however, to fall into a number of distinct classes of masses $M, M^{\prime}$, etc., the corresponding laws of distribution being denoted by $f, f^{\prime}$, etc. Let the number of stars of these types per unit volume be denoted by $\nu, \nu^{\prime}$, etc. Then

$$
\rho=\Sigma \nu M=\Sigma M \iiint f d u d v d w
$$

so that equation $(560)$ becomes

$$
\nabla^{2} V=-4 \pi \Sigma M \iiint f d u d v d w
$$

We shall now shew that values of $f, f^{\prime}, \ldots$ of the form (558) which are also such as to satisfy equation (562) will give a natural motion of stars.

233. The general characteristic equation satisfied by $f$ is equation (556). Let us multiply this by $u d u d v d w$ and integrate with respect to all values of $u, v, w$. We have

$$
\iiint \frac{\partial f}{\partial u} u d u d v d w=-\iiint f d u d v d w
$$

on integrating by parts, while similarly

$$
\iiint \frac{\partial f}{\partial v} u d u d v d w=0
$$

Hence the resulting equation is seen to be

$$
\begin{aligned}
\frac{d}{d t} \iiint f u d u d v d w+ & \frac{\partial}{\partial x} \iiint f u^{2} d u d v d w+\frac{\partial}{\partial y} \iiint f u v d u d v d w \\
& +\frac{\partial}{\partial z} \iiint f u w d u d v d w=\frac{\partial V}{\partial x} \iiint f d u d v d w
\end{aligned}
$$

* An alternative proof will be found in Monthly Notices R.A.S. 76 (1915), p. 78. 
We may however put

$$
\begin{aligned}
& \iiint f d u d v d w=\nu \\
& \iiint f u v d u d v d w=\nu \overline{u v}, \text { etc., }
\end{aligned}
$$

where $\overline{u v}$ denotes the mean value of $u v$ for stars of the first class at the point $x, y, z$. Thus equation (563) becomes

$$
=\frac{d}{d t}(\nu \bar{u})+\frac{\partial}{\partial x}\left(\nu \bar{u}^{2}\right)+\frac{\partial}{\partial y}(\nu \overline{u v})+\frac{\partial}{\partial z}(\nu \overline{u w})=\nu \frac{\partial V}{\partial x}
$$

When there is only one type of star, this and its two companion equations are simply the hydrodynamical equations of motion of the element $d x d y d z$ of the star-cluster. They could be derived directly from the equations of motion (557) by the methods of the Theory of Gases*. When there are several types of star we merely multiply the equations such as (564) by $M, M^{\prime}$, etc. and add, and the resulting equations are then seen to be the hydrodynamical equations of motion of the element $d x d y d z$.

Thus it appears that if equations such as (558) are satisfied by $f, f^{\prime}$, etc., then the hydrodynamical equations will be satisfied at every point of the starcluster in addition to the equation of continuity being satisfied. Every such solution will accordingly give a possible motion of the stars of a cluster in a field of potential $V$. In order that the field may be a purely gravitational field, $V$ must further be such as to satisfy equation (562), while we must still further have $V_{T}=0$ if the stars move purely under their mutual gravitational forces.

\section{Steady Motion}

234. The simplest problems of stellar dynamics naturally occur when the group of stars under consideration is supposed to be in a steady state. The steady state problem is the analogue of determining the configurations of equilibrium for a gravitating mass of gas and we shall at once find that there is a considerable similarity between the two solutions.

Analytically the characteristic of a steady state solution is that $f$ must be independent of the time; the integrals $E_{1}, E_{2}, \ldots$ which enter into $f$ must therefore not involve the time. Equations (557) reduce to

$$
\frac{d u}{\frac{\partial V}{\partial x}}=\frac{d v}{\frac{\partial V}{\partial y}}=\frac{d w}{\frac{\partial V}{\partial z}}=\frac{d x}{u}=\frac{d y}{v}=\frac{d z}{w}
$$

and one integral can be written down at once, namely the equation of energy

$$
E_{1} \equiv \frac{1}{2}\left(u^{2}+v^{2}+w^{2}\right)-V=\text { constant. }
$$

Jeans, Dynamical Theory of Gases (2nd Ed.), Equations (454), p. 180. 
235. As regards other integrals, equations (565) assume their simplest form when the cluster is spherically symmetrical, so that $V$ is a function of $r$ only. Denote $\partial V / \partial r$ by $R$, then $\partial V / \partial x=x R / r$ and the equations become

$$
\frac{d u}{\frac{x R}{r}}=\frac{d v}{\frac{y R}{r}}=\frac{d w}{\frac{z R}{r}}=\frac{d x}{u}=\frac{d y}{v}=\frac{d z}{w} .
$$

There are obviously three integrals,

$$
\begin{aligned}
& \varpi_{1} \equiv y w-z v=\mathrm{cons} . \\
& \varpi_{2} \equiv z u-x w=\mathrm{cons} . \\
& \varpi_{3} \equiv x v-y u=\mathrm{cons} .
\end{aligned}
$$

these integrals expressing that the moments of momentum per unit mass, $\varpi_{1}, \varpi_{2}, \varpi_{3}$, remain constant.

It is clear that with the most general value of $R$ there can be no other integrals, although with special values of $R$ there may be. For instance, if $R=\kappa r$, where $\kappa$ is a constant, there will be integrals of the form

$$
u^{2}-\kappa x^{2}=\text { cons., etc., }
$$

each particle describing an elliptic orbit about the centre.

Apart from very special and artificial cases such as this, the law of distribution in a spherically symmetrical cluster must be of the form

$$
f\left(E_{1}, \varpi_{1}, \varpi_{2}, \varpi_{3}\right) d u d v \tau d w d x d y d z
$$

Not every such law will give a possible cluster, for equation (562) remains to be satisfied. Since the cluster is supposed to be spherically symmetrical, the law of distribution must be invariant as regards change of axes, the origin being kept fixed. Now the only invariant of $\varpi_{1}, \varpi_{2}, \varpi_{3}$ is $\varpi_{1}^{2}+\varpi_{2}^{2}+\varpi_{3}^{2}$, whence it appears that the law of distribution in a spherically symmetrical cluster must be of the form

$$
f\left(E_{1}, \varpi_{1}^{2}+\varpi_{2}^{2}+\varpi_{3}^{2}\right)
$$

236. The next simplest solution occurs when the cluster is arranged symmetrically about an axis, say that of $z$, so that the figure is one of revolution. In this case there is only one general integral beyond the integral of energy, and this is $\varpi_{3}=$ cons. Thus the law of distribution must be of the form

$$
f\left(E_{1}, \varpi_{3}\right)
$$

237. Consider finally clusters which possess no symmetry at all, so that the only integral is that of energy, and the law of distribution must be

$$
f\left(E_{1}\right)
$$

Inserting for $E_{1}$ its value $\frac{1}{2}\left(u^{2}+v^{2}+w^{2}\right)-V$, it is clear from equation (561) that the density $\rho$ must be a function of $V$ only. 
Now this is exactly the same relation as that obtained in Chapter VII (equation (396)) in discussing the configurations of equilibrium of a compressible mass, namely

$$
\phi(\rho)=V+C
$$

In this equation different forms of the function $f$ corresponded to different relations between pressure and density. It is at once clear that the different laws of distribution $f$ in formula (569) correspond exactly to different relations between pressure and density for a compressible medium.

Further, the different possible configurations of a cluster of stars given by law (569) must be identical with those of different compressible masses in which the pressure is a function of the density. In particular, when no external forces act, these configurations must be spherically symmetrical.

This conclusion, however, is antagonistic to the hypothesis from which we started, namely that the cluster was to possess no symmetry at all : our search for asymmetrical clusters has merely led us back to a group of spherically symmetrical clusters which form only a sub-group of those already discovered in $§ 235$.

238. Thus it has now been found that, except for special isolated cases such as that mentioned in $\S 235$, the only possible configurations for a cluster of stars moving freely under their own gravitation in steady motion are those in which the stars either form asspherically symmetrical figure or a figure of revolution which is symmetrical about an axis.

For a spherically symmetrical cluster, the law of distribution must be

$$
f\left(E_{1}, \varpi_{1}^{2}+\varpi_{2}^{2}+\varpi_{3}^{2}\right)
$$

for a figure of revolution the law of distribution must be

$$
f\left(E_{1}, \varpi_{3}\right)
$$

Let us examine these laws in detail, paying special attention to their relation to observation in the case of our own universe, and also their relation to possible final states of the cluster of stars originating from a rotating nebula.

239. If $c^{2}$ stands for $u^{2}+v^{2}+w^{2}$ and $r^{2}$ for $x^{2}+y^{2}+z^{2}$, the law of distribution (571) may be expressed in the form

$$
f\left[\frac{1}{2} c^{2}-V, r^{2} c^{2}-(u x+v y+w z)^{2}\right] .
$$

If $a$ denote the angle between the radius $r$ and the direction of the velocity $c, u x+v y+w z=r c \cos \alpha$, so that $f$ may be put in the form

$$
f\left[\frac{1}{2} c^{2}-V, r^{2} c^{2} \sin ^{2} \alpha\right] .
$$

Thus at any point $x, y, z$ in space $f$ is a function of $c$ and $\alpha$. The velocities of the stars at this point are accordingly not distributed uniformly for all 
directions in space. If a velocity diagram be drawn at any point $x, y, z$, formed of lines representing the velocities of the stars near this point in magnitude and direction, this velocity diagram will not be spherically symmetrical; it will be a figure of revolution having the radius through the point as an axis of symmetry.

In the particular case in which this figure of revolution is very elongated in the direction of the radius, the majority of the stars would appear to be moving in directions only slightly inclined to the radius, and the motion might be interpreted as that of two streams of stars intermingled, each moving in a radial direction, but one moving inwards and the other outwards.

This brings to mind a suggestion made by H. H. Turner* to explain the observed "star-streaming" in our own universe. Turner supposed that the "star-streaming" might originate in the backwards and forwards motion of stars describing orbits of high eccentricity (nearly parabolic) about the centre of gravity of the universe. The question of the possibility of some such motion was investigated theoretically by Eddington $\dagger$, who found, by a method different from ours, that steady states of types included in formula (573) were possible. Eddington, however, did not notice that such motions were possible only in a strictly spherical universe. Our universe is almost certainly not spherical, being a lenticular or biscuit-shaped structure, and the star-streaming is almost certainly not along radii but in directions nearly tangential to radii. Thus it appears fairly certain that a formula such as (573) cannot express the observed stellar motions in our own universe.

Before leaving this formula, let us notice that the angular momentum of the whole system of stars about the axis of $z$

$$
=\Sigma M \iiint \iint f \varpi_{3} d u d v d w d x d y d z=0 .
$$

Since the angular momentum of a system moving solely under its own gravitational forces must remain constant, it follows at once that a system specified by formula (573) cannot possibly have originated out of a rotating nebula or out of any other system in which the angular momentum was not zero.

240. We pass now to the consideration of the type of motion expressed by formula (572). In this the total angular momenta about the axes of $x$ and $y$ are easily seen to be zero, but the angular momentum about the axis of $z$ is not zero. The plane of $x y$ is accordingly the invariable plane of the system, and the system can have originated out of a system in rotation, the axis of rotation having been parallel to the axis of $z$. 
Let us pass to cylindrical coordinates $\varpi, \theta, z$ and let the components of velocity at any point in these directions be denoted by $\Pi, \Theta, Z$. Then $\varpi_{3}=\varpi \Pi$, and the law of distribution (572) becomes

$$
f\left[\frac{1}{2}\left(\Pi^{2}+\Theta^{2}+Z^{2}\right)-V, \varpi \Theta\right] \ldots \ldots \ldots \ldots \ldots \ldots . .(574) .
$$

The velocities at any point are again not distributed uniformly for all directions in space, but the velocity diagram at any point will be a figure of revolution having the direction of $\theta$ increasing for axis. In other words starstreaming will take place, the direction being everywhere along the circles $\varpi=$ cons., $z=$ cons., which are circles coaxal with the axis of the whole universe.

This type of system, we have now seen, is the only type of system in a steady state which can have originated out of a rotating system. Thus if we assume, as we most reasonably may, that the cluster of stars generated out of a rotating nebula will ultimately assume a steady state, then this state must be one expressed by formula (574). In particular, if our universe is believed to be in a steady state, the hypothesis that it has originated out of a rotating nebula must fall unless the stellar motions are found to conform to a law of the type of (574).

\section{STELLAR MOTIONS IN THE GALACTIC UNIVERSE}

241. Let us examine the special problem presented by our own universe.

Charlier, who has made a special study of the distribution of stellar velocities, believes that the velocity surface is approximately an ellipsoid of revolution; in his opinion the axis is approximately, though not exactly, perpendicular to the radius vector to the centre of the system*. We have seen that if the system, whatever its origin, were in a steady state, the axes of the velocity surfaces would have to be either exactly radial or exactly perpendicular to the radius vector at each point. Charlier's result accordingly indicates that the system has not yet finally attained to a steady state, but that it is approaching a steady state of the type indicated by the law of distribution (574). And this steady state is, as we have seen, the one to which it would necessarily tend if it had originated, as we conjecture, out of a rotating nebula.

242. Let us try to estimate the length of time required for this final state to be reached. We have already supposed our universe to have a mass of $5 \times 10^{42} \mathrm{gms}$. and an equatorial radius of 2000 parsecs or $6 \times 10^{21} \mathrm{cms}$. The period of a star describing a circular orbit round the equator would be about $160,000,000$ years $\dagger$, and the period of description of any orbit by any star

* The Observatory, 40 (1917), p. 390, or Scientia, Aug. 1917.

† Eddington, in his Stellar Movements, using somewhat different data, obtains periods of $300,000,000$ years (p. 255) and of $0 \cdot 5^{\prime \prime}$ a century or $259,200,000$ years (p. 261). 
would obviously be of the same order of magnitude. This may be compared with the possible period of rotation of $370,000,000$ years for the Milky Way referred to in $\S 220$. Thus after $10,000,000$ years the path of the average star will have undergone a deflection of only about $22^{\circ}$ on account of the description of its orbit under the attraction of the universe as a whole, while encounters with near stars will, as we found in $§ 228$, have given it a crossvelocity out of its orbit of the order of 16 metres a second. This velocity, for a star moving with a velocity of $25 \mathrm{kms}$. a second, corresponds to a deflection of only about 2 '.

Thus it appears that after an interval of $10,000,000$ years the courses of the stars will be but little altered; their orbits over $10,000,000$ years are not far removed from the straight lines they would describe if gravitation were suddenly annihilated. It is clear that the approach to a final steady state is an excessively slow process.

We must, however, bear in mind that the foregoing calculations have been based upon numerical data derived from a consideration of the system in its present state. Our conjecture that the system may have evolved out of a rotating nebula of dimensions much less than those of the present system compels us to suppose that conditions must have been very different in the past. Adopting the conjectural figure arrived at in $\S 221$, we see that the period of description of an orbit in the earliest stages of a star's life must have approximated to 160,000 years, as compared with our estimated present value of about 160,000,000 years. Here we have immediately a shortening of the time-scale to about a thousandth part of its present value; what gravitation fails to accomplish now in 10,000,000 years may have been accomplished in 10,000 years when the system was young and the stars closely packed together.

243. A still more far-reaching change occurs when we turn back to nebular conditions.

In $\$ 220$ we estimated that the density of stars in the nebular arms may initially have lain between two limits. According to the first, stars were $\frac{1}{75}$ parsec apart, giving about 400,000 stars to the cubic parsec; according to the second, in which the stars were $\frac{1}{5}$ parsec apart, there would be 125 stars to the cubic parsec. Either star-density is very high compared with that of our present system which we have estimated as one star per 13 cubic parsecs. Thus the quantity $\nu$ which we have taken to be $10^{-56 \cdot 5}$ must be increased at least a thousand-fold, and perhaps a million-fold before our calculations can apply to our system in its earliest stages.

The effect on our previous calculations is profound. The times between successive collisions and the time required for a star to be deflected appreciably from its course by encounters with neighbouring stars are reduced enormously 
-instead of being measured in billions $\left(10^{12}\right)$ of years they must be measured only in millions. In a period which is, astronomically speaking, a short time, each star may be expected to have been knocked about considerably both by direct collisions and by close encounters with other stars. The only factor which makes a complete calculation of the final result impossible is that we do not know for how long the period in which the stars were close together must be supposed to have lasted.

244. If this period lasted indefinitely we know quite certainly what the final result would be-it would be a final steady state in which Maxwell's law of distribution of velocities and the law of equipartition of energy would hold. The final law of distribution would necessarily be of the general type found to be necessary for every cluster, namely

$$
f\left(E_{1}, \varpi_{3}\right)
$$

but Maxwell's law fixes the form of the function $f$. The appropriate form for a rotating system is known to be*

$$
f\left(E_{1}, \varpi_{3}\right)=C e^{-2 h\left[E_{1}+k \varpi_{3}\right]}=C e^{-h\left[M c^{3}-2 M V+2 k(x v-y w)\right]}
$$

where $C, h$ and $k$ are constants.

The shape of the cluster is determined by making $V$ satisfy equation (562). It is clear that this demands that the shape and arrangement shall be the same as that of a uniformly rotating mass of gas in isothermal equilibrium, the stars of types $M, M^{\prime}, \ldots$ playing the same part in the clusters as molecules of different kinds of gas. Now a uniformly rotating mass of isothermal gas cannot form a figure of equilibrium at all-its molecules merely fly off into space. It follows that a star-cluster cannot ever finally attain to the equipartition law expressed by equation ( 576$)$.

245. We must therefore suppose our system to have moved part way on the path towards equipartition but not to have attained it by the time that its expansion had become so great that all hope of finally attaining it had disappeared. We may suppose the law of distribution in our system to be approximately of the general type (575) and we may further suppose the equipartition law (576) to give a very rough approximation to the actual form of this law.

Let us examine some of the consequences of the law (576) with a view to testing whether any of them are fulfilled, even approximately, in our system.

The first property implied in the law of distribution (576) is one of correlation between a star's mass and its velocity, this being of the kind predicted by the well-known theorem of equipartition of energy. If $c, c^{\prime}$

\footnotetext{
* Jeans, Dynamical Theory of Gases (2nd Ed.), §§ 107, 113.
} 
denote typical velocities of stars of two types of masses $M, M^{\prime}$, then the law of equipartition requires that

$$
M \overline{c^{2}}=M^{\prime} \overline{c^{\prime 2}}
$$

where the bars over $c^{2}$ and $c^{\prime 2}$ denote that the means of these quantities are to be taken over a number of stars of the appropriate types; the stars included in these means may be selected in any way provided that this does not, directly or indirectly, imply a selection according to velocity. Clearly the law (577) requires that, statistically, the most massive stars shall move with the smallest velocities.

It appears to be generally accepted that the stars of greatest mass do, on the whole, move with the lowest velocities*; Halm $†$ has gone so far as to claim that the correlation between mass and velocity is actually that required by the equipartition law ( 577$)$. Whether we accept this result or not, it is an undoubted fact that there is a very marked correlation between a star's velocity and its spectral type, the $B$-type stars moving the most slowly, and so on+, while there is little doubt that $B$-type stars are on the whole the most massive.

246. A second property implied in the law (576) is one of correlation between mass and distance from the centre of the system; the most massive stars tend to remain in the more intense parts of the gravitational field while the lighter stars spread to greater distances, just as is the case with molecules of different masses in planetary atmospheres. Remembering that there is also a correlation between mass and spectral type, it appears that there ought to be correlation between spectral type and distance from the centre of the universe, the $M$-stars being statistically the most remote. Correlation of this kind has been found, but the $B$-stars, and apparently also the $A$-stars down at least to about $A 4$, appear to form an exception $\$$.

247. A third property implied in law (576) is that stars of similar type shall have the same average linear velocity relatively to the system as a whole, no matter what part of the system they are selected from. This is probably approximately true in our system; there appears to be no correlation between a star's distance and its velocity $\|$.

In $\S 221$ we imagined our system initially to have been a nebula of about 30 parsecs radius rotating in a period of about 160,000 years. The velocity of a point on the equator would be approximately $1200 \mathrm{kms}$. a sec., and the velocity of the ejected stars must at first have been comparable with this.

* See, for instance, Eddington's Stellar Movements, Chap. VIII, or Charlier, The Observatory, 40 (1917), p. 391.

† Monthly Notices R.A.S. 71 (1911), p. 634.

¥ Eddington, Stellar Movements, p. 154; Campbell, Stellar Motions, Chap. VI.

$\S$ Eddington, Stellar Movements, p. 167.

॥ Eddington, Stellar Movements, p. 161; Kapteyn, Amsterdam. Akad. Proc. 1911, pp. 528, 911. 
As the system of stars expanded these stellar velocities must have decreased, the tangential components in accordance with the law of conservation of angular momentum and the radial components on account of the gravitational attraction of the central mass. In the present state we may suppose orbits of an average of perhaps 2000 parsecs diameter to be described in about $160,000,000$ years, this rate af description of orbits would give linear velocities of the order of $25 \mathrm{kms}$. a second, which is of the order of magnitude of observed velocities.

Stars of different masses will describe orbits of different sizes. We have already seen that according to the equipartition law (576), the most massive stars will stay nearest to the centre of the universe; they will therefore describe the smallest orbits. As the period of an orbit is approximately independent of its size*, it follows that the most massive stars will move most slowly. If the law (576) were fully obeyed, the relation between mass and velocity would be that already obtained in equation (577).

248. A fourth property implied in the law (576) is that there is no starstreaming at any point or region of the system. The system rotates as a whole with a uniform angular velocity $\omega$, so that any selected set of comparison stars would rotate as a whole with this same angular velocity, and the only means of detecting the rotation of the system would be by some method such as that of Charlier explained in $\$ 221$. Relative to axes rotating with this angular velocity, the stellar velocities will, if law (576) is obeyed, be uniformly distributed over all directions in space, and the velocity diagrams will be uniformly spherical.

Thus the fact that star-streaming is observed is definite evidence that law (576) is not fully obeyed. On general principles we might expect the least massive stars to have advanced furthest towards equipartition. As we believe that there is correlation between spectral type and mass, there ought also to be correlation between spectral type and star-streaming, in the sense of $M$-type stars shewing star-streaming least. Such correlation is observed, with the $B$-type stars forming an exception.

249. A fifth property implied in the law (576), or indeed in any other steady-state law, is that the average radial velocity of the stars of any type measured from any point which is at rest relatively to the system as a whole shall be nil.

Campbell $\dagger$ has measured the radial velocities of 1060 stars and after freeing them from the solar motion finds that 586 are receding from the sun while only 474 are advancing towards it. Dividing the stars into first type

* If the stars were arranged in a spherical cluster of uniform density, the period would be entirely independent of the size of orbit.

+ Stellar Motions, Chap. VI. 
stars $(B$ to $F 4)$ and second type stars $(F 5$ to $M)$ it is found that there are 723 second type stars of which 371 are receding while 352 are advancing, the average velocity being found to be a velocity of recession of less than $1 \mathrm{~km}$. a second. Of the first type stars, however, 215 are receding while only 122 are advancing, and there is an average velocity of recession of $3: 33 \mathrm{kms}$. a second. Further analysis shews that the motion of recession may be attributed almost entirely to the $B$-type stars, 138 of these shewing an average velocity of recession of $4.93 \mathrm{kms}$. a second.

Thus although stars of types $A, F, G, K, M$ behave very approximately as required by the steady-state law (576), it is quite clear that the $B$-type stars do not, at least if the most direct and obvious interpretation is put upon the observations. Campbell and others are inclined to explain the observations away by supposing that there may be a systematic error in the spectroscopic determination of radial velocities, but our theory suggests another explanation -namely, that the universe is still expanding and that the $M$-type stars, being nearer to their steady state than the $B$-type stars, are expanding less rapidly.

250. We have discussed five properties which ought to be observed in our system if the final steady-state law (576) gave a tolerable approximation to the motion. Of these properties we found at least a strong tendency for two to be obeyed by all classes of stars. The remaining three were obeyed tolerably well by all classes of stars except the $B$-type stars and possibly some of the $A$-type stars.

Let us for the moment consider our universe as it would be if these latter stars were blotted out of existence altogether. Then we have a universe of which we can understand the mechanism well enough; the motion is in accordance with the laws of statistical mechanics and the system is exactly of the type we should expect to find formed as the final product of a rotating nebula.

To a first approximation, to which the $M$-stars conform particularly well, this universe is simply a mass of stars rotating in an equilibrium configuration, the angular velocity being everywhere uniform and of the order of $1^{\prime \prime}$ in 300 years. Superposed on to the rotational velocity (which does not come into our observational data, since the comparison stars share it with other stars) are individual velocities of separate stars; these, to our approximation, are distributed according to Maxwell's law, the mean velocity depending on a star's mass but not on its position in space. The shape of this universe is what we have called a pseudo-spheroid. The whole equilibrium is analogous to that of a rotating mass of gas, the stars forming the "molecules." Throughout the central portion, this "gas" is in isothermal equilibrium, although doubtless this condition changes at the boundary. In accordance with the laws of statistical mechanics, the heavier stars tend to congregate near the centre.

ग. c. 
This scheme gives a fair representation of the $M$-stars, but when we pass through $K, G, F$ and $A$-stars, the representation becomes less good. If we are right in believing the $M$-stars to be those of least mass we can find a reason for this. In the earlier stages of evolution of the system, when the stars were much more closely packed than now, encounters between the various stars were of frequent occurrence. The light $M$-stars would be easily and rapidly deflected from their paths by encounters with the heavier $K, G, F, A$-stars, but these more massive stars would only be slightly affected by encounters with $M$-stars. Thus we may naturally expect some departure from the equipartition law to be observed in the case of these heavier stars; we expect to find some trace remaining of their original motion along the nebular arms. The actual motion to be expected has been investigated; we found it to consist of a motion of "star-streaming," the directions of star-streaming forming circles round the axis of symmetry of the whole figure. This, according to Charlier, is the type of motion observed, and the amount of star-streaming, which is insignificant for the $M$-stars, becomes progressively greater as we pass through types $K, G, F, A$.

251. Thus the observed stellar motions would admit of a simple and natural interpretation if only the $B$-stars did not exist. The $B$-stars fail to carry on the sequence of the other types $K, G, F, A$ in almost every respect. No very satisfactory explanation of their anomalous behaviour suggests itself, but a conjecture may perhaps be risked.

Equation (543) suggests that the most massive stars may perhaps have been formed when the primitive nebula had its greatest density*, and therefore in the latest stages of its history. Thus, dividing the stars according to their present spectral types, the stars which are now $B$-stars may, statistically, have been created last. They may be the youngest type of stars. This conception may go some way towards reconciling Russell's theory of stellar evolution with older views to the relative ages of stars of different spectral types; we have already had occasion to notice ( $(201)$ that it is in no way inconsistent either with Russell's theory or with the results of our own theoretical investigations on stellar evolution.

Thus we may conjecture that the $B$-stars fail even to approximate to law (576) because they have not had time in which to begin to do so; they were created when the universe was already not far removed from its present state in which collisions and close encounters of stars are very rare. They have been acted on only by the main gravitational attraction of the universe as a

* The mass depends on $C^{3} \rho^{-\frac{1}{2}}$. For homologous contraction $\rho$ varies as $T^{3}$ or as $C^{6}$, so that the nuasses would all be uniform. But homologous contraction is an ideal process, occurring only if the physical properties of the gas remain unaltered with changes of temperature and derisity. It is possible that actually $T^{3}$ may have varied more rapidly than $\rho$, leading to the result stated. 
whole, and consequently their original motions persist to a much greater extent than is the case with other types of stars. Thus they still lie mainly in the galactic plane in which they were born; they are still, perhaps, expanding in this plane; to a large extent they still move in undissipated starclusters, perhaps even carrying wisps of uncondensed nebulous matter along with them.

Although some such conjecture naturally suggests itself, it has to be admitted that the subject is full of difficulty. No single hypothesis seems able to explain all the facts; for the present, apparently, we must be content to hold a number of self-contradictory hypotheses. Each of these hypotheses can, perhaps, give us a glimpse of part of the truth, but the time for welding them into one consistent whole has not yet come.

\section{GLOBULAR STAR-CLUSTERS}

252. Even the roughest of calculations makes it clear that the dimensions of globular clusters are much less than those of our galactic universe, while their star-density is vastly higher. Shapley* has made a special study of the bright cluster M3 (N.G.C. 5272) in Canes Venatici. He estimates its parallax to be $0.000074^{\prime \prime}$ with an error of less than 20 per cent.; it follows that the majority of the stars in the cluster are included within a sphere of 10 parsecs radius. Shapley estimates that such a sphere will contain at least 15,000 stars brighter than magnitude 20 . Each of these stars is at least two absolute magnitudes brighter than the sun.

Now in a sphere of the same radius surrounding our sun there are at most five stars of absolute magnitude two degrees brighter than the sun. As regards stars of this brightness, the stellar density in the cluster is 3000 times greater than that in the neighbourhood of our sun. We are not entitled to make a similar statement for stars of all kinds, but may notice that the stellar density of these bright stars in the cluster is far higher than our estimated stellar density for stars of all kinds in our galactic universe. The density of these bright stars in the cluster is about four per cubic parsec, as against our estimate of one per 13 cubic parsecs for stars of all kinds in our galactic universe.

Thus as regards stellar density the condition of this cluster approximates more closely to the earlier condition we have imagined for our universe than to its present condition, and the same is readily seen to be true for other clusters. To examine the effect on our calculations as to collision-frequency, etc., let us suppose the stellar density to be 1000 times greater than that assumed for our universe in $\$ 223$. Assuming the stars to be of diameter equal to that of Neptune's orbit we find that a star would experience material

* Astron. Soc. Pacific, No. 172 (1917); a later study of this and other star clusters will be found in Mt Wilson Contributions, No. 152. 
collision once in $4 \times 10^{9}$ years; assuming the masses to be equal to those in our system, encounters producing a deflection of more than $1^{\circ}$ would occur about once in $10^{8}$ years; the cumulative effect of encounters feebler than this produces a cross-velocity of $1 \mathrm{~km}$. a sec. in about $4 \times 10^{7}$ years; the "time of relaxation" $(\$ 228)$ is of the order of $3 \times 10^{10}$ years.

These estimates suggest that the typical globular star-cluster is hardly likely to have attained fully to the final steady state of equipartition of energy, but that this state is likely to be more closely approximated to than in our own system.

253. If we suppose the cluster to have formed out of a rotating nebula, the law of distribution of density and velocities must, as in $\$ 240$, be of the general form

$$
f\left(E_{1}, \varpi_{3}\right)=f\left[\frac{1}{2}\left(\Pi^{2}+\Theta^{2}+Z^{2}\right)-V, \varpi \Theta\right]
$$

Approximation to the final equipartition state will be shewn by the function $f$ approximating to the special form expressed by equation (576). This law of distribution can never be fully attained; as it is approached, the stars having the highest total energy escape from the main cluster and form runaway stars in space just as those molecules which are endowed with the highest total energy may escape from a planetary atmosphere and describe orbits in space. These stars carry with them an undue share both of energy and of angular momentum, with the result that the cluster contracts and rotates less rapidly; the cluster must continually approach, but never quite reach, a spherically symmetrical configuration.

The investigations of Pease and Shapley to which reference has already been made $(\$ 6)$ suggest that the majority of star-clusters still shew evidence of a flattened form, but the approximation to a globular configuration is nevertheless tolerably close. The approach to a spherical form will be indicated by the function $f\left(E_{1}, \varpi_{3}\right)$ depending less and less upon $\varpi_{3}$. In the final spherical form the law of distribution will reduce to the law $f\left(E_{1}\right)$ discussed in $§ 237$.

In this law the stars behave like the molecules of a gas; different forms of the function $f$ correspond to different relations between pressure and density in this supposed gas. In the final equipartition law, $f\left(E_{1}\right)$ reduces to the exponential form $C e^{-2 h E_{1}}$, and the corresponding law between pressure and density is that of an isothermal gas. With this law the density at a great distance from the centre falls off as $1 / r^{2}$. Thus the total mass is infinite or the central density infinitesimal; the law is not one which can ever be attained in an actual star-cluster.

254. Let us simplify the problem by limiting the possible relations between pressure and density in the supposed gas to those which correspond to adiabatic equilibrium; different laws are supposed (quite arbitrarily) to 
correspond to different values of $\gamma$ in the relation $p=\kappa \rho^{\gamma}$. The approach to the final equipartition state will be represented by $\gamma$ approaching the value $\gamma=1$ which corresponds to isothermal equilibrium, but the lowest value of $\gamma$ which is possible, short of the cluster scattering to infinity, is $\gamma=1 \frac{1}{5}$. Thus with our arbitrary supposition that different laws are adequatety represented by different values of $\gamma$, it appears that the value of $\gamma$ cannot fall below $1 \frac{1}{5}$, and the final law of distribution of density in a globular star-cluster would be the same as that in a gas in adiabatic equilibrium with $\gamma=1 \frac{1}{5}$, namely Schuster's law (cf. §149)

$$
\rho=\rho_{0}\left(1+r^{2} / a^{2}\right)^{-\frac{5}{2}}
$$

This is the law actually found in a number of globular star-clusters by Plummer and von Zeipel ( $(6)$. It is at best only approximate; indeed it appears that a law falling off at great distances as $r^{-4}$ would fit the distribution in many star-clusters still better*. This is hardly surprising. Our limitation of the various laws to those corresponding to adiabatic equilibrium was quite unjustifiable; it may lead to an approximate picture of the processes going on in a globular cluster but cannot be expected to reveal the whole truth.

* Monthly Notices R.A.S. 76 (1916), p. 567. 


\section{CHAPTER XI}

\section{THE EVOLUTION OF BINARY AND MULTIPLE STARS}

\section{THE PROCESS OF FISSION}

255. The motion of our hypothetical mass of nebulous matter has now been traced out through its earlier stages in which it formed a rotating nebula, and through its later stages in which this nebula condensed into stars. In the last chapter we considered the general nature of the motion to be expected in the cluster of stars so formed; the present chapter will be devoted to the further history of individual stars.

We have supposed that an individual star comes into existence as a condensation in a nebular arm. In this earliest period of its existence its mean density is very low, being perhaps of the order of $10^{-17}$ grammes per cubic centimetre, and its surrounding atmosphere is contiguous with that of the neighbouring stars. At this stage it shares in the rotation of the nebula of which it forms part, the period of this rotation being perhaps of the order of 160,000 years.

As time proceeds the arms of the nebula expand while individual stars contract, so that the stars become continually more distinct from one another until finally they may be regarded as entirely separate bodies, each describing its independent orbit under the gravitational attractions of the other stars. For purposes of numerical calculation, which must necessarily be very vague and inexact, let us suppose that the star starts its independent existence as a separate star when its linear dimensions have contracted to one-quarter, so that its mean density has increased 64-fold, and is now of the order of $64 \times 10^{-17}$. Let us also suppose that during this process the linear dimensions of the nebula have doubled, so that its period of rotation will have increased four-fold, to about 640,000 years. This will also be approximately the period of rotation of the average star when it first starts its existence as an independent body.

From these estimates, we find that the value of $\omega^{2} / 2 \pi \gamma \bar{\rho}$ for the star when it starts as an independent body will be of the order of 00035 . During the subsequent further contraction of the star, the conservation of angular momentum requires that $\omega$ shall increase (approximately) inversely as the square of the linear dimensions of the star and so as $\bar{\rho}^{\frac{2}{3}}$, whence it follows that $\omega^{2} / 2 \pi \gamma \bar{\rho}$ will increase approximately as $\bar{\rho}^{\frac{1}{3}}$. By the time the density of 
the star has increased by a further factor $10^{9}$ the value of $\omega^{2} / 2 \pi \gamma \bar{\rho}$ will have increased by a factor $10^{3}$ to 0.35 . This is just about the critical value at which a sharp edge forms $(\$ 205)$ and the mass begins again to disintegrate by throwing off matter from its equator.

Naturally no stress can be laid on these particular figures but they suggest that a star formed in the way we have imagined would begin to disintegrate, owing to rotational instability, as soon as it had contracted to a density of the order of $64 \times 10^{-17} \times 10^{9}$ or, say, $10^{-6}$.

256. Consider next the course of events after this density has been passed. A sharp edge forms and jets of matter are thrown off from the equator. Will these jets of matter condense into filaments in the way we have imagined the arms of spiral nebulae to condense?

The minimum mass per unit length for which a jet of gas can condense into a filament has been seen to be about $2 C^{2} / 3 \gamma(\$ 216)$. The velocity with which the matter is ejected can hardly be less than the velocity of effusion into a vacuum, say $\frac{1}{4} C^{*}$, and is likely to be greater. Thus condensation can only occur if the rate of ejection of matter is greater than $C^{3} / 6 \gamma$ grammes per second. Taking the very low value $C=4 \times 10^{5}$, the value of this quantity is found to be $1.6 \times 10^{23}$ grammes per second. Thus condensation is hardly to be expected unless the star ejects matter at this rate, which would correspond to a dissolution of the whole star in a few centuries.

This time is so small compared with what we believe to be the time of shrinkage of a star that the formation of condensed filaments can hardly be regarded as a probable, or even as a possible, event. If such filaments were formed, the mass would constitute a miniature spiral nebula of mass comparable with that of a single star, and the filaments might ultimately condense into a system of encircling planets. In some such way Arrhenius, See and others ( 16 ) have imagined our system of sun and planets to have been formed. The foregoing calculations make it very improbable that this process can ever take place in masses comparable only with those of the stars.

This conclusion is in accord with observation, for not a single spiral nebula is known which there is any reason to suppose lies within the confines of our galactic system. If such miniature spirals existed we should expect them to shew a preference for regions near the galactic plane. But the observed spiral nebulae, with remarkable unanimity, avoid this region: of the thousands of spirals which are known to exist, not a single one has been found within the galactic structure $†$.

257. If the ejected matter does not condense into filaments, it will form a surrounding atmosphere, and as the dissolution of the central mass 
progresses the atmosphere will continually increase at the expense of the central mass. This motion is one which it is exceedingly difficult to trace out dynamically, even in its main outlines. It seems possible that the atmosphere may in time condense into nuclei, and that these might ultimately form planets, but this has to be mainly a matter of conjecture. It is perhaps worth considering whether the planetary, and possibly also the ring nebulae, can be bodies of the type we have been considering*. We may also contemplate the possibility of planets being formed in this way although, as we shall see later, the planets of our solar system cannot have been so formed.

258. The equatorial ejection of matter will continue until a further critical density $\rho_{0}$ is reached, at which the pseudo-spheroidal figure for the nucleus becomes unstable and gives place to a pseudo-ellipsoidal form (cf. $§ 185)$. The new pseudo-ellipsoidal figure will eject matter only at its two pointed ends; it is perhaps worth considering spindle-shaped planetary nebulae such as N.G.C. 7009 in this connection (see Plate I). Ultimately the pseudo-ellipsoidal nucleus gives place to a pear-shaped figure and this will divide into two detached masses.

The final result of the process of disintegration will accordingly be a binary star, the two components rotating about one another in a more or less dense atmosphere of ejected matter, through which they will plough their way. This formation at once recalls Duncan's attempted explanation of the Cepheid variablest. At a later stage the atmospheres will condense round the two stars, leaving an ordinary binary star.

During the process of condensation, the greater part of the atmosphere is likely to condense round the more massive constituent, so that the light from the more massive star will be more screened than that from the lighter one. Temporarily the more massive component may shine less brightly than its smaller companion. This condition is observed in $\beta$ Lyrae, a spectroscopic binary in which the dark star has a mass about $2 \cdot 2$ times that of the bright star, the two masses being very nearly in contact; the explanation we have given of this condition was first suggested by Meyers + . If this is the true explanation, it would appear that $\beta$ Lyrae provides an instance of a binary star in the very earliest stages of its existence.

259. If the stars may be regarded as masses of ordinary gas, it is not difficult to obtain an estimate of the critical density $\rho_{0}$ before which fission cannot begin.

Discussing a mass of gas in adiabatic equilibrium, we found in Chapter VII that fission could not begin so long as $\gamma$, the ratio of the specific heats, was less than about $\mathbf{2 \cdot 2}$.

* See Plate I (p. 5) ; also Campbell, Science, 45 (1917), p. 538.

† Lick Obs. Bull. 6 (1911), 151. See also Campbell, Stellar Motions, p. 307.

‡ Astrophys. Journ. 7 (1898), p. 21. 
Since for an ordinary gas under ideal conditions, $\gamma$ is always less than $1 \frac{2}{3}$, this result indicates that fission cannot begin, at any rate for a mass of gas of uniform composition, until the density is so great that the ideal gas laws are substantially departed from. Koch* has obtained the experimental value $\gamma=2 \cdot 21$ for air at 100 atmospheres pressure and temperature $-79^{\circ} \mathrm{C}$., the corresponding density being about 180 times that of normal air, or say $\cdot 23$. Thus if a star were made of air in adiabatic equilibrium, the critical density $\rho_{0}$ would be something like $\frac{1}{4}$.

More generally we have seen that the critical density $\rho_{0}$, for a mass of uniform composition, must be one at which the gas laws are substantially departed from. Now according to Russell's theory of stellar evolution, for which as we have seen $(\S 200)$ there is a strong theoretical basis, a star is supposed to get continually hotter until the gas laws are substantially departed from, after which its temperature begins to decline. On this theory the point at which the gas laws are first substantially departed from may be approximately identified with the point of maximum temperature in the star's evolution, and this corresponds to spectral type $B$ for massive stars, but to a later spectral type for lighter stars.

The two estimates we have formed of the critical density $\rho_{0}$ are in fair agreement. Russell estimates the average density of "giant" $A$-type stars to be about $\frac{1}{10}$, so that $\frac{1}{4}$ is not an unreasonable estimate for the density of $B$-type stars.

It would now follow, from our preliminary theory in which a star is represented as an adiabatic mass of air, that

(i) no binary star which has formed by fission can have a density of less than about $\frac{1}{4}$,

(ii) no giant binary star can have been formed by fission,

(iii) the temperature of a binary star which has formed by fission must decrease as its evolution progresses.

260. The densities of eclipsing binaries can be estimated with very considerable accuracy. Shapleyt has computed the densities of 90 of these, the results being given in the following table in which densities are classified according to spectral type.

At once it appears that there are binaries of very low density, 33 out of the 90 having a density of less than $\frac{1}{10}$, and 4 having a density of less than $\frac{1}{1000}$. Further, in Shapley's table the division into giant and dwarf stars is quite marked, the entries in the table lying approximately thus : <; the

* Soc. Franç. de Physique, Recueil des Constantes, p. 321.

† Contributions from the Princeton Univ. Observatory, 3 (1915). I have omitted from the table three stars which Shapley states "should not be given much weight" and "probably deserve little consideration." 
stars on the lower branch are giant stars. The six stars which occupy the bottom right-hand corner must all be regarded as binary giant stars, of types $F, G$ and $K$ and of density less than $\frac{1}{100}$.

\begin{tabular}{|c|c|c|c|c|c|c|c|}
\hline $\log \rho$ & $\rho$ & $B$ & $A$ & $F$ & $G$ & $K$ & Total \\
\hline+0.5 to 0 & 1 to $3 \cdot 2$ & - & - & - & 2 & - & 2 \\
\hline 0 to -0.5 & 32 to 1 & - & 11 & 7 & 1 & - & $1 \overline{9}$ \\
\hline-0.5 to -1.0 & $\cdot 10$ to $\cdot 32$ & 8 & 24 & 3 & 1 & - & 36 \\
\hline-1.0 to $-1 \cdot 5$ & .032 to $\cdot 10$ & 5 & 13 & - & - & - & 18 \\
\hline$-1 \cdot 5$ to $-2 \cdot 0$ & $\cdot 01$ to $\cdot 032$ & 3 & 6 & 1 & 1 & - & 11 \\
\hline$-3 \cdot 0$ to $-4 \cdot 0$ & .0001 to $\cdot 001$ & - & - & - & 2 & 1 & 3 \\
\hline$-5 \cdot 0$ to $-6 \cdot 0$ & order of $2 \times 10^{-6}$ & - & - & - & 1 & - & 1 \\
\hline
\end{tabular}

The densities of 40 visual binaries have been estimated by Opik*, although the method does not admit of great accuracy. Of the 40 computed densities 32 are greater than one-tenth of the sun's density, say greater than $\cdot 14$, while 8 are less than this. The two lowest densities are 0.017 and 0.018 for the brighter components of $\epsilon$ Hydrae and $\xi$ Boötis. These densities are well below the limit which our theory allows for fissional binaries; moreover these two stars, being of spectral types $F 8$ and $K 5$, must both be "giants."

261. Thus observational material shews either that all binaries are not formed by fission or else that our estimate of the critical density, below which fission cannot occur, stands in need of revision.

This estimate was based upon the study of a particular model star, namely a mass of gas of uniform composition arranged in adiabatic equilibrium. Calculations of the temperature distribution in such model stars have been made by Emden $\dagger$, Eddington ${ }_{+}^{+}$and others. Assuming the two components of $\beta$ Lyrae to consist of monatomic hydrogen in adiabatic equilibrium $\left(\gamma=1 \frac{2}{3}\right)$, Emden calculates central temperatures of $6.8 \times 10^{6}$ and $4.2 \times 10^{6}$; if the matter is supposed to be diatomic hydrogen the temperatures are found to be $1.8 \times 10^{7}$ and $1.1 \times 10^{7}$. For a model star of mass $2.87 \times 10^{33}$ grammes and mean density 002 , supposed made of iron vapour of atomic weight 54 in adiabatic equilibrium with $\gamma=1 \frac{1}{3}$, Eddington finds a central temperature of $1.52 \times 10^{8}$. The essential point is that all such calculations give a central temperature of the order of $10^{7}$. Now this temperature is so high as to be quite inconsistent with the supposition that the gas is of uniform structure throughout - if our physical knowledge is to count for anything, it is almost certain that the properties and structure of gas at a temperature of $10^{7}$ will be widely different from those at the surface temperature of a few thousand degrees. 
262. The most plausible conjecture that can be made is perhaps that in the far interior of a star the atoms must be almost completely broken up into their constituent electrons*. In this case, the effective molecular weight would approximate, as Eddington has pointed out $\uparrow$, to 2. This limit would be almost independent of the chemical structure of the matter, since the number of electrons in all atoms except hydrogen is nearly equal to half the atomic weight.

This reduction of the effective molecular weight will greatly modify our numerical estimates. Assuming the star to be made of air (molecular weight about 30 ) we found in $\$ 259$ a critical density $\rho=\frac{1}{4}$. If other factors remained unaltered a reduction of molecular weight from 30 to 2 would reduce this critical density from $\frac{1}{4}$ to $\frac{1}{60}$. Other factors do not, however, remain unaltered. On our present tentative view of the interior structure of a star, the effective molecular weight $m$ must have its minimum value, nearly equal to 2 , at the centre of the star, and must gradually increase as we pass outwards towards the surface. Our critical value $\gamma=2 \cdot 2$ was determined on the assumption that $m$ had a uniform value throughout the star. It was found that a decrease of $m$ on passing outwards would increase the critical value of $\gamma$; in the same way a decrease of $m$ on passing inwards must decrease the critical value of $\gamma$. This might possibly result in a still further decrease of the critical density $\rho$.

Beyond this there are general physical considerations which require a still further adjustment of the critical density. Eddington + has pointed out the importance of radiation-pressure in the internal mechanism of a star. When there is extreme ionisation in a star's interior, Eddington's conclusions will need modification, and the disturbing effect of radiation-pressure will be less than that originally estimated but it will still be appreciable§. Finally the departure of our ordinary gas laws from the laws of a perfect gas, on which the relation between $\gamma$ and the density depends, arises from the "size" of the molecules and the extension of the field of force surrounding them. When the gas is highly ionised, we have to deal rather with the extension of the field of force round individual electrons and positive nuclei, and it is almost impossible even to guess at the density at which the departure from the gas laws becomes appreciable.

All these considerations suggest that our preliminary theoretical conclusions must be viewed at least with suspicion, so that there is certainly no ground for surprise that they have not proved to be confirmed by observation. 
263. We have seen that a double-star must be supposed to be born as the result of cataclysmic motion. The pear-shaped figure is unstable, so that as soon as it is formed dynamical motion ensues and fission results. The masses are at first projected away from one another with considerable velocity, but seem likely to settle down finally to describe steady orbits about one another.

In his researches on this problem, Sir G. Darwin supposed that the initial orbits would be strictly circular, but this was because he believed the process of fission to be a statical process and not a dynamical process, as we have seen it to be. According to Darwin's view, the changes in the star while fission was taking place were, initially at least, of a purely secular nature, and it was natural to suppose that the final result would be two masses rotating in actual contact and at rest relatively to one another.

We have seen that this cannot be the final result of fission for incompressible masses, because such a configuration would be statically unstable, as indeed was ultimately found by Darwin himself (cf. $\$ \S 64,65$ ). For a compressible mass, there is no reason why it should not be the final result of fission (cf. $§ 164$ ) although the intermediate processes would almost certainly be different from those imagined by Darwin, cataclysmic motion probably ensuing immediately the pear-shaped figure is formed, but possibly giving place to steady statical motion before actual fission occurs.

There being no longer any theoretical justification for supposing that the initial orbits will be strictly circular, we have to consider the possibility of the masses being thrown apart with appreciable radial velocities, and describing elliptic orbits about one another.

Consider for simplicity the ease in which the original star is supposed to divide into equal masses, and suppose that fission occurs when the centre of each mass is at a distance $r$ from the common centre of gravity. Let each star be supposed to have a radial velocity $v$ in addition to the tangential velocity $\omega r$ in space resulting from rotation. Each mass will describe approximately an elliptic orbit in space so that after the orbits are partially described the masses will again each be at a distance $r$ from their common centre of gravity, but are now approaching each other with a radial velocity $v$. A collision of some kind must occur, and since the masses will not be perfectly elastic, their velocity of recession after collision will be some velocity $v_{0}$ less than $v$, while the radial velocity $\omega r$ must, from the conservation of angular momentum, be the same as before. It follows that the new orbit will be of less eccentricity than the old, and the eccentricity will further diminish at each subsequent collision. We cannot argue that the eccentricity will be finally reduced to zero; a limiting value will be reached such that the masses just graze one another at periastron. 
264. Sir G. Darwin further supposed that immediately after fission the periods of rotation of the two masses and the period of revolution about one another would all three coincide, so that the system would rotate as a rigid body. There is no longer the same justification for this supposition when it is recognised that fission occurs only after cataclysmic motion.

We may however notice that only one vibration is unstable at the point of bifurcation at which cataclysmic motion begins; this vibration is one in which neither half of the mass gains upon the othèr either in rotation or revolution. When the elongation of the pear-shaped figure first takes place, the pointed end of the pear must, on account of conservation of angular momentum, lag somewhat behind the rotation of the blunter end, but any such difference of rotation produces a distortion which corresponds to a stable vibration: forces of restitution at once come into play and equalise the angular velocities. Similar forces of restitution will be in operation right up to the instant of fission, so that in the final system the rotations may be expected to agree with the revolution, both in period and in phase. The stars will accordingly rotate about one another like a rigid body except for the slight eccentricity of orbit discovered in the preceding section.

\section{Comparison with Observation}

265. These theoretical conclusions are borne out by observation on stars of the $\beta$ Lyrae type*. In stars of this type the light curve varies continuously, shewing that the masses must be either in actual contact or close to actual contact as in fig. 34 (p. 163). Any difference in the periods of rotation and revolution would shew itself in nonperiodicity of the light curve; of this there is no evidence whatever. The eccentricity of orbit is invariably small, being about 0.02 for $\beta$ Lyrae, $X$ Carinae and $R R$ Centauri, in which the separation calculated from the light curve is zero or negative (corresponding to imperfect fission), and being 0.03 for $U$ Pegasi in which the separation is excessively small.

The periods are short, varying from $14 \mathrm{~h}$. $32 \mathrm{~m}$. for $R R$ Centauri to 12.908 days for $\beta$ Lyrae.

\section{MOTION SUBSEQUENT TO FISSION}

\section{Tidal Friction}

266. Darwin has shewn the importance of tidal friction in the subsequent motion. As the stars shrink, the rate of rotation of each will increase in accordance with the conservation of angular momentum, so that the rotations of the separate masses will gain on their revolution about one another, and

* Campbell, Stellar Motions, Table XXXI. 
the arrangement will be of the type represented in fig. 44 . In such a configuration, each mass will exert a couple on the other in such a direction as to augment the motion of revolution already taking place. These couples

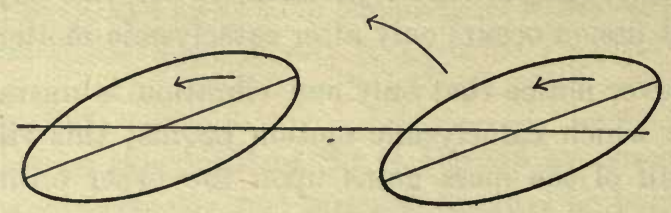

Fig. 44.

are the direct successors of the forces of restitution, mentioned in $\$ 264$, which tend to equalise the periods of rotation and of revolution. Let us investigate the effect of the couples on the orbits of the masses.

Suppose the original star of mass $M+M^{\prime}$ to divide into two components of masses $M, M^{\prime}$, each of which will describe an approximately elliptic orbit about the centre of gravity of the two. Let $e$ be the eccentricity and $a$ the semi-major-axis of the orbit of either mass relative to the other.

If the tidal friction couples were non-existent, there would be the usual two first integrals of the motion,

$$
\frac{M M^{\prime}}{M+M^{\prime}} r^{2} \dot{\theta}=h, \text { where } h^{2}=\frac{M^{2} M^{\prime 2}}{M+M^{\prime}} a\left(1-e^{2}\right)
$$

Energy $=E$, where $E=-M M^{\prime} / 2 a$

Let the couples produced by tidal friction be supposed to act for a short interval $d t$, each star exerting a couple $G$ on the other in the direction of rotation. The orbit will be disturbed and at the end of the interval $d t$ a new orbit will be described. The eccentricity and semi-major-axis of this may be denoted by $e+\dot{e} d t, a+\dot{a} d t$, in which $\dot{e}$ and $\dot{a}$ may be regarded as rates of increase during the action of the couple $G$.

These rates of change are readily found. From equation (580)

so that

$$
\begin{gathered}
\frac{1}{a}=-\frac{2 E}{M M^{\prime}} \\
\frac{1}{a^{2}} \frac{d a}{d t}=\frac{2}{M M^{\prime}} \frac{d E}{d t}=\frac{2}{M M^{\prime}} G \dot{\theta}
\end{gathered}
$$

Since $G$ must be supposed to act in the direction of $\theta$ increasing, $G \dot{\theta}$ will be positive, so that $d a / d t$ is positive. Tidal friction increases $a$.

By logarithmic differentiation of equation (579)

$$
\begin{aligned}
\frac{1}{1-e^{2}} \frac{d}{d t}\left(1-e^{2}\right) & =\frac{2}{h} \frac{d h}{d t}-\frac{1}{a} \frac{d a}{d t} \\
& =\frac{2}{h} G-\frac{2 a}{M M^{\prime}} G \dot{\theta}
\end{aligned}
$$


from equation (581). Using equations (579) this becomes

$$
\frac{2 G}{h}\left[1-\frac{a^{2}}{r^{2}}\left(1-e^{2}\right)\right] \text {. }
$$

Introducing the polar equation of the orbit in the form

$$
\frac{a\left(1-e^{2}\right)}{r}=1+e \cos \theta
$$

we find

$$
\begin{gathered}
\frac{d}{d t}\left(1-e^{2}\right)=\frac{2 G}{h}\left[\left(1-e^{2}\right)-(1+e \cos \theta)^{2}\right], \\
\frac{d e}{d t}=\frac{G}{h}\left[\cos \theta+e\left(1+\cos ^{2} \theta\right)\right] .
\end{gathered}
$$

As $G$ acts in the direction of $\theta$ increasing, $G / h$ will be positive, while $e\left(1+\cos ^{2} \theta\right)$ is necessarily positive. Tidal friction acts mainly when the masses are closest together-i.e. when $\cos \theta$ is nearest to +1 . Hence it is readily found that $G \cos \theta / h$ is preponderatingly positive and de/dt integrated through a whole orbit will be positive.

Thus tidal friction increases both $a$ and $e$, and as the evolution of a binary star progresses we ought, on the tidal-friction theory, to find-(i) increasing separation, (ii) increasing period, (iii) increasing eccentricity.

267. Campbell has attempted to test these conclusions with the help of

\begin{tabular}{|c|c|c|c|c|c|c|}
\hline Period & $\begin{array}{c}\text { Short } \\
\text { unknown }\end{array}$ & $\begin{array}{l}0-5 \\
\text { days }\end{array}$ & $\begin{array}{l}5-10 \\
\text { days }\end{array}$ & $\begin{array}{l}10 \text { days- } \\
1 \text { year }\end{array}$ & $\begin{array}{c}\text { Over } \\
1 \text { year }\end{array}$ & $\begin{array}{l}\text { Long } \\
\text { unknown }\end{array}$ \\
\hline No. of types $O, B$ & 8 & 15 & 10 & 14 & 1 & 0 \\
\hline$e=$ & - & 0.04 & $0 \cdot 10$ & 0.34 & - & - \\
\hline No. of type $A$ & 4 & 10 & 1 & 12 & 2 & 0 \\
\hline No. of type $F^{e=}$ & $\overline{0}$ & $\begin{array}{c}0 \cdot 04 \\
6\end{array}$ & $\overline{2}$ & $\begin{array}{c}0.55 \\
4\end{array}$ & $\overline{3}$ & $\overline{1}$ \\
\hline$e=$ & & 0.05 & - & $0 \cdot 15$ & $0 \cdot 44$ & $\overline{10}$ \\
\hline $\begin{array}{c}\text { No. of types } G-M \\
e=\end{array}$ & 0 & $\frac{0}{-}$ & $\frac{0}{-}$ & $\begin{array}{c}3 \\
0 \cdot 06\end{array}$ & $\begin{array}{c}9 \\
0 \cdot 38\end{array}$ & 13 \\
\hline $\begin{array}{l}\text { All types } \\
e=\end{array}$ & 12 & $\begin{array}{c}31 \\
0.04\end{array}$ & $\begin{array}{c}13 \\
0 \cdot 14\end{array}$ & $\begin{array}{c}33 \\
0 \cdot 36\end{array}$ & $\begin{array}{c}15 \\
0 \cdot 38\end{array}$ & 14 \\
\hline
\end{tabular}
material provided by his studies of spectroscopic and visual binaries*. The general summary of Campbell's classification of spectroscopic binaries is shewn in the following table:

In this table the values of $e$ are mean values for all the binaries for which the eccentricity can be calculated, no entry being inserted when the eccentricity is known only for a single star.

* "Second Catalogue of Spectroscopic Binary Stars," Lick Obs. Bull. No. 181 (1910); Stellar Motions, Chap. VIII. 
We notice in the last two lines that increasing period goes with increasing eccentricity, as it ought to do. The parallelism becomes still more striking when visual binaries are included: Campbell gives the following table:

\begin{tabular}{|c|c|c|c|}
\hline \multirow[t]{2}{*}{ Type of Star } & Number & Mean Period & Mean $e$ \\
\hline & 31 & . 2.59 days & $0 \cdot 04$ \\
\hline Spectroscopic & 13 & $6.90 \quad$ & $0 \cdot 14$ \\
\hline Binaries & 33 & $73 \cdot 5^{-}$ & $0 \cdot 36$ \\
\hline & 15 & 20.5 years & 0.38 \\
\hline Visual & 25 & $32 \cdot 8$ & 0.48 \\
\hline Binaries & 25 & $108 \cdot 1$ & 0.51 \\
\hline
\end{tabular}

in which the increase of eccentricity with period is very apparent.

Returning to the original table, it will be seen that the entries form roughly a slanting diagonal thus: - . Advancing spectral type goes with increasing period and eccentricity, and these according to the tidal friction theory increase with age. The inference drawn by Campbell and others is that, generally speaking, age and advancing spectral type go together*. The youngest binaries are of types $O, B$; then come types $A, F$, and finally types $G-M$. This would bring us back to our theoretical conclusion of $\$ 259$ that fission takes place at about $B$-type, but we shall immediately find reasons for modifying very considerably this interpretation of Campbell's table.

268. Increasing separation of the two components of a binary star, whether under tidal friction or otherwise, requires an increase in the orbital momentum of the system. So long as the system remains free from external disturbance, the total angular momentum of the system must remain constant, so that the increase of orbital momentum is necessarily gained at the expense of the rotational momenta of the cunstituent stars. When the masses of the two components are very unequal there is a large store of angular momentum in the rotation of the more massive one, and separation can proceed very far before this has all been transferred to orbital momentum. But, as Russell has pointed out $\dagger$, conditions are very different when the components are of approximately equal mass, as is the case with the majority of binary stars $(§ 2)$.

Consider a binary star whose components are of masses $M, M^{\prime}$. Allowing for the finite sizes of the components and for their distortion from the spherical shape, the force between them may be supposed to be

$$
\frac{M M^{\prime}}{r^{2}}(1+\zeta)
$$

* Campbell, Lick Obs. Bull. 181 (1910), p. 42 ; Stellar Motions, p. 269. Eddington, Stellar Movements, p. 178.

† Astrophys. Journ. 31 (1910), p. 185. 
as in $\S 62$. An elliptic orbit will be described only if $\zeta$ remains sensibly constant; in this case the "mean motion" $n$ is given by

$$
n^{2}=\frac{M+M^{\prime}}{a^{3}}(1+\zeta)
$$

this reducing to our former equation (121) when the orbit is circular.

The orbital momentum of the system is readily found to be

$$
\frac{M M^{\prime}}{\left(M+M^{\prime}\right)^{\frac{1}{2}}}(1+\zeta)^{\frac{1}{2}} l^{\frac{1}{2}}
$$

where $l$ is the semi-latus rectum, equal to $a\left(1-e^{2}\right)$, whence, on adding the rotational momenta, the total angular momentum is found to be

$$
\mathrm{M}=M k^{2} \omega+M^{\prime} k^{\prime 2} \omega^{\prime}+\frac{M M^{\prime}}{\left(M+M^{\prime}\right)^{\frac{1}{2}}}(1+\zeta)^{\frac{1}{2}} l^{\frac{1}{2}}
$$

Let us suppose that in the earliest stage of existence the components - rotate fairly close to one another with a common angular velocity in an approximately circular orbit of radius $R$. In this case $\omega=\omega^{\prime}=n$, and formula (583) becomes

$$
\mathrm{M}=\left[M k^{2}+M^{\prime} k^{\prime 2}+\frac{M M^{\prime}}{\left(M+M^{\prime}\right)} R^{2}\right](1+\zeta)^{\frac{1}{2}}\left(M+M^{\prime}\right)^{\frac{1}{2}} R^{-\frac{3}{2}}
$$

an equation which has already been given in $\S 64$.

269. Consider first the extreme case in which the masses are supposed homogeneous and incompressible. To obtain some idea of the ratio of division of $\mathbf{M}$ into its rotational and orbital parts, $I$ have calculated the ratios of the separate terms in $\mathbf{M}$ for Darwin's figures of closest approach, the data being those already tabulated in $\S 65$. The results prove to be as follows:

$$
\frac{M^{\prime}}{M}=0
$$

0.4

0.5

$1 \cdot 0$

Rotational mom. of $M^{\prime} \quad 0$

.039

$\cdot 046$

077

",$\quad M \quad 1$

$\cdot 160$

$\cdot 135$

.077

Orbital momentum

$\cdot 801$

$\cdot 819$

$\cdot 846$

Total $1.000 \quad 1.000 \quad 1.000 \quad 1.000$

With very few exceptions all known binary stars have values of $M^{\prime} / M$ lying between 0.4 and $1 \cdot 0$. Excluding the few systems for which $M^{\prime} / M$ is less than 0.4 , it appears that the orbital momentum must initially be at least 80 per cent. of the whole if the components move in circular orbits; it would of course be still greater if they moved in stable elliptical orbits.

Thus no matter for how long tidal friction or other similar tendencies act, the orbital momentum cannot, in the whole course of a binary star's history, 
increase to more than $1 \frac{1}{4}$ times its initial value. From formula (582) it follows that $(1+\zeta) l$ cannot increase to more than 1.56 times its initial value. For bodies at a considerable distance apart $\zeta=0$; for two similar ellipsoids in contact $\zeta=022$, which is the maximum value of $\zeta$. Thus in the whole course of evolution the value of $1+\zeta$ cannot decrease more than in the ratio 1.22:1. It follows that at the very most $l$ cannot increase in a ratio greater than $1.56 \times 1.22$ or 1.90 .

These calculations refer to a perfectly homogeneous mass. To study the effect of compressibility let us pass to the extreme case of matter so compressible that Roche's model ( $\$ 149)$ may be supposed to give an approximation to the arrangement of density. We may put $k^{2}=k^{\prime 2}=0$ and $\zeta=0$. The whole momentum is orbital, and the constancy of $\mathrm{M}$ requires that $l$ shall remain constant. Thus we may reasonably suppose that compressibility lessens the possible range of increase in $l$ and that the ratio of 90 per cent. just calculated for an incompressible mass is the maximum possible, always provided the mass ratio does not exceed $2 \frac{1}{2}: 1$, and that the system remains free from external disturbance.

270. Similar calculations can be made with respect to the period. Calling this $P$ we have

$$
P=\frac{2 \pi}{n}=\frac{2 \pi\left[l^{\frac{1}{2}}(1+\zeta)^{\frac{1}{2}}\right]^{3}}{\left(1-e^{2}\right)^{\frac{3}{2}}\left(M+M^{\prime}\right)^{\frac{1}{2}}(1+\zeta)^{2}} .
$$

As evolution progresses $l^{\frac{1}{2}}(1+\zeta)^{\frac{1}{2}}$, which is proportional to the orbital momentum, will increase, but not in a ratio greater than $1 \cdot 25: 1$ if $M / M^{\prime}<2.5$. Similarly $1+\zeta$ will decrease but not in a ratio beyond $1 \cdot 22: 1$. The factor $\left(1-e^{2}\right)^{\frac{3}{2}}$ will decrease to an unknown extent, and may decrease beyond limit.

Thus there is theoretically no limit to the increase of $P$, but large increases can only occur through $1-e^{2}$ becoming very small, so that a binary in which $P$ has increased largely must have an almost parabolic orbit. Observation has so far revealed no binary with a nearly parabolic orbit; the largest observed eccentricities are 0.90 found by Aitken for $\gamma$ Virginis and 0.88 found by Campbell for $\beta$ Arietis; for these $1-e^{2}=0.19^{\circ}$ and 0.23 respectively. The average values of $e$ for binaries of different types will be seen from Campbell's tables given on pp. 255,6. Campbell has catalogued $e$ for 75 spectroscopic binaries*; in only one case ( $\beta$ Arietis just mentioned) is $e$ greater than 0.80 ; similarly out of 50 visual binaries, $e$ has a value greater than 0.80 in only three cases ( $\gamma$ Virginis, $e=0.90 ; \gamma$ Androm. $B C, e=0.82 ; 99$ Herculis, $e=0.81$ ). Thus we may take $e=0.80$ as an upper limit for $e$ for the great majority of binaries; this makes $1-e^{2}=0.36$ and the maximum evolutionary decrease in $\left(1-e^{2}\right)^{\frac{3}{2}}$ may be taken to be one of $1: 0 \cdot 216$.

* I have excluded Cepheids in view of the uncertainty as to whether these really are binaries or not. 
With the various maximum figures which have now been mentioned, the greatest increase possible in $P$ is found to be one of 13.6 times. This is a maximum, and entirely abnormal increase; for most binaries $e$ is not greater than $\frac{1}{2}$, so that $\left(1-e^{3}\right)^{\frac{3}{2}}$ is not less than 0.65 , and the maximum possible increase in $P$ is one of 4.4 times.

271. Detailed calculations can be made for individual stars. For $a$ Centauri, $P=81.18$ years, $M=M^{\prime}$ (approximately) and $e=0.53$. The parallax is $0.76^{\prime \prime}$ and the semi-major-axis subtends an angle of $17.71^{\prime \prime}$, whence $a=3.5 \times 10^{14} \mathrm{cms}$. and $l=2.5 \times 10^{14} \mathrm{cms}$. Since $M=M^{\prime}$ it appears from the figures on p. 257 that the maximum possible increase in $l^{\frac{1}{2}}(1+\zeta)^{\frac{1}{2}}$ cannot be greater than $1: 0 \cdot 846$, so that $l(1+\zeta)$ cannot have increased by more than 40 per cent., and $l$ cannot have increased by more than 71 per cent. Thus in the very earliest stages of the star's history $l$ cannot have been less than 59 per cent. of its present value, say $1.5 \times 10^{14} \mathrm{cms}$., and $a$ cannot have been less than the same amount. The period, which is now $81 \cdot 18$ years, can never have been less than $20^{\circ} 4$ years.

272. Still assuming that the binary system may be supposed to have been free from external disturbances, a simple relation can be obtained between the dimensions of the present orbit of a binary star evolved by fission and those of the primitive nebula out of which the system originated.

Consider the primitive nebula of mass $M+M^{\prime}$ at the instant at which the pseudo-spherical form first became unstable. Let $r$ and $\bar{\rho}$ denote its mean radius and mean mass at this instant, so that $M+M^{\prime}=\frac{4}{3} \pi \bar{\rho} r^{3}$, and let $\theta$ denote the value of $\omega^{2} / 2 \pi \bar{\rho}$. The angular momentum at this instant is

$$
\mathbf{M}=\left(M+M^{\prime}\right) k^{2} \omega=\left(M+M^{\prime}\right)^{\frac{3}{2}} \frac{k^{2}}{r^{2}} r^{\frac{1}{2}}\left(\frac{3}{2} \theta\right)^{\frac{1}{2}} .
$$

After fission has taken place and the components have become thoroughly separated, the orbital momentum will be

$$
\frac{M M^{\prime}}{\left(M+M^{\prime}\right)^{\frac{1}{2}}} l^{\frac{1}{2}}
$$

Since this must always be less than $\mathbf{M}$, it follows that at any stage of the star's orbital motion

$$
\frac{l}{r}<\frac{\left(M+M^{\prime}\right)^{4}}{M^{2} M^{\prime 2}} \frac{k^{4}}{r^{4}} \times \frac{3}{2} \theta
$$

Suppose first that the primitive nebula is wholly incompressible. The figure is a Maclaurin spheroid at its point of bifurcation, so that $k^{2} / r^{2}=0.3838$ and $\theta=0 \cdot 18712$. Our inequality becomes

$$
\frac{l}{r}<\cdot 04135 \frac{\left(M+M^{\prime}\right)^{4}}{M^{2} M^{\prime 2}}
$$


For a compressible mass the value of $k^{2} / r^{2}$ is less, but the value of $\theta$ is greater. It is clear that $k^{2} / r^{2}$ will decrease much more rapidly than $\theta$, so that compressibility lessens our calculated factor 04135 - for instance for Roche's model it reduces to zero.

Thus the inequality (585) will be true independently of the compressibility of the mass.

For a binary in which the components are of very unequal mass

$$
\left(M+M^{\prime}\right)^{4} / M^{2} M^{\prime 2}
$$

will be very large, so that $l$ may be very large compared with $r$-the components can separate to a distance large compared with the dimensions of the primitive nebula. But for binaries in which $M / M^{\prime}<2 \frac{1}{2}$, the value of $\left(M+M^{\prime}\right)^{4} M^{2} M^{\prime 2}$ is less than 24.01 and our inequality (585) becomes

$$
l<\cdot 9928 r .
$$

Thus under no circumstances can the semi-latus rectum of a binary system in which $M / M^{\prime}<2 \frac{1}{2}$ exceed the mean radius of the primitive nebula at the instant at which the spheroidal form became unstable.

For a Centauri the present value of $l$ is $2.5 \times 10^{14} \mathrm{cms}$., subtending an angle of $12 \cdot 75^{\prime \prime}$. If it was generated by fission, the mean radius of the pseudo-spheroid just before elongation commenced must have been at least $2.5 \times 10^{14} \mathrm{cms}$., so that the major-axis must have been at least $6 \times 10^{14} \mathrm{cms}$., subtending (at its present distance) an angle of at least $30^{\prime \prime}$. The mass of the system being $3.8 \times 10^{33}$ gms., the mean density must have been less than $6 \times 10^{-11}$.

273. The results just obtained dispose at once of the hypothetical interpretation put upon Campbell's table on p. 255. So long as a binary star is regarded as a self-contained system, we have seen that, for the great majority of binaries, the linear dimensions and period of the orbit can only vary slightly through the whole course of the star's life. A binary star can no longer be supposed, as it grows older, to pass in turn through the different columns of that table; on the contrary, except in rare cases, it will remain continually in the same column. Its spectral type and eccentricity will vary as its evolution progresses, but the general order of magnitude of its period must remain perpetually the same. If we suppose spectroscopic binaries to have originated by fission, the problem of explaining why it is that shortperiod binaries are generally of small eccentricity of orbit and of early spectral type, the reverse being true of long-period binaries, admits of no answer so long as we regard a binary star as a self-contained dynamical system. 


\section{Equipartition of Energy}

274. Let us examine how the problem stands when account is taken of the forces from the other stars. We have seen that in the present state of the universe the forces from neighbouring stars may be neglected in a statistical discussion; the forces acting on a binary system from outside may be supposed to be those arising from the gravitational field of the universe as a whole.

Over the extent of any binary system, the potential of this gravitational field may be represented by a single second harmonic term, say

$$
\mu\left(x^{2}-\frac{1}{2} y^{2}-\frac{1}{2} z^{2}\right)
$$

the centre of gravity of the binary system being taken for origin. We may first examine whether the action of such a field is to increase the eccentricity and period of the orbit of the binary system.

The field may be regarded as the superposition of two fields, having potentials respectively

$$
-\frac{1}{2} \mu\left(x^{2}+y^{2}+z^{2}\right) \text { and } \frac{3}{2} \mu x^{2} \text {. }
$$

The first of these fields gives rise merely to a force of repulsion $\mu$ acting away from the centre of gravity of the star. So long as $\mu$ remains constant, the apsidal distances of the orbit naturally remain fixed; when $\mu$ increases, there is an increase in the dimensions and period of the orbit, but when $\mu$ decreases, there is a corresponding decrease. Thus any secular change in the dimensions and period of the orbit can depend only on $\mu^{2}$, which is so small as to be negligible.

The field of force of potential $\frac{3}{2} \mu x^{2}$ gives rise to a repulsive force $3 \mu x$ away from the plane of $y z$. As the line of apses of the orbit must be supposed to make all angles indifferently with the axis of $x$, it is readily seen that the average effect of these forces must be nil. Thus any secular change can be proportional only to the negligible quantity $\mu^{2}$.

It accordingly appears that in the present state of the universe, the general gravitational field can only have an infinitesimal effect upon the orbits of binary stars.

275. We have already had occasion to contemplate a past epoch in the history of the universe, in which the stars were much closer together than they now are. We have found reasons for supposing that at this time the stars were close enough to affect one another's orbits in space, to an appreciable degree, and it is natural to inquire in what way the close encounters of this epoch would influence the relative orbits of binary stars.

The problem now before us has a close analogy in the Kinetic Theory of Gases. In the present epoch the stars have been seen to behave like the 
molecules of a gas in which no collisions occur. In the past epoch that we have under consideration, they behaved like the molecules of a gas in which collisions occurred in the ordinary way. Single stars must have behaved like monatomic molecules and binary stars like diatomic molecules. There is however the welcome difference that we understand the dynamics of a binary star, whereas we do not yet understand the dynamics of a diatomic molecule. The tendency of encounters with other stars must have been towards establishing an internal distribution of energy such as would be in equilibrium with the translational energy of the stars. It will, however, be best to state the problem in a form which does not imply or presuppose any analogy with the Kinetic Theory of Gases.

276. Let $M, M^{\prime}$ be the masses of the two constituent stars of a binary system of total mass $M+M^{\prime}$. Let $u, v, w$ denote the components of the velocity of the centre of gravity in space, and let $r, \theta, \phi$ be polar coordinates of $M$ relatively to $M^{\prime}$. Then the whole kinetic energy of the system is

$$
\frac{1}{2}\left(M+M^{\prime}\right)\left(u^{2}+v^{2}+w^{2}\right)+\frac{1}{2} \frac{M M^{\prime}}{M+M^{\prime}}\left(\dot{r}^{2}+r^{2} \dot{\theta}^{2}+r^{2} \sin ^{2} \theta \dot{\dot{\phi}^{2}}\right) \ldots(586) .
$$

Following our view of the genesis of binary system, we suppose that when a double-star first comes into being, the value of $\dot{r}^{2}$ will be very small, while the value of $\dot{r}^{2}+r^{2} \sin ^{2} \theta \dot{\phi}^{2}$, the square of the tangential velocity, bears no relation at all to the translational velocity of the system as a whole. The theorem of equipartition of energy shews that the tendency of stellar encounters must be towards equalising the mean values of the different terms in formula (586). In the final steady state which would be attained after an infinite number of encounters, the mean square of the tangential velocity would be equal to twice the mean square of the radial velocity; it would also be equal to $\frac{2}{3}\left(M+M^{\prime}\right)^{2} / M M^{\prime}$ times the mean square of the velocity of translation in space.

Consider the description of an orbit of eccentricity $e$. Without loss of generality the plane of the orbit may be supposed to be $\phi=0$, and the equation of the orbit will be

$$
\frac{l}{r}=1+e \cos \theta .
$$

The motion will have the usual integral of momentum $r^{2} \dot{\theta}=h$, so that

$$
d t=r^{2} d \theta / h \text {. }
$$

Using a bar over a quantity to denote its mean value at all instants of the description of a complete orbit we readily find that

$$
\begin{gathered}
\overline{\dot{r}^{2}}=\frac{e^{2} h^{2}}{l^{2}} \sin ^{2} \theta=\frac{h^{2}}{l^{2}}\left[1-\left(1-e^{2}\right)^{\frac{1}{3}}\right], \\
\overline{r^{2} \dot{\theta}^{2}}=\frac{h^{2}}{l^{2}} \overline{(1+e \cos \theta)^{2}}=\frac{h^{2}}{l^{2}}\left(1-e^{2}\right)^{\frac{3}{2}} .
\end{gathered}
$$


The mean square of the tangential velocity will be equal to twice the mean square of the radial velocity, as required by the Theorem of Equipartition, when

$$
\left(1-e^{2}\right)^{\frac{3}{2}}=2-2\left(1-e^{2}\right)^{\frac{1}{2}}
$$

an equation whose root is

$$
\left(1-e^{2}\right)^{\frac{1}{2}}=\cdot 7709, \quad e=\cdot 6370 .
$$

Thus the general effect of stellar encounters is to decrease the eccentricity of orbits whose eccentricity is greater than 6370 , and to increase the eccentricity of orbits whose eccentricity is less than 6370 . If the constituents of binaries start life by moving in nearly circular orbits, the general effect of stellar encounters must be to increase the eccentricities of these orbits until they are ranged about a median value $e=\cdot 6370$. This, however, is not the arithmetic average of all eccentricities in the final equipartition state; it can be shewn that in the final state, the eccentricities would be distributed according to the law $2 e d e$, so that the average value of $e$ would be $\frac{2}{3}$.

If $C^{2}$ denote the mean value of $u^{2}+v^{2}+w^{2}$, the second equipartition condition will be satisfied if

$$
\frac{h^{2}}{l^{2}}\left(1-e^{2}\right)^{\frac{3}{2}}=\frac{2}{3} \frac{\left(M+M^{\prime}\right)^{2}}{M M^{\prime}} C^{2}
$$

In terms of the period $P$ of description of the orbit, this becomes

$$
\left[\frac{2 \pi \gamma\left(M+M^{\prime}\right)}{P}\right]^{\frac{2}{3}}\left(1-e^{2}\right)^{\frac{1}{2}}=\frac{2}{3} \frac{\left(M+M^{\prime}\right)^{2}}{M M^{\prime}} C^{2}
$$

If $M, M^{\prime}$ are fairly equal, the value of $\left(M+M^{\prime}\right)^{2} / M M^{\prime}$ will not differ greatly from 4. Moreover as equation (587) does not depend greatly on $e$ we may suppose $e$ to have its equipartition value 6370 . The equation now becomes very approximately

$$
P=\gamma\left(M+M^{\prime}\right) C^{-3} .
$$

We notice that for a massive slow-moving star, $P$ will be large; for a light and rapidly-moving star, $P$ will be small. Taking $M+M^{\prime}$ equal to the sun's mass, $2 \times 10^{33}$ grammes, and $C$ equal to $25 \mathrm{kms}$. a second, the value of the period is found to be almost exactly three months. For a binary star of nine times the sun's mass, say an average $B$-type binary star, the equipartition value of $C^{2}$ would be only one-ninth of that just assumed, and the equipartition value of $P$ would be 243 times that just calculated, or about 60 years.

277. It has now been seen that stellar encounters produce the same general effect as tidal friction, namely increases in the eccentricity, linear dimensions and period of the orbit. But, whereas tidal friction was found competent to produce only small proportional increases in the period and 
linear dimensions, stellar encounters increase these quantities until the period is measured in years, regardless of the value of the initial period.

Any group of stars which has experienced a large number of stellar encounters will have orbits in which the eccentricities are ranged according to the law 2 ede round a mean value of $\frac{2}{3}$, while the periods will depend on the mass of the star, being of the order of a year for stars of the average mass of $1 \cdot 7$ times the mass of the sun.

The interpretation of Campbell's table (p. 255) which now suggests itself is one which fits in, exactly and completely, with the conclusions we have already reached from a study of stellar motions (\$251). The stars which are now $B$-stars (including some $A$-stars) were the last to be born; they were born when the universe was already so far developed that close encounters were rare, and as a result the eccentricities and periods of their orbits differ only slightly from what they were when fission occurred. The stars of later type were born earlier; fission took place while close encounters were still comparatively frequent, so that some approximation at least towards equipartition has been attained; the periods are for the most part measured in years and the eccentricities have advanced appreciably towards the mean equipartition value $e=\frac{2}{3}$.

\section{The Genesis of Triple and Multiple Systems}

278. After the two components of a binary are fully separated, each will continue to shrink. If the angular momentum of each component were to remain constant, this would result in an increase of the value of $\omega^{2} / 2 \pi \bar{\rho}$ for each component, so that fission of the components might eventually take place. The angular momentum of each component will not in actual fact remain constant, being diminished to an unknown extent by tidal friction, but it is still possible for fission to take place, although of course not at such an early date as it would if tidal friction did not operate. Let us examine the conditions under which this second fission can occur.

Let us consider a limiting ideal case in which tidal friction is supposed to be wholly inoperative, so that the angular momentum of each component remains unaltered after fission has occurred. Suppose for simplicity that the masses are incompressible. The value of $\omega^{2} / 2 \pi \rho$ just after fission occurs will be the same for each component, being given in the last column of the table on p. 63 . If the components are equal this value is 0.0420 ; if they are in the ratio $2 \frac{1}{2}: 1$, the value is the nearly equal quantity 0.0435 . As shrinkage takes place the value of $\omega^{2} / 2 \pi \rho$ will increase for each component in such a way as to keep the angular momentum constant.

During this shrinkage the tidal influence of the components on one another continually decreases. After a time $\omega^{2} / 2 \pi \rho$ will attain to a value $0 \cdot 18712$. 
The figure of the component under consideration will now be a Maclaurin spheroid just about to give place to a Jacobian ellipsoid. The increase in $\omega^{2} / 2 \pi \rho$ since fission took place is one of about 4.3 times, whence it follows that the stars will now be separated by about 4 diameters and the tidal distortion of one on the other may legitimately be neglected. The increase of density in the mass under consideration will be about $(4 \cdot 3)^{3}$ or 79.5 . A further increase of density to $4: 3$ times this value is found to bring the figure to the critical Jacobian ellipsoid, after which fission of the component takes place and the star forms a triple system. The total increase in density since the first fission occurred is $79.5 \times 4.3$ or 342 times, so that the linear dimensions of the sub-system will be about one-seventh of those of the original system, while their periods will be in a ratio of about 18 to 1.

279. This calculation has neglected tidal friction altogether; it is clear that any action of tidal friction will postpone the formation of a sub-system and so will increase the inequality of dimensions, density and period between the two systems. The calculation has also supposed the masses to be incompressible; it is easily seen that compressibility will further increase the inequalities, for the ratio of rotational to orbital momentum in the original pair decreases with compressibility.

Thus the inequality we have calculated is the minimum possible. When triple systems form under natural conditions, the density at the second fission must be more than 342 times that at the first, and so on*.

With still further increase of density either component of the sub-system may again sub-divide, but this cannot happen until the original density is more than $(342)^{2}$ or 11,700 times that at the original fission, while the period of the final system must be less than $\frac{1}{18}$ times that of the sub-system of which it is part and less than $\frac{1}{342}$ times that of the main system.

A typical multiple system of the kind predicted by the rotational theory appears to be found in Polaris. This shews spectroscopically periods of 4 days and 12 years, while Courvoisier finds that the spectroscopic triple system is in orbital motion with a fourth visible star, the period being 20,000 years.

A typical visual system of the kind predicted by theory is illustrated in fig. 45, this being the star 1502 in Jonckheere's Catalogue $\dagger$. The figure is drawn to scale to represent the projection of the system on the celestial sphere,

D. $\dot{C}^{\mathrm{C}}$

Fig. 45.

* Russell, to whom the first investigation of this question is due, gives 380 as the minimum increase, the mass ratio in the first fission not being greater than $3: 1$ (Astrophys. Journ. 31, 1910, p. 196).

+ Memoirs R.A.S. Vol. 61 (1917). 
except that the distance $C c$ has been somewhat increased. The actual separations (epoch 1908.9) are

$$
C c=3 \cdot 10^{\prime \prime}, C D=22 \cdot 67^{\prime \prime}, A B=24 \cdot 17^{\prime \prime}, A C=235 \cdot 72^{\prime \prime} .
$$

280. Generally speaking, all that can be observed of a multiple system is its projection on the celestial sphere at a single instant of time. Even when the orbital elements of the close pair can be determined, it is still impossible to determine those of the wide pair. Thus effects of foreshortening and ellipticity of orbit make it impossible to decide whether any observed individual system conforms to the demands of theory or not.

In a statistical discussion, allowance can of course be made for foreshortening and ellipticity. A group of triple systems having the same ratio of their semiparameters $l_{2} / l_{1}$, and oriented at random in space, would shew projections on the celestial sphere such that the ratio $s_{2} / s_{1}$ of their observed separations ought to obey a definite law of distribution. The summarised results of an interesting statistical discussion of this kind by Russell* are shewn in the following table:

\begin{tabular}{|c|c|c|c|}
\hline \multirow{2}{*}{$\begin{array}{l}\text { Observed ratio } \\
\text { of separations }\left(s_{2} / 8_{1}\right)\end{array}$} & \multicolumn{3}{|c|}{ Number of systems } \\
\hline & Class I & Class II & $\begin{array}{c}\text { Theory } \\
\left(l_{2} / l_{1}=0 \cdot 09\right)\end{array}$ \\
\hline$>0.40$ & 1 & 5 & 1 \\
\hline 0.40 to 0.30 & 2 & - & 2 \\
\hline 0.30 to 0.20 & 3 & - & 3 \\
\hline $0 \cdot 20$ to 0.15 & 5 & - & $4 \frac{1}{2}$ \\
\hline 0.15 to 0.10 & 8 & 3 & $9 \frac{1}{2}$ \\
\hline 0.10 to 0.05 & $17 \frac{1}{2}$ & 2 & $16^{2}$ \\
\hline 0.05 to 0.025 & $14 \frac{1}{2}$ & 2 & 8 \\
\hline$<0.025$ & $13^{2}$ & 7 & 1 \\
\hline
\end{tabular}

The material for discussion consists of 74 triple or multiple systems given in Burnham's Catalogue; since multiple systems appear two or even three times in the list the total number of entries is 83. Class I consists of 64 systems in which the separation of the wide pair is less than 1000 years' proper motion, while Class II consists of 19 systems in which the separation of the wide pair is greater than this; this gives a rough classification according to the actual dimensions of the system. The last column gives the theoretical distribution of $s_{2} / s_{1}$ to be expected for 45 systems for which $l_{2} / l_{1}$ has the uniform value 0.09 .

It is clear that the systems in Class II do not conform at all to the theoretical law of distribution, while the systems in Class I conform closely down to a separation ratio of about $0 \cdot 05$. Those having a separation ratio of less

* Astrophys. Journ. 31 (1910), p. 200. 
than 0.05 shew an excess of about 6 systems in the last line but one, and of 12 in the last line of all. Doubtless these represent systems having a still smaller value of $l_{2} / l_{1}$.

Thus, so far as Class $I$ is concerned, the law of distribution $n f s_{2} / s_{1}$ is what might statistically be expected for a number of systems in which $l_{2} / l_{1}$ had values ranging from about 0.09 downwards. This distribution fully conforms to theoretical requirements.

The systems in Class II fall into two sharply defined groups. A group of 14 for which $s_{2} / s_{1}$ is less than $0 \cdot 15$ may very possibly have originated by fission, but we must look for some other origin for the group of 5 for which $s_{2} / s_{1}$ is greater than 0.40. Russell, following a suggestion of Moulton's, supposes that these may perhaps have been evolved from separate nuclei in the original nebula. There is no reason why, in the star-cluster motion we discussed in the last chapter, some pairs of stars should not end by permanently describing orbits about one another-indeed it would be contrary to all laws of statistical mechanics if this did not happen. Russell further makes the very reasonable suggestion that if we could extend our survey to systems of still greater linear extent we should find systems such as the 5 triple systems just discussed gradually grading into the moving star-clusters such as the Pleiades $(\S 6)$. On this view these triple systems are merely moving star-clusters consisting of three members, or of two members one of which has subdivided by fission.

281. Russell's investigation accordingly shews that it is possible that the majority of pairs of stars in orbital motion about one another at distances of less than about 1000 years' proper motion have originated by fission. It also assigns a limit of about 1000 years' proper motion to the dimensions of the orbits of systems which can have been generated by fission, and this, except for a projectional effect, and for changes which may have resulted from encounters with other stars, must also be a limit to the dimensions of the nebulae out of which binary systems evolved by fission ( $(272)$. Taking $25 \mathrm{kms}$. a second as an average stellar velocity* the distance represented by 1000 years' proper motion would be of the order of $8 \times 10^{16} \mathrm{cms}$. If a stellar mass of $35 \times 10^{33}$ gms. were spread through a sphere of diameter equal to this, the mean density would only be about $1.3 \times 10^{-17}$.

This density is of the order of magnitude of what we have supposed to be the density in the original rotating nebula; it is enormously less than the density at which we have computed $(\S 255)$ that fission might be expected to begin. Moreover, even after allowing for all uncertainties in our theoretical discussion of the density at which equatorial disintegration gives place to fission ( $(263)$, it seems impossible that matter of such low density as $10^{-17}$ could possibly break up by fission. Thus it seems unlikely that the fissional

* We take the total velocity in 3-dimensional space so as to eliminate the projectional effect just referred to. 
hypothesis can be maintained for systems whose separation is of the order of 1000 years' proper motion.

282. Another hypothesis suggests itself. We identify the density $1.3 \times 10^{-17}$ which corresponds to a separation of 1000 years' proper motion with the density in the arms of the original rotating nebula out of which the whole system of stars was evolved. As stars form out of the nuclei in these arms the majority will, as we have supposed, move as separate and independent systems, but there must necessarily be a number of cases in which two adjacent nuclei in the nebular arms remain permanently describing orbits about one another. Such a pair of stars is dynamically, so far as Russell's investigations go, indistinguishable from a pair which has evolved by fission. It has not evolved by fission but the relations between angular velocity, separation and mass are the same as if it had evolved by fission, and the distribution of momentum between rotational and orbital momentum is the same as if it had evolved by fission.

Thus we are led to conjecture that wide binary systems of separation less than about 1000 years' proper motion are the remains of adjacent nuclei in the original nebula which have never got out of one another's gravitational attraction; systems of separation greater than this may perhaps be pairs of stars which have fallen into orbits round one another in the random motion of those stars which had become properly separated. Close binary systems may no doubt have been evolved by fission, but at present it is difficult to draw the line between such systems and systems which have never formed a single mass.

The most direct evidence we have on this point is provided by the observed distribution of periods. We have found that, briefly speaking, encounters with other systems cause the periods to approximate, on the average, to about one year. Thus binaries with periods of less than one year probably (although not certainly) started life with still shorter periods; such binaries probably originated by fission. In the same way, binaries with periods greater than one year probably started life with still longer periods, so that the majority of these stars are likely to represent the relics of independent nuclei in the original nebula. Thus we may perhaps conjecture with some confidence that such binaries as a Centauri, discussed in $\$ 271,272$, and with it the vast majority of long-period visual binaries, have not been evolved by fission, but in more doubtful cases, such as $W$ Crucis which figures in the bottom line of Shapley's table (p. 250), the only reason for forming a decision is that supplied by dynamical theory, and this as we have seen leads to very indefinite results. 


\section{CHAPTER XII}

\section{THE ORIGIN AND EVOLUTION OF THE SOLAR SYSTEM}

283. The sequence of events to be expected in a mass of astronomical matter left solely to the influence of its own rotation has now been traced out with tolerable completeness.

Of the five uniformities of structure mentioned in our introductory chapter we have found that two fall naturally into their places in the scheme of evolution of a rotating mass, these two being the spiral nebulae and the binary and multiple stars. Two others, namely the planetary and ring nebulae and the globular and moving star-clusters, seem at least to be capable of explanation in terms of a rotational theory of evolution, although our interpretation of these formations was largely conjectural.

The fifth uniformity was that observed in the solar system, and for this no place has been found in the rotational scheme of evolution. It is true that we found $(\$ 257)$ that planets might possibly form out of the atmosphere thrown off equatorially from a rotating mass of gas, but several objections present themselves against any attempt to explain the origin of our solar system in this way-primarily the objection that the next stage in evolution ought to be for the central mass to break up into an ordinary binary star, whereas our sun and planets are not binary. Also the arrangement of the components of typical multiple stars such as can have been formed by rotation (cf. fig. 45, p. 265) does not in the least resemble that observed in the solar system.

Such considerations alone would throw doubt on a rotational theory of the evolution of our solar system. Beyond these there is the objection already referred to in $\$ 14$, that the total angular momentum of our system appears, at first sight at least, to be much too small for the system ever to have broken up by rotation. We proceed to examine this supposed objection in the light of the theoretical knowledge we have now obtained as to the conditions for rotational break-up to occur.

\section{THE ROTATIONAL THEORY}

284. For an incompressible and homogeneous mass of fluid, rotational break-up cannot commence until after the configuration has passed the Maclaurin-Jacobian point of bifurcation; at this

$$
\frac{\omega^{2}}{2 \pi \gamma \rho}=0.18712
$$


For compressible and non-homogeneous masses, rotational break-up can occur in two, and only two, ways-by fission or by equatorial ejection of matter. Fission can only begin after the configuration has passed the point of bifurcation at which the pseudo-spheroidal form gives place to the pseudoellipsoidal. The value of $\omega^{2} / 2 \pi \gamma \bar{\rho}$ at this point depends naturally on the structure of the mass. For a gas in adiabatic equilibrium, the possible limits have been found to be

$$
0 \cdot 18712<\frac{\omega^{2}}{2 \pi \gamma \bar{\rho}}<0.31
$$

The limits for equatorial break-up for a mass in adiabatic equilibrium have similarly been found to be

$$
0.31<\frac{\omega^{2}}{2 \pi \gamma \bar{\rho}}<0.36075 .
$$

In both these sets of limits the entry 0.31 is subject to considerable error, but this is immaterial to our present purpose. It seems quite certain that a mass of gas in adiabatic equilibrium cannot break up rotationally unless the value of $\omega^{2} / 2 \pi \gamma \bar{\rho}$ has exceeded the value $0 \cdot 18712$.

A natural mass of gas differs from a mass in adiabatic equilibrium in two respects-the quantity $k$, or $p / \rho^{\gamma}$, will not be constant throughout the mass, and the chemical structure will not be constant throughout the mass. For stability, $k$ must increase on passing outwards along a radius, and the heaviest molecules or atoms must sink towards the centre. Both of these departures from the adiabatic arrangement tend to increase the degree of central condensation of mass. The mass approaches more closely to Roche's model, and the critical value of $\omega^{2} / 2 \pi \gamma \bar{\rho}$ approaches more closely to the value 0.36075 . Thus we seem fully justified in supposing that no mass can break up rotationally until after $\omega^{2} / 2 \pi \gamma \bar{\rho}$ has exceeded the value $0 \cdot 18712$.

In particular, if our solar system has been formed by the rotational break-up of a primitive mass of any kind whatever, the value of $\omega^{2} / 2 \pi \gamma \bar{\rho}$ for this body must have been greater than 0.18712 before break-up commenced.

285. Babinet's criterion ( $(14)$ proceeds on the suppositions that the mass of this primitive body must have been equal to the total mass of the present solar system, and that the angular momentum of this body must have been equal to the total angular momentum, rotational and orbital, of the solar system.

Neither of these suppositions is altogether justifiable. The supposition that the angular momentum has remained constant requires us to suppose that our system has remained entirely undisturbed by encounters with other systems ever since its birth. This is in opposition to the results reached in the two last chapters, where we came to the conclusion that most stars, with the exception of $B$-type and some $A$-type stars, shew evidence of having experienced considerable disturbance by other systems; there is no reason 
why our solar system should be expected to have escaped this common fate. Close encounters with other systems are now so rare as to be negligible, but we have been led to suppose that conditions were widely different in the remote past, so that close encounters in a past epoch may have greatly altered the angular momentum of our system. There is also another possibility to be considered. If a wandering star were to enter into our system and carry off Jupiter by capture the total angular momentum of the system would be reduced to less than half, although the total mass would only be reduced to an insignificant extent. The occurrence of such an event in the past would invalidate entirely the supposition of the total angular momentum remaining constant.

286. Leaving this objection aside, let us follow Babinet in supposing that the primitive body out of which our system formed had a mass equal to the total mass, and angular momentum equal to the total angular momentum, of the present solar system. Let us proceed to investigate the possibility of the value of $\omega^{2} / 2 \pi \gamma \bar{\rho}$ for such a system having ever been greater than $0 \cdot 18712$.

The mass of the system is 1.0013 times that of the sun, and this, to within one per cent., will be $2 \times 10^{33}$ grammes. The angular momentum M arises mainly (cf. $§ 14$ ) from the orbital momenta of the four outermost planets, and these are known perfectly. About a sixtieth of the whole arises from the axial rotation of the sun, and this, depending on the sun's interior arrangement, is not known with great accuracy. But to within one per cent. we may take the whole moment of momentum of the system to be

$$
\mathrm{M}=3.3 \times 10^{50} \mathrm{C} . \text { G.S. units. }
$$

Let $r_{0}$ be the mean radius and $\bar{\rho}$ the mean density of the primitive mass before break-up, so that $M=\frac{4}{3} \pi \bar{\rho} r_{0}$. Let $k$ have been the radius of gyration, so that $\mathrm{M}=M k^{2} \omega$. From these relations it follows that, in any configuration whatever,

$$
\frac{\omega^{2}}{2 \pi \gamma \bar{\rho}}=\frac{2 M^{2}}{3 \gamma M^{3}} \frac{r_{0}^{3}}{k^{4}}
$$

or, inserting numerical values for $\mathrm{M}, M$ and $\gamma$,

$$
\frac{\omega^{2}}{2 \pi \gamma \bar{\rho}}=1 \cdot 34 \times 10^{8} \frac{r_{0}^{3}}{k^{4}}
$$

It follows that $\omega^{2} / 2 \pi \gamma \bar{\rho}$ can only have been as great as $0 \cdot 18712$ if $k^{4} / r_{0}{ }^{3}$ was less than $7 \cdot 16 \times 10^{8} \mathrm{cms}$. If $r_{0}$, the mean radius of the original nebula, is supposed to have been $n$ times the present radius of the sun, then $k^{2} / r_{0}{ }^{2}$ must have been less than $0 \cdot 101 / \sqrt{ } n$. For instance with $r_{0}$ equal to the radius of Neptune's orbit, $k^{2} / r_{0}{ }^{2}$ must have been less than 0.0013 . The exact value which ought to be assigned to $n$ can only be a matter of conjecture. It is 
probably large, but whatever reasonable value is assigned to $n$, the value of $0 \cdot 101 / \sqrt{ } n$ or $k^{2} / r_{0}^{2}$ comes out very small. Thus there must have been very extreme central condensation in the primitive mass.

General dynamical theory has shewn that there are two, and only two, distinct types of rotational break-up. The fissional break-up happens in a mass in which great variations of density do not occur, while the equatorial break-up happens in masses with considerable central condensation. We have seen that, if our system broke up by rotation, there must have been very extreme central condensation, so that we may be confident that the break-up, if ever it occurred, must have been by equatorial ejection*

The values of $\omega^{2} / 2 \pi \gamma \bar{\rho}$ for equatorial ejection range from about 0.31 to 0.36075. With central condensation as extreme as that we are now considering $\omega^{2} / 2 \pi \gamma \bar{\rho}$ must be very nearly equal to the latter value. Within an error of about one per cent. we may suppose it to be 0.36 .

287. We have now determined three numerical data,

$$
\mathrm{M}=3.3 \times 10^{50}, \quad M=2 \times 10^{33}, \quad \omega^{2} / 2 \pi \gamma \bar{\rho}=0.36,
$$

all probably accurate to within about one per cent. Equation (590) now determines the further value

$$
\frac{k^{4}}{r_{0}^{3}}=3.7 \times 10^{8} \mathrm{cms}
$$

This gives the following values for $k^{2} / r_{0}^{2}$, these still being exact to about one per cent. :

$$
\begin{array}{lll}
r_{0}=\text { Radius of present sun } & k^{2} / r_{0}{ }^{2}=0.072, \\
r_{0}=\quad \# \quad \text { orbit of earth } & k^{2} / r_{0}{ }^{2}=0.005, \\
r_{0}=\quad \Rightarrow \quad \# \quad \text { Neptune } & k^{2} / r_{0}{ }^{2}=0.00090 .
\end{array}
$$

Exact analysis has not so far sustained the objection of Babinet ( $\$ 14$ ) to Laplace's Theory. The smallness of the present angular momentum does not shew that the system cannot have broken up rotationally; it merely shews that the value of $k^{2} / r_{0}^{2}$ must have been very small if the system ever did break up rotationally. This necessity for extreme central condensation was, however, apparent to Laplace, and has been fully recognised by subsequent cosmogonists $\uparrow$. It should, however, be added that See and also Moulton and Chamberlin, starting apparently from the tacit assumption that

* For an adiabatic mass of gas, the transition occurs when $\gamma=2.2$ (about), $\gamma$ bere denoting (momentarily) the ratio of the two specific heats. The value of $k^{2} / r_{0}^{2}$ for a spherical mass in which $\gamma=1.66$ is 0.22 , for one in which $\gamma=2$ is 0.26 , and for one in which $\gamma=\infty$ is 0.40 . Thus it appears that for a mass in which $\gamma=2 \cdot 2$ the value of $k^{2} / r_{0}^{2}$ will be about $0 \cdot 29$. The flattening produced by rotation naturally increases $k^{2} / r_{0}{ }^{2}$, so that there is a wide margin of safety in supposing that $k^{2} / r_{0}^{2}=0 \cdot 101 n^{-\frac{1}{2}}$ corresponds to equatorial break-up.

+ Cf. Poincaré, Leçons sur les Hypothèses Cosmogoniques, p. 18. 
extreme central condensation is impossible, have arrived, naturally enough, at the conclusion that rotational break-up was also impossible. Such extreme condensation as is demanded by the rotational theory will be admitted to be highly improbable, but there seems to be no way of proving it to be impossible.

At the same time, as we shall now see, a slight change in the form of the argument brings to light considerations which suggest very strongly that Laplace's hypothesis must be abandoned, at any rate if we hold to the assumption that the angular momentum of the system has remained constant since its birth.

288. Small values of $k^{2} / r_{0}{ }^{2}$ can only mean that the matter in the outlying parts of the nebula is of density low compared with the mean density $\bar{\rho}$. Let $\rho_{e}$ denote the density of matter near the edge. The interior matter may then be supposed of density greater than $\rho_{e}$. The moment of inertia is

$$
M k^{2}=\iiint\left(x^{2}+y^{2}\right) \rho d x d y d z
$$

and, since $\rho>\rho_{e}$ except near the edge, this requires that

$$
M k^{2}>\rho_{e} \iiint\left(x^{2}+y^{2}\right) d x d y d z .
$$

For the figure corresponding to extreme central condensation ( $\$ 152)$, the integral is easily evaluated, and found to be equal to $0.52313 r_{0}{ }^{2}$ times the volume of the figure. It follows that

$$
0.523 \frac{\rho_{e}}{\bar{\rho}}<\frac{k^{2}}{r_{0}^{2}} .
$$

The table of $\S 287$ (opposite) now assigns upper limits to $\rho_{e} / \bar{\rho}$. We have

$$
\begin{array}{lll}
r_{0}=\text { Radius of present sun } & \rho_{e} / \bar{\rho}_{.}<0.137, \\
r_{0}=\quad " \quad \text { orbit of earth } & \rho_{e} / \bar{\rho}<0.009, \\
r_{0}=\quad " \quad \text { Neptune } & \rho_{e} / \bar{\rho}<0.0017 .
\end{array}
$$

289. We have seen that the method of break-up, if this occurred at all, must have been that of equatorial ejection, as imagined by Laplace and Roche. They imagined the next stage of evolution to be the formation of a ring. This, on account of the conservation of angular momentum, must have rotated with angular velocity $\omega$ practically equal to that of the original mass, and so given by $\omega^{2} / 2 \pi \bar{\rho}=0.36$. The theorem of Poincaré, quoted in $\S 210$, now assigns a lower limit to the mean density $\rho_{r}$ of this ring; it must be greater than $\omega^{2} / 2 \pi$, and therefore greater than $0 \cdot 36 \bar{\rho}$.

Thus for evolution to have taken place on the lines imagined by Laplace and Roche, $\rho_{r}$ must have been much greater than $\rho_{e}$; the ejected matter must have increased in density, and so contracted, before a ring could form. 
To make the matter definite, let us suppose the mean radius $r_{0}$ of the original nebula to have been equal to the radius of Neptune's orbit, about $4.5 \times 10^{14} \mathrm{cms}$. The total mass of the nebula being $2 \times 10^{33}$ grammes, the mean density $\bar{\rho}$ must have been $5.5 \times 10^{-12}$. From the table just given, $\rho_{e}$, the density in the outer regions of the nebula must have been less than 0.0017 times this, say less than $9 \times 10^{-15}$, whereas the density of the ejected matter when condensation began must have been greater than $0.36 \bar{\rho}$ and so greater than $2 \times 10^{-12}$. The ejected matter must have had a density more than 200 times as great as the density in the outer regions of the nebula.

If the ejected matter remained gaseous such an increase of density would be unthinkable. It will, however, be rememberred that we have already ( $\$ 211$ ) found reasons why the ring of matter imagined by Laplace could not be gaseous. For Laplace's hypothesis to be saved, it seems to be necessary to suppose that the ejected matter liquefied shortly after ejection so that the planets were born in a liquid, or possibly even in a solid state*.

This supposition is not objectionable in itself, but it leads into difficulties when we proceed to the consideration of the further stages of evolution. According to the Nebular Hypothesis, the planets shrunk further after their birth, until the rotation had increased to such an extent that a further break-up took place, resulting in the formation of satellites. Now if the planets were born in the fluid state, it is impossible to imagine a further shrinkage of anything like sufficient amount to effect a second break-up. Using the relation $\omega^{2} / 2 \pi \gamma \bar{\rho}=0 \cdot 36$, and assigning to $\bar{\rho}$ the value already assumed, namely $5 \cdot 5 \times 10^{-12}$, it is found that the period of rotation of the original nebula must have been about 35 years, and this must also have been approximately the period of rotation of the planets when first born. It is inconceivable that the planets, already fluid, should shrink until this period was reduced to a few hours, which is the period necessary for rotational break-up to occur in a fluid mass. Moreover, even if the inconceivable were to happen, if this shrinkage took place and the planets broke up further, the break-up of the fluid planets would necessarily be by fission into masses of comparable size, and the final formation of the planets would be that of a system of binaries of the well-known type.

290. For the foregoing reasons, it seems probable, although by no means certain, that we must abandon the Nebular Hypothesis of Laplace. Before abandoning the rotational theory altogether, we ought perhaps to consider the possibility, not contemplated by Laplace at all, of the ejected matter being localised in one or two streams, as we imagined it to be in the formation of the spiral nebulae.

* A modified form of the foregoing argument has been presented by Jeffreys (Monthly Notices R.A.S. 78 (1918), p. 424), who arrives at the same conclusion as that stated here. 
The main objection to this form of the rotational theory has been already stated ( $(253)$; for condensation to occur the emission of matter would have to be very rapid. Calculation shews that the mass of the earth must have been ejected in less than three years, and more probably in a few months*. A further objection is that even if jets of matter were emitted with sufficient rapidity to condense in the gaseous form, they would condense into masses very much greater than the planets of our system-we have seen that to obtain masses comparable with Uranus or Neptune the density must be about $10^{-9}$ which is about 200 times the mean density $\left(5.5 \times 10^{-12}\right)$ of the supposed primitive nebula, and 100,000 times the mean density just calculated for its outer regions. On this form of the hypothesis we are again brought to the conclusion that the planets must have started life in the solid or liquid state, and this is open to the objections mentioned in the last section.

Finally, an objection to any form of rotational theory is that the central mass ought to continue disintegrating until a double star is formed. Our sun has left off disintegrating and has not formed a double star, and the same is true of all the planets.

291. We may perhaps sum up as follows. Babinet's criterion in itself has not provided conclusive proof against the solar system having been formed by rotation, and could not in any case do so, for the whole criterion becomes inapplicable as soon as we admit the possibility of interaction between our system and external stars. But exact analysis has shewn that the present angular momentum is excessively low for a system which has broken up by rotation, so that after making full allowance for the possibility of this angular momentum having been reduced since the birth of the system, it still seems highly probable that our system was formed in some other way. Combining this with the circumstance that we have been unable to discover any process of rotational fission which would lead to a final formation resembling our solar system in the least degree, it becomes almost impossible to continue to believe that our system is the result of a rotational break-up. We have conjectured that spiral nebulae, star-clusters, binary and then multiple stars are formed by rotation; these complete the chain of rotational evolution, and there appears to be no room on this chain for systems like our own.

\section{THE TIDAL THEORY}

292. It being apparently impossible to explain the genesis of our system in terms of the evolution of a single mass rotating by itself in space, it is natural to examine whether it can be explained in terms of the interaction of two masses. This brings us at once to the tidal theory, which has already been investigated dynamically to a considerable extent.

* Monthly Notices R.A.S. 77 (1917), p. 197. 


\section{The Origin and Evolution of the Solar System}

[CH. XII

The general conception of the Tidal Theory ( $\$ 16)$ as applied to our solar. system is that a second mass has at some past period approached so close to our sun. as to break it up by intense tidal forces into a number of detached masses. As between the tidal and rotational theories, first appearances are all in favour of the tidal theory. We have found that the rotational theory applied to a mass comparable with that of our sun leads only to a binary star ( $\$ 258$ ) or perhaps ultimately to a triple or multiple system of a type which is well known and has certainly no resemblance to our solar system ( $\$ 278)$. The tidal theory on the other hand leads at once and naturally to the conception of a number of separate masses becoming detached from the primary mass and finally describing orbits about it.

A further general feature which favours the tidal theory may be noticed. A system in rotation, and consequently also a system which has broken up by rotation, has an invariable plane, which is perpendicular to the original axis of rotation. Such a system ought to remain symmetrical about this plane, and the axis of rotation of the central mass ought to remain perpendicular to this plane.

In the solar system over 98 per cent. of the angular momentum is orbital, the remainder arising almost entirely from the sun's rotation. Of the orbital momentum over 99.9 per cent. belongs to the outer planets, whose orbits all lie within $1 \frac{1}{2}$ degrees of the invariable plane-indeed the orbits of Jupiter, Saturn and Neptune, contributing $94: 3$ per cent. of this momentum, lie within $45^{\prime}$ of the invariable plane. The plane of the sun's rotation, on the other hand, lies about $6^{\circ}$ from this plane. The rotational theory fails to account for this distance between the plane of the orbits and that of the sun's rotation; the tidal theory explains it very naturally by supposing that the present invariable plane records the plane of passage of the tide-generating mass, while the present plane of the sun's rotation coincides approximately with that of the rotation of the original mass.

293. The details of tidal motion have been dynamically investigated for two models - for an incompressible mass of uniform density, and for Roche's model, representing the limit of non-uniform density. In each case the mass is found to break up into a number of separate masses, but the incompressible mass breaks up into masses of comparable size, while the very non-uniform mass breaks up only by the ejection of one or two streams of matter, which will probably condense into masses small compared with the central mass. Clearly these latter conditions give the closer approximation to those observed in our solar system, so that if our system has broken up tidally, it must have been far from homogeneous when the break-up occurred, and Roche's model may be expected to give the better picture of the process. 
The action between the two masses in a tidal encounter may be of varying degrees of rapidity. These we have classified as slow, intermediate, and transitory.

In a "slow" encounter, the changes are so leisurely that an equilibrium theory of the tides is supposed to give a good enough approximation. For these we found that break-up would occur if the secondary mass $\left(M^{\prime}\right)$ approached to within about $2 \cdot 2 \times\left(M^{\prime} / M\right)^{\frac{1}{3}}$ radii of the primary. This limiting distance was approximately the same both for the incompressible mass and for Roche's model, so that it may reasonably be expected to be about the same also for all intermediate types of structure.

At the other end of the scale come "transitory" encounters. Here the tidal forces are supposed to be impulsive; their function is to set the parts of the primary into relative motion and the break-up occurs in the subsequent motion of the primary under its own internal forces. Unfortunately it has so far only proved possible to work out the details of this motion for the incompressible model.

294. We have found $(\$ 130)$ that, with relative velocities of the order of present stellar velocities $(40 \mathrm{kms}$. a second), all encounters except the very closest ones may be classified as transitory - in the very closest ones, the action is still more rapid, but the forces may not be treated as impulsive because the primary has departed substantially from its original spherical shape before the tidal forces disappear. We have found that a transitory encounter will break up an incompressible mass if

$$
\left.R^{2}<\frac{M^{\prime} \gamma^{\frac{1}{2}}}{0.675 v \rho^{\frac{1}{2}}} \ldots \ldots \ldots \ldots \ldots \ldots \ldots \ldots \ldots . . . . . . .591\right),
$$

where $R$ is the periastron distance, $M^{\prime}$ the mass of the tide-raising body, $v$ the relative velocity, $\rho$ the density of the primary, and $\gamma$ the gravitation constant (now restored).

Our system is unlikely to have been broken up by a very massive star, for these are rare. It is likely to have been broken up by a star of mass rather above the average, for massive stars are more likely to effect a break-up than lighter ones. For definiteness, let us assign to $M^{\prime}$ a value equal to twice the sun's mass, or $4 \times 10^{33}$ grammes. Equation (591) becomes

$$
R<1.24 \times 10^{15} \times v^{-\frac{1}{2}} \rho^{-\frac{1}{4}}
$$

Taking $v=40 \mathrm{kms}$. a second and $\rho=5.5 \times 10^{-12}$, this being the density of our sun expanded to a sphere filling the orbit of Neptune, we find the limit for $R$ to be $4.05 \times 10^{14} \mathrm{cms}$., which is slightly less than the radius of Neptune's orbit.

Thus with a secondary of double the sun's mass, a relative velocity of $40 \mathrm{kms}$. a second would require actual grazing contact before tidal forces 


\section{8}

could break up a sun of density such that it just filled the present orbit of Neptune. Moreover since the critical value of $R$ only varies as $\rho^{-\frac{1}{4}}$, it is clear that even grazing contact would not suffice to break up a primitive sun of density less than this.

We are hardly free to suppose the secondary to have had a mass much greater than that already assumed, nor to suppose the primitive sun to have had a radius much less than that of Neptune's orbit. For, as we shall see immediately, either of these suppositions would result in encounters capable of effecting tidal break-up becoming excessively rare events. We accordingly retain the already assumed values $M^{\prime}=4 \times 10^{33}$ grammes, $\rho=5.5 \times 10^{-12}$, and examine the effect of assigning a smaller value to the relative velocity $v$.

With a relative velocity of only $4 \mathrm{kms}$. a second, formula (592) gives $1.28 \times 10^{15} \mathrm{cms}$. as the limit for $R$, but encounters with this velocity are no longer transitory; on taking $v=4 \times 10^{5}$ the calculation of $\S 130$ gives $1.6 \times 10^{16}$ as the closest distance of transitory encounters. Indeed the encounter is so far from transitory that we may expect the calculations for slow encounters to give a better approximation. Taking $M^{\prime} / M=2$, the critical value of $R$ for a slow encounter is $2 \cdot 78 r_{0}$ for an incompressible mass and $2 \cdot 87 r_{0}$ for Roche's model. Taking $r_{0}=4.5 \times 10^{14} \mathrm{cms}$. (the radius of Neptune's orbit), these limits are found to be $1.25 \times 10^{15}$ and $1.29 \times 10^{15} \mathrm{cms}$. respectively.

Thus with a primitive sun filling Neptune's orbit our calculations give the following critical distances for a mass double that of the sun, passing with a velocity of $4 \mathrm{kms}$. a second:

Incompressible mass, transitory $\quad R=1.28 \times 10^{15} \mathrm{cms}$.

$\begin{array}{ccc}\text { Roche's model " slow } & R=1.25 \times 10^{15} & \\ & \# & R=1.29 \times 10^{15}\end{array}$

The encounter we are here considering ( $R=$ about $1 \cdot 27 \times 10^{15}, v=4 \mathrm{kms}$. a second) is neither slow nor transitory, and the actual sun is not likely either to have been incompressible or to have conformed to Roche's model. But the calculated values of $R$ agree so closely among themselves that there is not likely to be much error in taking the limiting value of $R$ to be $1.27 \times 10^{15} \mathrm{cms}$., or about $\mathbf{2} \cdot 8$ times the radius of Neptune's orbit.

This value of $R$ corresponds to a velocity of $4 \mathrm{kms}$. a second. Higher velocities of course require closer approaches, a velocity of $40 \mathrm{kms}$. a second requiring as we have seen an approach to a distance of $4 \times 10^{14} \mathrm{cms}$., which represents grazing contact. On the other hand lower velocities do not permit of larger values of $R$, for these lower velocities give rise to slow encounters for which $R$ is independent of the velocity. Thus the largest value of $R$ for which tidal break-up can occur in a sun of density such that it fills a sphere of radius equal to Neptune's orbit is of the order of $1.27 \times 10^{15} \mathrm{cms}$. 
295. The average time between encounters at a distance equal to or less than $R$ is, as in formula (545),

$$
\frac{1}{\pi \nu R^{2} v} \text { seconds }
$$

In our present universe, $\nu$, the stellar density, is about $5 \times 10^{-56}$, while $v$ averages $40 \mathrm{kms}$. a second. The average interval between encounters at a distance less than $1.27 \times 10^{15} \mathrm{cms}$. is found to be about $10^{18}$ seconds or $3 \times 10^{10}$ years. This period is much longer than any reasonable estimate of the age of the universe. Moreover, of the encounters in question, only a few, namely those having small relative velocities, are likely to effect a tidal break-up. Thus tidal break-up is an excessively rare event, and only a small fraction of stars can ever experience it at all. .

This estimate of frequency of encounters which effect a tidal break-up has of course depended on the assumed density of the broken up star, which we took to be $5.5 \times 10^{-12}$, corresponding to a solar radius equal to the radius of Neptune's orbit. Greater densities would make tidal break-up still more improbable, the time interval varying as $\rho^{\frac{1}{2}}$ for transitory encounters and as $\rho^{\frac{2}{3}}$ for slow encounters. No reasonable density could make tidal break-up probable within astronomical time.

Thus if we suppose the constitution of our stellar universe to have been always as it now is, a tidal break-up would be an abnormal event: the $\grave{a}$ priori odds against our sun having broken up tidally would be so great that we might feel inclined to discard the tidal theory on the grounds of its inherent improbability.

We have, however, already had occasion to contemplate an earlier epoch in the evolution of our stellar universe, in which the stars were much closer than now, their relative velocities probably much smaller than now, and their densities very low. Making the appropriate alterations in the numerical data, the mean interval between tidal encounters is greatly reduced. Suppose that in this earlier stage the mass-density in space was of the order of $10^{-18}$ grammes per cubic $\mathrm{cm}$. Taking the mass of the average star to be 1.7 times that of the sun, the value of $\nu$, the number of stars per cubic $\mathrm{cm}$., would be $3 \times 10^{-52}$, or 10,000 times that previously assumed. The time-scale (593), which varies as $1 / \nu$, is reduced from $3 \times 10^{10}$ years to three million years by this change. The time-scale ought no longer to be compared with the whole supposed age of the universe, but rather with the duration of the epoch in which the stars were closely crowded together. Tidal break-up, even now, can hardly be considered a likely event, but it is considerably more probable than our former calculations would have shewn it to be, and the improbability of close encounters among the stars no longer provides adequate grounds for rejecting the tidal theory. 
296. Formula (593) shews that the frequency of encounters at a distance less than $R$ is proportional to $R^{2}$. It follows that tidal break-up of our sun, if ever it occurred, is likely to have been caused by an encounter in which the value of $R$ was not far below the maximum possible. In $\S 294$, assuming certain definite numerical data, we found the greatest value of $R$ at which tidal break-up could possibly be effected to be about $1.27 \times 10^{15} \mathrm{cms}$., the corresponding relative velocity at periastron being about $4 \mathrm{kms}$. a second. But a relative velocity as small as this must be excluded on dynamical grounds. The relative velocity at periastron must, except in the special case of a triple encounter, be greater than that due to a fall from infinity, and when the assumed masses $(\odot$ and $2 \odot)$ fall from infinity to a distance of $1.27 \times 10^{15} \mathrm{cms}$., they acquire a relative velocity of $7.9 \mathrm{kms}$. a sec. It now follows that the relative velocity is not likely to have been much greater than that due to a fall from infinity.

In general this velocity is given by $R v^{2}=2\left(M+M^{\prime}\right)$ while in $\S 130$ we saw that an encounter would be "transitory" if $2 M^{\prime} / R v^{2}$ was small. Clearly the encounter we are now considering is far from transitory and may perhaps, with fair accuracy, be treated as slow. If so, the critical value for $R$ is simply proportional to $r_{6}$, the sun's mean radius, and we see that the sun is most likely to have been broken up when its density was very low.

297. On the tidal theory, as we are now considering it, the planets must have been formed as condensations in an arm of matter thrown out from the sun towards a passing mass. In terms of the molecular velocity $C$ and mean density $\rho$ of the matter in this arm, the mass of each planet ought to be of the order of $\frac{1}{3} C^{3} \gamma^{-\frac{3}{2}} \rho^{-\frac{1}{2}}$, if its birth occurred in the manner considered in $\S 217$.

The calculations already made have shewn that our system is $\grave{a}$ priori most likely to have broken up when it was of low density and when our stellar universe was in the earliest stages of its existence. Let us conjecturally assume for the nebular arms a mean density $5.5 \times 10^{-13}$, this being one-tenth of that of our sun spread through a sphere of radius equal to that of the orbit of Neptune; let us assume a molecular velocity of $4 \times 10^{4}$, this being about that of hydrogen or oxygen at their boiling points. The mass of the resulting condensations is found to be about $10^{30}$ grammes - a mass intermediate between those of Jupiter and Saturn. It is clear that if our system contained, beyond the central sun, only planets of masses of the order of those of the two greatest planets, the tidal theory would provide a highly satisfactory explanation of the genesis of the system.

298. The tidal theory can only inspire confidence if it proves able to account for the small planets as well as for the large planets, and also if it can account for the satellites of the planets in the same way in which it accounts 
for the planets themselves. The systems of Saturn and Jupiter are so like that of the sun that any hypothesis which assigned different origins to the system and its sub-systems would be condemned by its own artificiality.

The first five satellites of Saturn all have masses comparabte with $5 \times 10^{23}$ grammes. Assuming these to have been formed by gaseous condensation, the range of molecular velocities $C=4 \times 10^{4}$ to $C=4 \times 10^{5}$ would give a range of density from 8 to $8,000,000$ if calculated by the method of $\S 284$. The obvious inference is that either these satellites were not formed by gaseous condensation, or that they are mere remnants of larger masses.' Similar considerations apply to the satellites of Mars and to some at least of the satellites of Jupiter. It is improbable that these satellites are all remnants of much larger masses; their present uniformity of size is opposed to any such hypothesis. Thus we are driven to supposing that they have been solid or liquid from their birth*.

299. This conclusion is quite independent of the tidal theory, or of any other theory of cosmogony. The small bodies we are considering are even now too small to retain an atmosphere; if they were suddenly transformed into a gaseous state, so that gravity was largely reduced at their surface, they would be still less able to retain an atmosphere, and their outer layers would rapidly dissipate into space. Whatever theory of cosmogony we hold, it seems comparatively certain that most of the asteroids, the majority of the satellites of the planets, and of course the particles of Saturn's rings, have been solid or liquid from birth.

It is fairly safe to assume that the satellites of Mars, Saturn and Jupiter originally formed part of these planets, for in each case the plane of rotation of the planet almost coincides with the plane of the orbits of the innermost planets. If the satellites had been "captured" or otherwise picked up from outside, it is improbable that they should have almost unanimously stumbled into the plane of rotation of the planet for the planes of their orbits. If we suppose that Jupiter and Saturn have always been gaseous we must suppose that solid or liquid satellites were born out of a gaseous planet. This might have happened if the outer layers were at a temperature not far above their boiling point; the jet of matter thrown out by tidal action might cool still further by radiation and immediately condense into a solid or liquid mass.

300. The satellites of the smaller planets are not so easily accounted for. To take a definite instance, the satellites of Mars are too small to have started life in the gaseous form; they seem likely to have originated out of Mars; Mars itself is too small to have formed as a gaseous condensation out of the same nebular arms as produced Jupiter and Saturn; and yet if the satellites 
of Mars were born in the liquid or solid state out of a liquid or solid primary, they ought to have been comparable in mass with that primary.

This apparent difficulty may arise largely from our having assumed that a body may be accurately labelled either as gaseous or as liquid or solid. The masses we have under consideration must all have been at low temperatures, and the pressure must have been considerable in their central regions. We must consider the possibility of formations which are liquid in their central regions where the pressure is highest, and gaseous in their outer regions. Such masses will be represented with tolerable accuracy by the composite Roche's model, of which the behaviour under tidal forces was discussed in $§ 162$.

We can now perhaps account for the formation of the system of Mars by supposing Mars to have started condensing in its central regions during or immediately after birth, and so assuming the structure of a dense nucleus surrounded by a light atmosphere. A further tidal cataclysm would result in a jet of the atmosphere being ejected, and if this immediately started to condense into the liquid state the final result might be two small planets of the kind observed.

The satellites of Uranus and Neptune may be explained in the same way. The earth-moon system admits of a similar explanation. but may also admit of explanation in terms of a wholly fluid earth ; pending further mathematical investigation it is hardly possible to say whether the masses of the earth and moon are too unequal for the system to have originated out of a wholly fluid mass. The question reduces ultimately to one of degree only; the earth at birth was probably more largely fluid than the planets whose satellites are relatively smaller.

301. The foregoing considerations have shewn that four at least of the eight planets must have been partially fluid at, or shortly after, their birth. If once this conclusion is admitted-and it seems inevitable on almost any theory of cosmogony - then there is no justification left for assuming, as we momentarily did in $\S 300$, that the two biggest planets, Jupiter and Saturn, were wholly gaseous at their birth, although the calculation of $§ 297$ shews it to be quite possible that Jupiter at least may have always been gaseous.

302. Let us examine how the tidal theory stands if we admit the possibility of all the planets having been partially fluid at birth.

We picture the primaeval sun throwing out a jet of matter under the influence of a passing star. The calculations of $\$ 296$ have already suggested that the conditions of the tidal encounter must have approximated to those we have described as "slow." The tide-generating star must have described an orbit passing within a sphere of radius equal to $2 \cdot 2\left(M^{\prime} / M\right)^{\frac{3}{3}}$ mean radii of the sun. As soon as the star came within this sphere the tidal ejection of 
matter must have begun. The rate of ejection of matter would be slow at first, it would increase to a maximum when the passing star was at its distance of closest approach, and would subsequently diminish to zero. The result ought to be a filament of matter of which the line density would be zero at each end and would increase to a maximum near the middle.

As this filament lost heat by radiation, the ends would experience the greatest fall of temperature, for the ratio of surface to mass would be greatest here. Thus liquefaction ought to commence near the ends, and after a time the ends of the filament might be mainly liquid while the middle region was still almost entirely gaseous. During this process of condensation, gravitational instability would result in the formation of furrows, leading to ultimate fission into separate masses.

We have already noticed ( $(217)$ that, when fission of this kind occurs, small masses are formed out of dense matter, and conversely. Thus those planets which formed near the ends of this filament, being formed out of dense matter, would be those of smallest mass, while the planets formed near the middle, mainly from uncondensed gas, would be of greatest mass. In this way the tidal theory readily explains the great inequality between the masses of Jupiter, Saturn and the other planets, while explaining at the same time why the two largest planets occur in the middle of the chain. The theory indicates that the smaller planets must have been mainly liquid or solid from their birth, while Jupiter and perhaps also Saturn may have always been almost entirely gaseous. We have already seen that the masses of these two larger planets are quite consistent with this view of their origin.

303. It is impossible to trace the early life of the planets with any precision. If it were not for the tangential velocity which they must have acquired from the gravitational attraction of the passing star, they must have all fallen back into the sun. If they were endowed with only a small tangential velocity, they would describe highly eccentric orbits; some would pass through the outer layers of the sun at perihelion and perhaps finally become merged in the sun's mass, others would pass near to the sun's surface while escaping actual collision. The tidal forces exerted on these planets by the sun might result in the creation of systems of satellites encircling the planets. This hypothesis accounts at once for the directions of revolution of the majority of the satellites, and explains why their orbital planes are, for the most part, close to the orbital planes of the corresponding planets.

We have already noticed that the least velocity that the tide-generating mass can have is that due to a fall from infinity, and this is $\sqrt{ } 2$ times the velocity for a circular orbit. Considerations of probability make it unlikely that the velocity was much greater than this minimum, for a much higher velocity would require an improbably close encounter. As an approximation, let us suppose that the tide-generating mass had a velocity of $10 \mathrm{kms}$. a second 
at a perihelion distance of $1.2 \times 10^{15} \mathrm{cms}$., this being only 22 per cent. more than the minimum velocity possible. The angular velocity of revolution at closest approach would be $8 \times 10^{-10}$. Let us suppose, again as a rough approximation, that the ejected filament was set in motion so that it rotated as a straight line with an angular velocity equal to half this, say $4 \times 10^{-10}$. Then a point at a distance $9 \cdot 4 \times 10^{14} \mathrm{cms}$. (or $2 \cdot 1$ times the radius of Neptune's orbit) from the sun's centre would have the velocity appropriate to the description of a circular orbit; planets formed at a distance less than this would describe eccentric orbits which would start by approaching nearer to the sun.

The innermost planets would describe the most eccentric orbits, for their velocity would differ most from that required for a circular orbit. Traces of this law may perhaps still be found in the solar system in which the eccentricity of orbit diminishes on the whole as we recede from the sun, Venus and the earth forming exceptions. See* has shewn that the action of a resisting medium surrounding the sun would be to diminish the eccentricities of the planetary orbits. This is shewn by the analysis already given in $\S 261$; to study the effects of a resisting medium we need only change the sign of the couple $G$. We find that the action of a resisting medium diminishes both the eccentricity and the major-axis of the orbit.

The diminution of eccentricity ought to be greatest nearest the sun, where the resisting atmosphere may have been supposed to be most dense. This might perhaps account for the smallness of the eccentricities of the orbits of Venus and the earth, but if so that of Mercury remains anomalously large. Similarly the diminution of major-axis ought to be greatest near to the sun. This would be in accordance with the comparative crowding of the planets near to the sun (Bode's Law), and with the corresponding phenomenon in the systems of Jupiter and Saturn.

304. The distance within which a planet must approach the sun for satellites to be created by tidal break-up depends on the density of the planet. If we are right in supposing that the small planets at the two ends of the series, say Neptune, Uranus, Venus and Mercury, condensed to an almost liquid state immediately after birth, then it seems highly improbable that these bodies can have been broken up tidally by the much less dense mass of the sun. We should expect these planets to have remained without satellites, or at least should expect their satellites not to have been born by tidal interaction with the sun. The high inclinations of the satellites of Uranus and the retrograde motion of the satellite of Neptune seem in any case to suggest some origin other than a tidal encounter with the sun. On the other hand, if we are right in supposing Jupiter and Saturn to have

* Researches on the Evolution of the Stcllar Systems, Vol. II, Chap. VII. See also Poincaré, Leģons sur les Hypothèses Cosmogoniques, Chap. VI. 
been mainly gaseous from their birth, we might naturally expect them to be surrounded by systems of satellites moving approximately in the planes of the orbits of their primaries. The genesis of the earth-moon system remains indefinite, although not inexplicable, but the satellites of Mars present something of a puzzle on the tidal theory, as indeed on any other theory of cosmogony.

305. This vague sketch of the tidal theory will, it is hoped, be read as an indication of the possibilities open to the tidal theory, rather than as an attempt to advocate the theory or to present it in a final form. The theory is beset with difficulties and in some respects appears to be definitely unsatisfactory. To the author it appears more acceptable than the rotational theory, or any other theory so far offered of the genesis of the solar system ; but an enormous amount of mathematical research appears to be needed before the theory can either be advocated with confidence or finally abandoned.

\section{THE TIME-SCALE}

306. Throughout our discussion of the various processes of astronomical evolution, the question of the time occupied by these processes has, intentionally, been kept in the background.

There are three main sources of information as to the time-scale on which the duration of these processes must be measured:-

I. Lord Kelvin, in a well-known calculation, shewed that the gravitational energy produced by the sun falling to its present configuration from a state of infinite rarity would only suffice to maintain the sun's present rate of radiation for about 20 million years. This estimate has been challenged on the grounds that the sun may have other sources of energy, radioactive energy in particular.

II. Geological investigations have given various estimates of the time which has elapsed since our earth assumed its present solid form. These are not altogether consistent with one another, and a number of early estimates must be definitely rejected in view of our recently gained knowledge of radioactive processes, but after excluding doubtful estimates there remains a number of apparently fairly reliable estimates which seem to converge to a period of the order of 250 million years for the age of our earth.

III. Some material for estimating the time-scale is provided by the present motions of the stars. The time of transition of a star across our galactic universe and back is of the order of $320,000,000$ years, and the period of motion round the milky way, or of rotation of the milky way, is of the same order of magnitude. The stars shew such uniformity of velocity and arrangement in different parts of space that we may reasonably suppose that 
they have moved several times across the universe before becoming so evenly arranged as they now are. For example, the assumption that each star has made ten journeys to-and-fro would give an age of the order of $3,200,000,000$ years.

Here we have three more or less conjectural estimates differing, roughly speaking, by successive powers of 10 . The last and largest estimate, being most closely allied to the subjects we have been discussing, may be considered first.

307. The estimate implicitly assumes that the period of motion across or round our universe has always been the same as now. Our investigations, on the contrary, have led us to conjecture that our system of stars may have only recently expanded to its present size; we have even found reasons for supposing that the expansion is not yet finished. If we estimate the age in terms of its completed orbits among the other stars, it is true that the last orbit is now being performed at the rate of one revolution (say) per $320,000,000$ years, but the first orbit may, we have supposed, have been completed in 160,000 years. The time of ten orbits will not be ten times 320 million years; it will be more nearly equal to the sum of ten terms of a geometrical progression beginning with 160,000 years and ending with 320 million years; this sum is 560 million years. Considering the uncertainty of all the numerical data, this estimate may be considered to be in agreement with the geological estimate, the estimate of 560 million years referring to the time since the primaeval rotating nebula begun to break up into stars, and the geological estimate of 250 million years referring to the period since the earth solidified.

308. The only discordant estiniate is seen to be that derived from the sun's radiation. Taking the solar constant to be 1.92 , the sun radiates away $3.8 \times 10^{33}$ ergs per second. The gravitational energy gained by the sun in contracting to a homogeneous mass of its present size is $2 \cdot 2 \times 10^{48} \mathrm{ergs}$, representing radiation for 18.3 million years at the present rate. It hardly appears probable that the sun can have other sources of energy comparable with its gravitational energy. Chemical energy is well known to be insignificant. Lindemann* has pointed out that radioactive energy will also be insignificant in comparison with gravitational. It is possible that other sub-atomic changes, unknown to us, may provide more energy than radioactive changes. If these changes consist of a mere rearrangement of electrons, it is found that each electron in the sun would have to fall through a sub-atomic difference of potential equal. to 3000 volts in order to set free as much energy as that set free by gravitational contraction, so that the possibility of extending the time-scale in this way seems remote. There remains, apparently as a last resource, the possibility of energy being created by the destruction of matter, as for instance by positive

* Nature, April 22, 1915.

† J. H. Jeans, Nature, August 2, 1917. 
and negative charges rushing together and annihilating one another. Taking the intrinsic energy of an electron to be $m C^{2}$, where $C$ is the velocity of light, the reduction of the sun's mass by only one per cent. would set free $1.8 \times 10^{52}$ ergs of energy, or sufficient to furnish radiation at the present rate for 150,000 million years.

309. Before either giving up the question as insoluble, or calling to our aid stores of energy such as that just mentioned, it will be well to examine how far the gravitational source of energy is really proved to be inadequate. The situation is that the total energy of contraction of our sun provides for radiation at the present rate-i.e. as a star of absolute magnitude $5 \cdot 0$-for only 18.3 million years. Let us consider the problem first in reference to the universe as a whole, and afterwards with special reference to our sun.

The energy set free by gravitational contraction varies as the square of the mass of the contracting body. Taking the average star to be of mass 1.7 times that of our sun, the energy lost in contracting to the radius of our sun -i.e. to a density of $2 \cdot 3$ - would be 2.9 times that generated by the contraction of our sun to its present size. Thus it would provide for radiation for 53 million years as a star of luminosity equal to our sun.

Our sun, however, is somewhat exceptionally bright. To estimate how far its brightness is above the average, we must limit ourselves to the nearest stars, for distant faint stars escape observation altogether. Of the nineteen stars of parallax greater than $0 \cdot 2^{\prime \prime}$ tabulated by Eddington*, only nine have luminosities greater than $\frac{1}{10}$, that of the sun being taken as unity, while nine have luminosities less than $\frac{1}{10}$, the nineteenth star being of estimated luminosity equal to $\frac{1}{10}$. Moreover no fewer than seven have luminosities less than $\frac{1}{100}$ suggesting that we ought to add to the least luminous stars others whose nearness has not been suspected on account of their faintness, such as the near star recently discovered by Barnard. Taking the luminosity of the average star to be $\frac{1}{10}$, we find that contraction provides for radiation at this rate for 530 million years, a period which agrees well enough with our other estimates of the age of the universe.

310. Thus as regards the universe as a whole, there is no difficult problem associated with the time-scale: the problem only arises in connection with special stars, and our sun happens to be one of these. Both Russell's theory and the theoretical investigation of Chap. VIII suggest that in the comparatively recent past our sun must have been radiating even more energy than at present. On the other hand, when we pass to the remote past, there is no justification for believing the rate of radiation to have been so great. There must be a long period between the stage at which a star is formed in the nebulous state and the period at which it 
bursts into incandescence as a giant $M$-star. During this period energy is radiated in the form of heat, but observation tells us nothing as to its amount. Theory, as developed in Chap. VIII, shews that the early heatradiation will be the same in amount as the later light-radiation provided the opacity $c$ remains constant. The opacity, however, is not likely to remain constant; the radiation in the interior of a luminous star is of very short wave-length*, comparable with that of soft $\mathrm{X}$-radiation, and matter might be expected to be much more transparent for such radiation than for the radiation which conveys the energy in the interior of a non-luminous star. Against this must be set the fact that a comparison of the total emission of a star with the temperature gradient in its interior shews that the matter of luminous stars must be very much more opaque than ordinary gaseous matter; Eddington + calculates an opacity such that light is reduced to $1 / e$ times its original intensity after passing through $\frac{1}{30} \mathrm{gm}$. per sq. $\mathrm{cm}$. of stellar matter. Imagine, however, that we could in some way suppose the mean opacity $c$ for the earlier dark sun to have been forty times as great as for the present sun. Then the rate of emission of radiation by the dark sun would have been only a fortieth of the present rate, and the time-scale may be extended about forty times. More precisely we may perhaps suppose that three-quarters of the total energy of contraction has been radiated from the sun in its luminous period, this lasting about 15 million years, while the remaining quarter was radiated in a non-luminous period lasting for about 200 million years.

Some such course seems to be the only one open if we accept the geological estimates of the earth's age, while refraining from introducing unknown sources of solar energy such as that suggested in $\S 308$. Our conjecture will not, however, satisfy those geologists who maintain that the earth has remained at a temperature near to its present temperature for a period of hundreds of millions of years.

\section{CONCLUSION}

It has not been part of our task to arrive at a conclusion; the time for arriving at conclusions in cosmogony has not yet come. Our object has rather been to consider different hypotheses in turn, pointing out and perhaps to some extent balancing the advantages and disadvantages of each, leaving it to future investigators, armed with more mathematical and observational knowledge than we at present possess, to pronounce a final decision.

In so far as one conclusion has seemed to us more probable than another, it has been something of the following kind. Some hundreds of millions of years ago all the stars within our galactic universe formed a single mass of excessively

* Eddington, Monthly Notices R.A.S. 77 (1917), p. 34.

+ l.c. p. 28. 



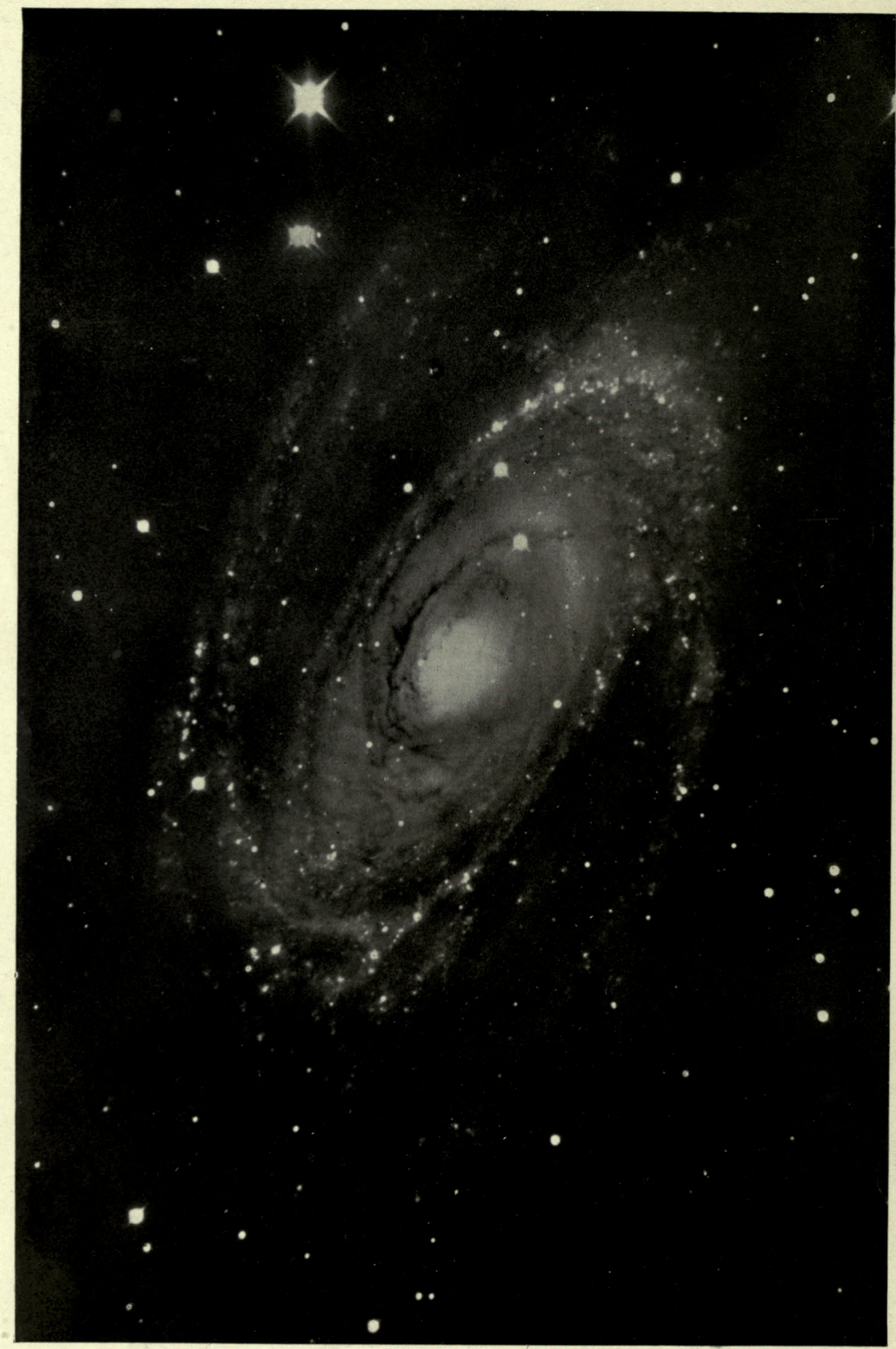

N.G.C. 3031

The Spiral Nebula M. 81 
tenuous gas in slow rotation. As imagined by Laplace, this mass contracted owing to loss of energy by radiation, and so increased its angular velocity until it assumed a lenticular shape, similar to that of the figures shewn on Plate II. After this, further contraction was a sheer mathematical impossibility and the system had to expand. The mechanism of expansion was provided by matter being thrown off from the sharp edge of the lenticular figure, the lenticular centre now forming the nucleus, and the thrown-off matter forming the arms, of a spiral nebula of the normal type. The long filaments of matter which constituted the arms, being gravitationally unstable, first formed into chains of condensations about nuclei, and ultimately formed detached masses of gas. With continued shrinkage, the temperature of these masses increased until they attained to incandescence and shone as luminous stars. At the same time their velocity of rotation increased until a large proportion of them broke up by fission into binary systems. The majority of the stars broke away from their neighbours and so formed a cluster of irregularly moving stars-our present galactic universe, in which the flattened shape of the original nebula may still be traced in the concentration about the galactic plane, while the original motion along the nebular arms still persists in the form of "star-streaming." In some cases a pair or small group of stars failed to get clear of one another's gravitational attractions and remain describing orbits about one another as wide binaries or multiple stars. The stars which were formed last, the present $B$-type stars, have been unusually immune from disturbance by their neighbours, partly because they were born when adjacent stars had almost ceased to interfere with one another, partly because their exceptionally large mass minimised the effect of such interference as may have occurred; consequently they remain moving in the plane in which they were formed, many of them still constituting closely associated groups of stars-the moving star-clusters.

At intervals it must have happened that two stars passed relatively near to one another in their motion through the universe. We conjecture that something like 300 million years ago our sun experienced an encounter of this kind, a larger star passing within a distance of about the sun's diameter from its surface. The effect of this, as we have seen, would be the ejection of a stream of gas towards the passing star. At this epoch the sun is supposed to have been dark and cold, its density being so low that its radius was perhaps comparable with the present radius of Neptune's orbit. The ejected stream of matter, becoming still colder by radiation, may have condensed into liquid near its ends and perhaps partially also near its middle. Such a jet of matter would be longitudinally unstable and would condense into detached nuclei which would ultimately form planets. The more liquid planets at the end of the chain would be those of smallest mass; the gaseous centre would form the larger planets Jupiter and Saturn. Owing to the orbital velocity which had been communicated to these planets by the

J. c. 
attraction of the passing star, they would not fall back into the sun but would describe elliptic orbits, passing fairly near to the sun's surface at their closest approach. As they passed relatively near to the sun, the same process as resulted in the formation of planets out of the sun, may have resulted in the formation of satellites out of the planets. It is not difficult to account for the systems of Jupiter and Saturn in this way, but the satellites of Neptune, Uranus, Mars and the earth are less easy to explain. The system which interests us most nearly, namely our earth-moon system, is just the one about which it is most difficult to come to any definite conclusion. For the earth-moon system is exceptional in the system of the planets, just as the solar system to which it belongs appears to be exceptional, and for ought we know may be unique, in the system of the stars. 


\section{INDEX}

\section{[The numbers refer to the pages.]}

A-type stars, 13, 14, 239, 241, 249, 270

a Centauri, 259, 260, 268

Absolute magnitudes, 14, 201, 202, 287

Adams (W. s.), 14

, and Joy, 14, 201, 202

Adiabatic equilibrium, gas in, $165,184,193$, 194, 204

, $\quad$ rotating gas in, 165, $184,185,203$

Andromeda, nebula (M. 31) in, 5, 217, 223

Angular momentum in binary stars, 257, 259

, $\quad$ in solar system, 15, 270, 271,275

Arrhenius, 17, 247

Asteroids, 1, 2, 281

Atmospheres of binary stars, 163,248

$$
\begin{array}{ll}
\text {, } \quad \text {, nebulae, 204 } \\
\text {, planets, 281, } 282
\end{array}
$$

B-type stars, 3, 13, 14, 239, 241, 242, 249, 263, 270

B Lyrae, 248, 250, 253

Babinet's criterion, 15, 270, 275

Bailey, 8

Bifurcation, points of, see Points

Binary stars, 2, 255

" , dynamics of, $46,134,137,162$, $187,246 \mathrm{ff}$.

, , genesis of, $246 \mathrm{ff} ., 289$

" , orbits of, $252,253 \mathrm{ff}$.

Bode's Law, 17, 284

Boss, 3, 9

Brown (F. G.), 7

Bryan (G. H.), 43

Campbell (w. W.), 3, 4, 5, 6, 9, 14, 204, 240, 241, $247,253,255,256,258$

, and Moore, 7

Canes Venatici, nebula (M. 51) in, 6, 215

Cataclysmic Motion, 23, $117 \mathrm{ff} ., 148,252$

Cepheid variables, $9,248,258$

Chamberlin, 16

,, and Moulton, 16, 131, 272

Charlier, 223, 229, 236, 239

Clausius, 181
Clusters, star, see Star-clusters

Coefficients of stability, 25, 30

Collisions between stars, 224, 237, 238

Compressible masses, dynamics of, $139,165,188$

Condensations in spiral nebulae, 17, 216, 247, 289

Contraction, energy generated by, 191, 285, 286

Cosmogony, theories of, 10, 269

Courvoisier, 265

Curtis, 221

Cylinders, rotating liquid, 102

Darwin (Sir G. H.), 11, 33, 39, 40, 46, 52 ff., 61 ff., $80,84,87,88,92,146,252,253$

Deflection, gravitational, of a star, 226, 229

Density of binary stars, 249,250

Dirichlet, 119 '

" at fission, $248,249,267$

Double stars, see Binary stars

Draper classification of Stellar spectra, 13

Duncan, 248

"Dwarf" stars, 13, 201, 202

Earth-moon system, 3, 282, 290

Eccentricity of orbits of binary systems, 25\%, $255,256,263,264$

in solar system, 2, 281

Eclipsing binaries, 249

Eddington, 188, 189, 222, 223, 229, 235, 236, $239,250,251$

Effusion of gas from a rotating gaseous mass, 247

Ellipsoidal configurations of gravitating masses, 35 ff., 65,137

Emden, 250

Equatorial ejection of matter from a rotating mass, $150 \mathrm{ff}$., 185, 186, 206, 207, 209, 210, $214,247,272,275$

Equipartition of Energy, in binary systems, 261 , , in star-clusters, $238 \mathrm{ff}$.

Faye, 18

Fission, the process of, $115,132,138,144,185$, $246 \mathrm{ff}$.

Fouché, 15

Friction, tidal, see Tidal friction

Frost, 3 
"Giant" stars, 13, 201, 202, 250

Globular star-clusters, 8, 9, 220, 221, 243

Halm, 239

Hertzsprung, 3, 9

Hinks, 9

Homologous contraction, 194, 242

Irregular Nebulae, 5, 6, 7, 204

Island universe hypothesis, 5, 203, 218, 220

Jacobian ellipsolds, 35,39 ff., 48, 84, 92, 105, $132,143,153$

Jeffreys (H.), 274, 281

Jonckheere, 3, 265

Jupiter, 280, 282, 283, 284

, $\quad$ satellites of, $2,3,136,138,281,284$

Kant, 10, 11

Kapteyn, 239

Keeler, 4, 6

Kelvin (Lord), 13, 15, 285 (see also Thomson and Tait)

Kinetic Theory of Gases, analogies with, 224, $229,230,232,241,261$

Kostinsky, 6, 223

Lane's Law, 192

Laplace, $10,11,210,213,215,272$ ff., 289

, 's law of density, 165

Liapounoff, $33,87,88$

Ligondès, 18

Lindemann, 286

Linear serles of equilibrium configurations, 20 , 35 ff., 142, 148

Lockyer (Sir J. Norman), 13

Ludendorff, 9

Luminosities of stars, 201, 287

M-type stars, 13, 14, 202, 239, 241, 288

Maclaurin's spheroids, 35, 38 ff., 48, 104, 143, 153

Mars, 281, 282

, satellites of, 136, 281, 282, 285

Masses of nebulae, 217

, , , stars, 222

Mass-ratio of binary stars, 3, 134

Maxwell, 12

Melotte, 8, 9

Mercury, 2, 284

Meyer (C. 0.), 39

Moulton (F. R.), 15, 16, 267, 272

Nebula in Andromeda (M. 31), 5, 217, 223

, , Canes Venatici (M. 51), 6, 215

" , Orion, 5, 6, 13, 204

, Ursa Major (M. 101), 6, 215, 216, 217
Nebulae, irregular, 5, 6, 7

, planetary, $5,6,7,8,204,248$

, ring, 5,248

, spindle-shaped, 5,248

, $\quad$ spiral, $4,5,6,7,215,216 \mathrm{ff} ., 247,289$

Nebular hypothesis, 10, 210, 213, 288

Neptune, 2, 275

, $\quad$ satellites of, $2,282,284$

O-type stars, 13, 255

Orion nebula, 5, 6, 13, 204

Pahlen, v. d., 4

Partial stability, 62

Pear-shaped figures of equilibrium, $78 \mathrm{ff} .$, 107, 126

Pease, 5, 6, 208, 214, 217 " and Shapley, 8, 221

Periods of binary systems, 253, 255, 256, 258, 260, 263, 264

Perrine, 4, 6

Planetary nebulae, see Nebulae

Planetesimal Theory, 16, 131

Planets, origin of, 11, 17, 248, 275, $280 \mathrm{ff}$., 289

Pleiades Cluster, 9, 13, 267

Plummer (H. C.), 8, 245

Poincaré, 18, 20, 21, 24, 33, 65, 79, 80, 87, 92, 117, 144, 188, 191, 209, 211, 272

Points of Bifurcation, general theory, 21, 92, 143

pecial cases of, 39,78 , $87,105,107,132,143$, 176

dynamical, 125, 157

Polaris, 265

Potential of ellipsoid, 36

," , distorted ellipsoid, 66, 87, 166

Pseudo-ellipsoids, 143

Pseudo-spheroids, 143

Pulsations in cosmogony, 17, 209

RR Centaurl, 253

Radiation from a mass of gas, 191, 198

, pressure of, 251

", solar, 285, 286

,, stellar, 198, 200

Radiative transfer of energy, 197

Radioactive energy, 285, 286

Relaxation, time of, 229

Resisting medium, effect of a, 284

Ring nebulae, 5, 248

Roche, 12, 46, 47, 48, 49, 54, 55, 136, 147, 209, 210

Roche's model, 12, 147, 184, 185, 208, 258, 270, 276

Rotation of gaseous masses, 203, 208, 215

, , planetary nebulae, 7,248 
Rotation of spiral nebulae, 6, 207, 217

Russell (H. N.), 3, 4, 13, 14, 201, 202, 249, 256, $265,266,267$

," 's theory of stellar evolution, 13, 201, 249

\section{Sanford (R. F.), 7}

Saturn, 2, 280, 282, 283

, rings of, $11,136,138$

, satellites of, $2,3,136,138,281,284$

Schuster, 146, 245

Schwarzschild, $31,65,80,87$

secular instability, 31, 32, 52

See (T. J. J.), 15, 17, 247, 272, 284

Shapley, 8, 9, 221, 243, 249

Slipher, 5, 6

Solar radiation, $\mathbf{2 8 5 ,} 286$

,, system, angular momentum of, 15, 270, 271,275

, , , origin of, 11, 17, 18, 138, $269 \mathrm{ff}$. Spectral type, 13, 256

Spectroscopic binaries, 3, 255

Spheroidal configurations

of a rotating mass, 35,137

of a tidally-distorted mass, 45, 83, 118, 137

Spindle-shaped nebulae, 5, 248

Spiral nebulae, see Nebulae

Stability, coefticients of, 25, 30

,, disappearance of, $22,32,41,45,50$

, exchange of, $22,25,32$

, of binary systems, $51,85,137,162$, 187

, " mass of gas at rest, 190, 199

", "rotating systems, $26,40,84,137$, 149,185

, " tidally-distorted masses, 45, 59, $83,137,153,186$

Star-clusters, globular, 8, 9, 220, 243
Star-clusters, moving, 9, 267

Star-streaming, 235, 240, 242, 289

Steady-motion in stellar dynamics, 232

Stellar dynamics, general theory, 224

", evolution, order of, 12, 13, 201, 202, 256

, radiation, 198,200

Sutherland, 17

Taurus Cluster, 3, 9

Temperature of stellar interiors, 250 , , , surfaces, 13

Thomson and Tait, 30, 38

Tidal action, dynamics of, 43, 83, 118, 137

, , theory of cosmogony, 16

, " , , solar system, 275

" friction, $53,62,253,263$

Triple and Multiple stars, 3, 4, 264

Truman, 6

Turner (H. H.), 10, 235

U Pegasi, 253

Uranus, 2, 275

, satellites of, 2, 282, 284

Ursa Major, cluster in, 9, 10

,, , nebula (M. 101) in, 6, 215, 216, 217

Van Maanen, 6, 8, 216, 223

Venus, 284

Virial, 188

Von der Pahlen, 4

Von Zeipel, 8, 245

W Crucis, 258

Wright, 5

X Carinae, 253

Young and Harper, 6 




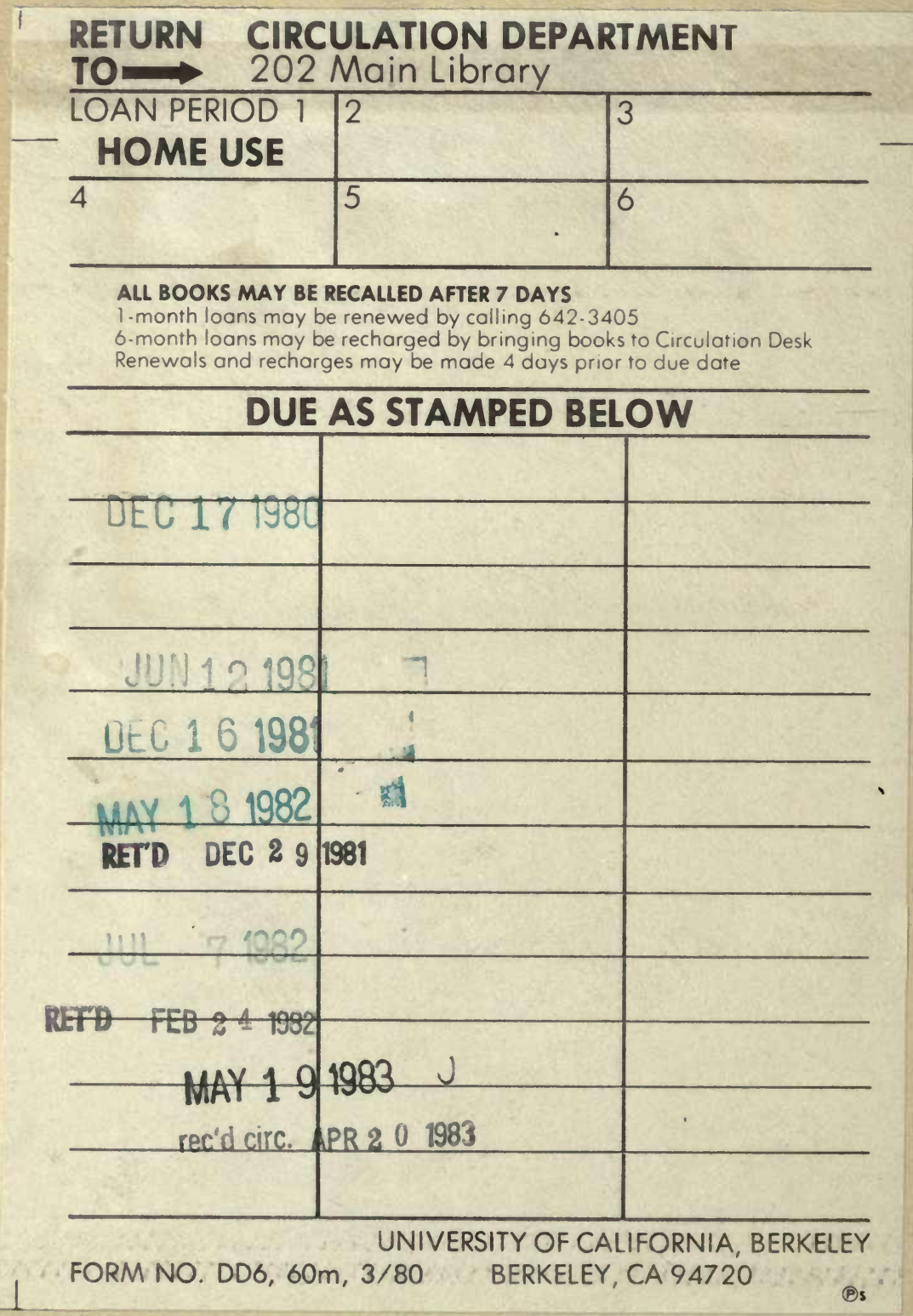



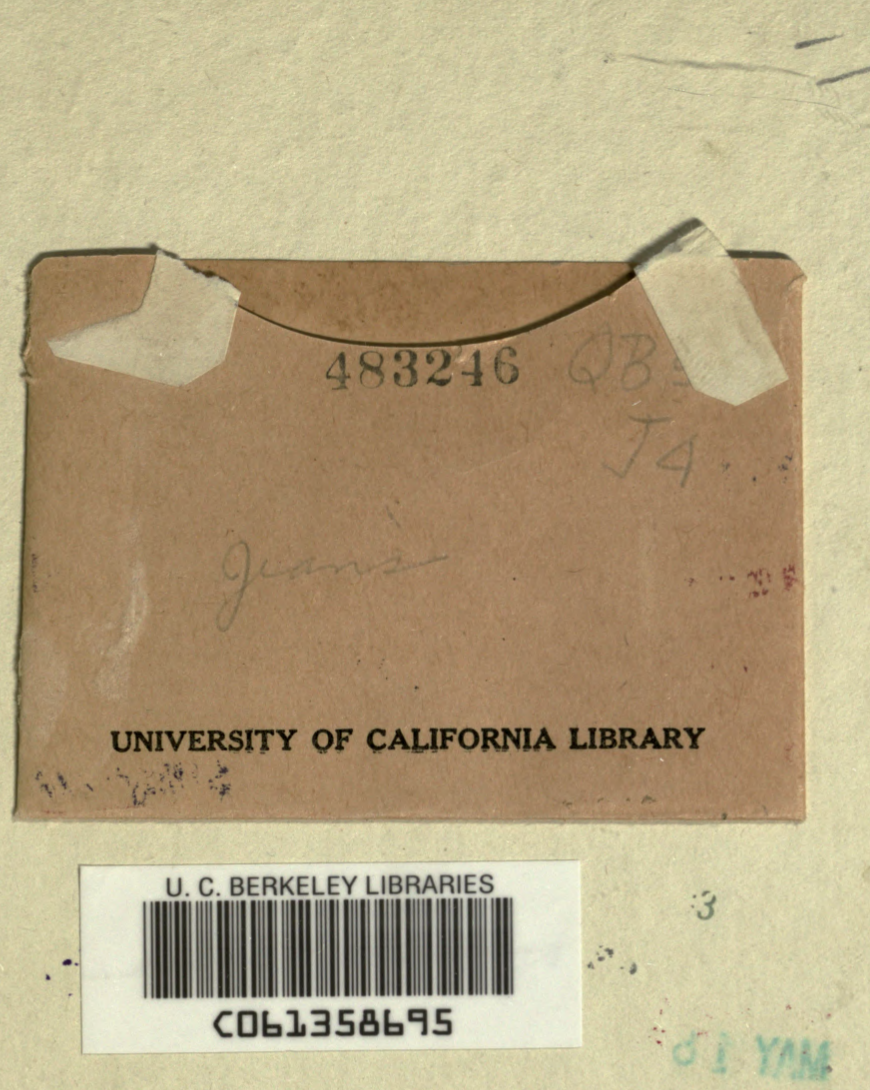

$\because$ 
